Albert Fleischmann

\title{
Stefan Oppl
}

Werner Schmidt

Christian Stary

Ganzheitliche

Digitalisierung

von Prozessen

Perspektivenwechsel - Design Thinking -

Wertegeleitete Interaktion 
Ganzheitliche Digitalisierung von Prozessen 
Albert Fleischmann - Stefan Oppl

Werner Schmidt · Christian Stary

\section{Ganzheitliche \\ Digitalisierung von \\ Prozessen}

Perspektivenwechsel - Design Thinking Wertegeleitete Interaktion 
Albert Fleischmann

InterAktiv Unternehmensberatung

Pfaffenhofen, Deutschland

Stefan Oppl

Johannes Kepler Universität Linz

Linz, Österreich
Werner Schmidt

Technische Hochschule Ingolstadt

Ingolstadt, Deutschland

Christian Stary

Johannes Kepler Universität Linz

Linz, Österreich

\section{(9) $(1) \Theta$}

ISBN 978-3-658-22647-3

ISBN 978-3-658-22648-0 (eBook)

https://doi.org/10.1007/978-3-658-22648-0

Die Deutsche Nationalbibliothek verzeichnet diese Publikation in der Deutschen Nationalbibliografie; detaillierte bibliografische Daten sind im Internet über http://dnb.d-nb.de abrufbar.

\section{Springer Vieweg}

(C) Der/die Herausgeber bzw. der/die Autor(en) 2018. Dieses Buch ist eine Open-Access-Publikation.

Open Access Dieses Buch wird unter der Creative Commons Namensnennung - Nicht kommerziell - Keine Bearbeitung 4.0 International Lizenz (http://creativecommons.org/licenses/by-nc-nd/4.0/deed.de) veröffentlicht, welche die nicht-kommerzielle Nutzung, Vervielfältigung, Verbreitung und Wiedergabe in jeglichem Medium und Format erlaubt, sofern Sie den/die ursprünglichen Autor(en) und die Quelle ordnungsgemäß nennen, einen Link zur Creative Commons Lizenz beifügen und angeben, ob Änderungen vorgenommen wurden. Die Lizenz gibt Ihnen nicht das Recht, bearbeitete oder sonst wie umgestaltete Fassungen dieses Werkes zu verbreiten oder öffentlich wiederzugeben.

Die in diesem Buch enthaltenen Bilder und sonstiges Drittmaterial unterliegen ebenfalls der genannten Creative Commons Lizenz, sofern sich aus der Abbildungslegende nichts anderes ergibt. Sofern das betreffende Material nicht unter der genannten Creative Commons Lizenz steht und die betreffende Handlung nicht nach gesetzlichen Vorschriften erlaubt ist, ist auch für die oben aufgeführten nicht-kommerziellen Weiterverwendungen des Materials die Einwilligung des jeweiligen Rechteinhabers einzuholen.

Das Werk einschließlich aller seiner Teile ist urheberrechtlich geschützt. Jede kommerzielle Verwertung, die nicht ausdrücklich vom Urheberrechtsgesetz zugelassen ist, bedarf der vorherigen Zustimmung des Autors und ggf. des Herausgebers. Das gilt insbesondere für Vervielfältigungen, Bearbeitungen, Übersetzungen, Mikroverfilmungen und die Einspeicherung und Verarbeitung in elektronischen Systemen. Der Verlag hat eine nicht-exklusive Lizenz zur kommerziellen Nutzung des Werkes erworben.

Die Wiedergabe von Gebrauchsnamen, Handelsnamen, Warenbezeichnungen usw. in diesem Werk berechtigt auch ohne besondere Kennzeichnung nicht zu der Annahme, dass solche Namen im Sinne der Warenzeichenund Markenschutz-Gesetzgebung als frei zu betrachten wären und daher von jedermann benutzt werden dürften.

Der Verlag, die Autoren und die Herausgeber gehen davon aus, dass die Angaben und Informationen in diesem Werk zum Zeitpunkt der Veröffentlichung vollständig und korrekt sind. Weder der Verlag noch die Autoren oder die Herausgeber übernehmen, ausdrücklich oder implizit, Gewähr für den Inhalt des Werkes, etwaige Fehler oder Äußerungen. Der Verlag bleibt im Hinblick auf geografische Zuordnungen und Gebietsbezeichnungen in veröffentlichten Karten und Institutionsadressen neutral.

Springer Vieweg ist ein Imprint der eingetragenen Gesellschaft Springer Fachmedien Wiesbaden GmbH und ist ein Teil von Springer Nature

Die Anschrift der Gesellschaft ist: Abraham-Lincoln-Str. 46, 65189 Wiesbaden, Germany 


\section{Vorwort}

„Die wichtigsten Innovationen sind jene, die das Denken verändern. “ Diesen Anspruch stellen wir mit diesem Buch, dem Aphorismus von Hans-Jürgen Quadbeck-Seeger, einem deutschen Chemiker, folgend. Unsere Ingredienzen:

„, Verständlichkeit ist die Höflichkeit eines Experten. “ Auch hier haben wir versucht, unserem Aphorismengeber treu zu bleiben. Das Werk soll alle Interessierten begeistern können. Daher muss es verständlich sein.

„Luxus: Kult um das Unnötige. “Wir wollen alle jene ansprechen, die das Wesen von Prozessen und ihre Nutzbarkeit im praktischen Tun begreifen wollen, ohne intensive Sprach- und Gebrauchsstudien, vielmehr mit praxistauglichen Konzepten unterfüttert. Studierende mögen den Textbuchcharakter des Buchs schätzen, PraktikerInnen die Beispiele, ForscherInnen und EntwicklerInnen die Konzeptdarstellungen und Theorieausflüge.

„Je größer das Projekt, desto stiller wird es begraben.“ Seit mehr als einem Jahrzehnt gibt es das Konzept und auch das Projekt. Es huldigt Einfachheit und Überschaubarkeit, ohne komplexe Zusammenhänge zu vernachlässigen. Die Treiber des Projekts verspüren steten Aufbruch. Es ist also Zeit, die Digitalisierung von Prozessen aus der Brille der Subjektorientierung zu betrachten.

„Abenteuertouristen zieht es zu Orten, wo sie nichts zu suchen haben. “ Der Blick lohnt sich, da sich eine Sicht auftut, die Nahe an unsere Wahrnehmung der Wirklichkeit kommt, Bestehendes schlüssig erweitert, und uns so neuen Handlungsspielraum verschafft. Auch unsere BegleiterInnen auf dem Weg zur Fertigstellung des Werks sind Abenteurer. Ihnen gebührt besonderer Dank:

- Christoph Moser - mit seinen Einsichten zur organisationalen Praxis

- Edith Rieß - sie hat uns geholfen, die Formatierung formschön zu gestalten 
- Sabine Kathke, Heike Jung, Sybille Thelen von Springer Vieweg und Ralf Gerstner von Springer für ihre Unterstützung seitens des Verlags zur Umsetzung unserer Ideen

- Jerome Geyer-Klingeberg von Celonis SE - zur Verdeutlichung der Prozesspraxis

„Innovationen sind keine Naturereignisse, wir müssen sie wollen und durchsetzen. “ - Ad multos multiplicatores, nicht nur in Ingolstadt, Pfaffenhofen, Steyr und Wien.

Albert Fleischmann

Stefan Oppl

Werner Schmidt

Christian Stary 


\section{Inhaltsverzeichnis}

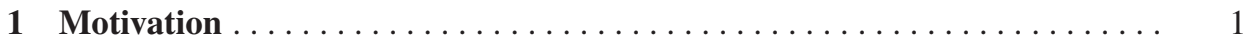

1.1 Geschäftsprozesse und Geschäftsprozessmanagement ........... 1

1.2 Blick auf die Welt, Strukturierung und Modellbildung ............ 3

1.3 Bestandteile einer Prozessbeschreibung . . ................ 5

1.4 Rahmenbedingungen für Prozessmodelle und Prozessinstanzen . . . . . . 6

1.5 Prozesskennzahlen............................. 7

1.6 Unterstützungskonzepte.......................... 8

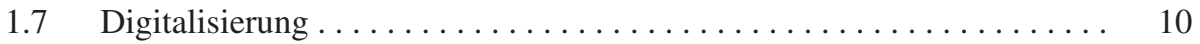

1.8 Prozess zum Erstellen von Prozessen $\ldots \ldots \ldots \ldots \ldots \ldots \ldots \ldots \ldots . \ldots \ldots$

1.9 Organisatorische und technische Implementierung. . . . . . . . . 13

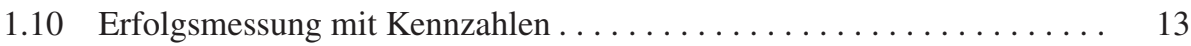

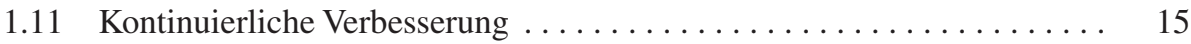

1.12 Unternehmensführung und Geschäftsprozessmanagement.......... 16

Literatur. . . . . . . . . . . . . . . . . . . . . . . . . . . . 17

2 Modelle............................................ 19

$2.1 \quad$ Modell und Wirklichkeit ............................. 19

2.2 Eigenschaften von Modellen ........................... 21

2.3 Modelle der Sozialwissenschaften..................... 23

2.4 Modelle der Betriebswirtschaft ...................... 28

2.5 Modelle der Wirtschaftsinformatik . . . . . . . . . . . . . . . . 36

2.6 Modelle der Informatik . . . . . . . . . . . . . . . . . . . . . . 48

$2.7 \quad$ Fazit: Modelle für Geschäftsprozesse $\ldots \ldots \ldots \ldots \ldots \ldots \ldots \ldots \ldots$

Literatur. . . . . . . . . . . . . . . . . . . . . . . . . . . . 67 


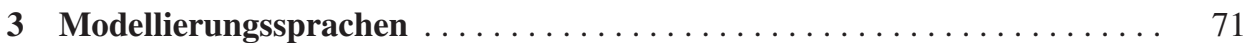

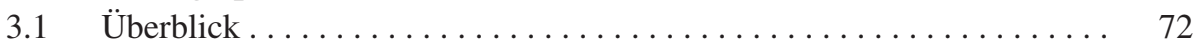

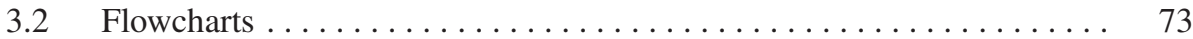

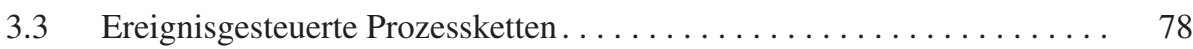

$3.4 \quad$ UML Aktivitätsdiagramme $\ldots \ldots \ldots \ldots \ldots \ldots \ldots \ldots \ldots \ldots \ldots \ldots$

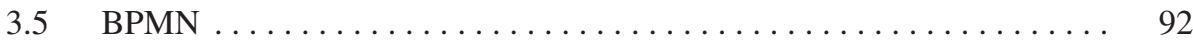

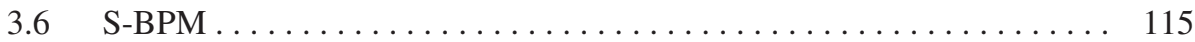

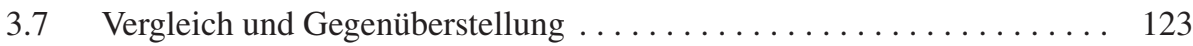

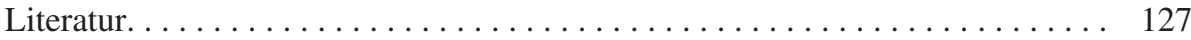

4 Vorgehensweise von der Modellbildung zur Digitalisierung . . . . . . . . . . 129

$4.1 \quad$ Einordnung in den Gesamtzusammenhang . . . . . . . . . . . . . . . . . 129

4.2 Aktivitätsbündel im Geschäftsprozessmanagement. . . . . . . . . . . . 130

4.3 Einführung in Design Thinking . . . . . . . . . . . . . . . . 141

4.4 Verbindung der Konzepte. . . . . . . . . . . . . . . . . . . . . . . . . 149

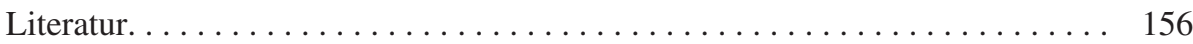

5 Vorbereitung der Prozessimplementierung $\ldots \ldots \ldots \ldots \ldots \ldots \ldots \ldots$

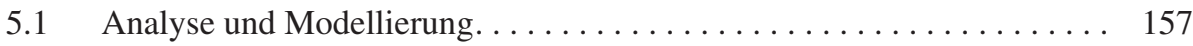

5.2 Qualitätskontrolle: Validierung und Optimierung . . . . . . . . . . . . . 192

Literatur. . . . . . . . . . . . . . . . . . . . . . . . . 197

6 Umsetzung . . . . . . . . . . . . . . . . . . . . . . . . . . . . . . . . 199

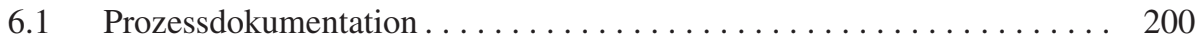

6.2 Verknüpfung von Elementen der Unternehmensarchitektur . . . . . . . . . 202

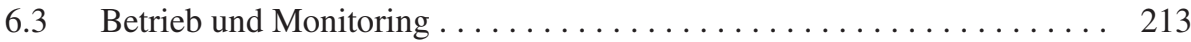

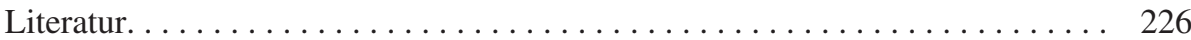

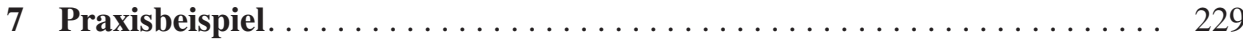

$7.1 \quad$ Ausgangsituation . . . . . . . . . . . . . . . . . . . . . 229

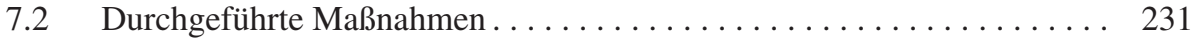

$7.3 \quad$ Erzielte Ergebnisse. . . . . . . . . . . . . . . . . . . . . . . 241

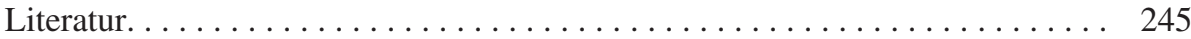

Sachverzeichnis . . . . . . . . . . . . . . . . . . . . . . . . . . . . . . . . . 247 


\title{
Über die Autoren
}

\begin{abstract}
Albert Fleischmann ist promovierter Informatiker und seit 1995 Inhaber der InterAktiv Unternehmensberatung in Deutschland. Er beschäftigt sich methodisch mit der Spezifikation, dem Design und der Implementierung paralleler Systeme, insbesondere mit Geschäftsprozessen, production control und embedded systems. Anwendungsgebiete sind smart city environments und Industrie 4.0. Für seine grundlegendenden Arbeiten erhielt er 1986 den IBM Outstanding Technical Achievement Award. Darüber hinaus ist Dr. Fleischmann seit vielen Jahren in der akademischen Bildungsarbeit tätig, sowie aktives Mitglied der Gesellschaft für Informatik und der IEEE.
\end{abstract}

Stefan Oppl ist Assoziierter Universitätsprofessor am Institut für Wirtschaftsinformatik - Communications Engineering an der Johannes Kepler Universität Linz, Österreich. Er beschäftigt sich in Forschung und Lehre mit der partizipativen und human-zentrierten Erhebung und Gestaltung von organisationalen Arbeitsprozessen. Die Unterstützung der Artikulation individueller Arbeitsbeiträge und der Abstimmung der unterschiedlichen Sichtweisen auf kollaborative Arbeit durch Aushandlungsprozesse der Gegenstand seiner gestaltungswissenschaftlichen und empirischen Forschungsarbeiten. Zudem beschäftigt er sich mit dem Umgang mit heterogenen Lernvoraussetzungen und -rahmenbedingungen in unterschiedlichen institutionellen und organisationalen Lernsituationen. Seine Arbeiten erscheinen in internationalen Zeitschriften im Bereich der Forschung an kollaborativen Informationssystemen und des technologieunterstützten Lernens.

Werner Schmidt ist Professor für Wirtschaftsinformatik, insbesondere Prozess- und IT-Management, an der Business School der Technischen Hochschule Ingolstadt. Er veröffentlicht Monografien, Tagungsbände und wissenschaftliche Artikel in diesen Fachgebieten, organisiert und leitet einschlägige Konferenzen und Workshops und ist regelmäßig Mitglied in mehreren Programmkomitees. Als Mitgründer und Vorstand des Institute of Innovative Process Management e. V. (www.i2pm.net) legt er großen Wert auf den Austausch zwischen Wissenschaft und Praxis. Dabei kommt ihm seine Berufserfahrung in Prozessmanagement- und Softwareentwicklungsprojekten ebenso zugute wie sein Engagement bei der Begleitung von IT-Unternehmen als Beiratsmitglied. 
Christian Stary ist zurzeit Ordentlicher Universitätsprofessor für Wirtschaftsinformatik und leitet den Schwerpunkt Communications Engineering sowie das Kompetenzzentrum Wissensmanagement an der Johannes Kepler Universität Linz, Österreich. Sein Wirkungskreis in der Forschung umfasst Lernunterstützungssysteme unter Berücksichtigung wissensbasierter und organisationsentwickelnder Techniken. Seine von ihm betreuten Projekte zielen auf intelligentes Design auf Basis von Beteiligtenbedürfnissen. Dabei spielt Prozessmanagement eine entscheidende Rolle neben erkenntnistheoretisch fundierter Entwicklung von Methoden. Seine Arbeit wird auf unterschiedlichen internationalen Konferenzen und Workshops zur disziplinübergreifenden Forschung in den Bereichen Entwicklung stakeholder-zentrierter sichtbar. Er engagiert sich in mehreren internationalen Vereinigungen, wie beispielsweise als Vorstandsvorsitzender im International Council on Knowledge Management. 
Geschäftsprozesse helfen, Organisationen zu strukturieren und zu betreiben. Sie stellen den Bezugsgegenstand von Geschäftsprozessmanagement dar und integrieren alle wesentlichen Elemente, um Anforderungen, welche an Organisationen gestellt werden, nachvollziehbar und strukturiert zu bearbeiten. Alle Beteiligten wissen somit, in welcher Form ihre Aufgaben zu bewältigen sind. Jedoch ist seitens der Stakeholder grundsätzliches Verständnis zu den heutigen Rahmenbedingungen der Geschäftswelt sowie zu Unternehmensstrukturen und deren Repräsentation erforderlich.

\subsection{Geschäftsprozesse und Geschäftsprozessmanagement}

Es gibt keine Organisation ohne Prozesse. Wenn Menschen etwas gemeinsam tun wollen, benutzen sie dafür die notwendigen Hilfsmittel und stimmen entsprechend dem gewünschten Ergebnis ihre Tätigkeiten aufeinander ab. Da solche Tätigkeiten nicht nur von Menschen ausgeführt werden können, sondern auch von Automaten und Computern müssen deren Aktivitäten in die Abstimmung ebenfalls einbezogen werden. Insbesondere in zumindest teilweise automatisierte Prozesse sind also verschiedene Typen von Handelnden involviert.

Ausgelöst wird ein Prozess durch ein Ereignis, das seinen Ursprung innerhalb oder außerhalb der Organisation haben kann wie etwa ein Dienstreiseantrag oder eine Kundenbestellung. Das abgestimmte und zielgerichtete Handeln als Reaktion auf ein solches Ereignis wird als Prozess bezeichnet. Handelt es sich bei der Organisation um ein Unternehmen spricht man von Geschäftsprozessen.

Es gibt kein Unternehmen ohne Geschäftsprozesse. Es gibt nur Unterschiede welchen Reifegrad diese haben. Die Reaktionen einer Organisation auf bestimmte Geschäftsereignisse können bei ihrem Auftreten immer von neuem abgestimmt werden oder 
es wird eine Vorgehensweise festgelegt, die dann in solchen Fällen immer wieder ausgeführt wird. Ereignisse der gleichen Art wie beispielsweise Bestellungen werden als Ereignisklasse bezeichnet. Eine vorab festgelegte Vorgehensweise für eine Ereignisklasse wird als ein Prozessmodell bezeichnet. Bei der Ausführung der im Modell festgelegten Tätigkeitsfolgen als Reaktion auf ein identifiziertes konkretes Ereignis, z. B. die Buchbestellung des Kunden Huber vom 20. Mai, handelt es sich um eine Prozessinstanz.

Jedes Unternehmen hat unabhängig von seiner Art des Geschäfts bestimmte Standardprozesse, die aber unternehmensindividuell ausgestaltet und zugeschnitten sein können. So verfügt beispielsweise jedes Unternehmen über einen Order-to-Cash-Prozess, mit dem es auf Geschäftsereignisse vom Kundenauftrag bis zum Zahlungseingang reagiert und diese durch Buchungen dokumentiert. Umgekehrt wird ein Beschaffungsprozess existieren mit Bestellungen zur Befriedigung eigener Bedarfe, dem konkreten Bezug (z. B. Wareneingang und Einlagerung) sowie der Bezahlung der Kreditoren. Weitere Beispiele sind Prozesse für die Personalbeschaffung oder die Logistik. Eine gebräuchliche Klassifikation unterteilt Prozesse nach ihrem Charakter in Management-, Kern- und Supportprozesse. Die Einordnung ist unternehmensspezifisch und hängt u. a. von der Branche ab.

Je klarer ein Unternehmen seine Geschäftsprozesse definiert und je konsequenter es diese im täglichen Geschehen umsetzt, umso leistungsfähiger ist es. Bei viele Unternehmen gründet ihre Wettbewerbsfähigkeit nicht (mehr) nur auf die Besonderheit ihrer Produkte, sondern auf die Güte der Geschäftsprozesse. Während beispielsweise das Geschäft eines Verlags in erster Linie durch seine Bücher bestimmt wird, bestimmt bei Amazon maßgeblich die Kundenerfahrung bei Suche, Auswahl, Kauf, Bezahlung, Lieferung und eventuelle Rückgabe von Produkten, also der reibungslose, kundenzentrierte Prozess den Erfolg.

Die Modelle für solche Prozesse sind laufend anzupassen oder komplett neu zu gestalten, weil sich die Reaktionen auf eine Ereignisklasse ändern können bzw. zusätzliche Reaktionen auf neue Ereignisklassen nötig werden. Die resultierenden Spezifikationen müssen außerdem in der Organisation und der IT-Infrastruktur umgesetzt werden, damit die Mitarbeiterinnen und Mitarbeiter im Tagesgeschäft Instanzen der Prozesse abarbeiten können. Dabei sind Rahmenbedingungen zu beachten wie die Effektivität, Effizienz und Compliance, also die Anforderungen, das gewünschte Ergebnis mit dem geringsten möglichen Ressourcenaufwand und unter Einhaltung gültiger externer und interner Regularien (z. B. Gesetzen) zu liefern. Für die Erledigung dieser Aufgaben hat sich das Geschäftsprozessmanagement (Business Process Management, BPM) etabliert. Es bezeichnet einen integrierten Managementansatz für Analyse, Design, Optimierung, Implementierung, Steuerung, Überwachung und Weiterentwicklung der Management-, Kern- und Supportprozesse im Unternehmen. In technischer Hinsicht schließt es auch die IT-Unterstützung dieser Teilaufgaben durch Werkzeuge z. B. für die Modellierung oder Ausführung (z. B. Process Engines) oder umfassendere BusinessProcess-Management-Systeme (BPMS) ein. 
Im Geschäftsprozessmanagement werden als Ausschnitt der Wirklichkeit ein Unternehmen und sein unmittelbares Umfeld betrachtet. In diesem Ausschnitt der Welt wünscht jemand eine Leistung von anderen in Form eines physischen Produkts, eines Service oder einer Kombination aus beidem. Die Leistung soll entsprechend den Anforderungen erbracht werden, der Wunsch nach ihr ist das Geschäftsereignis, auf das das Unternehmen nach seinen Vorstellungen im definierten Modell reagieren soll.

Beim Geschäftsprozessmanagement gilt es also ein Modell der Leistungserstellung zu definieren und auf die Abwicklung von Geschäftsfällen anzuwenden. Dies bedeutet die Wirklichkeit gemäß Modell anzupassen, also betroffene Ausschnitte der Wirklichkeit zu analysieren und diese Wirklichkeit zu verändern. Da diese Wirklichkeit und die angestrebten Veränderungen sehr komplex sind, werden im BPM mehrere Modellkonzepte aus der Sozialwissenschaften, der Betriebswirtschaft und der Informatik zusammengeführt und kombiniert.

In den folgenden Abschnitten umreißen wir eine Gesamtsicht auf das Prozessmanagement und erläutern diese dann in den weiteren Kapiteln im Detail. Ausgehend vom Blick der Beteiligten auf die Welt werden die vielfältigen Facetten des Geschäftsprozessmanagements dargestellt und eine Auswahl von Modellen vorgestellt, die sich dabei in unserer Praxis bewährt haben. Die Gestaltung solcher Modelle unterstützt den Weg von einer mehr oder weniger unstrukturierten oder unbefriedigenden Arbeitsweise zu einer Prozessabwicklung, die den Vorstellungen des Unternehmens und seiner Kunden entspricht.

Die überblicksartige Gesamtsicht entwickeln wir schrittweise, ausgehend von individuellen Perspektiven der Beteiligten auf ihre Arbeit in einem Prozess, deren Strukturierung und Harmonisierung über die Spezifikation in einem Modell und dessen Einbettung in die organisatorische und IT-Umgebung des Unternehmens bis hin zur gemeinsamen Bearbeitung von Prozessinstanzen in den dadurch entstehenden soziotechnischen Systemen. Eine entsprechend mitwachsende Abbildung zeigt am Ende unser umfassendes Verständnis des Geschäftsprozessmanagements.

\subsection{Blick auf die Welt, Strukturierung und Modellbildung}

Wie bereits erwähnt, gilt es für ein Unternehmen die interessierenden Geschäftsereignisse zu identifizieren und die dadurch ausgelösten Tätigkeiten zu definieren. Dazu ist der entsprechende Ausschnitt der Wirklichkeit zu identifizieren und genauer zu betrachten.

Der Ausschnitt wird bestimmt durch die Kunden, die eine Leistung fordern, und bildet für die an der Leistungserbringung beteiligte Gruppe von Mitarbeitern ${ }^{1}$ des Unternehmens die

\footnotetext{
${ }^{1}$ In der Folge wird das männliche Geschlecht für beide Geschlechter stellvertretend verwendet.
} 
sie unmittelbar betreffende und umgebende Wirklichkeit. Zum Erbringen der gewünschten Leistung müssen die Beteiligten direkt oder indirekt zusammenwirken.

Jeder leistet dabei in Abstimmung mit den anderen seinen Beitrag. Basierend auf seinem persönlichen Hintergrund bezüglich Ausbildung, Wissen, Wollen (Motivation), Erfahrungen und Vorlieben hat jedes Gruppenmitglied eine eigene Wahrnehmung des Prozesses und seines Kontextes. Es entwickelt seine individuelle Vorstellung, was sein Beitrag sein soll, wie er erbracht wird, welche Ereignisse mit welchen Tätigkeiten und vom wem zu berücksichtigen sind, in welcher Reihenfolge Teilschritte stattfinden, von wem welche Vorleistungen erwartet und für wen welche Vorleistungen erbracht werden.

Folglich besitzen alle Betroffenen ihr eigenes mentales ,Weltmodell“ des betrachteten Wirklichkeitsausschnitts (vgl. Abb. 1.1). Für die erfolgreiche Reaktion auf Geschäftsereignisse gilt es, die unterschiedlichen Wirklichkeiten der Beteiligten zu strukturieren und in ein konsistentes Prozessmodell für ein gemeinsames zielgerichtetes Tun zu überführen. Dies bedeutet, der Geschäftsprozess wird „,verabredet“, in dem die einzelnen, mehr oder weniger gut zusammenpassenden mentalen Modelle der involvierten Personen harmonisiert werden.

Dieses Zusammenführen der individuellen Vorstellungen der von einem Geschäftsprozess Betroffenen und die wechselseitige Abstimmung der verschiedenen Aspekte eines Geschäftsprozesses (vgl. Abschn. 1.3) ist selbst ein komplexer Prozess und zentraler Aspekt des Geschäftsprozessmanagements.

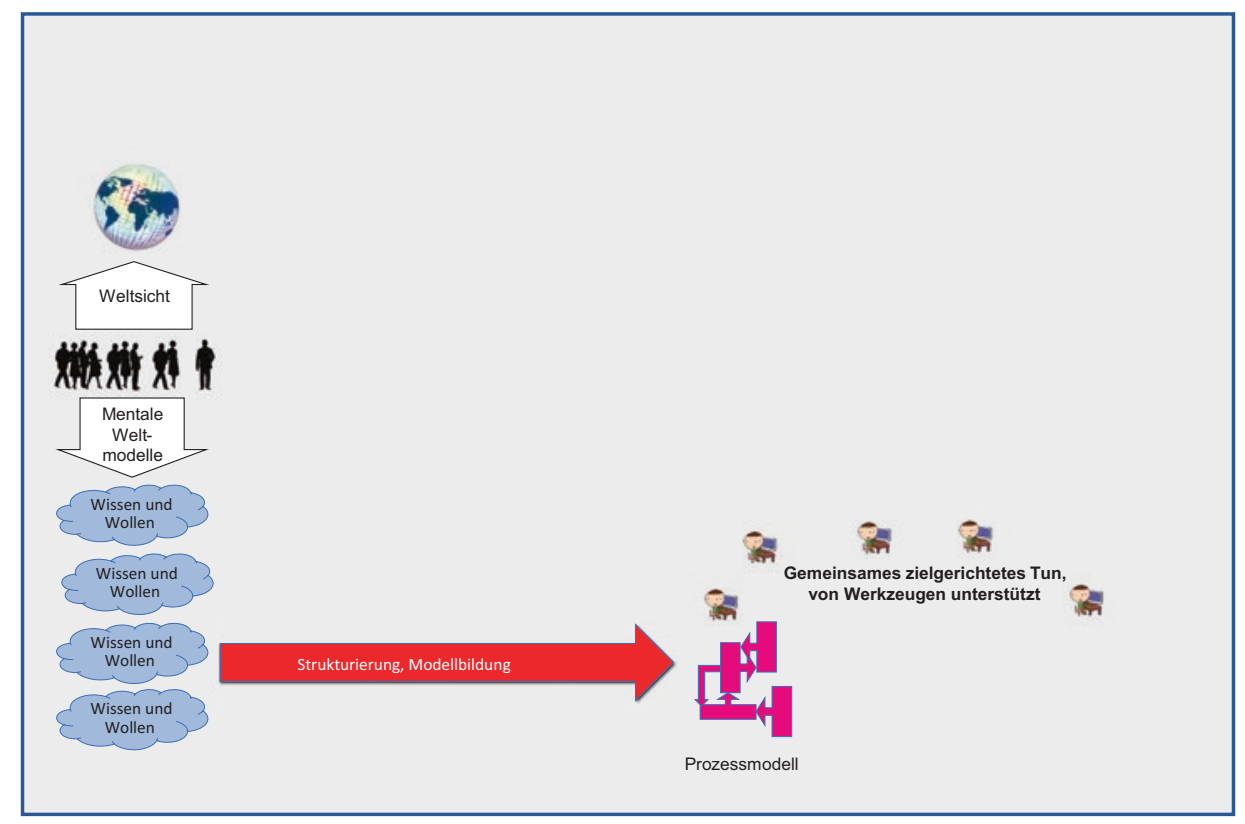

Abb. 1.1 Individuelle mentale Modelle der Beteiligten 


\subsection{Bestandteile einer Prozessbeschreibung}

Wir teilen eine Geschäftsprozessbeschreibung gedanklich in drei Abschnitte (vgl. Abb. 1.2). Der erste als Prozessstrategie bezeichnete Teil macht Aussagen zu Zweck, Auslöser, Inputs, Ende und Outputs des Vorgangs. Auslöser ist das Ereignis, das die Leistungserbringung auf Basis der Erwartungen des Initiators in Gang setzt, also eine Prozessinstanz erzeugt. Mit diesem Anstoß geht einher, dass der Initiator Informationen oder Gegenstände bereitstellt, welche entsprechend seiner Erwartungen bearbeitet werden sollen. Diese Inputs gilt es in die erwarteten Ergebnisse zu transformieren und diese dem definierten Empfänger zur Verfügung zu stellen. Damit schafft der Geschäftsprozess einen Wert, für den ein Kunde bezahlt.

Diese Außensicht eines Geschäftsprozesses wird ergänzt durch die Prozesslogik. Diese innere Perspektive beschreibt die involvierten Handelnden und deren abgestimmtes Zusammenwirken. Die Akteure führen Aktivitäten in einer sachlogisch und zeitlich sinnvollen Reihenfolge aus. Die Ergebnisse ihrer Aktionen übergeben sie zur Weiterbearbeitung an andere Handelnde bzw. am Ende an den vorgesehenen Empfänger.

Bei der Prozessrealisierung geht es um die Bereitstellung der Ressourcen für die Abarbeitung von Prozessinstanzen. Dies können Menschen, Maschinen und Softwaresysteme sein, welche als konkrete Realisierungen der involvierten Handelnden die diesen zugeordneten Aktivitäten übernehmen. Im Zeitalter der Digitalisierung synchronisieren Softwaresysteme (Process oder Workflow Engines) die Aktionen der Handelnden durch

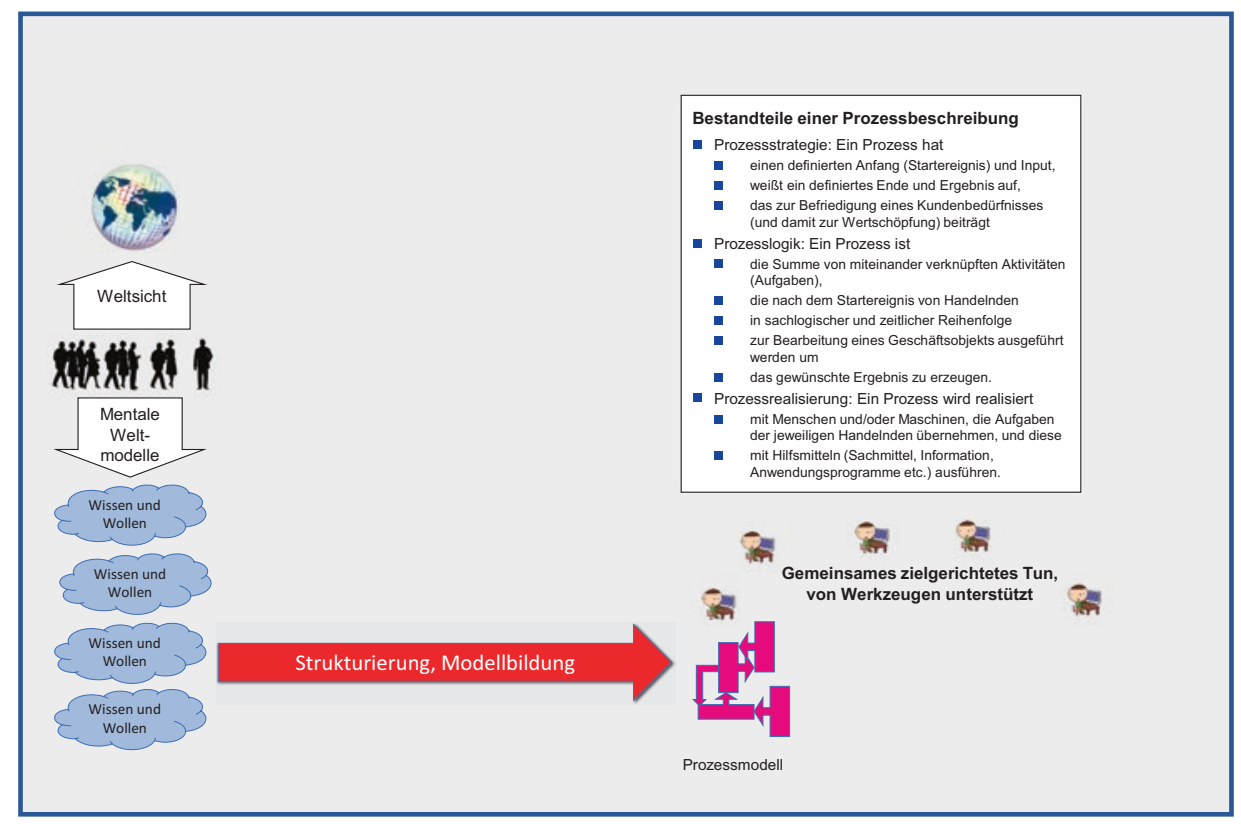

Abb. 1.2 Bestandteile einer Prozessbeschreibung 
Steuerung der zeitlichen und sachlogisch notwendigen Reihenfolge der Teilschritte gemäß dem Prozessmodell. Für die Erledigung ihrer einzelnen Aufgaben setzen die Handelnden gegebenenfalls Hilfsmittel ein, wie Informationen, Anwendungsprogramme oder Werkzeuge.

Im Rahmen der Prozessrealisierung ist dafür zu sorgen, dass auf Basis des als Muster definierten Modells durch entsprechende Ressourcenzuordnung mehrere Prozessinstanzen parallel und unabhängig voneinander ausgeführt werden können.

\subsection{Rahmenbedingungen für Prozessmodelle und Prozessinstanzen}

Das Geschäftsmodell beschreibt im Wesentlichen, wie ein Unternehmen in die Welt hineinwirkt und auf welche Art und Weise es dabei Erlöse erzielt und Gewinn erwirtschaftet. Von besonderer Bedeutung dabei sind das Kundenversprechen sowie die Ressourcen und Partner, mit denen dieses Versprechen eingelöst wird.

Die Unternehmensarchitektur beschreibt eine Maschinerie, mit der das Geschäftsmodell zum Leben erweckt werden soll. Als typisches Schichtenkonzept definiert sie fachliche und IT-Strukturen und verknüpft diese miteinander. Das Konzept des Business Engineering [1] sieht etwa auf einer strategischen Ebene die Geschäftsarchitektur mit der Definition von Zielen und Leistungen vor, die mit dem Geschäftsmodell verwoben ist. Auf der Ebene der Prozesse als Implementierungshilfsmittel der Strategie folgt die Prozessarchitektur mit Aufbau- und Ablauforganisation. Der Übergang zu den IT-Strukturen für die Unterstützung der Prozesse führt in die Ebene der Informationssysteme mit der Applikationsarchitektur und der IT-Architektur.

Die Geschäftsprozesse befinden sich also als zentraler Bestandteil der Unternehmensarchitektur in einer Art Sandwich-Position, welche ihre Beeinflussung durch andere Architekturelemente verdeutlicht. Beispielsweise kann eine gegebene und nur schwer änderbare Organisationsstruktur die Vorgehensweisen in Prozessen und die Art, wie mit externen Partnern zusammengearbeitet wird, mitbestimmen. Analog gilt dies für die Verfügbarkeit von Ressourcen. Aber auch horizontale Abhängigkeiten innerhalb der Ablauforganisation sind zu berücksichtigen, etwa wenn eine bestimmte Arbeitsweise im Bestellprozess Auswirkungen auf die Gestaltung der Zahlungsabwicklung hat.

Von „unten“ wirken Einflüsse nicht nur hinsichtlich der inhaltlichen Gestaltung der Prozessmodelle, sondern auch hinsichtlich Detailgrad und Genauigkeit. Für die Entwicklung von IT-Lösungen zur Prozessdigitalisierung gelten rigide Anforderungen an die Modelldefinition. Prozessteile, die mit IT-Unterstützung ausgeführt werden sollen, müssen genau und präzise spezifiziert sein.

Neben den exemplarisch erläuterten und in Abb. 1.3 ergänzten internen Rahmenbedingungen wirken auch noch externe Faktoren auf die Prozessgestaltung ein. Hier kann man als Beispiel Prüfschritte sehen, welche aufgrund von Compliance-Vorschriften in einen Ablauf aufgenommen werden müssen. 


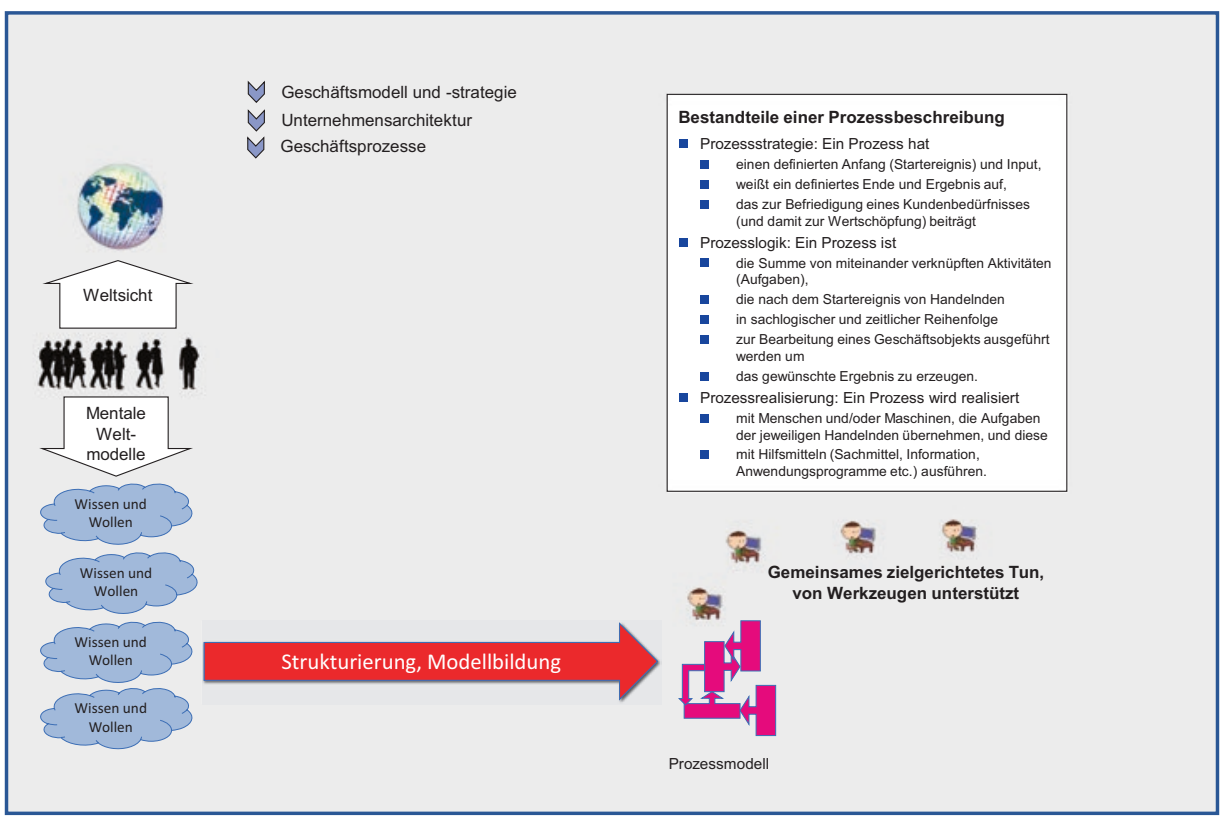

Abb. 1.3 Ergänzung um Rahmenbedingungen für die Prozessdefinition

\subsection{Prozesskennzahlen}

Die zu entwickelnden oder zu ändernden Prozesse haben das allgemeine Ziel, die Umsetzung des Geschäftsmodells und der zugehörigen Strategie zu unterstützen. Die Beziehung zwischen den Kennzahlen aus dem Geschäftsmodell (Key Performance Indicators, KPIs) und den Prozessen wird über Prozesskennzahlen (Process Performance Indicators, PPIs) hergestellt. Diese Prozesskennzahlen sind Verfeinerungen von Zielen aus dem Geschäftsmodell (vgl. Abb. 1.4).

Typische betriebswirtschaftliche Key Performance Indicators leiten sich aus Geschäftsmodell und -strategie ab und messen den Geschäftserfolg auf höheren Aggregationsebenen, z. B. Erlöse und Kosten auf Gesamtunternehmens-, Sparten-, Produktgruppenebene etc. Hier steht die Effektivitätsbetrachtung im Vordergrund („Doing the right things“). Mit den Geschäftsprozessen werden die Strategie umgesetzt und die Elemente der Unternehmensarchitektur zusammengeführt. Die damit verbundenen Process Performance Indicators zielen auf die Effizienz ab („Doing things right"). Sie stehen damit in engem Zusammenhang mit den Key Performance Indicators und leiten sich teilweise von diesen ab.

Beim Ableiten der Kennzahlen muss bereits geprüft werden, ob diese mit vertretbarem Aufwand in ausreichender Präzision gemessen werden können. Unter Umständen ergeben sich daraus auch Anforderungen an den zu entwickelnden Prozess, um die 


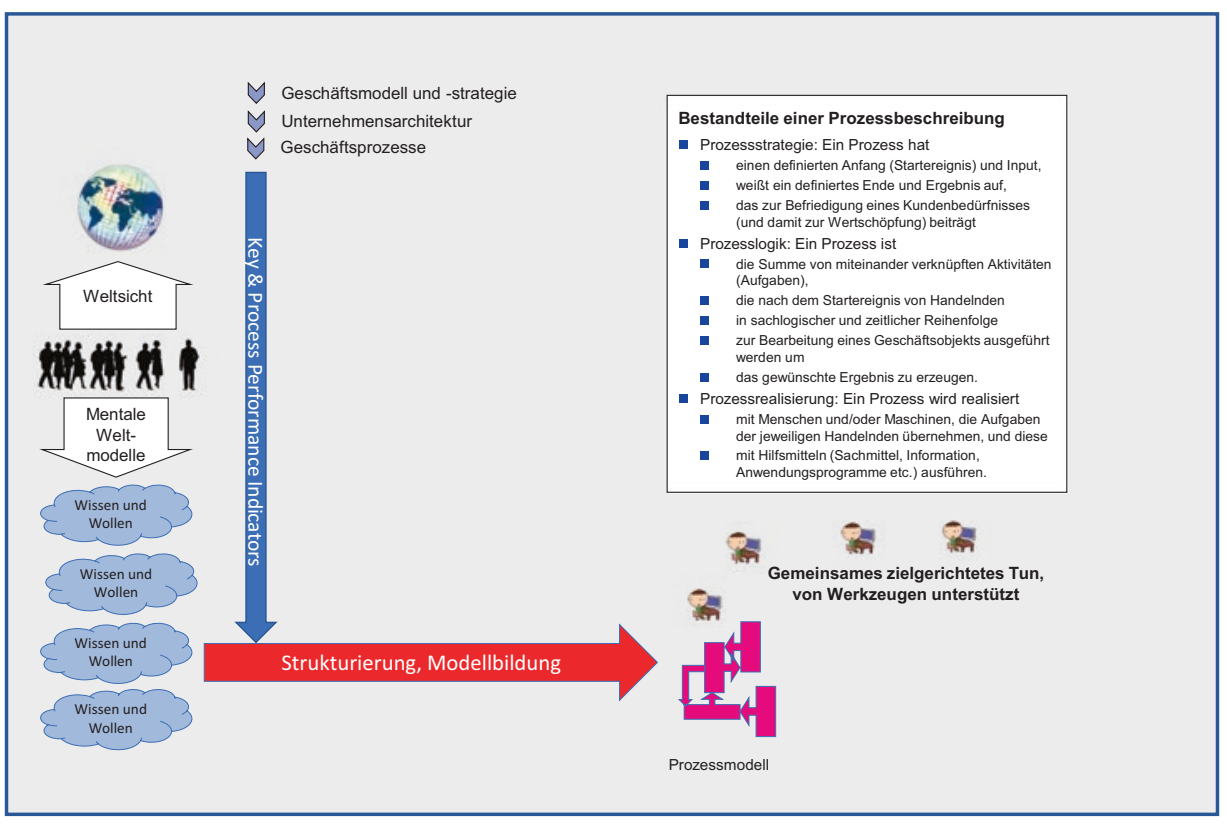

Abb. 1.4 Definition von Prozesskennzahlen

Kennzahlen direkt oder indirekt messen zu können. Ist unmittelbare Messung nicht möglich, können auch Ziele für Ersatzkennzahlen festgelegt und daraus Werte für den eigentlich gewünschten Performance Indicator abgeleitet werden.

Für die Prozesskennzahlen werden Zielwerte festgelegt, die es durch einen geänderten oder neu zu gestaltenden Prozess zu erreichen gilt. Während des ganzen Wegs von der Identifikation des Problems, bis hin zur Inbetriebnahme eines geänderten oder neuen Prozesses gilt es laufend $\mathrm{zu}$ plausibilisieren, ob mit dem entstehenden Prozess die angestrebten Ziele erreicht werden können.

\subsection{Unterstützungskonzepte}

Der Weg vom individuellen Wissen und Wollen, also von den mentalen Modellen der Beteiligten zu einem Prozessmodell, das auch zumindest in Teilen digitalisiert werden kann, ist komplex und aufwendig. Um Komplexität und Aufwand zu reduzieren, wurden Unterstützungskonzepte wie Frameworks, Vorgehensmodelle und Beschreibungssprachen entwickelt.

Die folgende Übersicht umfasst eine thematisch gruppierte Auswahl solcher Hilfsmittel, welche nach unseren Erfahrungen in der Praxis weit verbreitet sind. Sie sind in Abb. 1.5 eingefügt und werden in den Kapiteln zu Modellen (Kap. 2) und Modellierungssprachen (Kap. 3) genauer betrachtet. 


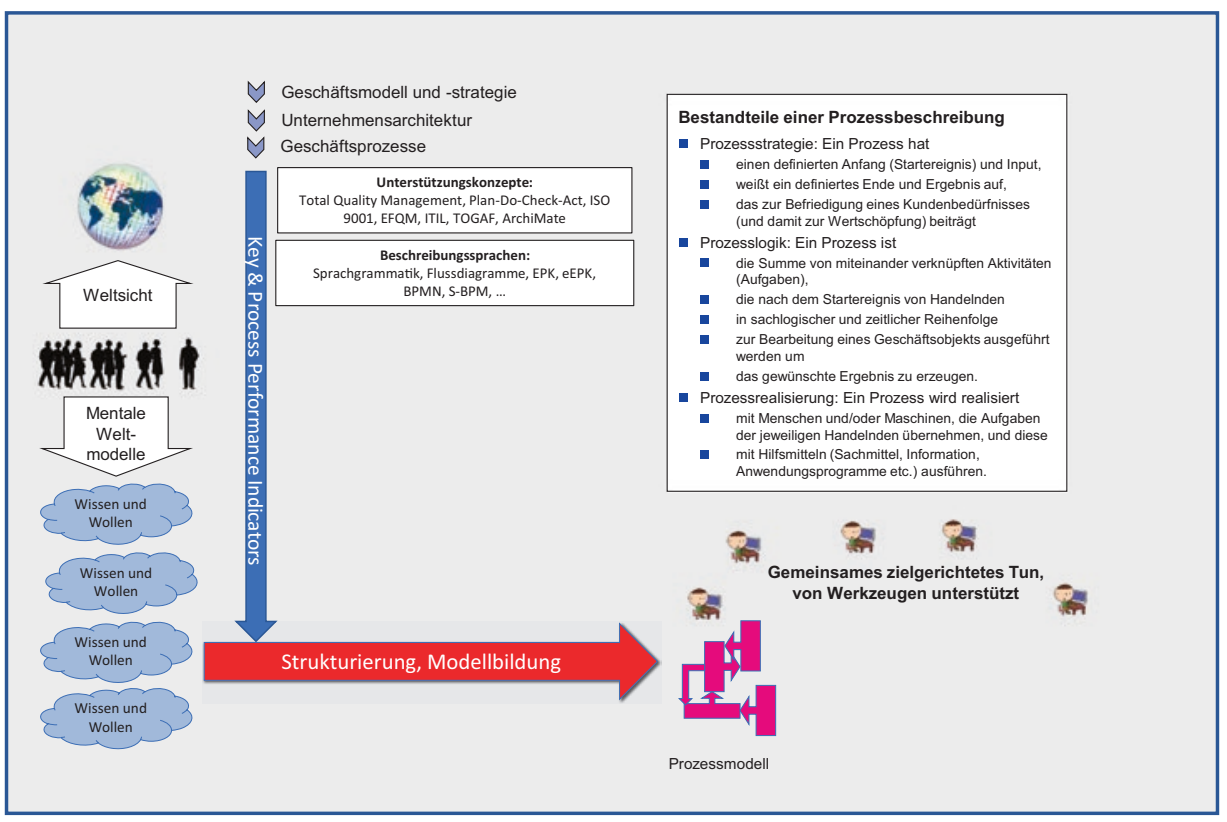

Abb. 1.5 Ergänzung um Konzepte zur Unterstützung der Prozessdefinition

Frameworks für das Qualitätsmanagement:

- Total Quality Management (TQM) TQM/PDCA,

- Deming-Zyklus (PDCA, Plan-Do-Check-Act)

- EN ISO 9001

- European Foundation for Quality Management (EFQM)

Frameworks für das Unternehmensarchitekturmanagement (Enterprise Architecture Management, EAM):

- Zachman-Framework

- The Open Group Architecture Framework (TOGAF)

- Architecture-Animate (ArchiMate)

Frameworks für IT-Management und IT-Governance:

- IT Infrastructure Library $\left(\right.$ ITIL $\left.^{\circledR}\right)$

- Control Objectives for Information and Related Technology (COBIT) 
Beschreibungssprachen für die Prozesslogik:

- Flussdiagramme

- Ereignisgesteuerte Prozessketten mit Erweiterung (EPK, eEPK)

- Business Process Model and Notation (BPMN)

- Subject-oriented Business Process Management (S-BPM)

\subsection{Digitalisierung}

Heute ist Digitalisierung das Schlüsselwort bei der Transformation der Wertschöpfung. Digitalisierung in der Wirtschaft oder allgemein in Organisationen heißt Digitalisierung von Geschäftsmodellen, Produkten und Services sowie von ganzen Prozessen oder Teilen davon. Bei Prozessen bedeutet dies jedoch nicht notwendigerweise Vollautomatisierung ohne jeglichen menschlichen Eingriff. So kann es beispielsweise sein, dass ein Programm, das einen Prozess steuert, bei entsprechender Notwendigkeit Aktionen einbindet, die von Menschen oder auch Cyber Physical Systems ausgeführt werden. Letztere bestehen aus miteinander kommunizierenden Geräten mit Software sowie mechanischen und elektronischen Komponenten.

In der Initiative Industrie 4.0 wird diese umfassende Betrachtung von Prozessen, d. h. die Kommunikation zwischen Menschen, Maschinen und Werkstücken angestrebt. Diese Aspekte müssen zum einen in den Prozessmodellen ausgedrückt werden können und zum anderen muss die Überführung eines Geschäftsprozessmodells in die digitale Ausführung so weit wie möglich unterstützt werden. Insbesondere wenn man die Aspekte des Qualitätsmanagements, also die laufende Verbesserung der Prozesse, in die Betrachtung einbezieht, müssen Prozessänderungen, die eine Änderung der Digitalisierung nach sich ziehen, schnell und mit möglichst geringem Aufwand umgesetzt werden können.

Die in den vorangegangenen Abschnitten beschriebenen Aspekte müssen bei der Modellbildung bereits mit einfließen, um die technische Implementierung von Prozessen zu erleichtern, ohne jedoch Implementierungsdetails vorweg zu nehmen (vgl. Abb. 1.6). Je präziser die Prozesse beschrieben sind, umso leichter fällt dies. Prozessabschnitte, deren Ablauflogik zum Zeitpunkt der Modellierung noch nicht präzise beschrieben werden können, sind entsprechend zu kennzeichnen. Diese Teile eines Prozesses können aber mit anderen geeigneten Methoden gemäß der gewünschten oder notwendigen Offenheit modelliert werden. Solche Prozessabschnitte können entweder mit Adaptive Case Management Methoden beschrieben werden oder, falls eine kommunikationsorientierte Beschreibungssprache verwendet wird, als Kommunikationsschleife. Letztere beendet einer der beteiligten Partner nach Erreichen eines entsprechenden Ergebnisses, ehe im Prozess fortgefahren wird.

Wichtig in diesem Kontext ist die Granularität, also der Detailgrad der Prozessbeschreibung. Aktivitäten sollten so fein geschnitten werden, dass man eindeutig festlegen kann, ob sie digitalisiert, teildigitalisiert (Mensch-IT, Physik-IT) oder manuell von 


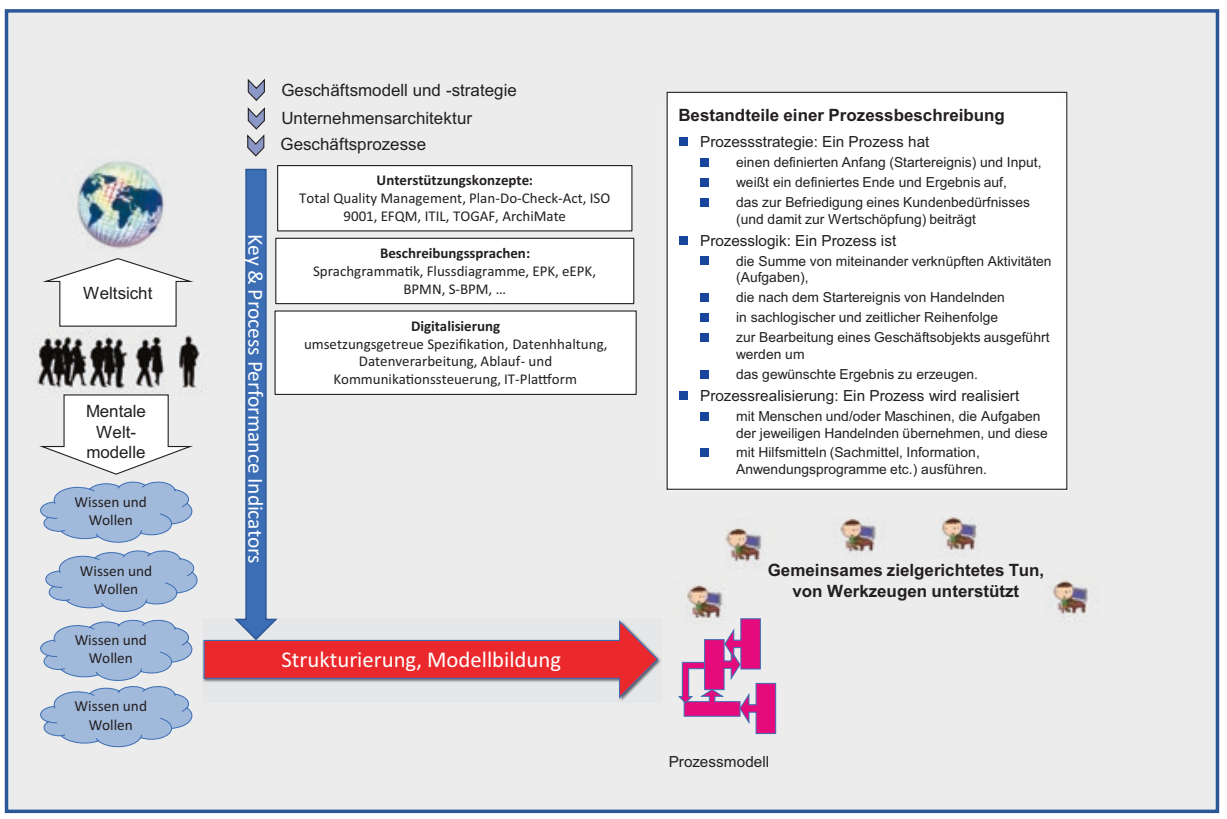

Abb. 1.6 Berücksichtigung von Digitalisierungsaspekten im Modell

Menschen ausgeführt werden. Der Zuschnitt sollte sich an den fachlichen Anforderungen orientieren und nicht an der Funktionalität eines möglicherweise schon vorhandenen IT-Systems. Notfalls muss ein derartiges System bei der Prozessumsetzung an dessen fachliche gewünschte Spezifikation angepasst werden.

\subsection{Prozess zum Erstellen von Prozessen}

Die Definition der Geschäftsprozesse kann nicht schematisch bzw. algorithmisch erfolgen, d.h., es gibt keine Software, die gefüttert mit dem Geschäftsmodell, der Unternehmensarchitektur, den Kennzahlen mit dazugehörigen Zielwerten und Unterstützungskonzepten, nach kurzer Zeit eine passende Prozessbeschreibung ausgibt.

Die Definition von Geschäftsprozessen ist ein intuitiver und kreativer Prozess. Deshalb werden v.a. zu Beginn von Geschäftsprozessmanagementaktivitäten auch Kreativitätstechniken und Methoden des Wissensmanagements wie Storytelling, World Café oder Value Networks eingesetzt.

So kann man sich beispielsweise des Design-Thinking-Ansatzes bedienen. Dabei handelt es sich um ein Konzept, bei dem interdisziplinäre Teams in einem die Kreativität fördernden Umfeld in einem iterativen Prozess zusammenarbeiten, um innovative Lösungen für eine Problemstellung zu entwickeln (vgl. Abschn. 4.3). Ein Kernpunkt ist dabei 
ein tiefes Verständnis für die Bedürfnisse und Motivationen von Menschen der Zielgruppe zu entwickeln und zu berücksichtigen. Design Thinking bietet eine umfangreiche Methodensammlung für die Nutzung in den einzelnen Schritten seines Vorgehens. Mit diesen Eigenschaften lässt sich der Ansatz auch für die Überarbeitung bzw. Neudefinition eines Geschäftsprozesses einsetzen. Unter Umständen lassen sich dabei außergewöhnliche Lösungen finden, die beim üblichen BPM-Vorgehen nicht entstanden wären.

Allerdings muss ein kreatives, innovatives Prozesskonzept auch detailliert ausgearbeitet und umgesetzt werden. Die kreative Gestaltung ist deshalb eingebettet in Bündel von Aktivitäten, die den Prozess letztlich Teil der realen Welt werden lassen. Als solche Aktivitätsbündel identifizieren wir Analyse und Modellierung, Validierung, Optimierung, organisatorische Implementierung, IT-Implementierung sowie Betrieb und Monitoring. Diese Aktivitätsbündel sind eine Weiterentwicklung bzw. Verfeinerung des Plan-Do-Check-Act-Kreislaufs. Sie werden meist kreisförmig angeordnet, was einen entsprechenden Durchlauf impliziert. Dies entspricht nicht immer dem Vorgehen in der Realität, weshalb wir die Aktivitätsbündel in Abb. 1.7 als lose vernetzte Waben darstellen. Dort sind die Phasen des Design-Thinking-Prozesses und die Aktivitätsbündel ergänzt. Beide Konzepte werden in Kap. 4 ausführlicher vorgestellt und miteinander in Beziehung gesetzt.

Umfangreiche und komplexe Prozessänderungen bedürfen meist Tätigkeiten aus mehreren Aktivitätsbündeln und werden als Projekt durchgeführt. Ein solches Projekt kann deshalb als ein Durchlauf (Prozessinstanz) des Prozesses zum Erstellen von Geschäfts-

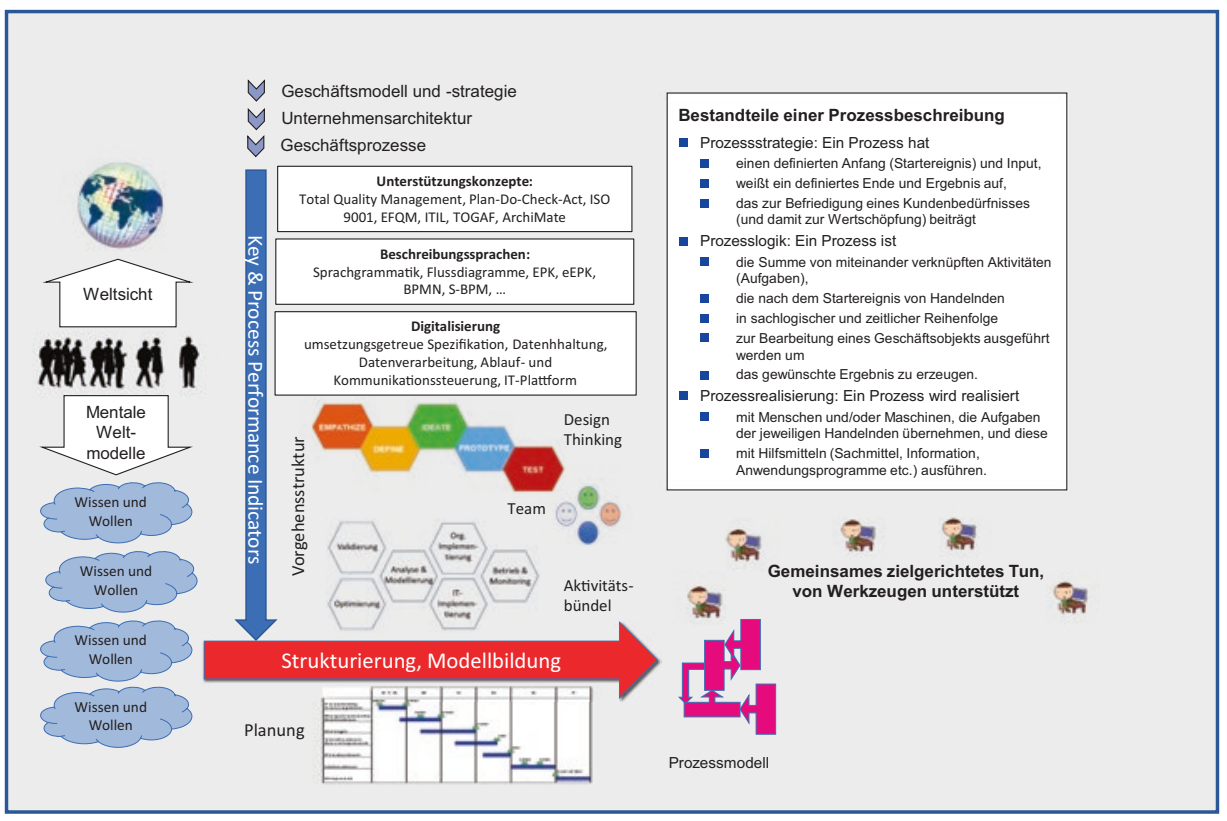

Abb. 1.7 Ergänzung um die Vorgehensstruktur und Planung für Prozessänderungen 
prozessen betrachtet werden. Dafür ist eine detaillierte Projektplanung zu erstellen mit den auszuführenden Tätigkeiten, Zuständigkeiten und Terminen (vgl. Abb. 1.7). Der Projektplan sollte dann entsprechend den Methoden des Projektmanagements abgearbeitet werden.

\subsection{Organisatorische und technische Implementierung}

Nach der Erstellung des Prozessmodells gilt es, das Modell in die Organisationsstruktur eines Unternehmens einzubetten. Es wird damit festgelegt, welche Tätigkeit von welcher Person oder Organisationseinheit ausgeführt wird. Diese Zuordnung muss nicht statisch sein, sondern kann von Instanz zu Instanz variieren. So kann beispielsweise der Einkaufsprozess für die Teile A und B die gleiche Ablauflogik haben, allerdings ist für den Einkauf der Teile A eine andere Einkaufsabteilung zuständig als für die Teile B. Prozessinstanzen für die Teile A tangieren also andere Organisationseinheiten (und ihnen zugeordnete Personen) als für Teile B. Diese Regeln sind so abzubilden, dass ein Prozess richtig mit der Aufbauorganisation verknüpft wird.

Neben Tätigkeiten, die durch Menschen ausgeübt werden, können im Prozess auch Aktivitäten vorkommen, welche Anwendungsprogramme oder IT-Services ausführen. Dazu sind solche Aktionen im Prozessmodell auf Funktionen von Softwarebausteinen abzubilden, welche diese dann zur Laufzeit ausführen. Wurde bei der Prozessmodellierung schon auf die mögliche Digitalisierung geachtet, so ist diese Abbildung mehr oder weniger unproblematisch.

Software kann auch die Abarbeitung von Vorgangsschritten steuern und dabei die im Modell spezifizierten Aufgaben den jeweils vorgesehenen Personen oder IT-Services als Akteur zuordnen. Softwaresysteme, die dies unterstützen, werden auch als Workflow-Systeme (Process Engine, Workflow Engine) bezeichnet. Im Idealfall können Prozessbeschreibungen direkt in Workflow-Systeme übernommen werden.

Nach der Einbettung in die Organisation und in die IT-Umgebung kann ein Prozess für die Abwicklung von Instanzen, also echten Geschäftsfällen verwendet werden, das Ziel ist erreicht. Abb. 1.8 zeigt den nun vervollständigten Weg von den individuellen mentalen Modellen mit dem Wissen und Wollen der Beteiligten zur gemeinsamen Bearbeitung von Prozessinstanzen.

\subsection{Erfolgsmessung mit Kennzahlen}

Bei der Ausführung von Instanzen eines Prozesses kann überprüft werden, ob die für die Prozesskennzahlen definierten Zielwerte erreicht werden. Dazu werden Istwerte für die festgelegten Key und Process Performance Indicators (KPIs und PPIs) gemessen, berechnet, gespeichert und mit den Zielwerten verglichen (vgl. Abb. 1.9). Dieser Vergleich kann in Realzeit oder über längere Zeitintervalle erfolgen. Eine Realzeitauswertung führt bei einer Zielabweichung zur sofortigen Einleitung geeigneter 


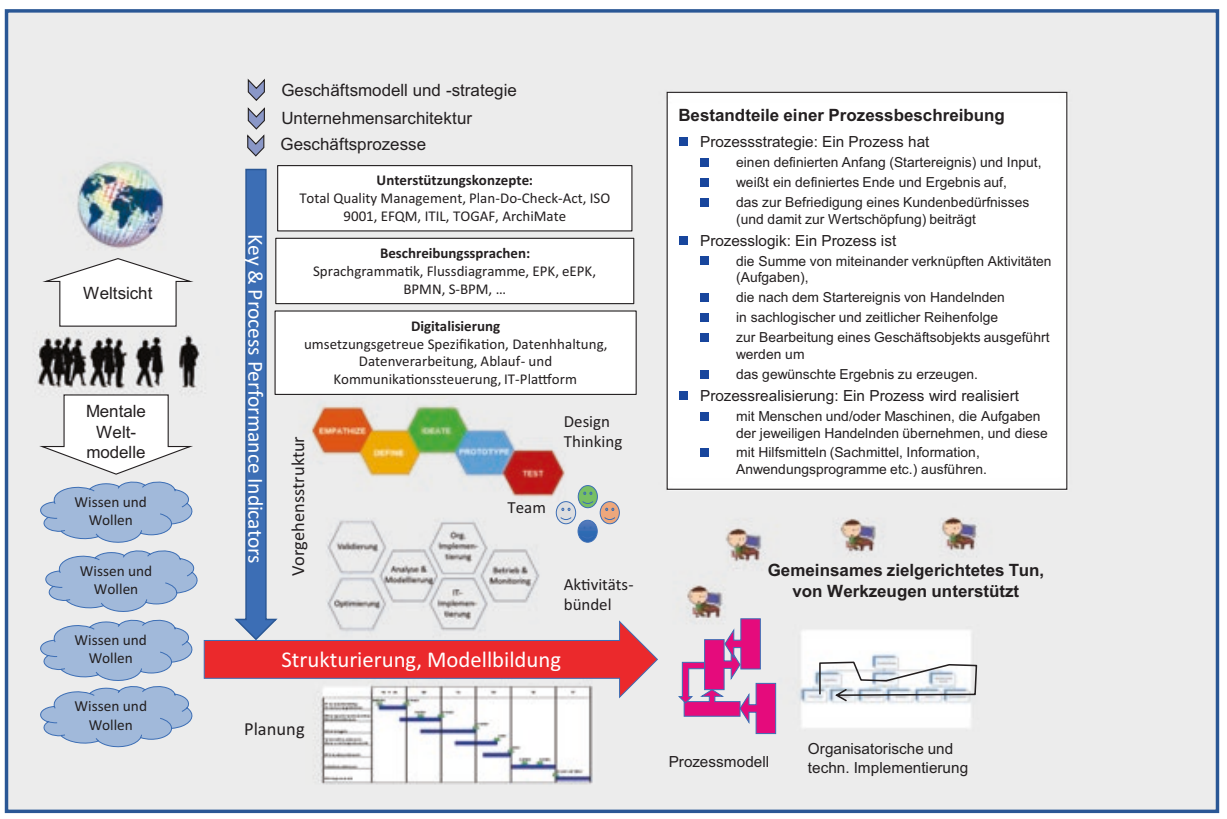

Abb. 1.8 Ergänzung um die Einbettung in die Organisation und IT-Umgebung

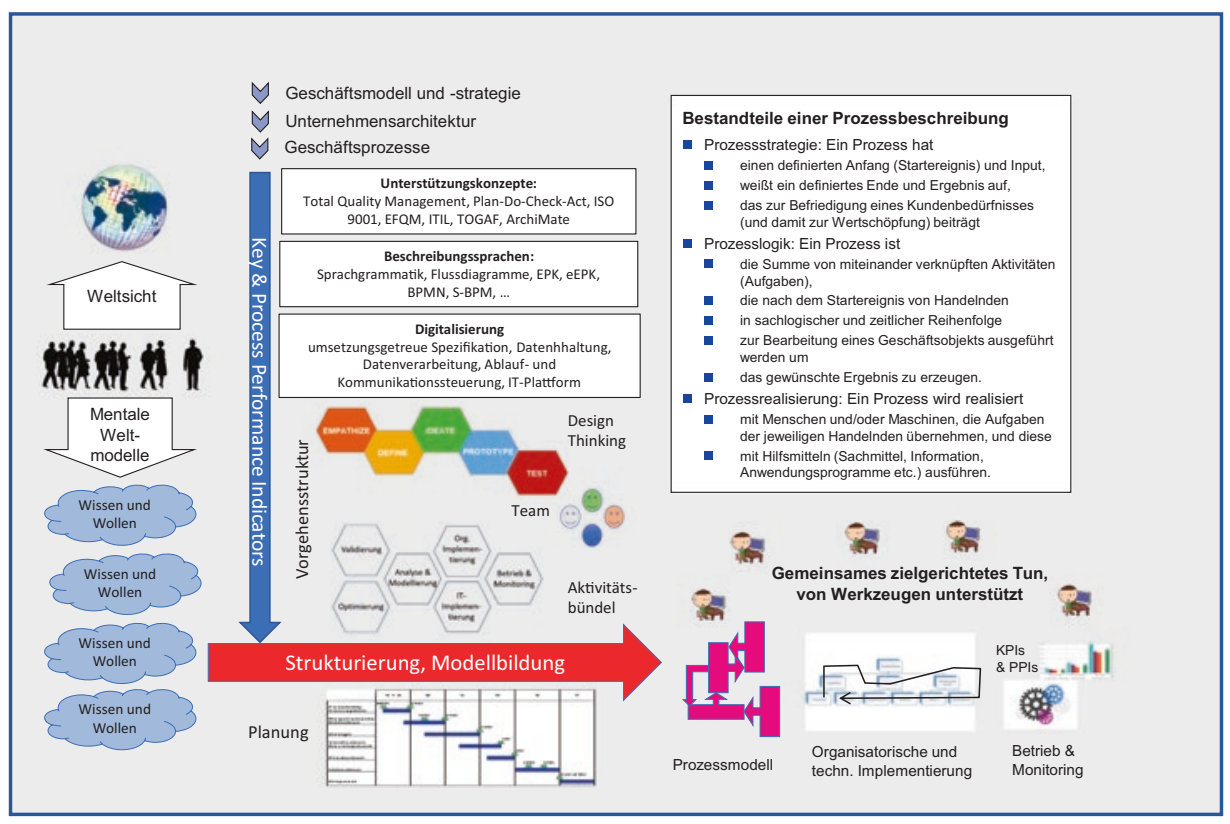

Abb. 1.9 Betrieb, Monitoring und Kennzahlen 
Gegenmaßnahmen. Eine Auswertung von Messwerten über längere Zeit zeigt dagegen mittel- bis langfristige Trends von Kennzahlen und kann entsprechende Änderungen auslösen. Auswertungsergebnisse werden u. a. in Prozesscockpits visualisiert.

\subsection{Kontinuierliche Verbesserung}

Prozesse sind nicht statisch, sondern unterliegen Veränderungen der in Abschn. 1.4 erläuterten internen und externen Rahmenbedingungen. Entwicklungen wie Geschäftsmodellmodifikationen, neue Wettbewerber, technischer Fortschritt oder Verschlechterungen bei gemessenen Prozesskennzahlen wie der Durchlaufzeit können Anpassungen eines Prozesses erfordern. Dazu sind passende Maßnahmen im Rahmen der in Abschn. 1.8 vorgestellten Aktivitätsbündel und Vorgehensweisen zu ergreifen.

Der Feedback-Pfeil in Abb. 1.10 deutet an, dass dazu die Beteiligten möglicherweise erneut divergierende Sichten des Realitätsausschnitts haben. Mit deren Harmonisierung in der beschriebenen Weise wird eine neue Instanz des Prozesses zur Erstellung von Prozessen gestartet.

Die kontinuierliche Verbesserung ist ein sehr wesentlicher Aspekt des Prozessmanagements. Durch laufende Anpassungen nähert man sich dem gewünschten Prozess immer mehr an. Allerdings können Veränderungen im Umfeld diese Annäherung beeinflussen. Der angestrebte Zustand ist also ein bewegliches Ziel (,moving target ${ }^{\text {‘). }}$

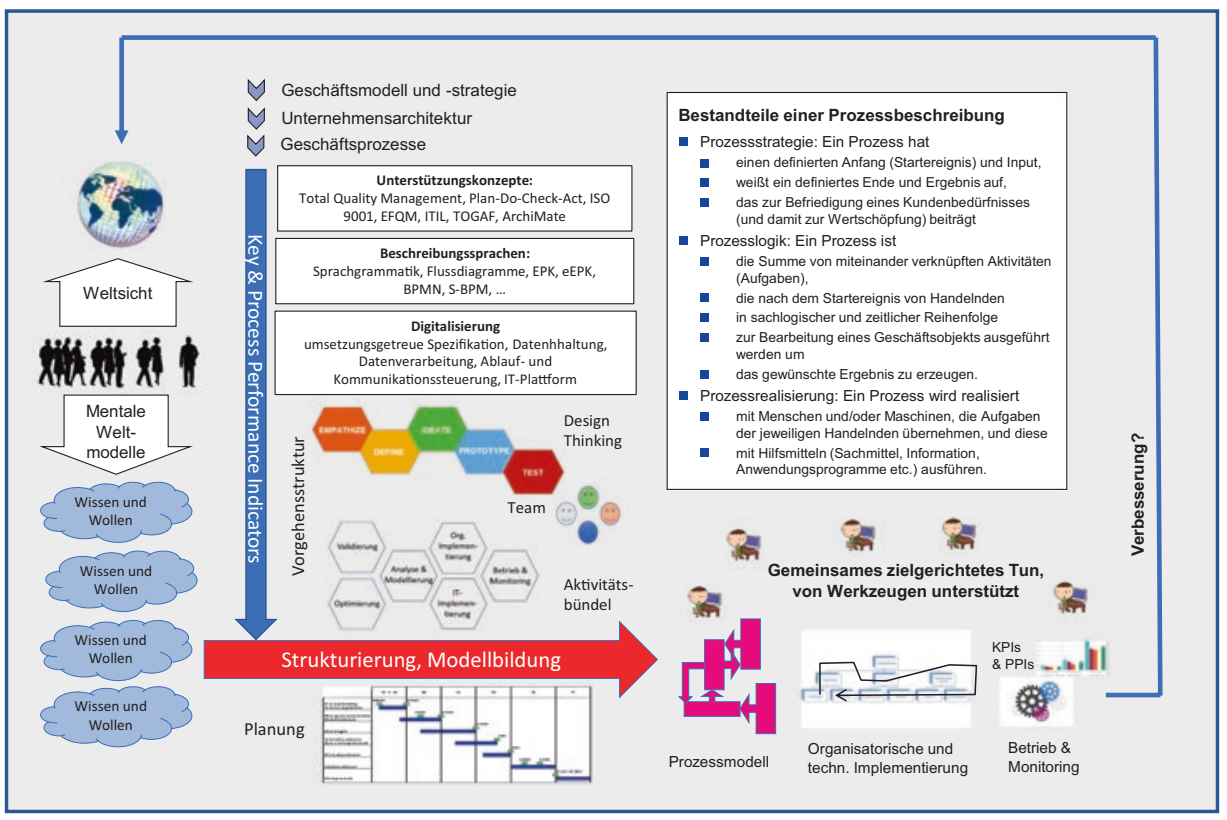

Abb. 1.10 Ergänzung um kontinuierliche Verbesserung 


\subsection{Unternehmensführung und Geschäftsprozessmanagement}

Die Unternehmensführung als Institution gestaltet das Unternehmen. Sie bestimmt maßgeblich das Geschäftsmodell, die Unternehmensstrategie und die Organisation. Geschäftsmodell und Strategie sollen zukünftige Erfolgspotenziale erschließen und damit die nachhaltige Existenz des Unternehmens sichern. Mit der Unternehmensarchitektur wird die Infrastruktur zur Ausschöpfung der Erfolgspotenziale geschaffen. Die Geschäftsprozesse und die in ihnen bearbeiteten Geschäftsobjekte (Daten) verknüpfen die fachliche mit der technischen Ebene der Unternehmensarchitektur.

Die Geschäftsprozesse sind Gegenstand der Digitalisierung, d. h. der informationstechnischen Unterstützung der Prozessausführung durch Menschen und Maschinen. In den letzten Jahren haben sich die diesbezüglichen Anforderungen deutlich vergrößert. So sollen in Geschäftsprozessen nicht nur Menschen und IT-Systeme, sondern auch „smarte“ Maschinen und Geräte interagieren können. Gemeint sind hochintegrierte Geschäftsprozesse im Kontext von Industrie 4.0 und Internet of Things, die sowohl menschliche Akteure, als auch einzelne Geräte und Maschinen zu einem gemeinsamen Ganzen integrieren. Die technischen Akteure werden dabei oftmals als „smart“ oder ,intelligent" bezeichnet.

Unternehmensführung als Prozess bezeichnet die Managementtätigkeiten bei der Schaffung und Ausschöpfung der Erfolgspotenziale. Im Kontext des Geschäftsprozessmanagements bedeutet sie das Management von soziotechnischen Systemen mit Menschen, die in Prozesse eingebunden sind, und mit Maschinen, die Menschen bei ihren Tätigkeiten unterstützen oder Folgen von Tätigkeiten autonom ausführen.

Trotz der zunehmenden Bedeutung der Digitalisierung steht der Mensch als Gestalter von soziotechnischen Systemen und Anwender der unterstützenden Technik im Mittelpunkt des Prozessmanagements. Nicht zuletzt aufgrund steigender Agilitätsanforderungen ist es heute Ziel, dass die Mitarbeiter, so weit wie möglich, autonom und selbstständig die operativen Prozesse gestalten (modellieren) können und diese danach ohne wesentliche Verzögerungen und zusätzlichen Aufwand direkt informationstechnisch unterstützt werden. Mit einem deutlichen Bekenntnis zur Prozessorientierung muss die Unternehmensleitung muss die Voraussetzungen dafür schaffen (,,Tone from the top“). Diese umfassen sowohl die notwendige Infrastruktur als auch ein Umfeld, das Menschen ermutigt, sich aktiv in die Prozessmanagementaktivitäten einzubringen.

Der Grad der Einbindung der Mitarbeiter ist geprägt vom Menschenbild und der damit verbundenen Führungsphilosophie der Unternehmensleitung (,Tone at the top“). Bei einem klassischen, eher hierarchisch geprägten Ansatz werden die Menschen und ihre Fähigkeiten als Ressource betrachtet, die Gegenstand der Handlungen von Führungskräften sind und schließlich Anweisungen ausführen. Eine derartige Managementphilosophie ist gekennzeichnet durch direkte Intervention der Unternehmensführung und folgt der Theorie X. Gemäß dieser Theorie wird etwaiger fehlender Motivation durch Androhung von Sanktionierung seitens der Unternehmensführung begegnet. 
Im mehr systemischen, d. h. ganzheitlichen Ansatz wie er dem St. Gallener Managementmodell zugrunde liegt, soll ein System geschaffen werden, das selbst und weitgehend selbstständig an der Gestaltung eines Geschäftsprozessmanagementsystems arbeitet. Alle Mitarbeiter sollen sich aktiv einbringen können. Dieser Managementstil folgt dem Menschenbild gemäß Theorie Y. Danach sind die wesentlichen Merkmale eines Menschen Freude an anspruchsvoller Arbeit, Selbstdisziplin, Verantwortung und Verstandeskraft.

Ergänzt wird dieses Menschenbild durch entsprechende Organisationstheorien. Diese haben den Zweck, das Entstehen, Bestehen und die Funktionsweise von Organisationen zu erklären. Organisationstheorien setzen implizit ein bestimmtes Menschenbild voraus. So geht der Taylorismus überwiegend von einem Menschenbild aus, das der Theorie X entspricht. Die systemische Organisationstheorie nach Luhmann macht hingegen keinerlei ethische Annahmen zu den Menschen in einer Organisation, sie nimmt nur an, dass diese miteinander kommunizieren. Der Schwerpunkt bei der Theorie des Kommunikativen Handelns liegt zwar auch auf der Kommunikation, aber durch Theorie und Vernunft soll die Welt verändert werden. Es wird davon ausgegangen, dass der Mensch von Natur aus einsichtig und offen für Argumente ist.

$\mathrm{Zu}$ den verschiedenen Menschenbildern gibt es passende Managementphilosophien und Organisationstheorien. Art und Einsatz von Methoden, Techniken und Werkzeuge müssen damit in Einklang stehen. Beispielsweise passt es nicht zusammen, die Einbindung von Mitarbeitern zu propagieren, wenn die Unternehmensführung dann deren Vorschläge nicht ernst nimmt oder gar nicht wahrnimmt. Bevor es an die Gestaltung von Prozessen geht, sollte sich ein Unternehmen deshalb bewusst machen, welches Menschenbild seine Führungs- und Unternehmenskultur prägt.

Wir denken, dass es insbesondere für die mit der Digitalisierung verbundenen Herausforderungen einer Ausrichtung zu Theorie Y bedarf, welche in der Praxis häufig zu Kulturwandel führen wird (müssen).

\section{Literatur}

1. Österle, H. und Winter, R. (2003), Business Engineering, in: Österle, H. und Winter, R. (Hrsg.), Business Engineering, 2. Auflage, Springer, Berlin. 
Open Access Dieses Kapitel wird unter der Creative Commons Namensnennung - Nicht kommerziell - Keine Bearbeitung 4.0 International Lizenz (http://creativecommons.org/licenses/ by-nc-nd/4.0/deed.de) veröffentlicht, welche die nicht-kommerzielle Nutzung, Vervielfältigung, Verbreitung und Wiedergabe in jeglichem Medium und Format erlaubt, sofern Sie den/die ursprünglichen Autor(en) und die Quelle ordnungsgemäß nennen, einen Link zur Creative Commons Lizenz beifügen und angeben, ob Änderungen vorgenommen wurden. Die Lizenz gibt Ihnen nicht das Recht, bearbeitete oder sonst wie umgestaltete Fassungen dieses Werkes zu verbreiten oder öffentlich wiederzugeben.

Die in diesem Kapitel enthaltenen Bilder und sonstiges Drittmaterial unterliegen ebenfalls der genannten Creative Commons Lizenz, sofern sich aus der Abbildungslegende nichts anderes ergibt. Sofern das betreffende Material nicht unter der genannten Creative Commons Lizenz steht und die betreffende Handlung nicht nach gesetzlichen Vorschriften erlaubt ist, ist auch für die oben aufgeführten nicht-kommerziellen Weiterverwendungen des Materials die Einwilligung des jeweiligen Rechteinhabers einzuholen. 
Im vorangegangenen Kapitel haben wir die vielfältigen Aspekte des Geschäftsprozessmanagements umrissen. Da in der Praxis häufig Modelle für die Beschreibung dieser Aspekte zum Einsatz kommen, gehen wir in den folgenden Abschnitten zunächst näher auf die Aufgaben und Eigenschaften von Modellen und Modellbildung ein. Danach stellen wir Beispiele von Modellen aus verschiedenen Fachgebieten vor, welche sich nach unserer Erfahrung in zahlreichen Geschäftsprozessmanagementprojekten als hilfreich erwiesen haben. Die Darstellung erhebt keinen Anspruch auf Vollständigkeit, kann jedoch dem Leser als Orientierung dienen, wenn er selbst Beschreibungsmodelle für sein individuelles Projekt auswählen muss.

\subsection{Modell und Wirklichkeit}

„Man muss die Welt nicht verstehen, man muss sich nur darin zurecht finden“. Im Internet wird dieser Satz Albert Einstein zugeschrieben. Wer versteht schon, was in der Welt alles geschieht? Wer weiß schon, wie sie funktioniert? Daher sollten wir uns um unsere Welt kümmern, um den Teil der Welt, der uns momentan wichtig ist. Wir sollten erkennen, dass wir uns unsere Welt täglich erschaffen bzw. konstruieren. Ein betrachteter Ausschnitt ist natürlich bestimmt von unseren subjektiven Interessen.

Wir entscheiden, welchen Ausschnitt der Welt wir betrachten wollen, und welche Aspekte uns darin wichtig erscheinen. Wir identifizieren dabei die für uns wesentlichen Artefakte und die für uns wesentlichen Beziehungen zwischen ihnen. Eine solche Abstraktion eines Ausschnitts der Wirklichkeit wird als Modell bezeichnet. Es kann auch sein, dass der betrachtete Ausschnitt der Wirklichkeit bereits ein Modell ist. Damit können Teile eines bereits existierenden Modells näher betrachtet werden. Dies wäre dann ein Modell eines Modells. Dieses Hochschrauben kann beliebig fortgesetzt werden. 


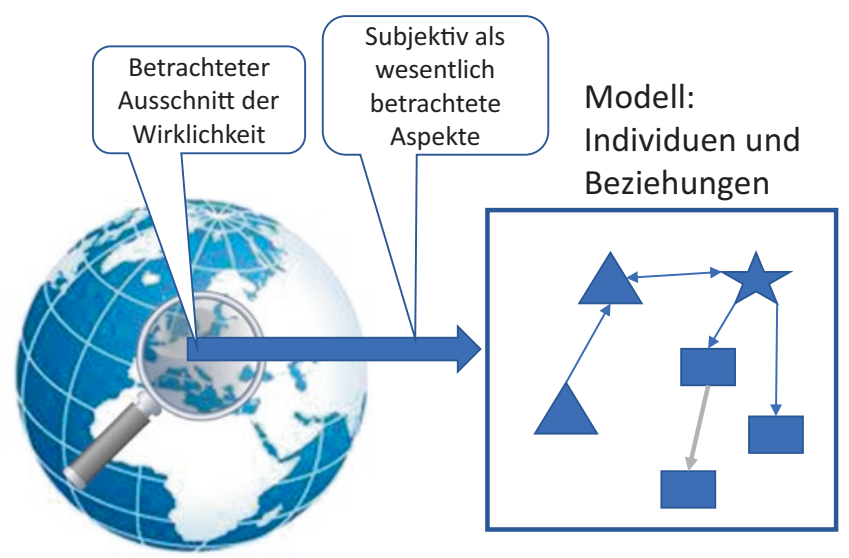

Abb. 2.1 Modellbildung

Jede Person hat eine eigene, subjektive Sicht auf die Welt oder einen Ausschnitt der Welt. Verschiedene Menschen betrachten den gleichen Ausschnitt der Wirklichkeit, kommen allerdings zu unterschiedlichen Modellen, da sie unterschiedliche Schwerpunkte setzen (vgl. Abb. 2.1).

Da Modelle nicht alle Aspekte der zugeordneten Wirklichkeit abdecken, gibt es durchaus Vorfälle in der Wirklichkeit, die durch ein Modell nicht abgedeckt sind und in ihm nicht nachvollziehbar sind. Diese nicht in einem Modell erklärbaren Phänomene bilden dessen Grenzen. Der Erschaffer eines Modells kann nun entscheiden, dass er dieses anpasst, weil er die bisher nicht abgedeckten Phänomene auch mit einbeziehen möchte, oder er legt fest, dass er diese nicht weiter betrachten möchte. Jedes Modell ist und bleibt eine Vereinfachung der Wirklichkeit, weshalb es immer Phänomene geben wird, die von einem Modell nicht erfasst werden.

Jeder Anwender muss entscheiden, ob das von ihm verwendete Modell für seine Versuche, sich in der Welt zurecht zu finden, ausreicht. Möchte man mit einem Modell alle Aspekte der Wirklichkeit abdecken, erreicht es die Komplexität der Wirklichkeit. Dann versteht man das Modell nicht mehr, so, wie man vorher die Wirklichkeit nicht verstanden hat. Der Sinn eines Modells, nämlich die Wirklichkeit besser begreifbar und handhabbar zu machen, wird nicht mehr erreicht.

Das Kreieren eines Modells und seine Verwendung sind subjektive Tätigkeiten, d. h. der Ersteller wählt die Eigenschaften der Wirklichkeitsabbildung nach seinen Vorstellungen aus. Allerdings ist es üblich, dass sich Gruppen von handelnden Personen, in weiterer Folge als Subjekte oder Akteure bezeichnet, auf ein Modell zur Betrachtung der Wirklichkeit einigen. Viele wissenschaftliche Schulen basieren auf solchen gemeinsamen Modellen der involvierten Forscher. 
Modellbildung ist eine wesentliche Tätigkeit in allen Wissenschaften, seien es Philosophie, Soziologie, Physik, Chemie, alle Ingenieurswissenschaften, Ökonomie usw. Die jeweiligen Modelle haben dabei verschiedene Aufgaben: Entweder bilden sie den betrachteten Ausschnitt ab, wie in den Naturwissenschaften üblich, oder sie dienen dazu, bestimmte notwendige Änderungen an dem betrachteten Ausschnitt der Wirklichkeit auszuprobieren (Simulationsmodell). Dies ist beispielsweise wichtig, um keine Menschenleben zu gefährden. Die Sicherheitsmodelle von Autos werden vor deren Serienproduktion in entsprechenden Tests auf ihre Eigenschaften hin überprüft. Dies geschieht nicht mit Menschen, sondern Modellen von Menschen, sogenannten Dummies. Dabei werden zwei Modelle miteinander kombiniert: Das Automodell, das die entsprechenden Sicherheitskonzepte umsetzt, und das Menschenmodell (Dummy), um die Verletzungsrisiken zu untersuchen.

Der in einem Modell erfasste Sachverhalt kann nun so lange angepasst werden, bis bei den mit dem Modell betrachteten Phänomenen das gewünschte Ergebnis erreicht wird. Beispielsweise werden dem Sicherheitsmodell eines Autos weitere Sicherheitskonzepte hinzugefügt, bis die gewünschte Reduktion des Verletzungsrisikos erreicht wird. Solche Modelle sind dann keine Abbilder der Wirklichkeit mehr, sondern stellen eine gewünschte Wirklichkeit dar. Die gewünschten Eigenschaften werden dann in die Wirklichkeit zurück transferiert, indem man beispielsweise die im Modell überprüften Sicherheitskonzepte in die Serienautos einbaut.

Ziel bei der Modellbildung ist immer, dass wir uns in der Welt zurechtfinden, oder gefahrlos ausprobieren können, wie eine entsprechende Änderung der Wirklichkeit sich auswirken würde. $\mathrm{Zu}$ verstehen, was die Welt im Innersten zusammenhält, wird uns wie es aussieht auf absehbare Zeit damit nicht gelingen. Das entsprechende Modell wäre dann die Welt, die es nicht gibt [1].

\subsection{Eigenschaften von Modellen}

Modelle dienen sowohl der abstrakten Darstellung der betrachteten Wirklichkeit im Sinne einer Erkenntnisfunktion als auch der Gestaltung der betrachteten Realität im Sinne eines Rückschlusses. Wie bereits angesprochen wird ein Modell so verändert, dass es einer gewünschten Wirklichkeit entspricht und dann als Bauplan für eine entsprechende Umgestaltung der Realität verwendet. In Naturwissenschaften wie der Physik haben Modelle überwiegend eine Erkenntnisfunktion, während sie in den Ingenieurwissenschaften und der Betriebswirtschaft die Gestaltung der Wirklichkeit unterstützen sollen [2].

Bei der Präzisierung des bisher verwendeten Modellbegriffs lehnen wir uns an Herbert Stachowiak an, der in seiner Modelltheorie die Merkmale und die daraus 
abgeleiteten Eigenschaften von Modellen genauer untersucht hat [3]. Demnach sind Modelle durch mindestens drei Merkmale gekennzeichnet [3]:

\section{Abbildung}

Ein Modell ist stets ein Modell von etwas. Es kann die Abbildung oder Repräsentation eines natürlichen oder künstlichen Originals sein, wobei dieses Original wiederum selbst ein Modell sein kann. Die Originale können auf natürlichem Wege entstanden, technisch produziert oder sonst wie einfach gegeben sein. Modelle können sehr unterschiedlich beschrieben bzw. repräsentiert werden:

- Mentale Modelle: Vorstellung im Kopf

- Verbale Modelle: Natürlich-sprachliche Beschreibung

- Grafische Modelle: Technische Zeichnungen oder sonstige Bilder

- Materielle Modelle: Modelle von Gebäuden

- Formale Modelle: Mathematische Modelle, Computerprogramme usw.

Ein Modell und das Original bilden eine Klasse von Attributen. Unter Attributen versteht man Merkmale und Eigenschaften von Individuen, Relationen zwischen Individuen, Eigenschaften von Eigenschaften, Eigenschaften von Relationen usw. Stachowiak überlässt dem modellierenden Subjekt, was ein Individuum ist. Er sieht Individuen als Attribute nullter Stufe. Mengen von Attributen können zu Klassen zusammengefasst werden und bilden dann Attribute der ersten Stufe. Diese Klassen können wieder zu einer nächsten Attributstufe zusammengefasst werden usw.

\section{Verkürzung}

Ein Modell erfasst im Allgemeinen nicht alle Attribute des betrachteten Originals, sondern nur diejenigen, die dem Modellschaffern bzw. Modellnutzern als relevant erscheinen.

Nicht alle Attribute des Originals werden von einem Modell erfasst. Bereits mit diesem Merkmal ist im weiteren Sinne eine pragmatische Betrachtungsdimension eingeführt worden. Im ,weiteren Sinne“ bedeutet hier, dass noch nicht spezifische pragmatisch-operationale Gesichtspunkte betrachtet werden, nach denen die Attributklassen, die in ein Modell eingehen sollen, selektiert werden. Diese erste Auswahl der Attribute ist intuitiv und willkürlich. Im engeren Sinn pragmatisch ist die Verkürzung erst dann, wenn die Absichten und operationalen Zielsetzungen des Modellerstellers bzw. Modellbenutzers die Auswahl der modellrelevanten Attribute beeinflussen. Diese Anpassungen an den beabsichtigten praktischen Gebrauch erfolgt erst im nächsten Schritt.

3. Pragmatismus

Nach der intuitiven Auswahl der Attribute wird überprüft, ob damit der beabsichtigte Zweck erreicht wird. Modelle sind ihren Originalen nicht eindeutig zugeordnet. Sie erfüllen eine Ersetzungsfunktion

- für bestimmte erkennende bzw. handelnde modellbenutzende Subjekte (für wen?). Modelle sind nicht nur Modelle von etwas, sie sind auch Modelle für jemanden. Dieser Jemand kann ein Mensch oder auch ein künstlicher Modellbenutzer wie 
z. B. ein Computerprogramm sein. Für einen Modellierer können Modelle dazu dienen, sich in der Welt zurecht zu finden, d. h. der Modellierer ist gleichzeitig auch Nutzer des Modells. Modellierer und Modellnutzer können aber auch zwei unterschiedliche Subjekte sein.

- innerhalb bestimmter Zeitintervalle (wann?)

Modelle erfüllen auch eine Funktion über die Zeit, d. h. ihre Nutzung ist auf einen bestimmten Zeitpunkt oder auf ein definiertes Zeitintervall bezogen. Innerhalb dieser Zeit können sich die betrachtete Wirklichkeit oder die Vorstellungen des Modellierers oder Modellanwenders derart verändert haben, dass zusätzliche Attribute in ein Modell einfließen sollen

- unter Einschränkung auf bestimmte gedankliche oder tatsächliche Operationen (wozu?).

Modelle werden zu einem bestimmten Zweck geschaffen, sei es, damit ein bestimmter Ausschnitt der Wirklichkeit besser verstanden werden kann, oder, um einen Bauplan für den Umbau der Wirklichkeit zu bekommen.

Bei der Erstellung eines Modells stecken Modellierende immer in einem gewissen Dilemma. Zum einen soll das Modell in ausreichendem Maße die gewünschten Aspekte der Wirklichkeit wiedergeben, wobei nicht klar definiert ist, was ausreichend ist; Zum anderen soll das Modell nicht zu komplex sein, damit es noch handhabbar bleibt. Dieser Zielkonflikt führt dazu, dass die meisten Modelle iterativ weiterentwickelt werden, bis sie wegen der steigenden Komplexität das Ende ihres Lebenszyklus erreichen, da sie nicht mehr handhabbar sind.

In den folgenden Abschnitten stellen wir Beispiele für Modelle vor, die Aspekte betrachten, welche explizit oder implizit in Modelle für Geschäftsprozesse einfließen. Wir haben die Beispiele gruppiert in Modelle aus den Sozialwissenschaften, der Betriebswirtschaftslehre, der Wirtschaftsinformatik und der Informatik. Die Einordnung in diese Gruppen ist teilweise nicht überschneidungsfrei, da insbesondere die Wirtschaftsinformatik als Querschnittsdisziplin Sachverhalte grundsätzlich aus mehreren Perspektiven betrachtet.

\subsection{Modelle der Sozialwissenschaften}

Geschäftsprozessmanagement hat mit Menschen und Maschinen zu tun. Es möchte deren Zusammenwirken unter Berücksichtigung von Zusatzanforderungen der technischen sowie der ökonomischen und ökologischen Machbarkeit organisieren. Insbesondere das Zusammenwirken und Zusammenleben der Menschen ist schon Jahrtausende lang Thema der Philosophie. Die Philosophie als die Lehre von den grundlegenden Bestimmungen und Strukturen des Lebens, der Welt und des Wissens versucht, die Welt und die menschliche Existenz zu ergründen, zu deuten und zu verstehen. Die ursprüngliche Bedeutung von Philosophie war die Lehre vom guten Leben. 
In diesem Sinn ist Philosophie der Versuch, das umfassende Modell unserer Welt zu kreieren was bisher noch nicht gelungen ist. Gesellschaftsphilosophen reduzieren den Blick und versuchen ein Modell der Gesellschaft als einen Ausschnitt der Wirklichkeit zu schaffen und damit deren Sinn und Wesen besser zu verstehen. Insbesondere beleuchten Gesellschaftsphilosophien das Verhältnis zwischen dem einzelnen Menschen und der Gemeinschaft sowie die Strukturen des Zusammenlebens. Sie gelten deshalb auch als Philosophievarianten, welche die Soziologie berühren. Sie sollen Soziologen dabei helfen, gesellschaftliche Vorgänge zu analysieren und Organisationsentwickler bei ihrer Arbeit unterstützen, und den ,normalen“ Menschen helfen, sich in der Welt zurecht zu finden. Es gibt zahlreiche Organisationstheorien, die in ihren Modellen auf unterschiedliche Aspekte fokussieren [4-7]. Die Frage, welche Organisationstheorie die Beste ist kann nicht beantwortet werden. Organisationstheorien repräsentieren Modelle und damit (nach Stachowiak) die zwar begründete, aber subjektive Sicht des Modellierers. Organisationstheorien setzen höchst unterschiedliche Schwerpunkte bei der Analyse von Organisationen und verfolgen unterschiedliche Zielsetzungen. Es gibt zu den jeweiligen Organisationstheorien empirische Untersuchungen, die Ergebnisse zugunsten oder Ungunsten einer Theorie liefern. Allerdings sind die verwendeten Untersuchungs- sind Analysemethoden umstritten [7].

Organisationstheorien liegen bestimmte Menschenbilder zugrunde, und die Ausgestaltung des Geschäftsprozessmanagements wird stark von dem in einer Organisation herrschenden Menschenbild geprägt. Deshalb stellen wir mit dem Taylorismus, der Habermas'schen Theorie des kommunikativen Handelns, und den sozialen Systemen von Luhmann drei Organisationstheorien mit ihren Menschenbildern in den folgenden Abschnitten vor ${ }^{1}$.

\subsubsection{Taylorismus und Fordismus}

Der Taylorismus führte das Experiment in die Managementlehre und -praxis ein, und zählt zu den Klassikern der Organisationslehre. Mit dem sogenannten Scientific Management erhielten Organisationen ein Instrument zu ihrer effizienten Gestaltung. Ein wesentliches Kennzeichen ist die Trennung zwischen planender Kopfarbeit und ausführender Handarbeit. Nach dem Tayloristischen Menschenbild sind Arbeiter dumm und faul und müssen deshalb strengen Regeln unterworfen werden. Diese Sicht beeinflusst auch heute noch oft implizit das Verhalten von Führungskräften.

Für Frederik Winslow Taylor waren die wichtigsten Ziele die Vervollkommnung der Produktionsmittel und Arbeitsverfahren, straffere Organisation und Zeitordnung des Arbeitsablaufes im Betrieb, sowie eine Neuordnung des Entlohnungssystems. Ein Kernelement des Taylorismus ist die Gestaltung von Arbeitsprozessen auf der Grundlage

${ }^{1}$ Ein Überblick über Organisationstheorien findet sich in [7]. 
von Zeit- und Bewegungsstudien. Eine Zerlegung des Produktionsprozesses in kleinste Arbeitsschritte, eine Entlastung der Arbeiter von geistigen Tätigkeiten, sowie eine Änderung des Lohnsystems sollen zu einer optimalen Nutzung der vorhandenen Leistungspotenziale führen.

Ziel ist die Steigerung der Produktivität menschlicher Arbeit. Dies geschieht durch die Teilung der Arbeit in kleinste Einheiten, zu deren Bewältigung keine, oder nur geringe Denkvorgänge zu leisten, und die aufgrund des geringen Umfangs bzw. Arbeitsinhalts schnell und repetitiv zu wiederholen sind.

Dem Taylorismus liegen folgende Kernprinzipien zugrunde:

- Arbeitsplanung

Die Planung der Arbeit wird von anderen Personen durchgeführt als deren Ausführung (Trennung von Hand- und Kopfarbeit). Damit wollte Taylor die Drückebergerei, die er den Arbeitern unterstellte, umgehen. Durch Zeit und Bewegungsstudien, die von den Kopfarbeitern ausgeführt wurden, sollte der geringste Bewegungs- und Zeitaufwand für einen Arbeitsschritt ermittelt werden.

- Leistungslohn

Aus diesen Zeit- und Bewegungsstudien ergab sich auch, was die Arbeiter in einer gewissen Zeit zu leisten hatten. Ein Bonus oder eine Prämie sorgten dafür, dass sich die als dumm und faul eingestuften Arbeiter auch tatsächlich bemühten, die vorgegebenen Leistungsdaten zu erreichen.

- Auswahl der am besten geeigneten Arbeiter

Durch eine entsprechende Auslese galt es einen erstklassigen Arbeiterstamm aufzubauen. Es wurden entsprechende Tests entwickelt und eingesetzt, um besonders fingerfertige und flinke Arbeiter zu identifizieren.

- Versöhnung zwischen Arbeitern und Management

Taylor glaubte, dass durch das von ihm entwickelte System die Produktivität so gesteigert werden kann, dass der Streit um die Verteilung des Gewinns zur Nebensache werden würde. Dadurch sollte der Konflikt zwischen Arbeitgebern und Arbeitnehmern aufgelöst werden.

Der Taylorismus betrachtet im Wesentlichen die Strukturierung der Arbeitsschritte, stellt jedoch nicht auf deren Reihenfolge ab. Diesen Aspekt ging Henry Ford an, der mit der Einführung des Fließbands die einzelnen Tätigkeiten koordinierte. Damit waren die Voraussetzungen für die Massenfertigung geschaffen, die das 20. Jahrhundert prägte. Das Fließbandprinzip wurde auch in die Verwaltung übertragen und beeinflusste stark das Geschäftsprozessmanagement. Flussdiagramme sind die Fließbandvorgaben für die Abwicklung von Verwaltungsaufgaben oder die „Produktion“ von Dienstleistungen. 


\subsubsection{Kommunikatives Handeln nach Habermas}

Im Gegensatz zum Taylorismus geht die Theorie des kommunikativen Handelns nach Habermas von einsichtigen Menschen aus, die durch die Kommunikation untereinander zu einem gemeinsamen vernünftigen Handeln kommen. Mit seinem Gesellschaftsmodell erklärt Habermas die Vorgänge in einer Gesellschaft, wie z. B. die Suche nach Wahrheit, nach Gerechtigkeit. Es ist also ein Modell, das alle angeht, denn die Themen Wahrheit und Gerechtigkeit betreffen sämtliche Mitglieder von Gesellschaften. Der zentrale Aspekt im Gesellschaftsmodell von Habermas ist das sogenannte kommunikative Handeln.

„Der Begriff des kommunikativen Handelns schließlich bezieht sich auf die Interaktion von mindestens zwei sprach- und handlungsfähigen Subjekten, die (sei es mit verbalen oder extraverbalen Mitteln) eine interpersonale Beziehung eingehen. Die Aktoren suchen eine Verständigung über die Handlungssituation, um ihre Handlungspläne und damit ihre Handlungen einvernehmlich zu koordinieren“ [8].

Nach Habermas gelingt es durch Kommunikation, dass der einzelne Mensch, der von sich aus nicht vernunftbegabt ist, diesen Mangel überwindet. Die Kommunikation zwischen Menschen wird zum intersubjektiven Handeln und zu einer möglichen Quelle der Vernunft. Kommunikatives Handeln heißt Handeln auf der Grundlage der Verständigung zwischen Menschen.

Habermas möchte damit Soziologen und Politikern ein Modell anbieten, das sie nutzen können, um die Gesellschaft zu analysieren bzw. zu gestalten. Dem einzelnen Menschen kann es dazu dienen, sich trotz der Komplexität heutiger Gesellschaften zurecht zu finden.

\subsubsection{Soziale Systeme nach Luhmann}

Ähnlich wie bei Habermas basiert das Gesellschaftsmodell von Luhmann auf Kommunikation. Die Unterschiede liegen darin, in wieweit Kommunikation und Handeln kombiniert sind. Luhmann lässt ausschließlich Kommunikation als konstituierenden Aspekt für Organisationen zu - Kommunikation findet nicht zwischen Menschen statt, sondern zwischen mindestens zwei informationsverarbeitenden Prozessoren. Damit sieht Luhmann die Kommunikation abstrakter. Nach ihm besteht die Gesellschaft nicht aus Menschen oder Teilen von Menschen. Sonst würde man etwas von der Gesellschaft abschneiden, wenn man etwas vom Menschen abschneidet. Der Körper eines Menschen (als biologisches System) mit einem Bewusstsein (psychisches System) ist zwar in vielen Fällen Voraussetzung für das Funktionieren eines sozialen Systems, also der Kommunikation, aber ein Mensch ist nicht das soziale System selbst. Luhmann macht in seiner Organisationstheorie keinerlei Aussagen zur Natur des Menschen, lässt also das Menschenbild offen. Menschen sind nur insofern Teil der Organisation, als sie miteinander kommunizieren.

Die Kommunikation zwischen den informationsverarbeitenden Prozessoren besteht aus den sogenannten Selektionen der Information, der Mitteilung und des Verstehens 


\begin{tabular}{|c|c|c|}
\hline & \multicolumn{2}{|c|}{$\begin{array}{l}\text { Zwei informationsverarbeitende Prozessoren } \\
\text { In der Regel Personen oder soziale Systeme }\end{array}$} \\
\hline & $\begin{array}{c}\text { Sender } \\
\text { Bei Luhmann: „Alter“ }\end{array}$ & $\begin{array}{c}\text { Empfänger } \\
\text { Bei Luhmann: „Ego“ }\end{array}$ \\
\hline Drei Selektionen: & $\begin{array}{l}\text { 1. Selektion der Information } \\
\text { 2. Selektion der Mitteilung }\end{array}$ & $\begin{array}{l}\text { 3. Selektion der Annahme/ des } \\
\text { Verstehens }\end{array}$ \\
\hline
\end{tabular}

Abb. 2.2 Luhmann'sches Kommunikationsverständnis

(siehe Abb. 2.2). Die ersten beiden Selektionen liegen beim Sender und die dritte beim Empfänger. Die Kommunikation als Stück mit mindestens zwei Akteuren in drei Akten ist eine unteilbare Einheit, nämlich die kleinste Einheit eines sozialen Systems und die elementare Operation der Gesellschaft [9]. Diese Sicht kann als Muster zur Definition der Kommunikation in Geschäftsprozessen dienen, vollkommen unabhängig von einem konkreten Menschenbild.

Sowohl Luhmann als auch Habermas stellen in ihrer Organisationstheorie also die Kommunikation in den Mittelpunkt der Betrachtung. Dass diese beiden bedeutenden Organisationstheoretiker den Kommunikationsaspekt der Organisation so stark betonen und ihre Theorien weitreichend akzeptiert sind, kann als Indiz gelten, Geschäftsprozesse primär kommunikationsorientiert zu betrachten.

\subsubsection{Organisationen}

Komplexe soziale Systeme können in kleinere soziale System gegliedert werden. Diese Struktur eines komplexen sozialen Systems wird als Organisationsstruktur bezeichnet. Nach welchen Kriterien die Aufteilung in kleinere soziale System erfolgt ist subjektiv und hängt von den jeweiligen Absichten ab. Entsprechend der Luhmann'schen Definition eines sozialen Systems kommunizieren die einzelnen sozialen Systeme innerhalb eines komplexeren sozialen Systems. Organisationsstrukturen sind also ein Modell eines komplexeren sozialen Systems.

In Abgrenzung zum weiten Verständnis des Begriffs Organisation bei Luhmann oder Habermas hat sich auch ein engeres Verständnis von Organisationen entwickelt. In der Betriebswirtschaft wird unter Organisation das formale Regelwerk eines arbeitsteiligen Systems verstanden. In der Organisationssoziologie bezeichnet sie eine besondere Form von sozialem Gebilde, die sich von anderen sozialen Gebilden wie beispielsweise Familien, Gruppen, Bewegungen oder Netzwerken unterscheiden lässt. Wesentliche Kennzeichen von Organisationen sind, dass Personen sich ihnen anschließen oder sie verlassen können.

Darüber hinaus besitzen sie einen Zweck auf den hin sie sich ausrichten. Organisationen haben Regelungen zur Arbeitsteilung wie Spezialisierung nach Verrichtung, 
Funktion, Objekten oder Raum bzw. entsprechende Mischformen. Diese Arbeitsteilung erfordert die Koordination der einzelnen Tätigkeiten. Als zentrales Instrument der Koordination gilt in der Organisationslehre die Hierarchie. Die hierarchische Koordination wird ergänzt durch Stäbe, Kommissionen, Arbeitskreise etc. Bei einmaligen oder erstmalig zu lösenden Problemen wird die Hierarchie durch eine Projektorganisation ergänzt.

\subsection{Modelle der Betriebswirtschaft}

Betriebswirtschaftslehre ist die Lehre von den wirtschaftlichen, organisatorischen, technischen sowie finanziellen Abläufen und Strukturen in Unternehmen. Damit ist auch das Geschäftsprozessmanagement ein Teil der Betriebswirtschaft. Denn Geschäftsprozesse dienen dazu die Wirtschaftlichkeit eines Unternehmens zu verbessern, und zwar mit all den zugehörigen Aspekten wie Kundenzufriedenheit, Mitarbeitermotivation, Einbindung von Partnern usw. Für die Strukturierung all dieser Aspekte und zur Analyse ihres Zusammenwirkens hat die Betriebswirtschaft Modelle entwickelt, die bei ihrer Anwendung in das Geschäftsprozessmanagement hineinwirken.

\subsubsection{Geschäftsmodell}

Ein Geschäftsmodell (Business Model) bezieht sich auf das Gesamtkonzept eines Unternehmens. Es repräsentiert modellhaft die Zusammenhänge, wie dieses für seine Kunden Mehrwert erzeugen und damit nachhaltig Erträge erzielen kann. Neben den angebotenen Produkten und Services geht es u. a. um die Struktur des Unternehmens, die Definition der Zielgruppen (Kunden) und deren Ansprache sowie um die Gestaltung der Geschäftsprozesse. Über dieses Verständnis hinaus gibt es für den Begriff des Geschäftsmodells eine Reihe weiterer Definitionen [10].

Ein Geschäftsmodell dient folglich dazu, den Zusammenhang zwischen dem Unternehmen als Handlungssystem und der Wertschöpfung zu verstehen. Es spiegelt wider, wie eine Firma funktioniert und welche Werte sie für bestimmte Zielgruppen erzeugt. Geschäftsmodelle werden im Rahmen einer Unternehmensgründung oder einer Neuausrichtung erstellt. Sie bestehen aus mehreren Teilmodellen, in denen beschrieben wird, welche Ressourcen (Materialien, Informationen usw.) einem Unternehmen als Eingangsgrößen zur Verfügung stehen (müssen), und wie diese Ressourcen bearbeitet und in vermarktungsfähige Produkte oder Dienstleistungen umgeformt werden, die dann zum Kunden transferiert werden, um entsprechende Einnahmen zu erzielen [11].

Geschäftsmodelle können mehreren Stakeholdern dienen. Die Unternehmensführung kann damit das eigene Geschäft besser verstehen, bestehende Stärken und Schwächen sowie Möglichkeiten zur Weiterentwicklung, Transformation und Verbesserung der Wettbewerbsposition erkennen. Für Investoren ist das Geschäftsmodell oft ein wichtiger Aspekt bei Anlageentscheidungen. 
Zur Erstellung von Geschäftsmodellen wurde eine Reihe von Instrumenten entwickelt. Das bekannteste ist der Business Model Canvas von Alexander Osterwalder [12], der in den letzten Jahren große Akzeptanz fand. Wie der Name sagt, geht der Business Model Canvas-Ansatz von einem Plakat aus, auf dem die betrachteten Aspekte des Geschäftsmodells visualisiert werden. Der Canvas liefert ein Raster für neun Geschäftsmodellaspekte, welches mit den konkreten Ausprägungen der Aspekte für das betrachtete Unternehmen gefüllt wird (vgl. Abb. 2.3). Im Zentrum steht das Werteversprechen (Produkt oder Dienstleistung).

Beim Ausfüllen gilt es jeweils eine Reihe von Fragestellungen zu den neun Aspekten zu beantworten. Die folgenden Ausführungen erläutern die Aspekte kurz und geben eine Auswahl wichtiger dazugehöriger Fragen wieder.

1. Kundensegmente, Zielgruppen:

Alle Personen oder Organisationen, für die das betrachtete Unternehmen Werte kreieren will.

$\mathrm{Zu}$ beantwortende Fragen sind $\mathrm{u}$. a.:

- Wer profitiert vom Produkt oder der Dienstleistung?

- Welche Kunden sind besonders wichtig?

2. Werteversprechen, Kundenutzen:

Für jedes Kundensegment gibt es ein eigenes Werteversprechen, den Kundennutzen. Dies ist eine auf die Bedürfnisse des jeweiligen Segments abgestimmte Kombination aus Produkt und Dienstleistung.

$\mathrm{Zu}$ beantwortende Fragen sind u. a.:

- Welchen Nutzen bzw. Wert hat das Angebot für die Kunden?

- Welche Kundenprobleme werden mit den angebotenen Produkten und/oder Dienstleistungen gelöst?

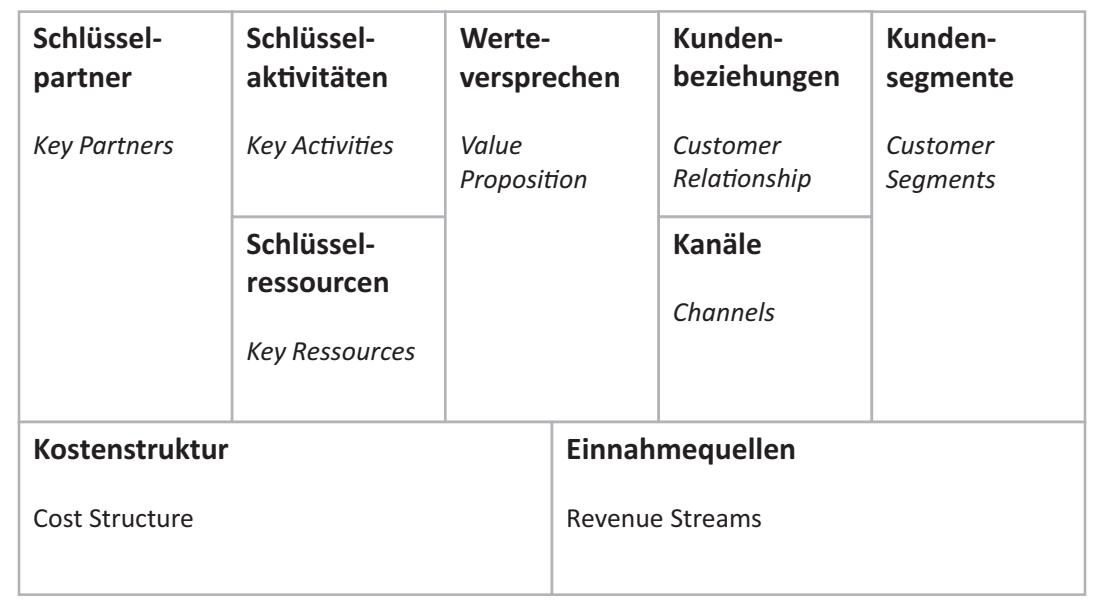

Abb. 2.3 Schema des Business Model Canvas 
3. Kanäle, Vertriebswege:

Dieser Faktor steht für die einzelnen Kanäle, über die mit den Kunden kommuniziert wird und ihnen die versprochenen Werte übermittelt werden. Die Vertriebskanäle bestimmen, wie die Interaktion mit den Kunden abläuft. Kommunikation, Distribution und Verkaufsstellen bilden Schnittstellen eines Unternehmens zu seinen Kunden. Die Wahrnehmung des Kunden an diesen Berührungspunkten ist dabei zentral und bestimmt den Eindruck, den ein Kunde von einem Unternehmen hat.

$\mathrm{Zu}$ beantwortende Fragen sind u. a.:

- Wie erfahren Kunden von den angebotenen Produkten und Dienstleistungen?

- Wie gelangen die Produkte/Dienstleistungen zum Kunden?

4. Kundenbeziehungen:

Hier wird beschrieben, welche Form des Umgangs mit den Kunden gepflegt wird.

Jedes Unternehmen sollte sich darüber Gedanken machen, welche Arten von Kundenbeziehungen es mit den verschiedenen Zielgruppen eingehen möchte. Dabei hängt die Gestaltung der Kundenbeziehungen nicht nur von der jeweiligen Zielgruppe ab, sondern auch von den damit verbundenen Zielen des Unternehmens (Neukundengewinnung, Bestandskundenpflege etc.).

$\mathrm{Zu}$ beantwortende Fragen sind u. a.:

- Welche Art von Beziehung erwarten die einzelnen Kundengruppen?

- Wie wird die Beziehung zu den Kunden organisiert?

- Was kostet die Pflege des Kundenkontakts und was bringt dieser Kunde?

5. Einnahmequellen, Erlösmodelle:

Das Unternehmen schafft mit seinem Angebot einen Mehrwert. Die zentrale Frage ist, wie viel der Kunde bereit ist, dafür zu bezahlen. Das Unternehmen trifft eine Entscheidung bezüglich der Preismodelle und der Preisstrategie (Einmalzahlung, Abonnement etc.).

$\mathrm{Zu}$ beantwortende Fragen sind u. a.:

- Wofür und wie viel sind Kunden wirklich bereit für das Angebot zu zahlen?

- Wie viel trägt jede der einzelnen Umsatzquellen zum Gesamtumsatz bei?

- Wie würden die Kunden gerne zahlen?

6. Schlüsselressourcen:

Zur Erstellung des Angebots sind in jeder Unternehmung bestimmte Ressourcen erforderlich. Diese können sich im eigenen Besitz befinden, aber auch gemietet oder von strategischen Partnern zur Verfügung gestellt werden.

$\mathrm{Zu}$ beantwortende Fragen sind $\mathrm{u}$. a.:

- Welche physischen Ressourcen (Räumlichkeiten, Produktionsmaschinen) werden benötigt, um ein Produkt oder einen Service erstellen und anbieten zu können?

- Welche intellektuellen Ressourcen (Wissen, Patente, Partnerschaften, Kundenstamm) werden benötigt?

- Welche personellen Ressourcen (Team) werden benötigt?

- Welche finanziellen Ressourcen (verfügbares Kapital, Sicherheiten) werden benötigt?

- Wie können die nötigen Ressourcen beschafft und vorgehalten werden? 
7. Schlüsselaktivitäten:

Schlüsselaktivitäten sind die für die Leistungserstellung und -verwertung nötigen Tätigkeiten, z. B. Produktion, Vertrieb.

$\mathrm{Zu}$ beantwortende Fragen sind u. a.:

- Welche Schlüsselaktivitäten müssen ausgeführt werden, um ein Produkt oder einen Service anbieten und damit den Kundennutzen realisieren zu können?

- Welche Aktivitäten für welche Vertriebskanäle?

- Welche Aktivitäten für welche Kundenbeziehungen?

8. Schlüsselpartner:

Schlüsselpartner sind Geschäftspartner, die wichtige Ressourcen für die Realisierung des Geschäftsmodells bereitstellen.

Mit ihnen gehen Unternehmen oft strategische Allianzen ein. Beispiele sind Lieferanten, Service Provider o. Ä.

$\mathrm{Zu}$ beantwortende Fragen sind $\mathrm{u}$. a.:

- Wer sind Schlüsselpartner und was tun diese für das Unternehmen?

- Welche Schlüsselressourcen werden von welchen Partnern zur Verfügung gestellt?

9. Kostenstruktur:

Die Kostenstruktur gibt Aufschluss über die wichtigsten Kostenfaktoren eines Geschäftsmodells.

$\mathrm{Zu}$ beantwortende Fragen sind $\mathrm{u}$. a.:

- Was sind die größten und wichtigsten Kostenfaktoren in dem Geschäftsmodell?

- Welche Schlüsselressourcen/Schlüsselaktivitäten sind die teuersten?

Aus den einzelnen Feldern eines Geschäftsmodells lassen sich Kennzahlen und die zugehörigen Zielwerte für Geschäftsprozesse ableiten. Umgekehrt können die Kennzahlen und Zielwerte den Entwurf der Prozesse beeinflussen. Liegt der Fokus auf niedrigen Preisen, werden Prozesse anders aussehen, als wenn das Geschäftsmodell eher auf hoher Qualität ausgerichtet ist.

\subsubsection{Balanced Scorecard}

Die Balanced Scorecard (BSC) wurde Anfang der 1990er-Jahre von Kaplan und Norton vorgestellt [13]. Sie ist ein Bindeglied zwischen dem Geschäftsmodell, der Strategiefindung und deren Umsetzung. Unter Strategie werden in der Wirtschaft klassisch die (meist langfristig) geplanten Verhaltensweisen der Unternehmen zur Erreichung ihrer Ziele verstanden.

Eine BSC beginnt bei der Vision und Strategie eines Unternehmens und definiert auf dieser Basis die kritischen Erfolgsfaktoren (KEF) mithilfe von Kennzahlen und dazugehörigen Zielwerten. Die Vision eines Unternehmens beschreibt das langfristige, ambitionierte Ziel, das eine Organisation bzw. Unternehmen anstrebt. Typische Visionen sind Formulierungen wie „Wir wollen Marktführer in unserem Marktsegment werden“ oder „Wir wollen das profitabelste Unternehmen in unserem Marktsegment werden“. 
Die Kennzahlen fördern die Zielsetzung und Leistungsfähigkeit in kritischen Bereichen der Strategie, um die Vision zu erreichen. Die BSC ist daher ein Management-System, das aus der Vision als Teil des Geschäftsmodells und der Strategie zur Umsetzung des Geschäftsmodells abgeleitet wird, und die wichtigsten Aspekte des Unternehmens widerspiegelt. Das BSC-Konzept unterstützt strategische Planung und Implementierung durch eine Bündelung der Maßnahmen aller Einheiten eines Unternehmens auf der Basis eines gemeinsamen Verständnisses seiner Ziele und durch einen leichteren Zugang zur Bewertung und Fortschreibung der Strategie.

Da traditionelles, rein auf finanzielle Kennzahlen aufgebautes Management den Anforderungen von Unternehmen im Informationszeitalter an effektive Planungswerkzeuge nicht mehr genügt, haben Kaplan und Norton für die BSC vier Perspektiven eingeführt, aus deren Blickwinkel die Aktivitäten eines Unternehmens umfassend bewertet werden können. Für jede Perspektive werden Ziele, Kennzahlen, Vorgaben und Maßnahmen definiert (vgl. Abb. 2.4).

\subsubsection{Total Quality Management und EFQM}

Der Begriff des Total Quality Management (TQM) steht für die Optimierung der Qualität von Produkten und Dienstleistungen eines Unternehmens in allen Funktionsbereichen und auf allen Ebenen durch Mitwirkung aller Mitarbeiter. Optimierung der Qualität bedeutet dabei, weder mit gegebenem Aufwand das höchste Qualitätsniveau zu erreichen, noch die Qualität ohne Rücksicht auf Kosten zu steigern. Vielmehr geht es

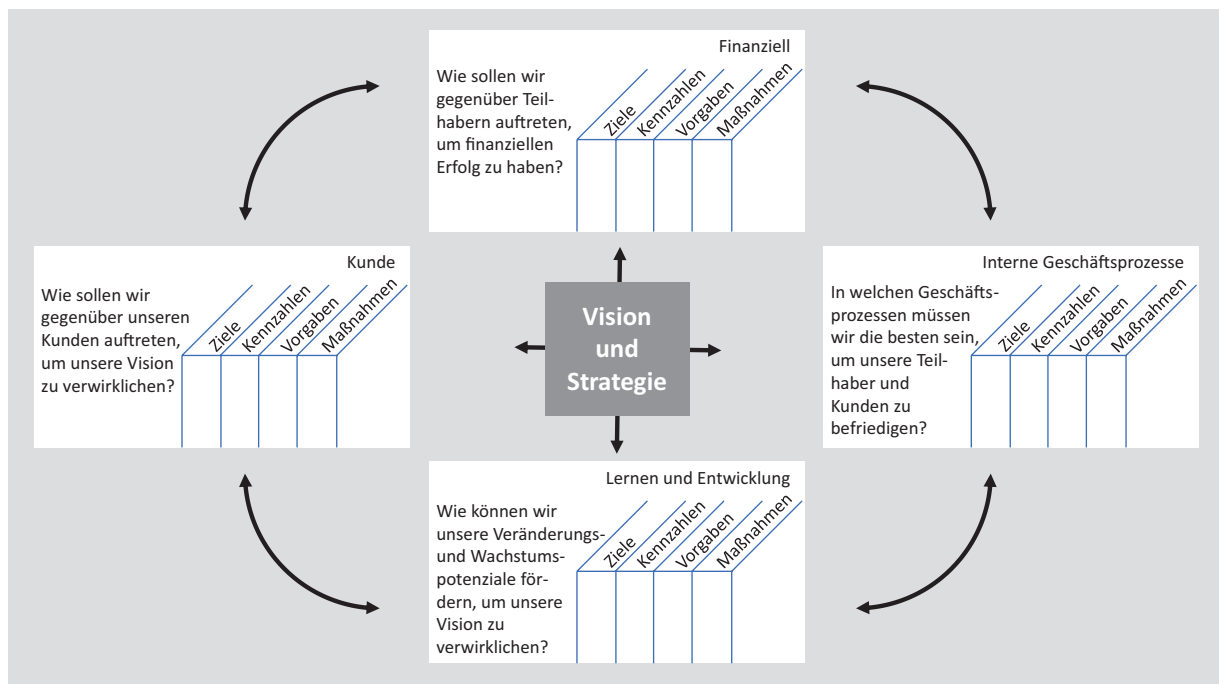

Abb. 2.4 Perspektiven der Balanced Scorecard [14] (Mit freundlicher Genehmigung von (C) Springer Fachmedien Wiesbaden GmbH 2010. All Rights Reserved) 
darum, auf die Interessen des Kunden zu fokussieren und Qualität an der Erfüllung seiner Anforderungen festzumachen.

Welche Anforderungen ein Unternehmen dabei an sich stellt, und durch welche Positionierung gegenüber dem Kunden es sich den größten und nachhaltigsten Geschäftserfolg verspricht, entscheidet die Führung eines Unternehmens selbst. Diese Positionierung ist nicht statisch. Erkenntnisse zu Kundenwünschen und zu den Vorgehensweisen zur Erfüllung dieser Wünsche erfordern eine permanente Anpassung des Unternehmens.

Um TQM zu etablieren bietet die European Foundation for Quality Management (EFQM) Organisationen Hilfestellung für den Aufbau und die kontinuierliche Weiterentwicklung eines umfassenden Managementsystems. Abb. 2.5 zeigt die Struktur des EFQM-Ansatzes. Diese Struktur dient zum einen als Werkzeug, um ein TQM aufzubauen und zum anderen dazu, durch ein umfassendes Bewertungssystem Verbesserungspotenziale zu ermitteln und den Geschäftserfolg zu steigern.

Als Befähiger gelten im EFQM-Modell die Methoden und Konzepte, die eingesetzt werden, um die Ergebnisse zu erreichen, die in der rechten Hälfte der Abbildung dargestellt sind. Die Prozentwerte in der Darstellung geben an, in wieweit die einzelnen Aspekte in die Gesamtbewertung eines Unternehmens einfließen.

Bei den Befähigern geht die EFQM davon aus, dass Prozesse den größten Einfluss auf die Ergebnisse auf der rechten Seite haben. Damit liefert das Modell gute Ansatzpunkte für die Identifikation von Kennzahlen und deren Zielwerte.

\section{Check, Act}

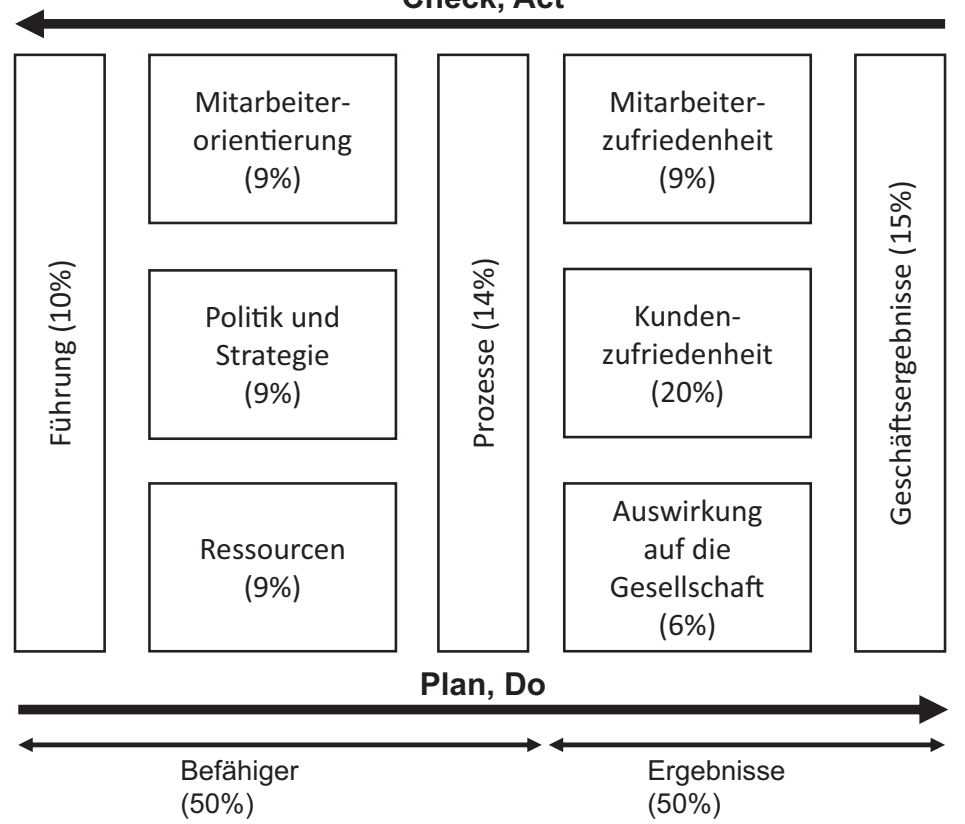

Abb. 2.5 EFQM-Struktur 
Neben der Möglichkeit ein Managementsystem aufzubauen bietet EFQM auch ein sehr ausgefeiltes Konzept, dessen Stand der Entwicklung zu bewerten. Durch einen umfangreichen Fragekatalog kann eine Rundumbewertung einer Organisation erfolgen. Die Bewertung kann durch Mitarbeiter der Organisation selbst oder durch externe Berater erfolgen. Die besten Organisationen in Europa erreichen bei einer solchen Bewertung rund 750 von maximal 1000 erzielbaren Punkten.

\subsubsection{EN ISO 9001}

Eine im Vergleich zu TQM abgeschwächte Form eines Qualitätsmanagements bildet der Standard EN ISO 9001. Er beschreibt Minimalanforderungen an ein Qualitätsmanagementsystem. Abb. 2.6 illustriert die Grundlagen des Standards.

Die Verantwortung der Leitung bedeutet, dass diese definiert, welche Kundenforderungen erfüllt werden und welche Qualitätspolitik dabei verfolgt wird. Die Umsetzung der Qualitätspolitik wird geplant und die entsprechenden Verantwortlichkeiten und Befugnisse in der Organisation dafür festgelegt. In der Verantwortung der Leitung liegt auch die Bewertung des QM-Systems in geplanten Abständen und insbesondere unter Berücksichtigung der Kundenrückmeldungen. Die Unternehmensführung muss außerdem die notwendigen Ressourcen wie Personal, Infrastruktur und eine adäquate Arbeitsumgebung bereitstellen.

Den Kern eines EN ISO 9001 konformen QM-Systems bilden die Prozesse zur Realisierung der Produkte und der zugehörigen kundenbezogenen Dienstleistungen. Aufgaben umfassen die Planung und Definition von geeigneten Prozessen für die Entwicklung und Herstellung von Produkten, die Beschaffung von Inputs usw. Die Hilfsmittel zur Überwachung der Produktherstellung und der Produktqualität müssen regelmäßig auf ihre Tauglichkeit überprüft werden. Die Ausführung der Prozesse muss durch Messungen

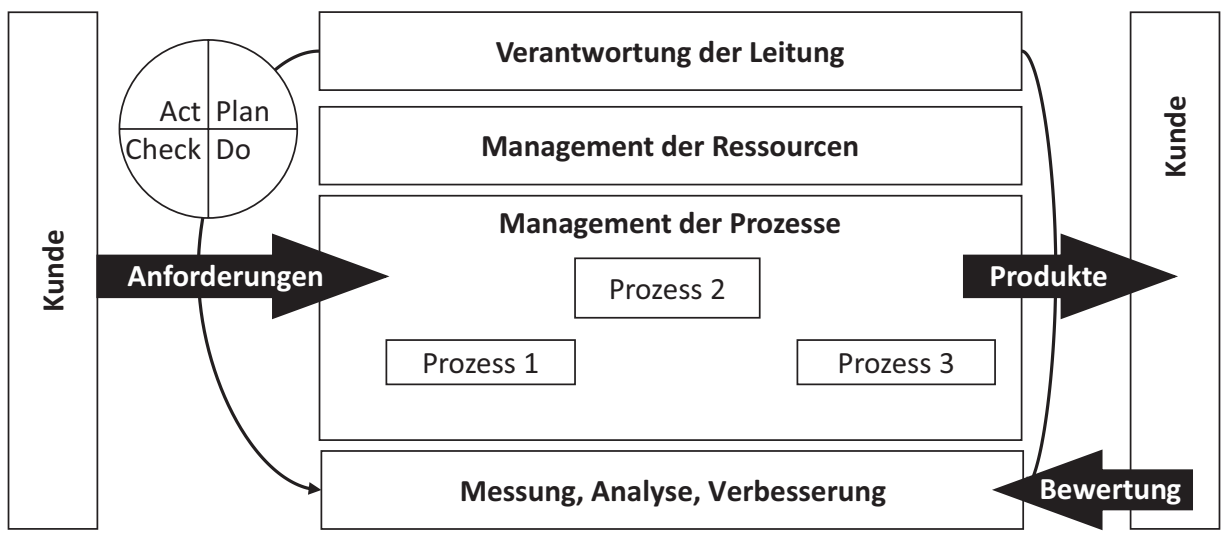

Abb. 2.6 EN ISO 9001 
und Analysen von Kennzahlen laufend überwacht werden, um bei Abweichungen entsprechende Verbesserungsmaßnahmen einleiten zu können.

EN ISO 9001 stellt also einen Rahmen für das Geschäftsprozessmanagement bereit. Die Ausführungen zeigen, dass es genau genommen keinen Unterschied zwischen Geschäftsprozess- und Qualitätsmanagement gibt. Ohne Geschäftsprozessmanagement gibt es kein Qualitätsmanagement und umgekehrt.

Die vergleichsweise niedrigeren Anforderungen von EN ISO 9001 äußern sich in der Tatsache, dass ein Unternehmen mit einem (nur) EN ISO 9001-konformen Qualitätsmanagementsystem etwa 300 Punkte bei einer EFQM Bewertung erreichen kann.

\subsubsection{Value Networks}

Das Konzept der Value Networks stammt von Verna Allee [15]. Unter einem Value Network versteht man Rollen und Personen, die untereinander sogenannte Tangibles und Intangibles austauschen. Tangible Wertflüsse sind materielle Wertflüsse zwischen Rollen und Personen und entsprechen dem Austausch von Waren, Dienstleistungen, Umsatzerlösen usw. Tangible Wertflüsse repräsentieren Transaktionen, die auf Verträgen basieren. Intangible Wertflüsse sind ein Zusatznutzen durch den Fluss von Wissen, sie sind nicht vertraglich fixiert oder kostenpflichtig. Intangible Wertflüsse sind z. B. strategische Informationen, Planungswissen sowie bestehende emotionale Komponenten wie gegenseitiges Vertrauen, gemeinsame Interessen, Wissensbedarf, Sicherheit usw.

Value Networks sollen Beteiligten und Organisationsentwicklern durch die Sichtbarmachung und gestalterische Handhabung wechselseitiger tangibler und intangibler Leistungsflüsse (Transaktionen) befähigen, soziale und fachliche Bezüge von Interaktion in organisationalen Systemen mit zu bestimmen bzw. aktiv zu gestalten. Abb. 2.7 zeigt

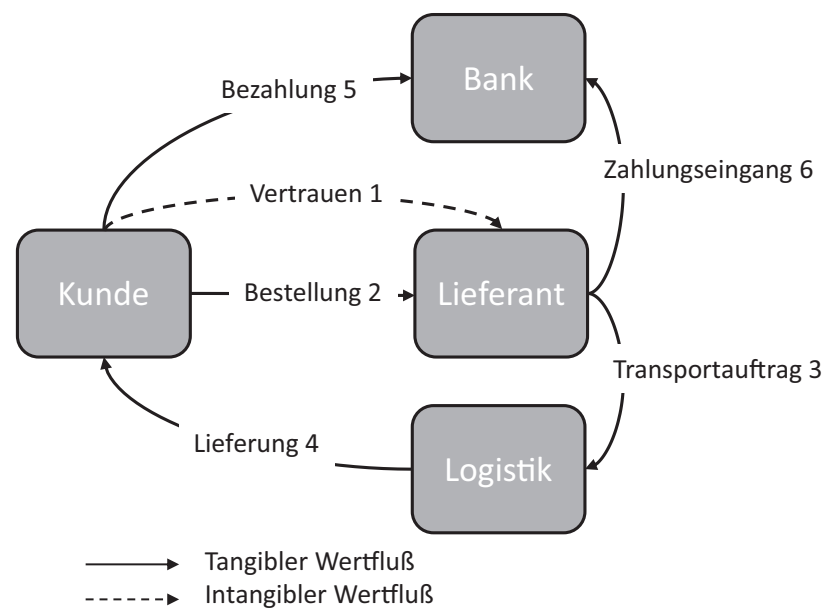

Abb. 2.7 Schema eines Value Network 
ein einfaches Value Network. Der Kunde gibt einen Wert Bestellung als einen tangiblen Wertfluss an den Lieferanten. Dieser tangible Wertfluss wird begleitet vom intangiblen Wertfluss Vertrauen. Der Kunde und die Logistikfirma sind mit dem tangiblen Wertfluss Lieferung verbunden usw. Die Nummern an den einzelnen Transaktionen drücken aus, in welcher Reigenfolge sie ausgeführt werden.

Organisationen erbringen Leistungen als Ergebnis ihrer Aktivitäten, die schließlich zur Wertschöpfung eines Unternehmens oder einer Institution beitragen. Um diese Leistungen zu erfassen und sichtbar zu machen, empfiehlt sich eine austauschorientierte Sicht auf Organisationen. Es ergibt sich dabei zumeist eine netzwerkartige Struktur, in der die Rollen innerhalb einer Organisation sowie deren Übertragungs- bzw. Kommunikationskanäle im Vordergrund stehen. Damit kann der Übergang zu einem kommunikationsorientierten Geschäftsprozessmanagement erfolgen.

\subsection{Modelle der Wirtschaftsinformatik}

Modelle der Wirtschaftsinformatik kombinieren Aspekte aus dem wirtschaftlichen und sozialen Bereich und der Informatik, um daraus Anforderungen für Informationssysteme abzuleiten. Die Modelle dienen überwiegend der Beschreibung von sozio-technischen Mensch-Maschine-Systemen. Die soziale Komponente deckt die Aspekte um die Mitarbeiter und Partner ab. Die technische Dimension betrifft die Sachverhalte der Informatik. Wichtig ist dabei, dass entsprechende Modelle die Wechselwirkung zwischen den beiden Domänen betrachten, insbesondere die Mensch-Maschine-Interaktion. Im Gegensatz zu rein technischen Systemen, die als deterministisch betrachtet werden, können sozio-technische Systeme aufgrund der Mitwirkung von sozialen Komponenten auch nicht-deterministisch, damit komplex, $\operatorname{sein}^{2}$.

Wir beschränken uns hier auf die Behandlung von Frameworks für Unternehmensarchitekturen und für IT-Management. Erstere entsprechen mit ihrer Verknüpfung der fachlichen und technischen Dimensionen von Organisationen genau dem Charakter der Wirtschaftsinformatik als Querschnittsdisziplin und sind von zentraler Bedeutung für das Prozessmanagement.

Frameworks für das IT-Management beziehen sich im Wesentlichen auf das IT-Service-Management und damit verbundene Aspekte wie Governance und Compliance. Die bekanntesten Vertreter sind die IT Infrastructure Library (ITIL ${ }^{\circledR}$ ) [17] und die Control Objectives for Information and related Technology (COBIT). Wegen seiner vergleichsweise weiten Verbreitung in der Praxis gehen wir näher auf ITIL $^{\circledR}$ ein und verweisen für weiterführende Informationen zu COBIT auf die einschlägige Website [18].

\footnotetext{
${ }^{2}$ Ausführliche Informationen zum Stand der Entwicklung und Forschung zum Thema Modellbildung in der Wirtschaftsinformatik finden sich beispielsweise in Franz Lehner; Modelle und Modellierung in der Wirtschaftsinformatik- Versuch einer Standortbestimmung [16].
} 


\subsubsection{Unternehmensarchitekturen}

Das Verständnis von Architektur im Kontext von Unternehmen deckt sich mit der ursprünglichen Bedeutung des Architekturbegriffs. Dieser bezeichnet in vielen Fachgebieten die grundlegende Organisation eines Systems mit seinen Komponenten und deren Beziehungen zueinander und zu ihrer Umgebung.

Konkret beschreibt und verknüpft eine Unternehmensarchitektur (Enterprise Architecture), wie bereits in Abschn. 1.4 angesprochen, die fachlichen und technischen Elemente des Unternehmens. Letztere umfassen insbesondere die IT-Landschaft. Sowohl die Gesamtarchitektur als auch ihre Teile werden mit Modellen beschrieben. Die Palette der dafür eingesetzten Modelltypen reicht vom Geschäftsmodell über Organigramme, Datenund Prozessmodelle auf der fachlichen Ebene bis hin zu Datenbankmodellen, Algorithmen und Programmen in der technischen Schicht.

Wie für Geschäftsmodelle gibt es auch für die Modellierung von Unternehmensarchitekturen zahlreiche Rahmenwerke, die den Verantwortlichen Orientierung geben und die Arbeit erleichtern sollen. Dirk Matthes [19] hat mehr als 40 Frameworks mit unterschiedlichem Detailierungsgrad, Schwerpunkt und Bekanntheitsgrad identifiziert.

Wir beschränken uns auf die Behandlung von vier einschlägigen Vertretern: Das Zachman-Framework und The Open Group Architecture Framework (TOGAF) mit seiner Ergänzung Architecture-Animate (ArchiMate) wurden in Umfragen wesentliche Rahmenwerke genannt [19]. Die Architektur Integrierter Informationssysteme (ARIS) ist im deutschsprachigen Raum in der Praxis weit verbreitet und hat signifikante Bedeutung im Rahmen des Prozessmanagements.

\subsubsection{Zachman-Framework}

Das von John A. Zachman 1992 in seiner erweiterten Form vorgestellte Framework stellt ähnlich dem Business Modell Canvas ein Strukturraster dar, das der Anwender mit den Fakten für sein Unternehmen anreichern muss. Es besteht aus einer Matrix mit verschiedenen Perspektiven in den Zeilen und Abstraktionen zu jeder Perspektive in den Spalten. Abb. 2.8 zeigt eine verdichtete Darstellung, ein detailliertes Bild findet sich auf der Website von Zachman International ${ }^{3}$.

Die Perspektiven in den Zeilen haben folgende Bedeutung:

- Planer: Zielsetzung des Unternehmens, externe Anforderungen und Einflüsse, Geschäftsmodell

- Besitzer: Anforderungen an Daten, Abläufe, Strukturen usw. zur Unternehmensführung

- Designer: Systementwurf und Systemstruktur zur Umsetzung der Anforderungen

\footnotetext{
${ }^{3}$ www.zachman.com.
} 


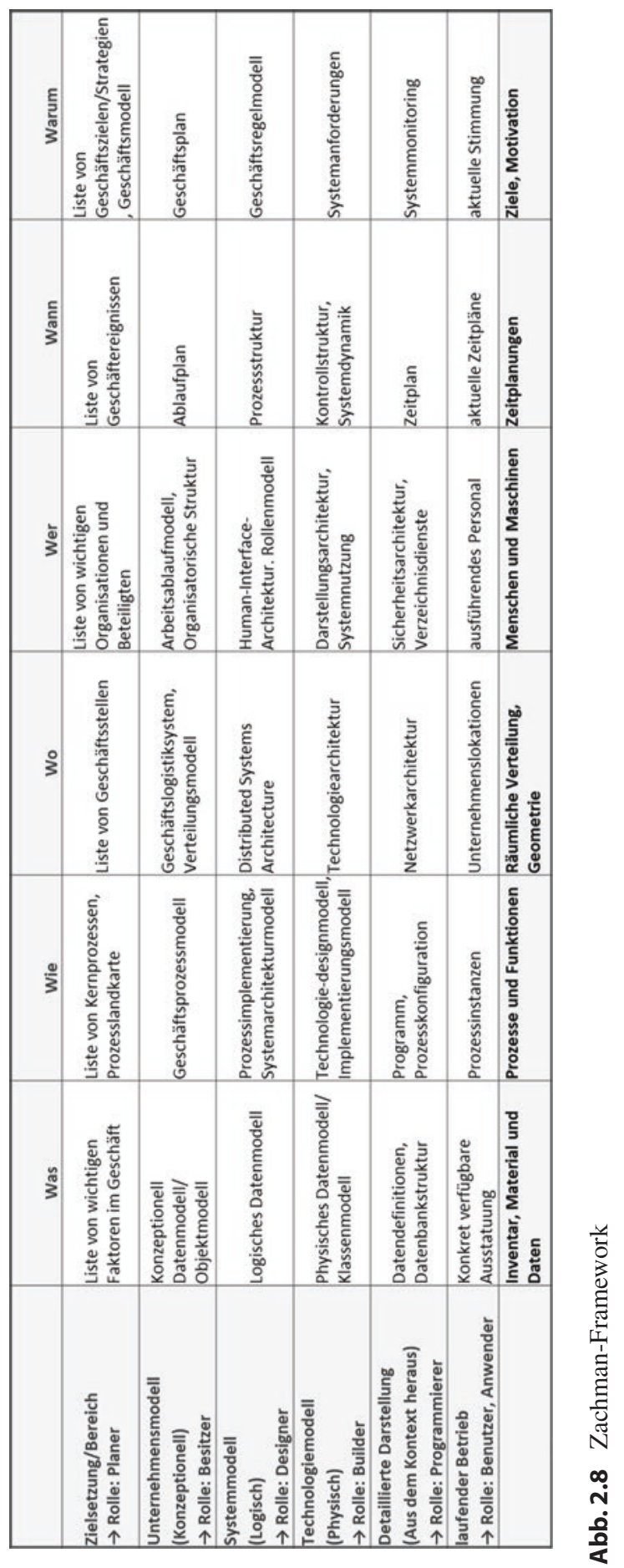


- Builder: Umsetzung des Systementwurfs

- Programmierer: Bereitstellen der technischen Infrastruktur

- Benutzer: Betriebsverantwortliche zur Sicherstellung der Funktionsfähigkeit

In den Spalten finden sich die Fragen, die das Unternehmen beantworten muss:

- Was (Inventar): Was für Objekte, Ausrüstungen, Daten, Informationen etc. werden benötigt?

- Wie (Funktionen und Prozesse): Wie arbeitet das Unternehmen, z. B. wie sehen die Geschäftsprozesse aus?

- Wo (Standorte, Netzwerk); Wo sind die Standorte des Unternehmens?

- Wer (Menschen): Wer sind die Menschen, die das Unternehmen am Laufen halten? Welche Geschäftseinheiten gibt es und wie sieht die Organisationsstruktur aus?

- Wann (Zeit): Wann werden Geschäftsprozesse instanziiert und ausgeführt? Was sind die Zeitpläne für das Geschäft?

- Warum (Motivation): Warum betreiben wir das Geschäft so wie wir es betreiben? Was sind die Treiber des Geschäfts? Hier fließen Aspekte des Geschäftsmodells ein.

Zachman sieht vor, dass für jede Zelle der Tabelle ein geeignetes Modell entwickelt wird. So gesehen handelt es sich bei seinem Framework um ein Modell für eine Menge von Modellen, die verschiedene Aspekte eines Unternehmens genauer betrachten.

Die Nutzer können bei den Reihen und Spalten durch andere Schwerpunktsetzung vom Original abweichen. Diese Flexibilität ist eine Stärke des Modellrahmens. Er enthält aber keine Vorgehensweise oder Methodik zur Definition einer konkreten Unternehmensarchitektur. Prozesse für deren Entwicklung oder Transformation müssen sich die Anwender anderweitig erschließen oder gänzlich selbst gestalten.

\subsubsection{The Open Group Architecture Framework (TOGAF)}

TOGAF ist das Rahmenwerk der Open Group zur Entwicklung von Unternehmensarchitekturen einschließlich der Geschäftsprozesse. Während das Zachman-Framework die zu betrachtenden Objekte betont und kaum Unterstützung für den Architekturentwicklungsprozess bietet, fokussiert TOGAF auf die Vorgehensweise zur Modellerstellung. Es stellt Methoden und Werkzeuge zur Verfügung, die bei Einführung, Erstellung, Gebrauch und Weiterentwicklung von Unternehmensarchitekturen helfen.

TOGAF unterscheidet bei der Beschreibung vier Teilarchitekturen:

- Business Architecture

Fachliche Aspekte der Unternehmensarchitektur

- Data Architecture

Logische und physische Strukturen der Daten und Ressourcen für deren Management. 
- Application Architecture

Verwendete Anwendungssysteme und deren Beziehungen untereinander sowie ihre Relevanz für das Geschäft des Unternehmens.

- Technology Architecture

Anforderungen an die Software und Hardware für das Management der Daten und die Ausführung der Anwendungssysteme. Dies schließt z. B. Laufzeitumgebungen, Netzwerke, Middleware und sonstige Betriebsinfrastrukturen ein.

Das TOGAF-Rahmenwerk setzt sich aus folgenden Komponenten zusammen:

- Architecture Development Method (ADM)

Methode und Vorgehensweise zur Entwicklung einer Unternehmensarchitektur

- ADM Guidelines and Techniques

Satz von Hilfsmitteln und Richtlinien, die die Anwendung des ADM unterstützen (z. B. Hilfsmittel für die iterative Verwendung des ADM)

- Architecture Content Framework

Strukturmodell um die Ergebnisse, die mit dem ADM erzeugt werden, einheitlich und konsistent zu definieren, strukturieren und darzustellen

- Enterprise Continuum

Modell zur Strukturierung eines möglichen Repository, das die jeweiligen Architekturen und die möglichen Lösungen wie Modelle, Muster, Architekturbeschreibungen etc. enthalten kann

- Reference Models

Grundmodelle, an denen sich spezifische Modelle für ein Unternehmen orientieren können. Dies sind das Technical Reference Model (TRM) und das Integrated Information Infrastructure Model

- Architecture Capability Framework

Verschiedenen Referenzmaterialien für die Entwicklung bestimmter ArchitekturmodelleDie Architecture Development Method (ADM) bildet als iteratives Prozessmodell den Kern von TOGAF (vgl. Abb. 2.9). Damit werden alle Architekturartefakte erzeugt. ADM kann auf mehreren Ebenen angewendet werden, sodass die Architekten unterschiedliche Detailierungsstufen der Unternehmensarchitektur definieren können. Mithilfe der anderen Komponenten werden die Ergebnisse dann beschrieben, strukturiert und abgelegt.

- Die Phasen der ADM sind:

Verschiedenen Referenzmaterialien für die Entwicklung bestimmter ArchitekturmodelleDie Architecture Development Method (ADM) bildet als iteratives Prozessmodell den Kern von TOGAF (vgl. Abb. 2.9). Damit werden alle Architekturartefakte erzeugt. ADM kann auf mehreren Ebenen angewendet werden, sodass die Architekten unterschiedliche Detailierungsstufen der Unternehmensarchitektur definieren können. Mithilfe der anderen Komponenten werden die Ergebnisse dann beschrieben, strukturiert und abgelegt. 


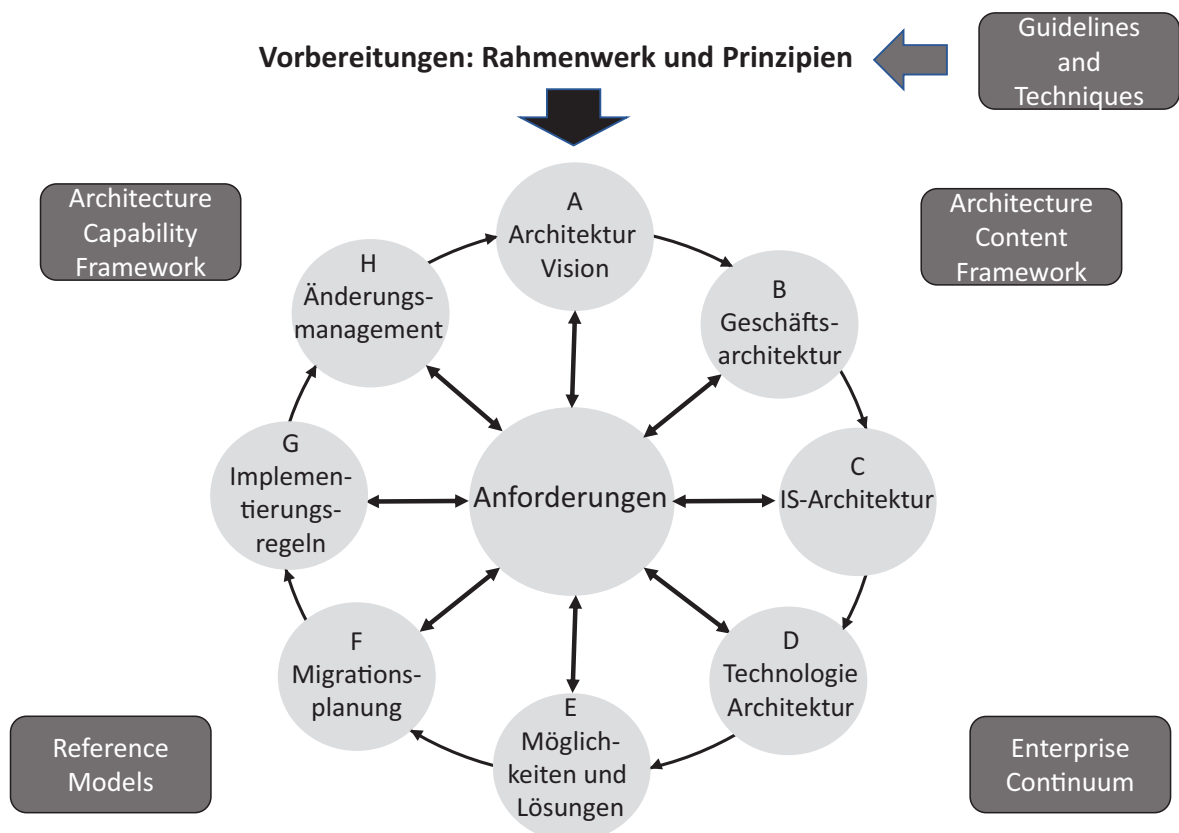

Abb. 2.9 Architecture Development Method von TOGAF

- Vorbereitungsphase (Preliminary Phase)

Hier werden das organisatorische Umfeld und die verwendeten Rahmenwerke, Methoden, Unterstützungswerkzeuge sowie wichtige Prinzipien festgelegt.

- Phase A Architekturvision (Architecture Vision)

Hier werden die Ziele und die Beteiligten bei der Aktualisierung der Unternehmensarchitektur festgelegt und eingebunden.

- Phase B Geschäftsarchitektur, Geschäftsmodell (Business Architecture)

Hier werden für die Geschäftsarchitektur der aktuelle und der gewünschte Zustand beschrieben. Die entscheidenden Unterschiede werden herausgearbeitet. Es werden die gewünschten Sichten festgelegt und die dazu geeigneten Werkzeuge ausgewählt.

- Phase C IS-Architektur (Information System Architecture)

Hier werden für die Anwendungs- und Informations-/Daten Architektur der aktuelle und der gewünschte Zustand beschrieben. Die entscheidenden Unterschiede werden herausgearbeitet. Dazu werden die konkreten Anwendungen und Datenmodelle verwendet.

- Phase D Technologiearchitektur (Technology Architecture)

Hier werden für die Technologiearchitektur der aktuelle und der gewünschte Zustand beschrieben. Die entscheidenden Unterschiede werden herausgearbeitet. Dazu werden die konkreten Hardwaresysteme beschrieben. 
- Phase E Möglichkeiten und Lösungen (Opportunities and Solutions)

Hier werden die Vorhaben festgelegt, welche die Transformation aus der Ist-Situation zum Sollzustand durchführen.

- Phase F Migrationsplanung (Migration Planning)

Hier wird die Überführung von einem Ist-Zustand in einen Sollzustand geplant.

- Phase G Implementierungsregeln (Implementation Governance)

Hier wird die Implementierung in den Sollzustand durchgeführt und überwacht.

- Phase H Änderungsmanagement (Architecture Change Management)

Hier werden Anforderungen und externe Einflüsse gesammelt, welche dann als Grundlage für den nächsten Durchlauf des ADM dienen.

- Anforderungen (Requirements Management)

Das Anforderungsmanagement treibt den ADM-Prozess kontinuierlich und steht deshalb im Zentrum.

\subsubsection{Architecture-Animate (ArchiMate)}

Architecture-Animate, kurz ArchiMate ${ }^{4}$, ist die Bezeichnung einer von der Open Group veröffentlichten offenen und unabhängigen Modellierungssprache für Unternehmensarchitekturen. Sie bietet Instrumente, die es Enterprise-Architekten ermöglichen, die Beziehungen zwischen Geschäftsbereichen und deren Entwicklung zu beschreiben, zu analysieren und zu visualisieren.

Die ArchiMate-Sprache ermöglicht die Beschreibung der Struktur und des Ablaufs von Geschäftsprozessen, von Organisationsstrukturen, Informationsflüssen, IT-Systemen und der technischen Infrastruktur. Die Beschreibungen helfen den Beteiligten, Veränderungen von Architekturelementen und deren Beziehungen zu konzipieren, die Folgen zu bewerten und zu kommunizieren. Abb. 2.10 zeigt das ArchiMate-Rahmenwerk.

Die ersten drei Spalten entsprechen den Grundkonzepten von ArchiMate:

- Passive Strukturelemente (Passive structure elements)

Passive Strukturelemente sind die Objekte, auf denen die Aktionen aus dem Verhalten (Verhaltenselemente) ausgeführt werden. Im Allgemeinen sind dies Informationsobjekte, aber auch physikalische Objekte können als passive Strukturelemente modelliert werden.

- Aktive Strukturelemente (Active structure elements)

Aktive Strukturelemente sind Elemente, die Handlungen ausführen können. Beispiele sind Menschen, Anwendungen, Rechnerknoten o. Ä. Die Handlungen können über Schnittstellen (Interfaces) angestoßen werden, die auch die Ergebnisse zur Verfügung stellen.

\footnotetext{
${ }^{4}$ http://www.opengroup.org/subjectareas/enterprise/archimate-overview.
} 


\begin{tabular}{|c|c|c|c|c|}
\hline & $\begin{array}{l}\text { Passive } \\
\text { structure }\end{array}$ & Behavior & Active Strucure & Motivation \\
\hline Strategy & ressourcen & & ressourcen & \multirow{6}{*}{$\begin{array}{l}\text { Stakeholders, } \\
\text { drivers, goals, } \\
\text { principles and } \\
\text { requirements }\end{array}$} \\
\hline Business & Business objects & $\begin{array}{l}\text { Business services, } \\
\text { functions and processes }\end{array}$ & Business actors and roles & \\
\hline Application & Data objects & $\begin{array}{l}\text { Application services, } \\
\text { functions and processes }\end{array}$ & $\begin{array}{l}\text { Application components and } \\
\text { interfaces }\end{array}$ & \\
\hline Technology & artifacts & $\begin{array}{l}\text { Technology services, } \\
\text { functions and processes }\end{array}$ & $\begin{array}{l}\text { Devices, system software, } \\
\text { communicatioon networks }\end{array}$ & \\
\hline Physical & material & & & \\
\hline $\begin{array}{l}\text { Implementation and } \\
\text { migration }\end{array}$ & deliverables & Work packages & platforms & \\
\hline
\end{tabular}

Abb. 2.10 ArchiMate Architectural Framework

- Verhaltenselemente (Behavior elements)

Verhaltenselemente repräsentieren die dynamischen Aspekte eines Unternehmens. Ein Service ist das von außen sichtbare Verhalten des Systems, das diesen Service erbringt. Die Services werden über die entsprechenden Schnittstellen verwendet. Schnittstellenereignisse stoßen die aktiven Strukturelemente an, welche dann die zugehörige Servicefunktion ausführen.

Diese drei Modellfragmente entsprechen den Grundelementen von natürlichen Sprachen: Subjekt, Prädikat bzw. Verb und Objekt. Sie werden auf insgesamt sechs Ebenen betrachtet:

- Strategieebene (Strategy Layer)

In der Motivation wird beschrieben, was ein Unternehmen erreichen möchte. In den Strategiekonzepten wird auf übergeordneter Ebene beschrieben wie ein Unternehmen seine Ziele erreichen möchte.

- Geschäftsebene (Business Layer)

Mit den Elementen des Business Layer können Produkte und Services beschrieben werden, die ein Unternehmen extern zur Verfügung stellt. Die Geschäftsebene zeigt, wie das Unternehmen diese Produkte und Services realisiert und soll bei der Analyse der Unternehmensstruktur helfen.

- Applikationsebene (Application Layer)

In der Anwendungsebene wird die Unterstützung der Geschäftsebene durch Anwendungen und Daten dargestellt.

- Technologieebene (Technology Layer)

Auf der Technologieebene wird die Infrastruktur beschrieben, welche benötigt wird, um Anwendungen zu realisieren. Dies sind im Wesentlichen die benötigten Hardwareund Softwarekomponenten. 
- Physikalische Ebene (Physical Layer)

In dieser Ebene wird auf das Zusammenwirken von IT und den physischen Komponenten wie Maschinen, Sensoren und Aktoren fokussiert.

- Implementierung und Migration (Implementation and Migration Layer)

Das Implementierungs- und Migrationskonzept beschreibt, wie eine definierte Architektur realisiert werden soll. Insbesondere werden darin die Arbeitspakete für die Umsetzung beschrieben.

Die Zellen der Tabelle enthalten also die Kernelemente für die aktiven und passiven Strukturen sowie für das Verhalten auf der jeweiligen Ebene.

Die Spalte Motivation beschreibt die Gründe für den Entwurf oder die Änderung eines Unternehmensmodells. Diese beeinflussen die Modellbildung und geben ihr die entsprechende Richtung.

ArchiMate wird als Ergänzung und Konkretisierung von TOGAF gesehen. TOGAF beschreibt den Prozess für die Definition und Beschreibung einer Unternehmensarchitektur (Unternehmensmodell), allerdings enthält es keine Beschreibungssprachen für die jeweiligen Teilmodelle. ArchiMate soll diese Lücke schließen. Es bietet neben dem gezeigten Rahmenwerk für die zu entwickelnde Architektur auch Sprachen, um die Aspekte in den einzelnen Ebenen auszudrücken. Abb. 2.11 zeigt wie die einzelnen Schritte in der Architecture Development Method von TOGAF mit den Schichten von ArchiMate zusammenhängen.

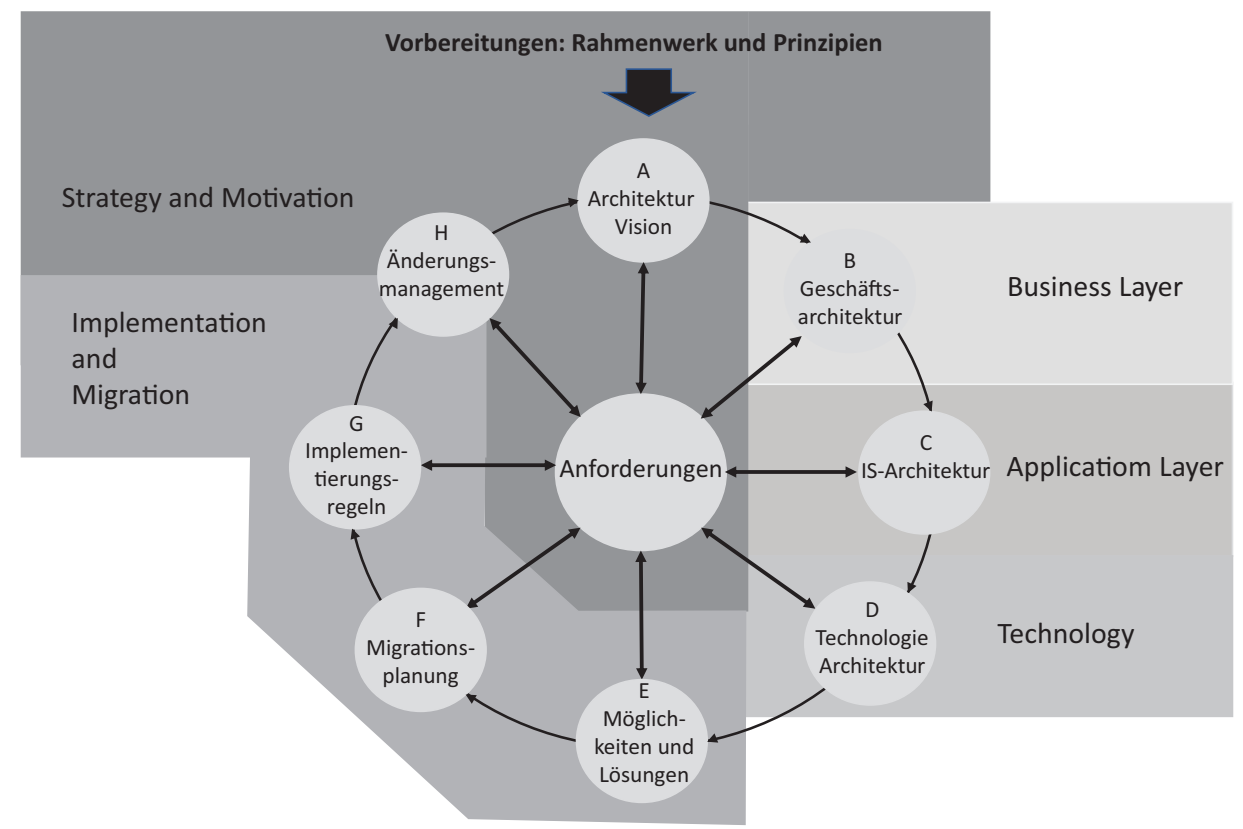

Abb. 2.11 Zusammenhang von ArchiMate-Ebenen und TOGAF ADM 


\subsubsection{Architektur Integrierter Informationssysteme (ARIS)}

Die Architektur integrierter Informationssysteme (ARIS) ist ein Rahmenwerk zur Definition von Unternehmensmodellen. Es umfasst die Daten-, Funktions-, Organisations-, Steuerungs- und Leistungssicht. Für jede Sicht sieht ARIS eine Reihe von Modelltypen für die Dokumentation vor.

- Datensicht:

Die Datensicht umfasst die betriebswirtschaftlich relevanten Geschäfts- oder Informationsobjekte und deren Beziehungen untereinander, das heißt alle Daten, die im Zusammenhang mit den Aktivitäten eines Unternehmens stehen. Informationsobjekte umfassen sowohl Zustände wie Artikel- oder Kundenstatus als auch Ereignisse wie „Kundenauftrag ist eingetroffen“ oder „Fertigungsauftrag wurde ausgelöst“. Einschlägiger Modelltyp ist das Entity Relationship Model (ERM).

- Funktionssicht:

Die Funktionssicht beschreibt die betriebswirtschaftlich relevanten Tätigkeiten (Funktionen, Aktivitäten) sowie ihre hierarchischen Beziehungen. Untergeordnete Funktionen sind Teilfunktionen der übergeordneten Funktion. Die Funktionen führen Operationen auf den in der Datensicht beschriebenen Objekten aus. Funktionen werden in der Praxis mit Funktionsbäumen modelliert.

- Organisationssicht:

In der Organisationssicht wird die Organisationsstruktur, das heißt die personellen Ressourcen, eines Unternehmens und deren typischerweise hierarchischen Beziehungen in einer Aufbauorganisation modelliert. Das Organigramm ist hierfür als Modelltyp üblich.

- Steuerungssicht:

Die Steuerungssicht stellt den zeitlich-sachlogischen Zusammenhang zwischen den einzelnen betrieblichen Tätigkeiten her. Sie führt die Daten-, Funktions- und Organisationssicht zusammen und nimmt damit eine zentrale, integrierende Rolle ein. Die Steuerungsschicht wird deshalb auch Prozesssicht genannt. Wesentlicher Modelltyp ist die Ereignisgesteuerte Prozesskette (EPK).

- Leistungssicht:

In der Leistungssicht werden die Eingaben und Ergebnisse des beschriebenen Geschäftsprozesses in der Regel mithilfe von Produktbäumen beschrieben.

Die Sichten, dafür typische Modelltypen und ihr Zusammenhang sind in Abb. 2.12 dargestellt. Das Bild macht auch deutlich, dass ARIS mit der integrierenden Steuerungssicht die Geschäftsprozesse, insbesondere mit der Abfolge der Aktivitäten, in den Mittelpunkt der Betrachtung stellt.

Orthogonal zu der durch die Sichten vorgenommenen Strukturierung differenziert ARIS in Anlehnung an das Software Engineering die Abstraktionsebenen Fachkonzept, DV-Konzept und Implementierung. Dies zeigt die Nähe der mit ARIS entwickelten Modelle zur Informationstechnik. Für die Lösung einer betrieblichen Problemstellung 


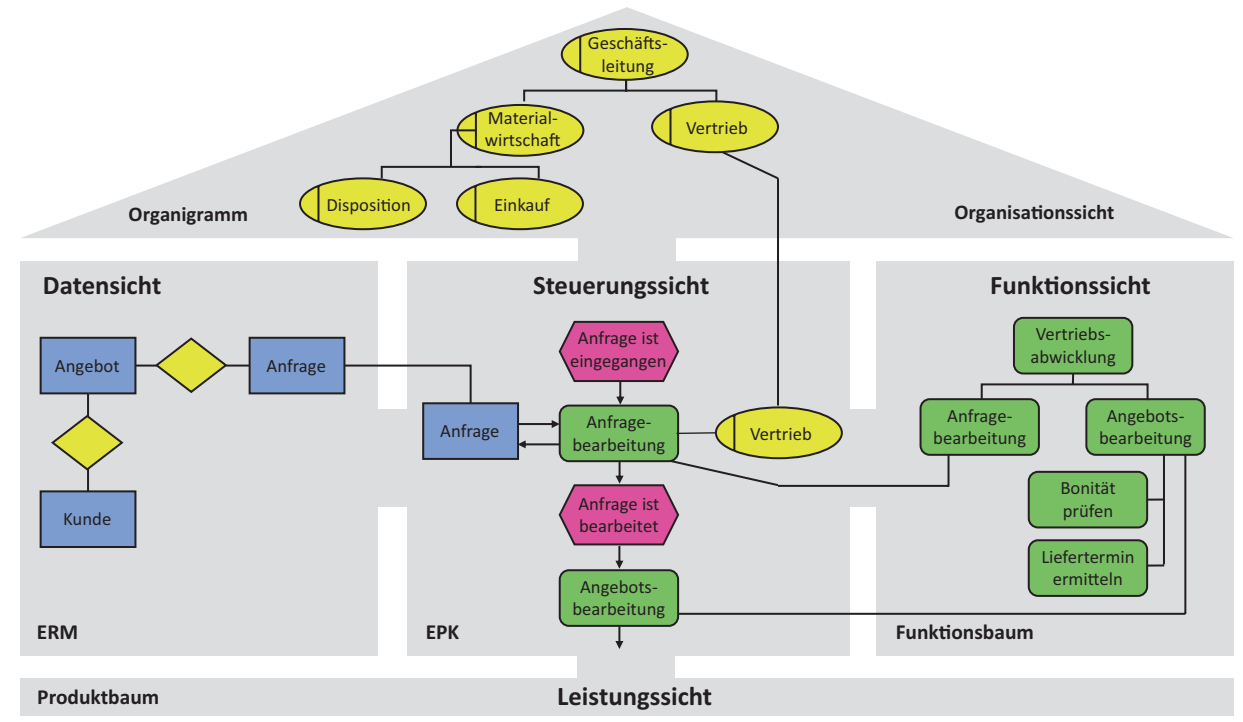

Abb. 2.12 Sichten und einschlägige Modelltypen in ARIS

wird ein fachliches Modell erstellt und in ein entsprechendes DV-Konzept (DV-Modell) überführt. Dieses dient schließlich als Basis für die konkrete technische Umsetzung.

- Fachkonzept:

Im Fachkonzept werden die Sachverhalte der betrieblichen Problemstellung beschrieben. Auf dieser Ebene werden Datenmodelle, Funktionsmodelle, Organigramme, Wertschöpfungsketten bzw. Ereignisgesteuerte Prozessketten (EPKs) und Produktmodelle eingesetzt.

- DV-Konzept:

Im DV-Konzept wird spezifiziert, wie das Fachkonzept DV-technisch umzusetzen ist. Auf dieser Ebene werden Datenbankmodelle (Datensicht), Struktogramme (Funktionssicht), Netztopologien (Organisationssicht) und Trigger-Mechanismen (Steuerungssicht) betrachtet. Der Zweck des DV-Konzepts ist eine Anpassung des Fachkonzepts an die Anforderungen der Informationstechnik.

- Implementierung:

Auf dieser Ebene wird das DV-Konzept in ein ausführbares Softwaresystem umgesetzt. Auf dieser Abstraktionsstufe werden Datenbeschreibungssprachen (Datensicht), Programme (Funktionssicht), Netzwerkprotokolle (Organisationssicht) und die Programmsteuerung (Steuerungssicht) betrachtet. 


\subsubsection{Framework für IT-Service-Management: ITIL}

Die IT Infrastructure Library $\left(\right.$ ITIL $\left.^{\circledR}\right)$ ist eine Sammlung vordefinierter Prozesse, Funktionen und Rollen, wie sie typischerweise in jeder IT-Infrastruktur mittlerer und großer Unternehmen vorkommen. Die praktische Zuweisung der Tätigkeiten erfolgt anhand von Rollen und Funktionen. Es handelt sich dabei um Good-Practice-Vorschläge, die an die Bedürfnisse des Unternehmens angepasst werden müssen. Inzwischen wurde die Sammlung ergänzt durch ISO 20.000:2005, ein ITIL $^{\circledR}$-basiertes Zertifizierungsmodell für Organisationen.

ITIL $^{\circledR}$ umfasst fünf Kernbände mit derzeit insgesamt 37 Kernprozessen. Abb. 2.13 zeigt die Struktur von ITIL $^{\circledR}$. Die fünf Kernbände orientieren sich am Service Live Cycle. Ausgehend von der Servicestrategie mit den Prozessen Strategiefindung, Finanzmanagement, Serviceportfoliosteuerung und Nachfragesteuerung wird über die Prozessgruppen der Serviceentwicklung und der Serviceinbetriebnahme schließlich die Dienstleistung mit den Prozessen der Gruppe Serviceerbringung erbracht. Die Prozeduren unterliegen der permanenten Verbesserung.

Weitere Bücher wie „Software Asset Management“, „Small-Scale Implementation“ oder „Building an ITIL ${ }^{\circledR}$-based Service Management Department“ ergänzen die Kernpublikationen. Damit bietet ITIL ${ }^{\circledR}$ umfassende Unterstützung bei der Entwicklung eines Prozesssystems für den IT-Bereich einer Organisation.
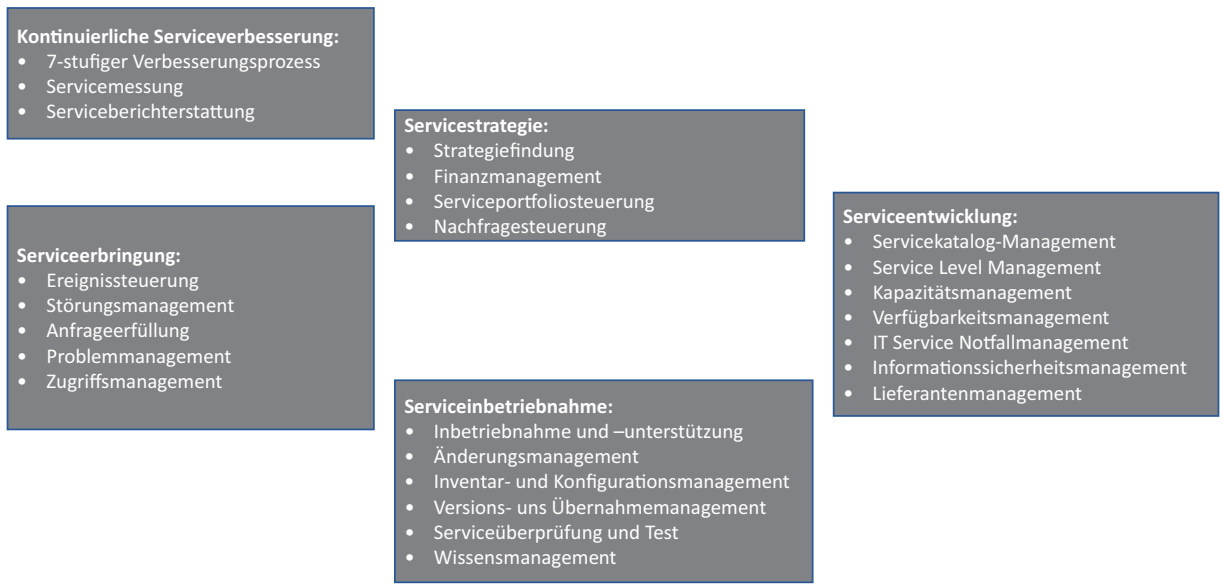

Abb. 2.13 ITIL ${ }^{\circledR}$-Prozessgruppen 


\subsection{Modelle der Informatik}

Modelle der Informatik beziehen sich auf Datenstrukturmodelle und Abläufe in Computersystemen sowie wesentliche Aspekte wie Sicherheit, Robustheit etc.

Diese Modelle dienen zum einen zur Abbildung eines betrachteten Realitätsausschnitts, um eine Aufgabe mithilfe der Informationsverarbeitung zu lösen. Sie beziehen sich auf ein abgegrenztes Problemfeld oder bestimmte Einsatzbereiche von Computersystemen. Hierunter fallen Modelle, die Daten und die darauf ablaufenden Operationen im Fokus haben, sowie Modelle, die mehr die Gesamtstruktur von komplexen Programmen, also deren Architektur, betrachten. Diese beiden Modellkategorien werden auch Modelle zum Programmieren im Kleinen bzw. Modelle zum Programmieren im Großen genannt.

Neben diesen zentralen Modellkategorien der Informatik gibt es weitere Modelle, die flankierende Aspekte betrachten, wie Zugriffsmodelle, Sicherheitsmodelle usw. Diese sind nicht Gegenstand der weiteren Ausführungen. Vielmehr erläutern wir in den folgenden Abschnitten einige für das Geschäftsprozessmanagement wesentliche Modellkonzepte.

\subsubsection{Information}

Information ist der zentralere Aspekt der Datenverarbeitung, die deshalb auch Informationstechnologie oder IT genannt wird. Information und Informationssysteme sind Modelle, die ein Objekt der realen Welt gemäß den Vorstellungen eines oder mehrerer Subjekte repräsentieren. Die Vorstellungen des Subjekts orientieren sich dabei am beabsichtigten Verwendungszweck. Beispielsweise werden Gegenstände in einem Lager durch ihre Eigenschaften beschrieben wie Teilenummer, Maße, Gewicht usw. Verschiedene Anwender verwenden unterschiedliche Ausschnitte aus dem Modell: Der Einkauf blickt auf Informationen wie Einkaufspreis und Bestellgrenze, während den Lagerarbeiter eher die Maße und der Lagerplatz interessieren.

Die Modellierung der Objektrealität kann deshalb als Interpretation durch die verwendenden Handelnden (Subjekte) wie Einkauf oder Lagerist verstanden werden. Information ist dann das Ergebnis und der Grund einer Interpretation; Sie kann aber auch selbst wiederum Objekt und damit Interpretations- und Modellierungsgegenstand sein. Dieses Verhältnis zwischen Subjekt, Information (Modell) und Original ist in Abb. 2.14 dargestellt:

Information erhält ihren Wert durch die Interpretation des Gesamtgeschehens durch die betrachtenden Subjekte. Diese Betrachtung läuft teils bewusst, größtenteils aber unbewusst ab. Die Informationsmenge wird dabei reduziert und dem jeweiligen Erkenntnisinteresse entsprechend gefiltert oder mit anderen Informationen verknüpft.

Daten unterscheiden sich von Informationen. Ein Datum (Einzahl von Daten) stellt zunächst nur eine Folge von Zeichen dar, deren Bedeutung nicht eindeutig ist. Die Zeichen können z. B. Zahlen, Buchstaben oder Symbole sein. In der Marketingabteilung 
eines Online-Shops findet sich beispielsweise die Zahlenfolge 0815. Diese Abfolge von Zeichen stellt zwar ein Datum dar, die Bedeutung ist allerdings nicht bekannt. Die Zeichenfolge selbst hat keine Bedeutung mit Ausnahme ihrer einzelnen Elemente.

Aus diesem Datum können aber Informationen entstehen, wenn bekannt ist, in welchem Kontext es steht. Durch eine Kombination mit anderen Daten wird eine Beziehung hergestellt, die interpretiert werden kann und Information entstehen lässt. Steht das Datum 0815 im Kontext „Kunde Max Muster, Artikel 0815“ kann die Marketingabteilung interpretieren, dass der Kunde Max Mustermann einen Artikel mit der Nummer 0815 bestellt hat.

Die Ergänzungen von Daten mit anderen Daten hängen vom Erkenntnisinteresse bzw. Nutzungsabsichten eines Subjekts ab. Ein Subjekt möchte mit den jeweils hergestellten Informationen meist einen Adressaten beeinflussen, z. B. zu einer Handlung bewegen. Information ist somit zu verstehen als „Aussagen, die den Erkenntnis- bzw. Wissensstand eines Subjektes (Informationssubjekt/-benutzer) über ein Objekt (Informationsgegenstand) in einer gegebenen Situation und Umwelt (Informationsumwelt) zur Erfüllung einer Aufgabe (Informationszweck) verbessern“ [20] (vgl. Abb. 2.14). Die Modellbildung ist demnach ein Teil des Informationsmanagements.

Informationen sind wichtig für Politiker, Führungskräfte in der Wirtschaft aber auch für jeden Bürger dieser Welt. Sie geben einen bestimmten Sachverhalt, der zu einem bestimmten Zeitpunkt galt, wieder und erlauben in der Regel eine Fortschreibung in die Zukunft. Sie dienen dazu politische, wirtschaftliche oder persönliche Entscheidungen zu treffen. Bei der Nutzung von Informationen stellt sich immer die Frage, wer sie erzeugt hat und welche Absichten derjenige damit verfolgt. Hier spielt folglich die Subjektivität von Modellen eine wesentliche Rolle.

\subsubsection{Entity-Relationship-Modell}

Um aus Daten Informationen zu generieren, müssen diese wie gezeigt mit anderen Daten kombiniert und die entsprechenden Beziehungen beschrieben werden. Dadurch entsteht ein Datenmodell. Die bekannteste Methode für die Beschreibung von Datenmodellen ist

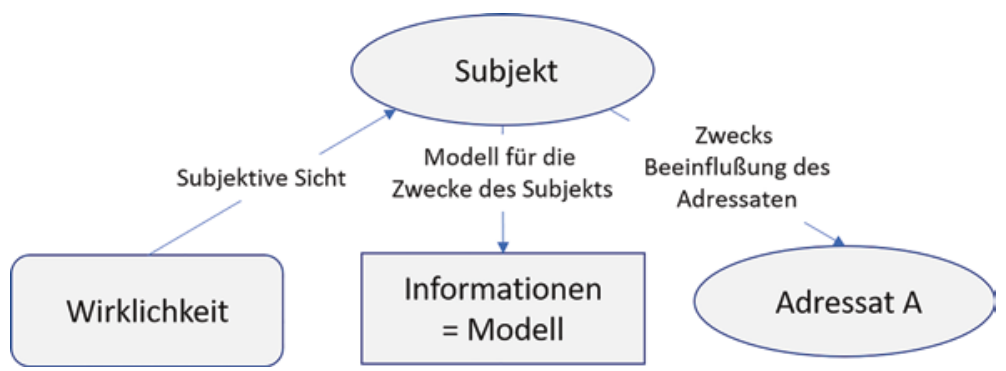

Abb. 2.14 Information ist „Modell-wovon-wozu-für wen“ [21] 


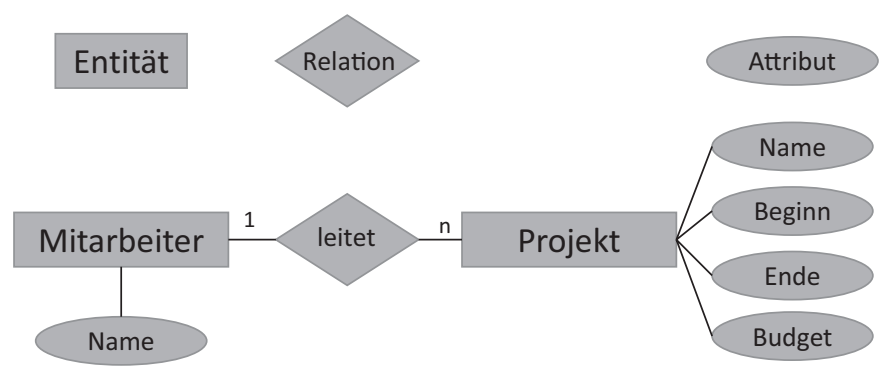

Abb. 2.15 Beispiel für ein Entity-Relationship-Diagramm

das Entity Relationship Model (ERM). Ein Entity-Relationship-Modell setzt sich aus drei Hauptelementen zusammen:

- Entitäten

Entitäten sind die Objektklassen, die in dem interessierenden Ausschnitt der realen Welt betrachtet werden.

- Beziehungen oder Relationen

Relationen beschreiben die Beziehungen zwischen Entitäten.

- Attribute

Attribute sind Eigenschaften innerhalb des Kontexts einer Entität.

Abb. 2.15 zeigt ein Beispiel für ein ERM. Aus ihm geht auch hervor, mit welchen Symbolen die Hauptelemente in der Regel ausgedrückt werden. Man findet jedoch auch ERM-Darstellungen mit Notationselementen der Unified Modeling Language (UML).

Das Diagramm beschreibt den folgenden Sachverhalt:

- Ein Mitarbeiter hat einen Namen. Ein Projekt hat einen Namen, einen Beginn, ein Ende und ein Budget. Die sogenannte Kardinalität drückt aus, dass ein Mitarbeiter mehrere Projekte leiten kann, aber ein Projekt nur von genau einem Mitarbeiter geleitet werden kann.

Im Modellierungskonzept der Klassifikation werden Objekte zu Objekttypen (entity sets), und Beziehungen zu Beziehungstypen (relationship sets) zusammengefasst. Diese Typen unterscheiden sich nach:

- Entitätstyp: Typisierung gleichartiger Entitäten (z. B. Mitarbeiter, Projekt)

- Beziehungstyp: Typisierung gleichartiger Beziehungen (z. B. Mitarbeiter leitet Projekt)

- Attributtyp: Typisierung gleichartiger Eigenschaften (z. B. Name für den Entitätstyp Mitarbeiter). Attribute oder Attributkombinationen, deren Wert(e) eine Entität eindeutig identifizieren, heißen identifizierende(s) Attribut(e) (z. B. identifiziert das Attribut Projektname den Entitätstyp Projekt) 
Terminierungselement

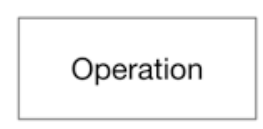

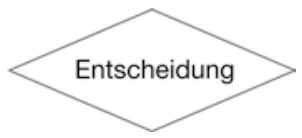

Abb. 2.16 Häufige Symbole in Flussdiagrammen

Zur Beschreibung besonderer Sachverhalte wie z. B. der angesprochenen Kardinalität umfassen ERMs noch weitere Konstrukte ${ }^{5}$.

\subsubsection{Fluss- oder Ablaufdiagramme}

Fluss- oder Ablaufdiagramme veranschaulichen eine Ausführungsreihenfolge von Tätigkeiten bzw. Aktionen und werden in zahlreichen Anwendungsbereichen verwendet. Sie können beschreiben, in welcher Reihenfolge Handlungen von Menschen oder anderen Akteuren ausgeführt werden sollen. Algorithmen oder Computerprogramme werden oft in Form von Flussdiagrammen (z. B. Programmablaufplänen) dokumentiert. Wegen der verbreiteten Anwendung von Flussdiagrammen haben sich zahlreiche Varianten herausgebildet, die spezielle Sachverhalte des jeweiligen Anwendungsbereichs berücksichtigen. Für die Datenverarbeitung wurde die Symbolik für Flussdiagramme in den Standards DIN 66.001:1983-12 bzw. ISO 5807:1985 festgelegt. Abb. 2.16 und 2.17 enthalten eine Auswahl häufig verwendeter Symbole und ein Beispiel für ein Flussdiagramm.

Bei der Erläuterung von ARIS in Abschn. 2.5.1.4 haben wir Ereignisgesteuerte Prozessketten (EPK) als Modelltyp für die Steuerungssicht angesprochen. Bei diesen EPKs handelt es sich um Flussdiagramme aus Sequenzen von Ereignisknoten, Funktionsknoten (Operationen) und Konnektoren. Pfeile als Kanten repräsentieren Kontrollflüsse zwischen den Symbolen. Funktionen und Ereignisse (mit Ausnahme von Start- und Endereignis) besitzen genau je eine eingehende und eine ausgehende Kante. Sollen Funktionen mehrere Ereignisse erzeugen oder mehrere Ereignisse eine Funktion auslösen, müssen Konnektoren wie ein exklusives Oder (XOR) zwischengeschaltet werden. Der Modellierer kann auch ausdrücken, wer eine Funktion mit welcher IT-Unterstützung ausführt und welche Daten dabei manipuliert werden. Dazu ordnet er den Funktionsknoten Symbole für Organisationseinheiten (z. B. Abteilungen, Stellen, Rollen), Informationsobjekte (Daten) oder Anwendungssysteme zu. Diese Elemente müssen in den entsprechenden Modelltypen spezifiziert sein. Man spricht dann von erweiterten Ereignisgesteuerten Prozessketten (eEPK).

${ }^{5}$ Details dazu finden sich in einschlägiger Literatur, z. B. bei [22]. 
Abb. 2.17 Flussdiagramm für Kontotransaktion

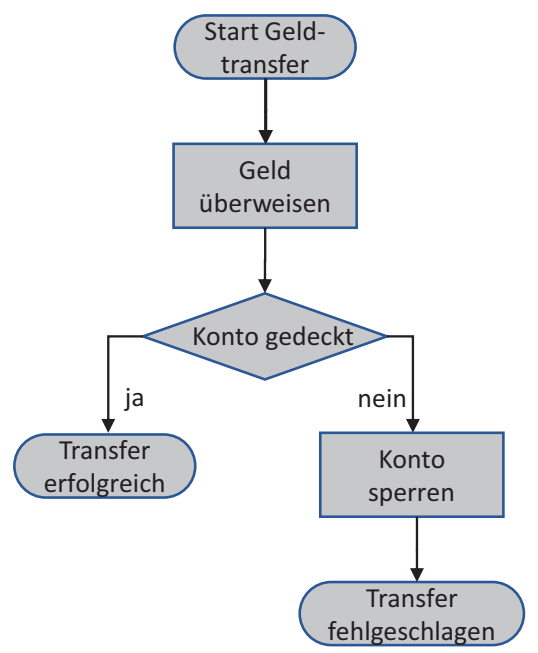

Den Zusammenhang, den eine eEPK als Instrument der Steuerungs- oder Prozesssicht mit den Elementen der anderen Sichten und Modelltypen herstellt, haben wir bereits in Abschn. 2.5.1.4 kurz angedeutet. Konkret äußert er sich folgendermaßen:

- Steuerungssicht

- Ein Ereignis ist ein Zustand, der vor oder nach einer Funktion auftritt. Das Symbol für ein Ereignis ist sechseckig.

- Eine Funktion (Prozess) ist eine Aktion oder Aufgabe, die auf ein Ereignis folgt. Funktionen werden durch Rechtecke mit abgerundeten Ecken symbolisiert.

- Konnektoren dienen zum Aufspalten (Split) oder Vereinigen (Join) des Kontrollflusses. Es stehen die drei Konnektoren UND, ODER und XOR zur Verfügung, die jeweils in einem kleinen Kreis mit dem entsprechenden Symbol dargestellt werden. Die Entscheidung, welcher Pfad nach einem Konnektor verfolgt wird, trifft die dem Konnektor vorangehende Funktion.

- Funktionssicht

- Die Funktionsknoten in der Steuerungssicht werden mit Knoten aus dem Funktionsbaum der Funktionssicht verknüpft - damit wird jeweils die auszuführende Aktivität spezifiziert.

- Datensicht

- Informationsobjekte sind Entitäten aus dem Datenmodell, die an Träger wie Dokumente oder sonstige Datenspeicher gebunden sind. Sie stellen und Inputs oder Outputs der Funktion dar, mit der sie durch eine gerichtete Kante verbunden werden. Das Symbol für ein Informationsobjekt ist ein Rechteck, der Charakter als Input oder Output bestimmt die Pfeilrichtung der Verbindungskante. 


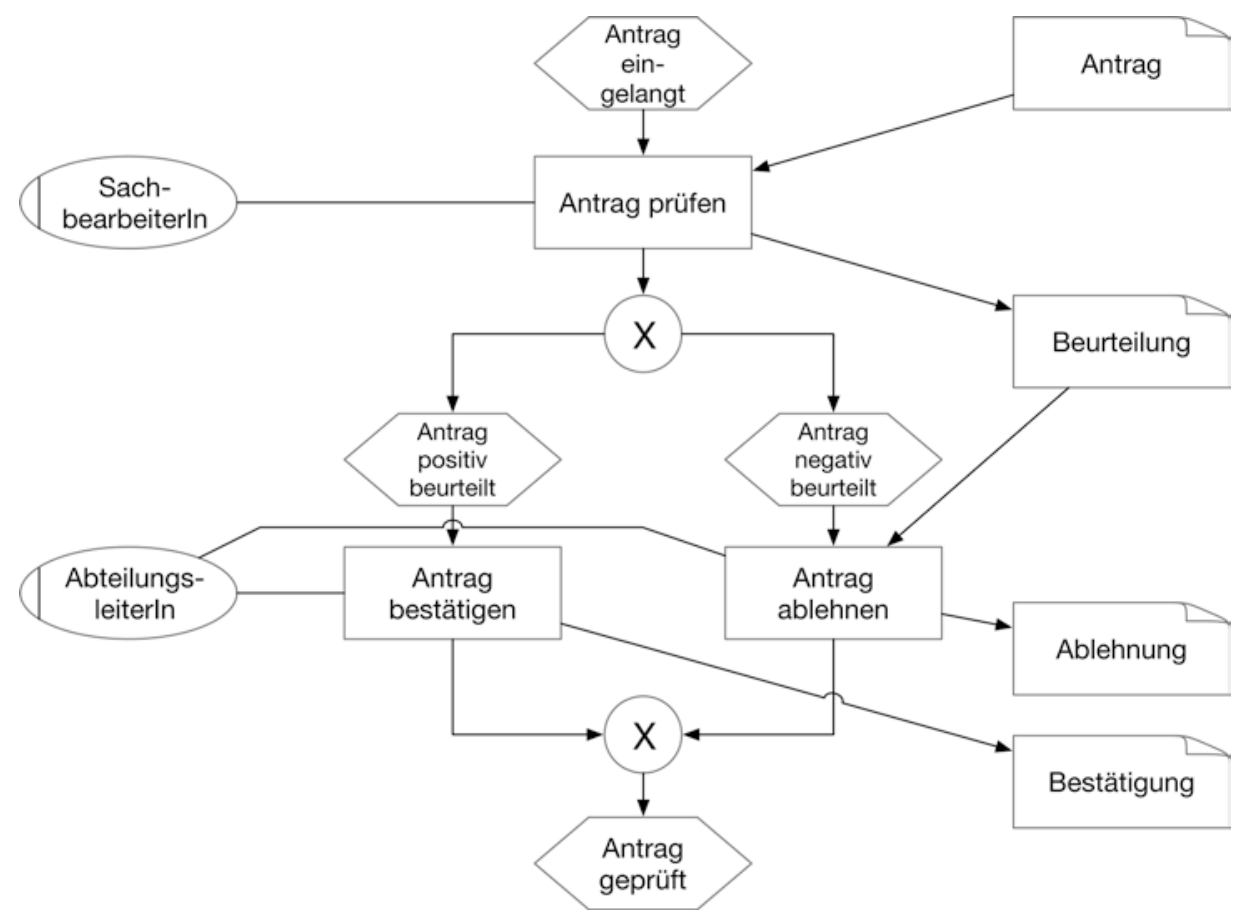

Abb. 2.18 Erweiterte Ereignisgesteuerte Prozesskette für Kontotransaktion

- Organisationssicht

- Organisationseinheiten zeigen an, welche Elemente aus dem für die Organisationssicht modellierten Organigramm die Aktivitäten (Funktionen) im Prozess ausführen. Organisationseinheiten werden durch ungerichtete Kanten mit Funktionen verbunden.

In Abb. 2.18 ist das Flussdiagramm aus Abb. 2.17 in der Notation einer erweiterten Ereignisgesteuerten Prozesskette zu sehen.

\subsubsection{Petrinetze}

Einer der ersten und auch am weitest verbreiteten Theorieansätze zur Beschreibung von Parallelität sind Petrinetze.

Petrinetze dienen der logischen Modellierung von Verhalten. Sie betrachten das Verhalten von Systemen, in der Regel von Informationssystemen unter folgenden Aspekten:

- Auszuführende Aktivitäten

- Vor- und Nachbedingungen einer Aktivität 
- Zustände aller Bedingungen (mögliche Ausprägung für jede einzelne Vor- bzw. Nachbedingung: Zustand einer Bedingung ist die Verteilung sogenannter Marken als Zustandsanzeige auf den Vor- bzw. Nachbereich)

- Anfangszustand (Anfangsmarkierung)

- Abläufe (mögliche Folgen von Aktivitäten)

Ein Petrinetz ist ein Gebilde, das formal und mathematisch präzise beschrieben wird als gerichteter Graph mit Knoten, die aus zwei disjunkten Teilmengen bestehen und mit Markierungen versehen sind. In der Schreibweise als Quadrupel gilt: Ein Petri Netz ist ein Quadrupel: $\mathrm{PN}=(\mathrm{S}, \mathrm{T}, \mathrm{K}, \mathrm{M}) \mathrm{mit}$

- $s$ \& S: Stellen (oder auch Plätze), zur Beschreibung von Zuständen und/oder Bedingungen, Puffer, Speicher oder Lager, im Graph kreisförmig. Sie dienen der Ablage von Information bzw. der Markierungen.

- $\quad \mathrm{t} \varepsilon$ T: Transitionen, beschreiben Zustandsübergänge, Ereignisse, Aktionen oder Tätigkeiten und sind im Graph strich-, balken- oder quaderförmig. Ihr Zweck ist die Verarbeitung von Information.

- $\mathrm{k} \varepsilon \mathrm{K}$ : Kanten sind ggf. gewichtete (d. h. mit Zahlen versehene) Verbindungen zwischen Stellen und Transition, im Graph als Pfeile dargestellt. Sie zeigen den Verlauf der Transitionen an.

- $\mathrm{m} \varepsilon$ M: Marken (Token), die den aktuellen Zustand des Petrinetzes wiedergeben. Jedes Netz hat einen Initialzustand, d. h. es gibt eine Anfangsmarkierung.

Gemäß obiger Definition gehen Kanten jeweils nur von Stelle zu Transition bzw. Transition zu Stelle. Eingabestellen einer Transition t sind Stellen, von denen Kanten zur Transition t laufen. Ausgabestellen einer Transition t sind Stellen, zu denen Kanten der Transition $\mathrm{t}$ führen.

Eine Transition kann schalten, wenn in jeder Eingabestelle mindestens eine Marke (Token) liegt. Schalten heißt, dass aus jeder Eingabestelle der schaltenden Transition ein Token abgezogen und jeder Ausgabestelle ein Token hinzugefügt wird.

Die folgenden Grafiken zeigen ein Petrinetz mit seinen jeweiligen Zuständen nach der Durchführung der Schaltoperationen. Im Startzustand in Abb. 2.19 hat nur die Stelle
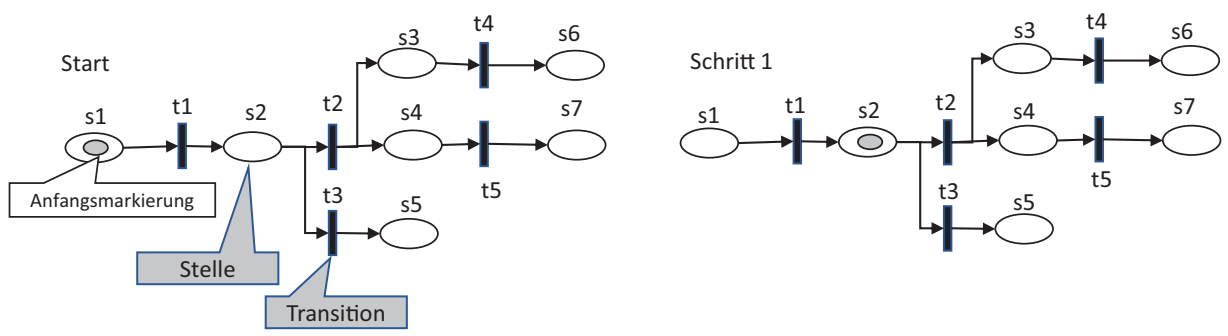

Abb. 2.19 Petrinetz I 
s1 eine Markierung, die Initial- oder Anfangsmarkierung. Die Stelle s1 ist die einzige Eingangsstelle der Transition t1, die deshalb schalten kann. Die Markierung wird aus s1 abgezogen und eine Markierung zur einzigen Ausgangsstelle s2 hinzugefügt. Die rechte Hälfte des folgenden Bildes zeigt den Zustand nach dem Schalten von $\mathrm{t} 1$.

Die Stelle s2 ist die Eingangsstelle der beiden Transitionen t2 und t3 (vgl. linke Seite von Abb. 2.20). Es könnte also die Transition $t 2$ oder $\mathrm{t} 3$ schalten, aber nicht beide. Welche Transition schaltet ist zufällig. Damit haben wir einen sogenannten nichtdeterministischen Zustand. Schaltet $\mathrm{t} 3$, wird der Stelle s5 ein Token hinzugefügt und ein Endzustand erreicht, da aus s5 keine Kante mehr herausführt. Schaltet die Transition t2, so wird den Stellen s3 und s4, die Ausgangsstellen von t2 sind, jeweils ein Token hinzugefügt. Den entsprechenden Zustand zeigt die rechte Hälfte von Abb. 2.20.

Nun kann entweder die Transition 44 oder 15 schalten. Hier könnte man folgern, dass diese beiden Transitionen gleichzeitig schalten. Dies ist aber in Petrinetzen nicht erlaubt. $\mathrm{Zu}$ einem Zeitpunkt darf nur genau eine Transition schalten. Es ist also keine echte Parallelität möglich. Es ist aber willkürlich, ob nun zuerst t4 oder t5 schaltet. In unserem Beispiel schaltet erst $\mathrm{t} 4$ und dann $\mathrm{t} 5$. Wenn beide Transitionen geschaltet haben, ist wieder ein Endzustand erreicht. Abb. 2.21 zeigt die entsprechenden Netzzustände.

Petrinetzmodelle erlauben die Analyse und Simulation von dynamischen Systemen mit nebenläufigen und nicht-deterministischen Vorgängen.

Bei der vorgestellten Art der Petrinetze handelt es sich um die Grundversion. Mit ihr lassen sich bestimmte Sachverhalte nicht beschreiben. Beispielsweise ist es nicht möglich, Prioritäten für Transitionen zu vergeben, weshalb z. B. die Rechts-vor-links-Regel an Verkehrskreuzungen nicht modelliert werden kann.

Um solche Defizite zu beheben wurden Erweiterungen eingeführt, mit denen man dem Modell weitere Aspekte der Wirklichkeit hinzuzufügen bzw. bestimmte Sachverhalte

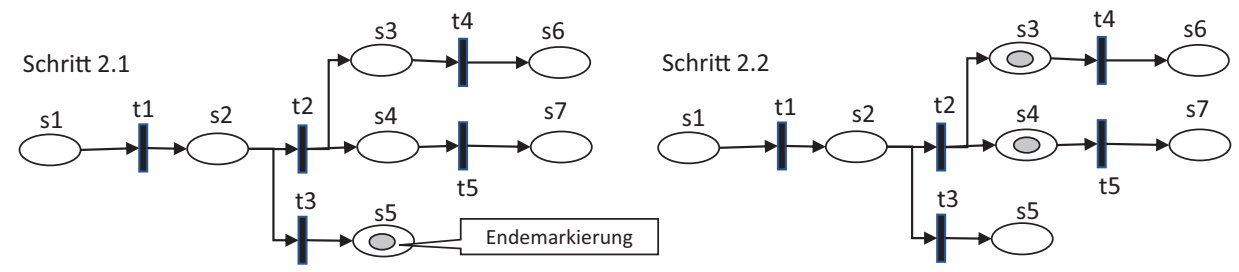

Abb. 2.20 Petrinetz II
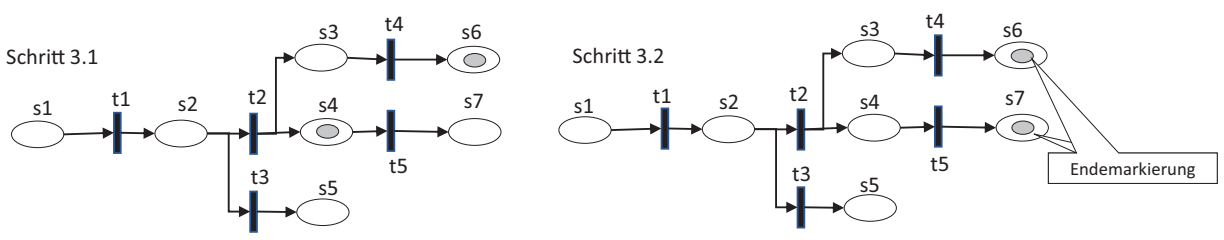

Abb. 2.21 Petrinetz III 
kompakter zu beschreiben kann. Beispiele sind mehrwertige, bunte oder mit Prioritäten versehene Petrinetze ${ }^{6}$.

\subsubsection{Calculus of Communicating Systems}

Der Calculus of Communicating Systems (CCS) wurde von Robin Milner im Jahr 1980 [24] publiziert. Dieser Kalkül erlaubt die formale Modellierung von parallelen kommunizierenden Systemen. Damit können vernetzte Systeme mit einer statischen Topologie beschrieben werden. Mit CCS können Eigenschaften von Programmen wie Verklemmungen, Verhaltensgleichheit etc. formal untersucht werden. CCS erlaubt die Beschreibung von folgender Aspekte:

- Kommunikation zwischen Akteuren über Kanäle

- Interaktion mit der Umgebung, d. h. Reaktivität

- Parallele Komposition

- Verbergen von Aktionen gegenüber der Umwelt (Information Hiding)

- Nicht-deterministische Verzweigungen

Der Ablauf eines Prozesses wird als Baum beschrieben, d. h. es gibt eine Wurzel, die den Anfangszustand darstellt und von der die einzelnen Äste ausgehen. Jeder der Äste ist markiert. Diese Markierungen repräsentieren die Aktionen, die ausgeführt werden, um von einem Zustand zum Folgezustand zu gelangen. Es wird zwischen beobachtbaren und nicht beobachtbaren Aktionen unterschieden. Nicht beobachtbare Aktionen können innerhalb eines Prozesses jederzeit ausgeführt werden, ohne dass dies Auswirkungen auf andere Prozesse hat. Prozesse haben keine gemeinsamen Variablen.

Um das Verhalten eines Prozesses zu beschreiben werden rekursive Ausdrücke verwendet. Innerhalb von Verhaltensausdrücken können Variablen verwendet werden, um andere Verhaltensausdrücke zu referenzieren.Die Verhaltensausdrücke werden gemäß folgender Syntax beschrieben, bei der Großbuchstaben Prozessnamen und Kleinbuchstaben Aktionen bezeichnen.

- Leerer Prozess:

$\varnothing$

- Aktion

Der Prozess a.P1 führt die Aktion a aus und verhält sich dann wie P1

- Prozessname

Mit dem Ausdruck A: = P1 erhält der Prozess P1 den Namen A. Da rekursive Definitionen erlaubt sind kann in dem Ausdruck P1 der Name A wieder enthalten sein.

${ }^{6}$ Einzelheiten zu solchen Erweiterungen finden sich in der verfügbaren Literatur, wie z. B. bei [23]. 
- Auswahl

Der Prozess P1 + P2 kann entweder mit dem Prozess P1 oder dem Prozess P2 fortgesetzt werden.

- Parallelkomposition

P1|P2 bedeutet, dass die Prozesse P1 und P2 parallel ausgeführt werden

- Umbenennung

$\mathrm{P} 1[\mathrm{~b} / \mathrm{a}]$ beschreibt den Prozess P1, in dem alle Aktionen mit der Bezeichnung a in $\mathrm{b}$ umbenannt werden.

- Restriktion

$\mathrm{P} 1 \backslash$ a bezeichnet den Prozess P1 ohne die Aktion a

Zueinander passende Ein- und Ausgabeaktionen in zwei unterschiedlichen Prozessen können sich synchronisieren und zu einer internen Aktion $\tau$ werden. Im Allgemeinen wird die Koaktion zu einer Aktion mit einem Querstrich über dem Aktionsnamen gekennzeichnet. Diese komplementären Aktionen sind die Sende- und Empfangsaktionen. Abb. 2.22 zeigt beispielhaft die Interaktion zwischen zwei Prozessen.

Das folgende Beispiel (siehe Abb. 2.23) zeigt, wie mit CCS ein einfacher Urlaubsantragsprozess beschrieben werden kann.

Im Prozess Mitarbeiter wird die Sendeaktion Urlaubsantrag ausgeführt (Aktionsname mit Überstrich). Danach wird auf die Nachrichten , abgelehnt ‘ oder , genehmigt ${ }^{\text {* }}$ gewartet. Der Manager empfängt die Nachricht Urlaubsantrag, die er entweder mit der

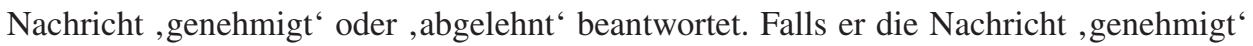
sendet, wird danach auch noch die Nachricht, genehmigter Urlaubsantrag ' gesendet. Diese Nachricht empfängt die Reisestelle. Der Austausch von Nachrichten erfolgt in CCS asynchron, d. h. ein Sender wartet so lange, bis der Empfänger die dazugehörige Empfangsaktion ausführt.

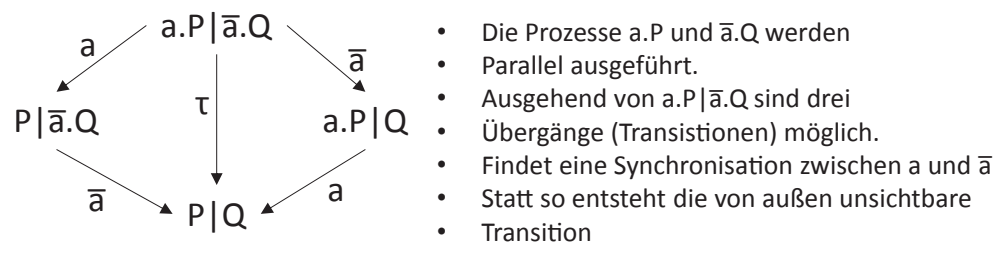

Abb. 2.22 Prozessinteraktion mit CCS

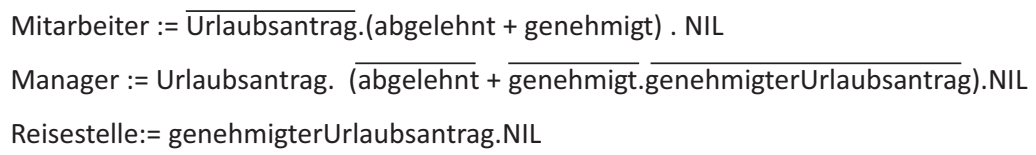

Urlaubsantragsprozess := Mitarbeiter | Manager | Reisestelle

Abb. 2.23 Beispielprozess in CCS 
$\mathrm{Zu}$ der ausgeführten informellen Interpretation gibt es auch formale Ableitungsregeln. Damit werden Prozessdefinitionen zugänglich für formale Untersuchungen.

\subsection{6 $\pi$-Kalkül}

CCS erlaubt nur statische Prozessstrukturen. Kommunikationsbeziehungen können nicht dynamisch verändert werden. Der $\pi$-Kalkül [25], ebenfalls von Robin Milner entwickelt, erlaubt die Darstellung von Prozessen mit wechselnden Strukturen. Es sind beliebige Verbindungen zwischen Komponenten darstellbar und diese Verknüpfungen können sich auch ändern bzw. neue können entstehen. Damit ist der $\pi$-Kalkül eine Erweiterung des CCS um Nebenläufigkeit. Die Notation im $\pi$-Kalkül orientiert sich weitgehend an der CCS-Notation. Das folgende Beispiel erläutert die Modellierungsmöglichkeiten des $\pi$-Kalküls (vgl. Abb. 2.24).

Der Agent (Prozess) P möchte den Wert 7 an Q über einen Link a senden. Allerdings soll der Wert indirekt über einen weiteren Agenten R übertragen werden.

Die einzelnen Schritte bei der Ausführung des Systems O sind in Abb. 2.25 sichtbar.

Die Prozesse P, R und Q werden parallel ausgeführt. Prozess P sendet über den Kanal b den Namen a und dann den Namen 7. Der Prozess P erhält über den Kanal b die beiden Namen. Dies bedeutet, alle x werden durch a ersetzt und alle z durch 7. In Abb. 2.25 ist dies das Ergebnis nach Schritt 2. Nun kann über den Kanal a der Name 7 gesendet werden, der vom Prozess Q dann angenommen wird. Damit wurde der Wert 7 über den Prozess R an den Prozess Q gesendet. Die Abb. 2.26 zeigt, wie durch den Ablauf des Systems O seine Struktur geändert wird.

Da der Kanalname a von P nach R gesendet wird, sind nach der Annahme der Nachricht die Prozesse R und Q über den Kanal a verknüpft. Damit wird deutlich, dass der $\pi$-Kalkül im Gegensatz zu CCS die Modellierung von Strukturänderungen zulässt.

- $\mathrm{P}:=\overline{\mathrm{ba}} \cdot \overline{\mathrm{b}} \overline{\mathrm{7}} \cdot \mathrm{P}^{\prime} \quad$ Prozess $\mathrm{P}$ sendet über den Kanal b den Wert a und nachfolgend den Wert 7 und verhält sich dann wie P'.

- $\quad \mathrm{R}:=\mathrm{b}(\mathrm{x}) \cdot \mathrm{b}(\mathrm{z}) \cdot \overline{\mathrm{x} z} .0$ Prozess $\mathrm{R}$ empfängt über den Kanal $\mathrm{b}$ jeglichen Wert. Dies bedeutet in $\mathrm{R}$ wird $\mathrm{x}$ und $\mathrm{z}$ durch die empfangenen Werte ersetzt.

- $\mathrm{Q}:=\mathrm{a}(\mathrm{x}) \cdot \mathrm{Q} \quad$ Prozess $\mathrm{Q}$ empfängt über den Kanal a einen beliebigen Wert für $\mathrm{x}$. Der empfangene Wert wird überall dort eingesetzt wo sich $\mathrm{x}$ befindet.

\section{- O:=P IRIQ Gesamtsystem O}

Abb. 2.24 Prozessbeschreibung mit $\pi$-Kalkül 
Abb. 2.25 Schritte bei der Ausführung des Systems O
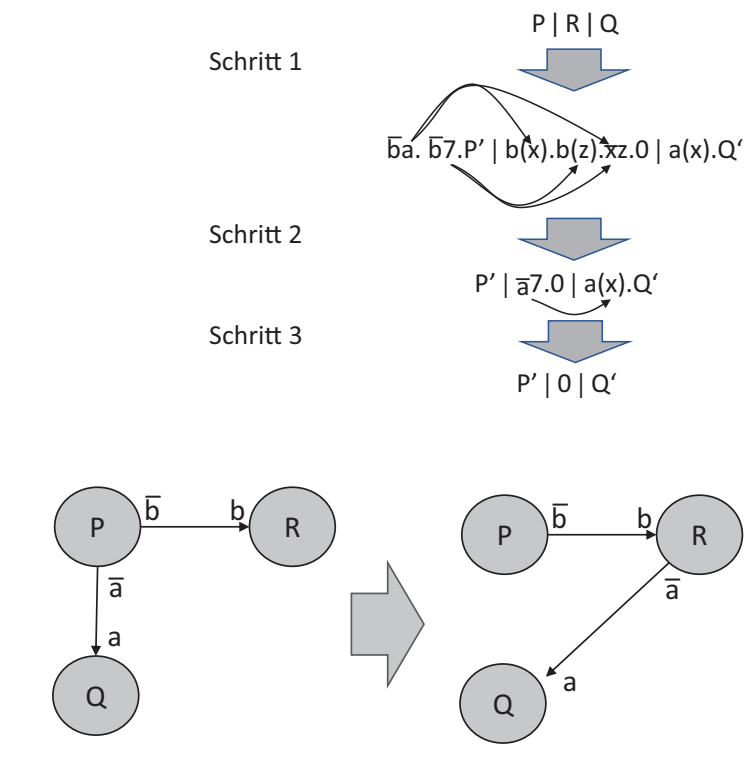

Abb. 2.26 Strukturänderung

\subsubsection{Communicating Sequential Processes}

Communicating Sequential Processes (CSP) ist eine Methodik zur Beschreibung von Interaktion zwischen kommunizierenden Prozessen. Die Idee hat Tony Hoare erstmals 1978 als imperative Sprache vorgestellt [26], dann zu einer formalen Algebra ausgebaut und 1985 mit der Veröffentlichung des Buchs mit dem Titel Communicating Sequential Processes berühmt gemacht [27].

In CSP ist wie in CCS die Anzahl der Prozesse statisch. Sie kann während der Laufzeit nicht geändert werden und zwischen Prozessen gibt es keine gemeinsamen Variablen. Stattdessen ,kennen“ sich die Prozesse und verständigen sich untereinander durch das Senden und Empfangen von Nachrichten. Zum Senden führt der Sendeprozess P das Ausgabekommando.

Q! (expr)

und der Empfängerprozess Q das Eingabekommando

P ? (vars)

aus. Ausgabe- und Eingabekommandos heißen korrespondierend, falls die Folge von Ausdrücken (expr) und die Folge von Variablen (vars) in der Anzahl und komponentenweise vom Typ übereinstimmen. Analog CCS und dem $\pi$-Kalkül basiert CSP auf einem ungepufferten Nachrichtenaustausch, bei dem der Sende- und der Empfangsprozess explizit genannt werden müssen.

Mit Q ! () und P ? () wird eine Nachricht ohne Inhalt gesendet. Solche Nachrichten werden als Signale bezeichnet und dienen einzig und allein der Synchronisation von Prozessen. Werden unterschiedliche Signale benötigt, so wird die Unterscheidung durch Typbezeichner der Form Q ! (Signal1) und P ? (Signal1) ausgedrückt. 
Neben dem oben beschriebenen unbedingten Nachrichtenaustausch gibt es die Empfangsanweisung innerhalb eines sogenannten Guarded Commands (Bewachte Anweisung). Eine bewachte Anweisung wird nur ausgeführt, wenn die vorangestellte Bool'sche Bedingung wahr ist. So wird der Formelsatz.

$$
\mathrm{x}>\mathrm{y} ; \mathrm{P} ?(\mathrm{z})->\mathrm{x}:=\mathrm{x}+\mathrm{y} ; \mathrm{y}:=\mathrm{z}
$$

nur ausgeführt, wenn $\mathrm{x}$ größer als $\mathrm{y}$ ist. Dann wird die Nachricht von $\mathrm{P}$ entgegengenommen, falls $\mathrm{P}$ bereit ist, die Nachricht zu senden.

Um auf Nachrichten von unterschiedlichen Sendern warten zu können, werden mehrere Guarded Commands zu einer Alternativanweisung zusammengeschlossen.

$$
[\mathrm{x}>\mathrm{y} ; \mathrm{P} ?(\mathrm{z})->\mathrm{x}:=\mathrm{x}+\mathrm{y} ; \mathrm{y}:=\mathrm{z} \| \mathrm{x}<\mathrm{y} ; \mathrm{Q} ?(\mathrm{z})->\mathrm{y}:=\mathrm{x}+\mathrm{y} ; \mathrm{y}:=\mathrm{z}]
$$

Im Fall $x>Y$ wird die Nachricht P?(z) erwartet, im Fall $x<y$ die Nachricht Q?(z). Alternativanweisungen können auch wiederholt ausgeführt werden. Syntaktisch wird dies durch einen der Alternativanweisung vorangestellten * ausgedrückt. Die Anweisung.

$*[\mathrm{x}>\mathrm{y} ; \mathrm{P} ?(\mathrm{z})->\mathrm{x}:=\mathrm{x}+\mathrm{y} ; \mathrm{y}:=\mathrm{z} \| \mathrm{x}<\mathrm{y} ; \mathrm{Q} ?(\mathrm{z})->\mathrm{y}:=\mathrm{x}+\mathrm{y} ; \mathrm{y}:=\mathrm{z}]$ wird solange ausgeführt, bis keine der Bedingungen mehr wahr ist - dann wird die Wiederholung beendet.

Die Konzepte bezüglich der Nebenläufigkeit von CSP dienen als Gestaltungsgrundlage der Programmiersprache Go.

\subsubsection{Abstract State Machines}

Eine abstrakte Zustandsmaschine (Abstract State Machine (ASM)) ist in der Informatik ein Modell zur formalen, operationellen Beschreibung von Algorithmen. Die Zustände einer abstrakten Zustandsmaschine sind allgemeine mathematische Strukturen. Der Erfinder des Modells ist Yuri Gurevich. Egon Börger hat die ASM für die praktische Anwendung weiterentwickelt [28].

Abstract State Machines (ASM) sind endliche Mengen von Transitionsregeln der Form.

If condition then action

mit denen die Zustände einer ASM geändert werden. Condition ist dabei ein beliebiger logischer Ausdruck und Action eine beliebige Handlung. In der Regel ist Action eine Wertzuweisung der Form $\mathrm{f}(\mathrm{t} 1, \ldots \ldots . \mathrm{tn}):=\mathrm{s}$. Die Bedeutung der Regel besteht darin, im gegenwärtigen Zustand die angegebene Regel auszuführen, wenn die angegebene Bedingung in diesem Zustand erfüllt ist. ASM-Zustände werden allgemein als beliebige Mengen beliebiger Elemente mit beliebigen darauf definierten Funktionen (Operationen) und Prädikaten definiert. Im Fall von Geschäftsobjekten sind die Elemente Platzhalter für Werte beliebigen Typs und Operationen wie Erzeugen, Duplizieren, Löschen oder algebraische Manipulationen von Objekten. 
Ein Berechnungsschritt einer ASM in einem bestimmten Zustand bedeutet, dass alle Actions, für die die Bedingung (Condition) wahr ist, gleichzeitig ausgeführt werden. Durch die simultane Ausführung kann von irrelevanten Reihenfolgen abstrahiert werden.

Mehrere ASMs können gleichzeitig ablaufen und über sogenannte Controlled bzw. Monitored Functions verknüpft werden. Eine gegebene ASM M kann Controlled Functions aktualisieren, sie kann aber nicht von anderen ASM aus der Umgebung von M verändert werden. Monitored Functions einer gegebenen ASM M können nur durch deren Umgebung aktualisiert werden. Durch die paarweise Verwendung von Controlled and Monitored Functions kann ein Netzwerk paralleler sich koordinierender ASMs aufgebaut werden.

\subsubsection{Objektorientierte Modelle}

Die bisher betrachteten Modelle der Informatik legen den Schwerpunkt entweder auf Daten oder Funktionen. Die Darstellung von Daten in einem ERM und Funktionen in einem Flussdiagramm ergänzen sich nur in entkoppelten Darstellungen - die Unterscheidung in Daten- und Funktionssicht bei ARIS macht dies deutlich. Die objektorientierte Modellbildung reduziert diese isolierten Sachverhalte nicht mehr auf ihre Einzelteile, sondern betrachtet sie als integriertes Ganzes, bei dem die einzelnen Komponenten miteinander verbunden und voneinander abhängig sind.

Unter einem objektorientierten Modell versteht man eine Sichtweise auf ein komplexes System, bei der dieses durch das Zusammenspiel von Objekten beschrieben wird. Durch diese Art der Modellbildung soll die Komplexität der Beschreibung für Sachverhalte, die in Software abzubilden sind, reduziert werden. Die Objektorientierung sieht die in der realen Welt vorkommenden Entitäten als Objekte. Ein Telefon ist ebenso ein Objekt, wie ein Fahrrad, ein Mensch oder ein Mitarbeiter. Solche Objekte setzen sich wiederum aus anderen Objekten zusammen wie z. B. Schrauben, Stangen, Armen, Füßen, Kopf o. Ä. Die Objekte werden, wie in der Modellbildung üblich, auf ihre in der jeweiligen Situation bedeutsamen Eigenschaften reduziert. So wird z. B. ein Mitarbeiter in einem Lohnabrechnungssystem auf Name, Adresse, Mitarbeiternummer, vereinbartes Einkommen, Steuerklasse usw. reduziert.

Die in einem Modell berücksichtigten Objekte werden nicht einzeln konzipiert. Für gleichartige Objekte wird ein grober Bauplan mit gleichartigen Eigenschaften erstellt. Beispielsweise modelliert man für eine Bibliotheksanwendung die Eigenschaften von Büchern, die für allen Büchern identisch sind. Solche allgemeinen Beschreibungen von Objekten werden in der Objektorientierung Klassen genannt. Mit diesen Klassen werden dann innerhalb des Modells die benötigten konkreten Objekte erzeugt (Instanzen).

Die Abbildung der Wirklichkeit in ein Modell erfolgt also zweistufig. Zuerst werden gleichartige Objekte der Wirklichkeit identifiziert und jeweils als Klasse beschrieben. Die einzelnen Objekte werden dann als sogenannte Instanzen einer Klasse erzeugt. Eine Klasse beschreibt somit die Struktur einer Menge gleichartiger Objekte. Abb. 2.27 zeigt eine Klasse-Objekt Beziehung, nach der das Buch „Subjektorientiertes 


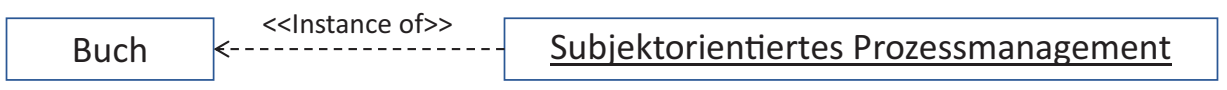

Abb. 2.27 Beziehung Klasse-Objekt

Prozessmanagement“ eine Instanz der Klasse Buch ist. Die verwendete Notation entstammt der Unified Modeling Language (UML). UML ist eine von der Object Management Group standardisierte Sprache zur Beschreibung objektorientierter Modelle.

Die Eigenschaften einer Klasse sind

- ihre Bestandteile und die in ihnen enthaltenen Daten und Informationen, auch Attribute genannt,

- die auf den Bestandteilen definierten Operationen und ihre Parameter (Methoden), mit denen ein Objekt manipuliert bzw. dessen Zustand abgefragt werden kann und

- Bedingungen, Voraussetzungen und Regeln, die die Objekte erfüllen müssen (Zusicherungen).

Abb. 2.28 zeigt ein Beispiel für die Beschreibung der Klasse Buch. Diese Klasse hat fünf Attribute. Das Attribut Seitenzahl hat eine Zusicherung, die besagt, dass die Seitenzahl positiv sein muss. Die Klasse erlaubt den Zugriff und die Manipulation ihrer Daten mit sechs Methoden. So können der Titel, die Autoren, die Seitenzahl, der Verlag und der Inhalt gesetzt werden (Set). Mit der Operation (Methode) AnzeigenSeite(Seite) kann der Inhalt der angegebenen Seite aus dem Buch ,,ausgelesen“ werden.

Ausgehend von einer solchen Klassendefinition können nun Objekte instanziiert werden. Mithilfe der Operationen können dann für jede dieser Instanzen die jeweiligen Attribute entsprechend gesetzt werden. Ein objektorientiertes Modell beschreibt neben den Definitionen der Klassen und der zugehörigen Objekte auch die Beziehungen der Klassen zueinander. Die Arten von Beziehungen sind:

- Vererbung

Eigenschaften können von einer Klasse zur nächsten weitergegeben werden. Man spricht dann von Vererbung. Die Klasse, die vererbt, heißt Oberklasse, und die Klasse, die erbt, Unterklasse. So ist eine Klasse Notizbuch Unterklasse der Klasse Buch. Der Klasse Notizbuch wird noch die Methode „Seiteneintrag (Seite, Inhalt)“ hinzugefügt. Damit kann auf der angegebenen Seite ein Text eingegeben werden; Ansonsten erbt die Unterklasse Buch alle Attribute und Methoden von der Klasse Buch. Abb. 2.29 zeigt die Vererbungsbeziehung zwischen Buch und Notizbuch. Der Dreieckspfeil zeigt von der Unterklasse zur Oberklasse.

- Assoziationen

Eine Assoziation ist eine Beziehung zwischen verschiedenen Objekten einer oder mehrerer Klassen. Dargestellt werden Assoziationen als einfache Linie zwischen zwei Klassen. Die Linie kann mit einem Namen (Bezeichnung) und Anzahlangaben versehen werden. Auch die assoziierten Klassen können Namen bezüglich der Beziehung 
Abb. 2.28 Beschreibung der Klasse Buch

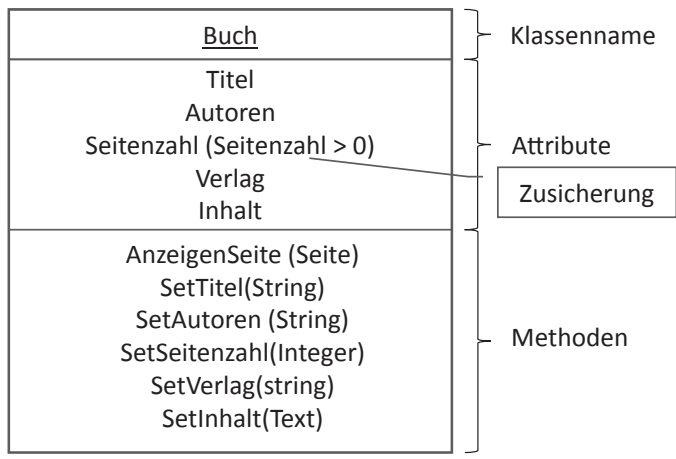

Abb. 2.29 Vererbung von Eigenschaften

Abb. 2.30 Assoziationen zwischen Objekten

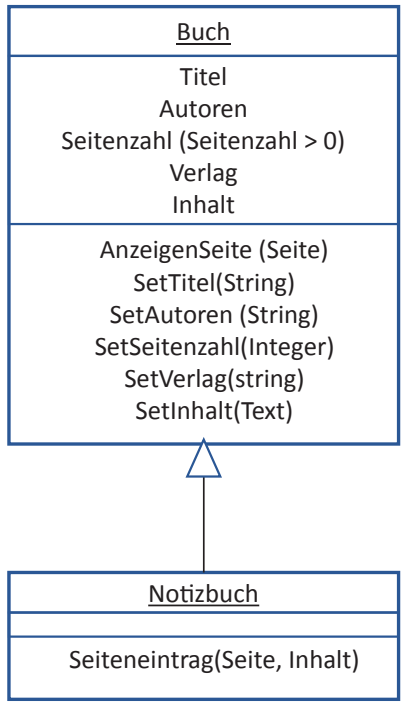

\begin{tabular}{|c|c|c|c|c|}
\hline Firma & $0 . .1$ & beschäftigt> & $0 .{ }^{*}$ & Persor \\
\hline
\end{tabular}

erhalten. Abb. 2.30 illustriert ein Beispiel für eine Assoziation. Eine Person (Arbeitnehmer) kann bei keiner oder einer Firma (Arbeitgeber) beschäftigt sein und eine Firma kann keine oder mehrere Personen beschäftigen. Assoziationen haben eine große Ähnlichkeit mit den Entity-Relationship-Diagrammen.

- Aggregationen

Aggregationen sind eine Variante von Assoziationen. Dabei handelt es sich ebenfalls um eine Beziehung zwischen zwei Klassen, jedoch mit der Besonderheit, dass die zwei Klassen zueinander in Beziehung stehen wie ein Teil zu einem Ganzen. Eine Aggregation setzt sich zusammen aus einer Menge von Einzelteilen. Abb. 2.31 zeigt ein Aggregationsbeispiel, bei dem ein Unternehmen aus Abteilungen, und eine Abteilung aus Mitarbeitern besteht. 


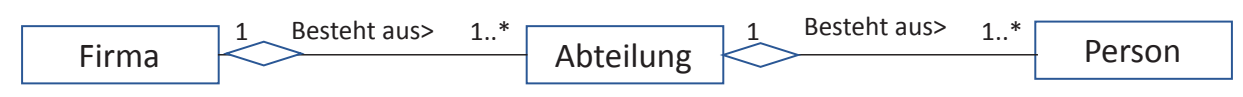

Abb. 2.31 Mehrstufige Aggregation

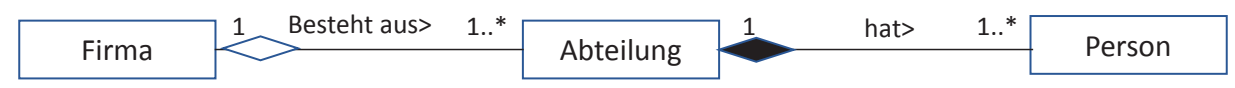

Abb. 2.32 Komposition

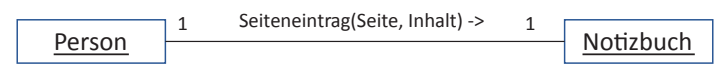

Abb. 2.33 Nachricht zwischen Objekten

- Komposition

Eine besondere Form der Aggregation ist die Komposition. Hier hängt das Ganze von der Existenz seiner Einzelteile ab. In Abb. 2.32 ist eine Änderung an der obigen Aggregation zu sehen. Eine Abteilung besteht nicht mehr, wenn ihr niemand angehört.

- Objekte kommunizieren miteinander, d. h. ein Objekt sendet Nachrichten an ein anderes Objekt. Die Nachrichten stoßen dann die zugehörigen Operationen an. Ein Objekt versteht also nur die Nachrichten, zu denen es auch die zugehörigen Operationen enthält. Abb. 2.33 zeigt die Kommunikation einer Person mit dem Notizbuch zum Eintragen einer neuen Notiz.

Die beschriebenen Konstrukte bilden den Nukleus des objektorientierten Modellierungsansatzes. Darüber hinaus existieren zahlreiche Erweiterungen zur Abbildung bestimmter Sachverhalte wie die sogenannten Behälterklassen ${ }^{7}$.

\subsubsection{Agenten-/Aktoren-orientierte Modelle}

Bislang gibt es keine standardisierte und allgemein akzeptierte Definition des Agentenkonzepts. Der Begriff wird abhängig von der Anwendungsdomäne im Detail unterschiedlich definiert [30]. All diesen Definitionen ist aber gemeinsam, dass ein Agent eine abgrenzbare Einheit ist, die in der Lage ist, die ihr vorgegebenen Aufgaben flexibel, interaktiv und autonom zu verfolgen. Oft wird der Begriff Aktor als synonym für den Begriff Agent benutzt. So definieren Lankhorst et al. [31] einen Business Actor als „,... an entity

${ }^{7}$ Weiterführende Informationen finden sich beispielsweise bei [29]. 
that is capable of performing behavior". Multiagentensysteme bestehen aus mehreren Agenten, die synchron oder asynchron Nachrichten austauschen. Multiagentensysteme können die Strukturen von Softwaresystemen abbilden oder auch der Modellbildung sozialer Systeme dienen. Je nach Anwendungssituation kann dann zwischen Softwareagenten und menschlichen Agenten unterschieden werden.

In gewisser Weise können die Prozesse in CCS, im $\pi$-Kalkül und in CSP als Multiagentensysteme betrachtet werden. Der Begriff Prozess wird dort analog zu den Begriffen Agent und Aktor verwendet.

Ein agentenorientiertes Modell beinhaltet also die Agenten, die Kommunikationspfade und die Nachrichten, die ausgetauscht werden. ${ }^{8}$

\subsection{Fazit: Modelle für Geschäftsprozesse}

Geschäftsprozessmodelle bilden den Wirklichkeitsausschnitt der Geschäftsprozesse ab. Das subjektive Verständnis des Geschäftsprozessbegriffs beeinflusst, welche Prozessaspekte als wesentlich erachtet werden und deshalb in den Modellen im Vordergrund stehen. Hier schlagen sich Absichten und Interessen des Modellerstellers nieder. Konsequenz sind zahlreiche Auslegungen des Geschäftsprozessbegriffs, von denen jede weder richtig noch falsch ist, sondern lediglich andere Betrachtungsschwerpunkte setzt.Als Beispiele dafür können folgende Definitionen des Terminus Geschäftsprozess gelten:

1. „Folge von Wertschöpfungsaktivitäten (Wertschöpfung) mit einem oder mehreren Inputs und einem Kundennutzen stiftenden Output“" [33].

2. „Ein Prozess ist die inhaltlich abgeschlossene, zeitliche und sachlogische Folge von Aktivitäten, die zur Bearbeitung eines betriebswirtschaftlich relevanten Objekts notwendig sind.“[34].

Beide Definitionen stellen auf die erforderlichen Aktivitäten und deren Folgen ab. Das erste Beispiel erwähnt zusätzlich den Input und den Output mit dem Kundennutzen, während in der zweiten Definition stattdessen die Bearbeitung der Geschäftsobjekte einbezogen wird.

Handelnde und benötigte Ressourcen fehlen dagegen in beiden Begriffsfassungen. Sie betrachten nicht, durch wen und womit die Aktivitäten ausgeführt werden. Es gibt keinen Bezug zur Organisation, in die ein Geschäftsprozess eingebettet ist, sowie dazu, welche IT-Anwendungen oder sonstige Ressourcen eingesetzt werden, um ihn auszuführen.Wir

${ }^{8}$ Eine Sammlung von agentenorientierten Modellierungssprachen und den dazugehörigen Vorgehensweisen findet sich in [32]. 
folgen deshalb in Anlehnung an Gerhard Schewe einem Begriffsverständnis, das auch diese fehlenden Aspekte berücksichtigt [33]:

1. Ein Prozess ist die Summe von miteinander verknüpften Aktivitäten (Aufgaben),

2. die von Handelnden (Menschen, Systeme als Aufgabenträger)

3. in sachlogischer und zeitlicher Reihenfolge

4. mit Hilfsmitteln (Sachmittel, Information)

5. zur Bearbeitung eines Geschäftsobjekts ausgeführt werden,

6. um ein Kundenbedürfnis zu befriedigen (und damit zur Wertschöpfung beizutragen),

7. und einen definierten Anfang und Input

8. sowie ein definiertes Ende und Ergebnis aufweisen.

Wie bereits in Abschn. 1.3 ausgeführt, formen wir die Bestandteile etwas um und gruppieren sie wie folgt:

9. Prozessstrategie: Ein Prozess hat

a) einen definierten Anfang und Input (Startereignis),

b) und weist ein definiertes Ende mit einem Ergebnis auf,

c) das zur Befriedigung eines Kundenbedürfnisses (und damit zur Wertschöpfung) beiträgt

10. Prozesslogik: Ein Prozess

a) ist die Summe von miteinander verknüpften Aktivitäten (Aufgaben),

b) die nach dem Startereignis von Handelnden

c) in sachlogischer und zeitlicher Reihenfolge

d) zur Bearbeitung eines Geschäftsobjekts ausgeführt werden um

e) das gewünschte Ergebnis zu erzeugen.

11. Prozessrealisierung: Ein Prozess wird realisiert

a) mit Menschen und/oder Maschinen, die Aufgaben der jeweiligen Handelnden übernehmen, und diese

b) mit Hilfsmitteln (Sachmittel, Information, Anwendungsprogramme etc.) ausführen.

Mit diesem Verständnis von Geschäftsprozessen wird der Bezug der in diesem Kapitel beschriebenen Modelle aus unterschiedlichen Domänen zum Geschäftsprozessmanagement deutlich. Abb. 2.34 zeigt entsprechend den integrativen Charakter der Geschäftsprozessmodelle.Die Modelle von Habermas und Luhmann befassen sich mit Aspekten der gesellschaftlichen Systeme und Organisationen. Sie beschreiben welche Komponenten und Beziehungen eine Organisation ausmachen und wie Menschen darin positioniert sind. Komplexe Organisationen können beispielsweise anhand betrieblicher Funktionen, Leistungsspektrum, geografischer Aspekten oder Kombinationen davon in Teilorganisationen strukturiert werden. Das Ergebnis ist das Organigramm. 


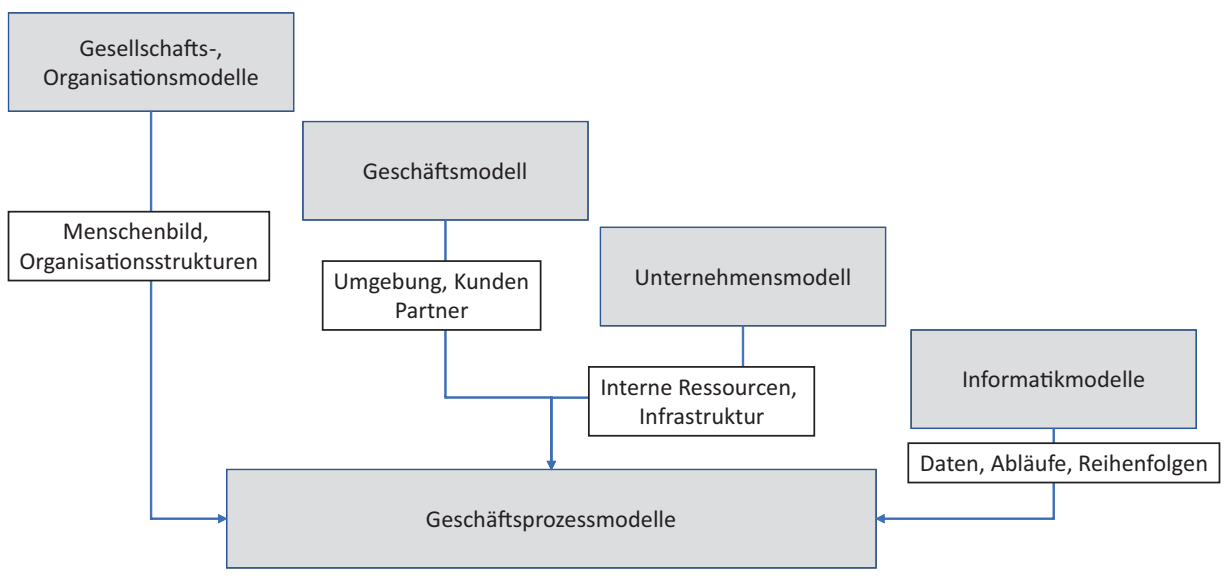

Abb. 2.34 Integration verschiedener Modelle durch Geschäftsprozessmodelle

Geschäftsmodelle betrachten unter anderem die Aspekte Kunden, Lieferanten, Partner und Wertschöpfung und blicken damit zunächst auf die externen Leistungsbeziehungen. Insbesondere mit Wertversprechen (Produkte und Services), Aktivitäten und Ressourcen stellen sie aber auch die Verbindung zur stärker nach innen gerichteten Unternehmensarchitektur her. In dieser sind auf der fachlichen Ebene die Aufbauorganisation mit den personellen Ressourcen, die Prozesse und die logischen Geschäftsobjekte modelliert. Die Verknüpfung mit der technischen Schicht der Unternehmensarchitektur führt zu den Modellen aus der Informatik. Diese beschreiben etwa Datenstrukturen, Kontrollflüsse und Algorithmen für Programme sowie die Gestalt und das Zusammenwirken weiterer informations- und kommunikationstechnischer Komponenten, die für die Ausführung gewünschter Aktionen im Rahmen der Prozessunterstützung und -automatisierung nötig sind.

\section{Literatur}

1. Markus Gabriel, Warum es die Welt nicht gibt, Ullstein Taschenbuch (7. Januar 2015)

2. O. Siemoneit; Eine Wissenschaftstheorie der Betriebswirtschaftslehre; Dissertationsschrift, Universität Stuttgart; 2010

3. Herbert Stachowiak, Allgemeine Modelltheorie, Springer Verlag, Wien New York, 1973, S. 131

4. Alfred Kieser, Peter Walgenbach; Organisation; Schäffer-Pöschl Verlag, Stuttgart 2007

5. David Crowther, Miriam Green; Organisational Theory; Chartered Institute of Personnel Development, London 2004

6. Georg Schreyögg; Organisation; Gabler Verlag, 5. Auflage, Wiesbaden 2008

7. A. Kieser, Mark Ebers; Organisationstheorien; Kohlhammer Verlag; Stuttgart 2006, S. 68 
8. Jürgen Habermas, Theorie des kommunikativen Handelns Band 1,Auflage 2014, Suhrkamp Taschenbuch Wissenschaft, Frankfurt am Main 1981, S. 128

9. Margot Berghaus; Luhmann leicht gemacht; Böhlau Verlag, 2. Auflage, Köln 2004 und Niklas Luhmann; Soziale Systeme, Suhrkamp Verlag, Frankfurt 1984

10. Christian Scheer, Thomas Deelmann, Prof. Dr. Peter Loos; Geschäftsmodelle und internetbasierte Geschäftsmodelle - Begriffsbestimmung und Teilnehmermodell; Working Papers of the Research Group Information Systems \& Management, Universität Mainz 2003

11. Bernd W. Wirtz, Oliver Schilke and Sebastian Ullrich, Strategic Development of Business Models Implications of the Web 2.0 for Creating Value on the Internet, Long Range Planning 43 (2010) 272-290, Elsevier 2010

12. Alexander Osterwalder, Yves Pigneur; Business Model Generation; John Wiley \& Sons, Hoboken 2010

13. Kaplan R.S. Norten D.P.; (1997) Balanced Scorecard; Schaeffer-Poeschl Verlag

14. Springer Gabler Verlag (Herausgeber), Gabler Wirtschaftslexikon, Stichwort: Balanced Scorecard, online im Internet: http://wirtschaftslexikon.gabler.de/Archiv/1856/balanced-scorecard-v8.html. letzter Zugriff am 22.02.2018

15. V. Allee; Value Networks and true nature of collaboration; Meghan Kiffer Press; Tampa Fl. 2015

16. Wächter H. (eds) Selbstverständnis betriebswirtschaftlicher Forschung und Lehre. Gabler Verlag, Wiesbaden

17. ITIL Best Practice; https://www.axelos.com/best-practice-solutions/itil; Last Access February 2018

18. COBIT 5; http://www.isaca.org/COBIT/Pages/COBIT-5-german.aspx. Last Access February 2018

19. Dirk Matthes; Enterprise Architecture Kompendium; Springer Verlag, Berlin Heidelberg 2011, Seite 5

20. Szyperski, N.; Informationsbedarf. In E. Grochla (Hrsg.), Handwörterbuch der Organisation, S. 904. Verlag C.E. Poeschel, Stuttgart, 2. Auflage, 1980

21. Steinmüller, W.: Eine sozialwissenschaftliche Konzeption der Informationswissenschaft. Nachrichten für Dokumentation, 23 (1981) Nr. 2, 69-80

22. P. P.-S. Chen: Entity-Relationship Modeling - Historical Events, Future Trends, and Lessons Learned (PDF-Datei; 417 kB). In: M. Broy, E. Denert (Hrsg.): Software Pioneers: Contributions to Software Engineering. Springer-Verlag, Berlin 2002.

23. Reisig W.; Petrinetze: Modellierungstechnik, Analysemethoden, Fallstudien; Vieweg+Teubner Verlag; Auflage: 2010 (15. Juli 2010)

24. Robin Milner, A Calculus of Communicating Systems; Lecture Notes in Computer Science, Springer 1980

25. Robin Milner, Communicating and Mobile Systems: the $\pi$-calculus; Cambridge University Press; 1999

26. C.A.R. Hoare, Communicating Sequential Processes, Communications of the ACM, Vol. 21, Nr. 8, 1978

27. C.A.R. Hoare, Communicating Sequential Processes, Prentice Hall, Englewood Cliffs, New Jersey, 1985

28. E. Börger, R. Stärk; Abstract State Machines; Springer Verlag 2003

29. G. Booch et al.; Object-Oriented Analysis and Design with Applications; Third Edition, Addison-Wesley: Boston MA; 2007.

30. Stan Franklin, Art Graesser; Is It an Agent, or Just a Program?: A Taxonomy for Autonomous Agents; Institute For Intelligent Systems, University of Memphis, Memphis, TN 38152, USA; see https://pdfs.semanticscholar.org/288d/7952b6648.749fcbdcedabedf8f43cf7fda52.pdf. Letzter Zugriff September 2017 
31. Marc Lankhorst et al.; Enterprise Architecture at Work, Fourth Edition; Springer Verlag, Berlin 2017

32. Massimo Cossetino, et al.; Handbook on Agent-Oriented Design Processes, Springer Verlag, Berlin, 2014 und G. Wieß, R. Jakob; Agentenorientierte Softwareentwicklung.; Springer Verlag, Berlin, 2005

33. Gerhard Schewe, Geschäftsprozess, Gabler Wirtschaftslexikon. http://wirtschaftslexikon.gabler.de/Definition/geschaeftsprozess.html\#definition. letzter Zugriff 31.7.2017

34. Jörg Becker, Geschäftsprozessmodellierung, Enzyklopädie der Wirtschaftsinformatik, Online. Lexikon, http://www.enzyklopaedie-der-wirtschaftsinformatik.de/lexikon/daten-wissen/ Informationsmanagement/Information-/index.html. letzter Zugriff 31.7.2017

Open Access Dieses Kapitel wird unter der Creative Commons Namensnennung - Nicht kommerziell - Keine Bearbeitung 4.0 International Lizenz (http://creativecommons.org/licenses/ by-nc-nd/4.0/deed.de) veröffentlicht, welche die nicht-kommerzielle Nutzung, Vervielfältigung, Verbreitung und Wiedergabe in jeglichem Medium und Format erlaubt, sofern Sie den/die ursprünglichen Autor(en) und die Quelle ordnungsgemäß nennen, einen Link zur Creative Commons Lizenz beifügen und angeben, ob Änderungen vorgenommen wurden. Die Lizenz gibt Ihnen nicht das Recht, bearbeitete oder sonst wie umgestaltete Fassungen dieses Werkes zu verbreiten oder öffentlich wiederzugeben.

Die in diesem Kapitel enthaltenen Bilder und sonstiges Drittmaterial unterliegen ebenfalls der genannten Creative Commons Lizenz, sofern sich aus der Abbildungslegende nichts anderes ergibt. Sofern das betreffende Material nicht unter der genannten Creative Commons Lizenz steht und die betreffende Handlung nicht nach gesetzlichen Vorschriften erlaubt ist, ist auch für die oben aufgeführten nicht-kommerziellen Weiterverwendungen des Materials die Einwilligung des jeweiligen Rechteinhabers einzuholen.

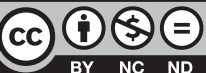


Mithilfe von Modellierungssprachen wird festgelegt, mit welchen Konzepten ein Ausschnitt der menschlich wahrgenommenen Realität beschrieben werden kann und wie diese zueinander in Beziehung gesetzt werden können. Modellierungssprachen stellen also das Vokabular und die Grammatik zur Verfügung, die benötigt werden, um Sachverhalte der menschlich wahrgenommenen Realität in Modellen abbilden zu können. Sie werden in der Folge strukturiert betrachtet.

Die Verwendung einer bestimmten Modellierungssprache schafft einen definierten Ausgangspunkt für die Modellbildung, auf den sich alle beteiligten Akteure - seien sie aktiv an der Erstellung oder passiv als Konsumenten beteiligt - beziehen können. Akteure können hier einerseits Personen sein, denen durch die Modellierungssprache ein gemeinsames, definiertes Vokabular zur Verfügung gestellt wird, um sich ein gemeinsames Verständnis über den Modellgegenstand zu bilden. Andererseits können Akteure auch Computersysteme sein, denen durch eine in der Modellierungssprache exakt spezifizierte Semantik von Modellelementen die Gelegenheit geboten wird, Modelle automationsgestützt weiter zu verarbeiten, um sie etwa als Grundlage für Workflow-Unterstützung einzusetzen.

Von der Wahl der Modellierungssprache hängt also ab, was in einem Modell überhaupt abgebildet werden kann, und was nicht sichtbar werden kann, weil die Modellierungssprache keine Konzepte dafür anbietet. Die Wahl der Modellierungssprache beeinflusst auch die Verwendbarkeit des Modells für unterschiedliche Zielgruppen: Während manche Modellierungssprachen eher zur Unterstützung der Kommunikation menschlicher Akteure konzipiert wurden, und dem entsprechend manchmal auch eine „,vage“ Abbildung von Sachverhalten ermöglichen, gibt es andere Modellierungssprachen, die für eine exakte Spezifikation von Sachverhalten konzipiert sind und deren Anwendungsfeld eher in der Aufbereitung von Modellen für die Verwendung in IT-Systemen liegt. Die Wahl einer Modellierungssprache ist also abhängig 
von der jeweiligen Zielsetzung der Modellbildung und damit ein wesentlicher Schritt hin zu einer erfolgreichen Unterstützung jener Aktivitäten, in denen die Modellierung eingebettet ist.

Im vorliegenden Abschnitt wollen wir die Grundlage für die sach- und personengerechte Auswahl einer passenden Modellierungssprache schaffen und stellen dazu unterschiedliche Sprachen mit deren jeweiligen Zielsetzungen und Sprachelementen vor. Der Fokus liegt hier auf der Abbildung von Geschäftsprozessen, weshalb alle im der Folge behandelten Sprachen im weiteren Sinne die Abbildung von Verhalten von Akteuren in Organisationen ermöglichen. Im Verständnis darüber, was nun ein Akteur sein kann und wie dieses Verhalten beschrieben wird, unterscheiden sich die Sprachen fundamental. Dies ist deren Entstehungsgeschichte und den jeweiligen Zielsetzungen geschuldet. Die Anordnung der einzelnen Ansätze folgt einer historischen Perspektive, um die Zusammenhänge zwischen den Sprachen und deren Entstehung deutlich hervortreten zu lassen. Für jede der Sprachen betrachten wir abschließend, wie sich diese zur Abbildung von Prozessen entsprechend unserer Definition eignen und wo ihre Abbildungsschwerpunkte bzw. Lücken liegen ${ }^{1}$.

\section{1 Überblick}

Flowcharts sind die älteste bis heute gebräuchliche Repräsentationsform von Modellen des Kontrollflusses in einem Computerprogramm. Aufgrund der Generalität ihrer Sprachelemente werden sie auch für Zwecke der Modellierung von Abläufen in Organisationen eingesetzt und sind hier deshalb als Einstieg in die grafische Prozessmodellierung zu betrachten. Sie weisen das Konzept der Verzweigung im Kontrollfluss zur Abbildung von alternativen Abläufen auf.

eEPKs (,erweiterte Ereignisgesteuerte Prozessketten“) stellten in Europa lange Zeit einen de-facto Industriestandard für die Geschäftsprozessmodellierung dar. Sie umfassen neben der Modellierung von Abläufen auch Elemente, mit denen Verantwortlichkeiten, Daten oder Leistungen modelliert werden können und sorgen damit dafür, dass Geschäftsprozesse gemeinsam mit ihrem organisationalen Kontext in Modellen abgebildet werden können. Darüber hinaus erlauben sie die explizite Modellierung von parallelen Aktivitäten und gehen damit bezüglich der Abbildung des Arbeitsablaufs über die Ausdrucksstärke von Flowcharts hinaus.

UML Aktivitätsdiagramme sind historisch gesehen eine Weiterentwicklung von Flowcharts und stellen heute als Teil der Unified Modeling Language (UML) den de-facto Standard zur Abbildung von Kontrollflüssen in Software dar. Auch sie ermöglichen die

\footnotetext{
${ }^{1}$ Die fachliche Grundlage zu diesem Kapitel findet sich in folgenden Quellen; www.iso.org, [1, 2].
} 
Abbildung von parallelen Abläufen. Anhand von Aktivitätsdiagrammen führen wir die Strukturierung von Modellen durch Partitionierung und Schachtelung ein und zeigen damit, wie Modelle auch anhand von Verantwortlichkeiten und nicht nur ausschließlich anhand des Arbeitsablaufs strukturiert werden können.

BPMN (Business Process Modelling and Notation) ist der heute meist eingesetzte Standard zur Abbildung von Geschäftsprozessen. Ausgehend von mehreren unterschiedlichen Modellierungssprachen - unter anderem von Aktivitätsdiagrammen - wurde eine Sprache definiert, die explizit zur Abbildung von Geschäftsprozessen geeignet sein sollte und mit deren Hilfe Modelle für unterschiedliche Zielsetzungen - von der Kommunikationsunterstützung bis hin zur Ausführung in Workflowmanagement-Systemen - erstellt werden können sollten. Aus Sicht der Sprachkonzeption ist die BPMN vor allem hinsichtlich ihrer Möglichkeiten zur kompakten Abbildung von komplexen Abläufen (etwa Ausnahmebehandlungen) interessant.

S-BPM (Subject-oriented Business Process Modeling) ist ein Modellierungsansatz, der die in einem Geschäftsprozess involvierten Akteure und deren Interaktionsverhalten ins Zentrum der Modellbildung stellt. Die sich daraus ergebende Modellierungssprache zeichnet sich durch einen geringen Umfang von Sprachelementen bei gleichzeitig umfassender Ausdrucksstärke zur Abbildung von Geschäftsprozessen aus. Sie wird hier einerseits als Vertreterin eines nicht vorrangig ablauforientierten Herangehens an die Geschäftsprozessmodellierung vorgestellt und bildet außerdem in der Sprachkonzeption eine Alternative zur BPMN und ihrem sehr umfangreichen Satz an Modellierungselementen.

\subsection{Flowcharts}

Flowcharts (oder deutsch: Flussdiagramme bzw. ursprünglich Programmablaufpläne) ermöglichen es, einfache, sequenzielle Prozesse abzubilden. Ein sequenzieller Prozess zeichnet sich dadurch aus, dass zu keinem Zeitpunkt mehr als eine Aktivität gleichzeitig durchgeführt wird - parallele Abläufe können also nicht abgebildet werden. Erstmals beschrieben wurden Flowcharts im Rahmen der industriellen Produktionsplanung in den 1920er-Jahren. Ende der 1940er-Jahre wurden sie für die Beschreibung von Prozessen in der aufkommenden Informationstechnologie adaptiert. Seit Mitte der 1960er-Jahre stellen sie eine standardisierte Repräsentationsform für Programmabläufe dar. Bis heute sind sie ein Mittel der Wahl, um Abläufe in Computerprogrammen oder auch Prozesse in Organisationen darzustellen, solange deren Komplexität die Ausdrucksstärke eines Flowchart nicht übersteigt.

Die Einschränkung der Ausdrucksstärke von Flowcharts ist deren historischen Entwicklung geschuldet. Sowohl in der industriellen Produktionsplanung als auch in frühen Computersystemen war es nicht notwendig, parallele Abläufe abbilden zu können. 
Durch die eingeschränkten zur Verfügung stehenden Ressourcen (in Computern: nur eine CPU bzw. nur ein Prozessorkern) war es schlicht nicht notwendig, Sprachelemente zur Abbildung etwa von parallelen Abläufen zur Verfügung zu stellen.

\subsubsection{Notationselemente}

Flowcharts kommen heute in vielen unterschiedlichen Varianten vor, die sich vor allem in der verwendeten Notation (d. h. der graphischen Darstellung) unterscheiden. Die Semantik der Sprachelemente ist aber allen gemein: Grundsätzlich wird eine beliebige Anzahl von Operationen (also Aktivitäten, dargestellt als Rechtecke) definiert. Diese werden mittels gerichteten Verbindungen (dargestellt als Pfeile) in die Reihenfolge gebracht, in der sie ausgeführt werden sollen. Terminierungselemente (dargestellt als abgerundete Rechtecke) kennzeichnen den Beginn und das Ende eines Prozesses (siehe Abb. 3.1).

Ein wesentliches Mittel der Beschreibung von Prozessen - sowohl in einem Computerprogramm als auch in Organisationen - stellt die Abbildung von alternativen Operationen dar. Die Auswahl einer Alternative ist üblicherweise abhängig von einer Bedingung, die geprüft werden kann - in einem Computerprogramm könnte dies das Überschreiten eines Grenzwertes in einer Variablen sein, in einer Organisation etwa das Vorhandensein eines bestimmten Dokuments oder die Entscheidung einer für die Ausführung des Ablaufs verantwortlichen Person. Alternativen werden in Flowcharts durch Verzweigungen abgebildet (dargestellt als Rauten), die durch einen eingehenden Pfeil von der vorhergehenden Operation und mit zwei ausgehenden Pfeilen mit den alternativ ausgeführten nachfolgenden Operationen verbunden sind.

Die Einschränkung auf genau zwei (und nicht mehrere) nachfolgende Operationen ist ebenfalls der Herkunft von Flowcharts aus der Abbildung von Computerprogrammen geschuldet, da diese eine Bedingung üblicherweise ausschließlich binär (d. h. als wahr oder falsch) auswerten können. Soll eine Bedingung auf mehr als zwei Ausprägungen geprüft werden, so müssen in Flowcharts mehrere Verzweigungen kaskadiert eingesetzt werden. Die ausgehenden Verbindungen werden mit der jeweiligen Ausprägung beschriftet, bei der nach der Prüfung der Bedingung das Programm fortgesetzt wird. Eine wiederholte Ausführung von Operationen (etwa solange noch Dokumente vorhanden sind, die bearbeitet werden müssen) wird abgebildet, in dem mit einer ausgehenden Verbindung zu einer früheren Operation zurückgesprungen wird. Die andere ausgehende
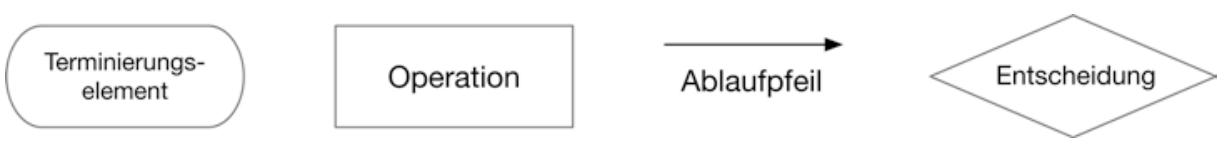

Abb. 3.1 Notationselemente von Flowcharts 
Verbindung leitet dann zu jenem Ablaufteil über, der ausgeführt wird, sobald die wiederholte Ausführung abgeschlossen ist.

Neben diesen Grundelementen bieten Flowcharts in manchen Varianten noch spezielle Sprachelemente, die vor allem der Abbildung von spezifischen Operationen im Anwendungsgebiet dienen (bei Computersystemen etwa Ein- oder Ausgabeoperationen). Für das konzeptionelle Verständnis von Flowcharts und vor allem deren Anwendung in der Geschäftsprozessmodellierung sind diese jedoch nicht relevant.

Im Folgenden betrachten wir die Verwendung der Notationselemente anhand von Beispielen aus der Geschäftsprozessmodellierung. Die verwendete Notation orientiert sich an den durch das ANSI bzw. die DIN in den 1960er-Jahren festgelegten Symbolik.

\subsubsection{Beispiele}

Dieses Beispiel (siehe Abb. 3.2) zeigt einen Prozess mit einer einzelnen Operation, in der ein Antrag (über den wir hier nicht mehr wissen) bearbeitet wird. Der Prozess endet, nachdem die Bearbeitung des Antrags abschlossen wurde.

Im obenstehenden Beispiel (siehe Abb. 3.3) ist der Prozess um eine Entscheidung erweitert. Der Antrag wird geprüft, das Ergebnis dieser Prüfung ermöglicht das Treffen einer Entscheidung über dessen Bestätigung oder Ablehnung. Das Bestätigen bzw. Ablehnen selbst sind wiederum Operationen. Die ausgehenden Verbindungen werden zusammengeführt, nachdem die alternativen Zweige abgeschlossen sind.

Ist es nun notwendig, eine Entscheidung zu treffen, die mehr als zwei mögliche Ausgänge hat, muss dieser Sachverhalt in Flowcharts mittels kaskadierter Entscheidungselemente dargestellt werden. Dies ist im obenstehenden Beispiel (siehe Abb. 3.4) zu sehen. Offensichtlich handelt es sich bei unseren Anträgen um Investitionsbegehren. Die erste Entscheidung prüft nun, ob die Investition weniger als EUR 1000,- kostet. Ist dies der Fall, wird der Antrag direkt bestätigt. Ist dies nicht der Fall, kommt es zu einer

Abb. 3.2 Einfaches

Flowchart

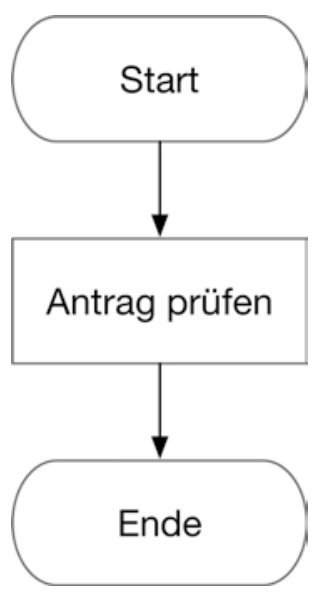




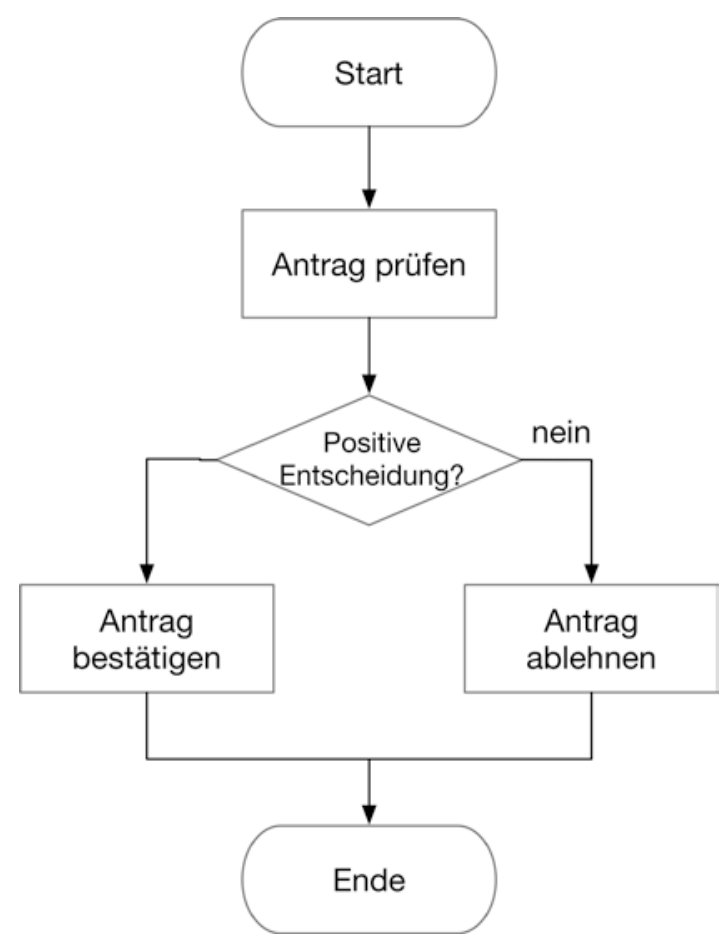

Abb. 3.3 Flowchart mit Entscheidung

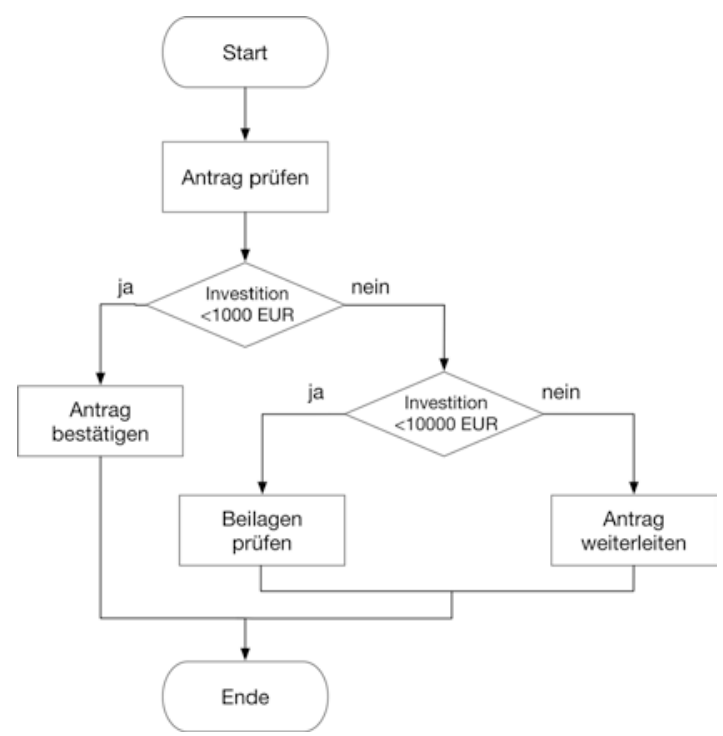

Abb. 3.4 Flowchart mit kaskadierten Entscheidungen 
zweiten Entscheidung. Diese prüft, ob die Antragssumme kleiner als EUR 10.000,- ist. Wenn dies der Fall ist, liegt die Antragssumme zwischen EUR 1000,- und EUR 9999,-, was zu einer Prüfung der Beilagen zum Antrag führt. Wenn die Antragssumme nicht kleiner als EUR 10.000,- ist, also EUR 10.000,-- oder mehr beträgt, wird der Antrag weitergeleitet. Wir erfahren hier nichts über das Ziel der Weiterleitung, da Flowcharts dafür keine Notationselemente anbieten.

Wie bereits erwähnt, können Verzweigungen auch genutzt werden, um Teile eines Prozesses wiederholt abzuarbeiten. Dazu wird die Entscheidung am Ende des zu wiederholenden Teils eingefügt und ein ausgehender Zweig vor die erste Operation des zu wiederholenden Teils zurückgeführt. Der andere ausgehende Zweig führt den Prozess nach der wiederholten Abarbeitung weiter. Im obenstehenden Beispiel (siehe Abb. 3.5) werden also solange Anträge bearbeitet, solange noch weitere Anträge vorhanden sind.

Wichtig ist hier $\mathrm{zu}$ verstehen, dass zumindest ein Antrag bearbeitet werden muss, bevor es zu einer Entscheidungsprüfung kommt. Soll der Prozess enden können, wenn gar keine Anträge vorliegen, müsste eine zusätzliche Entscheidung am Beginn des Prozesses eingefügt werden (,Anträge vorhanden?“), von der eine ausgehende Verbindung („nein“) direkt zum Ende des Prozesses führt. Die andere ausgehende Kante (,ja“) würde zum bereits abgebildeten Prozessablauf weiterführen.

\subsubsection{Einordnung}

Flowcharts bieten eine einfache Möglichkeit, Geschäftsprozesse hinsichtlich der logischen Abfolge der enthaltenen Aktivitäten abzubilden. Andere Aspekte eines Geschäftsprozesses, wie Daten oder Verantwortlichkeiten, sind im Sprachumfang nicht vorgesehen und können deshalb nicht abgebildet werden.

Die Abbildung von parallelen Abläufen ist ebenfalls nicht möglich. Dies ist ein maßgeblicher Grund dafür, dass Flowcharts zum Teil von aktuelleren Sprachen wie UML

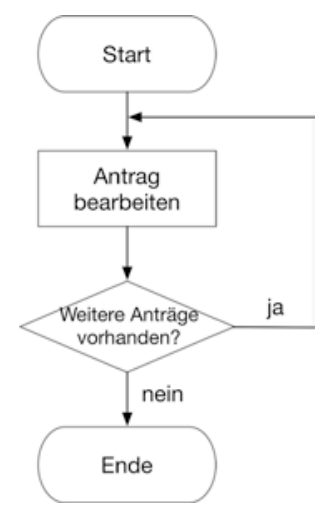

Abb. 3.5 Flowchart mit Schleife 
Aktivitätsdiagrammen oder BPMN abgelöst werden, die dafür Konstrukte anbieten. Da Flowcharts keine Verantwortlichkeiten abbilden lassen, ist auch die Abbildung von Kommunikationsvorgängen nicht möglich - diese Einschränkung wurde ebenfalls durch modernere Sprachen adressiert, die wir in den folgenden Abschnitten behandeln.

\subsection{Ereignisgesteuerte Prozessketten}

Ereignisgesteuerte Prozessketten (EPKs) waren lange Zeit in Europa der de-facto Standard zur Abbildung in Geschäftsprozessen in der industriellen Praxis. Sie wurden als Teil des in Abschn. 2.5.1.4 bereits vorgestellten ARIS-Konzeptes spezifiziert und dienen dort als Mittel zur Abbildung von Modellen der Steuerungssicht einer Organisation - also jener Sicht, die sich mit den Abläufen in einer Organisation und der dort stattfindenden Verknüpfung zwischen deren unterschiedlichen Ressourcen beschäftigt. Ressourcen können hier im Speziellen aus aktiver Sicht die handelnden Organisationsmitglieder bzw. -einheiten, sowie aus passiver Sicht die benötigten und manipulierten Daten sein.

Die EPK verknüpft die Funktionen, die eine Organisation ausführen kann, auf Basis von auftretenden Ereignissen miteinander. Das grundlegende Abbildungsprinzip ist dabei, dass eine Funktion immer durch ein Ereignis ausgelöst wird - dass also vor einer Funktion immer ein Ereignis modelliert werden muss, um feststellen zu können, ob mit der Ausführung einer Funktion begonnen werden kann. In der umfassenderen Variante „erweiterte EPK“ (eEPK) sind den Funktionen die für deren Ausführung relevanten Elemente der anderen ARIS-Sichten zugeordnet. Insbesondere können aus der Organisationssicht die ausführenden Akteure, Rollen oder Organisationseinheiten zugeordnet werden, aus der Datensicht die relevanten Dokumente oder Datenobjekte. Wenn eine Funktion eine abrechenbare Leistung erbringt, so kann diese durch Elemente der Leistungssicht abgebildet werden.

Neben der Abbildung von Entscheidungen im abgebildeten Ablauf ermöglicht die EPK auch die Abbildung von parallelen Abläufen in einen Geschäftsprozess. Dafür stellt sie zusätzliche Notationselemente zur Verfügung, die sich an den Operatoren der Bool'schen Logik orientieren - mittels UND-Konnektoren können parallel ablaufende Prozesszweige abgebildet werden, der XOR-Konnektor dient dazu, Entscheidungen abzubilden, bei denen genau eine Alternative gewählt werden muss (dies entspricht dem Entscheidungselement bei Flowcharts). Mit dem ODER-Konnektor können Prozesse abgebildet werden, bei denen aus einer oder mehreren Alternativen gewählt werden kann.

\subsubsection{Notationselemente der EPK}

Das Grundelement zur Beschreibung von Geschäftsprozessen ist bei EPKs, ähnlich wie bei Flowcharts, die Funktion (bei Flowcharts: Operation). Die Abfolge der Funktionen in einem Prozess wird aber nicht ausschließlich über Verbindungspfeile festgelegt. Durch 
den Einsatz von Ereignissen wird der Ablauf hier genauer spezifiziert. Jede Funktion wird durch ein Ereignis ausgelöst und erzeugt selbst ein oder mehrere Ereignisse. Ein Prozess wird also durch eine Folge von Ereignissen und Funktionen dargestellt, wobei sich Ereignisse und Funktionen immer abwechseln. Bei der Benennung muss hier darauf geachtet werden, dass Funktionen einen Vorgang beschreiben (etwa „Antrag prüfen“), während Ereignisse einen Zustand beschreiben (etwa „Antrag bestätigt“ oder „Antrag abgelehnt").

Eine EPK beginnt und endet immer mit einem Ereignis. Ereignisse, die einen Prozess auslösen, sind Start-Ereignisse. Ereignisse, die den Abschluss eines Prozesses beschreiben, sind Ende-Ereignisse. Folgeprozesse können durch Ende-Ereignisse eines vorangegangenen Prozesses ausgelöst werden, d. h. ein Ende-Ereignis kann in einem anderen Prozess ein auslösendes Start-Ereignis darstellen.

Durch den Einsatz unterschiedlicher Konnektoren können nun in Kombination mit Ereignissen verschiedene Ablaufvarianten eines Prozesses innerhalb eines Modells abgebildet oder auch die Möglichkeit zur parallelen Ausführung von Funktionen (wenn diese nicht voneinander abhängig sind) dargestellt werden (siehe Abb. 3.6). Es lassen sich der UND-, ODER- und XOR-Konnektor unterscheiden.

Wird ein UND-Konnektor mit mehreren ausgehenden Verbindungen verwendet, so bedeutet dies, dass alle ausgehenden Pfade parallel durchlaufen werden. Diese werden dann in der Regel an einer zeitlich bzw. kausal nachgelagerten Stelle wieder mit einem weiteren UND-Konnektor zusammengeführt. Die darauf folgende Funktion wird erst ausgeführt, wenn alle der zusammengeführten Pfade abgeschlossen sind.

Ein ODER-Konnektor mit mehreren ausgehenden Kanten zeigt an, dass ein oder mehrere der folgenden Pfade parallel durchlaufen werden. Diese Pfade werden zumeist an einer späteren Stelle wiederum mit einem ODER-Konnektor zusammengeführt, wobei der dann folgende Prozessschritt erst zu einem Zeitpunkt durchgeführt wird, wenn genau die Pfade, die bei der entsprechenden ODER-Verzweigung ausgewählt wurden, abgearbeitet sind. Wichtig ist hier, dass die zu aktivierenden Pfade zum Zeitpunkt des Eintreffens beim Konnektor ausgewählt werden müssen. Hinter jedem ODER-Konnektor muss sich auf jedem Pfad ein Ereignis befinden, das durch die vorangegangene Funktion ausgelöst werden könnte. Es werden jene Pfade aktiviert, deren erste Ereignisse tatsächlich eingetreten sind.

Ein XOR-Konnektor steht schließlich für ein ,exklusives Oder“ bzw. ein ,entweder, oder“. Die Verwendung eines XOR-Konnektors mit mehreren ausgehenden Kanten bedeutet, dass bei der Durchführung des Prozesses genau einer der folgenden Pfade

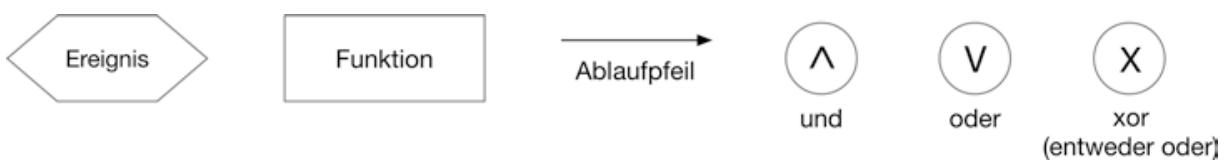

Abb. 3.6 Notationselemente von EPKs 
ausgewählt wird. Er eignet sich also zur Abbildung von einander ausschließenden Alternativen in der Prozessabarbeitung. Bei der Zusammenführung dieser Pfade mit einem XOR-Konnektor wird der folgende Schritt dann ausgeführt, wenn der ausgewählte Pfad fertig durchlaufen wurde. Auch bei einem XOR-Konnektor muss sich auf jedem Pfad ein Ereignis befinden, das durch die vorangegangene Funktion ausgelöst werden könnte. Diese Ereignisse müssen einander ausschließen. Es wird jener Pfade aktiviert, dessen erstes Ereignis tatsächlich eingetreten ist. Im Gegensatz zu Flowcharts ist es hier möglich, auch mehr als zwei Alternativen zu beschreiben, solange die eingesetzten Ereignisse einander ausschließen.

\subsubsection{Beispiele zur EPK}

Wir verwenden hier vorerst die gleichen Beispiele, wie sie für Flowcharts angegeben wurden, um die Unterschiede in der Notation zu visualisieren.

Dieses Beispiel (siehe Abb. 3.7) zeigt einen Prozess mit einer einzelnen Funktion, in der ein Antrag (über den wir hier nicht mehr wissen) bearbeitet wird. Der Prozess endet, nachdem der Antrag geprüft wurde.

Im obenstehenden Beispiel (siehe Abb. 3.8) ist der Prozess um eine Entscheidung erweitert. Der Antrag wird geprüft, das Ergebnis dieser Prüfung ermöglicht das Treffen einer Entscheidung auf Basis dessen positiver oder negativer Beurteilung. Das Bestätigen bzw. Ablehnen selbst sind ebenfalls Funktionen. Die ausgehenden Verbindungen werden mit einem XOR-Konnektor zusammengeführt, nachdem die alternativen Zweige abgeschlossen sind.

Die Darstellung von Entscheidungen, die mehr als zwei mögliche Ausgänge haben, ist hier nun einfacher möglich als bei Flowcharts (siehe Abb. 3.9). Dem XOR-Konnektor werden in diesem Fall drei Ereignisse nachgelagert, die sich alle auf die Investitionssumme beziehen und einander ausschließen.

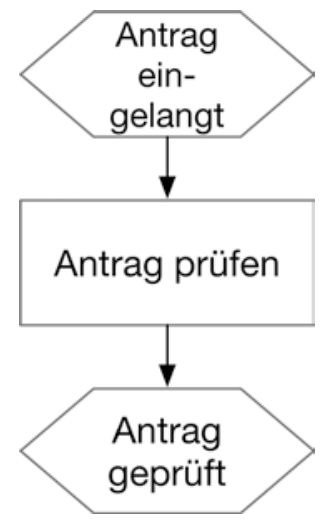

Abb. 3.7 Einfache EPK 


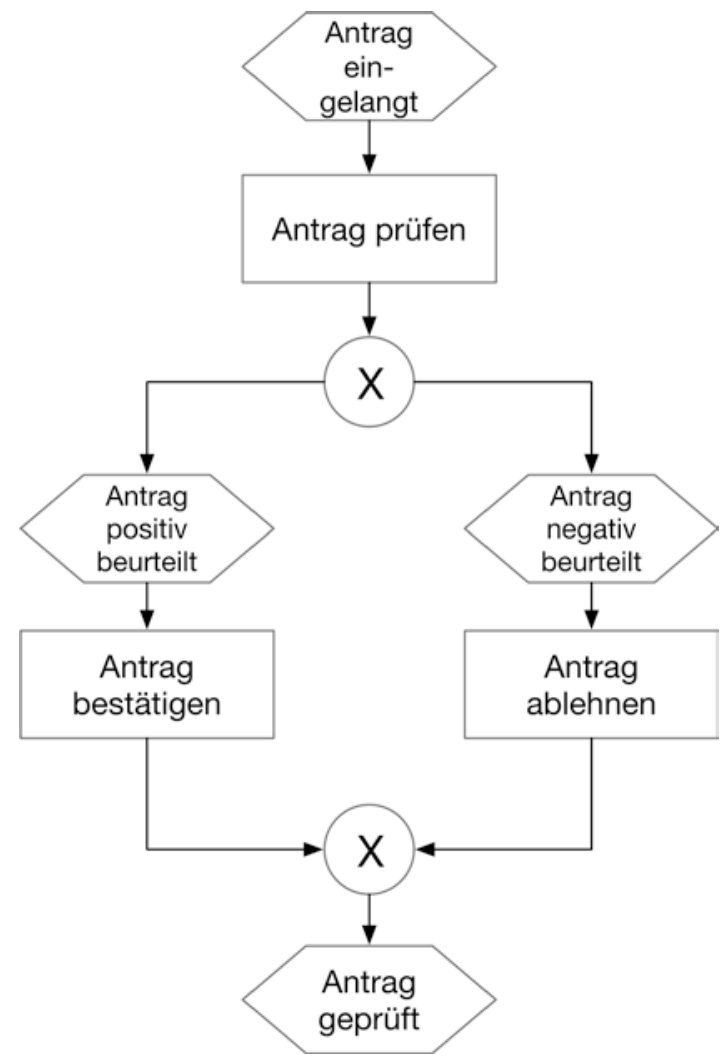

Abb. 3.8 EPK mit Verzweigung

Der XOR-Konnektor kann auch genutzt werden, um Teile eines Prozesses wiederholt abzuarbeiten. Dazu wird der Konnektor am Ende des zu wiederholenden Teils eingefügt und ein ausgehender Zweig auf das Ereignis zurückgeführt, das den zu wiederholenden Teil auslöst. Der andere ausgehende Zweig führt den Prozess nach der wiederholten Abarbeitung weiter und muss dementsprechend zu einem Ereignis führen, das den Abbruch der Wiederholung auslöst. Im obenstehenden Beispiel (siehe Abb. 3.10) werden also solange Anträge bearbeitet als Anträge vorhanden sind.

Der UND-Konnektor kann zum Einsatz kommen, wenn zwei Funktionen unabhängig voneinander ausgeführt werden können. Der im obigen Beispiel (siehe Abb. 3.11) modellierte Prozess ist also nur korrekt, wenn die Beilagen tatsächlich unabhängig vom Antrag geprüft werden können. Ist dies nicht der Fall, müssten die beiden Funktionen sequenziell angeordnet werden. Aus Sicht der Modellerstellung ist hier anzumerken, dass dem UND-Konnektor im Gegensatz zu den anderen Konnektoren nicht unmittelbar Ereignisse folgen müssen, da ja keine Entscheidung getroffen wird, sondern in jedem Fall alle ausgehenden Zweige aktiviert werden. 


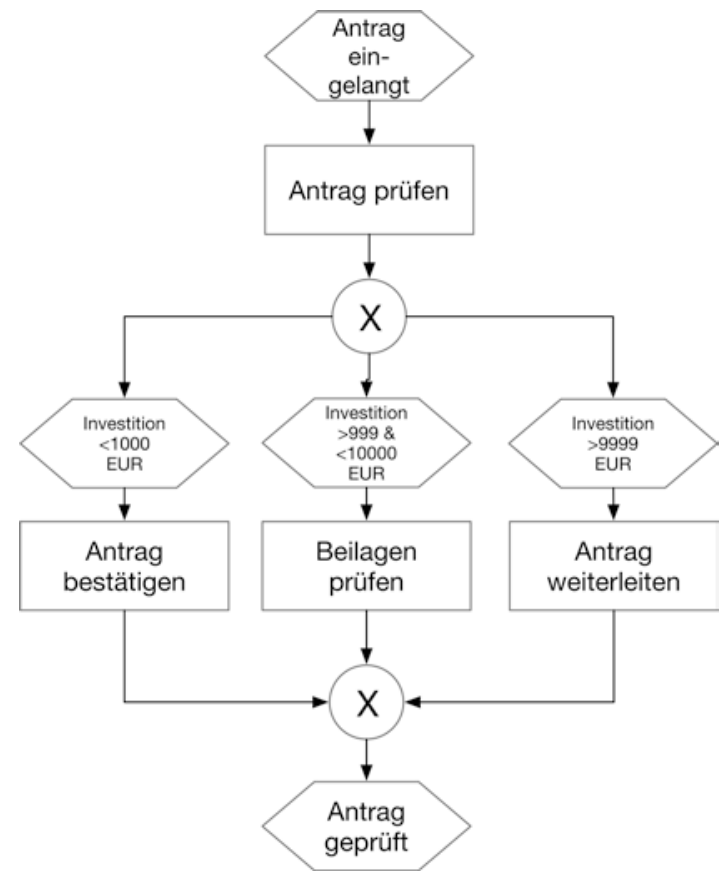

Abb. 3.9 EPK mit Verzweigung in drei Alternativen

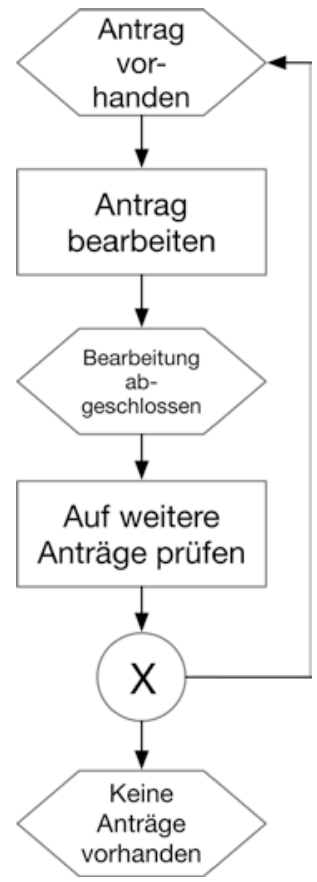

Abb. 3.10 EPK mit Schleife 


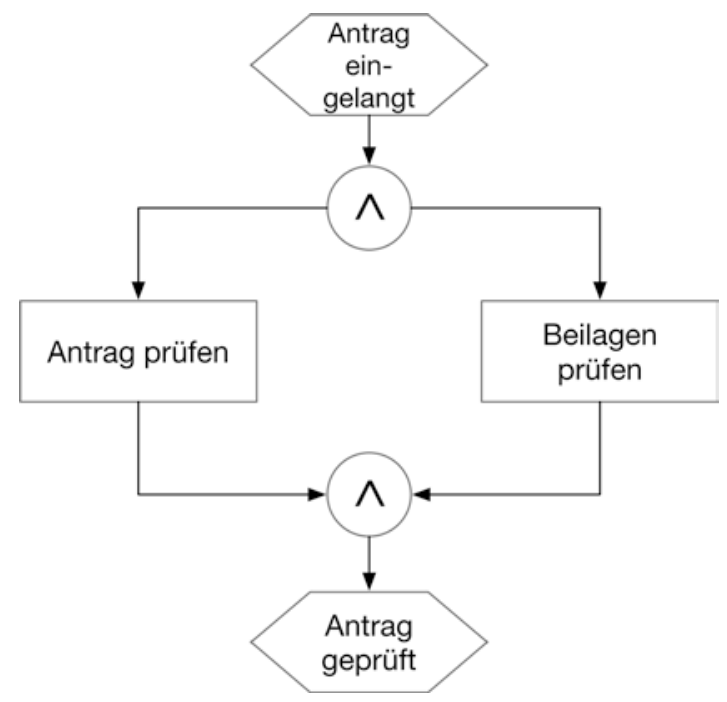

Abb. 3.11 EPK mit UND-Konnektor und parallel ablaufenden Zweigen

Die obige Abbildung (siehe Abb. 3.12) zeigt ein Beispiel für den Einsatz des ODER-Konnektors. Hier gehen wir davon aus, dass ein Antrag Angebote oder Stellungnahmen oder beides enthalten kann (aber zumindest Angebote oder Stellungnahmen enthalten muss). Wären Angebote und Stellungnahmen vollkommen optional (könnten also beide fehlen), würde deren grundsätzliche Notwendigkeit mit einem vorgelagerten XOR-Konnektor und entsprechenden Ereignissen zu prüfen sein. Alternativ könnte nach dem ODER-Konnektor ein zusätzlicher Zweig mit einem Ereignis „Antrag alleine genügt" eingefügt werden. In beiden Fällen muss darauf geachtet werden, dass die Bedingung, dass sich Ereignisse und Funktionen auf allen Pfaden durch den Prozess abwechseln, nicht verletzt wird. Falls nicht anders möglich, muss dies durch eine „Dummy“-Funktion, die keine Aktivität verursacht, gewährleistet werden.

\subsubsection{Ergänzende Notationselemente der eEPK}

Die eEPK ergänzt nun den in einer EPK abgebildeten Geschäftsprozess um Information über dessen Ausführungskontext. Insbesondere werden hier den Funktionen Verantwortlichkeiten und Ressourcenbedarf zugeordnet. Die grundsätzlichen Regeln einer EPK bleiben wie oben beschrieben in Kraft. Die zusätzlichen Elemente können Funktionen zugeordnet werden. Ereignisse sind nicht betroffen. Wir verzichten an dieser Stelle auf eine vollständige Beschreibung der möglichen Elemente und führen nur die gängigsten an (siehe Abb. 3.13).

Verantwortlichkeiten werden durch Organisationale Einheiten abgebildet. Solche Einheiten sind üblicherweise nicht konkrete Personen, sondern werden abstrakt durch 


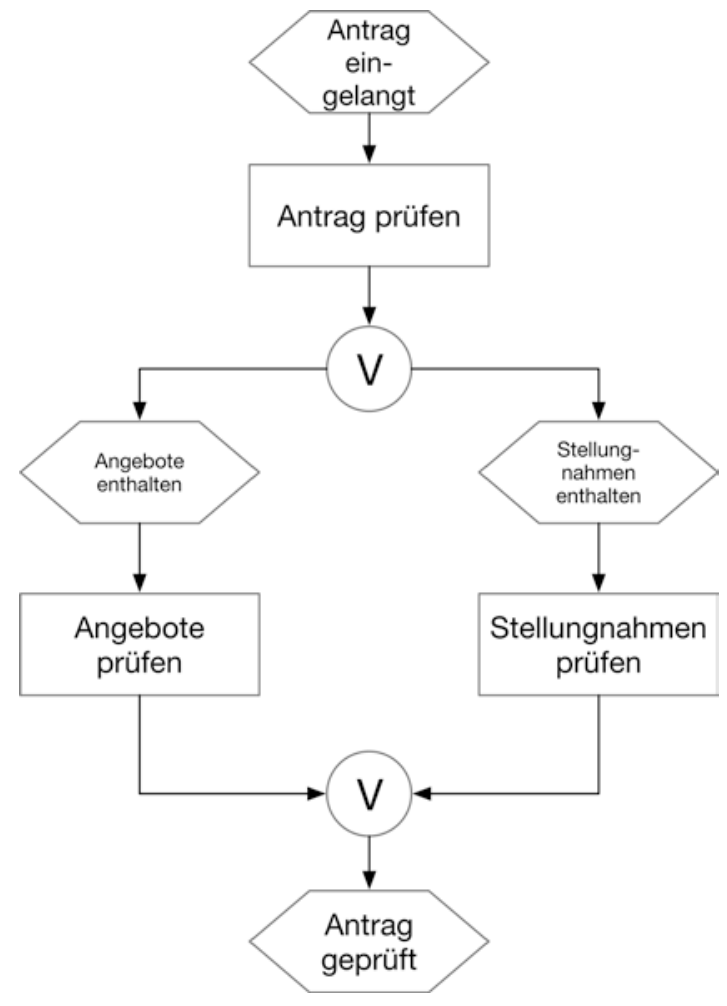

Abb. 3.12 EPK mit ODER-Konnektor und optional parallel ablaufenden Zweigen

Org. Einheit

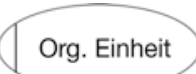

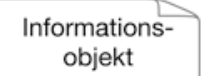

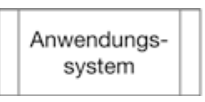

$\overrightarrow{\text { Datenfluss }} \quad \overrightarrow{\text { Zuordnung }}$

Abb. 3.13 Zusätzliche Notationselemente in eEPKs

die Benennung von Rollen (etwa: Geschäftsführung) oder Abteilungen (etwa: Finanzbuchhaltung) angeführt. Dadurch wird gewährleistet, dass die Spezifikation eines Prozesses von der Verfügbarkeit konkreter Personalressourcen unabhängig ist und erst zur Durchführungszeit konkrete Personen zugewiesen werden müssen. Die Zuordnung zu einer Funktion erfolgt durch ungerichtete Linien. Eine organisationale Einheit kann so mehreren Funktionen zugeordnet werden. Auch ist es möglich, organisationale Einheiten mehrfach anzuführen, wenn die Prozessdarstellung dadurch übersichtlicher wird.

In ähnlicher Form werden Anwendungssysteme modelliert. Sie kennzeichnen die Notwendigkeit, bei der Ausführung einer Funktion ein bestimmtes IT-System einzusetzen (z. B. ein ERP-System oder eine Datenbank). Sie werden Funktionen ebenfalls mit ungerichteten Linien zugeordnet. 
Informationsobjekte werden dazu verwendet, um die Datenverarbeitung in einem Geschäftsprozess darzustellen. Ein Informationsobjekt kann beliebig umfassend sein (also ein einzelner Wert ebenso wie ein vollständiges Dokument) und wird einer Funktion immer mittels einem gerichteten Pfeil zugeordnet, der den Datenfluss beschreibt. Endet der Pfeil bei der Funktion, bedeutet dies, dass das Informationsobjekt zur Durchführung der Funktion benötigt wird. Endet der Pfeil beim Informationsobjekt, so bedeutet dies, dass es durch die Funktion erzeugt oder verändert wird. Ein Informationsobjekt kann dabei mehrere ein- und ausgehende Verbindungen haben, wodurch sowohl dessen Entstehung als auch dessen Verwendung in einem Geschäftsprozess beschrieben werden kann.

\subsubsection{Beispiele zur eEPK}

Zur Illustration von eEPKs ergänzen wir eines der oben angeführten Beispiele um den stattfindenden Datenfluss sowie die benötigten Personal-Ressourcen. Anwendungssysteme würden analog zur Verwendung von organisationalen Einheiten modelliert werden (siehe Abb. 3.14).

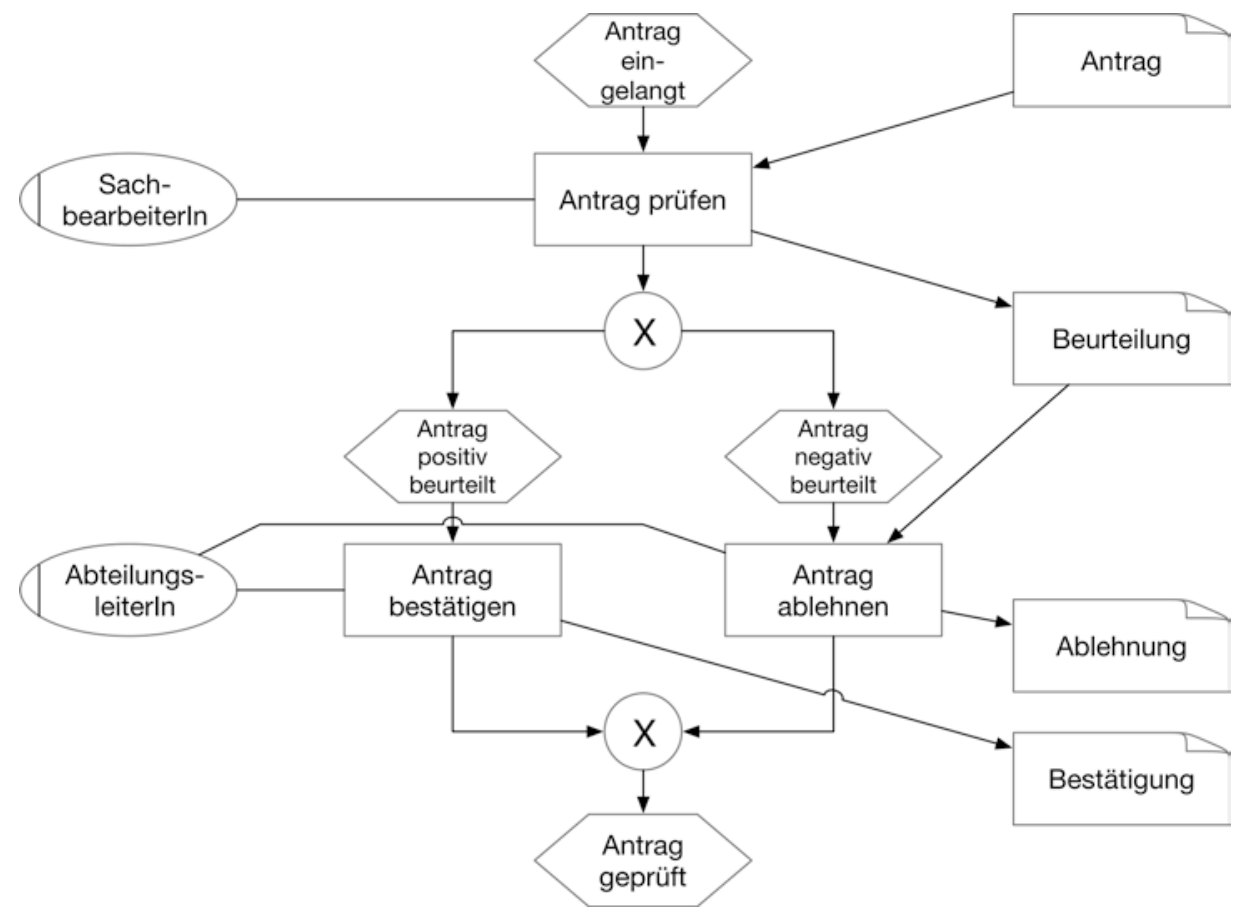

Abb. 3.14 Beispiel für eine eEPK 
Hinsichtlich des Datenflusses erkennen wir nun, dass zum Prüfen des Antrags der eigentliche Antrag vorhanden sein muss. Diese Prüfung führt nicht nur zur positiven oder negativen Beurteilung eines Antrags im Prozessablauf, sondern auch zu einem Informationsobjekt, in dem die Beurteilung gespeichert ist. Im Falle einer negativen Beurteilung wird dieses Informationsobjekt benötigt, um die Ablehnung zu erstellen (wir können also annehmen, dass die Ablehnung eine inhaltliche Begründung enthält). Im Falle der Bestätigung des Antrags wird das Datenobjekt „Beurteilung“ nicht mehr benötigt - wir können also annehmen, dass in diesem Fall keine weitere Begründung erfolgt.

Hinsichtlich der Verantwortlichkeiten erkennen wir nun, dass mehrere organisationale Einheiten am Prozess beteiligt sind. Während die Antragsprüfung durch einen Sachbearbeiter erfolgt, ist für die endgültige Bestätigung oder Ablehnung der Abteilungsleiter zuständig. Wichtig ist hier, dass die Ereignisse, auf Basis derer die Entscheidung getroffen wird, durch die Funktion „Antrag prüfen“ ausgelöst werden, für die der Sachbearbeiter zuständig ist.

\subsubsection{Einordnung}

Die EPK bietet umfassendere Möglichkeiten zur Abbildungen von Geschäftsprozessen als Flowcharts. Gemein ist ihnen die Orientierung an den Abläufen innerhalb einer Organisation als primäres Strukturierungsmerkmal des Geschäftsprozesses (d. h. alle im Modell abgebildete Information ist an der Beschreibung des Prozessablaufs verankert). Dies ist zwar naheliegend, wenn ein organisationaler Prozess beschrieben wird, aber - wie wir später sehen werden - nicht unbedingt die einzige Möglichkeit. Andere Modellierungssprachen nutzen Akteure oder Daten als primäre Strukturierungsmerkmale, an denen alle anderen Informationen verankert werden und machen so Aspekte eines Prozesses sichtbar, die in (e)EPKs nur implizit abgebildet werden können (wie etwa der Übergang von Verantwortlichkeiten im Prozess und der dabei notwendigen Kommunikation zwischen den Akteuren).

Die Notwendigkeit, Funktionen und Ereignisse einander immer abwechseln zu lassen, führt in EPKs zu sehr umfangreichen und zum Teil nur schwer verständlichen Modellen. Außerdem birgt sie das Risiko, Modellierende bei der Erstellung der Modelle dazu zu verleiten, Trivial-Ereignisse zu formulieren, die dem Modell keine Information hinzufügen (etwa: Funktion: „Aufgabe ausführen“, Ereignis: „Aufgabe ausgeführt“). Korrekt eingesetzt, bietet diese Systematik aber Vorteile: Einerseits können Prozesse exakter beschrieben und abgegrenzt werden als etwa mit Flowcharts, andererseits erlaubt die EPK eine Verknüpfung zwischen der Sicht auf die Fähigkeiten einer Organisation (ihrer Funktionen) und der Sicht darauf, wie sie mithilfe ihrer Fähigkeiten auf externe Reize oder Ereignisse innerhalb der Organisation selbst reagiert. So können organisationale Fähigkeiten generisch beschrieben und mehrfach in Prozessen eingesetzt werden, wodurch Ineffizienzen durch Replikation vermieden werden.

Aus pragmatischer Sicht hat sich in der Praxis jedoch gezeigt, dass sowohl die Spezifikation generischer Funktionen als auch prozessspezifischer Ereignisse nicht immer 
in der notwendigen Gesamtheit umsetzbar ist. Modernere Ansätze, wie die im folgenden behandelten Aktivitätsdiagramme oder die BPMN, kennen deshalb zwar nach wie vor das Konzept von Ereignissen, setzen diese aber nur ein, wenn tatsächlich auf einen externen Reiz (wie eine eingehende Nachricht, einen Fehler, oder einen Zeitablauf) eingegangen werden soll.

In Abgrenzung zu den Modellierungssprachen mit technisch orientierter Entstehungsgeschichte (wie Flowcharts oder die im nächsten Abschnitt beschriebenen Aktivitätsdiagramme) verfolgen die eEPK und das umgebende ARIS-Framework als ein ursprünglich aus der Betriebswirtschaftslehre stammendes Konzept einen umfassenderen Ansatz zur Beschreibung von Geschäftsprozessen. Die Berücksichtigung von Daten, Verantwortlichkeiten, aber auch Zielen oder Leistungen (die hier nicht diskutiert wurden) ermöglicht insgesamt eine umfassende Modellierung von Geschäftsprozessen, die bis heute Einfluss auf die Gestaltung von Modellierungssprachen zur Abbildung organisationaler Phänomene (wie Geschäftsprozessen oder Unternehmensarchitekturen) hat.

\subsection{UML Aktivitätsdiagramme}

Das Aktivitätsdiagramm wurde als Teil der UML (Unified Modeling Language) definiert, die eine Sammlung von Diagrammen enthält, die zur Spezifikation von Softwaresystemen geeignet sind. Das Aktivitätsdiagramm dient dabei analog zum Flowchart zur Abbildung des Verhaltens eines Softwaresystems, stellt aber ob seiner jüngeren Entstehungsgeschichte auch Elemente zur Abbildung verteilter und paralleler Prozessabläufe zur Verfügung. Wie das Flowchart ist auch das Aktivitätsdiagramm zur Abbildung organisationaler Abläufe, also von Geschäftsprozessen, geeignet. Während dieses bis heute zu diesem Zweck eingesetzt wird, hat sich der Fokus im Bereich der Geschäftsprozessmodellierung stark hin zur BPMN (Business Process Modeling and Notation) verschoben. Diese wurde vom gleichen Standardisierungsgremium wie die UML spezifiziert und hat viele Elemente des Aktivitätsdiagramms übernommen. Die BPMN fokussiert explizit auf die Anforderungen der Geschäftsprozessmodellierung und der dort abzubildenden organisationalen Aspekte, die wir bereits im Rahmen der EPKs diskutiert haben.

\subsubsection{Notationselemente}

Ein Aktivitätsdiagramm beschreibt per Definition immer eine Aktivität, die sich aus einzelnen Aktionen zusammensetzt (,Aktivität“ wird hier also analog zu „Prozess“ verwendet). Eine Aktion entspricht einer Operation bei Flowcharts oder einer Funktion bei EPKs (siehe Abb. 3.15).

Eine Aktivität beginnt üblicherweise mit einem Startknoten und endet mit einem Endknoten (analog zu den Terminierungselementen bei Flowcharts). Zwischen diesen Knoten werden die enthaltenen Aktionen angegeben und durch Ablaufpfeile in die 
Abb. 3.15 Notationselemente von Aktivitätsdiagrammen

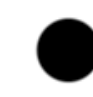

Startknoten

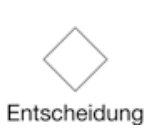

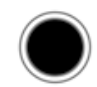

Endknoten

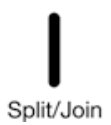

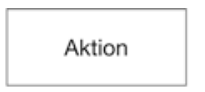

Ablaufpfeil

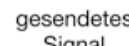

Signal

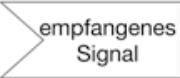

Datenobjekt

Abb. 3.16 Notationselemente zur Abbildung von Verantwortlichkeiten und Datenflüssen in Aktivitätsdiagrammen

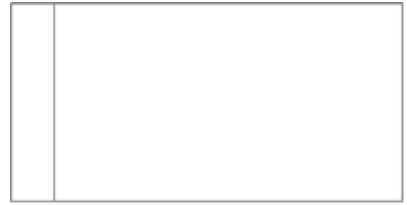

Partition

durchzuführende Reihenfolge gebracht. Zur Beeinflussung des Ablaufs ist es möglich, Entscheidungselemente einzufügen. Entscheidungen können beliebig viele ausgehende Zweige haben, deren Aktivierungsbedingungen sich einander ausschließen müssen. Die Bedingungen werden an den ausgehenden Verbindungen angeführt. Die Semantik des Entscheidungssymbols entspricht dem XOR in der EPK - für den ODER-Konnektor gibt es in Aktivitätsdiagrammen keine Entsprechung.

Um voneinander unabhängig parallel ausführbare Prozessteile abzubilden, bietet das Aktivitätsdiagramm das Split/Join-Element. Zum Aufspalten des Ablaufs eingesetzt, kann es beliebig viele ausgehende Verbindungen haben, die alle gleichzeitig aktiviert werden. Die so angelegten Zweige sollten durch ein Join wieder zusammengeführt werden. Der Ablauf wird erst fortgesetzt, sobald alle Zweige abgearbeitet sind.

Signale dienen der Kommunikation zwischen Prozessteilen in unterschiedlichen Aktivitäten (also in unterschiedlichen Diagrammen) oder innerhalb einer Aktivität, wenn Information für spätere Prozessteile bereitgestellt werden soll. Sie werden wie Aktionen in den Kontrollfluss eingebaut. Modelle müssen nicht immer vollständige Signalpaare (also gesendete und empfangene Signale) enthalten, sondern können auch Signale für nicht abgebildete Prozesse bereitstellen (also nur ein gesendetes Signal enthalten), oder dieses von einem nicht abgebildeten Prozess entgegennehmen (also nur ein empfangendes Signal enthalten). Empfangene Signale können außerdem eine Aktivität auslösen und damit den Startknoten in einem Diagramm ersetzen.

Das Aktivitätsdiagramm bietet auch Elemente, um Verantwortlichkeiten und Datenflüsse abzubilden (siehe Abb. 3.16). Verantwortlichkeiten werden mittels Partitionen abgebildet. Partitionen sind Elemente, die Teile eines Aktivitätsdiagramms umschlieBen und damit festlegen, dass alle Elemente, insbesondere Aktionen, die von ihnen umschlossen sind, in die Verantwortlichkeit der angeführten organisationalen Einheit (oder bei Softwaresystemen der Systemkomponente) fällt. Wenn Partitionen eingesetzt 
Abb. 3.17 Einfaches

Aktivitätsdiagramm

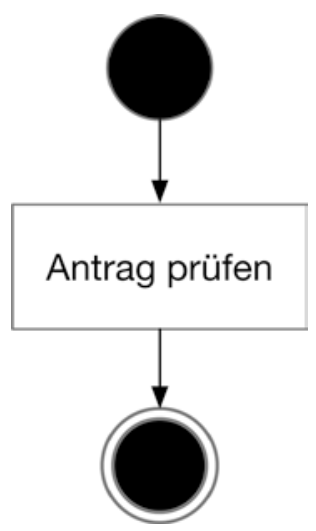

werden (was nicht verpflichtend ist), so sollten alle Elemente des Aktivitätsdiagramms von genau einer der abgebildeten Partitionen umschlossen werden. Überlappungen sind nicht erlaubt, Aktionen außerhalb einer Partition sollten vermieden werden.

Datenobjekte werden in Aktivitätsdiagrammen direkt im Kontrollfluss, und zwar zwischen Aktionen modelliert. Sie können damit (nur) dafür eingesetzt werden, den Informationsfluss zwischen zwei aufeinanderfolgenden Aktionen abzubilden. Wird ein Datenobjekt erst später im Prozessablauf wieder benötigt, so müsste dieses im Kontrollfluss über alle dazwischenliegenden Aktionen weitergegeben oder mittels eines Signals übergeben werden.

\subsubsection{Beispiele}

Um die Unterschiede und Gemeinsamkeiten zu den zuvor diskutierten Modellierungssprachen herauszuarbeiten, bedienen wir uns hier wieder der bereits verwendeten Beispiele.

Dieses Beispiel (siehe Abb. 3.17) zeigt eine Aktivität mit einer einzelnen Aktion, in der ein Antrag (über den wir hier nicht mehr wissen) bearbeitet wird. Die Aktivität endet, nachdem die Bearbeitung des Antrags abschlossen wurde.

Im obenstehenden Beispiel (siehe Abb. 3.18) ist der Prozess um eine Entscheidung mit drei möglichen Ausgängen erweitert, die einander ausschließen. Der Antrag wird geprüft, das Ergebnis dieser Prüfung ermöglicht das Treffen einer Entscheidung über die weitere Bearbeitung.

Auch hier können wir Verzweigungen nutzen, um Teile eines Prozesses wiederholt abzuarbeiten (siehe Abb. 3.19). Dazu wird die Entscheidung am Ende des zu wiederholenden Teils eingefügt und ein ausgehender Zweig zu einer schließenden Entscheidung vor die erste Operation des zu wiederholenden Teils zurückgeführt (eine schließende Entscheidung dient der Zusammenführung und hat mehrere eingehende und 


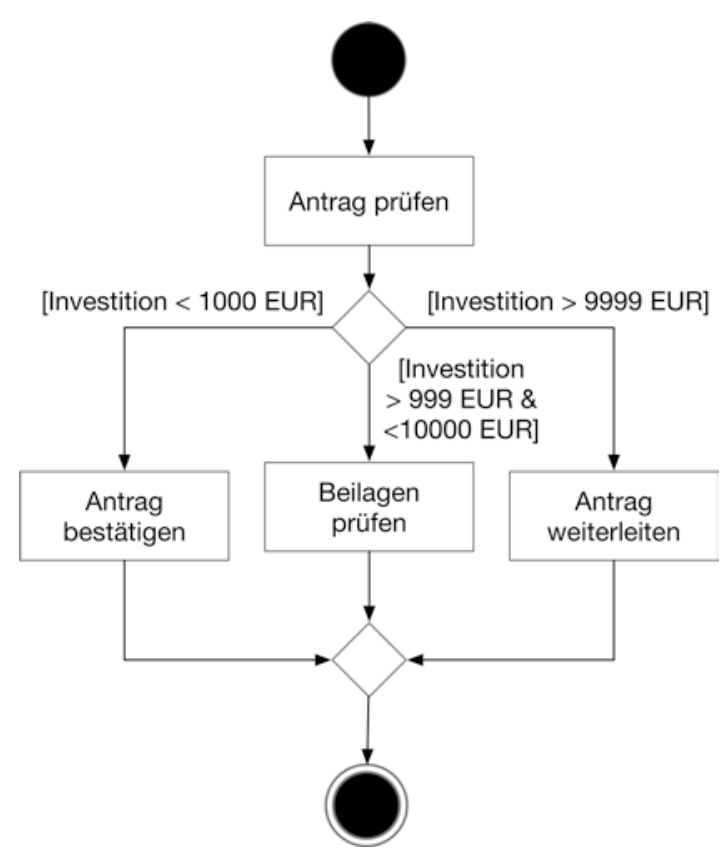

Abb. 3.18 Aktivitätsdiagramm mit Verzweigung (drei Zweige)

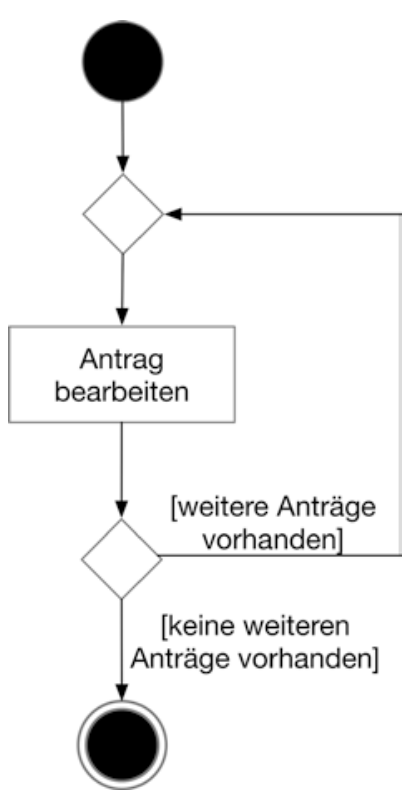

Abb. 3.19 Aktivitätsdiagramm mit Schleife 


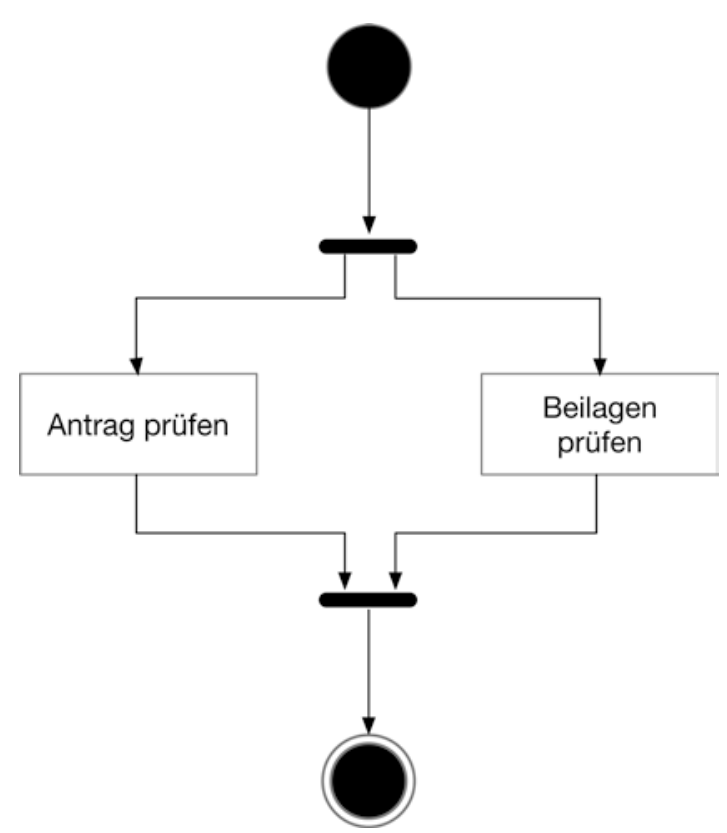

Abb. 3.20 Aktivitätsdiagramm mit parallel ablaufenden Zweigen

nur eine ausgehende Verbindung). Der andere ausgehende Zweig der öffnenden Verzweigung führt den Prozess nach der wiederholten Abarbeitung weiter.

Das Split/Join-Element kann zum Einsatz kommen, wenn zwei Aktionssequenzen unabhängig voneinander ausgeführt werden können. Der im obigen Beispiel (siehe Abb. 3.20) modellierte Prozess ist also nur korrekt, wenn die Beilagen tatsächlich unabhängig vom Antrag geprüft werden können. Ist dies nicht der Fall, müssten die beiden Funktionen sequenziell angeordnet werden. Beim zusammenführenden Join-Element wird auf den Abschluss beider eingehender Zweige gewartet, bevor der Prozess fortgesetzt wird.

Das obige Beispiel (siehe Abb. 3.21) zeigt den Einsatz von Partitionen, Datenobjekten und Signalen. Mittels Partitionen werden die Verantwortlichkeiten im Prozess abgebildet. Dessen Auslösung durch ein empfangenes Signal bildet explizit ab, dass die Ausführung erst dann startet, wenn ein Antrag einlangt (das hatten wir bei Flowcharts und den anderen Beispielen zum Aktivitätsdiagramm bislang immer implizit angenommen, aber im Gegensatz zu EPKs nie im Modell abbilden können). Gesendete Signale werden eingesetzt, um die Bestätigung oder Ablehnung an den Empfänger (der hier nicht abgebildet ist) zu übermitteln. Die Beurteilung des Antrags wird nur im Falle einer negativen Beurteilung als Datenobjekt zur Aktion „Antrag ablehnen“ übergeben - wir können folglich annehmen, dass die Ablehnung eine inhaltliche Begründung enthält. Im Falle der Bestätigung des Antrags wird das Datenobjekt „Beurteilung“ nicht mehr benötigt - wir können also annehmen, dass in diesem Fall keine weitere Begründung erfolgt. 


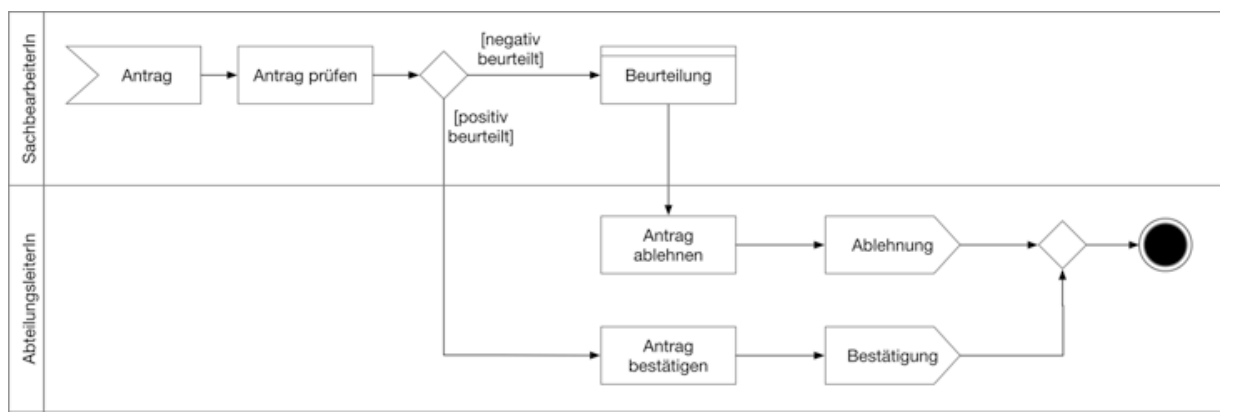

Abb. 3.21 Aktivitätsdiagramm mit Partitionen und Datenobjekten und Signalen

\subsubsection{Einordnung}

Aktivitätsdiagramme vereinen mit gewissen Einschränkungen die Einfachheit der Flowchart-Notation mit der Ausdrucksstärke von EPKs. Sie erlauben es, die Behandlung von Daten im Prozess darzustellen und führen mit Partitionen ein Mittel zur übersichtlichen Abbildung von Verantwortlichkeiten ein. Die Verfügbarkeit von Signalen erlaubt im Gegensatz zu den bisher behandelten Sprachen erstmals eine Abbildung von Kommunikationsvorgängen zwischen Prozessbeteiligten oder mit der Umwelt des abgebildeten Prozesses.

Das Fehlen eines Elements, das dem ODER-Konnektor in der EPK entspricht, stellt zwar eine Einschränkung dar, die allerdings in der Praxis selten schlagend wird, da im realen Umfeld zumeist einander ausschließende Alternativen oder vollständig voneinander unabhängige Ausführungszweige vorkommen. Insgesamt stellen Aktivitätsdiagramme also ein geeignetes Mittel zur Abbildung von Geschäftsprozessen dar, vor allem wenn die Zielgruppe für die Modellverwendung einen informationstechnischen Hintergrund hat und mit der Notation bereits vertraut ist. Für andere Zielgruppen ist aufgrund der flexibleren Einsetzbarkeit und der höheren Ausdrucksstärke für Geschäftsprozessmodellierung die BPMN vorzuziehen, die wir im nächsten Abschnitt behandeln werden.

\subsection{BPMN}

Die BPMN - Business Process Modeling (and) Notation - wurde 2002 bei IBM entwickelt und nachfolgend von der BPMI (Business Process Management Initiative) veröffentlicht. Ziel war es, der Vielzahl an Prozessmodellierungssprachen, die im akademischen Bereich und in der industriellen Praxis eingesetzt wurden, einen universell verwendbaren Standard entgegen zu setzen. Dieser sollte die wesentlichen Eigenschaften der gängigsten Sprachen übernehmen und es ermöglichen, neben der Dokumentation von Geschäftsprozessen auch Modelle zu erstellen, die unmittelbar zur IT-unterstützten 
Ausführung geeignet sind. Die BPMI wiederum wurde 2005 von der OMG (Object Management Group) übernommen bzw. fusionierte mit dieser. Dadurch wurde BPMN zum OMG-Standard und ergänzt damit die bereits erwähnte UML (Unified Modelling Language).

Im dritten Quartal 2010 wurde der neue Standard BPMN 2.0 veröffentlicht. Dieser Standard umfasst unterschiedliche Diagrammtypen: das Choreografie-, das Konversations- und das Kollaborationsdiagramm. Im Folgenden betrachten wir die grundlegenden Elemente der BPMN 2.0, die die Abbildung von Geschäftsprozessen auf fachlicher Ebene ermöglichen.

Die BPMN konzentriert sich auf Geschäftsprozesse, welche sie als eine zeitlich logische Abfolge von Aktivitäten (Aufgaben) darstellt und hinsichtlich der organisationalen Verantwortlichkeiten strukturiert. Die Darstellung von Daten ist nur ansatzweise und im Kontext von Prozessabläufen vorgesehen.

\subsubsection{Notationselemente zur Modellierung von Abläufen}

Prozessdiagramme, die mit der BPMN erstellt wurden, werden „Business Process Diagrams (BPD)“ genannt. Das BPD orientiert sich in seinen Kernelementen an Aktivitätsdiagrammen, die um Elemente ergänzt sind, die eine potenziell komplexe Ablaufsteuerung in Geschäftsprozesse abbildbar machen (siehe Abb. 3.22).

Im Prinzip müssen in einem Prozess bestimmte Dinge getan werden (Aufgaben), möglicherweise aber nur unter bestimmten Bedingungen (Gateways), und es können Dinge passieren (Ereignisse). Diese drei Flussobjekte werden über Sequenzflüsse miteinander verbunden, jedoch nur innerhalb eines Pool bzw. einer Lane. Pools und Lanes sind Konstrukte, um Verantwortlichkeiten in verteilten Geschäftsprozessen darzustellen. Sie werden im Folgenden genauer betrachtet. Falls eine Verbindung über Pool-Grenzen hinweg erfolgt, wird diese mittels Nachrichtenflüssen modelliert, die wir später detaillieren werden.

Ein Prozess besteht aus Aufgaben (Tasks). Nach dem Start eines Prozesses (durch ein Ereignis) folgt eine Aufgabe der anderen bis der Prozess endet (durch ein Ereignis). Aufgaben können dabei atomar sein (also nicht weiter verfeinert sein), oder als Subprozess vorliegen. In diesem Fall wird eine Aufgabe durch ein eingebettetes weiteres BPD verfeinert, d. h. dessen detaillierter Ablauf dargestellt. Dieser detaillierte Ablauf

Abb. 3.22 Grundlegende Notationselemente in BPMN

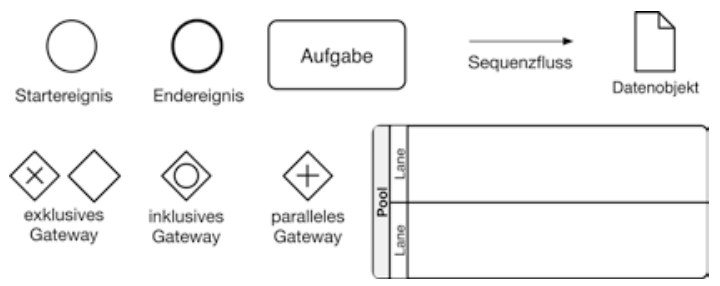


kann ,,versteckt“ werden und wird durch ein ,,+ “-Symbol am unteren Rand der Aufgabe repräsentiert.

Ein Prozess beginnt mit einem Start-Ereignis und endet in einem End-Ereignis. Die BPMN bietet eine Vielzahl an Möglichkeiten, Ereignisse zu definieren, die einen Prozess auslösen, abschließen oder dessen Verlauf beeinflussen können. Diese werden wir später näher behandeln.

An dieser Stelle ist wichtig zu betonen, dass ein Prozess mit einem oder mehreren Start-Ereignissen beginnen kann und auf jedem Pfad durch den Prozess (siehe Sequenzfluss und Gateways weiter unten) mit einem oder mehreren End-Ereignissen abgeschlossen werden kann. Von jedem Startereignis muss es einen durchgängigen Sequenzfluss zu mindestens einem Endereignis geben. Aufgaben, Gateways oder Zwischen-Ereignisse dürfen keine Endpunkte im Prozess sein, benötigen also auch immer mindestens einen ausgehenden Sequenzfluss.

Ein Gateway ist eine Abzweigung im Kontrollfluss. Das exklusive (oder XOR-)Gateway benötigt zu jedem ausgehenden Kontrollfluss eine Bedingung, die sich lt. Standard immer auf das Ergebnis einer unmittelbar vorangehenden Aufgabe beziehen muss.

Das parallele (oder AND-)Gateway verfolgt alle ausgehenden Kontrollflüsse unabhängig voneinander und parallel weiter. Die verzweigten Kontrollflüsse können separat mit Endereignissen beendet oder wieder explizit mit einem weiteren parallelen Gateway zusammengeführt werden. Der Kontrollfluss setzt nach dieser Zusammenführung erst fort, wenn alle eingehenden Kontrollflüsse abgeschlossen sind (entsprechend dem Split/Join-Konzept bei Aktivitätsdiagrammen).

Das inklusive (oder OR-)Gateway kann einen oder mehrere Pfade weiterverfolgen, wobei zur Pfadauswahl jeweils (wie beim exklusiven Gateway) eine Bedingung angeführt werden muss. Diese Bedingung muss zum Zeitpunkt der Entscheidung bereits prüfbar sein, die notwendigen Daten müssen also in einer der vorangegangenen Aufgaben erzeugt worden sein.

Für Entscheidungen, die nicht auf Basis bereits existierender Daten getroffen werden können, bietet sich die Verwendung des ereignisbasierten Gateways an. Dieses benötigt in jedem ausgehenden Zweig unmittelbar nach dem Gateway ein Ereignis (z. B. eintreffende Nachricht oder Timer). Es wird dann jener Zweig (und nur dieser) aktiviert, dessen Ereignis zuerst eintritt. Dieses werden wir genauer behandeln, wenn wir die Verwendung von Ereignissen diskutieren.

\subsubsection{Beispiele zur Modellierung von Abläufen}

Um die Unterschiede und Gemeinsamkeiten zu den zuvor diskutierten Modellierungssprachen herauszuarbeiten, bedienen wir uns hier wieder der bereits verwendeten Beispiele.

Dieses Beispiel (siehe Abb. 3.23) zeigt einen Prozess mit einer einzelnen Aufgabe, in der ein Antrag (über den wir hier nicht mehr wissen) bearbeitet wird. Der Prozess endet, nachdem die Bearbeitung des Antrags abschlossen wurde. 


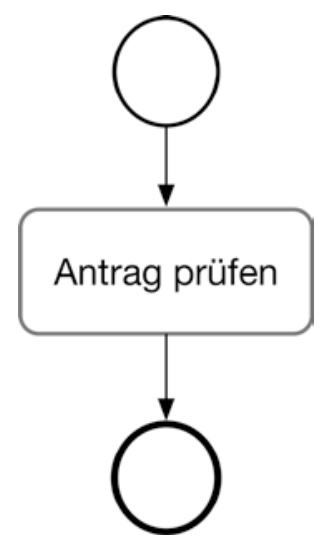

Abb. 3.23 Einfaches BPMN-Modell

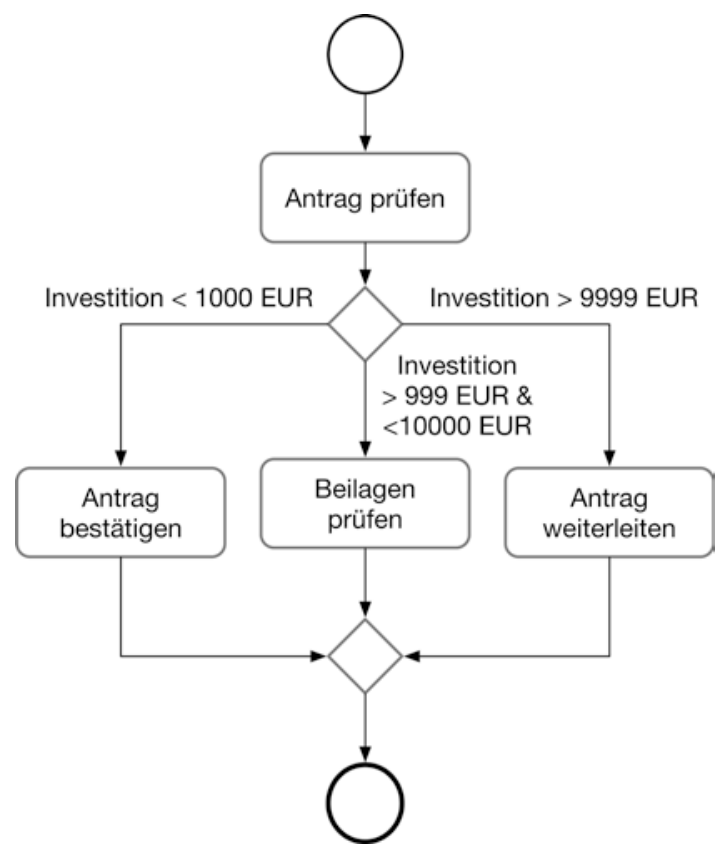

Abb. 3.24 BPMN-Modell mit Verzweigung (3 Zweige)

Im obenstehenden Beispiel (siehe Abb. 3.24) ist der Prozess um eine Entscheidung mit drei möglichen Ausgängen erweitert, die einander ausschließen. Der Antrag wird geprüft, das Ergebnis dieser Prüfung ermöglicht das Treffen einer Entscheidung über die weitere Bearbeitung. In der BPMN ist wichtig, dass die Daten, die als Grundlage einer 


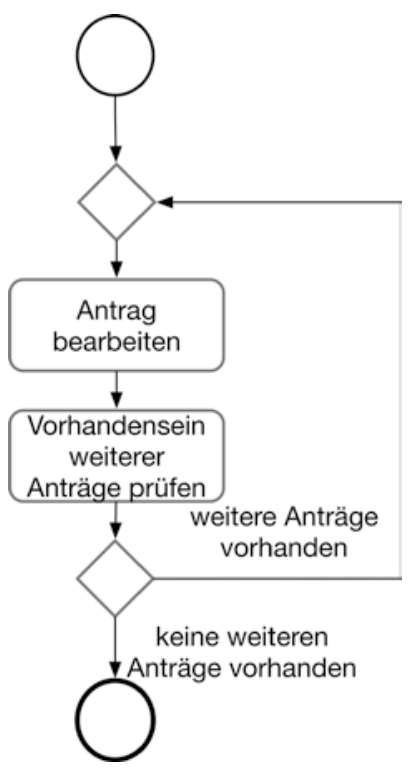

Abb. 3.25 BPMN-Modell mit Schleife

Entscheidung verwendet werden, vor dem Gateway explizit erzeugt oder empfangen werden.

Auch hier können wir Verzweigungen nutzen, um Teile eines Prozesses wiederholt abzuarbeiten (siehe Abb. 3.25). Dazu wird die Entscheidung am Ende des zu wiederholenden Teils eingefügt und ein ausgehender Zweig zu einer schließenden Entscheidung vor die erste Operation des zu wiederholenden Teils zurückgeführt (eine schließende Entscheidung dient der Zusammenführung und hat mehrere eingehende und nur eine ausgehende Verbindung). Der andere ausgehende Zweig der öffnenden Verzweigung führt den Prozess nach der wiederholten Abarbeitung weiter. Die Anforderung, die Entscheidungsgrundlage explizit vor dem Gateway zu schaffen, ist hier durch die zusätzliche Aufgabe abgebildet, das Vorhandensein weiterer Anträge zu prüfen.

Das parallele Gateway kann zum Einsatz kommen, wenn zwei Aufgaben-Sequenzen unabhängig voneinander ausgeführt werden können. Der im obigen Beispiel (siehe Abb. 3.26) modellierte Prozess ist also nur korrekt, wenn die Beilagen tatsächlich unabhängig vom Antrag geprüft werden können. Ist dies nicht der Fall, müssten die beiden Funktionen sequenziell angeordnet werden. Beim zusammenführenden Gateway wird auf den Abschluss beider eingehenden Zweige gewartet, bevor der Prozess fortgesetzt wird.

Das obige Beispiel (siehe Abb. 3.27) zeigt den Einsatz von Pools, Lanes und Datenobjekten. Mittels Lanes werden die Verantwortlichkeiten im Prozess abgebildet. Mit den bislang eingeführten Elementen der BPMN können wir Kommunikationsvorgänge nicht abbilden. Die in Aktivitätsdiagrammen verfügbaren Signale können deshalb vorerst nicht 


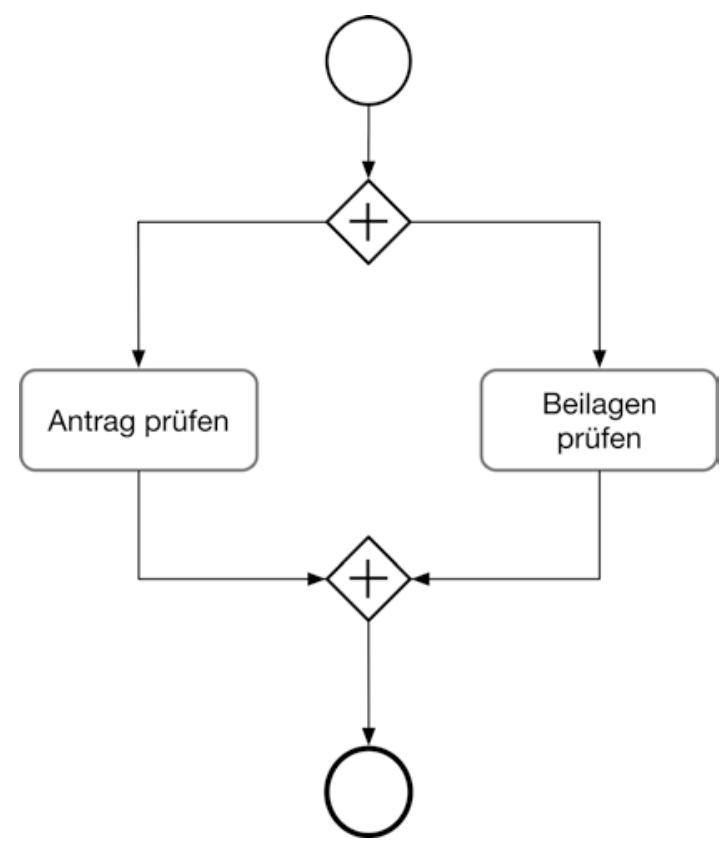

Abb. 3.26 BPMN-Modell mit parallel ablaufenden Zweigen

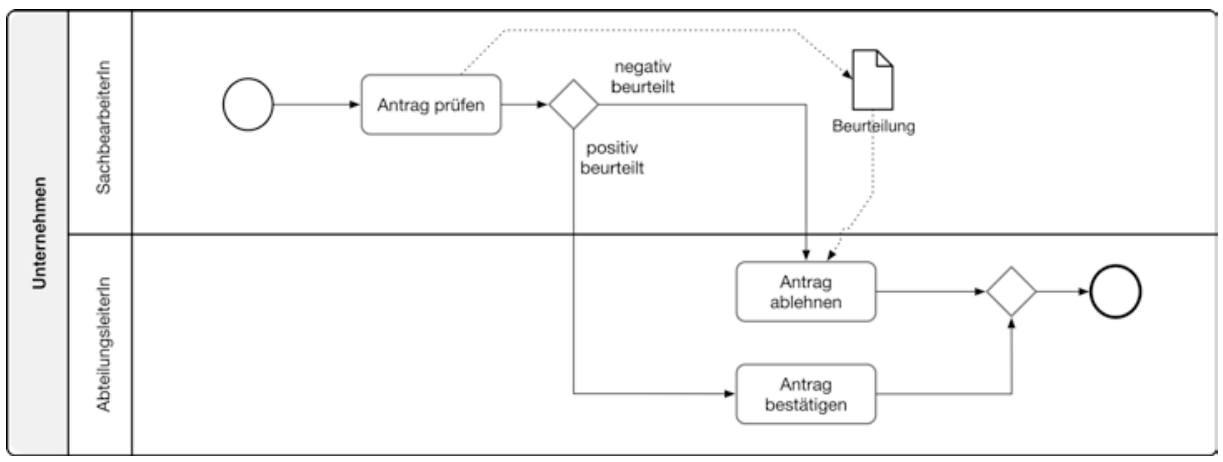

Abb. 3.27 BPMN-Modell mit Pools, Lanes und Datenobjekten

abgebildet werden, weswegen das Beispiel hier erneut unspezifischer wird. Der Einsatz von Nachrichten-Ereignissen, die wir im übernächsten Abschnitt einführen werden, wird diesen Mangel aber wieder beheben. Die Beurteilung des Antrags wird nur im Falle einer negativen Beurteilung als Datenobjekt zur Aufgabe „Antrag ablehnen“ übergeben - wir können also annehmen, dass die Ablehnung eine inhaltliche Begründung enthält. Im Fall der Bestätigung des Antrags, wird das Datenobjekt „Beurteilung“ nicht mehr benötigt wir können also annehmen, dass in diesem Fall keine weitere Begründung erfolgt. 


\subsubsection{Notationselemente zur Ablaufsteuerung mit Ereignissen}

Ein Unterscheidungsmerkmal von BPMN zu allen bislang behandelten Sprachen ist das hier sehr detailliert und umfassend ausgeführte Ereignis-Konzepte, das eine umfassende Kontrolle des Prozessablaufs ermöglicht.

Ereignisse geben an, dass etwas geschehen ist und sind damit Zeitpunkte im Gegensatz zu Aufgaben, die einen gewissen Zeitraum in Anspruch nehmen. Bis jetzt haben wir nur Start- und Endereignisse eingeführt, im Folgenden werden Start-, Zwischen- und Endereignisse noch einmal ausführlich beschrieben (siehe Abb. 3.28).

Ereignisse werden immer mit einem Kreis und üblicherweise einem Symbol dargestellt. Einfache Kreise kennzeichnen Startereignisse, doppelte Kreislinien Zwischenereignisse und dicke Kreislinien Endereignisse. Ist kein Symbol angegeben, handelt es sich um ein untypisiertes (Blanko-)Ereignis, das in der Regel nur am Beginn oder Ende eines Prozesses oder als auslösendes Zwischenereignis zu finden ist.

\subsubsection{Startereignisse}

Startereignisse geben den Auslöser für einen Prozess oder Teilprozess an. Häufig wird das unbestimmte (Blanko-)Ereignis verwendet, wenn sich der Auslöser aus dem Zusammenhang ohnehin ergibt oder wenn der Auslöser nicht näher bekannt ist.

Wird ein Prozess aufgrund eines Zeitpunkts oder einer Zeitspanne oder eines periodisch stattfindenden Ereignisses ausgelöst, wird zusätzlich das Symbol der Uhr verwendet. Ein Brief-Symbol wird verwendet, wenn eine Nachricht den Prozess auslöst (siehe Abb. 3.29).

Darüber hinaus gibt es noch Symbole für Bedingungen - der Prozess wird nur ausgeführt, wenn die angegebene Bedingung erfüllt ist. Ein Signal ist ein Zeichen, durch das der Prozess gestartet wird. Das Fünfeck als Symbol kennzeichnet mehrere mögliche Startereignisse, wobei nur eines der Ereignisse eintreten muss, um den Prozess zu starten (siehe Abb. 3.30).

Ein Prozess muss kein einzelnes Startereignis haben, Prozesse können auch mehrere alternative Startereignisse haben.

Abb. 3.28 Beispiele für Start-, Zwischen- und Endereignisse

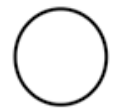

Startereignis

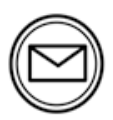

Zwischenereignis

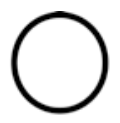

Endereignis

Abb.3.29 Grundlegende BPMN-Startereignisse
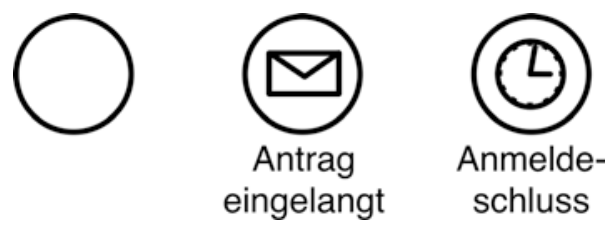
Abb. 3.30 Komplexe BPMNStartereignisse

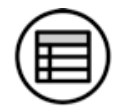

Auslastung $>90 \%$

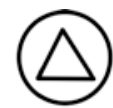

Onlinesystem Reinigungsintervall erreicht down

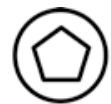

oder

Filter verschmutzt

Abb. 3.31 Grundlegende BPMN-Endereignisse
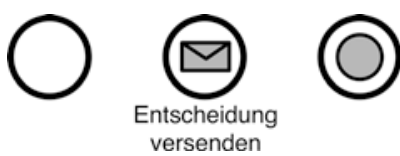

versenden

\subsubsection{Endereignisse}

Mit einem Endereignis werden Prozesse beendet, wobei es mit Ausnahme des zeitbezogenen Symbols die Bedingung und den parallelen mehrfachen Auslöser die gleichen Symbole gibt wie bei Startereignissen.

Zusätzlich zu den verschiedenen Arten von Endereignissen gibt es ein Terminierungs-Endereignis (schwarz gefüllter Kreis mit dicker schwarzer Umrandung), das den gesamten Prozess sofort beendet, d. h. die gesamte Prozessinstanz beendet, unabhängig davon, ob andere Sequenzen innerhalb des Prozesses zur gleichen Zeit noch durchlaufen werden oder nicht. Ein Standard-Endereignis beendet immer nur jenen Prozesszweig, in dem es eingefügt ist. Eventuell weitere, noch laufende Prozesszweige werden weiter ausgeführt (siehe Abb. 3.31).

Prozesse können, wie bereits bei den Startereignissen erklärt, mehrere Endereignisse haben. Ein Prozess ohne Endereignis ist unvollständig.

\subsubsection{Zwischenereignisse \& das ereignisbasierte Gateway}

Zwischenereignisse können an irgendeiner Stelle in einem Prozess verwendet werden und werden durch einen Kreis mit doppelter Umrandung dargestellt. Sie werden modelliert, wenn in einem Prozess ein für andere (Prozesse) relevantes Zwischenergebnis erreicht wird, oder innerhalb eines Prozesses auf ein Ereignis reagiert wird, etwa auf eine eingehende Nachricht oder den Ablauf eines bestimmten Zeitraums.

Gateways können auch ereignisbasiert sein, wenn ein oder mehrere Ereignisse zum Durchlaufen von verschiedenen Pfaden führen. Dabei kann die Modellierung mit einem ereignisbasierten Gateway erfolgen.

Die folgende Abbildung (siehe Abb.3.32) zeigt die Prozessmodellierung eines Bewerbungsvorgangs mit Ereignissen. Abhängig davon, ob eine Einladung oder eine Absage eintrifft oder eine Deadline von 2 Wochen abläuft, werden unterschiedliche Wege im Prozess beschritten. Das ereignisbasierte Gateway ist damit das einzige Gateway, bei dem zum Zeitpunkt der Prüfung des Gateways die dafür notwendigen Daten noch nicht vorliegen müssen. Ein ereignisbasiertes Gateway blockiert den Kontrollfluss so lange, bis eines der unmittelbar nachgelagerten Ereignisse eintritt und verfolgt dann den jeweiligen Zweig weiter. 


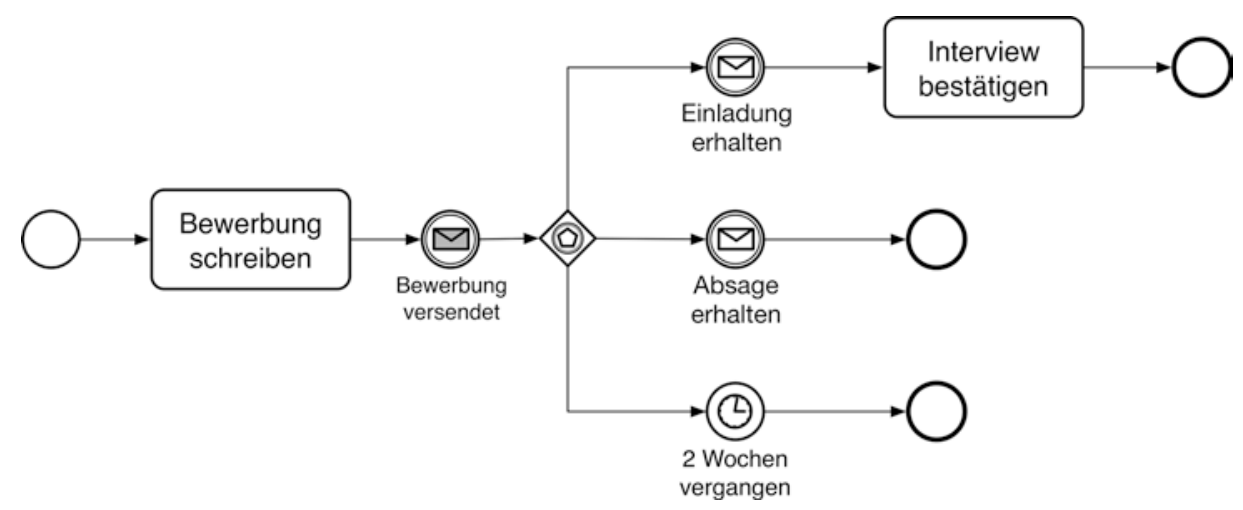

Abb. 3.32 Beispiel für den Einsatz des ereignisgesteuerten Gateways

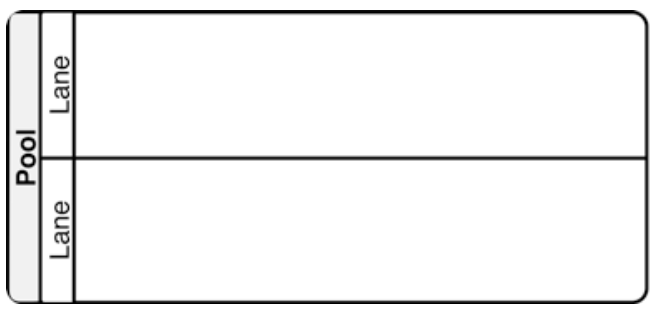

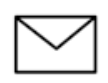

Nachricht
Nachrichten-

fluss

Abb. 3.33 Notationselemente zur Abbildung von Kommunikation in BPMN

\subsubsection{Notationselemente zur Modellierung von Kommunikation}

Die BPMN unterstützt auch die Modellierung verteilter Geschäftsprozesse. Die BPMN rückt zwar klar den Prozess bei der Modellierung in den Fokus (wie etwa auch ein Flowchart, eine EPK oder ein Aktivitätsdiagramm), aber auch den ausführenden bzw. beteiligten Personen eines Prozesses wird Beachtung geschenkt (siehe Abb. 3.33). Die dafür zur Verfügung stehenden Modellierungselemente werden in diesem Abschnitt betrachtet.

Ein Pool stellt eine Unternehmung oder eine Organisationseinheit in einer Unternehmung dar, wie z. B. eine Abteilung. Jede (Swim-)Lane in einem Pool repräsentiert eine prozessbeteiligte Person bzw. Rolle, die diesem Pool zugeordnet ist.

BPMN bietet die Möglichkeit, das Zusammenspiel von zwei oder mehreren Prozessen aufzuzeichnen. Zur Darstellung von Kollaborationen sind die eben erwähnten Pools und Lanes notwendig, wobei für alle an einem Prozess beteiligten Personen oder Gruppen eine eigene Lane erforderlich ist, für jeden Prozess bzw. jede Organisationseinheit, die für diesen Prozess verantwortlich ist, ein eigener Pool. In jedem Pool entstehen so eigene, individuelle Prozesse mit separaten Start- und Endereignissen. Dennoch kann es 
vorkommen, dass die einzelnen Prozesse von anderen Prozessen stark beeinflusst werden. Dies kann durch Nachrichtenflüsse modelliert werden.

Nachrichtenflüsse geben an, wenn zwischen verschiedenen Prozessen Daten ausgetauscht werden. Innerhalb eines Prozesses (Pool) kann daher kein Nachrichtenfluss stattfinden. Somit ist kein Nachrichtenfluss innerhalb einer Lane und zwischen einzelnen Lanes möglich. Sequenzflüsse zeigen an, welche Aktivitäten in welcher Reihenfolge ausgeführt werden. Sie dürfen im Gegensatz zu Nachrichtenflüssen nur innerhalb eines Pool stattfinden können und nicht zwischen unterschiedlichen Prozessen (Pools).

An Nachrichtenflüsse können zusätzlich Nachrichten angebracht werden, die hier als Datenelemente eingesetzt werden und eine genauere Spezifikation der übermittelten Information enthalten.

Nachrichtenflüsse dürfen einerseits von Pools und Aktivitäten ausgehen und dort enden, andererseits können sie explizit von Sende-Ereignissen abgeschickt und durch Empfangs-Ereignisse empfangen werden. Der erstgenannte Fall ist für die beschreibende Modellierung von Geschäftsprozessen sinnvoll, bei der ein Kommunikationsvorgang zwar erfasst werden soll, aber nicht notwendigerweise exakt beschrieben werden muss. Eine von einer Aktivität ausgehende Nachricht wird irgendwann im Zuge der Aufgabenausführung gesendet - der genaue Zeitpunkt bleibt unklar. Eine an einem Pool endende Nachricht sagt nur aus, dass die repräsentierte Organisation diese Nachricht empfängt, aber nicht, welche Aktivität sie auslöst. Dies kann sinnvoll sein, wenn externe Organisationseinheiten modelliert werden sollen, deren detailliertes Verhalten unbekannt ist. Nur durch die Verwendung von expliziten Sende- und Empfangsereignissen ist eine exakte Spezifikation von Kommunikationsvorgängen möglich.

\subsubsection{Beispiele zur Modellierung von Kommunikation}

Im Folgenden ergänzen wir das Beispiel, das wir zur Demonstration des Einsatzes von Ereignissen verwendet haben, um den dort nicht abgebildeten Kommunikationspartner, also das Unternehmen, an das eine Bewerbung gerichtet wird.

Das obenstehende Beispiel (siehe Abb. 3.34) zeigt zwei Prozesse (je einen pro Pool), die über Nachrichtenflüsse miteinander verknüpft sind. Der Prozess des Unternehmens wird durch eine eingehende Bewerbung gestartet, die hier im ersten Nachrichtenfluss abgebildet ist. Nach der Prüfung kann die Entscheidung getroffen werden, ob eine Einladung ausgesprochen oder eine Absage erteilt werden soll. Im oberen Pool wartet der Bewerber auf eine Antwort - allerdings maximal 2 Wochen lang. Durch das ereignisbasierte Gateway wird jener Prozesszweig aktiviert, dessen Ereignis zuerst eintritt. Die jeweils zusammengehörigen Sende- und Empfangs-Ereignisse sind über einen Nachrichtenfluss gekoppelt. Wichtig ist hier, dass Nachrichtenflüsse immer 1:1-Beziehungen abbilden - dass also eine gesendete Nachricht genau einmal empfangen werden kann und dass ein empfangendes Ereignis auf genau eine Nachricht reagieren kann. 


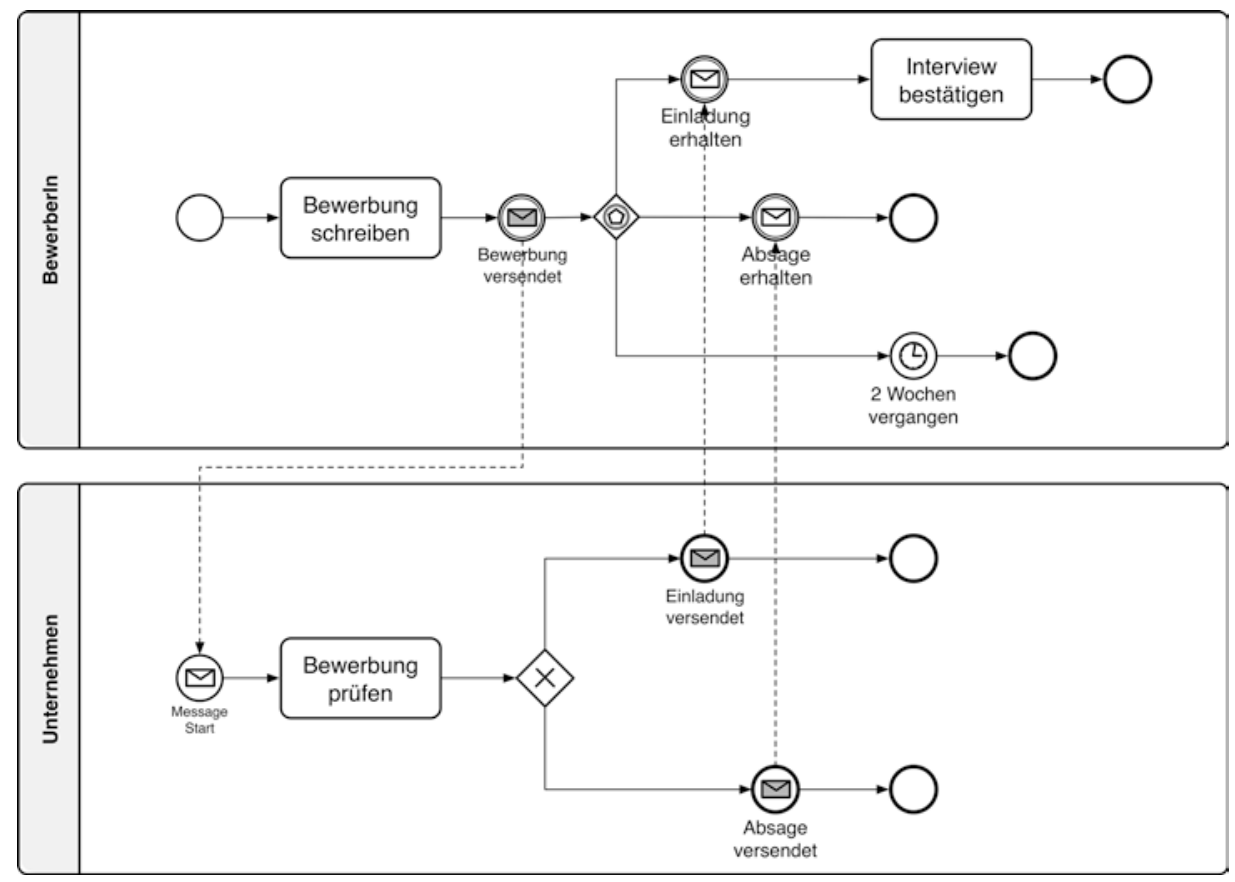

Abb. 3.34 Beispiel von verteilt ablaufenden Prozessen mit Kommunikationsvorgängen

Das obenstehende Beispiel (siehe Abb. 3.35) zeigt nun, wie die BPMN verwendet werden kann, um ausschließlich Kommunikationsvorgänge abzubilden. Die Pools werden hier als Blackbox verwendet, das in ihnen enthaltene Verhalten ist nicht abgebildet und bleibt unbekannt. Wir sehen lediglich, dass Nachrichten ausgetauscht werden, wobei die Reihenfolge der Nachrichten nicht definiert ist. Nachdem hier die näher beschreibenden Ereignisse zum Versenden und Empfangen fehlen, ergänzen wir das Modell um Nachrichten-Elemente, um die Kommunikation nachvollziehen zu können. Ein weiterer Unterschied ist der im oberen Pool angebrachte Modifikator, der eine parallele Mehrfachausführung des im oberen Pool enthaltenen Prozesses kennzeichnet. Dies bedeutet, dass der Prozess im unteren Pool mit mehreren, parallel unabhängig voneinander eintreffenden Bewerbungen umgehen können würde bzw. müsste.

Leere und gefüllte Pools können auch beliebig kombiniert werden. Wollten wir z. B. den Prozess der Bearbeitung einer eingehenden Bewerbung abbilden, könnten wir den Pool „BewerberIn“ unspezifisch belassen, da wir deren Verhalten nicht kennen (und es auch nicht relevant ist), aber wissen müssen, dass wir von dort eine Bewerbung erhalten können und dass wir unsere Antwort dann wieder dorthin adressieren. 


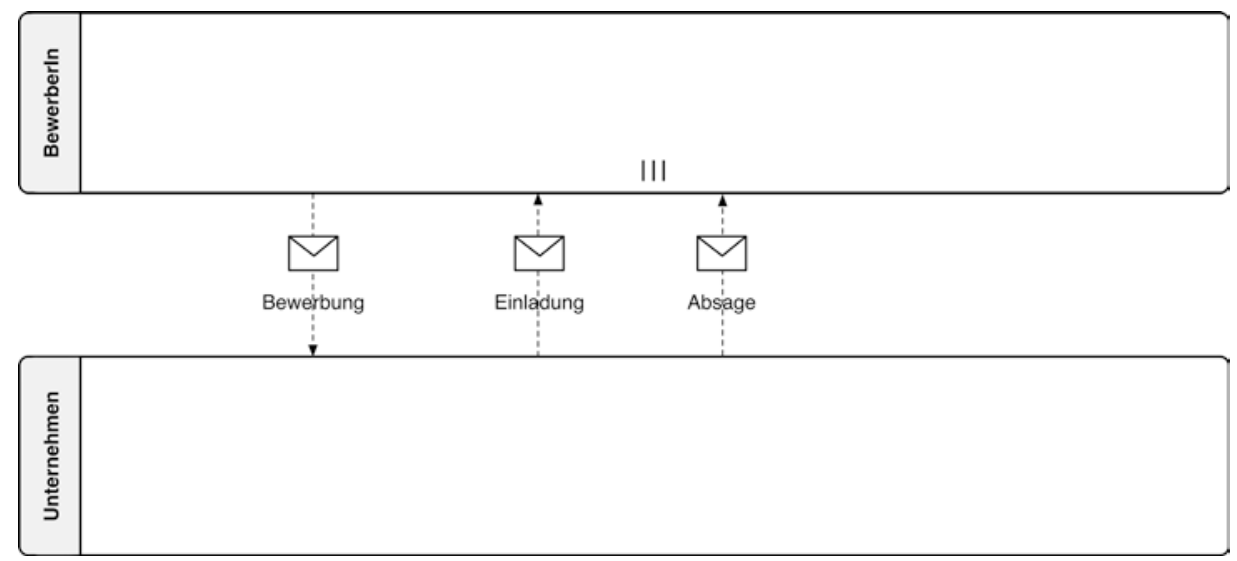

Abb. 3.35 Ausschließliche Abbildung von Kommunikationsvorgängen in BPMN

\subsubsection{Notationselemente zur Modellierung komplexer Sachverhalte}

Die BPMN bietet mithilfe der bislang vorgestellten Notationselemente die Möglichkeit, Geschäftsprozesse aus Sicht der durchführenden organisationalen Einheiten darzustellen. Die BPMN lässt dabei die Freiheit, Prozessmodelle vage zu halten oder Teile davon unspezifiziert zu lassen, wenn diese für die Zielsetzung der Modellierung nicht relevant erscheinen. In manchen Fällen soll aber ein Prozess möglichst exakt gefasst und in all seinen vorstellbaren Varianten und möglicherweise auftretenden Ausnahmefällen abgebildet werden. Dies ist etwa notwendig, wenn das Modell die Grundlage für die IT-basierte Unterstützung der abgebildeten Arbeitsprozesse dienen soll. Werden hier Aspekte weggelassen oder verkürzt abgebildet, ergibt sich eine Diskrepanz zwischen dem realen Arbeitsprozess und den auf dem Modell basierenden Unterstützungsmaßnahmen, was letztendlich zu nicht zufrieden stellenden Werkzeugen und Workarounds führt. Dieser Abschnitt beschreibt jene Notationselemente der BPMN, die komplexe Ablaufbeschreibungen ermöglichen. Aufgrund der Vielfalt der abbildbaren Szenarien führen wir Beispiele hier direkt bei der Beschreibung der jeweiligen Elemente an.

\subsubsection{Varianten der Aktivitätsmodellierung}

Im folgenden Abschnitt werden Besonderheiten der Modellierung von Aktivitäten sowie deren Modellierung als Subprozesse genauer erklärt.

\subsection{Subprozesse}

Prozesse können mittels Subprozessen modelliert werden. Diese Methode wird meistens verwendet, um bei der Modellierung von großen, umfangreichen Prozessen den Überblick behalten zu können. Subprozesse können dabei diagrammatisch minimiert werden. Dann ist der Prozess nur mehr als kleine Aufgabe im gesamten Prozess zu erkennen und durch ein kleines Plus gekennzeichnet. 

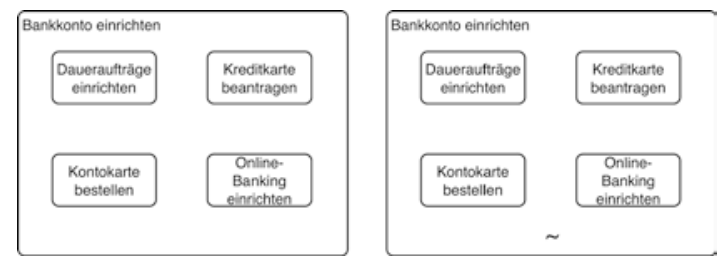

Abb. 3.36 Beispiele für Sub-Prozesse mit parallel (links) oder ad-hoc (rechts) auszuführenden Aktivitäten
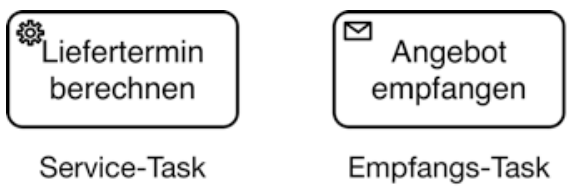

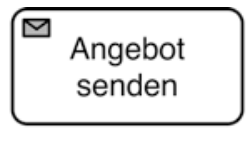

Sende-Task

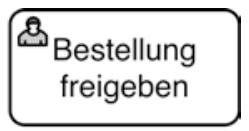

Benutzer-Task

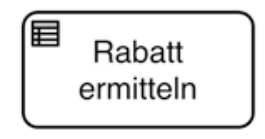

Geschäftsregel-Task

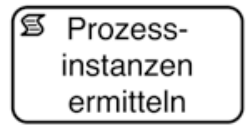

Skript-Task

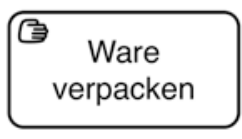

Manueller Task

Abb. 3.37 Unterschiedliche Aktivitätstypen

Subprozesse erlauben aber auch die Zusammenfassung mehrerer Aktivitäten in einen Durchführungskontext, ohne deren genaue Reihenfolge festzulegen. Das rechte Modell (ein Ad-hoc Unterprozess) in der nachfolgenden Abbildung (siehe Abb. 3.36) gibt nur an, dass beliebig viele der eingebetteten Aktivitäten durchgeführt werden können, macht aber keine Aussage über deren Zusammenhänge. Das linke Modell legt fest, dass alle vier Subaktivitäten ausgeführt werden müssen, bevor der Subprozess abgeschlossen ist. Es trifft keine Aussage über die Reihenfolge oder sonstige Zusammenhänge - die Aktivitäten können parallel ausgeführt werden.

\subsection{Aktivitätstypen}

Aufgaben-Typen beschreiben den Charakter einer Aufgabe, also welchen Zweck eine Aufgabe erfüllt. Dabei wird unterschieden nach Service, Empfang, Sendung, Benutzer, Geschäftsregel, Skript, manuell und unbestimmt (Abbildung von links nach rechts; siehe Abb. 3.37). Diese Modifikatoren müssen nicht unbedingt verwendet werden, konkretisieren aber die Semantik eines Prozessmodells.

\subsection{Ausführungsverhalten von Aktivitäten}

Aktivitäten können unterschiedliche Markierungen haben, die das Ausführungsverhalten von Aktivitäten beschreiben. Aktivitäten oder gesamte Prozesse oder Teilprozesse können dabei als Schleife, parallel, sequentiell, ad hoc oder als Kompensation ausgeführt werden (siehe Abb. 3.38). 


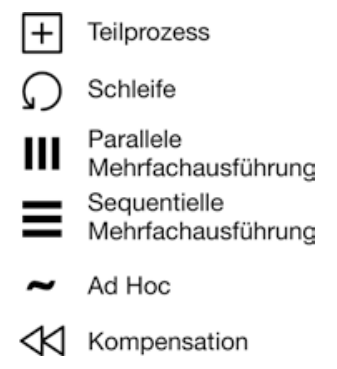

Abb. 3.38 Modifikatoren zur Abbildung unterschiedlichen Ausführungsverhaltens

Bei einer Schleife kann eine Abbruchbedingung zusätzlich zum Symbol angegeben werden. Ist die Abbruchbedingung erreicht, wird die Aktivität oder der betreffende Prozess nicht mehr länger ausgeführt und die jeweilige übergeordnete Sequenz weiter ausgeführt. Kann ein Prozess parallel ausgeführt werden, so wird dies durch drei vertikale Striche gekennzeichnet. So kann beispielsweise der Prozess „Bewerbung prüfen“ für mehrere eingelangte Bewerbungsunterlagen von mehreren Bearbeitern durchgeführt werden. Ist nur eine parallele Ausführung nicht möglich, die einzelnen Fälle aber unabhängig voneinander, so handelt es sich um eine sequenzielle Mehrfachausführung, die durch drei horizontale Striche gekennzeichnet wird.

Bei Ad-Hoc-Prozessen ist die genaue Reihenfolge der enthaltenen Aktivitäten unbekannt und ergibt sich erst während der Ausführung des Prozesses. Solche Prozesse werden über eine Tilde gekennzeichnet (wie oben bereits dargestellt). Es müssen auch nicht alle Aktivitäten abgearbeitet werden.

Kompensationsaktivitäten werden im Rahmen der Transaktionsmodellierung verwendet und werden weiter unten beschrieben.

\subsubsection{Ereignistypen}

Die BPMN bietet zur detaillierten Ablaufsteuerung eine Vielzahl von unterschiedlichen Events in verschiedenen Ausprägungen an. Während wir diese im Folgenden im Detail behandeln werden, soll hier ein Überblick gegeben und der systematische Aufbau von Events dargestellt werden (siehe Abb. 3.39).

Grundsätzlich können Events in drei unterschiedlichen Varianten vorkommen, die wir oben bereits eingeführt haben:

- Start-Ereignisse lösen neue Prozessinstanzen aus, starten also die Ausführung eines Prozesses. Sie sind immer ,empfangend“, reagieren also auf Stimuli von außen (etwa eingehende Nachrichten, Zeitabläufe etc.).

- End-Ereignisse sind Ereignisse, die beim Beenden einer Prozessinstanz ausgelöst werden. Sie sind immer ,sendend“, lösen also potenziell Stimuli für andere Prozesse bzw. Prozessteile aus.

- Zwischen-Ereignisse treten innerhalb des Sequenzflusses auf, haben also sowohl eine eingehende als auch eine ausgehende Kante (Ausnahme: Link-Event, siehe weiter unten). 


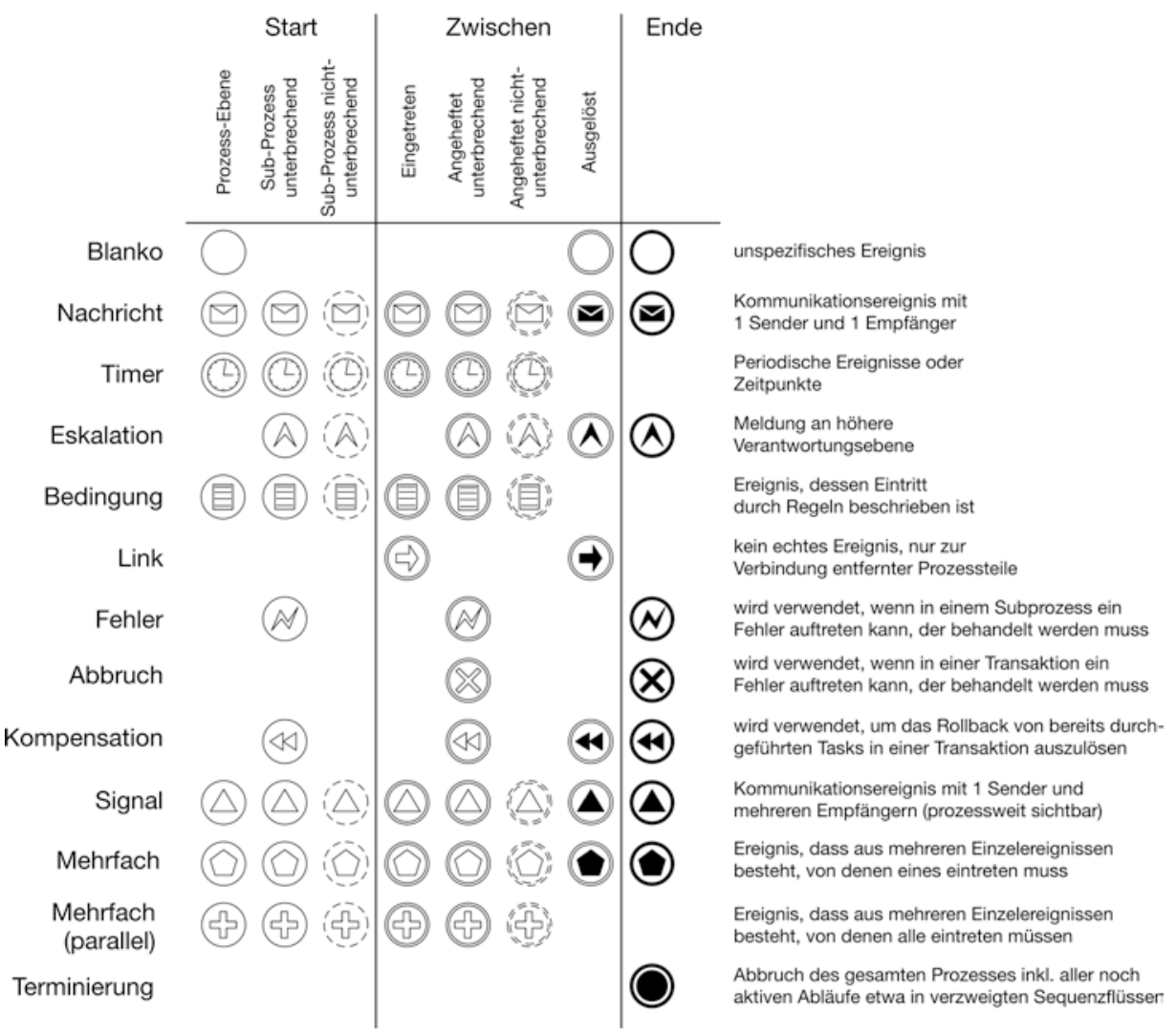

Abb. 3.39 Vollständiger Satz an BPMN-Ereignis-Typen

Start-Ereignisse lassen sich darüber hinaus hinsichtlich ihres Einsatzortes unterscheiden. Sie können zum Auslösen eines gesamten Prozesses oder zum Auslösen von Subprozessen verwendet werden. Der zweite Fall wird als „Ereignis-Teilprozess“ bezeichnet und kann mittels „unterbrechend“ und ,nicht-unterbrechend“ spezifiziert werden. Im eher üblichen Fall wird durch ein „unterbrechendes“ Startereignis gekennzeichnet, dass der Kontrollfluss vollständig an den Subprozess übergeben wird, innerhalb des jeweiligen Pool also alle anderen Aktivitäten unterbrochen werden. „Nicht-unterbrechende“ Ereignis-Teilprozesse werden beim Eintreffen des jeweiligen Ereignisses gestartet, ohne dass die Durchführung der aktuell innerhalb eines Pool ausgeführten Aktivitäten unterbrochen wird. Damit kann etwa auf Ereignisse reagiert werden, die nicht im Hauptprozess eines Pool behandelt werden sollen oder können, deren Auftreten aber eine Reaktion nach sich ziehen soll, ohne dass der Hauptprozess beeinträchtigt wird (etwa Kundennachfragen über die Status einer Auftragsbearbeitung, während dieser Auftrag gerade im Rahmen des Hauptprozesses bearbeitet wird). 
Zwischenereignisse existieren grundsätzlich in einer „empfangenden“ und einer ,sendenden“ Variante. Die „empfangende“ Variante (eingetretenes Ereignis) wartet auf das Eintreffen des spezifizierten Ereignisses und blockiert dabei den Sequenzfluss. Dieser kann also nicht fortgesetzt werden, bis das Ereignis eingetreten ist. Die „sendende“ Variante (ausgelöstes Ereignis) kennzeichnet das Eintreten bestimmter Ereignisse in einem Prozess (oder auch zwischen Prozessen aus unterschiedlichen Pools). Ereignisse treten bei vollständig modellierten Prozessen üblicherweise reziprok auf, d. h. dass zu einem eingetretenen Event auch ein auslösendes Event existiert.

Empfangende Zwischenereignisse existieren auch in einer ,angehefteten“ Form. Diese Ereignisse werden an Aktivitäten ,,angeheftet“ (d. h. grafisch an deren (Unter-) Kante angebracht) und kennzeichnen, dass während der Durchführung der Aktivität auf das jeweilige Ereignis reagiert werden können soll. Die Reaktion wird durch einen aus dem angehefteten Event ausgehenden Sequenzfluss spezifiziert, der zu den jeweils durchzuführenden Aktivitäten führt. Das angeheftete Zwischenereignis existiert dabei wiederum in einer unterbrechenden und einer nicht-unterbrechenden Form. Die unterbrechende Form bricht die Ausführung der so gekennzeichneten Aktivität ab und setzt den Sequenzfluss ausschließlich über das angeheftete Event fort. Die nicht-unterbrechende Form erlaubt die weitere Ausführung der so gekennzeichneten Aktivität, parallel dazu wird aber der vom Event ausgehende Sequenzfluss ausgelöst.

Die auslösenden Ereignisse, die zum Eintreten von angehefteten Events führen, können von außen kommen (etwa von anderen Pools eintreffende Nachrichten) oder auch von innerhalb der Aktivität, sofern diese durch einen Subprozess detailliert wird. So kann ein Fehler bei der Ausführung des Subprozesses zu Aktivitäten im Hauptprozess führen, etwa das Dokumentieren des Fehlers und einer Eskalation zu Vorgesetzten.

Nachrichten- und Timer-Ereignisse haben wir bereits in der grundlegenden BPMN-Modellierung verwendet. Die übrigen Ereignistypen betrachten wir nun im Rahmen ihrer jeweiligen Einsatzgebiete.

\subsubsection{Das Link-Event}

Bei komplexeren oder umfangreicheren Abläufen wird die Nachverfolgung von Sequenzflüssen durch das Diagramm manchmal schwierig. Einander kreuzende Sequenzflüsse oder Sequenzflüsse mit vielen Richtungsänderungen erschweren die Lesbarkeit und sind der Verständlichkeit und Übersichtlichkeit abträglich (siehe Abb. 3.40).

In derartigen Fällen kann das Link-Event verwendet werden. Im Gegensatz zu den übrigen Events bildet es semantisch kein echtes Ereignis ab, sondern dient lediglich als Konnektor zwischen zwei Sequenzflüssen, die weit voneinander entfernt liegen. Die Kopplung erfolgt dabei über die Bezeichnung des auslösenden und des empfangenden Events. Es muss immer eine 1:1-Zuordnung bestehen - eine implizite Parallelisierung durch ein auslösendes und mehrere gleichnamige empfangende Link-Events sind folglich nicht zulässig.

Link-Events sollten zur Erhöhung der Übersichtlichkeit trotzdem nur in nicht anders auflösbaren Fällen genutzt werden, da das Suchen zusammengehöriger Link-Events im 
Abb. 3.40 Beispiel für den Einsatz des Link-Events

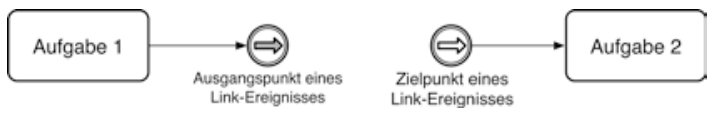

Aufwand das Nachverfolgen komplexer Sequenzflüsse sogar übersteigen kann (sofern keine Werkzeugunterstützung durch Sprungfunktionalitäten oder optische Markierung zusammengehöriger Events besteht). Eine alternative Anordnung von Aktivitäten oder Lanes ist üblicherweise die bessere Wahl.

\subsubsection{Verwendung von Signalen}

Nachrichten können in BPMN ausschließlich zur Kommunikation zwischen Pools verwendet werden. Des Weiteren hat eine nachrichtenbasierte Kommunikation immer exakt zwei Endpunkte, kann also immer nur genau einen Sender mit genau einem Empfänger verbinden. Wenn eine Information global innerhalb einer Kollaboration zur Verfügung gestellt werden soll, und dies unabhängig von Poolgrenzen passieren soll, können Signale verwendet werden. Signale können (als Zwischen- oder End-Ereignisse) im Prozess ausgelöst werden und stehen dann sowohl innerhalb des Pool als auch in allen anderen Pools der gleichen Kollaboration zur Verfügung.

Signale können dazu eingesetzt werden, alle Pools einer Kollaboration etwa über den Abbruch eines der abgebildeten Prozesse zu informieren. So können alle anderen noch ausgeführten Prozesse für sich ihre Prozesse zum Abschluss bringen und es bleiben keine „Prozessleichen“ zurück, die nicht mehr abgeschlossen werden können, weil etwa aufgrund eines Prozessabbruchs eines Kommunikationspartners eine erwartete eingehende Nachricht nicht mehr ankommt.

\subsubsection{Behandlung von Ausnahmen und Unterbrechungen}

Aktivitäten, also Aufgaben und Prozesse, können durch bestimmte Ereignisse abgebrochen oder unterbrochen werden. Dies wird durch Ereignissymbole gekennzeichnet, die der jeweiligen Aktivität angeheftet sind. Wird durch das eintretende Ereignis die Aktivität abgebrochen, so wird dies durch zwei durchgehende äußere Kreislinien gekennzeichnet. Wird die Aktivität nicht unterbrochen, so wird dies durch zwei gestrichelte Kreislinien dargestellt. Im untenstehenden Beispiel reagieren wir auf das Eintreten eines Timer-Ablaufs. Wir können also modellieren, dass die Ausführung der Aufgabe nicht länger als eine bestimmte Zeitspanne dauern darf (links) oder dauern sollte (rechts), und dass bei Zeitablauf in irgendeiner Form reagiert werden muss. Die Reaktion ist in dem vom angehefteten Ereignis ausgehenden Sequenzfluss zu modellieren. Auf dieselbe Art und Weise können auch ungeplante Ereignisse wie Fehler oder Eskalationen modelliert und dargestellt werden (siehe Abb. 3.41).

\subsection{Beispiel: Nicht-unterbrechende Timerevents}

Die folgende Abbildung (siehe Abb. 3.42) stellt dar, dass der Teilprozess, der die Aktivitäten der Bearbeitung einer Bestellung in einem Fast Food Restaurant abbildet, nicht länger als 5 min dauern soll. Dauert die Bearbeitung der Bestellung länger als 5 min, soll 


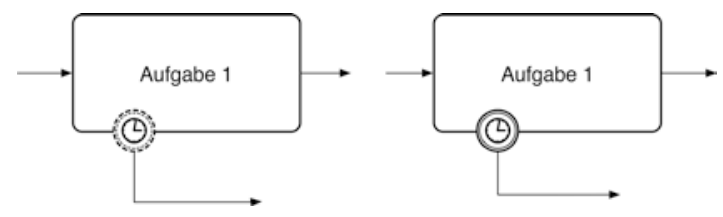

Abb. 3.41 Beispiel für angeheftete Ereignisse (links: nicht unterbrechend, rechts: unterbrechend)

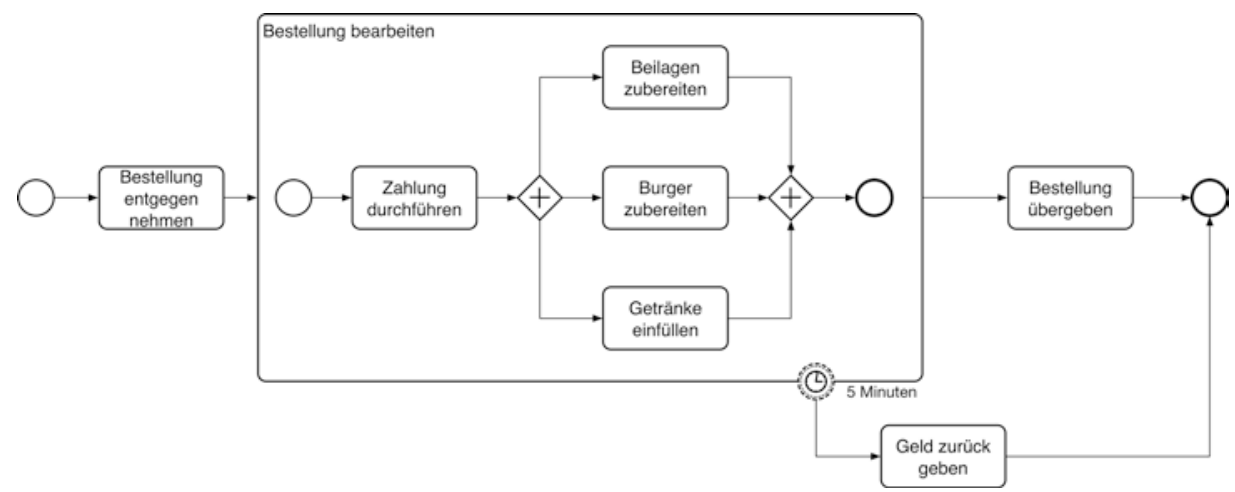

Abb. 3.42 Beispiel für die Verwendung von nicht-unterbrechenden Timer-Events

der Kunde das Geld zurückerhalten. Die Bestellung soll trotzdem noch fertig bearbeitet werden. Im Fall eines unterbrechenden Events würde der Kunde lediglich sein Geld zurückerhalten, nicht aber das bestellte Essen.

\subsubsection{Terminierung von Prozessen}

Sequenzflüsse in einem Prozess werden üblicherweise mit einem Endereignis abgeschlossen. Endereignisse beenden jedoch nur die Ausführung des jeweiligen Sequenzflusses. Falls parallel noch weitere Sequenzflüsse aktiv sind, etwa, weil sie durch eine paralleles oder inklusives Gateway geöffnet wurden oder weil sie durch angeheftete Events ausgelöst wurden, werden diese weiterhin ausgeführt. Um einen (Sub-)Prozess vollständig und sofort zu beenden (also die Ausführung in allen Zweigen zu beenden), existieren mehrere Möglichkeiten.

\subsection{Das Terminate-Event}

Das Terminate-Event bricht alle aktiven Zweige eines Prozesses innerhalb eines Pool unmittelbar und sofort ab. Prozesse in anderen Pools sind nicht betroffen und sollten deshalb ggf. vor dem Abbruch durch das Senden eines Signals vom Abbruch in Kenntnis gesetzt werden. 


\subsection{Das Error-Event}

Das Error-Event zeigt das Auftreten eines semantischen Fehlers an und wird üblicherweise in Subprozessen eingesetzt. Es bricht die Ausführung des Subprozesses ab, der Fehlergrund kann dem Event als Parameter mitgegeben werden. Durch angeheftete empfangende Ereignisse kann auf diese Fehler im übergeordneten Prozess reagiert werden und können entsprechende Aktivitäten ausgelöst werden. Angeheftete Fehlerereignisse sind immer unterbrechend, brechen also die Ausführung des Subprozesses (inkl. aller gegebenenfalls noch aktiver Sequenzflüsse in Zweigen, in denen kein Fehler aufgetreten ist) ab. Als „schwächere“ Variante kann auch das „Eskalationsereignis“ in identischer Weise verwendet werden. Dieses existiert auch in einer nicht-unterbrechenden Form und erlaubt damit die Fortsetzung der Bearbeitung des Subprozesses, in dem das Problem aufgetreten ist.

Falls beim Abbruch von Subprozessen die Auswirkungen von bereits durchgeführten Aktivitäten rückgängig gemacht werden müssen, kann auf die im nächsten Abschnitt vorgestellte Transaktionsbehandlung wie in der BPMN vorgesehen zurückgegriffen werden.

\subsubsection{Transaktionen}

In BPMN existiert auch die Möglichkeit, Transaktionen in einem Prozess abzubilden. Von einer Transaktion spricht man, wenn eine Menge von Aufgaben eines Prozesses als Gesamtheit vollständig oder gar nicht ausgeführt werden sollen. Insbesondere sollen bei Scheitern einer Aufgabe bestimmte andere bereits abgeschlossenen Aufgaben wieder rückgängig gemacht werden. BPMN kennt das Konzept transaktionaler Subprozesse in Kombination mit Kompensierungsereignissen und -aktivitäten. Kompensierung bedeutet, dass bereits ausgeführte Prozessschritte dadurch zurückgerollt werden, indem konkrete Gegenmaßnahmen durch einen weiteren Prozessschritt eingeleitet werden.

In einem Transaktionssubprozess (in BPMN gekennzeichnet durch eine doppelte Umrandung) wird jeder Aufgabe über ein angeheftetes Kompensierungszwischenereignis (gekennzeichnet durch ein „Zurückspulen“-Symbol) eine Kompensierungsaufgabe zugeordnet. Bricht die Transaktion ab, so wird für jede Aufgabe, die bereits erfolgreich abgeschlossen wurde, die jeweilige Kompensierung ausgeführt. In diesem Zusammenhang kommt das Abbruch-Event (gekennzeichnet durch „X“) ins Spiel. Als auslösendes Endereignis in einem Transaktionssubprozess bewirkt es dessen Abbruch und damit das Auslösen der Kompensierungen. Wenn es an die Transaktion angeheftet wird, ist es ein empfangendes Zwischenereignis und bestimmt es den weiteren Ablauf des Prozesses nach dem Abbruch der Transaktion. Durch das Konzept der Kompensierung kann eine Transaktion auch dann noch zurückgerollt werden, nachdem sie eigentlich schon erfolgreich abgeschlossen wurde. Außerhalb der Transaktion kann ein auslösendes Kompensierungsereignis verwendet werden, um die Kompensierung der referenzierten Transaktion nachträglich auszulösen.

Die nachfolgende Abbildung (siehe Abb. 3.43) illustriert diese Konzepte anhand eines Reisebuchungsprozesses. 


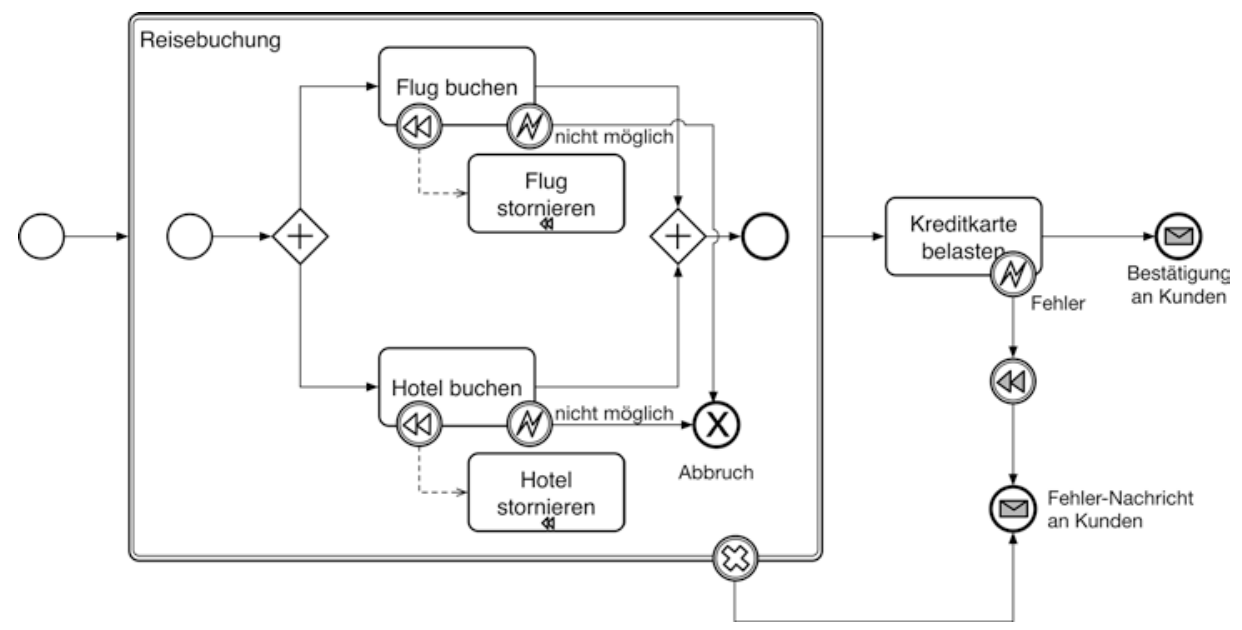

Abb. 3.43 Beispiel für einen Transaktions-Subprozess

Eine Reisebuchung besteht aus einer Flug- und einer Hotelbuchung, die potenziell parallel vorgenommen werden können. Ist eine der Buchungen nicht möglich, so muss die andere Buchung (falls sie schon ausgeführt wurde) wieder storniert werden. Ein Fehler bei einer einzelnen Buchung löst also den Abbruch der Transaktion aus und führt dazu, dass der Prozess mit einer Fehlernachricht an den Kunden reagiert. Außerhalb der eigentlichen Transaktion führt ein Fehler bei der Belastung der Kreditkarte zur Stornierung der gesamten Buchung.

\subsubsection{Ereignisgesteuerte Sub-Prozesse}

Ereignisgesteuerte Sub-Prozesse sind eine Alternative zur Behandlung von auftretenden Ereignissen in (Sub-)Prozessen. Während an Subprozessen angeheftete Events die Reaktion auf diese in den umgebenden Prozess ,hinaustragen', kann diese durch den Einsatz von Ereignisgesteuerten Sub-Prozessen lokal gehalten werden.

Die folgende Abbildung (siehe Abb. 3.44) zeigt ein Beispiel für einen timer-gesteuerten nicht-unterbrechenden Subprozess. Es greift das oben bereits verwendete Szenario der Zubereitung einer Bestellung in einem Fast-Food-Restaurant wieder auf.

Die Events, mit denen ereignisgesteuerte Subprozesse ausgelöst werden können, sind identisch mit jenen, die an einen Subprozess angeheftet werden können. Beide Varianten können unterbrechend und nicht-unterbrechend sein. Semantisch unterscheiden sie sich wie erwähnt lediglich in der Art der Behandlung des Ereignisses - nämlich lokal innerhalb des Subprozesses oder außerhalb im umschließenden Prozess. Je nach Anwendungsfall kann die eine oder die andere Variante zu sinnvollen und/oder übersichtlichen Darstellungsformen führen. 


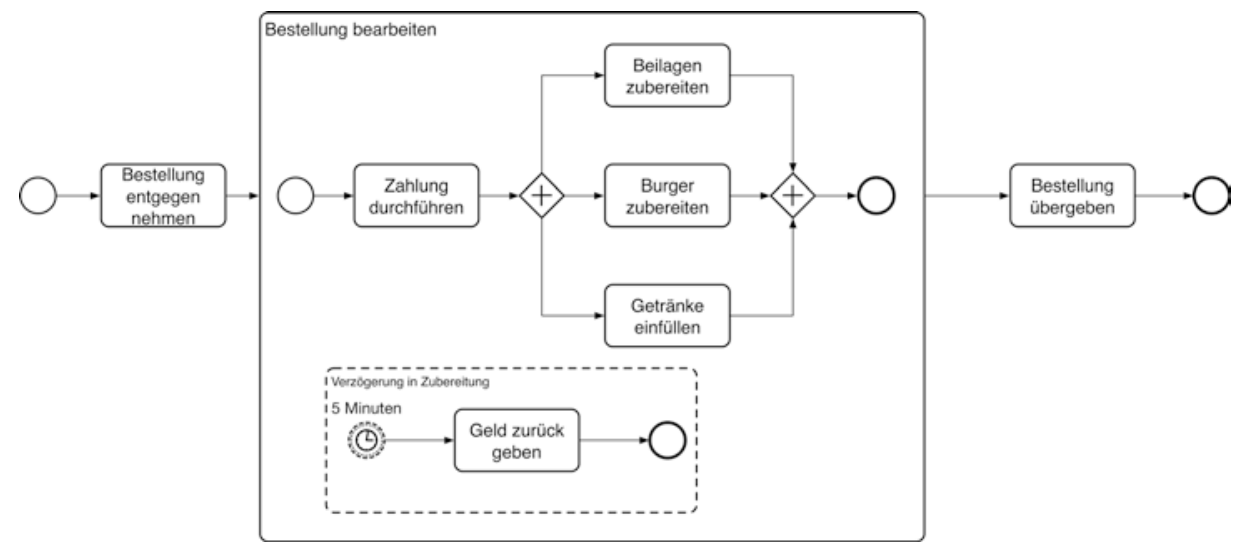

Abb. 3.44 Beispiel für den Einsatz eines nicht-unterbrechenden ereignisgesteuerten Sub-Prozesses

\subsubsection{Choreografiediagramme}

Die explizite Modellierung von Choreografien in Choreografie-Diagrammen wurde in der BPMN 2.0 neu eingeführt. Bei einer Choreografie handelt es sich um den Ablauf von Nachrichtenaustauschen zwischen unterschiedlichen Akteuren. Eine Choreografie ist damit eine andere Sicht auf eine Kollaboration, bei der die Reihenfolge der Nachrichtenübermittlung unabhängig von den Prozessen der einzelnen Akteure dargestellt wird.

Zwar kann man einer Darstellung der Kommunikation mittels zugeklappten, d. h. leeren, Pools entnehmen, welche Nachrichten zwischen welchen Partnern ausgetauscht werden, doch lassen sich die genaue Reihenfolge, bedingte Nachrichtenflüsse oder Schleifen nicht daraus ersehen. So ist im bislang verwendeten Bewerbungsbeispiel beispielsweise nicht gezeigt, dass nach dem Eintreffen eines Angebots beim Kunden entweder die Einladungs- oder die Absage-Nachricht gesendet wird. Dies lässt sich mit einem Choreografie-Diagramm visualisieren.

Die folgende Abbildung (siehe Abb. 3.45) enthält die Choreographie zu dem oben bereits als Kollaboration gezeigten Beispiel eines Bewerbungsprozesses. Hier sind die Nachrichtenaustauschvorgänge in den Mittelpunkt gerückt. Eine sogenannte Choreographie-Aktivität (Choreography Activity) repräsentiert den Austausch einer oder mehrerer Nachrichten zwischen zwei oder mehreren Partnern. Im einfachsten Fall entspricht sie dem Senden einer einzigen Nachricht von einem Partner zu einem anderen.

Jede Choreografie-Aktivität wird von einem der beteiligten Partner ausgelöst, indem er die erste Nachricht sendet. Dieser auslösende Partner wird am oberen oder unteren Rand der Choreografie-Aktivität in einem hellen Feld eingetragen. Die Namen des oder der weiteren Beteiligten werden am anderen Rand in einem dunkleren Feld eingetragen. Wer oben und wer unten eingetragen wird, ist dem Modellierer freigestellt. Normalerweise wird man bei mehreren Choreografie-Aktivitäten zwischen denselben Partnern die 
Abb. 3.45 Beispiel für den Einsatz von ChoreographieAktivitäten

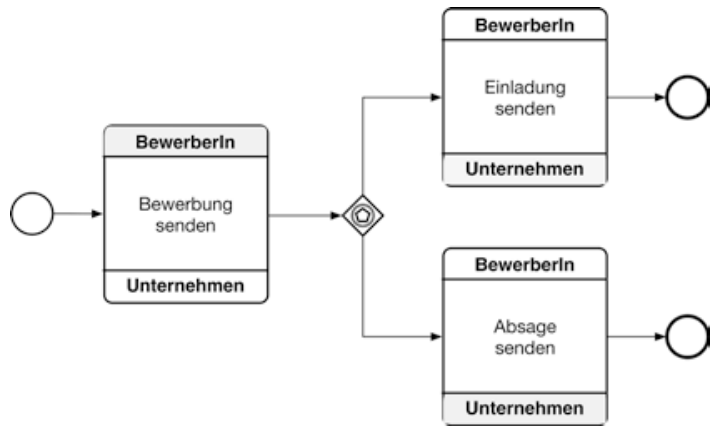

Anordnung beibehalten. Wenn man zusätzlich noch eine Kollaboration modelliert, ist es naheliegend, die vertikale Anordnung der Pools zugrunde zu legen. Entsprechend sind in den Choreografie-Aktivitäten der Abbildung entweder der Kunde oben und die Werbeagentur unten oder aber die Werbeagentur oben und die Grafiker unten eingezeichnet.

Choreografie-Aktivitäten mit mehr als zwei Partnern kommen in diesem Beispiel nicht vor. Hierfür kann man oben bzw. unten mehrere Partner-Felder eintragen. Dabei ist aber immer nur ein Feld hell hinterlegt, da nur einer der Partner den Nachrichtenaustausch durch eine initiale Nachricht in Gang setzen kann.

Für die Choreografie-Aktivitäten ist im Choreografie-Diagramm ein Sequenzfluss definiert. Seine Modellierung entspricht im Wesentlichen der Sequenzfluss-Modellierung von Prozessen. Allerdings sind gewisse Elemente der Prozessmodellierung im Zusammenhang mit der Choreografie-Modellierung nicht sinnvoll und daher auch nicht zulässig. So gibt es z. B. keine Nachrichten- Ereignisse innerhalb eines Sequenzflusses, da der Nachrichtenaustausch per Definition Teil der Choreografie-Aktivitäten ist. Entsprechend folgen beispielsweise auf das ereignisbasierte Gateway in der Abbildung keine Ereignisse, sondern Choreografie-Aktivitäten. Hierbei wird der Pfad gewählt, dessen Choreografie-Aktivität als erste durch die jeweilige auslösende Nachricht gestartet wird.

Will man wissen, welche Nachrichten in jeder Choreografie-Aktivität ausgetauscht werden, so können diese wiederum in Form kleiner Briefsymbole hinzugefügt und mit dem jeweiligen Partnerfeld verbunden werden. Die Briefe sind ebenso wie die beteiligten Partner farblich gekennzeichnet. Ein helles Briefsymbol steht für die Nachricht, mit der eine Choreografie-Aktivität ausgelöst wird. Die Briefsymbole der anderen Nachrichten sind dunkler dargestellt.

\subsubsection{Einordnung}

Die BPMN hat sich in den letzten Jahren auch in der industriellen Praxis durchgesetzt. Durch ihren umfassenden Satz an Sprachelementen eignet sie sich für viele Anwendungsbereiche von der Dokumentation bis hin zur automationsgestützten Ausführung von Geschäftsprozessen in Organisationen. Der umfangreiche Sprachschatz 
wird gleichzeitig aufgrund der gesteigerten Komplexität als Manko der BPMN gesehen. Insbesondere die Vielzahl an Ereignistypen mit zum Teil nur schwer zu unterscheidenden Bedeutungen führt zu gesteigertem Aufwand beim Erlernen der Sprache.

Der mit der möglichen Komplexität der entstehenden Modelle und der damit einhergehenden erschwerten Verständlichkeit wird üblicherweise dadurch begegnet, dass in geeigneten Anwendungsfällen ein reduzierter Sprachumfang verwendet wird. Zur deskriptiven Dokumentation von Geschäftsprozessen ist es üblicherweise nicht notwendig, den vollständigen Satz an Ereignissen und komplexeren Aufgabentypen zu verwenden. Erst wenn ein Prozessmodell etwa durch Simulation validiert oder zur Ausführung gebracht werden soll, müssen die Modelle um Information zu Ausnahmefällen o. Ä. angereichert werden. In diesen Fällen kann von den einfacheren Modellen ausgehend eine Detaillierung und Ergänzung vorgenommen werden.

Die BPMN ist eine der wenigen Sprachen zur Geschäftsprozessmodellierung, die explizit auf Kommunikationsvorgänge zwischen beteiligten Akteuren eingeht und deren Abbildung ermöglicht. Bei der Definition der Sprache war der Ausgangspunkt zur Spezifikation von Kommunikationsflüssen allerdings die Kopplung technisch getrennter Informationssysteme. Die BPMN geht implizit davon aus, dass es innerhalb eines Pool (also zwischen Lanes) nicht notwendig ist, sich um die Kommunikation zwischen Akteuren zu kümmern, weil alle Zugriff auf die gleiche Informationsinfrastruktur haben. Zwischen Pools werden Nachrichtenflüsse modelliert, die im Rahmen der Ausführung dazu dienen, die Abbildung der im Ausgangspool verwendeten Datenstrukturen auf jene des Ziel-Pool zu beschreiben.

Ein Nachrichtenfluss entspricht also im Wesentlichen semantisch einer Datenübergabe von einem Informationssystem zu einem anderen und ist dem entsprechend immer ein Kommunikationsvorgang mit genau einem Sender und genau einem Empfänger - mehrere Empfänger können beispielsweise nicht mit einer einzelnen Nachricht angesprochen werden. Während dieser Mechanismus auch zur Abbildung von nicht-technischer Kommunikation eingesetzt werden kann, ist seine Ausdrucksstärke jedoch beschränkt. Insbesondere kann etwa eine Kommunikation zwischen zwei oder mehreren Akteuren ohne klar abgrenzbare Nachrichten nur auf Umwegen und mehrdeutig modelliert werden. Diese Einschränkung ist jedoch dem Anspruch der Ausführbarkeit der erstellen Prozesse geschuldet und existiert in dieser Form auch in anderen kommunikationsorientierten Ansätzen.

Die BPMN fokussiert des Weiteren auf Prozessen mit einem vollständig spezifizierbaren Kontrollfluss. Dies stößt an Grenzen, sobald Prozessteile fallspezifisch und nicht vorab im Detail beschreibbar sind. Für derartige Prozesse haben sich in den letzten Jahren unterschiedliche Ansätze gebildet, die entweder auf eine deklarative Modellierung der Ausführungsbedingungen von Prozessteilen abzielen, oder die Kommunikationsvorgänge zwischen den beteiligten Akteuren in den Mittelpunkt stellen. Als Beispiel für letztere Kategorie betrachten wir im nächsten Abschnitt die subjektorientierte Geschäftsprozessmodellierung (S-BPM). 


\subsection{S-BPM}

Ein subjektorientiertes Prozessmodell beschreibt im Gegensatz $\mathrm{zu}$ anderen Modellierungsansätzen Geschäftsvorgänge primär aus Sicht von miteinander kommunizierenden Handelnden oder Systemen. Es erfasst, welche Arbeitsschritte eines Geschäftsprozesses durch wen bzw. welches System mit welchen Hilfsmitteln ausgeführt werden, welches Ergebnis dadurch erzeugt wird und für wen dieses bestimmt ist.

Bei der Modellierung nach dem subjektorientierten Ansatz stehen die Subjekte als Repräsentanten für an einem Prozess beteiligte Handelnde bzw. ausführende Systeme im Mittelpunkt der Betrachtung. Es wird im Wesentlichen beschrieben, wer in welcher Form miteinander kommuniziert und wie die einzelnen Akteure auf erhaltene Nachrichten reagieren. Die Beschreibung der Kommunikation erfolgt durch die Definition der Nachrichten, die zwischen den Subjekten ausgetauscht werden. Das Verhalten der Subjekte wird jeweils separat durch Zustandsdiagramme beschrieben, wobei drei unterschiedlichen Zustandstypen zum Einsatz kommen: Ein Subjekt kann auf eine Nachricht warten, eine Nachricht senden oder etwas tun, ohne mit anderen Subjekten zu kommunizieren. Der letztere Zustandstyp wird als Funktionszustand bezeichnet und wird verwendet, um das eigentliche Verhalten, also die Aktivitäten eines Subjekts zu beschreiben.

\subsubsection{Notationselemente}

Bei der Modellierung nach dem subjektorientierten Ansatz stehen die Subjekte als Repräsentanten für an einem Prozess beteiligte Handelnde bzw. ausführende Systeme im Mittelpunkt der Betrachtung. Die Modellierung eines Prozesses läuft im Wesentlichen in folgenden Schritten mit zunehmendem Detaillierungsgrad ab: zuerst wird das Interaktionsdiagramm erstellt, in dem die Subjekte und deren Nachrichtenaustausch modelliert werden (siehe Abb. 3.46). In einem weiteren Schritt wird das Verhalten jedes Subjektes in einem separaten Verhaltensdiagramm beschrieben.

Für das Interaktionsdiagramm werden zuerst die an einem Prozess beteiligten Subjekte festgelegt. Ein Subjekt ist eine aktive Einheit, muss aber nicht unbedingt ein menschlicher Akteur sein. Auch technische Systeme können Subjekte sein, solange sie eine aktive Rolle im Prozess einnehmen. Subjekte sind immer abstrakt zu beschreiben, d. h. nicht für konkrete Personen oder Maschinen, sondern auf Basis der notwendigen zu erfüllenden Aufgaben im Prozess festzulegen (also etwa „Antragsprüfer“, und nicht „Hr. Müller").

Abb. 3.46 Notationselemente des S-BPM

Interaktionsdiagramms

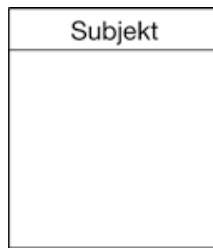



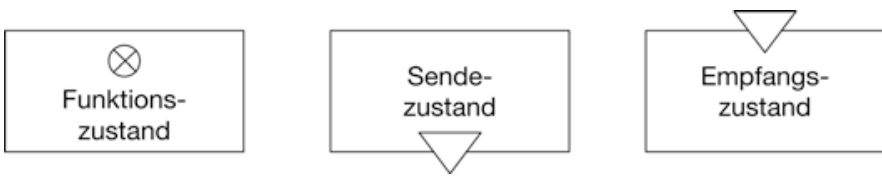

[Bedingung für Zustandsübergang]

Abb. 3.47 Notationselemente des S-BPM Verhaltensdiagramms

Zwischen den Subjekten werden Nachrichten ausgetauscht. Im Interaktionsdiagramm wird nur festgelegt, welche Nachrichten existieren und wer jeweils Sender und Empfänger ist. Die Reihenfolge der Nachrichten wird hier noch nicht festgelegt (siehe Abb. 3.47).

Für jedes Subjekt wird im Verhaltensdiagramm beschrieben, in welcher Reihenfolge es Nachrichten sendet und empfängt bzw. interne Funktionen ausführt. Die einzelnen Zustände werden mit Verbindungen zueinander in Beziehung gesetzt, welche die Zustandsübergänge beschreiben. Deren Verwendung hängt vom jeweils eingesetzten Zustandstyp ab:

- Zu jedem Funktionszustand wird beschrieben, was im jeweiligen Verhaltensschritt zu tun ist. Die Endbedingungen eines Funktionszustandes entsprechen den ausgehenden Verbindungen, die vom jeweiligen Funktionszustand ausgehen. Wenn die Funktion zu unterschiedlichen Ergebnissen führen kann, können also verschiedene Folgezustände aktiviert werden. Dadurch wird die Abbildung von alternativen Verhaltensweisen möglich.

- In einem Sendezustand wird eine Nachricht an einen Empfänger übermittelt. In ihm wird so lange verharrt, bis der Empfänger in der Lage ist, die Nachricht entgegenzunehmen. Wer die Nachricht empfangen soll und welche Nachricht übermittelt wird, wird an der ausgehenden Kante des Sendezustands beschrieben.

- In einem Empfangszustand wird so lange verharrt, bis eine der Nachrichten eingetroffen ist, die der Empfangszustand entgegennehmen kann. Da auf unterschiedliche Nachrichten reagiert werden kann, kann je nach Typ der eingetroffenen Nachricht auch ein unterschiedlicher Folgezustand aktiviert werden. Dazu werden wiederum mehrere ausgehende Verbindungen verwendet, an denen beschrieben wird, welche Nachricht von welchem Absender zum entsprechenden Zustandsübergang führt. Auf diese Weise wäre es auch möglich, auf die gleiche Nachricht von unterschiedlichen Absendern unterschiedlich zu reagieren.

\subsubsection{Beispiele}

Um die Unterschiede und Gemeinsamkeiten zu den zuvor diskutierten Modellierungssprachen herauszuarbeiten, bedienen wir uns hier wieder der bereits verwendeten Beispiele. In den ersten Beispielen findet keine Kommunikation statt, wir fokussieren deshalb auf das Verhaltensdiagramm des jeweils einzigen beteiligten Subjektes.

Dieses Beispiel (siehe Abb. 3.48) zeigt einen Prozess mit einer einzelnen Aufgabe, in der ein Antrag (über den wir hier nicht mehr wissen) bearbeitet wird. Der Prozess endet, 
Abb. 3.48 Einfacher

Prozess als S-BPM

Verhaltensdiagramm

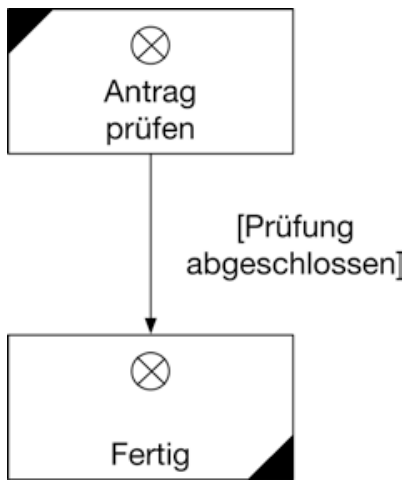

nachdem die Bearbeitung des Antrags abschlossen wurde. Ein Verhaltensdiagramm muss immer einen Startzustand haben, der üblicherweise durch ein Dreieck in der linken oberen Ecke gekennzeichnet wird. Außerdem muss ein Endzustand existieren, der durch ein Dreieck in der rechten unteren Ecke gekennzeichnet wird. Um das Verhalten des Subjektes vollständig zu beschreiben, benötigen wir einen Zustandsübergang, der kennzeichnet, unter welchen Bedingungen wird den Zustand „Antrag prüfen“ verlassen können. Deshalb fügen wir hier einen funktionslosen Zustand „Fertig“ ein, den wir als Endzustand kennzeichnen.

Im obenstehenden Beispiel (siehe Abb. 3.49) ist der Prozess um eine Entscheidung mit drei möglichen Ausgängen erweitert, die einander ausschließen. Der Antrag wird geprüft, das Ergebnis dieser Prüfung ermöglicht das Treffen einer Entscheidung über die weitere Bearbeitung. Im Falle einer Investitionssumme ab 10.000 EUR wird der Antrag weitergeleitet. Dies kennzeichnen wir durch einen Sendezustand und spezifizieren an der ausgehenden Verbindung, wer den Antrag erhalten soll. Damit der Prozess vollständig beschrieben ist, müsste an dieser Stelle auch ein Verhaltensdiagramm für den Vorgesetzten erstellt werden.

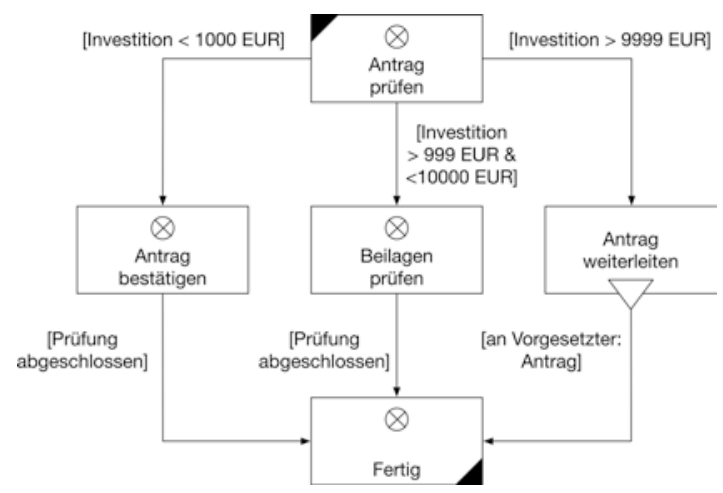

Abb. 3.49 S-BPM Verhaltensdiagramm mit Verzweigung (3 Zweige) 
Auch hier können wir Teile eines Prozesses wiederholt abarbeiten (siehe Abb. 3.50). Dazu wird eine Verbindung am Ende des zu wiederholenden Teils eingefügt, mit einer Wiederholungsbedingung versehen und zum ersten Zustand des zu wiederholenden Teils zurückgeführt. Die andere ausgehende Verbindung führt den Prozess nach der wiederholten Abarbeitung weiter.

Das obige Beispiel zeigt nun einen Prozess mit zwei Subjekten. Die erste Abbildung (siehe Abb. 3.51) zeigt das Interaktionsdiagramm. Die beiden folgenden Modelle (siehe Abb. 3.52) zeigen die Verhaltensdiagramme von Sachbearbeiter und Abteilungsleiter. Die positive oder negative Beurteilung wird jeweils als Nachricht übermittelt. Die Begründung zur Beurteilung wird nur im Falle einer negativen Beurteilung als Datenobjekt zur Aufgabe „Antrag ablehnen“ übergeben. Im Fall der Bestätigung des Antrags wird das Datenobjekt „Beurteilung“ nicht mehr benötigt - wir können also annehmen, dass in diesem Fall keine weitere Begründung erfolgt.

\subsubsection{Erweiterte Formen der Kommunikationsmodellierung}

Durch den Fokus von S-BPM auf die Abbildung von Kommunikationsvorgängen erlaubt diese eine umfassendere und flexiblere Beschreibung derselben als sämtliche zuvor betrachteten Modellierungssprachen. Insbesondere erlaubt die S-BPM die Abbildung

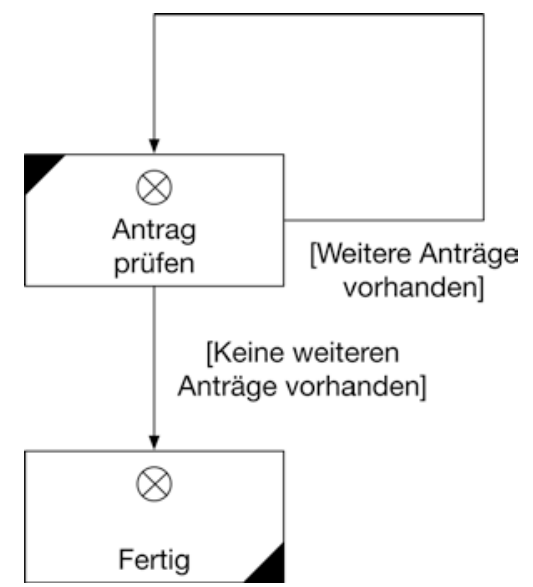

Abb. 3.50 S-BPM Verhaltensdiagramm mit Schleife

Abb. 3.51 S-BPM

Interaktionsdiagramm

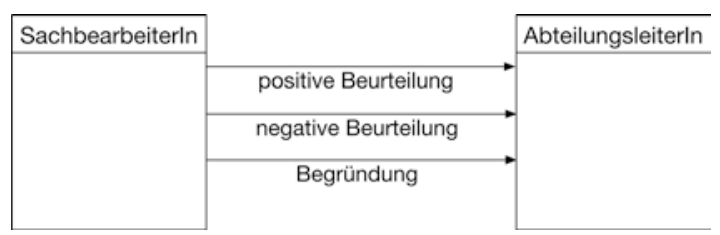



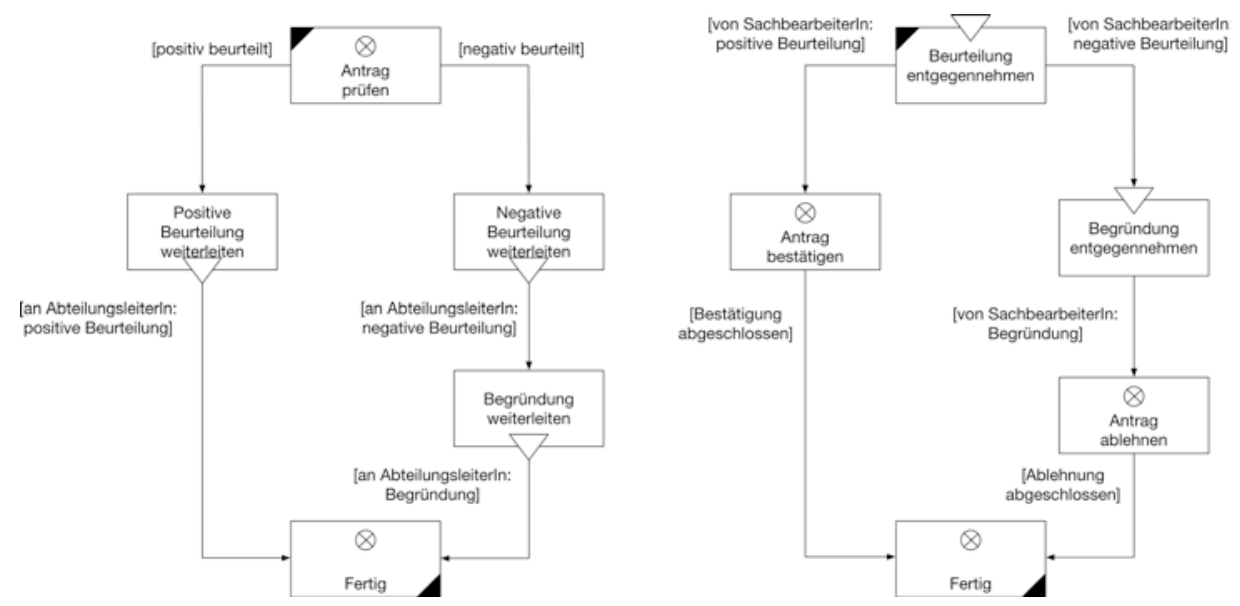

Abb. 3.52 S-BPM Verhaltensdiagramme zum obigen Interaktionsdiagramm (links: Verhalten Sachbearbeiter, rechts: Verhalten Abteilungsleiter)

von komplexen Kommunikationsszenarien durch den Einsatz von Inputpools sowie die detaillierte Beschreibung der in Nachrichten ausgetauschten Daten durch Geschäftsobjekte - wie nachfolgend erklärt.

\subsubsection{Inputpools}

Ein Inputpool dient einem Subjekt quasi als Postkasten, in dem eingehende Nachrichten gespeichert werden, bis sie im Verhaltensdiagramm benötigt werden. Im Gegensatz zu einem einfachen Postkasten ist ein Inputpool aber konfigurierbar. Es kann festgelegt werden, wie viele Nachrichten welchen Typs zwischengespeichert werden können. Wenn der Inputpool entsprechend seiner Konfiguration nicht in der Lage ist, eine Nachricht entgegenzunehmen, so muss der Sender im Sendezustand warten, bis die Nachricht zugestellt werden kann. Dadurch lassen sich unterschiedliche Kommunikationsszenarien abbilden.

Wird der Platz im Inputpool für einen bestimmten Nachrichtentyp auf 0 reduziert, so muss der Sender immer warten, bis der Empfänger bereit ist, die Nachricht entgegenzunehmen. Man spricht dann von synchroner Kommunikation. Wenn der Inputpool so konfiguriert ist, dass er Nachrichten zwischenspeichern kann, muss der Sender nicht warten, bis der Empfänger in jenem Zustand ist, in dem er die Nachricht annehmen kann. Man spricht dann von asynchroner Kommunikation (dies ist die einzige Art der Kommunikation, die in der BPMN abgebildet werden kann). Darüber hinaus erlauben Inputpools, Nachrichten in beliebiger Reihenfolge entgegen zu nehmen. Die Nachrichten müssen also nicht in jener Reihenfolge abgearbeitet werden, in der sie eintreffen, sondern können entsprechend der Bedürfnisse des Empfängers verarbeitet werden.

Inputpools haben keine grafische Entsprechung in der S-BPM, sondern sind ein Konzept der Ausführungssemantik. Sie werden für jedes Subjekt textuell bzw. in einem 
Konfigurationswerkzeug beschrieben. Wenn keine Inputpools definiert werden, so hat die Standardkonfiguration unbegrenzt viele Speicherstellen für beliebige Nachrichten. Das Kommunikationsverhalten entspricht also den Nachrichtenflüssen der BPMN (asynchrone Kommunikation).

\subsubsection{Geschäftsobjekte}

Geschäftsobjekte dienen der Spezifikation jener Dinge, die zur Leistungserbringung in einem Geschäftsprozess benötigt werden. Es sind also die Dinge, die in einem Prozess verwendet werden und können Daten genauso umfassen wie physische Ressourcen. Geschäftsobjekte sind passiv, d. h. sie initiieren keine Interaktionen oder Aktionen. Geschäftsobjekte werden von Subjekten bearbeitet und können Nachrichten zugeordnet werden, um diese hinsichtlich ihres Inhalts näher zu spezifizieren.

Wie für Inputpools gibt es auch für Geschäftsobjekte keine grafische Entsprechung in der Notation der Modellierungssprache und sind ebenfalls Konzepte der Ausführungssemantik und daher von der technischen Ausführungsumgebung abhängig. Sie werden deshalb üblicherweise tabellarisch angegeben. Eine Grundstruktur von Geschäftsobjekten besteht aus einem Bezeichner, aus Datenstrukturen und Datenelementen. Der Bezeichner eines Geschäftsobjektes ergibt sich aus dem Geschäftsumfeld, in dem es eingesetzt wird. Beispiele sind Dienstreiseantrag, Bestellung, Lieferschein, Rechnung etc.

Geschäftsobjekte setzen sich aus Datenstrukturen zusammen, deren Komponenten einfache Datenelemente eines bestimmten Typs (z. B. Zeichenkette oder Zahl) oder selbst wieder Datenstrukturen sein können.

Um das Verständnis sicherzustellen bzw. zu erleichtern empfiehlt es sich, die Bedeutung der Datenelemente näher zu beschreiben, insbesondere dann, wenn sich diese nicht zweifelsfrei aus den Bezeichnern ableiten lässt.

Die folgende Abbildung (siehe Tab. 3.1) zeigt ein Beispiel für einen Dienstreiseantrag. Dieser besteht unter anderem aus der Datenstruktur ,Daten zum Antragsteller (Mitarbeiter) mit den Datenelementen für Name, Vorname und Personalnummer und der Struktur „Daten zur Dienstreise“ mit den Datenelementen für Beginn, Ende und Zweck der Reise.

In vielen Fällen ändert sich die Semantik eines Geschäftsobjekts während der Prozessausführung, etwa wenn ein Lieferschein in eine Rechnung überführt wird. Für ein Geschäftsobjekt können deshalb mehrere verschiedene Zustände definiert werden. Bei einem Wechsel des Status werden nur die Datenstrukturen bzw. Datenelemente des vorherigen Status übernommen, die der neue Status benötigt, und bei Bedarf neue Komponenten hinzugefügt oder nicht mehr benötigte entfernt. Damit ist gewährleistet, dass ein Subjekt nur diejenigen Datenelemente für seine Arbeit zur Verfügung bekommt, die es dafür wirklich benötigt. Dies erleichtert die Einhaltung von Datenschutzbestimmungen.

Im Beispiel des Dienstreiseantrags kann aus dem ursprünglichen Status „Reiseantrag“ des Geschäftsobjekts der Status „Dienstreisebuchung“ abgeleitet werden. Dabei werden insbesondere Datenelemente mit internen Angaben wie Personalnummer, Vergütungs- 
Tab. 3.1 Datenstruktur des Geschäftsobjekts ,DR-Antrag ' (Dienstreiseantrag)

\begin{tabular}{|c|c|c|c|c|}
\hline Datenstruktur & Bedeutung & Datentyp & Kann/Muss & Wertebereich/Default \\
\hline \multicolumn{5}{|c|}{ Daten zum Antragsteller } \\
\hline Name & Nachname & Character & M & $\ldots$ \\
\hline Vorname & Vorname & Character & M & $\ldots$ \\
\hline Personalnummer & $\ldots$ & Integer & M & $\ldots$ \\
\hline Organisationseinheit & $\ldots$ & $\ldots$ & K & $\ldots$ \\
\hline Vergütungsgruppe & $\ldots$ & $\ldots$ & $\mathrm{K}$ & $\ldots$ \\
\hline \multicolumn{5}{|l|}{ Daten zur Reise } \\
\hline Reisebeginn & $\cdots$ & Date & M & $\begin{array}{l}\text { innerhalb/Jahres ab akt. } \\
\text { Datum/akt. Datum }\end{array}$ \\
\hline Reiseende & $\cdots$ & Date & M & $\begin{array}{l}\text { Reisebeginn plus/Jahr/ } \\
\text { Reisebeginn }\end{array}$ \\
\hline Internationale Reise & $\ldots$ & Boolean & $\mathrm{K}$ & $\mathrm{j} / \mathrm{n} ; \mathrm{n}$ \\
\hline Reiseziel (Ort/Land) & $\ldots$ & Character & M & $\ldots$ \\
\hline Reisegrund & $\ldots$ & Character & M & $\ldots$ \\
\hline $\begin{array}{l}\text { Gewünschter Vor- } \\
\text { schuss }\end{array}$ & $\ldots$ & Integer & $\mathrm{K}$ & $\ldots$ \\
\hline \multicolumn{5}{|c|}{ Daten zur Genehmigung } \\
\hline Genehmigung & $\begin{array}{l}\text { Genehmigungs- } \\
\text { vermerk }\end{array}$ & Boolean & M & $\mathrm{j} / \mathrm{n} ; \mathrm{n}$ \\
\hline Kostenstelle & $\ldots$ & Integer & M & $\ldots$ \\
\hline $\begin{array}{l}\text { Gewünschter Vor- } \\
\text { schuss }\end{array}$ & $\ldots$ & Integer & $\mathrm{K}$ & $\ldots$ \\
\hline
\end{tabular}

gruppe, Reisegrund und die komplette Datenstruktur zur Genehmigung entfernt, welche beispielsweise bei der Einschaltung eines Reiseagenten für die Buchung das Unternehmen nicht verlassen sollen und auch nicht außerhalb relevant sind. Dafür wird, wie in der folgenden Abbildung (siehe Tab. 3.2) gezeigt, eine neue Datenstruktur „Daten zur Buchung“ eingefügt. Sie enthält Datenelemente, mit denen die Reisestelle gegenüber dem Reiseagenten eine Frist für den spätesten Eingang der Buchungsbestätigung setzen und bestimmte Hotelketten vorgeben kann, mit denen beispielsweise Rahmenverträge bestehen.

\subsubsection{Einordnung}

Im Gegensatz zu den anderen bislang besprochenen Modellierungssprachen gibt es in der S-BPM kein einzelnes Diagramm, das einen Geschäftsprozess vollständig beschreibt. Vielmehr wird für jedes Subjekt ein separates Verhaltensdiagramm erstellt. Diese werden 
Tab. 3.2 Geschäftsobjekt, DR-Antrag ' im Status, Dienstreisebuchung ‘

\begin{tabular}{l|l|l|l|l}
\hline $\begin{array}{l}\text { Datenstruktur/ } \\
\text { Datenelement }\end{array}$ & Bedeutung & Datentyp & Kann/Muss & Wertebereich/Default \\
\hline Daten zum Antragsteller & Nachname & Character & M & $\ldots$ \\
\hline Name & Vorname & Character & M & $\ldots$ \\
\hline Vorname & $\ldots$ & Date & M & $\begin{array}{l}\text { innerhalb/Jahres ab } \\
\text { akt. Datum/akt. Datum }\end{array}$ \\
\hline Daten zur Reise & $\ldots$ & Date & M & $\begin{array}{l}\text { Reisebeginn plus/Jahr/ } \\
\text { Reisebeginn }\end{array}$ \\
\hline $\begin{array}{l}\text { Reisebeginn } \\
\text { Reiseende }\end{array}$ & $\ldots$ & Character & M & $\ldots$ \\
\hline $\begin{array}{l}\text { Reiseziel (Ort/Land) } \\
\text { Daten zur Buchung }\end{array}$ & $\ldots$ & Character & M & $\ldots$ \\
\hline Vertragshotelketten & Genehmigungs-vermerk & Date & K & $\ldots$ \\
\hline $\begin{array}{l}\text { Frist für Buchungs- } \\
\text { bestätigung }\end{array}$ & $\ldots$ & Date & M & $\mathrm{j} / \mathrm{n}$ \\
\hline Buchungsbestätigung & $\ldots$ & & & \\
\hline
\end{tabular}

durch ein Interaktionsdiagramm miteinander verknüpft, in dem ihr Nachrichtenaustausch beschrieben ist. Dadurch ermöglicht S-BPM eine lose Kopplung von Prozessteilen und eine einfachere Veränderbarkeit des Verhaltens eines Subjektes, solange dessen Kommunikationsschnittstelle, also der Satz an empfangenen und gesendeten Nachrichten und deren Reihenfolge, unverändert bleibt.

Die Verwendung von Zustandsdiagrammen zur Beschreibung des Verhaltens eines Subjekts stellt ebenfalls einen grundlegenden Unterschied zu den anderen bislang behandelten Sprachen dar. Ein Zustandsdiagramm beschreibt - wie im Namen bereits enthalten - den Zustand eines Systems (hier: eines Subjektes - dies kann genauso ein Mensch wie eine Maschine sein) und die Ereignisse, die zu einem Zustandsübergang führen. Ein Subjekt kann sich immer nur in genau einem Zustand befinden - es ist deshalb per Definition nicht in der Lage, Vorgänge parallel auszuführen. Vielmehr arbeiten alle Subjekte parallel und unabhängig voneinander. Dies bedingt ein Umdenken bei der Modellierung, da Konstrukte wie UND-Konnektoren (in EPKs), Split/Joins (in Aktivitätsdiagrammen) oder parallele Gateways (in BPMN) nicht zur Verfügung stehen. Gleichzeitig führt dieser Modellierungsansatz zu einfacheren, kompakteren Modellen und einem vor allem im Gegensatz zur BPMN deutlich reduzierten Sprachumfang, was der Verständlichkeit der Modelle zuträglich ist. 


\subsection{Vergleich und Gegenüberstellung}

Die hier betrachteten Modellierungssprachen sind unterschiedlich ausdrucksstark und haben aufgrund ihrer historischen Entwicklung unterschiedliche Schwerpunkte in der Abbildung von Aspekten der Geschäftsprozessmodellierung. Dieser Abschnitt versucht, diese Unterschiede nochmals systematisch anhand der im letzten Kapitel vorgestellten Prozessdefinition darzustellen und so die Handhabbarkeit der Sprachen ihrer Ausdrucksstärke gegenüberzustellen. Dabei ziehen wir die Semantik der vorgestellten Modellierungselemente als Ausgangspunkt heran.

Der Referenzpunkt der folgenden Betrachtungen ist die Prozessdefinition aus dem letzten Kapitel, die wir hier der Einfachheit halber nochmals anführen:

1. Prozessstrategie: Prozesse haben

a) einen definierten Anfang und Input (Startereignis),

b) und weisen ein definiertes Ende mit einem Ergebnis auf,

c) zur Befriedigung eines Kundenbedürfnisses (und damit zur Wertschöpfung beizutragen),

2. Prozesslogik: Ein Prozess

a) ist die Summe von miteinander verknüpften Aktivitäten (Aufgaben),

b) die nach dem Startereignis von Handelnden

c) in sachlogischer und zeitlicher Reihenfolge

d) zur Bearbeitung eines Geschäftsobjekts ausgeführt werden um

e) das gewünschte Ergebnis zu erzeugen.

3. Prozessrealisierung: Ein Prozess wird realisiert

a) In dem Menschen und/oder Maschinen die Aufgaben der jeweiligen Handelnden übernehmen, und diese

b) mit Hilfsmitteln (Sachmittel, Information, Anwendungsprogramme etc.) ausführen

Auf Basis dieser Definition können die Konzepte identifiziert werden, die in einem Prozessmodell abbildbar sein müssen, um Prozesse vollständig (entsprechend obiger Definition) abbilden zu können. Die folgende Tabelle (siehe Tab. 3.3) zeigt diese Konzepte. Mehrfach vorkommende Konzepte werden nur beim erstmaligen Auftreten genannt wie z. B. „Ergebnis“. Bei Konzepten, die unterschiedlich konkret angeführt sind, werden nur die konkreteren Konzepte betrachtet - z. B. „miteinander verknüpfte Aktivitäten“ als allgemeinere Formulierung von ,sachlogische und zeitliche Reihenfolge“.

Ordnet man nun die Modellierungselemente der betrachteten Sprachen diesen Konzepten zu, so ergibt sich folgende Tabelle (siehe Tab. 3.4):

$\mathrm{Zu}$ erkennen ist, dass die konzeptionelle Abdeckung über die Sprachen hinweg variiert. Darüber hinaus zeigt sich, dass nicht alle Sprachen alle Konzepte in gleichem Umfang bzw. in der der gleichen Ausdrucksstärke behandeln. Die Zuordnung der Modellierungselemente zu den Konzepten ermöglicht einen ersten Ansatzpunkt für die Einschätzung ihrer Ausdrucksstärke. 
Tab. 3.3 Konzepte der Geschäftsprozessdefinition

\begin{tabular}{l|l}
\hline Definitionsteil & Konzept \\
\hline $1 \mathrm{a}$ & Anfang \\
\hline & Input \\
\hline $1 \mathrm{~b}$ & Ende \\
\hline & Ergebnis \\
\hline $1 \mathrm{c}$ & Kundenbedürfnis \\
\hline $2 \mathrm{a}$ & Aktivitäten/Aufgaben \\
\hline $2 \mathrm{~b}$ & Startereignis \\
\hline $2 \mathrm{c}$ & Handelnder \\
\hline & Sachlogische Reihenfolge \\
\hline $2 \mathrm{~d}$ & Zeitliche Reihenfolge \\
\hline $3 \mathrm{a}$ & Geschäftsobjekt \\
\hline & Mensch \\
\hline $3 \mathrm{~b}$ & Maschine \\
\hline & Sachmittel \\
\hline & Information \\
\hline & Anwendungsprogramm \\
\hline
\end{tabular}

Nur bedingt erkennbar werden in dieser Übersicht die unterschiedlichen Zugänge in der Abbildung der sachlogischen und zeitlichen Zusammenhänge. Dennoch unterscheiden sich in diesem Punkt die Sprachen wesentlich: Flowcharts bieten keine Möglichkeit zur Abbildung paralleler Abläufe, in EPKs ist lediglich eine starke Kopplung von parallel verlaufenden Aktivitätszweigen vorgesehen, indem diese innerhalb eines Prozesses mittels UND- bzw. ODER-Operator verknüpft werden. UML Aktivitätsdiagramme und BPMN bieten die gleichen Mechanismen (unter anderen Namen), erlauben aber auch eine lose Kopplung von Prozessen bzw. Prozessteilen mittels Signalen (bei Aktivitätsdiagrammen) bzw. Nachrichtenflüssen (bei BPMN).

Insbesondere letzterer Mechanismus ermöglicht eine detaillierte Beschreibung von Kommunikationsabläufen von grundsätzlich unabhängigen Prozessteilen. Eine Einschränkung liegt in der notwendigen Vorabfestlegung von eindeutigen Zuordnungen zwischen Sendern und Empfängern von Nachrichten. Die S-BPM bietet einen ähnlichen Kommunikationsmechanismus, ist aber in diesem Punkt flexibler (insbesondere bei Verwendung der in der grafischen Darstellung der Sprache nicht abgebildeten Inputpools). Eine Beschreibung von parallel ablaufenden Prozessteilen ist in der S-BPM nur durch die Verteilung derselben auf unterschiedliche Subjekte möglich - innerhalb eines Subjektes kann immer nur ein Funktionszustand aktiv sein, es können also ausschließlich alternative Zweige im Verhalten eines Subjektes dargestellt werden. 


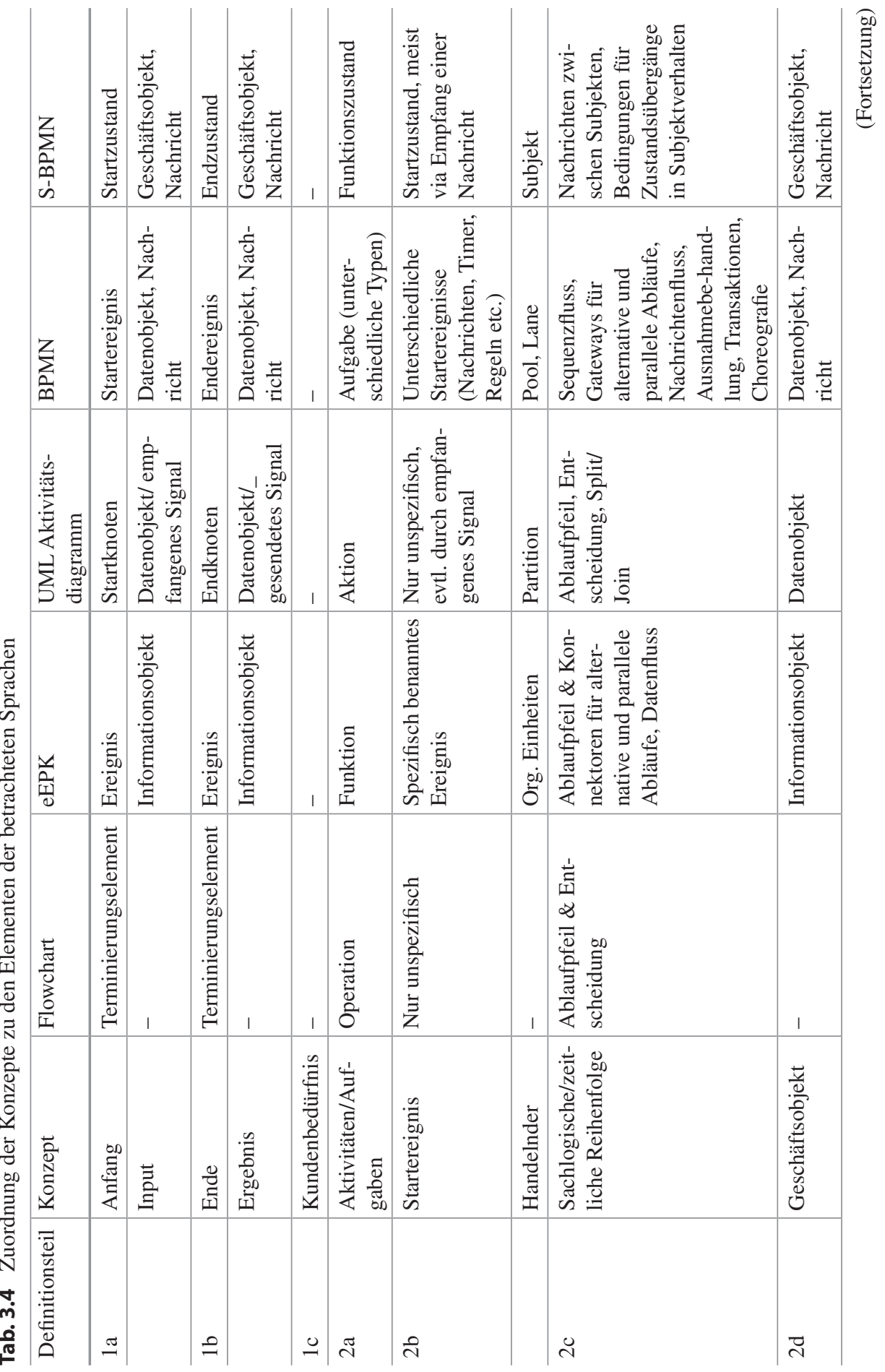




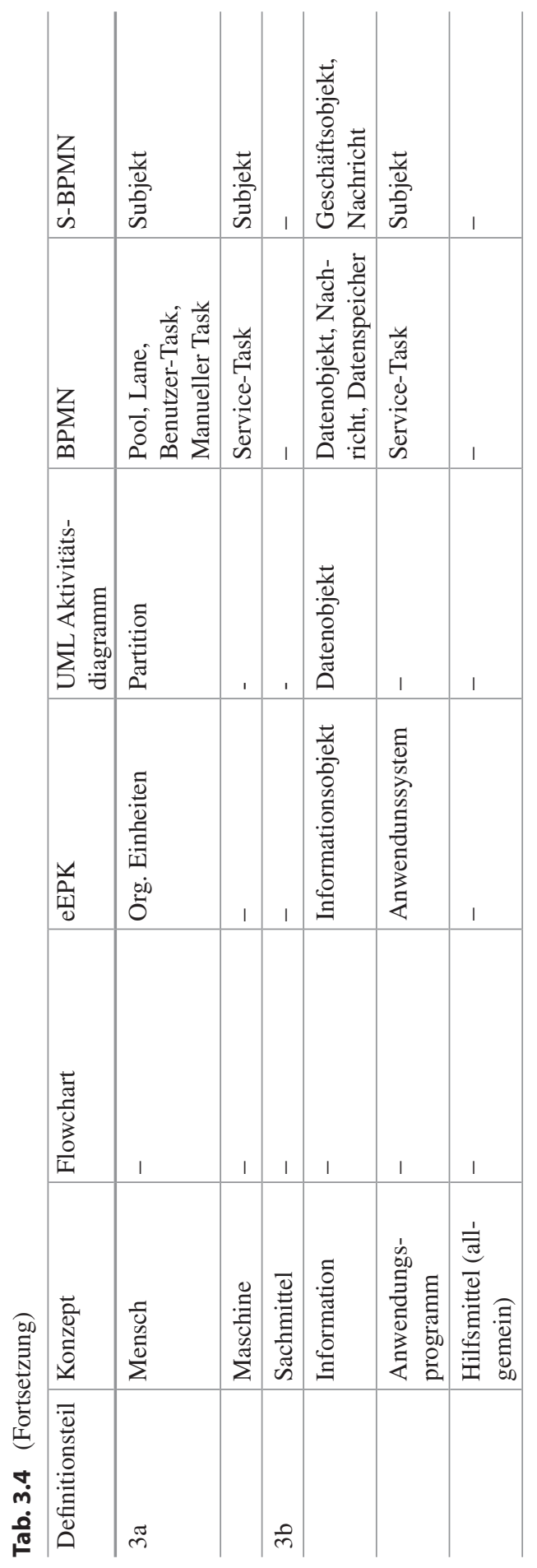


Generell bietet die BPMN die größte Flexibilität in der Wahl der Darstellungsform eines Prozesses. Durch die Vielzahl an Modellierungselementen können auch komplexe Zusammenhänge kompakt dargestellt werden, was jedoch höhere Ansprüche an das Sprachverständnis der Modellnutzenden stellt. Einen anderen Ansatz verfolgen hier Aktivitätsdiagramme oder die S-BPM, die auf einem kompakten Satz an Modellierungselementen aufbauen. Dies führt bei komplexen Zusammenhängen zu umfangreichen Modellen, die hohe Ansprüche an die Modellnutzenden hinsichtlich des Modellverständnisses stellt. Die S-BPM reduziert die unmittelbar sichtbare Komplexität von Modellen durch die Verteilung eines Prozesses auf unterschiedliche Subjekte. Während dies zwar zu einfach erfassbaren Teilmodellen führt, stellt es gleichzeitig höhere Ansprüche an Modellnutzende bei der Erfassung der Gesamtzusammenhänge innerhalb des Prozesses.

Bei der Auswahl einer für eine gegebene Aufgabenstellung und Zielgruppe geeigneten Modellierungssprache ist dem entsprechend nicht nur auf den Abbildungsgegenstand (also den betrachteten Geschäftsprozess) Bezug zu nehmen, sondern auch auf die bekannten oder vermuteten Kompetenzen der Modellierenden und Modellnutzenden. Eine grundlegende Unterscheidung kann zwischen den Sprachen getroffen werden, die den Aktivitätsfluss ins Zentrum der Betrachtung rücken (wie die FlowCharts und die EPK), und jenen, die die Handelnden eines Prozesses und deren Kommunikation in den Vordergrund stellen (wie die S-BPM).

Die BPMN und Aktivitätsdiagramme eignen sich grundsätzlich für beide Abbildungsarten, wobei die BPMN ausdruckstärkere Mittel zur Abbildung von Kommunikationsabläufen bietet. Die endgültige Auswahl einer Modellierungssprache nach Festlegung des grundlegend verfolgten Abbildungsansatzes (Aktivitäts- vs. Kommunikationsfluss) ist letztendlich abhängig von den Präferenzen der Modellierenden bzw. Modellnutzenden.

\section{Literatur}

1. Scheer, A. W. (2003). ARIS - Business Process Modeling (3rd ed.). Springer; www.omg.org

2. Fleischmann, A., Obermeier, S., Schmidt, W., Stary, C., \& Börger, E. (2011). Subjektorientiertes Prozessmanagement. Hanser Verlag 
Open Access Dieses Kapitel wird unter der Creative Commons Namensnennung - Nicht kommerziell - Keine Bearbeitung 4.0 International Lizenz (http://creativecommons.org/licenses/ by-nc-nd/4.0/deed.de) veröffentlicht, welche die nicht-kommerzielle Nutzung, Vervielfältigung, Verbreitung und Wiedergabe in jeglichem Medium und Format erlaubt, sofern Sie den/die ursprünglichen Autor(en) und die Quelle ordnungsgemäß nennen, einen Link zur Creative Commons Lizenz beifügen und angeben, ob Änderungen vorgenommen wurden. Die Lizenz gibt Ihnen nicht das Recht, bearbeitete oder sonst wie umgestaltete Fassungen dieses Werkes zu verbreiten oder öffentlich wiederzugeben.

Die in diesem Kapitel enthaltenen Bilder und sonstiges Drittmaterial unterliegen ebenfalls der genannten Creative Commons Lizenz, sofern sich aus der Abbildungslegende nichts anderes ergibt. Sofern das betreffende Material nicht unter der genannten Creative Commons Lizenz steht und die betreffende Handlung nicht nach gesetzlichen Vorschriften erlaubt ist, ist auch für die oben aufgeführten nicht-kommerziellen Weiterverwendungen des Materials die Einwilligung des jeweiligen Rechteinhabers einzuholen. 


\section{Vorgehensweise von der Modellbildung zur Digitalisierung}

\subsection{Einordnung in den Gesamtzusammenhang}

In den vorangegangenen Kapiteln haben wir gezeigt, dass das Geschehen in Organisationen (Unternehmen, Verwaltungen etc.) auf Modellen aus unterschiedlichsten Disziplinen basiert. Geschäftsmodelle, Unternehmensarchitekturen mit Modellen für Leistungen (Produkte und Services), Aufbauorganisation, Prozesse, Daten und IT-Infrastruktur beschreiben, in welchem Bereich ein Unternehmen tätig ist, wie es dies bewerkstelligt, in welchen Austauschbeziehungen mit Partnern es steht, von welcher technischen Infrastruktur es unterstützt wird usw.

Bei den Modellierungssprachen haben wir uns auf Ansätze und Notationen für die Spezifikation von Geschäftsprozessen und damit die fachliche Gestaltung betrieblicher Vorgänge konzentriert. Im Zuge der Digitalisierung sind diese Vorgänge soweit möglich und wirtschaftlich sinnvoll mit Informations- und Kommunikationstechnik abzubilden. Sowohl den dafür geeigneten inkrementellen Verbesserungen bestehender Prozesse als auch grundlegenden Prozessinnovationen liegen kreative Gestaltungsleistungen zugrunde, die von den fachlichen Modellen zu ausführbaren Systemen führen sollen. Deshalb beschäftigen wir uns im folgenden Kapitel zunächst mit den typischen Aktivitäten des Geschäftsprozessmanagements. Mit dem Ansatz des Design Thinking beleuchten wir dann einen methodischen Ansatz, um kreativ Neuartiges hervorzubringen und komplexe Probleme zu lösen, ehe wir beide Konzepte miteinander in Beziehung setzen. 


\subsection{Aktivitätsbündel im Geschäftsprozessmanagement}

\subsection{1 Überblick}

In Abschn. 1.8 haben wir bereits angesprochen, dass die Gestaltung von Geschäftsprozessen bis hin zu ihrer Ausführung als Instanzen bei der Abwicklung konkreter Geschäftsvorfälle („operatives Geschäft“) selbst einen Prozess darstellt. Dieser wird häufig als Geschäftsprozessmanagementzyklus mit Phasen wie Strategie, Entwurf, Implementierung und Controlling verstanden [1].

In der Praxis sind die Teilaktivitäten aber oft nicht klar voneinander abzugrenzen. Wir sehen diese deshalb weniger als Kreis mit sequenzieller Abfolge, sondern eher miteinander vernetzt und verwoben, wie es die Wabenstruktur in Abb. 4.1 andeuten soll. Die Darstellung zeigt außerdem, dass wir die Aufgaben etwas stärker differenzieren und als Aktivitätsbündel Analyse und Modellierung, Validierung, Optimierung, organisatorische Implementierung, IT-Implementierung sowie Betrieb und Monitoring unterscheiden.

Obwohl die übliche Darstellung als Zyklus suggeriert, dass bei Prozessmanagementvorhaben alle Aktivitätsbündel als Sequenz durchlaufen werden, hängen deren Auswahl und Reihenfolge von der konkreten Situation, z. B. vom Reifegrad eines Prozesses ab. Abschn. 4.2.2 bis 4.2.7 erläutern die Aktivitäten anhand eines Beispielprozesses. Dabei werden die Schritte zunächst komplett und auch in der angegebenen Reihenfolge durchlaufen. Ein solches Szenario ist etwa bei der erstmaligen Gestaltung oder einer kompletten Reorganisation eines Prozesses realistisch. Anschließend diskutieren wir in Abschn. 4.2.8 mehrere Szenarien für Verbesserungen, die sich aus den Erfahrungen im dem laufenden Betrieb der ursprünglich gestalteten Prozessumgebung ableiten lassen. Sie verdeutlichen die situativ unterschiedlichen Pfade durch die Aktivitätsbündel bei der Weiterentwicklung des Prozesses.

Abb. 4.1 Aktivitätsbündel im Geschäftsprozessmanagement

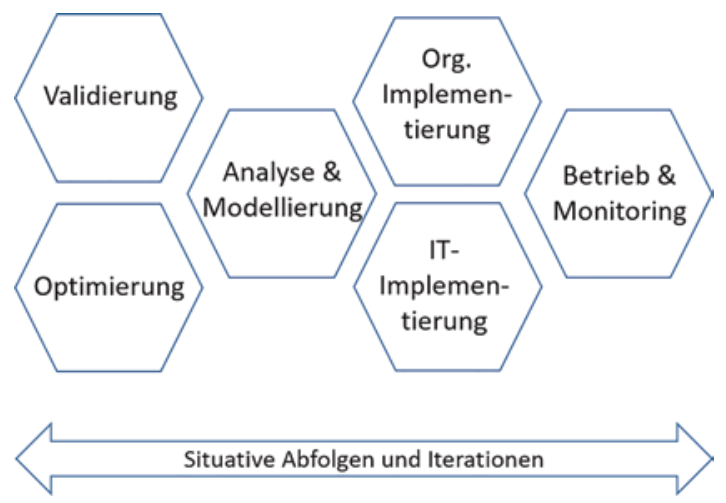




\subsubsection{Analyse und Modellierung}

Die Analyse dient der Erhebung von Informationen, warum ein Prozess existiert bzw. implementiert werden soll, welche Ziele eine Organisation mit diesem im Rahmen ihrer Strategie verfolgt, und wie in diesem aktuell gearbeitet wird. Ziele sind die Dokumentation und die Gewinnung von Anhaltspunkten für Verbesserungen. Die Modellierung verwendet u. a. die Ergebnisse der Analyse und befasst sich mit der Gestaltung der zukünftigen Arbeitsweise, also mit Prozessveränderungen und -innovationen. Wenn dazu weitere Informationen nötig sind, wechseln die Beteiligten wieder in den Analysemodus, um diese zu erheben, und agieren danach wieder gestaltend. Analyse und Modellierung lassen sich deshalb nicht scharf voneinander abgrenzen. Auch Validierung und Optimierung finden in der Regel bereits hier statt, wenn die Beteiligten das Modell iterativ nach besten Wissen und Gewissen und unter Berücksichtigung von in der Analyse erkannten Schwachstellen entwickeln und dabei Lösungsmöglichkeiten probieren und diskutieren.

Neben der Betrachtung von Rahmenbedingungen wie strategischer Bedeutung, Zielen und Risiken geht es bei Analyse und Modellierung im Wesentlichen darum zu analysieren bzw. zu spezifizieren (vgl. Abschn. 1.3),

- welche Handelnden (z. B. Menschen, Maschinen),

- welche Aktivitäten,

- nach welchen Geschäftsregeln (Business Rules),

- an welchen Geschäftsobjekten (z. B. an bestimmte Träger gebundene Informationen, physische Gegenstände)

- mit welchen Hilfsmitteln (z. B. IT-Systeme) ausführen und

- in welcher Weise sie dabei interagieren, um die gewünschten Prozessziele und -ergebnisse zu erreichen.

Zur Entwicklung von Prozessmodellen auf Basis dieser Erkenntnisse bedient man sich der in Kap. 3 vorgestellten Modellierungssprachen mit den dazugehörigen grafischen Notationen.

Bei der Analyse und Modellierung wird meist auch bereits eine wesentliche Voraussetzung für das operative Prozesscontrolling in der Betriebsphase geschaffen. Neben den bereits genannten Prozessattributen werden dafür Leistungsparameter (Kennzahlen), insbesondere Process Performance Indicators (PPIs), definiert, in einem Messgrößensystem systematisiert und mit Zielwerten versehen [2]. Typische Beispiele für PPIs sind Durchlaufzeit, Ausbringungsmenge pro Zeiteinheit, Fehlerrate, Kundenzufriedenheit o. Ä. Die PPIs und die dafür geplanten Soll-Werte bilden die Basis für das Geschäftsprozessmonitoring, also die operative Prozesskontrolle während der Ausführung (vgl. Abschn. 4.2.7 und 6.3.3). 


\section{Analyse und Modellierung im Fallbeispiel}

Als Fallbeispiel verwenden wir den stark vereinfacht dargestellten Vorgang der Kreditantragsbearbeitung bei einer Bank. Dort gehen Anträge von Interessenten auf Gewährung eines Immobiliendarlehens ein. Vor der Erstellung eines Angebots prüfen Sachbearbeiter die Bonität des jeweiligen Kunden und die Werthaltigkeit der zu beleihenden Immobilie. Ist das Ergebnis beider Prüfungen positiv, fertigt der Sachbearbeiter ein Angebot an mit Daten wie Darlehenssumme, Zins- und Tilgungssatz und Laufzeit. Beträgt der Darlehensbetrag unter $200.000 €$ unterschreibt er das Angebot und schickt es dem Kunden. Im anderen Fall muss er erst noch die Zustimmung seiner Abteilungsleitung und bei mehr als 500.000 € die des Vorstands einholen. Ergeben sich aus der Bonitätsprüfung oder der Objektprüfung Anhaltspunkte für Risiken, nimmt ein Sachbearbeiter Kontakt mit dem Interessenten auf, um die weitere Vorgehensweise, z. B. eine Verminderung der Darlehenssumme, zu abzustimmen. Im Rahmen einer Analyse des Prozesses wurden diese Informationen erhoben und strukturiert (vgl. Tab. 4.1).

Im vorliegenden Fall verwenden wir die in Kap. 3 beschriebene Modellierungssprache S-BPM zur Darstellung des Prozesses, da dieser in einer stark interaktionsorientierten Beschreibung vorliegt. Grundsätzlich wäre die Abbildung mit jeder anderen Modellierungssprache, die die Abbildung von Verantwortlichkeiten erlaubt (etwa eEPK oder BPMN) ebenso möglich.

Tab. 4.1 Merkmale des Beispielprozesses und dafür ermittelte Information

\begin{tabular}{l|l}
\hline Prozessmerkmal & Erhebungsergebnis \\
\hline Akteure/Rollen & $\begin{array}{l}\text { Interessent, Sachbearbeitung Immobilienkredit, Abteilungsleitung } \\
\text { Immobilienkredit, Vorstand }\end{array}$ \\
\hline Aktivitäten & $\begin{array}{l}\text { Kreditantrag stellen, Kreditantrag prüfen (Vollständigkeit), Kundenbonität } \\
\text { prüfen, Finanzierungsobjekt schätzen, Finanzierungskonditionen ermitteln, } \\
\text { Angebot erstellen, Angebot genehmigen, Angebot versenden }\end{array}$ \\
\hline Geschäftsobjekte & Kreditantrag mit Anlagen, Kreditangebot \\
\hline Geschäftsregeln & $\begin{array}{l}\text { Kreditangebote über 200.000 € müssen von der Abteilungsleitung } \\
\text { genehmigt werden, über 500.000 € vom Vorstand. }\end{array}$ \\
\hline Hilfsaktionen & $\begin{array}{l}\text { Kunde - Bank (Posteingang), Sachbearbeitung Immobilienkredit - } \\
\text { Abteilungsleitung Immobilienkredit, Abteilungsleitung Immobilienkredit - } \\
\text { Vorstand, Sachbearbeitung Immobilienkredit - Kunde }\end{array}$ \\
\hline Prozesskennzahlen & $\begin{array}{l}\text { Internet-Portal der Bank (Webformular), Bank-System, Workflow-System, } \\
\text { SCHUFA-Webformular, E-Mail, Telefon }\end{array}$ \\
& $\begin{array}{l}\text { Beobachtung des Verhaltens von Instanzen mithilfe von: } \\
\text { Durchlaufzeit vom Eintreffen eines Kreditantrags bis zum Versand eines } \\
\text { Angebots (Ziel: durchschnittlich max. 3 Tage) } \\
\text { Häufigkeit pro Woche inklusive Verteilung } \\
\text { Ablehnungsquote von Anträgen seitens Bank } \\
\text { Ablehnungsquote von Angeboten seitens Interessenten }\end{array}$ \\
\hline
\end{tabular}


Zusammen mit weiteren Informationen dienten diese Angaben als Basis für die Modellierung des Vorgangs. Die folgenden Bilder zeigen einen Auszug des zugehörigen Prozessmodells unter Verwendung der Modellierungssprache S-BPM (vgl. Abschn. 3.6). Abb. 4.2 stellt die involvierten Subjekte (Handelnde) und die im Prozessablauf ausgetauschten Nachrichten dar.Die Kommunikationsstruktur (Subject Interaction Diagramm, SID) enthält noch keine Reihenfolgen, in denen die jeweiligen Nachtrichten ausgetauscht werden. Diese werden im Verhalten der Subjekte beschrieben. Abb. 4.3 und 4.4 zeigen das Verhalten des Subjekts „Interessent“ und auszugsweise das Verhalten des Subjekts „Sachbearbeitung Immobilienkredit“.

Die nicht abgebildeten Verhaltensspezifikationen der Subjekte „Abteilungsleitung Immobilienkredit“" und „Vorstand“ sind analog zu beschreiben. Diese Subjekte würden in den Prozess involviert werden, wenn die gewünschte Kreditsumme 200.000 bzw. 500.000 Euro übersteigt. Diese Fälle sind in Abb. 4.4 nicht ausmodelliert - sie wären zusätzliche Zweige nach dem Zustand „Freigabe notwendig“.

\subsubsection{Validierung}

Unter Validierung verstehen wir im BPM-Kontext die Überprüfung, ob der gestaltete Prozess den vom (externen) Kunden und Prozesseigner erwarteten Output beispielsweise in Form eines Service oder Produkts erzeugt. Diese Frage nach der Effektivität bezieht

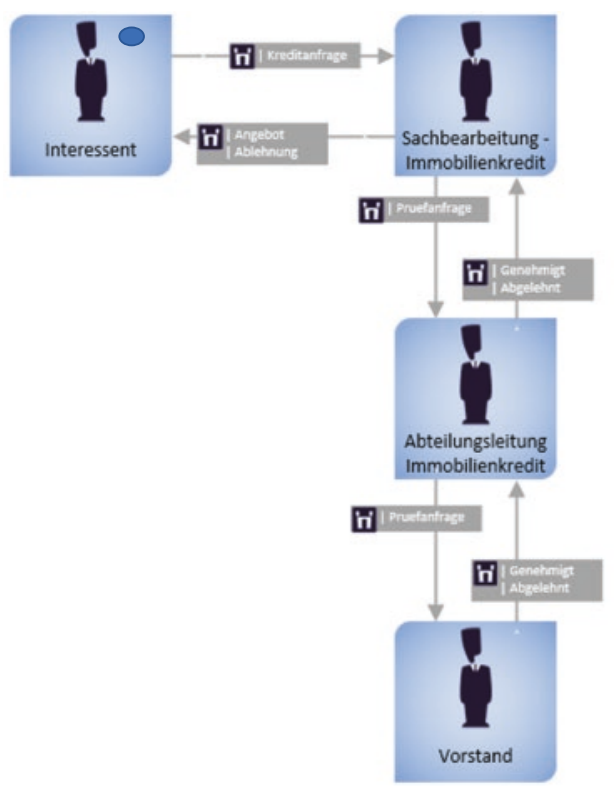

Das Bild zeigt die Kommunikationsstruktur für die Beantragung eines Kredits. Die Kommunikation wird initiiert vom Handelnden „Interessent" (Subjekt). Dieses Subjekt sendet die Nachricht Kreditanfrage an das Subjekt "Sachbearbeitung Immobilienanfrage“ und erhält die Nachrichten „Angebot“ oder "Ablehnung".

Entsprechend den Prozessanforderungen tauschen die anderen Subjekte die notwendigen Nachrichten aus. Die Kommunikationsstruktur impliziert noch keine Reihen-folge in der die Nachrichten ausgetauscht werden.

Abb. 4.2 Kommunikationsstruktur der Handelnden im Prozess Kreditanfrage 


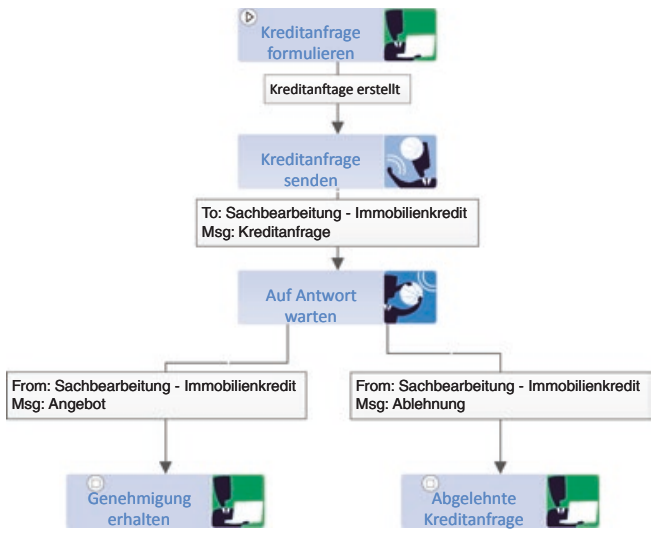

Das Bild zeigt das Verhalten des Subjekts „Interessent". Im Startzustand (markiert durch ein Dreieck im Kreis) wird eine Kreditanfrage formuliert. In der Regel geschieht dies durch das Aus-füllen eines entsprechenden Formulars. Die Inhalte des Formulars wären Bestandteil der Spezifikation des Geschäftsobjekts „Kreditanfrage“. Nachdem die Kreditanfrage erstellt wurde, wird diese an das Subjekt "Sachbearbeitung Kreditanfrage" gesendet. Danach wird auf eine Antwort vom Subjekt "Sachbearbeitung Immobilienkredit" gewartet. Dies kann entweder die Nachricht „Angebot" oder „Ablehnung" sein. In beiden Fällen ist das Subjektverhalten beendet durch erreichen der jeweiligen Endzustände die als solche durch Punkte gekennzeichnet sind.

Abb. 4.3 Verhalten des Subjekts „Interessent“

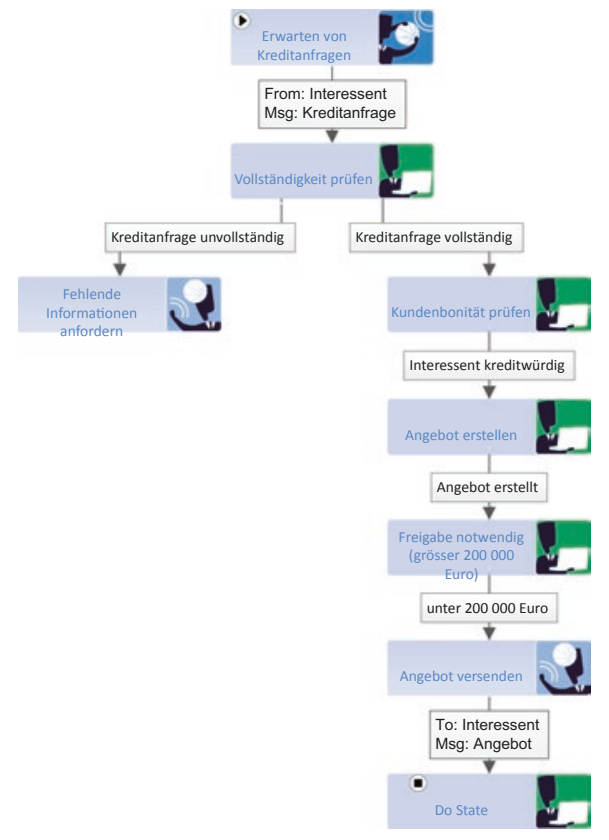

Das Bild zeigt einen Auszug der Verhaltensbeschreibung des Subjekts "Sachbearbeitung Immobilienkredit“. Dieser Auszug zeigt nur den einfachen positiven Fall.

Im Startzustand (Zustand markiert mit Dreieck im Kreis) empfängt das Subjekt die Nachricht „Kreditanfrage“ vom Subjekt „Interessent“. Mit dieser Nachricht erhält das Subjekt das ausgefüllte Formular (Geschäftsobjekt) mit den Daten der Kreditanfrage. Im nächsten Zustand wird geprüft ob diese Daten vollständig sind. Wenn nein wird eine entsprechende Rückfrage an das Subjekt „Interessent" gesendet. Dieser Zweig (Linker teil des Bildes) ist in der Verhaltensbeschreibung nicht weiter beschrieben.

Sind die Angaben vollständig wird die Kreditwürdigkeit des Interessenten geprüft. Ist der Interessent kreditwürdig wird ein Angebot erstellt. Liegt der gewünschte Kredit unter 200 Tsd. Euro wird die Nachricht „Angebot“ an das Subjekt Interessent gesendet.

Abb. 4.4 Verhalten des Subjekts „Sachbearbeitung Immobilienkredit“

sich auch schon auf die Ergebnisse von Teilschritten, also Prozessbeteiligte der eigenen Organisation als Kunden. So gilt es beispielsweise zu evaluieren, ob ein vorgelagerter Prozessschritt alle Informationen liefert, die ein Bearbeiter bei seiner Teilaufgabe für eine Entscheidung (z. B. Genehmigung) benötigt. Wir haben bereits angesprochen, dass ein Modell schon während seiner schrittweisen Entwicklung immer wieder in Teilen 
der Validierung unterzogen wird. Objekt der Validierung ist darüber hinaus das komplett fertiggestellte Prozessmodell, dessen Effektivität sichergestellt sein sollte, ehe es informationstechnisch umgesetzt wird (vgl. Abschn. 4.2.6). Sonst werden Fehler erst spät entdeckt und verursachen entsprechend hohe Kosten für ihre Beseitigung.

\section{Validierung im Fallbeispiel}

Das Prozessmodell für den Beispielvorgang wurde während seiner Entwicklung sowie abschließend validiert. Dabei wurde zum Beispiel zunächst festgestellt, dass im ursprünglichen Kreditantrag kein Feld für das Beschäftigungsverhältnis des Antragstellers vorgesehen war und somit eine wichtige Angabe zur Risikobeurteilung fehlt. Dies führte zur Erweiterung des Geschäftsobjektes und zu einem positiven Validierungsergebnis bei der entsprechenden Iteration.

\subsubsection{Optimierung}

Während die Validierung die Sicherstellung der Effektivität von Geschäftsprozessen zum Ziel hat, geht es bei der Optimierung um die Effizienz. Prozesseffizienz lässt sich über Prozessattribute zur Ressourcenbeanspruchung wie Dauer und Kosten fassen. Optimierung bedeutet, eine im Hinblick auf solche Prozessparameter optimale Gestaltung eines Prozesses zu finden. Wesentliche Ansatzpunkte sind Verbesserungen der ablauforganisatorischen und aufbauorganisatorischen Gestaltung sowie der IT-Unterstützung. Die Optimierung ist demnach streng genommen kein eigenständiges Aktivitätsbündel, sondern bedient sich der Modellierung, der organisatorischen Implementierung und der IT-Implementierung (vgl. Abschn. 4.2.2, 4.2.5 und 4.2.6).

Eine bekannte Methode für den Vergleich von Alternativen im Ablauf oder beim Ressourceneinsatz ist die Simulation. Mit ihrer Hilfe lassen sich für eine größere Menge von Prozessinstanzen (Aufträge, Fertigungsstücke etc.) quantitative Aussagen über die Entwicklung von Prozessparametern gewinnen. Die Simulation ermöglicht die Bewertung eines Prozessmodells mit einer bestimmten Kombination von Parametern. Dies können deterministische oder auch stochastische, durch Wahrscheinlichkeitsverteilungen beschriebene Größen sein. Durch Parameteränderungen und alternative Prozessgestaltungen können verschiedene Gestaltungsoptionen in ihrem Verhalten analysiert werden. Damit lassen sich Erkenntnisse über Engpässe bzw. Ineffizienzen und die Sensitivität von Parametern gewinnen. Der Aufwand für die Erweiterung eines Prozessmodells und für die Beschaffung notwendiger Informationen, um Simulationen durchführen zu können, kann beträchtlich sein.Eine Schwierigkeit bei der Optimierung besteht darin, dass die relevanten Attribute oft interdependent und in ihrer Veränderung einander gegenläufig sind. So kann es sein, dass eine Prozessalternative relativ zu einer anderen zwar eine geringere Durchlaufzeit, dafür aber höhere Kosten aufweist. Die Entscheidung für eine Alternative hängt damit auch von der Priorität der Prozessziele ab. 


\section{Optimierung im Fallbeispiel}

Das Modell konnte bereits bei seiner Entstehung optimiert werden, und zwar durch die Parallelisierung der zunächst sequenziell geplanten Schritte zur Prüfung der Kundenbonität und der Werthaltigkeit der Immobilie.

\subsubsection{Organisatorische Implementierung}

Validierte Prozesse müssen für den Produktivbetrieb in die bestehende und gegebenenfalls neu zu gestaltende organisatorische Umgebung eingebettet werden. Dies erfordert i. d. R. eine Anpassung der sie umgebenden Ablauf- und Aufbauorganisation.Ein einzelner Prozess ist meist Teil einer gesamten Wertschöpfungsumgebung (Wertschöpfungskette, Wertschöpfungsnetz), in die er sich nahtlos einfügen muss. Im Hinblick auf die ablauforganisatorische Integration in die Prozesslandkarte sind deshalb insbesondere die Schnittstellen mit anderen Prozessen zu betrachten. Dies kann dazu führen, dass an Schnittstellen eines vor- oder nachgelagerten Prozesses Änderungen durchzuführen sind. Solche Sachverhalte sind im Regelfall bereits in den vorgelagerten Aktivitätsbündeln berücksichtigt. Bei der Implementierung darf es deshalb letztlich nur noch um die zeitliche Synchronisation der Produktivsetzung gehen. Damit ist gemeint, dass Prozesse, die über Schnittstellen verbunden sind, gleichzeitig erneut produktiv gesetzt werden müssen, wenn sich an einer Schnittstelle eine Veränderung ergeben hat, die auch beim Partnerprozess Modifikationen nötig gemacht hat.

Die aufbauorganisatorische Einbettung umfasst die Zuordnung von konkreten Handlungsträgern, also letztlich Menschen als Stellen- oder Rolleninhaber, zu den im Modell abstrakt spezifizierten Akteuren. Herausforderung ist dabei u. a. die Berücksichtigung des organisatorischen Kontexts beim Einsatz von Workflow Engines. Diese müssen etwa zur Laufzeit dynamische Vertreterregelungen ebenso auflösen können wie die Tatsache, dass Personen im selben Prozess verschiedene Rollen bekleiden können. Beispielsweise kann eine vorgesetzte Person im Urlaubsantragsprozess der Genehmiger von Urlaubsanträgen der eigenen Mitarbeiterinnen und Mitarbeiter sein, selbst aber auch Antragsteller für den eigenen Urlaub sein, den wiederum der eigene Vorgesetzte genehmigen muss. Für die korrekte Steuerung einer Instanz durch die Bearbeitungsstellen und schritte muss die Software also Organisationswissen besitzen.Bei der organisatorischen Einbettung sind weitere qualitative und quantitative Aspekte zu berücksichtigen. So ist darauf zu achten, dass die Mitarbeiterinnen und Mitarbeiter die für die Ausführung des modellierten Verhaltens nötigen Qualifikationen (Skills) besitzen oder durch Schulungen erwerben können. Adäquate Qualifikation ist nicht nur Voraussetzung für eine erfolgreiche Arbeit im aktuell gültigen Prozess, sondern fördert auch Impulse für Verbesserungen seitens der Prozessbeteiligten.

Die Anzahl der Personen, die den abstrakten Akteuren im Modell zugeordnet werden, beeinflusst die Kapazität für die Bearbeitung von Prozessinstanzen und wirkt sich damit auf Parameter wie die Durchlaufzeit aus. 
Tab. 4.2 Potenzielle und konkrete Besetzung von Rollen

\begin{tabular}{l|l|l}
\hline Akteur/Rolle & Anzahl gesamt & Zugeordnet gemäß Personaleinsatzplan \\
\hline Sachbearbeitung Immobilienkredit & 9 & 5 Kräfte in Vollzeit \\
\hline Abteilungsleitung Immobilienkredit & 1 & $\begin{array}{l}\text { 1 Leiter } \\
1 \text { Stellvertreter (Leiter Konsumentenkredit) }\end{array}$ \\
\hline Vorstand & 3 & $\begin{array}{l}\text { 1 Ressortleiter Privatkunden } \\
1 \text { Ressortleiter Geschäftskunden } \\
1 \text { Ressortleiter Anlagemanagement }\end{array}$ \\
\hline
\end{tabular}

\section{Organisatorische Implementierung im Fallbeispiel}

Den identifizierten und modellierten Rollen wurde die in Spalte 2 in Tab. 4.2 aufgeführte Zahl von Mitarbeiterinnen und Mitarbeitern zugeordnet, welche die Rolle aufgrund ihrer Qualifikation prinzipiell besetzen können. Die dritte Spalte enthält die tatsächliche eingesetzte Kapazität (kurzfristige Ausfälle z. B. wegen Krankheit sind nicht berücksichtigt). Die Abteilungsleitungen für Immobilien- und Konsumentenkredite vertreten sich gegenseitig sowohl in disziplinarischen als auch in fachlichen Angelegenheiten. Dies gilt analog für die Vorstände.

\subsubsection{IT-Implementierung}

Die meisten Prozesse sind ohne IT-Unterstützung nicht wirtschaftlich auszuführen. Insbesondere wenn ein hoher Automatisierungsgrad eines Ablaufes angestrebt wird, erlangt die Qualität der Abbildung in der IT hohe Bedeutung. Aber auch oder gerade an Stellen, wo der menschliche Bearbeiter involviert ist (z. B. Eingaben tätigen, Entscheidungen treffen), muss viel Wert auf die bedarfsgerechte Gestaltung der IT-Systeme gelegt werden.

Die IT-bezogene Implementierung eines Prozesses bedeutet, ihn als IT-gestützten Workflow unter Integration einer geeigneten Benutzeroberfläche, der Ablauflogik und der beteiligten IT-Systeme abzubilden. Dazu gilt es zunächst, die mehr oder weniger formale Modellbeschreibung (vgl. Kap. 3) in eine von einer Workflow Engine interpretierbare Sprache, d. h. ein ablauffähiges Programm zu überführen. Damit kann die Engine die Abarbeitung einer Prozessinstanz zur Laufzeit gemäß dem Modell steuern. Für die Erledigung einzelner Teilaufgaben bei der Abarbeitung sind meist eine ganze Reihe von Softwareapplikationen und - Services in den Ablauf zu integrieren. Typische Beispiele sind ERP-Transaktionen und Dokumenten- und Content-Management-Systeme. Ausgiebige Tests der implementierten Lösungen müssen die Qualität der Prozessunterstützung durch IT sichern.

\section{IT-Implementierung im Fallbeispiel}

Tab. 4.3 zeigt die wesentlichen Elemente der IT-Umgebung, welche zur Unterstützung des Prozesses und seiner Teilschritte aufgebaut wurde, zusammen mit ihren wichtigsten prozessrelevanten Funktionen. 
Tab. 4.3 Realisierte IT-Umgebung des Prozesses

\begin{tabular}{l|l}
\hline IT-System/-Service & Auswahl für den Prozess wesentlicher Funktionen \\
\hline Portal der Bank & $\begin{array}{l}\text { Stellt Informationsmaterial zur Finanzierung und elektronisches Formular } \\
\text { zur Erfassung des Kreditantrags durch den Kunden oder einen Sachbe- } \\
\text { arbeiter Immobilienkredit zur Verfügung } \\
\ldots\end{array}$ \\
\hline Workflow Engine & $\begin{array}{l}\text { Instanziiert Vorgang beim Speichern des Antrags durch den Kunden im Portal. } \\
\text { Steuert die Instanzen gemäß Modell und bindet dabei bei Bedarf Benutzer } \\
\text { und andere Systeme oder Services ein. } \\
\text { Zeichnet Log-Daten zu den Vorgängen auf. } \\
\text { Erzeugt Meldungen und Berichte auf Basis der Log-Daten } \\
\ldots\end{array}$ \\
\hline Banksystem & $\begin{array}{l}\text { Verwaltet Kunden } \\
\text { Kategorisiert Kunden (Scoring) } \\
\text { Ermittelt Konditionen } \\
\text { Erzeugt Angebote } \\
\ldots\end{array}$ \\
\hline
\end{tabular}

\subsubsection{Betrieb und Monitoring}

Implementierte Prozesse gehen nach der Abnahme in den Echtbetrieb (Going Live). Dies bedeutet, dass die Prozessbeteiligten sie in der aufgebauten organisatorischen und informationstechnischen Umgebung im Tagesgeschäft in Form von Instanzen ausführen.

Für die Gewinnung von Information für das bewusste Management der Prozesse ist die Beobachtung ihres Verhaltens im laufenden Betrieb nötig. Dieses Monitoring nimmt Messdaten auf und berechnet daraus Ist-Werte für die bei der Analyse und Modellierung definierten Process Performance Indicators. Ein sofortiger Vergleich mit definierten Soll-Größen führt bei Abweichungen zu Eskalationen entlang der Managementhierarchie und gegebenenfalls zu kurzfristigen Maßnahmen. Mittel- und längerfristige Auswertungen lassen strukturelle Verbesserungsmöglichkeiten erkennen. Die Analyse des Prozessverhaltens und möglicher Abweichungen lässt Rückschlüsse auf Ursachen zu und löst Rückkopplungen in andere Aktivitätsbündel aus.

\section{Betrieb und Monitoring im Fallbeispiel}

Seit Freigabe des Prozesses arbeitet die Bank Kreditanträge der Interessenten in der beschriebenen Form und Umgebung ab. Das Monitoring für das vergangene Quartal ergab folgende durchschnittliche Zahlen:

- Interessenten haben 50 Anträge pro Woche gestellt

- Die Bank hat 20\% davon abgelehnt, die Hälfte davon wegen mangelnder Bonität

- Zu den restlichen Anträgen erhielt der Interessent ein Angebot innerhalb von 4 Tagen

- In 30\% der Fälle hat der Interessent das Angebot angenommen und einen Vertrag abgeschlossen 
Da Mitbewerber mit sehr kurzen Bearbeitungszeiten werben, nimmt die Bank an, dass die Dauer von 4 Tagen bis zum Erhalt eines Angebots bei sonst vergleichbaren Konditionen einer der Gründe ist, warum Kunden keinen Vertrag abschließen. Sie weicht auch signifikant vom vorab formulierten Ziel von 3 Tagen ab.

\subsubsection{Verbesserungsszenarien}

Die folgenden Szenarien zeigen, wie mit weiterführenden Analysen der Monitoring-Ergebnisse nach Ursachen für die lange Durchlaufzeit geforscht, und für Verbesserungsmaßnahmen (Optimierung) in passende Aktivitätsbündel verzweigt werden kann. Davon hängt im Einzelfall der weitere Pfad durch die Aktivitätsbündel ab, also welche Tätigkeiten anschließend nötig sind, ehe der umgestaltete Prozess produktiv gesetzt werden kann. Die Ausführungen beschränken sich zur Vereinfachung jeweils auf eine Maßnahme. In der Realität wird man meist mehrere Optimierungsmöglichkeiten parallel verfolgen.

\subsubsection{Verbesserungsszenario 1}

Die Betrachtung der Häufigkeitsverteilung für den Anfall der Kreditanträge hat ergeben, dass montags 25, dienstags 15, mittwochs 6 und donnerstags und freitags je 2 Anträge vorliegen. Dies könnte daran liegen, dass Interessenten am Wochenende vermehrt Immobilien besichtigen, Kaufentscheidungen treffen und die Finanzierung angehen. Die Auswertung der Liegezeit bis zur Bearbeitung durch die Sachbearbeitung Immobilienkredit zeigt, dass dort zu Wochenbeginn wegen des hohen Aufkommens ein Engpass vorliegt. Bei der derzeit vorgehaltenen Kapazität von 5 Sachbearbeitern in Vollzeit beträgt die durchschnittliche Liegezeit 2 Tage. Um diese und damit die Durchlaufzeit zu verringern, könnte im Rahmen der organisatorischen Implementierung an den Montagen und Dienstagen zusätzliche Sachbearbeitungskapazität, etwa in Form verfügbarer Halbtageskräfte eingesetzt werden. In diesem Fall ändert sich der Prozess nicht, es sind keine weiteren Aktivitäten nötig.

\subsubsection{Verbesserungsszenario 2}

Eine genauere Analyse hat ergeben, dass die hohe durchschnittliche Durchlaufzeit von den Anträgen mit Beträgen zwischen $200.000 €$ und 500.000 Mio. € verursacht wird, weil die Liegezeit bis zur Genehmigung durch die Abteilungsleitung relativ zu den anderen Anteilen der Gesamtdauer sehr hoch ist. Dies liegt daran, dass Abteilungsleitung und Stellvertretung z. B. wegen häufigen Dienstreisen nicht regelmäßig für Genehmigungen zur Verfügung stehen.Der interne Prozessberater der Bank schlägt eine Änderung der Geschäftsregel zur Genehmigung vor. Zukünftig soll es den Sachbearbeitern erlaubt sein, für Beträge bis $500.000 €$ Angebote selbst zu unterschreiben und zu verschicken. Diese Reorganisation berührt mehrere Aktivitätsbündel. Zunächst erfordert sie eine Änderung des Modells, da die Genehmigungsschleife über die Abteilungsleitung entfällt. Die Modelländerung bedarf der anschließenden Validierung, um sicherzustellen, dass der geänderte Ablauf (immer noch) zum gewünschten Ergebnis führt. Im Rahmen der 
IT-Implementierung muss die Modifikation des Modells auch in die Workflow-Software überführt und getestet werden.Durch den Wegfall der Genehmigungen ändert sich der Aufgabenzuschnitt bei der Abteilungsleitung. Bei der Sachbearbeitung bleiben die Aufgaben zwar gleich, jedoch erhöhen sich Kompetenz und Verantwortung. Diesen Änderungen ist in der organisatorischen Implementierung Rechnung zu tragen, etwa durch aktualisierte Aufgabenbeschreibungen und möglicherweise durch Qualifikation der Sachbearbeiter mithilfe von Schulungen z. B. zur umfangreicheren Risikoabschätzung. Dieses Szenario greift im Vergleich zum Fall 1 massiv in die Art ein, wie im Prozess gearbeitet wird, und erfordert deshalb wesentlich umfangreichere Aktivitäten.

\subsubsection{Verbesserungsszenario 3}

Die Bank holt Bonitätsdaten über die Antragsteller bei der Schutzgemeinschaft für allgemeine Kreditsicherung (SCHUFA) ein. Hierzu übertragen die Sachbearbeiter Immobilienkredit die nötigen Kundendaten aus dem Kreditantrag in das Web-Formular der SCHUFA und die von dort gelieferten Abfrageergebnisse in das Bank-System zur weiteren Verarbeitung im bankeigenen Scoring. Die Sachbearbeiter berichten vom zeitraubenden Umkopieren der Daten durch Copy \& Paste, den Fehlern die dabei entstehen, und den dadurch verursachten Nacharbeiten.

Um die Digitalisierung weiter voranzutreiben entscheidet die Bank ohne weitere Analysen, zukünftig anstatt der Internetauskunft der SCHUFA deren Web-Service zu nutzen. Dieser kann in den Workflow so integriert werden, dass die Process Engine ihn auf Knopfdruck des Sachbearbeiters aufruft und ihm die Kundendaten als Parameter übergibt. Der Service liefert das Ergebnis automatisch zurück an die Process Engine, die es in das Banksystem überträgt.Zu behandelnde Aktivitätsbündel beschränken sich in diesem Fall auf die IT-Implementierung, in deren Rahmen die entsprechenden Softwareanpassungen mit anschließenden Tests durchzuführen sind, ehe die Produktivsetzung erfolgt. Die Arbeitsweise der Beteiligten ändert sich nur geringfügig, es sind keine Qualifizierungsmaßnahmen nötig. Der Wegfall der manuellen Datenübertragung entlastet sie von stupiden, zeit- und damit kostenintensiven und fehleranfälligen Routinetätigkeiten. Der intensivere IT-Einsatz spart Bearbeitungs- und Durchlaufzeit sowie Kosten und erhöht die Kundenzufriedenheit.

\subsubsection{Verbesserungsszenario 4}

In Abschn. 4.2.4 haben wir beschrieben, dass bei der Modellierung die Kundenbonitätsprüfung und die Objektprüfung bewusst parallelisiert wurden, um Durchlaufzeit zu sparen. Die Analyse hat gezeigt, dass die Bank pro Woche fünf Anträge aus Bonitätsgründen ablehnt. In diesen Fällen haben aber Sachbearbeiter bereits Aufwand für die parallele Wertprüfung der betreffenden Immobilien betrieben. Diesen einzusparen könnte zunächst wieder dafür sprechen zuerst die Bonität zu prüfen und nur bei positivem Ergebnis die Wertprüfung durchzuführen. Nötig wäre eine Modelländerung mit Validierung und Anpassung der Workflow-Anwendung.

Die Rückführung der arbeitsteiligen Prüfung würde aber wieder die Durchlaufzeit erhöhen und zu einem Zielkonflikt führen. Deshalb gilt es weiter zu überlegen, ob sich 
der Aufwand für die Wertprüfung durch Automatisierung vermindern lässt, sodass eine unnötige Prüfung nicht mehr ins Gewicht fällt. Denkbar ist z. B., dass das Banksystem um Funktionen zur Bewertung erweitert wird. Es könnte dann nach automatischer Übernahme von Parametern aus dem Kreditantrag (Art, Größe, Baujahr, Adresse etc.) und angereichert mit Vergleichsinformationen (bankeigene Erfahrungswerte und Richtwerttabellen) und Geoinformationen (Infrastruktur mit Schulen, Einkaufsmöglichkeiten, Verkehrsanbindung etc.) einen Wertindex errechnen. Dieser beschleunigt die vom Sachbearbeiter vorgenommene finale Werteinschätzung. Bei dieser Möglichkeit könnte die parallele Ausführung der Schritte bestehen bleiben.

Anstelle einer Modelländerung wären die Zusatzfunktionalität in der IT-Implementierung zu realisieren und zu testen sowie die Sachbearbeiter im Umgang mit der Softwareerweiterung zu schulen.

\subsection{Einführung in Design Thinking}

\subsubsection{Wesen}

Design Thinking (DT) ist ein methodischer Ansatz, kreativ und gestalterisch tätig zu werden, um Neuartiges hervorzubringen und komplexe Probleme zu lösen. Es zeichnet sich durch Beschreiten neuartiger Wege zur lösungsorientierten Gestaltung aus. Problemstellungen können besser gelöst werden, indem bei fortlaufenden Iterationen und dem „Begreifbarmachen“ durch Prototypen die Bedürfnisse der (potenziellen) Nutzer in den Vordergrund gestellt werden. Über dieses grundlegende Verständnis von Design Thinking herrscht Einigkeit in Praxis und Wissenschaft ${ }^{1}$. Das Spektrum, das der Ansatz abdeckt, wird dagegen nicht einheitlich gesehen.

Es gibt beispielsweise Interpretationen als Denkweise, Prozess und Werkzeugkasten [4]. Eine empirische Untersuchung belegt die Wahrnehmung im Kontinuum zwischen den beiden Polen Werkzeugkasten und Mindset (vgl. Abb. 4.5) [5].

Dies ist einerseits auf die unterschiedlichen Wurzeln zurückzuführen, andererseits aber auch eine Folge der dem Konzept inhärenten, stetigen, erfahrungsgeleiteten Weiterentwicklung und Anpassung in unterschiedlichen Kontexten. „Die ständige Weiterentwicklung ist ein wichtiger Teil von Design Thinking“ [6]. konstatiert Larry Leifer, einer der Protagonisten des Ansatzes an der d.school in Stanford, und betont: „Wenn Design Thinking eines Tages ein festgeschriebenes Manifest herausgeben sollte, würde es sich damit selber unkenntlich machen“ [6, S. 9].

Der Ansatz geht auf David Kelley von der Design-Agentur IDEO und die Professoren Larry Leifer und Terry Winograd von der Universität Stanford zurück. Insbesondere letztere stellten bei der Ingenieursausbildung fest, dass bei der Entwicklung marktfähiger Produkt weniger die rein technischen, sondern vielmehr nutzerbezogene Aspekte

${ }^{1}$ Eine Übersicht über Definitionen findet sich z.B. bei [3]. 
Tool(box) Method/Process/Protocol Methodology Mindset

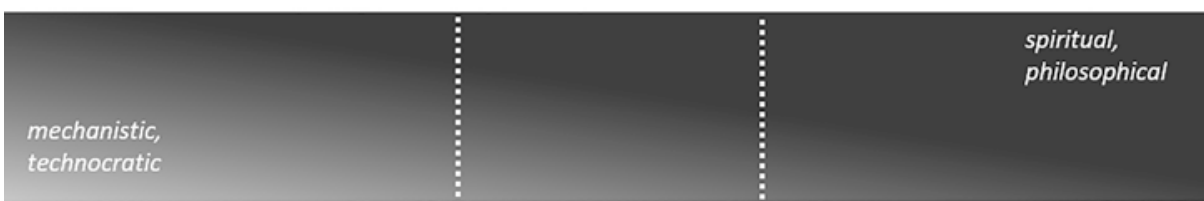

Tool(box)

Tools with clear rules and instruction manuals, e.g.: Empathy Map, POV, MadLib, Brainstorming Rules, Stakeholder Map, etc. Many tools come with steps how to apply them. The more complex these steps become, the more they are perceived as selfcontained methods.

\begin{abstract}
Method/Process/Protocol A means or manner of procedure to systematically get things done and know when to apply which tool (with ist sub-steps) to the situation at hand. Often understood as a (semi-) ordered sequence of actions, for example the $x$ steps in 'the' design thinking process, waterfall model, or other process representations.
\end{abstract}

\section{Methodology}

Combining and mastering a set of appropriate methods and methodologies, i.e. the principles, practices, and procedures of different knowledge domains (e.g., ethnographic research + industrial design + creativity methods etc.), which might constitute a coherent whole for an application context at hand. Examples might be Lean Start-up, Six Sigma or Design Thinking itself.

\section{Mindset}

A guiding stance or attitude, which influences ways of reasoning. As such it shapes the selection and development of appropriate methodologies, methods and tools. The frequent application of the latter three might influence the mindset and vice versa.

Abb. 4.5 Verständnis von Design Thinking

im Mittelpunkt stehen sollten. Diese Erkenntnis führte etwa ab den 1980er-Jahren zur Entwicklung des DT-Konzepts und manifestiert sich bis heute im Stanford-Kurs zum Mechanical Engineering 310 - Design Innovation (me310.stanford.edu). Zur weiteren Verbreitung in Forschung, akademischer Lehre und betrieblicher Praxis trug maßgeblich Hasso Plattner mit seiner Unterstützung der nach ihm benannten Einrichtungen des d.school Institute of Design an der Universität Stanford und der School of Design Thinking an der Universität Potsdam (HPI D-School) bei.

Aufgrund der Herkunft war DT ursprünglich vorwiegend auf die Entwicklung von physischen Produkten bezogen. Es findet aber mittlerweile vielfältige Anwendung in unterschiedlichen Gebieten wie bei der Entwicklung von Services oder ganzer Geschäftsmodelle, und gewinnt auch vermehrt in der Organisationsgestaltung und im Geschäftsprozessmanagement an Bedeutung.

\subsubsection{Kernelemente}

Kernelemente sind eine Mischung aus Mindset, Vorgehensweisen und konkreten Einrichtungen wie Arbeitsräumen. Dies spiegelt sich in der groben Einteilung in die drei „Ps“ wider, nämlich in die Bereiche People (Menschen), Process (Prozess) und Place (Arbeitsumgebung).

Design Thinking beginnt mit der Schaffung tiefer Empathie für die Betroffenen. Es identifiziert die optimale Lösung im Überlappungsbereich von Wünschen der Menschen (menschlich-psychologischer Aspekt), der Machbarkeit (technologischer Aspekt) und 
Wirtschaftlichkeit (geschäftlicher Aspekt) [7]. Die zu entwickelnde Innovation soll etwas sein, das

- die Menschen wirklich mögen (desirability),

- aus technologischer und prozessualer Sicht machbar ist (feasibility) und

- aus wirtschaftlicher Sicht erfolgreich ist (viability).

Um dies zu erreichen durchläuft ein interdisziplinäres Team (People) in variablen, Kreativität fördernden Räumlichkeiten (Place) einen Prozess (Process) mit vielen Iterationen, wobei eine Vielzahl von Methoden zum Einsatz kommen kann.

\subsubsection{People}

Die Fokussierung auf den Menschen erfolgt in zweierlei Hinsicht:

Zum einen stehen Vertreter der Zielgruppe der Innovation, d. h. Kunden oder Anwender, mit ihren Bedürfnissen im Vordergrund. Empathie für sie zu entwickeln, sich in ihre Lage im betrachteten Kontext zu versetzen und somit ein tiefes Problemverständnis zu erlangen, ist der Grundstein für erfolgreiche Innovation und nimmt breiten Raum in dem im Abschn. 4.3.2.2 beschriebenen Prozess ein.

Zum zweiten stellt Design Thinking stark auf die am Projekt beteiligten Menschen als Einzelpersonen und als Team ab. Es sieht vor, die Diversität in interdisziplinären Teams für die Erhöhung der Ergebnisqualität zu nutzen. Teammitglieder sollen „T-Shaped“, d. h. sowohl Experten als auch Generalisten sein. Als Experten sind sie tief verwurzelt in ihrem Fachgebiet und bringen die entsprechende Expertise ein (senkrechter Strich des T). Damit kann auch die fachliche Vertretung einer Interessengruppe (Stakeholder) einhergehen (z. B. Vertrieb, Produktion, IT). Der Blick aus unterschiedlichen Perspektiven auf eine Problemstellung und die Synthese von Know-how und Erfahrungen aus verschiedenen Domänen hilft oft, neuartige Lösungsansätze zu entwickeln.

Die Eigenschaft als Generalisten ist Voraussetzung dafür, von der eigenen Perspektive in die von anderen Beteiligten zu wechseln und offen zu sein für die Zusammenarbeit an den (fachlichen) Schnittstellen (waagrechter Strich des T, ,Wir“-Denken) [8, S. 122 ff.]. Lewrick et al. plädieren deshalb für interdisziplinäre versus multidisziplinäre Teams, weil erstere wirklich kollektiv Ideen hervorbringen und hinter diesen stehen, wogegen die Mitglieder multidisziplinärer Teams bei der Lösungsfindung oft die eigene Perspektive überbewerten. Letzteres führt eher zu Kompromisslösungen, die nicht von allen hundertprozentig mitgetragen werden [8]. Ein interdisziplinäres Team ist idealerweise heterogen zusammengesetzt, nicht nur aus Vertretern möglichst vieler Fachgebiete, sondern auch unterschiedlicher Altersgruppen, Nationalitäten und Geschlechter.

Der Erfolg eines Design-Thinking-Projektes wird maßgeblich durch die individuellen Eigenschaften der Mitglieder dieses Teams und der daraus erwachsenden gemeinschaftlichen Arbeitskultur und Denkweise bestimmt. Im Zentrum steht dabei, dem Menschen als Ausgangspunkt aller Aktivitäten hohe Wertschätzung und Empathie entgegenzubringen, sowohl den Kolleginnen und Kollegen im Team als auch den Benutzern 
bzw. Kunden. Dazu sollen Eigenschaften kommen wie Kooperationsfähigkeit, Neugier, Experimentierfreude, integratives Denken und Optimismus der Teammitglieder. Ein weiterer Erfolgsfaktor ist die Begleitung des Teams durch eine mit dem Prozess und dem Methodeneinsatz erfahrene Person als Facilitator. Diese gibt Orientierung über das jeweilige Stadium im Prozess und welche Instrumente dort am besten $\mathrm{zu}$ verwenden sind, ohne jedoch inhaltlich zu intervenieren.

\subsubsection{Process}

Design Thinking folgt einem Vorgehensmodell mit einer Reihe von Schritten. Zwar haben sich mit der Zeit leicht unterschiedliche Varianten des sogenannten Mikrozyklus mit alternativen Bezeichnungen der Phasen herausgebildet, welche aber inhaltlich letztlich kaum voneinander abweichen. Wir orientieren uns an dem Modell der d.school in Stanford, das dem Design-Thinking-Prozess fünf auch als Arbeitsmodi bezeichnete Phasen zugrunde legt (vgl. Abb. 4.6).

Gekennzeichnet sind alle Modelle vom Wechsel zwischen divergentem und konvergentem Betrachten, Denken und Agieren, sowohl beim Verstehen des Problemraums und Gestalten des Lösungsraums. Der Übergang zwischen der Ausweitung des kreativen Rahmens mit stetig zunehmender Informationsmenge und dem Fokussieren durch Eingrenzung wird als Groan Zone bezeichnet, also als eine Stelle wie ein „knarzendes“ Scharnier [8, S. 28 f.]. Auch die bewusste Iteration ist ein grundlegendes Element im DT-Prozess, das sich in dem Motto „fail early, fail often“ bzw. „fail forward“ in einer offenen Fehlerkultur äußert. Es beschreibt die Vorstellung, dass ideale Lösungen nur durch mehrfaches frühzeitiges Experimentieren, Testen und Berücksichtigen des Feedbacks der Zielgruppe gefunden werden können, was zu mehr oder weniger umfangreichen neuen Durchläufen früherer Modi führen kann. Die mehrfachen Iterationen sollen als sogenannter Makrozyklus vom Problemverständnis zur Konkretisierung einer Lösungsvision führen und in einem Umsetzungsplan münden [8, S. 37 f.].

In allen Prozessphasen gilt das Prinzip „Be visual \& show“, was bedeutet, dass Gedanken, Ideen, Ergebnisse visuell und plastisch dokumentiert und vorgeführt werden sollen, etwa durch Post-its mit Stichworten und Zeichnungen, Mindmaps, Process Maps,

Abb. 4.6 Design-ThinkingProzess nach Stanford d.school

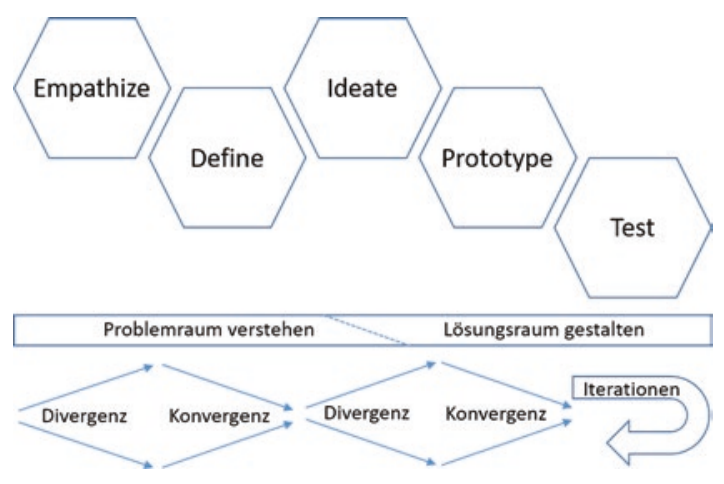


greifbare Prototypen etc. Aufgrund dieses Prinzips wird manchmal auch vorgeschlagen, besser von Design Doing anstatt von Design Thinking zu sprechen.

Für die erfolgreiche Arbeit eines Teams entlang des Prozesses hat sich eine Reihe von Regeln und Tipps (Rules of Engagement) als hilfreich erwiesen, von denen einige auch generell bei Gruppenarbeiten zu beachten sind. Sie sollten zu Beginn eines Projekts kommuniziert und im weiteren Verlauf vom Facilitator bei Bedarf in Erinnerung gerufen werden:

- Nutzerorientierte Denk- und Arbeitsweise (Entwicklung vom Empathie)

- Keine Vorgabe oder Beschränkung von Denkweisen, Lösungswegen oder Lösungen (Offenheit, Autonomie; „Go for quantity“)

- Konzentration auf das Thema (Fokussiertheit), daher auch keine Ablenkung durch Handys, Computer, iPads, Smart Watches etc., es sei denn sie sollen bewusst z. B. für Recherchen oder zur Prototyperstellung eingesetzt werden

- Aktive Teilnahme jedes Teammitglieds (eigene Artikulation, diszipliniertes Zuhören (,One conversation at a time“) und Aufbauen auf Ideen anderer)

- Förderung der Entwicklung unterschiedlicher Perspektiven und wilder Ideen (,Encourage wild ideas")

- Keine Wertungen bei der Ideengenerierung (,Defer judgement“, „No killer phrases“)

- Zeitlimitierung (,Time Boxing“), um Verliebtheit in eine bestimmte Idee zu vermeiden

Die folgenden Abschnitte beschreiben jede Phase kurz zusammen mit einer tabellarischen Auswahl an Methoden und Werkzeugen, welche dort zum Einsatz kommen. Ausführlichere Erläuterungen dazu finden sich beispielsweise im Bootcamp Bootleg der d.school [9] und bei [8, S. 36 ff.].

\section{Empathize (Empathie aufbauen)}

Empathie ist das Herzstück eines menschen-zentrierten Designprozesses. Sie zu entwickeln heißt, ein tiefes Verständnis für die Mitglieder der Zielgruppe aufzubauen im Hinblick auf die Problemstellung und deren Kontext. Ziel ist es zu verstehen, warum und wie die Menschen Dinge tun, wie sie über die Welt denken und was ihnen wichtig ist und sinnvoll erscheint, sowie etwas über ihre körperlichen und emotionalen Bedürfnisse zu erfahren. Dies bedeutet die Nutzer zu beobachten, ihnen zuzuhören, mit ihnen zu interagieren, sich in ihre Situation einzudenken und einzufühlen und damit in ihre bewusste und unbewusste Welt von Gefühlen, Werten und Bedürfnissen einzutauchen (engage, observe, immerse). Dies ist die Grundvoraussetzung, um Innovationen in die richtige Richtung zu lenken.

Ein wesentliches Instrument zur Dokumentation des Verständnisses für die Zielgruppe sind die sogenannten Personas. Sie stellen fiktive Kunden oder Benutzer dar und verkörpern deren unterschiedliche Ziele, Verhaltensweisen, Bedürfnisse und Eigenschaften mit Relevanz für die zu entwickelnde Lösung. 
Tab. 4.4 Methoden und Werkzeuge für die Phase „Empathize“

\begin{tabular}{l|l}
\hline Aktivität/Thema & Ausgewählte Methoden bzw. Werkzeuge \\
\hline $\begin{array}{l}\text { Problemkontext erschließen und gemein- } \\
\text { schaft-liches Verständnis dafür entwickeln }\end{array}$ & $\begin{array}{l}\text { Brain Dump } \\
\text { Business Process and Value Maps, Concept } \\
\text { Maps, Business Model Canvas }\end{array}$ \\
\hline Problemkontext strukturieren & Structuring/Clustering frameworks \\
\hline $\begin{array}{l}\text { Zielgruppe (Nutzer, Kunde) identifizieren und } \\
\text { verstehen }\end{array}$ & $\begin{array}{l}\text { User Profile Canvas mit } \\
\text { Persona } \\
\text { Jobs-to-be-done } \\
\text { Gains \& Pains } \\
\text { Use Cases }\end{array}$ \\
\hline Zielgruppe (Nutzer, Kunde) verstehen & $\begin{array}{l}\text { Needfinding-Gespräch, Interview for Emp- } \\
\text { athy (jeweils inkl. Vorbereitung), z. B. mit } \\
\text { W-Fragen (What? How? Why? Who?) nach } \\
\text { der AEIOU-Methode (Activities, Environment, } \\
\text { Interaction, Objects, User) } \\
\text { Empathy Map } \\
\text { Future User }\end{array}$ \\
\hline
\end{tabular}

Im Modell des Hasso-Plattner-Instituts umfasst „Empathize“ die Phasen „Verstehen“ und „Beobachten“. Tab. 4.4 zeigt gängige Instrumente für die in der Phase enthaltenen Aktivitäten.

\section{Define (Problemstellung definieren)}

In diesem Modus geht es darum, auf den Erkenntnissen aus dem Modus „Empathize“ aufzubauen, sie zu teilen und zusammenzuführen, zu strukturieren, zu gewichten und zu interpretieren. Diese Synthese dient dazu, die Personas für idealtypische Nutzer zu prüfen und weiterzuentwickeln und gegebenenfalls die Perspektiven verschiedener Stakeholder einzunehmen. Ergebnisse sind ein weiter vertieftes Verständnis für die Benutzer und den Problemraum sowie eine konkretisierte, aussagekräftige Problemstellung (Design Challenge). Letztere schlägt sich in einem einzigen Satz nieder, welcher als sogenannter Point of View (POV) die Frage für die anschließende Phase der Ideengewinnung bildet [8, S. 73]. In der Praxis werden unterschiedliche POV-Fragen verwendet. Eine typische Formulierung ist die „How might we?“-Frage, also beispielsweise „Wie könnten wir [User, Kunde] helfen, [ein bestimmtes Ziel] zu erreichen?“ [8, S. 74].

Im Modell des Hasso-Plattner-Instituts entspricht „Define“ der Phase „Standpunkt definieren“. In der folgenden Tab. 4.5 sind übliche Hilfsmittel für die in der Phase enthaltenen Aktivitäten aufgeführt.

\section{Ideate (Ideen gewinnen)}

Das Ziel der Ideengewinnung ist es einen breiten Lösungsraum zu erarbeiten, also möglichst viele und auch möglichst unterschiedliche Ideen $\mathrm{zu}$ entwickeln und $\mathrm{zu}$ visualisieren. Ausgangspunkt ist die Point-of-View-Frage, jedoch gehen sämtliche bisher 
Tab. 4.5 Methoden und Werkzeuge für die Phase „Define“

\begin{tabular}{l|l}
\hline Aktivität/Thema & Ausgewählte Methoden bzw. Werkzeuge \\
\hline Erkenntnisse teilen & Story share and capture (Storytelling) \\
\hline Erkenntnisse interpretieren, Schlüsse ziehen & $\begin{array}{l}\text { Saturate and group } \\
\text { Empathy Map } \\
\text { Customer/User Experience Journey (mit } \\
\text { actions, mindset, touch points, pain points, } \\
\text { moments of truth) }\end{array}$ \\
\hline Zielgruppe (Nutzer, Kunde) noch besser ver- & $\begin{array}{l}\text { Persona } \\
\text { stehen }\end{array}$ \\
& $\begin{array}{l}\text { Composite Character Profile } \\
\text { Power of Ten } \\
\text { 2x2 Matrix } \\
\text { Why-How-Laddering }\end{array}$ \\
& $\begin{array}{l}\text { Point of View } \\
\text { 360-Grad-Betrachtung } \\
\text { A day in the life of ... }\end{array}$ \\
\hline
\end{tabular}

erarbeiteten Erkenntnisse in diese Phase ein, also beispielsweise auch User Profile Canvas, Empathy Map und Customer/User Experience Journey. Grundlegendes Instrument ist das Brainstorming, das durch Kreativitätstechniken und gezielte Aufgaben (z. B. Ideengenerierung zu bestimmten Funktionen) weiter und mehrfach stimuliert werden kann. Auch die Gestaltung erster „Low-Fidelity“-Prototypen und deren Test können weitere Denkanstöße für Lösungen liefern und Iterationen auslösen.

Der Methodeneinsatz in diesem Modus soll dazu befähigen, über offensichtliche Lösungen hinauszugehen und damit das Innovationspotenzial erhöhen, indem die kollektiven Perspektiven und Stärken des Teams genutzt werden. Unerwartete Lösungsrichtungen sollen aufkommen können und zur Menge und Unterschiedlichkeit der Ideen beitragen. So entsteht eine Vielzahl von Ideen, welche sortiert, verdichtet und bewertet werden. Beim gesamten Vorgehen sollte streng zwischen der Generierung und der Bewertung der Ideen getrennt werden, um nicht früh den kreativen Flow zu einzuschränken.

Im Modell des Hasso-Plattner-Instituts entspricht „Ideate“ der Phase „Ideen finden“. Gängige Instrumente für die in diesem Modus enthaltenen Aktivitäten sind Tab. 4.6 zu entnehmen.

\section{Prototype (Prototypen erstellen)}

Das Prototyping greift die am höchsten bewerteten Ideen aus der Ideenentwicklung auf und bearbeitet sie weiter. Dabei wird der Grundsatz des Design Thinking umgesetzt, Sachverhalte, Produkte und Ergebnisse möglichst früh zu visualisieren und anhand greifbarer Modelle mit potenziellen Nutzern zu testen, zu diskutieren und mit dem erhaltenen Feedback weiterzuentwickeln. Prototypen werden also erzeugt, um zu lernen, offene Fragen und Unstimmigkeiten zu klären, eine Konversation bzw. einen Diskurs zu beginnen 
Tab. 4.6 Methoden und Werkzeuge für die Phase „Ideate“

\begin{tabular}{l|l}
\hline Aktivität/Thema & Ausgewählte Methoden bzw. Werkzeuge \\
\hline Ideen generieren (iterativ) & $\begin{array}{l}\text { Generelles Brainstorming auf Basis des POV (z. B. How might } \\
\text { we?) mit Stimulierung durch Kreativitätstechniken } \\
\text { gezieltes Brainstorming (kritische Funktionalitäten, Bench- } \\
\text { mark, Dark Horse, Funky Prototype) } \\
\text { Power of Ten, Bodystorming } \\
\text { Quick \& Dirty Prototyping }\end{array}$ \\
\hline Ideen sortieren, verdichten & $\begin{array}{l}\text { Swap Sort, } 2 \times 2 \text { Matrix } \\
\text { Concept-/Systems-/Mind-Maps, Ideensteckbriefe }\end{array}$ \\
\hline Ideen bewerten und priorisieren & Vier-Kategorien-Methode; Post-it-Voting, Spend your budget \\
\hline
\end{tabular}

und schnell und noch günstig Sackgassen zu erkennen. Neben der Veränderung kann das Feedback auch zum kompletten Verwerfen und damit zu einer grundlegenden Iteration in weiter zurückliegenden Phasen führen. Dieses Vorgehen wird auch mit dem Slogan „Love it, change it or leave it" umschrieben.

Prototyping transferiert Ideen aus dem Kopf in die physische Welt. Ein Prototyp kann demnach alles sein, was eine physische Form annimmt und der Maxime „don't tell me, show me!" folgt: eine Wand mit Post-it-Notizen, ein Rollenspiel, ein Raum, ein Objekt, ein Storyboard oder auch beliebige Kombinationen verschiedener Ausdrucksmittel.

Die Granularität des Prototyps sollte dem Projektfortschritt entsprechen. In den frühen Stadien eines Projektes sollten Prototypen entstehen, die schnell anzufertigen und kostengünstig sind (Low Fidelity, Quick \& Dirty), aber schon nützliches Feedback von Benutzern und Kollegen hervorrufen. In späteren Stadien sollten die Prototypen verfeinert werden und bestimmte Fragestellungen eingehend untersuchen lassen. Sie dienen dazu, Empathie zu vertiefen, zu testen, weitere Ideen und Inspiration zu gewinnen.

Im Modell des Hasso-Plattner-Instituts entspricht „Prototype“ der Phase „Prototyp entwickeln“. Die folgende Tab. 4.7 zeigt gängige Instrumente für die in dieser Phase enthaltenen Aktivitäten.

\section{Test (Prototypen testen)}

Wie im vorherigen Abschnitt bereits ausgeführt, ist Testen eng mit Prototyping verknüpft. Die Empfehlung „Prototype as if you know you're right, but test as if you know you're wrong." beschreibt eine Denkhaltung, welche diesen Zusammenhang verdeutlicht. Testen bietet die Chance, qualitatives Feedback zu den prototypischen Lösungen zu erhalten, sie besser zu machen, weiter über die Nutzer zu lernen und damit die Empathie für sie zu vertiefen. Der Testmodus ist ein iterativer Lernmodus, in dem die Prototypen in den Kontext des potenziellen Benutzers gestellt und von diesem benutzt und bewertet werden. Wichtige Prinzipien dabei sind: Zeigen und nicht reden, Erlebnisse schaffen und dem Benutzer Vergleiche ermöglichen.

Das Feedback bei den Tests kann nicht nur zu Veränderungen, sondern auch zum kompletten Verwerfen und damit zu einer grundlegenden Iteration über weiter zurückliegende Phasen führen. Dieses Vorgehen wird auch mit dem Slogan „Love it, change it, or leave 
Tab. 4.7 Methoden und Werkzeuge für die Phase „Prototype“

\begin{tabular}{l|l}
\hline Aktivität/Thema & Ausgewählte Methoden bzw. Werkzeuge \\
\hline Prototypen erstellen & $\begin{array}{l}\text { Low-Fidelity-Prototypen z. B. aus Bastelmaterial (Lego, Knetmasse etc.) } \\
\text { Rollenspiele, Storytelling, Storyboards } \\
\text { Wireframes, Screen-Design-Tools } \\
\text { Shooting and editing video }\end{array}$ \\
\hline
\end{tabular}

Tab. 4.8 Methoden und Werkzeuge für die Phase „Test“

\begin{tabular}{l|l}
\hline Aktivität/Thema & Ausgewählte Methoden bzw. Werkzeuge \\
\hline Feedback einholen & Feedback Grid \\
& „I like, I wish, What if?““ \\
& A/B-Tests mit digitalen Tools \\
\hline
\end{tabular}

it" umschrieben [8, S. 34]. Prinzipiell ist bei jeder Iterationsschleife unabhängig von deren Umfang zu reflektieren, welche vorherigen Ergebnisse (z. B. Personas, User/Customer Experience Journey) aufgrund des Feedbacks angepasst werden müssen.

Im Modell des Hasso-Plattner-Instituts entspricht „Test“ der Phase „Testen“. Tab. 4.8 enthält Instrumente für die in der Phase ausgeführten Aktivitäten.

\subsubsection{Place}

Für die Arbeit der interdisziplinären Teams in den beschriebenen Modi gilt es eine Kreativität fördernde Umgebung, sogenannte Make oder Creative Spaces, zu schaffen. Dies bezieht sich insbesondere auf die Verfügbarkeit, Größe und Einrichtung von Räumlichkeiten als auch auf Hilfsmittel und Materialien für Visualisierung und Prototypengestaltung. Es geht vor allem darum, den Teams, frei und dauerhaft verfügbare Arbeits-, Interaktions-, Entspannungs- und Lagerbereiche, flexible Möbel mit Rollen, beschreib- und abwischbare Flächen (Wände, Tische, Tafeln), sowie gute und schnelle Zugänge zu Informationen (Internet, Bibliotheken etc.), Werkzeugen, Arbeitsmaterialien und Verpflegung bereitzustellen [7, S. 216 ff.]. Auch Beleuchtung, Belüftung und Klimatisierung sind wichtige Rahmenbedingungen.

In der Praxis räumt man vor allem bei länger andauernden Projekten den Teams mitunter auch die Möglichkeit ein, die Umgebung selbst zu gestalten (z. B. Möbel selbst bauen). Doorley und Witthoft haben Anleitungen und Erfahrungen u. a. bei der Gestaltung von Kreativitätsumgebungen für die d.school publiziert [10].

\subsection{Verbindung der Konzepte}

\subsection{1 Überblick}

Wie gezeigt zielt Design Thinking auf die Innovation von Produkten und Services, Geschäftsmodellen und -prozessen ab. Im Mittelpunkt stehen Benutzerzentriertheit, Kreativität und Agilität in einem experimentellen, iterativen Prozess, den interdisziplinäre Teams durchlaufen. 
Das Prozessmanagement verfolgt mit der agilen und kreativen Prozessneu- oder -umgestaltung unter Berücksichtigung von Kundenbedürfnissen eine vergleichbare Zielsetzung. Im Folgenden stellen wir Bezüge zwischen den Konzepten her und diskutieren die Erfolg versprechende Nutzung von Design-Thinking-Elementen für das Prozessmanagement.

Wir legen dabei besonderes Augenmerk auf die Digitalisierung von Prozessen, das heißt auf den sinnvollen Einsatz von Informations- und Kommunikationstechnik für die Prozessverbesserung und -innovation. Prototypen und endgültige Lösungen sind damit immer Workflow-Anwendungen unterschiedlichen Automatisierungsgrades.

\subsubsection{Benutzerzentriertheit}

Traditionelle BPM-Ansätze beziehen zwar bei der Prozessanalyse in der Regel die Beteiligten mit Interviews und Workshops ein. Im Vordergrund der aktivitäts- und ablaufbezogenen Interviewfragen, Kartenabfragen oder Beobachtungen stehen dabei jedoch die ,harten“ Fakten der Arbeit im Prozess mit den in Abschn. 4.2.2 aufgeführten Merkmalen. Aspekte wie das Verstehen von Motivation, Denkweisen und Werten der Benutzer, die für die Entwicklung von Empathie im Design Thinking ausdrücklich betont werden, bleiben hier weitgehend unberücksichtigt. Daran haben auch neuere Konzepte wie Social BPM bisher wenig geändert.

Eine interessante Brücke kann der Ansatz des Subjektorientierten Business Process Management (S-BPM) schlagen, das bereits zu Beginn dieses Kapitels im Fallbeispiel verwendet wurde. Er stellt die Subjekte als Handelnde im Prozess in den Mittelpunkt der Betrachtung. Mit der dazugehörigen Methodik und Sprache (vgl. Abschn. 3.6) sowie geeigneten Werkzeugumgebungen können Vertreter von am Prozess beteiligten Subjekten nicht nur als Befragte oder Beobachtete, sondern als aktive Designer an der iterativen Lösungsentwicklung mitwirken. Sie spezifizieren dabei nicht nur explizit das Verhalten des von ihnen repräsentierten Subjekts und dessen Interaktionen mit anderen Beteiligten, sondern können durch die Ausführung des resultierenden Modells sofort das Ergebnis ihrer Gestaltung erproben und verändern. Bei all dem können sie implizit auch die oben angesprochenen ,weichen“ Faktoren einbringen.

\subsubsection{Agiler Prozess mit Iterationen}

Umfangreichere Prozessmanagementvorhaben werden in der Praxis häufig nach wie vor mit traditionellen Projektmanagementmethoden in klar definierten Phasen mit Meilensteinen durchgeführt, vergleichbar dem Wasserfallmodell bei der Softwareentwicklung.

Dies bedeutet, dass der Weg von der Analyse über die Gestaltung des fachlichen Modells und dessen organisatorische und informationstechnische Umsetzung bis zu einer lauffähigen Workflow-Anwendung viel Zeit in Anspruch nimmt. Außerdem steigt 
damit die Wahrscheinlichkeit, dass die IT-Lösung von den sich weiterentwickelnden Bedürfnissen und Wünschen der Benutzer abweicht.

Insbesondere für die Prozessdigitalisierung ist es deshalb von Vorteil den agilen, iterativen Prozess des Design Thinking zu adaptieren. Dies eröffnet die Möglichkeit der steigenden Dynamik hinsichtlich der Entstehung neuer Prozesse und der Veränderungen bestehender Vorgänge, etwa aufgrund neuer oder geänderter Geschäftsmodelle wie Servitization, gerecht zu werden. Für die weiteren Überlegungen stellen wir die Modi des Design Thinking den Aktivitätsbündeln im Prozessmanagement gegenüber (vgl. Abb. 4.7).

In Verbindung mit den Ausführungen in Abschn. 4.2.2 und 4.3.2.2 zeigt die Abbildung, dass DT stärker zwischen Problemverständnis und Lösungsgestaltung trennt. Letztere beginnt erst mit Ideate. Davor wird ausgiebig die Ist-Situation beleuchtet und beispielsweise auf dem Weg über Personas in der Customer/User Experience Journey dokumentiert und visualisiert, ehe mit der Point-of-View-Fragestellung der Ausgangspunkt für die Ideengenerierung formuliert wird.

Beim Prozessmanagement ist die Problemstellung dagegen in der Regel bereits zu Beginn klar formuliert. Bei der Erneuerung bestehender Prozesse leitet sie sich meist aus dem Wunsch nach verbesserter Prozess-performance ab (z. B. Durchlaufzeitverkürzung). In der Praxis werden deshalb meist nur diesbezügliche Schwachstellen des Ist-Zustands dokumentiert und Analyseinformation genutzt, um, wie auch bei einem neuen Prozess, gleich ein neues Soll-Modell zu entwickeln und zu visualisieren. Der kreative, gestalterische Teil beginnt damit früher als beim Design Thinking und ist tendenziell mit weniger Information als Ausgangsbasis unterfüttert. Er ist stärker analytisch (z. B. durch Performance Indicators) getrieben als durch die im Rahmen der Empathieentwicklung

BPM-Aktivitätsbündel
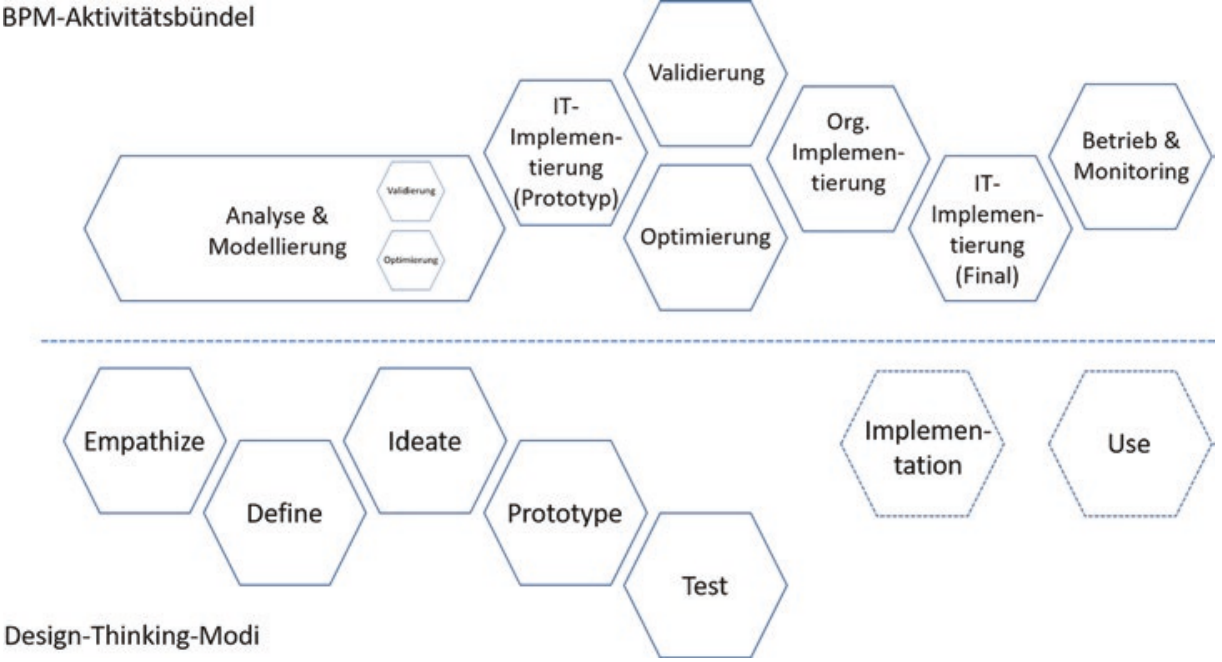

Abb. 4.7 Zuordnung von Design-Thinking-Modi und BPM-Aktivitätsbündeln 
im Design Thinking identifizierten ,weichen“ Faktoren. Unabhängig von der etwas unterschiedlichen konkreten Ausgestaltung lässt sich das Aktivitätsbündel Analyse \& Modellierung den DT-Modi Empathize, Define und Ideate zuordnen.

Für eine umfassendere Erfassung des Problemkontextes und die dadurch erzielbare Ausweitung des Lösungsspektrums für die Prozessinnovation bietet sich der Einsatz bewährter DT-Instrumente an. Insbesondere bei der Entwicklung eines neuen Prozesses können die Teammitglieder z. B. mit einem Brain Dump zum Problemumfeld und der Diskussion der Ergebnisse ihren Betrachtungshorizont erweitern und ein gemeinsames Verständnis entwickeln.

Mithilfe von Personas für die Prozessbeteiligten in ihren jeweiligen Rollen sowie mit Interviews und Beobachtungen lassen sich Customer/User Experience Journeys beschreiben.

Sind Mitwirkende am Prozess selbst Teammitglieder können diese selbst ihre Erfahrungen ebenfalls als Journey visualisieren. Damit erweitert sich die Informationsbasis über die klassischen, objektiven Prozesscharakteristika hinaus um die Perspektive der Nutzer. Dieses breitere Fundament für das Entwickeln von Lösungsideen sollte den höheren Aufwand rechtfertigen.

In Abschn. 4.2.2 haben wir dargelegt, dass bereits bei der Analyse und Modellierung von Prozessen Überlegungen zur Effektivität und Effizienz zumindest von Modellteilen angestellt werden. Dies gilt insbesondere, wenn die späteren Anwender dies wie bei der Subjektorientierung selbst tun. Deshalb sind die Aktivitätsbündel Validierung und Optimierung dem DT-Modus Test zugeordnet, erstrecken sich aber auch über Empathize, Define und Ideate.

Mit dem Fokus auf Prozessdigitalisierung entspricht dem Modus Prototype beim Prozessmanagement das Aktivitätsbündel der IT-Implementierung. Das Prototyping im Design Thinking erhebt den Anspruch schnell und mit einfachen Mitteln, mithin kostengünstig, einen vorführbaren Prototyp zu erzeugen, um rasch Feedback vom Benutzer einzuholen (Modus Test) und dieses zu verwerten. Mit der Übertragung dieses ,fail early, fail often“-Prinzips auf das Prozessmanagement muss das Team in die Lage versetzt werden, das Modell mit geringem Aufwand ausführbar zu machen. Im Vordergrund steht also die Erzeugung eines funktionalen Prototyps in Form von Software, der die Anwender erfahren und erleben lässt, wie ihre Arbeit mit der IT-Lösung aussehen würde.

Die Zuordnung zur IT-Implementierung sollte aber für den Prototyp nicht Programmierung bedeuten. Vielmehr muss es im Sinne der schnellen Iterationen möglich sein, den Prototyp aus dem Modell automatisch zu generieren und im Aktivitätsbündel Validierung die Nutzer erproben zu lassen. Anschließende Modelländerungen aufgrund des Feedbacks führen auf demselben Weg zu einem neuen Prototyp, so lange, bis eine Version gefunden ist, welche die Anwender zufriedenstellt. Solch kostengünstiges und frühzeitiges Prototyping verhindert möglicherweise aufwendigere Nacharbeiten bei der späteren Realisierung der echten Laufzeitumgebung. Mit einer vergleichbaren Vorgehensweise kann das Design der Benutzungsschnittstelle nach den Prinzipien des User Experience Design erfolgen. 
Da das jeweilige Prozessmodell ja nicht nur die Basis für Prototypen, sondern in seiner letztlich verabschiedeten Version auch für die angestrebte Workflow-Anwendung ist, umfasst das Aktivitätsbündel IT-Implementierung auch deren Realisierung in der in Abschn. 4.2.6 beschriebenen Weise. Wenn dafür über die modellbasierte Workflow-Steuerung hinausgehende Software entwickelt werden muss, bietet sich im Sinne der benutzerzentrierten, agilen Vorgehensweise SCRUM als Software-Entwicklungsmethode an.

Im Zusammenhang mit der IT-Implementierung gilt es auch zu entscheiden, inwieweit man die von Software-Start-ups oft verwendete Strategie der Minimum Viable Products verfolgen möchte. Dies würde bedeuten, Software mit minimaler Funktion den Kunden bzw. Anwendern nicht nur prototypisch, sondern auch produktiv zur Verfügung zu stellen, um deren Feedback für die Weiterentwicklung einzuholen. Bei IT-Lösungen für geschäftskritische Prozesse könnte dies riskant sein, anderseits kann es durch frühe Gewöhnung von Kunden an Features möglicherweise einen Vorsprung gegenüber Mitbewerbern eröffnen.

Wie in Abschn. 4.2.5 und 4.2.7 erläutert, ist das Modell auch in die Organisation einzubetten (Organisatorische Implementierung), ehe der Prozess produktiv gesetzt werden kann (Betrieb \& Monitoring).

Im Design Thinking folgen vergleichbare Schritte für die Implementierung z. B. eines Produkts auf Basis eines akzeptierten Prototyps, sowie für die Nutzung, die jedoch nicht mehr zu den Modi im engeren Sinn zählen (gepunktete Formen in Abb. 4.7).

\section{Fazit}

Um den Anforderungen der Digitalisierung von Prozessen gerecht zu werden sollte ein Prozessmanagementvorgehen Design-Thinking- und Prozessmanagementkonzepte kombinieren.

Es muss geeignet sein, Prozesse und deren Änderungen rasch sowohl fachlich als auch in der IT abzubilden und dabei in kurzen Iterationszyklen die Anwender adäquat einzubinden, um die entstehende Lösung deren Vorstellungen anzunähern.

Neben den für die Design-Thinking-Modi Empathize, Define und Ideate nutzbaren Instrumente sind dafür insbesondere einfach handhabbare Methoden und Werkzeuge nötig, mit denen das Team und/oder die Prozessbeteiligten selbst

1. individuell unterschiedliche mentale Modelle der Arbeit artikulieren können

2. diese verschiedenen mentalen Modelle harmonisieren können

3. daraus Lösungsideen und konkrete Lösungsvorschläge in Form von Modellen entwickeln können

4. diese Modelle automatisch in ausführbare Prototypen überführen und testen können

5. freigegebene Modelle mit begrenztem Aufwand in Workflow-Anwendungen für den Live-Betrieb überführen können

Ein Beispiel für ein Konzept zur Unterstützung von 1) und 2) ist Compare/WP (vgl. Abschn. 5.1.2). Die Anforderungen 3), 4) und 5) werden beispielsweise vom S-BPM-Ansatz und darauf basierenden BPM-Tools abgedeckt [11, 12]. 


\subsubsection{Interdisziplinäres Team}

Die Bedeutung der Interdisziplinarität des von einem Facilitator begleiteten Teams beim Design Thinking haben wir im Abschn. 4.3.2.1 ausgeführt.

Auch BPM-Projekte werden üblicherweise von Teams durchgeführt. Wir unterscheiden dabei vier Rollen:

Governors (Treiber und Verantwortliche) setzen die Rahmenbedingungen. Diese umfassen im Wesentlichen den Betrachtungsbereich (Scope), also die Abgrenzung des im Projekt bearbeiteten Prozesssystems sowie, konkret auf die Analyse und Modellierung bezogen, die Methodik und die Werkzeuge.

Actors (Arbeitshandelnde) sind die gegenwärtigen oder zukünftigen Akteure, welche die im zu verändernden oder zu entwickelnden Prozess zur Laufzeit die Aktionen in den Instanzen ausführen. Sie sind demnach die Träger konkreten ausführungsbezogenen Prozesswissens für ihren Anteil an der Erstellung des Prozessergebnisses, wissen also welche Teilschritte sie in welcher Reihenfolge erledigen, welche Informationen und Hilfsmittel sie dazu benötigen und mit wem sie dafür interagieren.

Experts (Spezialisten) unterstützen die anderen Rollen mit methodischem und fachlichem Wissen. Es sind beispielsweise Domänenexperten, die im betreffenden Fachgebiet über Expertise verfügen und einbringen können, welche über die der Actors hinausgeht. Methodenexperten helfen den Beteiligten, insbesondere den Actors, bei der Artikulation und Harmonisierung ihrer mentalen Modelle sowie bei der Umsetzung mit einer der in Kap. 3 vorgestellten Modellierungssprachen. Für die technische Implementierung der fachlichen Modelle werden schließlich IT-Experten hinzugezogen. Experten in den unterschiedlichen Feldern werden bei Bedarf auch von außerhalb der eigenen Organisation als externe Berater involviert.

Facilitators (Entwicklungsbegleiter) moderieren und koordinieren das Vorgehen und die Zusammenarbeit der Beteiligten. Sie sorgen beispielsweise dafür, dass Actors die Schnittstellen zwischen ihren Arbeitsschritten abstimmen und identifizieren für besondere Problemstellungen bei Bedarf geeignete Experten und binden sie ein. Bei alldem motivieren und überprüfen die Facilitators die Einhaltung der von den Governors gesetzten Rahmenbedingungen.

Beim traditionellen phasenorientierten Vorgehen im BPM wirken bei Analyse, Modellierung, Validierung und Optimierung unter der Projektleitung als Facilitator zunächst meist Actors und Experts als fachliche Inputgeber und Methodenexperten zusammen. Die Implementierung der verabschiedeten fachlichen Modelle übernehmen dann IT-Experten. Als Nachteile dieses Vorgehens haben wir bereits in Abschn. 4.4.3 die mitunter lange Dauer und dadurch bedingte Abweichung von den Bedürfnissen der Stakeholder identifiziert. 
Seit einiger Zeit verbreitet sich deshalb der BizDevOps-Ansatz, der den im Zuge der Digitalisierung steigenden Agilitätsanforderungen Rechnung tragen will. Er strebt eine vergleichsweise engere Verzahnung von Fachabteilung (Business, Biz), IT-Entwicklung (Development) und IT-Betrieb (IT Operations) $\mathrm{an}^{2}$. Dem agilen Team gehören von Beginn an Vertreter aller Bereiche in den beschriebenen Rollen an, welche die Prozesslösung gemeinsam gestalten. Das Konzept kann damit sowohl das Business-IT-Alignment verbessern als auch das Enabling durch IT fördern. Ersteres bedeutet, dass der Abdeckungsgrad der Bedürfnisse der Fachabteilungen durch passende IT-Services steigt. Beim Enabling gibt die IT Impulse für die Nutzung von Informations- und Kommunikationstechnik für Geschäftsmodell- und Geschäftsprozessinnovationen.

Wie das Design Thinking sieht der BizDevOps-Ansatz also ein interdisziplinäres Team vor. Herausforderung ist es in solchen Teams, das „Wir“-Denken unter „T-shaped“ Personen zu etablieren. Dies liegt daran, dass Linieneinheiten, denen die Beteiligten entstammen (verschiedene am Prozess beteiligte Fachabteilungen, IT-Entwicklung, IT-Betrieb), unterschiedliche Ziele verfolgen und diesen oft tendenziell höhere Priorität einräumen als einem gemeinschaftlich zu erreichenden Ziel (innovative oder verbesserte Prozesslösung). Hinzu kommt, dass die Kreativität möglicherweise durch Involvierung der fachlichen Domänenexperten behindert wird.

Einerseits kennen Prozessbeteiligte oft Schwachstellen existierender Prozesse und Lösungsvorschläge dafür. Andererseits sind sie eventuell ,betriebsblind“ und in der Betrachtung des Problem- und insbesondere des Lösungsraums zu eingeschränkt. Bei der Rekrutierung sollten demnach nicht nur Diversitätsaspekte wie Geschlecht, Alter und kultureller Hintergrund eine Rolle spielen. Vielmehr ist auf eine sinnvolle Balance zwischen Domänenexperten und Teammitgliedern zu achten, die themenfremde fachliche Hintergründe aufweisen und kein ausgeprägtes Eigeninteresse am Aussehen einer Lösung haben.

Die Schwerpunktverschiebung kann man davon abhängig machen, ob das Ziel eher eine Prozessverbesserung ist, bei der Erfahrungen und Inputs der mit dem bestehenden Prozess vertrauten Personen hilfreich sein können. Steht dagegen eine radikalere Prozessinnovation im Vordergrund, könnten diese möglicherweise eher limitierend wirken. Selbstverständlich sollten auch bei der ursprünglichen Zielsetzung der Verbesserung Ideen für eine fundamentale Innovation des betrachteten Prozesses nicht außer Acht gelassen werden.

\footnotetext{
${ }^{2}$ Bei der Betrachtung über Unternehmensgrenzen hinweg könnte man noch Partner im Wertschöpfungsnetzwerk ergänzen wie Lieferanten, Kunden oder Logistikdienstleister (Network Partners) und sinngemäß von NetBizDevOps sprechen.
} 


\section{Literatur}

1. Allweyer (2009), Geschäftsprozessmanagement: Strategie, Entwurf, Implementierung, Controlling, W3L, Herdecke.

2. Schmelzer H, Sesselmann W (2013) Geschäftsprozessmanagement in der Praxis - Kunden zufriedenstellen, Produktivität steigern, Wert erhöhen. 8. Auflage, München: Carl Hanser Verlag, S. $265 \mathrm{ff}$.

3. Schallmo (2017): Design Thinking erfolgreich anwenden. Springer Gabler.

4. Brenner, W. et al. (2016): Design Thinking as Mindset, Process and Toolbox, In: Design Thinking for Innovation, Brenner, W. \& Uebernickel (eds.) (pp. 3-21).

5. Schmiedgen, J., Rhinow, H., Köppen, E., Meinel, C. (2015): Parts without a whole?: The current state of Design Thinking practice in organizations, Technische Berichte des Hasso-Plattner-Instituts für Softwaresystemtechnik an der Universität Potsdam Nr. 97, Univ.-Verl., Potsdam, S. 47.

6. Leifer, L. (2012): Über Design Thinking, Bad Guys, Experimente, Jagd und organisationalen Wandel. In: OrganisationsEntwicklung, Nr. 2, 2012, (pp. 8-13, hier S. 13).

7. Uebernickel, F., Brenner, W., Pukall, B., Naef, T., Schindlholzer, B., (2015): Design Thinking Das Handbuch, Frankfurter Allgemeine Buch, Frankfurt am Main.

8. Lewrick, M., Link, P., Leifer, L. (Hrsg.), (2017): Das Design Thinking Playbook - Das Handbuch, Vahlen, München.

9. https://dschool.stanford.edu/resources/the-bootcamp-bootleg. Letzter Zugriff am 05.01.2018.

10. Doorley, S., Witthoft, S. (2012), Make Space: How to Set the Stage for Creative Collaboration, John Wiley \& Sons, Hoboken.

11. Fleischmann, A., Schmidt, W., Stary, C. (2013): Subject-oriented BPM = Socially Executable BPM, Proceedings of the 15th IEEE Conference on Business Informatics (CBI 2013), Vienna, IEEE Computer Society, pp. 399-406.

12. Fleischmann, A., Borgert, S., Elstermann, M., Krenn, F., Singer, R. (2017): An Overview of S-BPM-oriented Tool Suites, Proceedings of the $9^{\text {th }}$ S-BPM ONE, Darmstadt.

Open Access Dieses Kapitel wird unter der Creative Commons Namensnennung - Nicht kommerziell - Keine Bearbeitung 4.0 International Lizenz (http://creativecommons.org/licenses/ by-nc-nd/4.0/deed.de) veröffentlicht, welche die nicht-kommerzielle Nutzung, Vervielfältigung, Verbreitung und Wiedergabe in jeglichem Medium und Format erlaubt, sofern Sie den/die ursprünglichen Autor(en) und die Quelle ordnungsgemäß nennen, einen Link zur Creative Commons Lizenz beifügen und angeben, ob Änderungen vorgenommen wurden. Die Lizenz gibt Ihnen nicht das Recht, bearbeitete oder sonst wie umgestaltete Fassungen dieses Werkes zu verbreiten oder öffentlich wiederzugeben.

Die in diesem Kapitel enthaltenen Bilder und sonstiges Drittmaterial unterliegen ebenfalls der genannten Creative Commons Lizenz, sofern sich aus der Abbildungslegende nichts anderes ergibt. Sofern das betreffende Material nicht unter der genannten Creative Commons Lizenz steht und die betreffende Handlung nicht nach gesetzlichen Vorschriften erlaubt ist, ist auch für die oben aufgeführten nicht-kommerziellen Weiterverwendungen des Materials die Einwilligung des jeweiligen Rechteinhabers einzuholen.

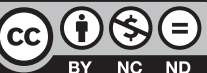




\section{Vorbereitung der Prozessimplementierung}

Ziel der Prozessvorbereitung im Sinne einer nachfolgenden Implementierung ist eine präzise Spezifikation von Prozessen mit der Beschreibung der Prozessstrategie und der Prozesslogik. Die Vorbereitung umfasst die Aktivitätsbündel auf der rechten Seite des offenen Zyklus, dies sind die Analyse, die Modellierung, die Validierung und die Optimierung (siehe Abb. 5.1). Das Ergebnis dieser Aktivitätsbündel ist eine Prozessbeschreibung, die präzise genug für die Umsetzung ist. Die Vorbereitung teilt sich in die Aktivitäten Analyse mit Modellierung, Validierung und Optimierung auf. Diese Aktivitäten werden nicht in einer strengen Reihenfolge durchgeführt, sondern die jeweiligen Tätigkeitsschwerpunkte können zwischen den Aktivitäten häufig wechseln. Die Abbildung zeigt die Einordnung der Vorbereitung in das gesamte Prozessmanagementmodell.

In den folgenden Abschnitten werden ausgewählte Methoden für diese Aktivitätsbündel vorgestellt.

\subsection{Analyse und Modellierung}

Analyse und Modellbildung kann nicht scharf getrennt werden. Schwerpunkt der Analyse sind die strategischen Aspekte von Prozessen, während in der Modellierung auf die Prozesslogik fokussiert wird. In der Analyse wird also der Anfang, mit dem zugehörigen Input, das Ende mit dem erzeugten Output und das damit befriedigte Kundenbedürfnis klargelegt. In der Analyse werden auch der Rahmen und die wesentlichen Aspekte der Prozesslogik abgesteckt.

In der Praxis wird allerdings bei der Überarbeitung von Prozessen in dieser Phase kaum die Prozesslogik des Ist-Zustands explizit beschrieben. Die Analyse der aktuellen Prozesslogik erfolgt im Rahmen der Definition des gewünschten Sollprozesses. Der ausschließliche Bezug zur Ist-Situation macht in der Regel wenig Sinn und ist auch für alle 
Abb. 5.1 Einordnung der Vorbereitung in das Prozessmanagementmodell

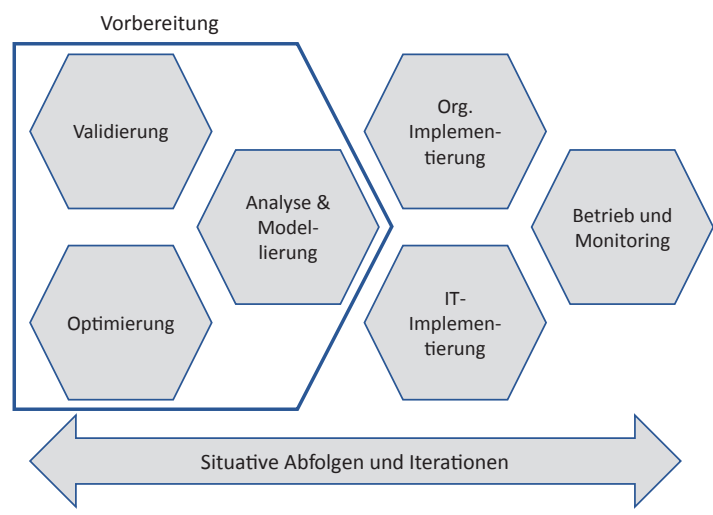

Beteiligten zumeist unangenehm zu dokumentieren - Was wurde in letzter Zeit „falsch“ gemacht?

Solange man das Werkzeug, natürliche Sprache" benutzt, befindet man sich eher in der Aktivität Analyse als Modellierung. Der Übergang von der natürlichen Sprache $\mathrm{zu}$ einer formaleren Prozessmodellierungssprache entspricht dem Übergang in Modellierungsaktivitäten. Der Modellierung kann eine mehr oder weniger intensive Analysemethode vorausgehen. In Extremfällen wird unmittelbar ein Prozessmodell ohne vorangehende natürlich sprachliche Analyse erstellt. Allerdings ist zu empfehlen, dass zumindest die strategischen Aspekte des betrachteten Prozesses bekannt und definiert sind.

In der Folge werden Richtlinien zur Artikulation und Abstimmung von prozessrelevantem Wissen vorgestellt, welche methodisch und werkzeugtechnisch unterstützt werden können. Ein wesentliches Element stellt dabei das Verständnis von Rollen dar, welche die Beteiligten als relevant für die Abwicklung von Prozessen erachten. Darüber hinaus empfiehlt sich, die Austauchbeziehungen zwischen Akteuren zu betrachten, zur weiteren Gestaltung von Prozessen deren Qualität zu bewerten und daraus gegebenenfalls Änderungspotenzial abzuleiten.

\subsubsection{Allgemeines zu Artikulation und Abstimmung}

Wissen über Arbeitsabläufe und organisationale Prozesse ruht in den meisten Fällen in den Köpfen von Handlungsträgern. Daher kommt einer kontextsensitiven strukturierten Erhebung und Analyse entscheidende Bedeutung zu. Die Erhebung dient der Artikulation von Erfahrungswissen und wird in den meisten Fällen im Rahmen der Modellierung durchgeführt. Wird sie allerdings bereits vorab bearbeitet, dann kann in GPM-Projekten mit der Vielfalt von Ansätzen zur Aufgaben- oder Problembewältigung strukturierter umgegangen werden. Allerdings spielen in diesem Zusammenhang die Abstimmung 
und der Abgleich unterschiedlicher Ansätze eine wesentliche Rolle. Deren Unterstützung trägt wesentlich $\mathrm{zu}$ einer integrationsfähigen Lösungsbildung trotz hoher Vielfalt und individueller Zugänge zur Aufgabenerfüllung bei. In diesem Abschnitt wird daher auf die Erhebungs- und Aushandlungsmethoden sowie Instrumente eingegangen, welche Individuen Artikulation im Rahmen kollektiver Reflexions- und Aushandlungsprozesse ermöglicht.

Wie Menschen ihre Arbeit durchführen, wie sie auf Vorgaben oder Abweichungen reagieren und wie sie mit anderen kooperieren, wird wesentlich von ihrer Wahrnehmung der organisationalen Realität geprägt. Die Interpretation der wahrgenommenen Rahmenbedingungen sowie die Ableitung der als adäquat erachteten Reaktion von Akteuren kann mit der kognitionswissenschaftlichen Theorie der mentalen Modelle erklärt werden. Diese Theorie kann auch als Grundlage verwendet werden, um von den operativ tätigen Personen ausgehende Lern- und Veränderungsprozesse in Organisationen zu erklären. In diesem Abschnitt bildet sie deshalb die Grundlage für die Ableitung von Maßnahmen, die Arbeitende in die Lage versetzen sollen, sich ihrer Arbeitsabläufe und der diese prägenden organisationalen Zusammenhänge und Rahmenbedingungen bewusst zu werden.

Das Konzept der ,,mentalen Modelle“ wird verwendet, um zu erklären ,wie Menschen die Welt verstehen - genauer: wie sie ihr Wissen benutzen, um sich bestimmte Phänomene der Welt subjektiv plausibel zu machen [1]“. Mentale Modelle sind dabei Erklärungsmodelle der Welt, die von Menschen auf Basis von Alltagserfahrungen, bisherigem Wissen und darauf basierenden Schlussfolgerungen gebildet werden. Ein mentales Modell wird vom jeweiligen Individuum als Basis verwendet, um die Welt zu verstehen und gegebenenfalls Vorhersagen über deren Verhalten zu bilden [1].

Das mentale Modelle prägende Wissen kann auf Alltagserfahrung basieren oder durch Vermittlung oder Instruktion begründet werden. Die Modifikation und Erweiterung der eigenen Wissensbasen und die (Weiter-) Entwicklung der kognitiven Fähigkeiten, die für die Ableitung von Schlussfolgerungen notwendig sind, bezeichnet Seel als „Lernen“. Lernen ist ,mit der Verarbeitung individueller Erfahrungen mit sowie vermittelter Information über die Welt, ihre Struktur und Evidenz verbunden und kann als ein Prozess permanenter konzeptueller Veränderungen verstanden werden."[1] Lernen setzt damit die Fähigkeit und Bereitschaft voraus, ,,vermittelte Weltauffassungen zu verstehen, zu akzeptieren und sodann den eigenen gedanklichen Konstruktionen zugrunde zu legen"[1].

Die Veränderung mentaler Modelle über Arbeitsprozesse weist zwei grundlegende Schwierigkeiten auf. Bei bereits als nicht adäquat erkannten mentalen Modellen besteht grundsätzlich die Bereitschaft zur Veränderung (im Sinne einer Anpassung des mentalen Modells an die als verändert wahrgenommene Umweltbedingungen), die Herausforderung besteht allerdings darin, an die notwendige Information $\mathrm{zu}$ gelangen und adäquat dargeboten zu bekommen. Eine weitere Schwierigkeit ergibt sich in Situationen, in denen nicht alle involvierten Individuen die Situation als ,problematisch“ wahrnehmen und deshalb keine grundlegende Bereitschaft zeigen, ihre der Arbeit zugrunde liegenden Annahmen (also ihre mentalen Modelle) zu verändern. Dies tritt vor allem in Situationen auf, in denen die kollaborative Reflexion nicht aus einer allgemein 
wahrgenommenen Problemsituation heraus durchgeführt, sondern entweder mit rein planendem Charakter angestoßen wird, oder welche nur von einzelnen beteiligten Individuen als ,problematisch“ wahrgenommen werden.

Diesen Problemen kann mit einer expliziten Unterstützung des Reflexionsprozesses begegnet werden. Eine derartige Unterstützung muss sicherstellen, dass Artefakte zur Repräsentation der individuellen mentalen Modelle geschaffen werden, die dann die Grundlage für die gegenseitige Verständlichmachung der jeweiligen Sichten auf den Arbeitsprozess bedienen kann. Derartige Artefakte können dazu dienen, Aspekte eines Arbeitsprozesses abzustimmen und sicherzustellen, dass die in Artefakten kodierte ostensive Sicht auf einen Arbeitsprozess durch darauf aufbauendes performatives subjektives Handlungswissen in der Arbeitspraxis umgesetzt werden kann. Aus methodischer Sicht muss dazu sichergestellt werden, dass alle am realen Arbeitsprozess beteiligten Personen organisatorisch und methodisch in der Lage sind, sich am kollaborativen Lernprozess zu beteiligen. Dies bedingt vor allem, dass sie die verwendeten Ausdrucksformen verstehen und aktiv einsetzen können. Dies ist wiederum eine Lernherausforderung, die explizit adressiert werden muss.

Eine in den Bildungswissenschaften weithin akzeptierte Möglichkeit zur Externalisierung und Abstimmung mentaler Modelle ist die Bildung konzeptioneller Modelle. Gleichzeitig können derartige Modelle die Grundlage für die Spezifikation von Arbeitsprozessen und die Konfiguration von Arbeitsunterstützungssystemen bilden, sofern sie sich einer formal spezifizierten Semantik bedienen (wie etwa die BPMN oder S-BPM). Konzeptionelle Modelle stellen also ein Mittel dar, Arbeitende in die Lage zu versetzen, ihre Arbeit zu reflektieren, abzustimmen, die Ergebnisse dieser Abstimmungsprozesse für die Dritte zugänglich zu machen und diese im Rahmen der existierenden Systemgrenzen zur Unterstützung der eigenen Arbeitsabläufe nutzbar zu machen.

Modelle sind Abbildungen der Realität, die zu einem bestimmten Zweck gebildet werden. Modelle repräsentieren nie das reale Phänomen als Ganzes, sondern enthalten nur jene Aspekte der Realität, die vom Modellbildenden als relevant für die jeweilige Zielerreichung erachtet werden. Für die Modellbildung stellt sich damit die Frage nach der Definitionsmacht dieser Modelle und der durch sie abgebildeten sozialen Realität. Sofern ein Modell nicht nur einen das modellbildende Individuum betreffenden Zweck erfüllt, sondern von anderen Personen genutzt wird, beeinflusst das Modell die mentalen Modelle dieser Personen und damit auch deren Verhalten.

Die aktive Involvierung operativ Tätiger in die Spezifikation von Arbeitsprozessen stellt deshalb eine Möglichkeit für deren selbstermächtigte Gestaltung ihrer Arbeit dar. Dazu ist es jedoch notwendig, dass operativ Tätige in die Lage versetzt werden, derartige Modelle zu verstehen, selbst zu gestalten, und deren Wirkung auf ihre Arbeitsprozesse abschätzen zu können. In aktuellen Ansätzen wird hingegen nach wie vor von der Notwendigkeit eines Prozess-Analysten ausgegangen, der die Sichtweisen der Arbeitenden in ein Prozessmodell übersetzt. Dies kann zu Abweichungen zwischen dem realen Arbeitsprozess und dessen Modellrepräsentation führen. Außerdem nimmt diese Vorgehensweise den operativ tätigen Personen die Möglichkeit im Sinne des 
modellbasierten Lernens ihre mentalen Modelle zu schärfen und mit jenen der anderen Beteiligten abzustimmen.

Um operativ Tätige in die Lage zu versetzen, derartige Modelle zu verstehen, muss das Erlernen von grundlegenden Ansätzen zur Erstellung und Interpretation konzeptioneller Modelle Gegenstand der Aus- oder Weiterbildung sein. Arbeitende müssen jene Modelle erkennen können, die den Systemen zugrunde liegen, in die sie eingebettet sind. Darüber hinaus sollen sie die Implikationen von externen oder selbst durchgeführten Änderungen an diesen Modellen abschätzen und Interventionen dementsprechend planen können.

Dazu müssen folgende Punkte methodisch unterstützt werden:

- die Ermöglichung der individuellen Artikulation eigener mentaler Modelle über Arbeit, um eine individuelle Reflexion zu ermöglichen und dadurch Lücken und Inkonsistenzen individuell wahrnehmbar zu machen, sowie zu verhindern, dass Sichtweisen einzelner Personen nicht berücksichtigt werden und diese in der Folge keinen Bezug zu ihrer Arbeitsrealität herstellen können

- die Unterstützung der Vereinbarung eines gemeinsamen Vokabulars, um unterschiedliche Verständnisse von Begriffen zu identifizieren und sich in der Folge eindeutig über die gegenständliche Arbeit austauschen zu können, und zu verhindern, dass das gleiche reale Phänomen mit unterschiedlichen Begriffen bezeichnet wird - oder dass umgekehrt der gleiche Begriff für unterschiedliche reale Phänomene verwendet wird

- die Unterstützung der Entwicklung eines gemeinsamen Verständnisses über die kollaborative Arbeit, um eine Grundlage für die Reflexion der individuellen mentalen Modelle anzubieten, und damit in Verbindung stehend,

- eine Unterstützung bei der Identifikation und Auflösung von konfliktionierenden Sichtweisen, um Unterschiede in jenen mentalen Modellen, die die Kollaboration zwischen Akteuren unmittelbar betreffen, sichtbar zu machen und deren Abstimmung zu ermöglichen.

In den folgenden Abschnitten werden einige Methoden gezeigt, mit denen diese Anforderungen umgesetzt wurden.

\subsubsection{Compare/WP}

Die oben beschriebenen Anforderungen werden in der Methode „CoMPArE/WP“ exemplarisch umgesetzt. CoMPArE/WP steht für „Collaborative Multi-perspective Articulation and Elicitation of Work Processes“. Bei der Anwendung von CoMPArE/WP wird anhand der Reflexion über einen realen kollaborativen Arbeitsprozess Bewusstsein über die Zusammenarbeit in einem konkreten Einzelfall geschaffen. Aufgrund ihrer Verankerung an konkreten Arbeitsprozessen eignet sich die Methode auch als Mittel zur Organisationsentwicklung. Die Kooperationsform der Methode wird grundsätzlich durch 
deren Durchführung mit einer Kartenlegetechnik festgelegt. Die teilnehmenden operativ Tätigen sind die wesentlichen Akteure und führen die Komponenten eigenverantwortlich durch, wobei Artikulations- und Nachfrage-Rollen wechseln können. Die konkrete Ausgestaltung der Kooperation ist in den Komponenten unterschiedlich und deshalb in der dort angeführten Prozedur beschrieben. Zur Unterstützung der Durchführung steht ein Facilitator bereit, der jedoch nicht inhaltlich interveniert.

Die Methode soll bei der Artikulation und Abstimmung von mentalen Modellen über Arbeit unterstützen und gleichzeitig grundlegende Fähigkeiten zu deren Ausdruck in konzeptionellen Modellen vermitteln. Die Kombination diese beiden Teilziele hat Auswirkungen auf das Rahmenwerk der Methode. Aus Sicht der Vermittlung von Modellierungskompetenz ist es sinnvoll, die notwendigen Fähigkeiten schrittweise mit steigender Komplexität einzuführen. Aus Sicht der Artikulationsunterstützung ist ein Vorgehen in drei Komponenten argumentierbar. Die Abb. 5.2 gibt eine Übersicht über diese drei Komponenten.

Komponente 1 dient der Findung eines gemeinsamen Verständnisses darüber, wo und wie der abzustimmende Arbeitsprozess beginnt und endet, sowie der Findung eines gemeinsamen Vokabulars. Komponente 2 dient der Artikulation und Reflexionen des jeweiligen individuellen Arbeitsbeitrages. Alle Teilnehmer erstellen hier individuell und ohne Interaktion mit anderen ein strukturiertes Modell ihrer Sichtweise auf ihren jeweiligen Arbeitsbeitrag. Durch die einheitlich strukturierte Darstellung der individuellen Beiträge ist in Komponente 3 eine kollaborative Abstimmung derselben möglich. Durch diese Abstimmung sollen konfliktionierende Sichtweisen aufgedeckt werden eine gemeinsame Sicht auf den gesamten Arbeitsprozess entstehen.
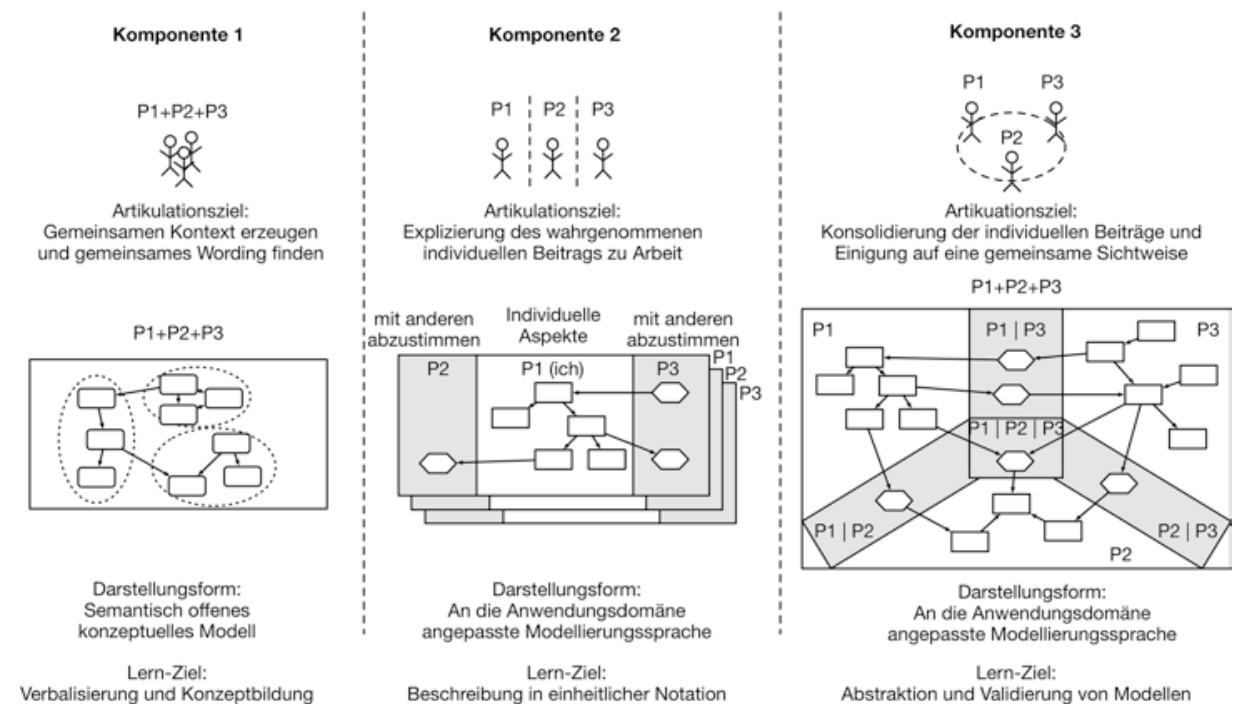

Abb. 5.2 Drei Komponenten von CoMPArE/WP 
In diesen Komponenten sind die Ziele der Kompetenzentwicklung im Modellierungsbereich zu verankern. In Komponente 1 ist die Verbalisierung von mentalen Modellen und die darauf aufbauende Konzeptbildung zu vermitteln. In Komponente 2 muss das Beschreiben der verbalisierten Inhalte mittels eines vorgegebenen Kategorienschemas und einer Notation vermittelt werden. Dabei muss festgelegt werden, welche Elemente des Kategorienschemas in der Verantwortlichkeit des artikulierenden Individuums stehen und welche als Verhandlungsgegenstände bei der Abstimmung in Komponente 3 validiert und gegebenenfalls abstrahiert werden müssen bzw. zur Disposition stehen.

Konkrete Umsetzung von Komponente 1. Nicht alle Teilnehmer haben notwendigerweise ein gemeinsames Verständnis über die Konzepte, mit denen sie ihre Arbeit beschreiben. Um die vorhandenen mentalen Modelle so weit abzustimmen, dass ein gemeinsames Vokabular eine Zusammenarbeit ermöglicht, kann kollaboratives Concept Mapping eingesetzt werden. Zusätzlich kann ebenfalls nicht davon ausgegangen werden, dass ein gemeinsames Verständnis über die Grenzen des abzustimmenden Arbeitsprozesses gegeben ist. Auch hier kann Concept Mapping zur Klärung beitragen. Neben der inhaltlichen Dimension ermöglichen Concept Maps einen niederschwelligen Einstieg in die Welt der konzeptuellen Modellierung, da sie die Bedeutung von Modellelementen nicht vorgeben, sondern diese während der Modellierung durch die beteiligten Personen festlegen lassen. Dies erleichtert die Abbildung der individuellen mentalen Modelle in die explizite Repräsentation und vermeidet die Notwendigkeit, neben der Abstimmung mit den anderen beteiligten Personen auch noch eine Übersetzung auf ein Modell mit formal definierte Semantik durchführen zu müssen.

Im Rahmen dieser Komponente werden die Teilnehmer aufgefordert, alle relevanten Aspekte der Arbeitsumgebung zu beschreiben, in die der zu reflektierende Arbeitsprozess eingebettet ist. Dies erfolgt, indem jeder Aspekt individuell einzeln auf einer Karte notiert wird. Bei der Zusammenführung der individuell gesammelten Aspekte werden die Karten reihum einzeln auf einer gemeinsamen Arbeitsfläche angeordnet. Die Aspekte können zueinander in Beziehung gesetzt werden. Karten mit unterschiedlichen Begriffen für den gleichen Aspekt werden überlappend angeordnet. Hierarchische oder kausale Beziehungen zwischen Aspekten können durch das explizite Zeichen von Verbindungen, aber auch durch die räumliche Anordnung der Karten dargestellt werden.

Das Beispiel in der folgenden Abb. 5.3, das in diesem Abschnitt durchgängig zur Erläuterung der Methode herangezogen wird, zeigt eine Concept Map mit relevanten Aspekten für die Beantragung eines Urlaubs in einem Unternehmen. Die Aspekte wurden durch räumliche Anordnung zueinander in Beziehung gesetzt. Die überlappenden Elemente zeigen Aspekte, die von mehreren Teilnehmern genannt und mit unterschiedlichen Begriffen bezeichnet werden.

Konkrete Umsetzung der Komponenten 2 und 3. Die Komponenten 2 und 3 fokussieren auf die Artikulation und Abstimmung des wahrgenommenen Ablaufs eines Arbeitsprozesses und der darin ablaufenden Interaktion. Die Modellbildung in Komponente 2 erfolgt dabei durch alle beteiligten Personen individuell, ohne Interaktion mit Anderen. So werden Überlagerungseffekte vermieden und unterschiedliche Sichtweisen 
Abb. 5.3 Concept Map zum Thema Beantragung eines Urlaubs

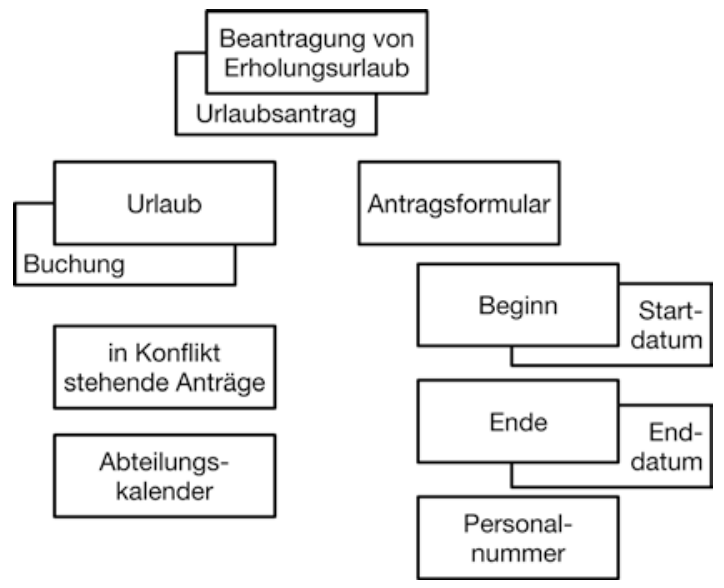

für die nächste Komponente explizit aufgedeckt. Die beteiligten Personen beschreiben dabei, welche Arbeitsschritte sie aus ihrer Sicht zur Erreichung des Arbeitsziels beitragen, mit wem sie interagieren und in welcher Form diese Interaktion erfolgt.

Die Aushandlung einer gemeinsamen Sichtweise in Komponente 3 und die damit einhergehende Erstellung eines gemeinsamen Models erfolgt wiederum mittels einer strukturierten Vorgehensweise, die komplexere Modellierungsaufgaben heranführen soll und eine einheitlich aufbereitete Modell-Repräsentation gewährleistet. Dabei werden die zuvor erstellten Modelle weiterverwendet. Das Strukturierungsschema trennt jene Modellaspekte, die in der Verantwortung der jeweiligen Arbeitenden bleiben, von jenen, die Gegenstand der Aushandlung sind.

Zur Darstellung der Arbeitsprozesse wird in diesem Schritt eine strukturierte Darstellungsform mit vorab spezifizierter Semantik verwendet. Diese ist an den gängigen Kategorie-Schemata zur Beschreibung kollaborativer Arbeitsprozesse orientiert (siehe Abb. 5.4). Zum Einsatz kommen dabei die Kategorien WER, WAS und AUSTAUSCH (M3). WER (blau in der Abbildung) bezieht sich auf die Akteure im Arbeitsprozess, WAS (rot in der Abbildung) wird verwendet, um aktive Beiträge im Rahmen des
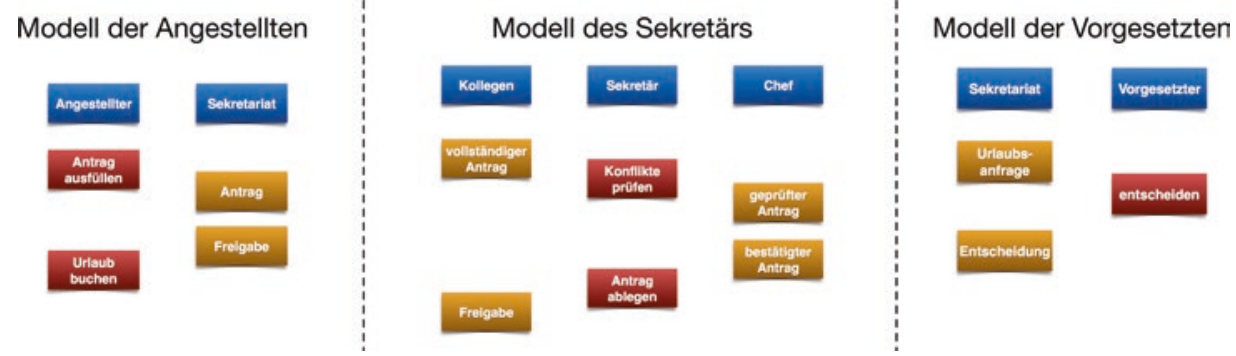

Abb. 5.4 Individuelle Modelle in der vorgestellten Notation 
Arbeitsprozesses zu beschreiben. AUSTAUSCH (gelb in der Abbildung) wird im Kontext kollaborativer Arbeitsprozesse verwendet, um die Weitergabe oder den Austausch von Informationen oder Material zwischen Akteuren im Rahmen der eigenen Tätigkeiten zu charakterisieren. Im Sinne der einfacheren Verwendbarkeit werden diese Kategorien nicht exakt spezifiziert und lassen bewusst Interpretationsspielraum im konkreten Einsatz - so kann ein WER Element etwa eine konkrete Person, eine Rolle, eine Abteilung oder eine gesamte Organisation darstellen. WAS-Elemente verbleiben in der Verantwortung der einzelnen Teilnehmer, WER und AUSTAUSCH-Elemente sind in Komponente 3 Gegenstand der Abstimmung und sollten zu einem gemeinsamen Verständnis hin entwickelt werden.

Individuelle Artikulation In Komponente 2 beschreiben die beteiligten Personen individuell mithilfe der Elemente, WAS sie im Arbeitsprozess beitragen, WER mit ihnen interagiert, und in welcher Form dieser AUSTAUSCH stattfindet. Zur Unterstützung des Artikulationsprozesses wurde ein Strukturierungsschema entwickelt, dass die erstellten Modelle in einer einheitlichen Form aufbereitet und deren Zusammenführung im nächsten Schritt ermöglicht. Das Strukturierungsschema gibt - wie in der obenstehenden Abbildung ersichtlich - die räumliche Anordnung der Modellelemente vor.

Operativ Tätige repräsentieren sich selbst durch ein WER-Element, unter dem die wahrgenommenen Beiträge zum Arbeitsprozess als sequenziell angeordnete WASElemente platziert werden. Für alle anderen Akteure, mit denen eine Interaktion wahrgenommen wird, wir ein weiteres WER-Element platziert, unter dem jeweils die Interaktion durch AUSTAUSCH-Elemente näher spezifiziert wird. Deren vertikale Positionierungen bestimmen, ob eine eingehende Ressource erwartet wird (Platzierung oberhalb des davon abhängigen WAS-Elements), oder ob diese zur Verfügung gestellt wird (Platzierung oberhalb des erzeugenden WAS-Elements).

Die obenstehende Abbildung zeigt drei individuelle Modelle $\mathrm{zu}$ dem oben beschriebenen Beispiel-Prozess, die entsprechend diesem Strukturierungsschema erstellt wurden. Im Beispiel ist ersichtlich, dass es an dieser Stelle zu inhaltlich divergenten Repräsentationen vor allem im Bereich der AUSTAUSCH-Elemente kommen kann (vgl. „Antrag“ vs. „vollständiger Antrag“ in der obenstehenden Abbildung). Diese Divergenzen werden in Komponente 3 explizit sichtbar und sind dann Gegenstand der Aushandlung einer gemeinsamen Sichtweise.

Kollaborative Abstimmung Grundlage der kollaborativen Abstimmung sind die in Komponente 2 erstellten individuellen konzeptionellen Modelle. Die folgende Abb. 5.5 zeigt exemplarisch einen Abstimmungsprozess für zwei der im Beispiel repräsentierten Akteure. Die gemeinsame Modellbildung erfolgt wiederum auf einer gemeinsamen Arbeitsoberfläche (siehe folgende Abbildung Mitte). Jener Teilnehmende, welche auch den realen Arbeitsprozess auslöst, beginnt mit der Beschreibung der eigenen Beiträge zum Arbeitsprozess und fügt der Oberfläche die entsprechenden Modelle-Elemente hinzu (Schritte 1-2 in der folgenden Abbildung). 


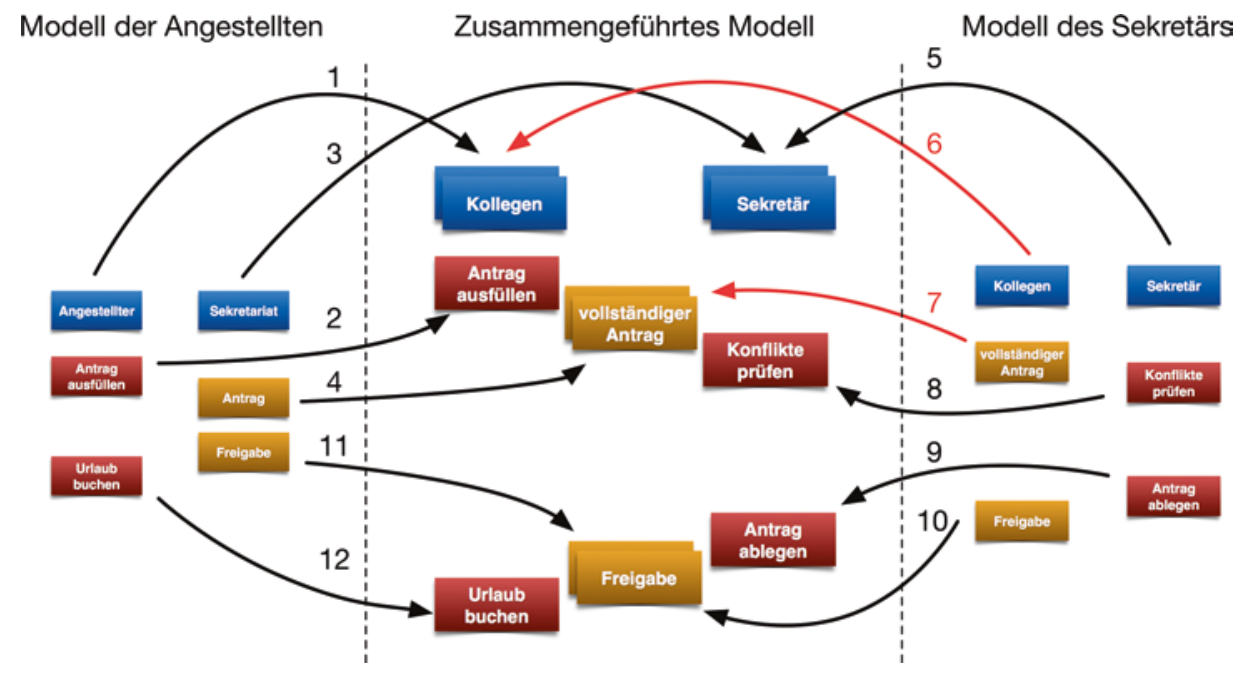

Abb. 5.5 Kollaborative Abstimmung

Die übrigen Teilnehmenden intervenieren hier nur nachfragend zur Vermeidung von Missverständnissen oder zur Offenlegung von Unklarheiten. Eine aktive Beteiligung der anderen erfolgt, sobald das erste AUSTAUSCH Element zum Einsatz kommt (Schritte 3-4). Sofern eine grundlegend gemeinsame Sichtweise auf den Arbeitsprozess existiert, sollte an dieser Stelle einer der Teilnehmenden ein entsprechend zuzuordnendes AUSTAUSCH-Element einbringen können (Schritte 5-7).

Ist dies der Fall, so wird der Beschreibungsprozess durch diese Person fortgesetzt (ab Schritt 8). Bei einer grundsätzlichen Passung, die sich jedoch in der Bezeichnung des Elementes - etwa durch unterschiedliche Abstraktionsebenen - unterscheidet, muss diese mehrfache Bezeichnung aufgelöst werden oder die semantische Äquivalenz der beiden Elemente durch überlappendes Anordnen dargestellt werden (z. B. Schritt 7). Falls kein zuzuordnendes Element vorhanden ist, wird eine grundsätzlich divergierende Repräsentation sichtbar. Diese kann auf mangelndes Relevanzbewusstsein eines Austausches zurückzuführen sein. Dies bedeutet, dass dem angesprochenen Teilnehmenden die Interaktion zwar bewusst war, aber im Kontext des Arbeitsprozesses als nicht relevant erschien. Falls eine wahrgenommene Interaktionserfordernis eines Teilnehmenden nicht erwidert wird, muss es jedoch zu tiefer gehenden Aushandlungsprozessen kommen.

Der initiale Abstimmungsprozess endet, sobald alle Teilnehmenden ihre individuellen Modelle erläutert und zum gemeinsamen Modell hinzugefügt haben. Dieser Externalisierungs-Phase folgt eine kollaborative Reflexionsphase, im Rahmen derer der Arbeitsprozess anhand des gemeinsamen Modells durchgegangen und hinsichtlich seiner Passung auf die individuellen Sichtweisen der Beteiligten diskutiert wird. Etwaige notwendige Modifikationen werden an dieser Stelle nach einer Konsensbildung der jeweils Betroffenen durchgeführt. 
Das Ergebnis der Anwendung der Methode stellt nun eine konsensuale Repräsentation des kollaborativen Arbeitsprozesses dar. Aufgrund der eingeschränkten Ausdrucksstärke der eingesetzten Modellierungssprache ist an dieser Stelle die Abbildung von ansonsten in Prozessbeschreibungen üblichen - Arbeitsvarianten oder Entscheidungen nicht möglich. Die Entscheidung für eine semantisch auf wenige Elemente beschränkte Modellierungssprache fiel aufgrund didaktischer Kriterien, da empirische Belege zeigen, dass unerfahrene Modellierende ihre Sichtweisen auf einen Arbeitsprozess initial einfacher narrativ anhand eines konkreten Falles beschreiben können.

Entscheidungen hinsichtlich der konkreten Umsetzung des Arbeitsprozesses sind bei der fallbasierten Beschreibung bereits getroffen. Damit ist eine explizite Repräsentation derselben im Rahmen der Modellierung nicht notwendig. Eine vollständige Beschreibung des Arbeitsprozesses bedingt somit eine mehrfache Durchführung der Methode oder deren Erweiterung um weitere Verfeinerungsschritte, die aber an dieser Stelle nicht näher betrachtet werden sollen.

Im Sinne der Bildung von Modellierungskompetenz liegt der Fokus in Komponente 1 auf der Hinführung zur für die Modellbildung notwendige Abstraktion und Konzeptualisierung von Wahrnehmungen der realen Welt. In Komponente 2 erfolgt die durch Strukturhilfen angeleitete Darstellung und Reflexion der eigenen Arbeitswahrnehmung in konzeptionellen Modellen und deren Beschreibung mittels vorgegebener Strukturelemente. Komponente 3 fokussiert in der Folge auf Modell-Verständnis (der anderen individuellen Modelle), -Interpretation (hinsichtlich deren Auswirkungen auf das eigene Modell) und -Aushandlung (der gemeinsam vertretbaren Sicht) von Modellinhalten, wodurch letztendlich die Kompetenz zur selbstermächtigenden Beeinflussung von Arbeitsprozessen vermittelt wird.

Zur Auseinandersetzung mit der Wirkung einer konkreten Anwendung der Methode muss der Begriff der „,Selbstermächtigung“ gefasst werden. „Selbstermächtigung“ kann nach Liebert (2015) folgendermaßen verstanden werden: ,[Der] Begriff der Selbstermächtigung [...] fordert [...] dazu auf, sein Schicksal in die eigene Hand zu nehmen, seine individuellen Ansprüche nicht nur zu formulieren, sondern auch - aktiv - durch ,Selbsttätigkeit‘ umzusetzen -, auch wenn dies bedeutet, gegen die eingespielten Regeln und etablierten Strukturen der institutionellen Ordnungen zu verstoßen.“

Die Methode soll nun zur „Selbstermächtigung“ im Sinne dieser Definition führen. Der Anspruch, Arbeitende in die Lage zu versetzen, ,individuelle Ansprüche [...] zu formulieren“, ist das grundlegende Gestaltungsziel der vorgeschlagenen Methode. Die Zielsetzung, dazu zu befähigen, ,diese [Ansprüche] auch - aktiv - durch Selbsttätigkeit umzusetzen“ wird im Kontext durch Arbeitsbeeinflussungssystem gesteuerter Arbeitsprozesse insofern entsprochen, als dass operativ Tätige dazu befähigt werden, die konzeptionelle Modellierung von Arbeitsprozessen als jene „Sprache“, die zu Konfiguration existierender Arbeitsbeeinflussungssystemen verwendet wird, nicht nur zu verstehen, sondern auch selbst zum Einsatz zu bringen. Dies soll dazu führen, dass diese sozio-technischen Systeme - wie zu Beginn argumentiert - als Arbeitsunterstützungssysteme einsetzen zu können, die den Arbeitsprozess und die zur Zielerreichung 
notwendige Kollaboration für die handelnden Individuen erleichtern können. Die Befähigung, diese „Umwidmung“ vorantreiben zu können, bildet die Grundlage dafür „die institutionelle Ordnung [...] infrage zu stellen und in letzter Konsequenz durch neue Formen der ,freien' Selbstorganisation zu überwinden.“

Die Akzeptanz existierender organisationaler und technischer Rahmenbedingungen und der Unabdingbarkeit ihres weiteren Einsatzes, die - wie in der Einleitung dargestellt - einen Ausgangspunkt dieser Methode bildet, stellt gleichzeitig die größte Herausforderung bei der Ermöglichung von Selbstermächtigung im organisationalen Kontext dar. Die Verwendung dieser Unterstützungssysteme ermöglicht nicht nur eine Fremdsteuerung, sondern auch eine Selbstdisziplinierung im Sinne der Erreichung vorgegebener Ziele, die mit der Verfügbarkeit technischer Unterstützung bei Planung und (Selbst- wie Fremd-)Kontrolle sogar erleichtert wird.

Die Verwendung der Werkzeuge des klassischen Geschäftsprozessmanagements zur Bildung von Reflexions- und Gestaltungskompetenz von Arbeitsprozessen birgt darüber hinaus für operativ Tätige das Potenzial, die entwickelten Kompetenzen auch zur Optimierung von Arbeitsprozessen aus betriebswirtschaftlicher Perspektive einzusetzen bzw. dazu angehalten zu werden. Während dies nicht per-se dem Konzept der Selbstermächtigung entgegensteht, kann gleichzeitig auch nicht von einer Kohärenz der Interessen beider Gestaltungsperspektiven ausgegangen werden. Im Sinne einer nachhaltigen Verankerung der selbstermächtigten Gestaltung von Arbeitsprozessen in Organisationen muss diese Kohärenz aber angestrebt und auch explizit sichtbar gemacht werden. Dies stellt eine große Herausforderung im Kontext des Einsatzes von Geschäftsprozessmodellierung zur Organisationsentwicklung dar und muss als solche auch vor und während des Einsatzes der vorgestellten oder ähnlicher Methoden der akteurszentrierten Geschäftsprozesserhebung und -modellierung berücksichtigt werden.

\subsubsection{Bewusstmachen von prozessrelevantem Veränderungspotenzial}

In der Folge wird zunächst die Value Network Analysis vorgestellt, wie sie im Wissensmanagement zur Bearbeitung von Leistungsbeziehungen zwischen vernetzten Akteuren eingeführt wurde, ehe ihr Potenzial für die Prozessanalyse- und -modellierung detailliert wird.

\subsubsection{Value Network Analysis}

Betrachten wir, wie eingangs erwähnt, die Wertschöpfung von Organisationen, und damit die Ebene der leistungsbezogenen Austauschbeziehungen zwischen Akteuren, so können im Rahmen von Arbeitsvorgängen tangible von intangible Austauschbeziehungen im Netzwerk von Akteuren unterscheiden werden. Tangibler Austausch wird durch Energieund Materialflüsse bestimmt. Intangibler Austausch, wie Wissen, verweist auf kognitive Prozesse und handlungsleitende Information. Werden nun TeilnehmerInnen und 
Austauschbeziehungen beschrieben, kann so die Struktur einer Organisation oder eines Netzwerks erfasst werden.

Austauschbeziehungen stellen die molekulare Ebene ökonomischer Aktivitäten dar. Somit besteht Wertschöpfung nicht nur aus tangiblen Transaktionen, sondern auch aus darüber hinausreichenden, intangiblen Transaktionen. Diese beziehen sich vor allem auf den kognitiven Austausch, da nachhaltiger Erfolg einer Organisation auf Informationsaustausch, Wissensteilung und offenen kognitiven Pfaden, die situationsgerechte Entscheidungsfindung (und damit erfolgreiches Bestehen einer Organisation) ermöglichen, beruht. Wissen und intangible Elemente verhalten sich allerdings anders als physische Ressourcen im Geschäftsleben, sodass sie nicht als tangibel betrachtet werden können. Sie konstituieren aufgrund ihrer Nähe zu lebenden Systemen eine eigene Kategorie von Austausch, und unterscheiden sich vom tangiblen Austausch mit Bezug zu Gütern, Dienstleistungen und Erträgen.

Tangibler Austausch ist nach Allee [2] definiert über Transaktionen, die Güter, Dienstleistungen oder Erträge beinhalten, z. B. physische Güter, Verträge, Rechnungen, Lieferund Empfangsbestätigungen, Anfragen, Aufforderungen, Bietereinladungen, Zahlungen. Wesentlich dabei ist, dass wissensintensive Produkte und Dienstleistungen, welche Erträge bewirken und für die Zahlungen als ein Teil einer Dienstleistung oder eines Produkts geleistet werden bzw. aufgrund einer vertraglichen Verpflichtung zu leisten sind, auch als tangible Transaktionen betrachtet werden.

Intangibler Austausch von Wissen und Leistung unterstützt Kernprozesse und damit die klassische Wertschöpfungskette, unterliegt aber keinerlei vertraglicher Verpflichtung. Intangibles sind ,Extras“ bzw. (kleine) Aufmerksamkeiten, die Personen einander zukommen lassen, um Beziehungen aufzubauen und Abläufe ohne Störungen bzw. angenehm ablaufen zu lassen. Intangible Transaktionen inkludieren den Austausch strategischer Information, Planungswissen, fachlich-operatives Wissen, gemeinsame Planungsaktivitäten, kollaborative Gestaltung, und policy-Entwicklung. Intangible Transaktionen sind folglich nicht vertraglich vereinbarte Leistungen zum Nutzen oder zur Unterstützung von Organisationen bzw. ihrer Mitglieder. Sie können von einer Person oder Gruppe zu anderen ausgeweitet werden, indem beispielsweise ein Experte für eine bestimmte Zeit von einer Organisationseinheit zur Mitarbeit aufgefordert wird, für den dies Prestige bedeutet. Anerkennung hilft oft in der Beziehungsarbeit, sodass intangible benefits echte Motivationsfaktoren für rege Beteiligung und aktive Teilnahme an Gruppenaktivitäten darstellen.

Intangibles stellen das Kernstück allen menschlichen Handelns dar, und bestimmen somit auch sozio-ökonomisches Handeln. Intangible Transaktionen werden bewusst gesetzt. Sie können herbeigeführt und erkannt werden. Soll verstanden werden, wie intangibles Wert generieren, ist zunächst zu verstehen, wie sie als negoziables in ökonomischen Austauschbeziehungen sichtbar werden und wirken. Sie sind oft nicht unmittelbar sichtbar, vielmehr , verpackt` in Dienstleistungen oder Produkten. Ein typisches 
Beispiel ist, Verständnis für eine Kundensituation aufzubauen (intangibel), bevor eine Leistung (tangibel) angeboten wird. Für eine Praxisgemeinschaft ist unmittelbar der störungsfreie Gang von Prozessen von Bedeutung. So sind jene Transaktionen wesentlich, welche (auch) mittels intangibles gewährleisten, dass ein gemeinsamer Zweck des Handelns sichergestellt ist. Es gilt dies nun methodisch zu berücksichtigen.

In einem Value Network werden mittels komplexer dynamischer Austauschvorgängen zwischen zwei oder mehreren Individuen, Gruppen oder Organisationen tangible und intangible Werte generiert, die den Gegenstand der reflektierenden Gestaltung darstellen.

\subsubsection{Holomapping}

Die auf Vernetzung beruhende Sichtweise zur organisationalen Wertgenerierung bringt eine neue Form der Organisationsmodellierung mit sich, jeder Austausch einen Mechanismus bzw. ein Medium als enabler für Transaktionen erfordert. Diese können Arbeitsmittel wie e-mail oder face-to-face-Interaktionen in Communities of Practice sein. Typische intangibles betreffen, wie oben bereits angesprochen, Wissen zur Informationsgewinnung von Kunden und Feedback zu (Produkt-)Entwicklungen.

Die Darstellung tangibler und intangibler Austauschprozesse in einem Diagramm mit Flusselementen erlaubt die Dynamik von lebenden Systemen abzubilden. Zunächst werden die Teilnehmer oder Rollen (auch Gruppen, Teams oder Organisationseinheiten, aber keine technischen Hilfsmittel) dokumentiert - sie bilden die Knoten des Netzwerks und werden oval visualisiert. Die Teilnehmer senden oder ergänzen sogenannte deliverables an andere Teilnehmer. Pfeile zeigen die Richtung an, welche die deliverables im Verlauf einer bestimmten Transaktion nehmen, bezeichnet mit dem jeweiligen deliverable.

Transaktionen oder Aktivitäten werden als gerichtete Kanten (Pfeile) dargestellt, wobei der Ursprung bei einem Teilnehmer und das Ende bei einem anderen Teilnehmer zu sein hat. Der Pfeil zeigt Bewegung an und gibt die Richtung vor, in die etwas zwischen zwei Teilnehmern geschieht. Im Gegensatz zu Teilnehmern, welche zeitstabil sind, sind Transaktionen zeitlich begrenzt und flüchtig. Sie besitzen einen Startpunkt, eine Dauer und einen Abschluss.

Deliverables hingegen sind echte ,Dinge", die sich von einem Teilnehmer zu einem anderen bewegen. Ein deliverable kann materiell (tangible) sein, wie ein Dokument oder ein Tisch, oder ideell (nicht am Materie gebunden), wie eine Nachricht oder eine Anforderung, die nur verbal überbracht wird. Deliverables können auch intangibel sein, wie beispielsweise Wissen über einen bestimmten Sachverhalt (kognitiv) oder ein Gefallen (sozial/emotional). Pfeile sind immer nur in eine Richtung zulässig - sie umfassen eine einzige Transaktion. Beidseitige gerichtete Pfeile sind bedeutungslos, vielmehr verunmöglichen sie eine Analyse der Abläufe und Austauschbeziehungen.

Ein Austausch tritt dann auf, sobald eine Transaktion in einem deliverable resultiert, das zurückkommt. Er muss in der praktischen Handlungswelt in Organisationen nicht zwangsläufig gegeben sein. Tritt er allerdings auf, kann sich ein Value Network etablieren, mit Transaktionen als molekulare Elemente der Wertegenerierung. 
Wesentlich ist im Rahmen von Veränderungsprozessen die Ermächtigung der Beteiligten, und somit die Erstellung der Kommunikationslandkarte mit tangiblen und intangiblen Beziehungen (Holomap) durch die betroffenen Rollenträger, ebenso wie die Bearbeitung der erhobenen Daten durch diese im Rahmen der 3 Value Network-Analysen.

Im Rahmen der Wissensgenerierung bzw. Wissenserhebung zu Beginn der Bearbeitung der Netzwerkstruktur überlegt jeder einzelne Teilnehmer seine Rolle, welche er dann den anderen Teilnehmern mitteilt. So werden Beziehungen und Wechselwirkungen zwischen den einzelnen Rollen, die oft nicht bekannt sind, explizit und klarer. Die Rollen werden als Knoten symbolisiert, der Austausch von materiellen oder ideellen Werten wird in Form von Linien dargestellt, die die Rollen miteinander verbinden. Die Modellierung bildet die Basis für die anschließenden Analysen zur Wissensauswertung und -verarbeitung.

\subsubsection{Exchange Analysis}

Ausgangsbasis für die Exchange Analyse stellt die Holomap dar. Wie bereits erwähnt, beziehen sich Tangibles (materielle Wertflüsse) im Netzwerk dabei auf den materiellen Austausch zwischen Personen (typischerweise Waren, Dienstleistungen und Umsatzerlöse). Sie repräsentieren Transaktionen, die auf Verträgen basieren. Intangibles (nicht-materielle, ideelle Wertflüsse) basieren im Unterschied zu tangibles auf Wissen oder einem bestimmten Zusatznutzen. Sie sind nicht vertraglich fixiert oder kostenpflichtig. Oft erhobene intangibles sind strategische Informationen, Prozess- oder Planungswissen sowie bestehende emotionale Komponenten wie gegenseitiges Vertrauen, gemeinsames Interesse, Wissensbedarf, Sicherheit etc. - siehe auch Abb. 5.6 mit Holomap zu Customer Service.

Die Exchange-Analyse untersucht ein Value Network auf seine Schlüssigkeit, Robustheit und Nachhaltigkeit. Sie gibt Einblick über die aktuelle Struktur und Dynamik des Netzwerks. Folgende Fragestellungen sollen die Exchange-Analyse unterstützen: Wie fließen die Werte durch die Organisation? Zeichnet sich eine bestimmte Logik ab? Ist das Verhältnis des Austauschs von materiellen und ideellen Werten ausgeglichen oder

Abb. 5.6 Auszug einer Holomap zu Customer Service

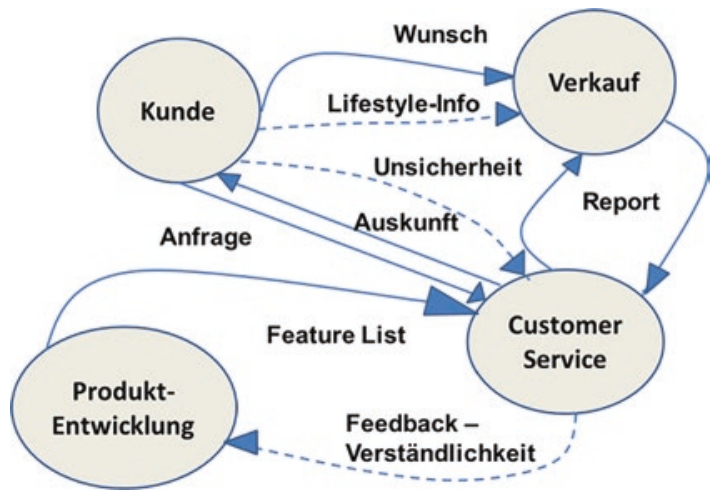


überwiegt eine bestimmte Art des Austausches? Zeigt das Muster im Value Network reziproke Wertflüsse auf oder gibt es beispielsweise Teilnehmer, die mehr Wertflüsse erhalten als bereitstellen? Gibt es im Netzwerk ineffektive Verbindungen, die Wertflüsse nicht weitergeben?

Durch diese Fragen soll überprüft werden, ob das Netzwerk seinen Zweck erfüllt, fehlende Endknoten oder Verknüpfungen zu erkennen sind, und wie die Struktur des Netzwerkes optimiert werden kann. Sie gewährleisten einen generellen Überblick über Wertschöpfung und Wertverlust. Die Exchange Analyse soll als Anregung zum Dialog dienen, komplexe Systeme zu verstehen und Systemdenken [3] fördern. Die Exchange-Analyse zu Customer Service, etwa in der Annahme, es handelt sich um die Organisation facebook, zeigt mehrere Befunde: Customer Service ist aus Sicht der Produktentwicklung tangible Senke - es empfängt nur Feature List. Der Verkauf bekommt zwar Lifestyle-info, aber keine vertrauensbezogene Information. Die Übersetzung von Unsicherheit lastet auf Customer Service zu Verkauf und zu ProduktEntwicklung. Diese erste Auswertung kann ein Indiz für die in Abbildung gezeigte Informationsgebarung sein, wo Features zwar eine bestimmte Form der Rückkoppelung ermöglichen, die aber seitens der NutzerInnen gegebenenfalls mit Anfragen, die Unsicherheit widerspiegeln, quittiert wird.

\subsubsection{Impact Analysis}

Die Impact-Analyse (Wirkanalyse) untersucht die Auswirkung jedes einzelnen WerteInput auf die Teilnehmer und legt so ihren Fokus auf die Empfangenden von WerteInputs. Diese Analyse zeigt also auf, welcher Input welche Reaktionen und Aktivitäten auslöst und wie sich dieser auf die materiellen und ideellen Vermögensgegenstände der betroffenen Empfänger auswirkt. Anschließend werden Kosten und Nutzen von Werte-inputs mit niedrig, mittel oder hoch bewertet.

Um einen besseren Überblick über diese Fragen zu erlangen, werden für jeden einzelnen Empfänger von Werte-inputs die Antworten in eine Tabelle eingetragen und die Ist-Situation analysiert. Die Tabelle zeigt die Impact-Analyse auf Basis der gewonnenen Erkenntnisse aus der Exchange-Analyse eines Customer Service-Mitarbeiters. In der Tabelle wird abgebildet, von wem Input für welche Aktivitäten kommt und welche Auswirkungen in Form materieller oder ideeller Werteflüsse wahrgenommen werden. Wesentlich für die Abschätzung von Veränderungspotenzial sind die Spalteneinträge zu den allgemeinen Kosten und Risiken sowie zum Nutzen von dem angesprochenen Input.

Die Daten der initialen Auswertung (Exchange-Analyse) stellen also eine Basis für die beiden weiteren Analysen dar, wobei aus der Sicht von erhaltenem Input (Impact-Analyse) und übermitteltem Output (Value Creation-Analyse) wertebasiert detaillierte Auswertungen von Transaktionen erfolgen und so Erkenntnisse für Veränderungsprozesse mit sich bringen.

So wird beispielsweise, wie in der Tabelle ersichtlich, ausgeführt, wann Features eine gelungene Form der Informationsrückkoppelung ermöglichen, um etwa Anfragen zu vermeiden, die Unsicherheit seitens der Nutzer widerspiegeln. Dies, selbst wenn der 
derzeitige Nutzen der Darstellung von Features seitens des Customer Service aufgrund mangelnder Verständlichkeit als gering eingeschätzt wird.

Aus den Einträgen der Tabelle zur Feature List wird ebenfalls der für die Wertschöpfung relevante Bezugspunkt ersichtlich, und zwar die Qualität der Auskunftsleistung an Kunden durch Customer Service-Mitarbeiter.

Aufbauend auf der Ist-Analyse können darauf folgend strategische Perspektiven abgeleitet werden und die Tabelle noch einmal im Vergleich dazu hinsichtlich ihrer geplanten, strategischen Aktivitäten (Soll-Analyse) ausgefüllt werden. Im gegenständlichen Fall kommt beispielsweise einer kundenorientierten Auskunftsleistung erhöhte Bedeutung zu.

\subsubsection{Value Creation Analyse}

Die Value Creation-Analyse analysiert wie Werte bestmöglich erschaffen, erhöht und eingesetzt werden können. Wie die Impact-Analyse betrachtet die Value CreationAnalyse auch die einzelne Rolle im Bezug zum gesamten System. Der Unterschied zur Impact-Analyse besteht darin, dass im Gegensatz zum Input diesmal der Sender oder Produzent von Output in seiner Rolle und mit seinen diesbezüglichen Aktivitäten betrachtet wird (siehe Abb. 5.7).

Jeder einzelne Sender von Werte-outputs wird analysiert, wie im Ist-Zustand Mehrwert und Wertezuwachs zum bestehenden Werte-output realisiert wird. Auch hierzu wird eine Kosten/Nutzenabschätzung vorgenommen.

Bei der in der Tabelle gezeigten Value Creation-Analyse (oder Wertschöpfungsanalyse) wurde anhand erzielter Arbeitsergebnisse analysiert, wie Werte bestmöglich erschaffen, erhöht und eingesetzt werden können. Die Tabelle zeigt die Analyse auf Basis der Daten der Exchange-Analyse eines Kundenberaters.

\begin{tabular}{|c|c|c|c|c|c|c|}
\hline Customer Service & & $\begin{array}{l}\text { Welche } \\
\text { Aktivitäten } \\
\text { lōst der Input } \\
\text { aus? }\end{array}$ & $\begin{array}{l}\text { Auswirkungen auf die Kosten } \\
\text { und materiellen } \\
\text { Vermögensgegenstände des } \\
\text { Empfängers }\end{array}$ & $\begin{array}{l}\text { Auswirkungen auf die } \\
\text { immateriellen } \\
\text { Vermögensgegenstände des } \\
\text { Empfängers }\end{array}$ & $\begin{array}{l}\text { Wie hoch sind die } \\
\text { allgemeinen } \\
\text { Kosten / Risiken } \\
\text { des Inputs? }\end{array}$ & $\begin{array}{l}\text { Wie hoch ist der } \\
\text { allgemeine Nutzen } \\
\text { dieses Inputs? }\end{array}$ \\
\hline $\begin{array}{l}\text { Was erhalten wir } \\
\text { (Deliverables) }\end{array}$ & $\begin{array}{l}\text { Kommt von } \\
\text { welcher Rolle? }\end{array}$ & Aktivität & WertmäBige Auswirkung & Immaterielle Auswirkung & Kosten / Risiko & Nutzen \\
\hline Anfrage & Kunde & Auskunft & $\begin{array}{l}\text { Hoher Aufwand, da } \\
\text { Öbersetzungsleistung Anfrage -> } \\
\text { Feature erforderlich }\end{array}$ & $\begin{array}{l}\text { Ũbermittlung von } \\
\text { diesbezüglichem Feedback } \\
\text { zur Verstăndlichkeit, um für } \\
\text { die Produktentwicklung zu } \\
\text { lernen }\end{array}$ & $\begin{array}{l}\mathrm{H} \text { bei unklaren } \\
\text { Anliegen } \\
\text { / } \mathrm{H} \text { bei neuen } \\
\text { Anforderungen }\end{array}$ & $\begin{array}{l}\text { M, da nicht immer } \\
\text { hoch, wenn kein } \\
\text { konstruktives } \\
\text { Feedback möglich }\end{array}$ \\
\hline $\begin{array}{l}\text { Verunsicherung } \\
\text { (Intansibel) }\end{array}$ & Kunde & $\begin{array}{l}\text { Report an } \\
\text { Verkauf }\end{array}$ & Notwendigkeit zu handeln & $\begin{array}{l}\text { Negativ besetzte Beziehung } \\
\text { zu Kunden }\end{array}$ & $\mathrm{H} / \mathrm{N}$ & $\begin{array}{l}\text { H, da } \\
\text { Unmittelbarkeit zu } \\
\text { handeln evident }\end{array}$ \\
\hline Report & Verkauf & Bearbeiten & $\begin{array}{l}\text { Wissensaufbau zu Kunden } \\
\text { (Customer Knowledge } \\
\text { Management) }\end{array}$ & $\begin{array}{l}\text { Erreichung guter } \\
\text { Kundenbeziehung durch } \\
\text { Wissen über Kunden }\end{array}$ & $\begin{array}{l}\text { M, abhăngig von } \\
\text { Inhalt / M }\end{array}$ & $\begin{array}{l}\text { H, da wesentlicher } \\
\text { Teil von } \\
\text { Informationsfluss }\end{array}$ \\
\hline Feature List & $\begin{array}{l}\text { Produkt- } \\
\text { Entwicklung }\end{array}$ & Bearbeiten & $\begin{array}{l}\text { Ermöglichung kundengerechter } \\
\text { Auskunftsleistungen }\end{array}$ & $\begin{array}{l}\text { Wissensgewinn ũber } \\
\text { Produkt }\end{array}$ & $\begin{array}{l}\text { M, da für Produkt } \\
\text { jedenfalls zu } \\
\text { erstellen / M, da } \\
\text { Verständlichkeit } \\
\text { gegebenenfalls } \\
\text { bei Technik- } \\
\text { Lastigkeit niedrig }\end{array}$ & $\begin{array}{l}\mathrm{H} \text { bei } \\
\text { verstāndlicher } \\
\text { Information, sonst } \\
\mathrm{N}\end{array}$ \\
\hline
\end{tabular}

Abb. 5.7 Teil einer Impact Analyse zu Customer Service 
An Leistungsindikatoren können aus den Analysen die Anforderungen, die an den Verkauf sowie die Produktentwicklung gestellt werden, gefiltert werden. Die daraus entwickelte Strategie kann mit der Aussage, Verfügbarkeit bzw. Transparenz von Information “ umrissen werden. So könnte als Veränderungsmaßnahme beschlossen werden, das identifizierte Wertschöpfungspotenzial im Value Network durch Erweiterung der tangiblen Informationsflüsse zwischen allen funktionalen Einheiten zu steigern.

Nach der Darstellung der Ist-Situation ermöglicht auch die Value Creation-Analyse strategische Perspektiven abzuleiten. Hier sollte sich die Frage gestellt werden, welche Möglichkeiten in Zukunft noch genützt werden sollen, um Wertoptimierung beim Werteoutput zu generieren. Hinsichtlich der konkreten Frage ,Was soll getan werden, um eine Wertsteigerung, -ausweitung oder -optimierung von Output zu erzielen?` wird die Tab. 5.1 zur Analyse der Soll-Situation vergleichend ausgefüllt.

Wesentlich zur Entscheidungsunterstützung sind die Einträge in die Spalten ,Kosten/ Risiken“ und ,Nutzen'. Hier kann sich die Einschätzung vor allem bei Kosten und Risiken bei der Soll-Situation relativieren, wenn beispielsweise Inhalte in Schulungen aufgenommen werden sollte, da es Know-How zur Erstellung entsprechender Unterlagen sowie der Bereitstellung effektiver Vermittlungsformate im Netzwerk gibt (etwa im Kontext ,Report‘- dritter Tabelleneintrag in der Tabelle). Eine Soll-Aufstellung berücksichtigt zumeist Wissen über die Machbarkeit bzw. Verfügbarkeit von Ressourcen und damit verbundene Aufwände zur Umsetzung von Maßnahmen, und zwar ohne eine diesbezügliche Entscheidung vorwegzunehmen (siehe Auswertung).

\subsubsection{Auswertung}

Für die Auswertung werden in Tabellenform (Impact-Analyse, Exchange-Analyse, Value Creation-Analyse) tangibles und intangibles nach ihrer Bedeutung für die jeweilige(n)

Tab. 5.1 Teil der fallbezogenen (Customer Service) Value Creation-Analyse

\begin{tabular}{l|l|l|l|l}
\hline Output des Senders & Output Adressat & $\begin{array}{l}\text { Wertsteigerung, } \\
\text { Mehrwert der Aktivität }\end{array}$ & Kosten/Risiken & Nutzen \\
\hline $\begin{array}{l}\text { Feedback- } \\
\text { Verständlichkeit }\end{array}$ & Produkt-Entwicklung & $\begin{array}{l}\text { Kundenorientierter } \\
\text { Zugang } \\
\text { Berücksichtigung der } \\
\text { Machbarkeit }\end{array}$ & $\mathrm{H} / \mathrm{H}$ & $\mathrm{H}$ \\
\hline Auskunft & Kunde & $\begin{array}{l}\text { Bedürfnisgerechter } \\
\text { Umgang } \\
\text { Wäre auch für } \\
\text { potenzielle Kunden } \\
\text { interessant }\end{array}$ & $\mathrm{H} / \mathrm{H}$ & $\mathrm{H}$ \\
\hline Report & Verkauf & $\begin{array}{l}\text { Gewinnung von } \\
\text { Kundendaten } \\
\text { wäre auch für Schulung } \\
\text { interessant }\end{array}$ & $\mathrm{H} / \mathrm{H}$ & $\mathrm{H}$ \\
\hline
\end{tabular}

Bei Kosten/Risiken und Nutzen: $\mathrm{N}=$ niedrig $\mathrm{M}=$ mittel $\mathrm{H}=$ hoch 
Rolle(n) bewertet. Anhand dieser Bewertungen werden Auswirkungen auf Beziehungen sichtbar und es können gezielt Maßnahmen gesetzt werden.

Bislang wurde mit der Durchführung der Exchange-Analyse Einblick über die aktuelle Struktur und Dynamik des Netzwerks geschaffen. Die Durchführung der Impact-Analyse erlaubt allen Teilnehmern ihre eigenen Rollen im Netzwerk kontextsensitiv zu erschließen. Die Impact-Analyse ermöglicht einen Überblick, welche Auswirkungen jede einzelne Wertetransaktion auf die Teilnehmer hat. Die Value CreationAnalyse erlaubt hingehen zu befinden, wie Werte bestmöglich geschaffen, erhöht und eingesetzt werden können, und gegebenenfalls auf andere Rollen wirken bzw. diese miteinbeziehen sollten. Anhand der abgeleiteten Leistungsindikatoren kann nun eine Strategie entwickelt werden, um das identifizierte Wertschöpfungspotenzial im Value Network zu steigern.

Typische Ergebnisse einer Auswertung zur Steigerung der Wertschöpfung umfassen die Berücksichtigung fehlender Austauschbeziehungen, also beispielsweise auf den gegenständlichen Fall bezogen:

- Konkrete Anfragen an Kunden, um deren Anliegen (insbesondere jene, welche zur Verunsicherung von Kunden führen) aus Sicht der Produktentwicklung und des Verkaufs besser verstehen zu lernen - Customer Service nimmt hier eine Mittlerrolle ein, wodurch auch der tangible Report aufgewertet werden und das Feedback konkrete Hinweise zur Verbesserung bzw. Schaffung von Features beinhalten kann.

- Feedback von Kunden, das sowohl den zukünftigen Umgang als auch den bisherigen Umgang mit Features, also im Besonderen die Produktentwicklung betrifft. Sobald die Verständlichkeit der Darstellung explizit adressiert wird, kann ein diesbezüglicher Informations(rück)fluss zur Produktentwicklung (und auch zum Verkauf) sichergestellt werden - eine weitere Maßnahme, die den kundengerechten Zugang zum Produkt erleichtert.

Beide Maßnahmen zeigen die Notwendigkeit vernetzter Darstellung von Austauschbeziehungen. Es ist nicht unbedingt die Unmittelbarkeit von Austauschbeziehungen, sondern vielmehr die Verkettung von Austauschbeziehungen, die Mehrwert bewirken kann. Würde beispielsweise die Produktentwicklung (z. B. Softwaredesigner) direkt mit Kunden beginnen zu kommunizieren, wäre zunächst eine bestimmte Gesprächsbasis herzustellen. Derartige Maßnahmen könnten für den zu erreichenden strategischen Zweck sogar kontraproduktiv sein und die bestehende Verunsicherung aufseiten der Kunden noch erhöhen, und damit dem angestrebten Ziel zuwider laufen.

Die Ergänzungen des Netzwerks (siehe Abb. 5.8) erlauben somit eine kontextsensitive Systembetrachtung der Wertschöpfung durch materielle und ideelle Leistungen, die zwischen den beteiligten Akteuren fließen sollten. Die vervollständigte Value Network Map, wie in der Abbildung gezeigt, bildet die Grundlage für die weitere Entwicklungsplanung. Sie zeigt die Anfrage an Kunden sowie das Feedback zur Verständlichkeit erstmals als tangible deliverable, wodurch sich die Informiertheit der Beteiligten durch die Transparenz 
Abb. 5.8 Adaptiertes Netzwerk

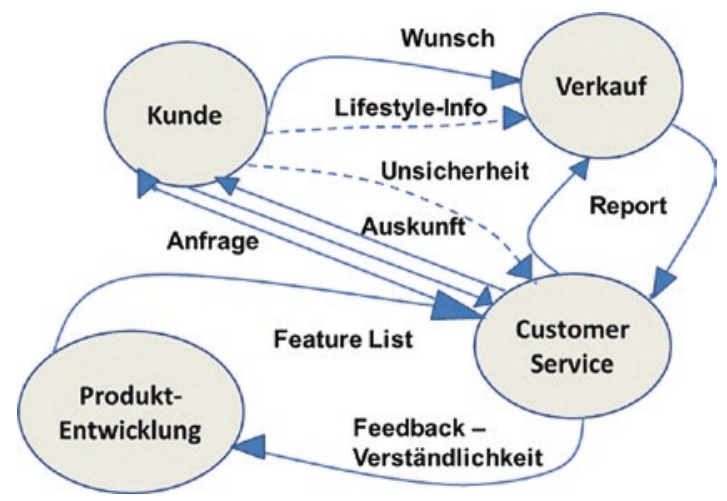

und Verfügbarkeit von Information erhöhen soll. Langfristig anzustreben ist als intangibler Austausch zwischen Kunden und Customer Service ein durch wechselseitige Sicherheit geprägter Umgang, der sich mit Loyalität der Kunden zur Organisation ausdrücken lässt. Erst durch den offenen Austausch an Information wird nachhaltiges Customer Knowledge Management möglich.

Value Networks betrachten Systeme in ihrer Gesamtheit und berücksichtigen deren Komplexität. Sie ermöglichen die ganzheitliche Identifikation von materiellen als auch ideellen Werten. Letztere bestimmen jedenfalls indirekt die Qualität materieller Austauschbeziehungen und sind folglich bei der Entwicklung sozio-technischer Systeme mit zu berücksichtigen. Der durch Value Networks einnehmbare Fokus auf die beteiligten Individuen fördert die Sinnstiftung und Motivation derselben zur Beteiligung an Reflexion und aktiven Mitgestaltung. Die vorgestellten Erhebungen und Analysen erlauben die Explikation einzelner Rollen und deren direkt oder indirekt wahrgenommener Beitrag zur Wertschöpfung eines organisationalen Systems durch Akteure. Dies erleichtert die Rollenklärung sowie das Verständnis von Zusammenhängen, da diese mittels Wertaustauschbeziehungen grafisch in Holomaps visualisiert, und somit rollenspezifisch rückgekoppelt werden. Sie stellen somit einen viablen Ausgangspunkt partizipativer Gestaltung sozio-technischer Systeme dar.

\subsubsection{Potenziale für Prozessanalyse und -modellierung}

Für die Gestaltung von Prozessen und deren Modellierung lassen sich aus der Value Network Analysis mehrfach Potenziale schöpfen:

- Die Value Network Analysis erlaubt die Repräsentation einer Situation, wie sie von Handlungsträgern wahrgenommen wird. Die Darstellung der Situation erfolgt anhand von Rollen, die durch Knoten eines Netzes von Akteuren repräsentiert werden. Da jeder Rollenträger diese Analyse durchführen kann, können individuell wahrgenommene Interaktionsmuster mit der Wahrnehmung anderer Rollenträger gegenübergestellt und abgeglichen werden. Somit erlaubt die Value Network Analysis 
die strukturierte Feststellung von unterschiedlich wahrgenommenen Interaktionsmustern zwischen Handlungsträgern aus deren Sicht, wobei die unterschiedlichen Interaktionsmuster auch kumuliert dargestellt werden können.

- Die Erfassung und Darstellung der Ist-Situation stellt einen Referenzpunkt dar, von dem aus Veränderungspotenziale erschlossen werden können. Damit können für alle Beteiligten nachvollziehbar Änderungen von Interaktionen auf der Basis einer gemeinsamen Ausgangslage diskutiert werden.

- Die Value Network Analysis erlaubt die Bestimmung von Rollen, welche nicht zwingend funktional in einer Organisation, etwa im Organigramm, zu finden ist. Die Rollenbestimmung orientiert sich an den Kommunikations- und Interaktionsmustern, welche im Rahmen der Aufgabenerfüllung und dem wahrgenommenen Organisationsgeschehen als relevant erachtet werden. Somit rücken die fachliche und die Interaktionsebene in den Vordergrund.

- Im Rahmen der Erarbeitung von Veränderungsvorschlägen, werden anstelle von Forderungen einzelner Rollenträger Vorschläge an andere im Sinne individueller Angebote an das Kollektiv gemacht. Eine derartige Vorgangsweise stellt den Adressaten frei, dieses Potenzial zu nutzen. Sämtliche Angebote werden im Kontext ihrer Herkunft dargestellt und bezüglich ihrer organisationalen Wirksamkeit von den jeweilig vorschlagenden Handlungsträgern bewertet und können darauf aufbauend im Kollektiv abgestimmt werden.

- Interaktionsbeziehungen werden differenziert nach vertraglich verpflichtenden Leistungen (tangibles) und nicht nur aus der jeweiligen Rolle nach vertragsmäßig geregelten Leistungen (intangibles). Damit wird bereits evident, in welcher Form die Bewältigung von Aufgaben erfolgt, ob eher nach formellen Grundsätzen oder Interaktionen, welche eine erfolgreiche Prozesserfüllung begünstigen, oder nach informellen Grundsätzen. Gleiches gilt für Änderungsvorschläge, die ebenfalls in formelle Strukturen (als Teil von funktionalen Rollenbeschreibungen) oder auf informeller Ebene angesiedelt (als freiwilliger Beitrag) sein können.

- Die Veränderung einer Ist-Situation in einen Soll-Zustand kann auf mehreren Wegen erfolgen: i) eine informelle (intangible) Interaktion wird formeller Teil einer Rolle (tangible); ii) eine formelle (tangible) Interaktion wird weggelassen oder informeller Teil einer Rolle (intangible); iii) eine tangible bzw. intangible Beziehung wird neu eingeführt und ergänzt bestehende Interaktionsmuster.

- Interaktionsbeziehungen sind direkt überführbar in konkrete Prozessschritte, da sie zeitlich aneinandergereiht die Erfüllung von Aufgaben durch die Rollenträger repräsentieren. Somit kann aus einer Holomap ein Prozessmodell abgeleitet werden, welches den Austausch von Leistungen zwischen Akteuren (im Gegensatz zu linearisierten Funktionsschritten) in den Mittelpunkt stellt.

Insgesamt stellt die Value Network Analysis eine diagrammatisch/tabellarische Technik zur Organisationsentwicklung mit dem Ziel von Prozessdefinitionen bzw. ausführbaren Prozessen dar, welche eine Abbildung von Interaktionsstrukturen auf kommunikationsorientierte 
Ansätze wie S-BPM direkt unterstützt. Sämtliche Informationen werden dabei aus der Sicht von beteiligten bzw. verantwortlichen Rollenträgern generiert [4, 5, 6, 7].

\subsubsection{Strukturierte Aktivsätze}

Im Folgenden wird eine Vorgehensweise beschrieben, die ausgehend von natürlich sprachlichen Beschreibung zu einem formalen Verhaltensmodell führt. Diese Vorgehensweise orientiert sich an den Aktivsätzen von natürlichen Sprachen. Der Ablauf wird an Hand des Projekts Poly Energy Net (NET), einem vom Deutschen Bundesministerium für Wirtschaft und Energie geförderten Ansatz, eingeführt. In diesem Projekt galt es eine Lösung für ein sich selbst organisierendes verteiltes Energieversorgungssystem zu entwickeln. In den folgenden Abschnitten wird gezeigt, wie die dazugehörige Software entwickelt wurde, und zwar ausgehend von einer allgemeinen Beschreibung bis hin zu einem präzisen Modell, das dann in einer ersten Stufe auf Basis von Prozessspezifikationen in ein Programm umgesetzt wurde.

\subsubsection{Allgemeines}

Die in der Folge vorgestellten Schritte, die von einer informellen Beschreibung eines Prozesses zu einem formalen Modell des Ablaufs führen, müssen nicht in der angegebenen Reihenfolge ausgeführt werden. Die Schritte dienen vielmehr dem Ziel einer präzisen Prozessbeschreibung. Stellt sich in einem Schritt heraus, dass in einem vorangegangenen Schritt etwas vergessen wurde, oder stellt es sich heraus, dass es vorteilhafter ist, Teilabläufe anders zu gestalten, so wird diese Änderung in dem momentan bearbeiteten Schritt aufgenommen. Diese Änderung wird nicht in den vorangegangenen Beschreibungen nachgezogen. Um einen ersten Entwurf einer Prozessbeschreibung zu erstellen, können die im vorangegangenen Kapitel beschriebenen Vorgehensweisen aus Design Thinking eingesetzt werden.

Alle Dokumente der vorangegangenen Beschreibungen sind nach Abschluss einer detaillierteren Beschreibung standardmäßig obsolet und nicht mehr gültig, ausgenommen, in einer Beschreibung wird explizit darauf hingewiesen, dass eine vorangegangene Beschreibung noch gültig ist (z. B. als Übersichtsdokument). Erfahrungsgemäß gelingt es, nicht mehrere Dokumente konsistent zu halten. Es sollte daher nur ein gültiges Dokument geben, sodass nach Abschluss der Vorbereitung ein formales Verhaltensmodell zur Verfügung stehen kann. Änderungen sollten in der Regel nur noch an diesem Modell vorgenommen werden.

Bei der Erstellung eines Prozessmodells kann auch mit einem beliebigen Schritt begonnen werden. So können beispielsweise bei einer natürlich sprachlichen Beschreibung nur Aktivsätze verwendet werden. Damit fallen die Schritte, welche eine Beschreibung in beliebiger Form in eine aktive Beschreibungsform transformieren, weg. Es kann auch mit der Identifizierung der Handelnden begonnen und mit der Detaillierung jedes Handelnden fortgesetzt werden, einschließlich der Kommunikation mit anderen Handelnden. Die konkrete Vorgehensweise hängt von den Umständen und den Vorlieben der Beteiligten ab. 


\subsubsection{Natürlich sprachliche Beschreibung der Prozesse}

Das Prozessgeschehen wird mehr oder minder strukturiert in natürlicher Sprache erzählt. Es gibt keinerlei Vorgabe hinsichtlich der Struktur des zu erstellenden Dokuments oder des $\mathrm{zu}$ verwendenden Vokabulars. Die Ersteller einer Prozessbeschreibung können ihren Vorlieben folgen. Das Bild (siehe Abb. 5.9) zeigt einen Textauszug aus der groben Anforderungsbeschreibung an das Energiemanagementsystem.

Die anfängliche freie Verwendung der natürlichen Sprache erfordert von den Beteiligten keine speziellen Methodenkenntnisse. Die Erfordernis solcher Kenntnisse könnte eine große Hürde für das Einbinden von Betroffenen aus den verschiedenen Fachbereichen darstellen.

Textuelle Beschreibungen können durch geeignete Bilder ergänzt werden. Das folgende Bild (siehe Abb. 5.10) zeigt eine funktionale Struktur des zu erstellenden Systems. Eine solche funktionale Struktur ist eine erste Annäherung an die Spezifikation eines Prozesssystems.

Aufbauend auf diese mehr strukturellen Beschreibungen kann eine erste ablauforientierte Spezifikation erstellt werden. Einen Auszug einer Ablaufbeschreibung zeigt das folgende Bild (siehe Abb. 5.11).

\section{Das holare Modell}

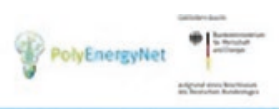

- Das holare Modell ist ein logisches System, das sich in der realen Welt auf ein physikalisches System abbilden lässt.

- Das gesamte Holare Versorgungssystem setzt sich aus Holonen zusammen.

- Innerhalb jedes Holons wird zu jedem Zeitpunkt gleich viel Energie bereitgestellt (.erzeugt") wie verbraucht.

- Holone bestehen aus Holaren (energetischen) Elementen. Dies sind:

- holare Erzeugungs- und Verbrauchselemente

- holare Verbindungselemente

- holare Umwandlungselemente

- holare Speicherelemente

- holare Wachter.

- Alle holaren Elemente besitzen eine physikalische Instanzilerung.

- Holare Elemente besitzen die Möglichkeit, mit mindestens einem Holonmanager zu kommunizieren. Sie sind mit maximal einem Holonmanager (zu einem holaren Objekt) verbunden..

- Holonmanager konnen über Steuersignale das Verhalten (z. B. Erzeugung. Verbrauch) von holaren Elementen beeinflussen. Alle holaren Elemente, die mit dem gleichen Holonmanager kommunizieren konnen, bilden ein Holares Objekt.

- In jedem Holon gibt es eine Instanz Holonkoordinator, die mit Holonmanagern im eigenen Holon und den Holonkoordinatoren in anderen Holonen kommunizieren kann.

- Holare Objekte können existieren, ohne einem Holon angeschlossen zu sein.

- Holare Elemente können existieren, ohne einem Holonmanager zugeordnet zu sein (frei fliegende Holare Elemente-

- Alle Holonmanager und Holonkoordinatoren besitzen eine physikalische Instanziierung.

- Holone konnen sich dynamisch nach bestimmten Holon-Regeln zu neuen Holonen zusammenschließen oder in kleinere Holone zerfallen. Die Umsetzung der Regeln erfolgt durch Holonkoordinatoren im Zusammenspiel mit Holonmanagern.

- Die Regeln fur die Holonbildung orientieren sich an logischen Erfordernissen und Rahmen-bedingungen, die durch die physikalische Instanzierung der holaren Elemente bestimmt sind.

- Holone konnen sich auflosen. Dies ist der Fall, wenn es keinen Holonkoordinator gibt.

- Es gibt auch, nicht-holare* Elemente, das sind solche, die entweder keine Ansteuerbarkeit oder keine Verbindung zum energetischen System besitzen.

Abb. 5.9 Anforderungen an ein Holares Energiemanagementsystem 


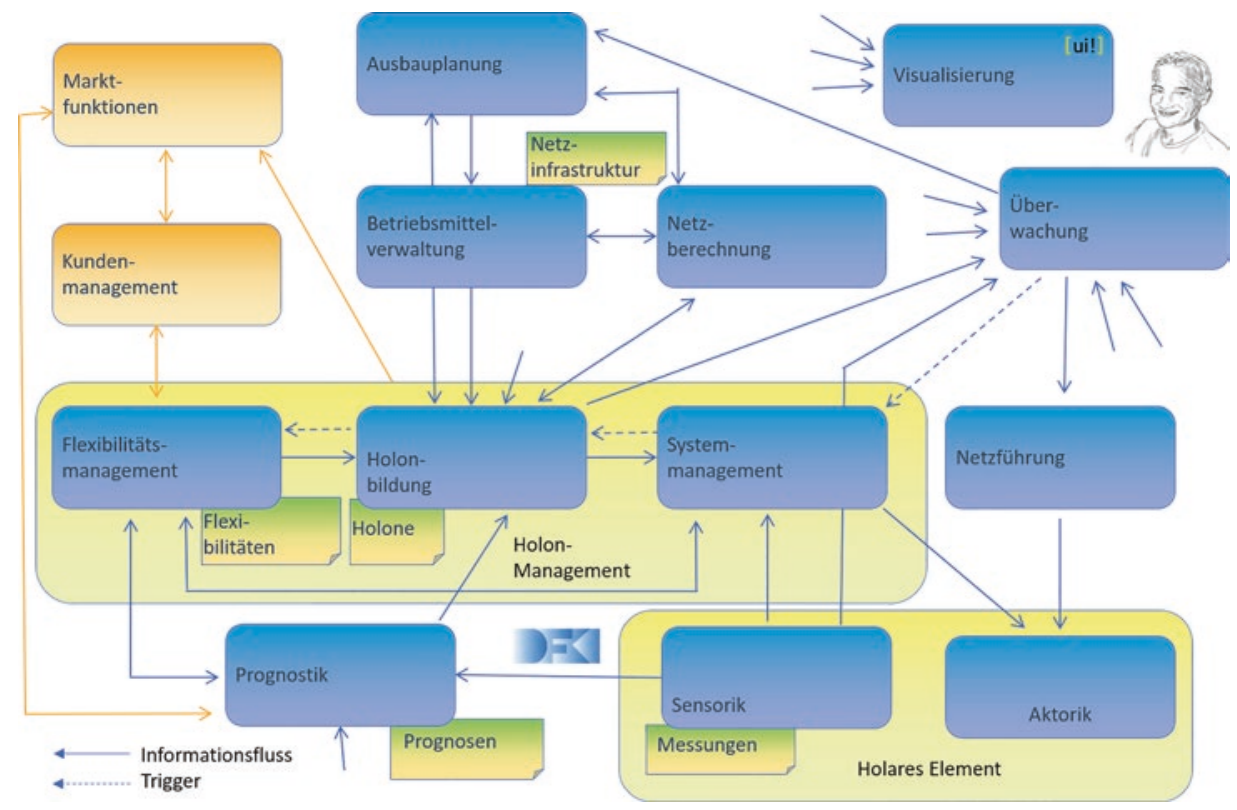

Abb. 5.10 Funktionsblöcke eines Holaren Energiemanagementsystems

im holaren System

Wegen des Ausfalls des Ortsnetztrafos (im Beispiel A) kann ein einzelner Netzabschnitt im Niederspannungsbereich nicht mehr durch das ūbergeordnete

Mittelspannungsnetz versorgt werden. Erkannt wir dies durch Messen und Melden eines Spannungsabfalls auf nahe Null in dem betroffenen Netzabschnitt (vgl,

을 PMU in Abb. 2). Dabei wird angenommen, dass die Verteilerschiene in der Ortsnetzstation intakt ist und lediglich der Trafo als solcher ausgefallen ist.

Leitstand bzw. Netzüberwacher melden das Ereignis „Spannungsabfall” automatisch an die Leitstelle, die mit einem weitestgehend durch automatisiertes Messen und Fernwirkung geprägten Prozess eine schnellstmögliche Versorgung (ggf. Minimalversorgung einiger Verbraucher) anstößt.

Durch automatisches Abfragen von Messwerten (bei den PMUs im Netz) und sonstigen Fehlerinformationen ( $z$. B. von den beteiligten Ortsnetzstationen) werden von der Leitstelle aus der Ort und soweit möglich die Ursache des Fehlers identifiziert. Im aktuellen Fall wird festgestellt, dass der Trafo bei $A$ ausgefallen ist. Die Leitstelle informiert einen oder mehrere Holon-Manager (HM) bzw. Holon-Koordinatoren im betroffenen Netzgebiet.

Die betroffenen Holon-Koordinatoren und Holon-Manager treten untereinander in Verbindung und verhandeln (auch mit benachbarten Holon-Koordinatoren) eine Neubildung von Holonen, die eine Versorgung von möglichst vielen Verbrauchern auch ohne den defekten Ortsnetztrafo ermŏglicht. Ziel ist eine solche Versorgure (auch ohne Netzersatzanlage) solange aufrechtzuerhalten, bis die defekten Teile der Ortsnetzstation ausgetauscht sind.

Bei der in Abb. 2 skizzierten Situation kann angenommen werden, dass vom Holon-Management der Leitstelle die folgende Neubildung vorgeschlagen wird (vgl. Abb. 3):

Die holaren Leitungen sind wie folgt zu schalten:

Der Schalter 4 ist zu schließen und verbindet somit die zuvor vom nun defekten Trafo versorgte Verteilschiene in der Ortsnetzstation A zu versorgen Durch Offnen bzw. Schließen der Schalter 5, 6, und 7 entsteht eine vom Rest des Systems abgetrennte Insel.

Durch Öffnen bzw. Schließen der Schalter 8 - 11 entsteht eine vom Rest des Systems abgetrennte Insel.

- Es werden folgende Holone gebildet:

Die durch Schalter 6 und 7 abgetrennte Insel bildet Holon C und versorgt sich selbst. Abgesehen von einer hăuslichen PV-Anlage ist die einzige Versorgungsquelle das BHKW, das als „Notstromaggregat” für einen sehr kritischen Verbraucher vorgehalten wird. Das Holon ist so zu betreiben, dass letzteres stets vollversorgt ist; weitere Verbraucher können nur eine Minimalversorgung erwarten.

(NB: Sollte diese Versorgungssituation nicht aufrechterhalten werden können, würde Holon C vermutlich bald in kleinere, teils sehr schlecht versorgt Holone zerfallen oder ein Teil der Verbraucher würde dem Holon A zugeschlagen werden.)

- Die durch Schalter 8 und 11 abgetrennte Insel bildet Holon $B$ und versorgt sich selbst. Hier ist eine weitgehende Vollversorgung zu erwarten, da es im Holon leistungsfăhige Erzeige (großes BHKW, Freiflächen-PV-Anlage, Quartiersspeicher) gibt.

- Alle anderen Holaren Elemente bilden Holon A. Abgesehen von einem kleineren BHKW und wenigen hăuslichen PV-Anlagen und zugehörigen hăuslichen Speichern sichert die Ortsnetzstation B die Versorgung.

Wenn die Leitstelle diese Holonbildung akzeptiert, veranlasst sie die entsprechenden Schaltvorgănge und Einstellungen

Das Betriebspersonal wird informiert, făhrt zur Ortsnetzstation und trennt den defekten Trafo von der Verteilerschiene.

Die durch die durch die Holon-Bildung herbeigeführte Situation erlaubt bei ausreichend vorhandener Energiequellen (Sonne, Gas) eine optimale, weitestgehend

volle Versorgung aller Verbraucher, bis eine Ersatztrafo installiert bzw. der bestehende Repariert werden kann.

NB: Sollte eine Versorgung wie durch die Holon-Neubildung herbeigeführt, nicht möglich sein, wird dies als Fehlersituation erkannt und es wird eine neue

Holonbildung angestoßen. Als ultima ratio würde auch hier eine Netzersatzanlage zum Einsatz kommen

$\omega$ Nach Beseitigung des Fehlers wird durch das Betriebspersonal vor Ort unter Einbezug der Netzleitstelle der Normalzustand (Trafo versorgt die Verteilschiene in der ( Ortsnetzstation A) wieder hergestellt,

Die Messung in der Leitstelle erkennt, dass ein neuer, starker Versorger zur Verfügung steht, und stößt eine Neubildung von Holonen an.

NB: Diese dürfte mit hoher Wahrscheinlichkeit wieder die Situation mit den Holonen A und B vor Eintritt des Use-Case herbeiführen.

Abb. 5.11 Dynamik eines Holaren Managementsystems 
Ergänzt wird diese Ablaufbeschreibung durch eine Illustration der Auswirkungen auf ein holares Energienetz. In der obigen Abbildung wird auf einige Elemente (Schalter) im folgenden Bild (siehe Abb. 5.12) verwiesen.

Die am Anfang verwendeten Werkzeuge für eine erste Anforderungsdefinition und Ablaufspezifikation sind nicht strukturiert. Es können einfach Texte und ergänzende Zeichnungen beliebiger Art verwendet werden. In den nachfolgenden Schritten wird diese unschematische Beschreibung eines Prozesses Zug um Zug in eine präzise Ablaufbeschreibung überführt.

\subsubsection{Prozessbeschreibungen in Aktivform}

Informelle Prozessbeschreibung in natürlicher Sprache enthalten sehr oft Passivsätze (siehe auch obige Ablaufbeschreibung). Passivsätze enthalten allerdings keine direkte Aussage über den Ausführenden einer Aktion. Passivsätze werden dann verwendet, wenn der Ausführende einer Aktion nicht wesentlich ist. Allerdings ist dies bei Prozessen nicht gegeben. Prozessbeschreibungen müssen den Ausführenden einer Handlung enthalten. Es gilt nun alle Passivsätze in Aktivsätze umzuwandeln. Dazu müssen zunächst die aktiven Elemente identifiziert werden. Aktive Elemente können Menschen, Softwaresysteme die automatisch ablaufen und bestimmte Aktivitäten ausführen, physikalische Systeme oder beliebige Kombinationen aus diesen Grundelementen sein. So kann in unserem Beispiel die Leitstation eine Kombination aus Software, Menschen und elektrischen Systemen sein. Die Software fordert einen Bediener auf einen bestimmten Messwert abzulesen und ihn einzugeben. Die Software veranlasst abhängig vom eingegebenen Messwert, dass ein Schalter geschlossen wird.
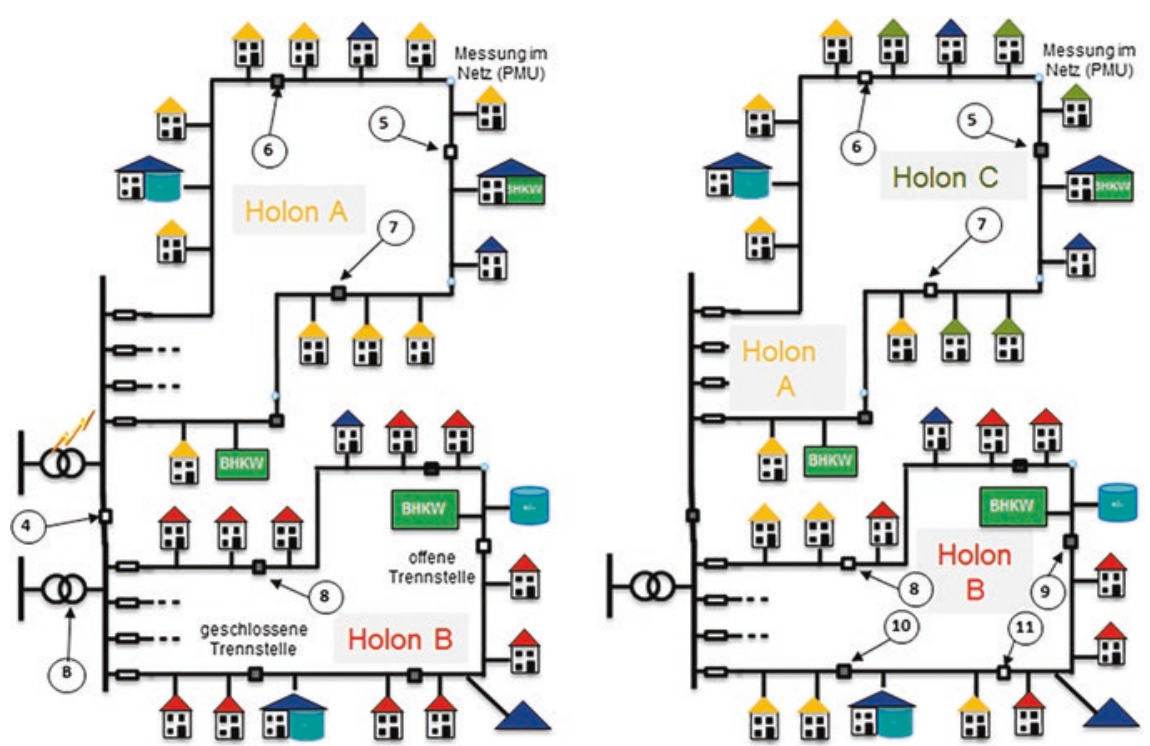

Abb. 5.12 Beispiel eines Holaren Energiemanagementsystems (Quelle: B.A.U.M.) 
Um zu vermeiden, dass eine Prozessbeschreibung zu sehr vom organisatorischen und technischen Umfeld abhängig ist, werden abstrakte Handelnde eingeführt. Solche abstrakte Handelnde sind Entitäten, die Nachrichten senden, empfangen oder interne Tätigkeiten ausführen. Das folgende Bild (siehe Abb. 5.13) zeigt das Funktionsdiagramm mit zugeordneten aktiven Elementen. Diese abstrakten aktiven Elemente werden als Subjekte bezeichnet, in Anlehnung an die Subjekte in Aktivsätzen. Subjekte sind die Rollen in Prozessen.

Die Aufgaben der identifizierten Subjekte können für ein besseres Verständnis wie in Tab. 5.2 gezeigt kurz beschrieben werden.

Durch die Einführung von Handelnden in Form von Subjekten können nun alle Passivsätze durch entsprechende Aktivsätze ersetzt werden. Damit wird die Prozessbeschreibung vollständiger. Das folgende Bild (siehe Tab. 5.3) zeigt eine Prozessbeschreibung mit Aktivsätzen in Tabellenform. Die Nummerierung der Sätze gibt bereits den Kontrollfluss wider. Die Nummer der Nachfolgeaktion wird in der so benannten Spalte angegeben. Hängt die Nachfolgeaktion von bestimmten Ergebnissen der Aktion $\mathrm{ab}$, wird diese Bedingung in der Spalte Nachfolgeaktion mit beschrieben. Abhängig von der gültigen Bedingung kann es eine andere Nachfolgeaktion geben.

Eine Tabelle mit dem Kontrollfluss eines Prozesses kann der Ausgangspunkt für ein kontrollflussorientiertes Prozessmodell sein. Die einzelnen Handelnden sind die Swim Lanes in BPMN bzw. in einer Swim-Lane-orientierten EPK oder in UML-Zustandsdiagrammen.

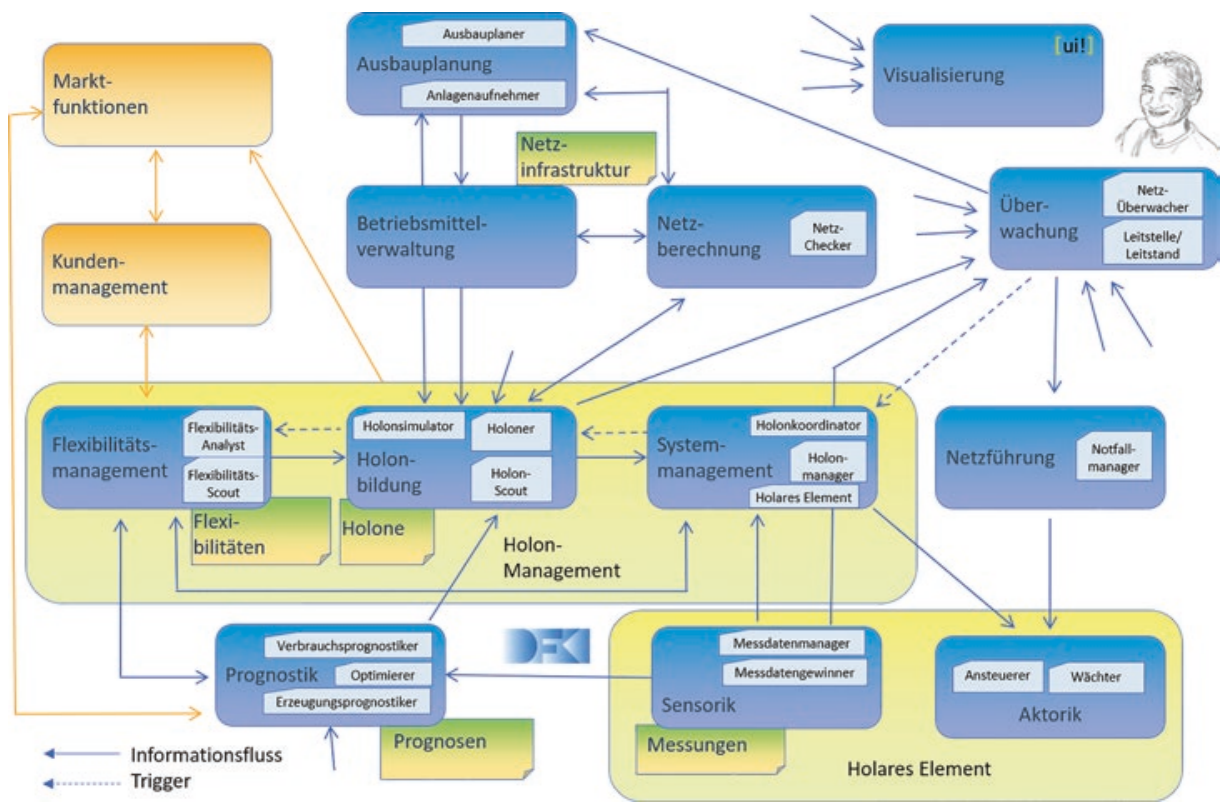

Abb. 5.13 Subjektorientierte Betrachtung eines Holaren Energiemanagementsystems 


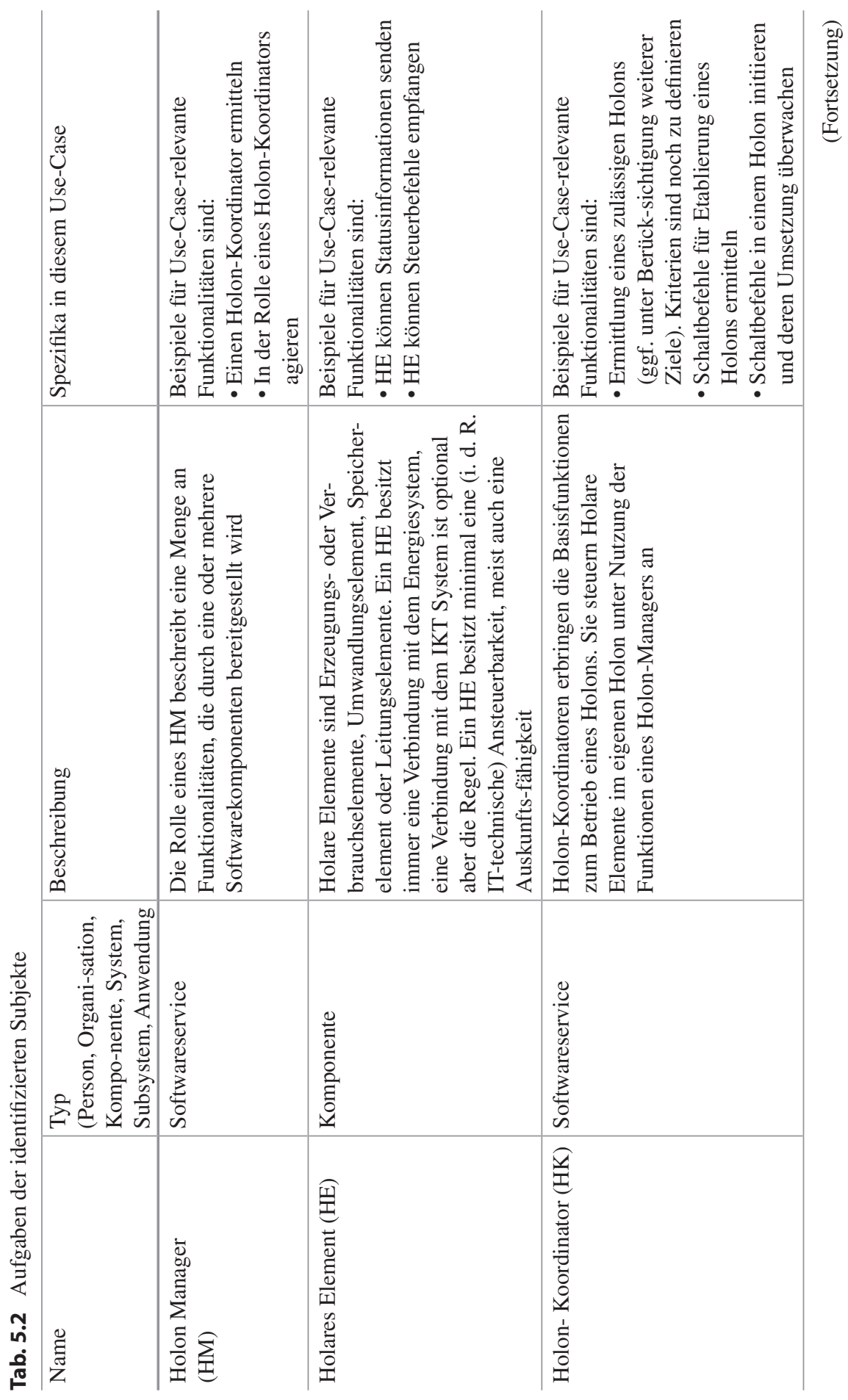




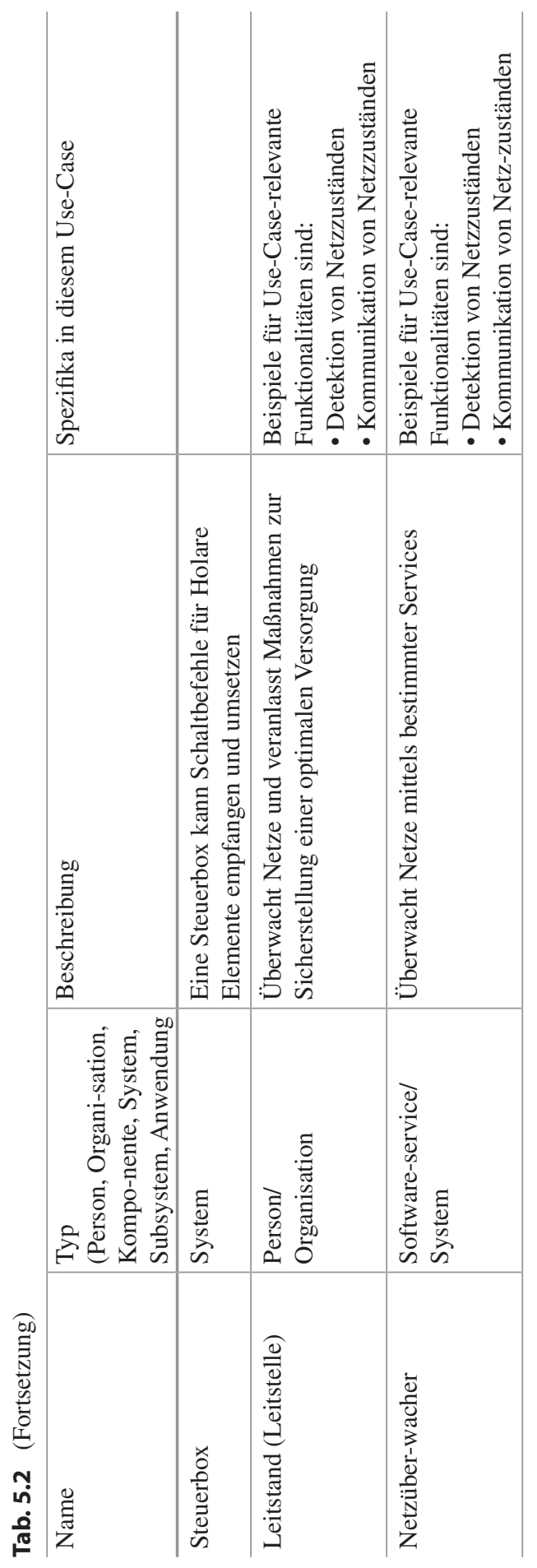


Tab. 5.3 Ablauf im Holaren System

\begin{tabular}{|c|c|c|c|}
\hline Nr. & Handlung & Hinweise & Nach-folge-aktion \\
\hline 1. & $\begin{array}{l}\text { Netzüberwacher stellt Spannungs- } \\
\text { abfall im Niederspannungsnetz fest }\end{array}$ & $\begin{array}{l}\text { Nutzt dafür Funktionen aus der } \\
\text { Funktionsgruppe „Messen“ }\end{array}$ & 2 \\
\hline 2. & $\begin{array}{l}\text { Netzüberwacher meldet die } \\
\text { Situation an Leitstelle }\end{array}$ & & 3 \\
\hline 3. & $\begin{array}{l}\text { Leitstelle identifiziert und lokalisiert } \\
\text { den Fehler }\end{array}$ & $\begin{array}{l}\text { Nutzt dafür Funktionen aus den } \\
\text { Funktionsgruppen „Überwachen“ } \\
\text { und „Messen“ (Bemerkung: Was } \\
\text { passiert wenn Leitstelle den Fehler } \\
\text { nicht identifizieren kann?) }\end{array}$ & 4 \\
\hline 4. & $\begin{array}{l}\text { Leitstelle informiert Holon- } \\
\text { Manager (HM) bzw. Holon- } \\
\text { Koordinatoren im betroffenen } \\
\text { Netzgebiet }\end{array}$ & $\begin{array}{l}\text { Achtung: hier kann ein single- } \\
\text { point-of-failure bzw. Engpass } \\
\text { vorliegen; ist ggf. durch einen } \\
\text { dezentralen Mechanismus zu } \\
\text { ersetzen }\end{array}$ & 5 \\
\hline 5. & $\begin{array}{l}\text { (sofern angesprochen) Holon- } \\
\text { Koordinatoren treten mit Holon- } \\
\text { Managern in den betroffenen } \\
\text { Holonen in Verbindung }\end{array}$ & $\begin{array}{l}\text { Nutzt dafür Funktionen } \\
\text { aus der Funktionsgruppe } \\
\text { (Holonmanagement) }\end{array}$ & 6 \\
\hline 6. & $\begin{array}{l}\text { (sofern kein Holon-Koordinator } \\
\text { tätig wird) Holon-Manager } \\
\text { identifizieren einen HM, der die } \\
\text { Koordinationsaufgabe übernimmt }\end{array}$ & & 7 \\
\hline 7. & $\begin{array}{l}\text { Holon-Koordinator und betroffene } \\
\text { Holon-Manager ermitteln eine } \\
\text { plausible neue Holon-Konstellation }\end{array}$ & $\begin{array}{l}\text { Nutzt dafür Funktion „Holonbil- } \\
\text { dung“ aus der Funktionsgruppe } \\
\text { „Holonmanagement“" }\end{array}$ & 8 \\
\hline 8. & $\begin{array}{l}\text { Holon-Koordinator meldet den } \\
\text { errechneten Vorschlag an die } \\
\text { Leitstelle }\end{array}$ & & 8 \\
\hline 9. & $\begin{array}{l}\text { Holon-Koordinator geht in Zustand } \\
\text {,alert“ und wartet auf Rückmeldung } \\
\text { von der Leitstelle }\end{array}$ & & 10 \\
\hline
\end{tabular}

Allerdings sollten diese Modellierungsmethoden nur verwendet werden, wenn in einem Prozess keine asynchronen Ereignisse wie z. B. die Möglichkeit Bestellungen zu ändern vorkommen, und die Parallelität der Handelnden nicht modelliert werden soll. Darüber hinaus sollte die Anzahl der Handelnden nicht zu hoch sein.

Swim Lane-Darstellungen sind in der Regel flach, d. h. es gibt keine Hierarchien von Swim Lanes. Eine größere Anzahl als fünf Swim Lanes führt zu unübersichtlichen Darstellungen. So hat ein Service Prozess mindestens die Swim Lanes Kunde, Call Center, First Level Support, Abrechnung und gegebenenfalls Kundenrückmeldung. Die Erfahrung zeigt, dass in realen Prozessen in der Regel etwa 10 Handelnde und mehr 
involviert sind. Unübersichtlich werden Swim Lane Diagramme auch dann, sobald der Kontrollfluss mehrere Swim Lanes kreuzen muss, um in eine andere Swim Lane zu wechseln.

Bei organisations- oder unternehmensübergreifenden Prozessen in einem verteilten Umfeld sind Kontrollflüsse keine handhabbare Darstellung. In einer verteilten Umgebung sind Nachrichten die anschaulichere Art die Zusammenarbeit von einzelnen Handelnden zu modellieren.

\subsubsection{Tabellarische rollenorientierte Beschreibung}

Im letzten Schritt vor der eigentlichen Prozessmodellierung wird die Prozessbeschreibung entsprechend der Handelnden strukturiert. Alle Sätze mit denselben Handelnden als Subjekt werden in einer Tabelle zusammengefasst. Dazu kann es notwendig sein, dass die Prozessbeschreibung mit Interaktionen zwischen Subjekten ergänzt werden muss. Phrasen wie ,,informiert Subjekt xy“ oder ,tritt in Verbindung mit“ usw. werden ersetzt durch Sende und Empfangsaktionen (siehe Tab. 5.4). Der Satz 2 „Netzüberwacher meldet Situation an Leitstelle“ im Bild in Abschn. 5.1.4.3. wird in eine Sende und eine Empfangsaktion umgewandelt. Im folgenden Bild entspricht dies der Aktion 2 (Netzüberwacher sendet Status-Schwarz an Leitstand“ im Tabellenabschnitt für den Netzüberwacher. Das Gegenstück dazu ist der Satz Nr. 1 im Tabellenabschnitt Leitstand.

Nach dem Erstellen der Verhaltenstabellen kann ein formales Modell in einer geeigneten Modellierungssprache abgeleitet werden. Es sollte dabei eine Sprache verwendet werden, in der die für wichtig erachteten Aspekte des betrachteten Prozesses anschaulich und präzise ausgedrückt werden können. In unserem Beispiel haben wir S-BPM ausgewählt. Abb. 5.14 zeigt in der linken Hälfte die Netzwerkstruktur des betrachteten Prozesses. Die Rechtecke mit den abgerundeten Ecken stellen die beteiligten Handelnden dar. Die Pfeile zwischen den Handelnden sind mit den ausgetauschten Nachrichten beschriftet. Die Zahlen an den Nachrichtennamen entsprechen den Satznummern in der obigen Tabelle.

Das Diagramm rechts neben der Prozessstruktur zeigt das Verhalten des Subjekts Holonkoordinator. Die Kreise mit den Buchstaben E und S sind Kommunikationszustände. Übergänge von Kreisen mit einem $\mathrm{E}$ sind mit den in diesem Zustand erwarteten Nachrichten beschriftet. Die Übergänge nach S-Zuständen sind mit den in diesem Zustand gesendeten Nachrichten beschriftet. Alle anderen Übergänge definieren lokale Operationen auf lokalen Daten.

\subsubsection{Prozessmodellierung}

\subsubsection{Auswahl der Modellierungssprache}

In der Folge werden exemplarisch einige Faktoren angeführt, welche die Auswahl einer geeigneten Modellierungsnotation bzw. -sprache erleichtern sollen. Dazu zählen die Rahmenbedingungen eines BPM-Vorhabens, die Handhabbarkeit der Sprache sowie die Unterstützung der nachgelagerten Aktivitäten wie Validierung [8, 9, 10, 11, 12]. 
Tab. 5.4 Ablauf im Holaren System (Präziser)

\begin{tabular}{|c|c|c|c|c|c|c|}
\hline Nr. & $\begin{array}{l}\text { Subjekt } \\
\text { (Akteur) }\end{array}$ & Prädikat & Objekt & $\begin{array}{l}\text { Indirektes } \\
\text { Objekt }\end{array}$ & Ergebnis & $\begin{array}{l}\text { Weiter } \\
\text { bei }\end{array}$ \\
\hline & Netzüberwacher & & & & & \\
\hline \multirow[t]{2}{*}{1} & \multirow[t]{2}{*}{ Netzüberwacher } & \multirow[t]{2}{*}{$\begin{array}{l}\text { Misst } \\
\text { Spannung im }\end{array}$} & \multirow[t]{2}{*}{$\begin{array}{l}\text { Niederspan- } \\
\text { nungsnetz }\end{array}$} & & $\begin{array}{l}\text { Spannungs- } \\
\text { abfall }\end{array}$ & 2 \\
\hline & & & & & $\begin{array}{l}\text { Spannungs- } \\
\text { anstieg }\end{array}$ & 3 \\
\hline 2 & Netzüberwacher & Sendet & $\begin{array}{l}\text { (1) Status- } \\
\text { Schwarz }\end{array}$ & $\begin{array}{l}\text { An Leitstand } \\
\text { (Leitstelle) }\end{array}$ & Gesendet & 1 \\
\hline \multirow[t]{2}{*}{3} & Netzüberwacher & Sendet & $\begin{array}{l}\text { (10) Status- } \\
\text { Grün }\end{array}$ & $\begin{array}{l}\text { An Leitstand } \\
\text { (Leitstelle) }\end{array}$ & Gesendet & 1 \\
\hline & Leitstand & & & & & \\
\hline 1 & Leitstand & Empfängt & $\begin{array}{l}\text { (1)Status- } \\
\text { meldung }\end{array}$ & $\begin{array}{l}\text { Von Netzüber- } \\
\text { wacher }\end{array}$ & Empfangen & 2 \\
\hline \multirow[t]{2}{*}{2} & \multirow[t]{2}{*}{ Leitstand } & \multirow[t]{2}{*}{$\begin{array}{l}\text { Identifiziert } \\
\text { und lokalisiert }\end{array}$} & \multirow[t]{2}{*}{ den Fehler } & & $\begin{array}{l}\text { Fehler } \\
\text { gefunden }\end{array}$ & 3 \\
\hline & & & & & $\begin{array}{l}\text { Fehler nicht } \\
\text { gefunden }\end{array}$ & $?$ \\
\hline 3 & Leitstand & Sendet & (2) Status & $\begin{array}{l}\text { An* Holon- } \\
\text { Koordinatoren }\end{array}$ & Gesendet & 4 \\
\hline 4 & Leitstand & Empfängt & (5) Vorschlag & $\begin{array}{l}\text { Von* Holon- } \\
\text { Koordinator }\end{array}$ & & 5 \\
\hline 5 & Leitstand & Prüft & Vorschlag & & Angenommen & 6 \\
\hline 6 & Leitstand & Sendet & $\begin{array}{l}\text { (6) V-Ange- } \\
\text { nommen }\end{array}$ & $\begin{array}{l}\text { An* Holon- } \\
\text { Koordinatoren }\end{array}$ & & 7 \\
\hline
\end{tabular}

Bezüglich Rahmenbedingungen interessiert vor allem die Frage, Was sind die Eigenschaften des zu modellierenden Sachverhalts?' Bestimmend für die Auswahl einer Modellierungssprache sollten vor allem die folgenden Eigenschaften eines Sachverhalts sein:

- Asynchrone Ereignisse: Liegen derartige Ereignisse vor, dann sollte eine entsprechende Modellierungssprache die Möglichkeit bieten, die damit verbundene Parallelisierung von Prozessen oder Prozessschritten derart abzubilden, dass gegebenenfalls eine Ausführung die Parallelität widerspiegelt. Dies bedeutet, dass die Modellierungssprache Konstrukte zur Darstellung asynchroner Ereignisse enthält, welche diese zeitliche Qualität explizit enthält. Damit kann zur Ausführungszeit entsprechend eine Laufzeitumgebung konfiguriert werden. 

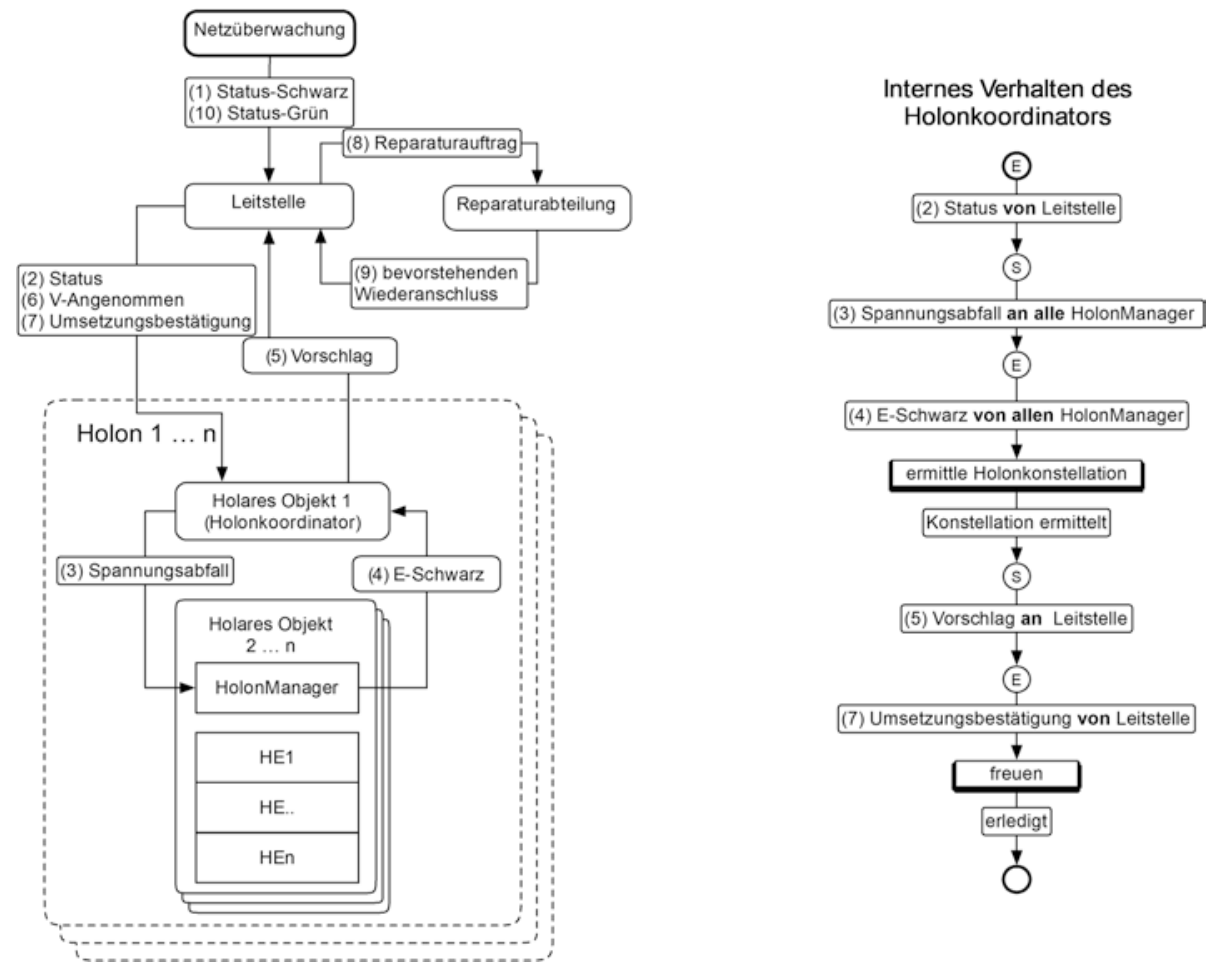

Abb. 5.14 Verhaltensbeschreibung des Subjekts Holonkoordinator

- Organisations- und unternehmensübergreifend: Ist ein Sachverhalt nicht nur für eine bestimmte Organisation oder deren Einheit, sondern auch für vernetzte Partner relevant, die außerhalb der unmittelbaren Tätigkeitsbereiche liegt, wie beispielsweise in der Zulieferindustrie, dann sollten ausgewiesene Modellierungsmechanismen oder Sprachkonstrukte zur Verfügung stehen, welche eine Kapselung organisationsexterner Sachverhalte ermöglicht. Damit wird eine Einbettung externer Akteure einer Organisation möglich, ohne im Detail deren Abläufe kennen und repräsentieren zu müssen.

- Anzahl der Handelnden: Diese Kenngröße scheint auf den ersten Blick nicht unbedingt relevant für die Auswahl einer Modellierungssprache. Sie gewinnt allerdings an Bedeutung, wenn es zum einen um die Komplexität von Prozessen geht, und zum anderen um die Verständlichkeit der Modelle. Schließlich spielt auch die Instanz-Modellbeziehung in diesen Faktor herein. Besteht eine Vielzahl an handelnden Akteuren, dann sollte eine Notation auch Modellierungsmechanismen oder Sprachkonstrukte aufweisen, welche diese sowohl sichtbar macht, als auch aggregiert oder mittels anderer Perspektiven (z. B. Funktionssicht) zugänglich macht. 
Bezüglich Handhabbarkeit interessiert vor allem die Frage, Wie soll der Sachverhalt beschrieben werden?` Bestimmend für die Auswahl einer Modellierungssprache sollten vor allem die folgenden Eigenschaften bzw. Qualität einer Modellierungssprache sein:

- Anzahl der Symbole: Die Anzahl der Symbole kann zum einen mächtige Modelle einfach überschaubar gestalten lassen, aber zum anderen eine unerwünschte Verkürzung von Sachverhalten mit sich bringen.

- Definition der Symbole: Der Gebrauch der Symbole sollte eindeutig, d. h. objektiv nachvollziehbar für Modellierende sein. Dies erleichtert die Effektivität und Effizienz bei der Erstellung von Modellen.

- Verfügbarkeit der Werkzeuge zur Modellbeschreibung: Die Verfügbarkeit von digitalen Werkzeugen zur einfachen und korrekten Darstellung von Sachverhalten bestimmt die Gebrauchstauglichkeit einer Modellierungssprache mit. Ein Syntaxeditor bei diagrammatischen Sprachen hilft beispielsweise, syntaktisch korrekte Modelle zu erstellen sowie umfangreiche Sachverhalte gebrauchstauglich zu strukturieren.

- Möglichkeiten der Hierarchisierung von Modellen: Diese Eigenschaft erlaubt, vernetzte Sachverhalten aus einer top-down Perspektive zu erschließen. Dadurch wird üblicherweise die Lesbarkeit von Modellen erleichtert und damit die Verständlichkeit abgebildeter Sachverhalte für nicht unmittelbar Beteiligte erhöht.

Bezüglich Unterstützung weiterer Aktivitäten interessiert vor allem die Frage, Wie ist die Unterstützung des Modells für nächste Schritte?`Für die Auswahl einer Modellierungssprache sollten in diesem Kontext vor allem die folgenden Features einer Modellierungssprache betrachtet werden:

- Validierungswerkzeuge: Kann ein Modell validiert werden? Dies bedeutet, dass mittels eines Werkzeugs festgestellt werden kann, ob die Notation und damit alle verwendeten Sprache im Sinne der Syntax der Sprache sowie im Sinn der Intention des abgebildeten Sachverhalts korrekt verwendet wurden.

- Optimierungswerkzeuge: Kann ein Prozess mithilfe seines Modells optimiert werden? Die Optimierung ist der erstmaligen Modellierung und Validierung nachgelagert und zielt auf die optimale Verteilung von Aufgaben und optimale Nutzung von Ressourcen ab. So soll mittels eines Werkzeugs festgestellt werden können, ob die Notation und damit alle verwendeten Sprachen die Optimierung von modellierten Prozessen durch entsprechende Konstrukte der Sprachen oder durch spezielle Mechanismen zulässt bzw. aktiv (etwa durch Vorschläge oder Referenzmodelle) unterstützt.

- Werkzeuge für die organisatorische Einbettung: Kann die Integration eines modellierten Prozesses in eine Organisation mithilfe eines Werkzeugs realisiert werden? Dieser Schritt ist der erste zur Implementierung von Prozessen und erfordert, dass mittels eines Werkzeugs bestimmte werden kann, welche Aufgaben- oder Rollenträger bzw. welche Organisationseinheiten den abgebildeten Sachverhalt in der Praxis ausführen können sollen. Auf dieser Basis kann danach entsprechende Zuordnung zur organisationalen Umsetzung von Prozessmodellen getroffen (und nach Bedarf wieder geändert) werden. 
- Werkzeuge für die technische Einbettung: Kann die Integration eines modellierten Prozesses in eine (informations-)technische Infrastruktur bzw. Informationssystemarchitektur mithilfe eines Werkzeugs realisiert werden? Dieser Schritt ist der zweite erforderliche zur Implementierung von Prozessen und bedarf der Bestimmung mittels eines Werkzeugs, welche technischen Systems den abgebildeten Sachverhalt in der Praxis ausführen können. Auf dieser Basis kann danach entsprechende Zuordnung zur technischen Umsetzung von Prozessmodellen getroffen (und nach Bedarf wieder geändert) werden.

- Werkzeuge für die Inbetriebnahme und den Betrieb: Können die Inbetriebnahme und der Betrieb eines modellierten Prozesses in einer Organisation mithilfe eines Werkzeugs unterstützt bzw. sichergestellt werden? Dieser Schritt ist erforderlich, sobald ein Prozess ,produktiv“ geschaltet wird, d. h. nach seiner organisationalen und technischen Implementierung in den operativen Betrieb einer Organisation überführt bzw. in deren operativen Praxis wirksam wird. In diesem Kontext sollte ein Werkzeug helfen, die Einführungsphase eines modellierten Prozesses zu unterstützen, also etwa die Reihenfolge festzulegen, welche Prozessschritte in welcher Reihenfolge in den operativen Betrieb übernommen werden. Ein entsprechendes Werkzeug sollte auch die Überwachung des Einsatzes von implementierten Prozessen unterstützen und so helfen. den operativen Betrieb einer Organisation sicherzustellen und deren Weiterentwicklung effektiv zu unterstützen. Letzteres kann etwa durch Annotation an Prozessmodellen, welche Ausführungshindernisse markieren, erfolgen.

\subsubsection{Modellierung durch Konstruktion}

Bei der Konstruktion eines Prozessmodells beginnt die Modellierung mit einem ,leeren Blatt Papier“. Mit den Informationen aus der Prozessanalyse wird Schritt für Schritt der Prozess beschrieben. Die erforderlichen Aktivitäten umfassen, abhängig vom gewählten Ansatz, mehrere Aktivitäten:

- Beschreibung der Prozesse mit ihren Beziehungen (Prozessnetzwerk)

- Identifikation des zu beschreibenden Prozesses

- Identifikation der an dem Prozess beteiligten Akteure bzw. Systeme

- Festlegen der zwischen den Akteuren bzw. Systemen ausgetauschten Informationen im Rahmen des Kontroll-, Daten- und Nachrichtenflusses zur Bearbeitung von Geschäftsfällen. Dies inkludiert auch die Umsetzung von Geschäftsregeln, da sie das Verhalten von Akteuren und Systemen unmittelbar beeinflussen.

- Beschreiben des Verhaltens der einzelnen Akteure bzw. Systeme, insbesondere durch Funktionsschritte und deren zeitlich-kausalem Zusammenhang entsprechend dem modellierten Sachverhalt.

- Definition der Geschäftsobjekte bzw. Daten und deren Verwendung.

Diese Aktivitäten werden entsprechend der ausgewählten Sprache in einer bestimmten Reihenfolge gesetzt und führen dem entsprechend zu unterschiedlich detaillierten Darstellungen von Sachverhalten. 


\subsubsection{Modellierung durch Restriktion}

Neben der Modellierung durch Konstruktion gibt es grundsätzlich noch die Möglichkeit der Modellierung durch Restriktion. Dabei wird von allgemeinen Prozessmodellen ausgegangen. Ein typisches Beispiel ist der kommunikationsorientierte Zugang, wie bei S-BPM verfolgt. Im universalen Prozessmodell kann jeder an einem Prozess beteiligte Akteur bzw. System an jedes andere beteiligte System bzw. jeden anderen Akteur jederzeit eine Nachricht senden bzw. von diesem empfangen.

Diese Nachricht hat den allgemeinen Namen ,Nachricht' und kann als Geschäftsobjekt beliebige Medien übertragen. Das Ergebnis ist ein Universalprozess, der durch die Anzahl seiner Subjekte gekennzeichnet ist. Diese werden als Kästen mit Subjekt 1..n gekennzeichnet. Deren wechselseitige Interaktionsmöglichkeiten werden durch vorab gesetzte Pfeile zwischen den Subjekt-Kästchen gekennzeichnet. Daraus resultiert für jedes Subjekt ein gleichartiges initiales Verhalten.

Im Rahmen der Modellierung durch Restriktion wird in folgenden Schritten zu einem detaillierten Sachverhalt vorgegangen: i) Anzahl der Subjekte und Subjektbezeichner bestimmen, ii) Kommunikationspfade reduzieren; iii) Nachrichtentypen spezifizieren, iv) Verhalten der Subjekte spezifisch anpassen, v) Geschäftsobjekte spezifizieren und verfeinern.

Werden andere, etwa funktionsorientierte Ansätze gewählt, dann können grundsätzliche Strukturen verallgemeinert, etwa in Form von Referenzmodellen, zur Verfügung gestellt werden. Dabei werden auch verhaltensorientiere Muster zum Einsatz kommen, etwa vorangegangene Ereignisse zu Funktionen oder Bedingungen, die nach Abarbeiten einer Funktion den weiteren Verlauf von Geschäftsprozessen bestimmen.

\subsubsection{Kombination}

Während bei der Konstruktion von Prozessmodellen die Modellierung mit einem „leeren Blatt Papier" beginnt, und um Informationen aus der Prozessanalyse Schritt für Schritt erweitert wird, wird bei der Modellierung durch Restriktion von einer verallgemeinerten Struktur von Prozessmodellen und deren Bestandteile ausgegangen. Ein typisches Beispiel einer Kombination beider Ansätze stellt der Fall dar, der dem ,middle-out ‘ Ansatz entspricht. Eine Instanz dieses Falls ist, mit einer Konstruktion zu beginnen, und sobald ein (wiederkehrendes) Muster auftritt, ein Referenzmodell zum Einsatz zu bringen und zu reduzieren bzw. zu konkretisieren.

Weitere Anwendungsinstanzen wären ein Mustervergleich sowie der Start mit wiederkehrenden (Routine-)Prozessen. Letztere kehren den obig genannten Fall um, und erlauben, spezielle Ausprägungen von Prozessverläufen in generalisierte Prozessarchitekturen einzubetten. Der Mustervergleich hingegen stellt eine Art Kontrollvorgang dar, wobei mittels eines verallgemeinerten Musters die Vollständigkeit bzw. Korrektheit eines Modells überprüft werden kann. 


\subsection{Qualitätskontrolle: Validierung und Optimierung}

Die Validierung steht in enger Beziehung zur Modellierung. In der Modellierung wird der Prozessablauf entsprechend der Zielsetzung beschrieben, Dies bedeutet, dass während der Modellierungstätigkeit folgernde die Frage mitschwingt: ,Entspricht das Modell den gesetzten qualitativen und quantitativen Zielen? Die Prüfung, ob ein Prozessmodell den gesetzten Zielen entspricht, wird als Validierung bzw. Optimierung bezeichnet. Somit laufen während der Modellierung permanent Validierung und Optimierung mit. Nach der Entscheidung die Modellierung abzuschließen, ist wird nun abschließend geprüft, ob das Gesamtmodell den gesetzten Zielen entspricht.

Eine Validierung zeigt, ob der Prozess alle Anforderungen erfüllt und die beabsichtigten Ergebnisse erreicht. Wesentlich ist für einen Prozess auch ob damit die gewünschten Ergebnisse mit dem geringsten möglichen Aufwand erreicht werden. Die Qualitätskontrolle bei Geschäftsprozessen hat also zwei wesentliche Aufgaben. Sie soll die Effektivität und die Effizienz von Prozessen prüfen. Effektivität bedeutet, dass der Prozess die an ihn gestellten Anforderungen erfüllt, d. h. das gewünschte Ergebnis (Output) liefert. Effizient ist der Prozess dann, wenn dieser mit möglichst geringem finanziellen und zeitlichen Mitteleinsatz ausgeführt werden kann, um das gewünschte Ergebnis zu liefern. Dies soll durch die Optimierung erreicht werden.

Diese Qualitätskontrollen müssen möglichst frühzeitig einsetzen, und zwar bevor IT-Systeme aufwendig entwickelt und die späteren Anwender geschult werden. Das folgende Bild (siehe Abb. 5.15) fasst die einzelnen Aspekte der Validierung und Optimierung zusammen. Die Überprüfung eines Prozessmodells wird durch entsprechende Werkzeuge und Referenzmodelle unterstützt. Für die Validierung gibt es manuell Hilfsmittel wie z. B. Checklisten oder Rollenspiele, während für die Optimierung Simulationssoftware eingesetzt werden muss. Die Ergebnisse der Überprüfung werden aufbereitet und in ToDo-Listen zur Abarbeitung eingetragen, d. h. es wird erneut mit Modellierungsaktivitäten gestartet. Dieser Zyklus wiederholt sich, bis die Überprüfung zu einem als gut genug betrachteten Ergebnis führt.

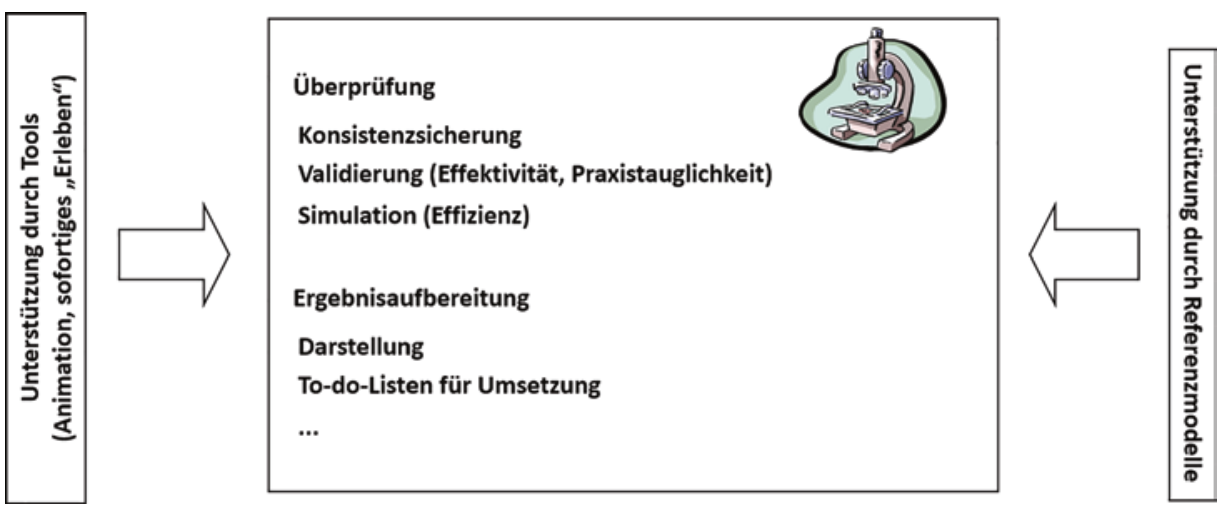

Abb. 5.15 Struktur der Qualitätssicherung eines Prozessmodells 


\subsubsection{Validierung}

Voraussetzung für die Validierung ist ein Modell, das den zu repräsentierenden Sachverhalt wiedergibt. Es wird überprüft, ob das Modell das erwartete Ergebnis gemäß den spezifizierten Qualitätsmerkmalen liefert, und ob der Prozess zur Erreichung der Ziele des Unternehmens beiträgt. Dieser Aspekt wird als semantische Richtigkeit bezeichnet. Diese ergibt sich aus dem Konsens der Führungskräfte sowie der Fach- und Methodenexperten, die das Modell als zutreffend erachten.

Von der semantischen Richtigkeit ist die syntaktische Gültigkeit abzugrenzen, welche die Einhaltung der festgelegten Beschreibungsregeln betrifft, d. h. sind die Beschreibungsmittel entsprechend der Vorgaben zur Modellierungssprache eingesetzt.

\subsubsection{Manuelle Prozessvalidierung}

Das folgende Bild (siehe Abb. 5.16) zeigt eine allgemeine Vorgehensweise bei der manuellen Validierung. Hier wird mithilfe von Checklisten die Prozessdokumentation überprüft. Die Prozessdokumentation umfasst die Beschreibung der Ziele, Inputs, Ergebnisse, auslösende Ereignisse und natürlich das Modell des Prozesses. Die Prozessdokumentation soll von allen betroffenen Parteien eines Prozesses an Hand der Checklisten überprüft werden.

Die Befunde der einzelnen Parteien werden konsolidiert und in einem gemeinsamen Workshop geklärt sowie gemeinsam die notwendigen Überarbeitungen festgelegt. Dieser Zyklus wiederholt sich bis gemeinsam entschieden ist, dass keine weiteren Überarbeitungen mehr notwendig mehr sind.

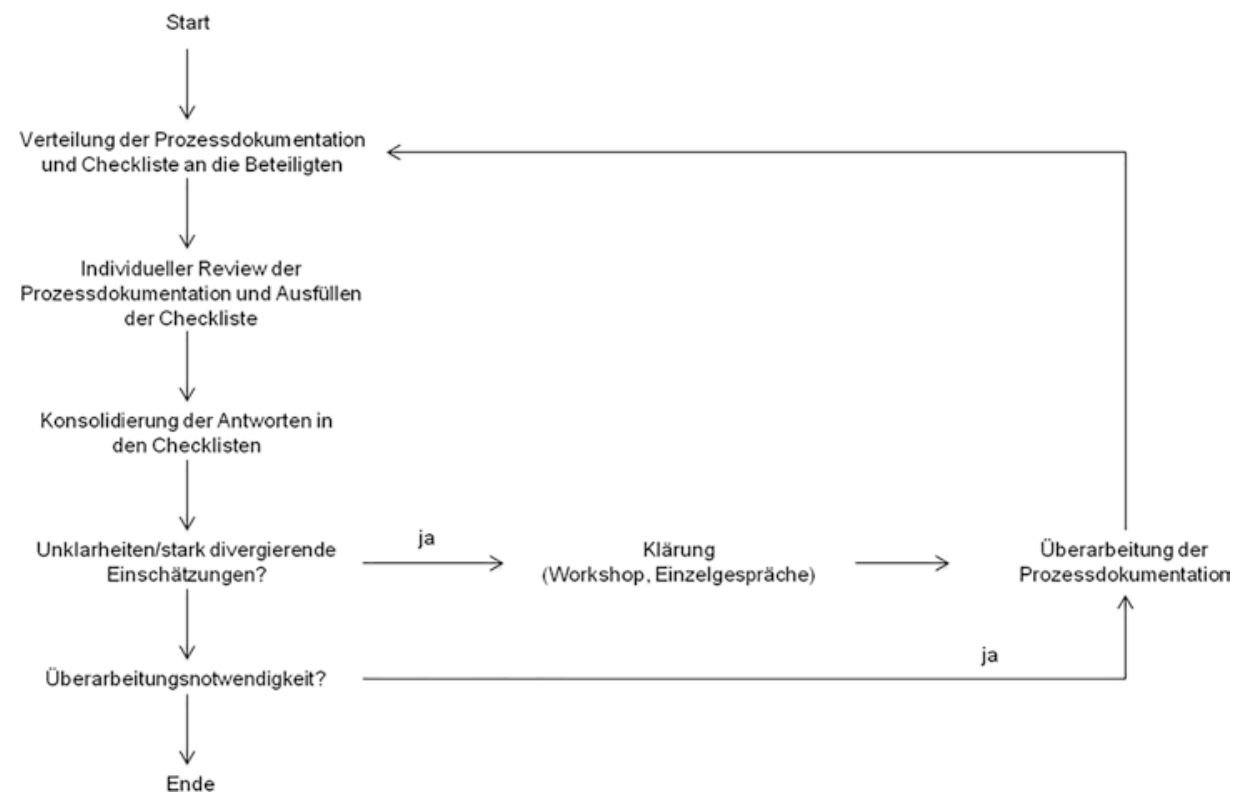

Abb. 5.16 Ablauf einer manuellen Prozessprüfung 
Zur Vorbereitung eines Reviews wird die Prozessbeschreibung und eine Checkliste, nach der die Prozessbeschreibung geprüft werden soll, kommuniziert. Diese Checkliste enthält Fragen, die die Gutachter bezüglich des Prozesses beantworten sollen.

Beispiele für solche Fragen sind:

- Unterstützt der Prozess die Ziele des Unternehmens?

- Sind die Ziele des Prozesses definiert?

- Ist der Nutzen des Prozesses in der Zielsetzung klar beschrieben und ist ersichtlich, welche Wertschöpfung er für wen liefert?

- Welche Risiken birgt der Prozess?

- Ist ein Prozessverantwortlicher (Process Owner) benannt?

- Sind die Befugnisse des Prozessverantwortlichen (Process Owner) festgelegt und sind diese ausreichend?

- Gibt es Kennzahlen, mit denen die Zielerreichung bewertet werden kann?

- Sind die Messverfahren für die Kennzahlen eindeutig festgelegt?

- Werden die Zielwerte für die Kennzahlen des Prozesses systematisch festgelegt und liefern sie eine Aussage über den Wertbeitrag des Prozesses?

- Unterstützt der Prozess die Politik und Strategie des Unternehmens bzw. der ITOrganisation?

- Ist der Prozessablauf beschrieben?

- Sind die Eingaben und Ergebnisse des Prozesses beschrieben?

- Ist klar, wer (Organisationen, Rollen, Personen) welche Eingaben liefert und welche Ergebnisse entgegennimmt?

- Werden die Beschreibungskonventionen für Prozesse eingehalten?

- Ist definiert, wer für die einzelnen Schritte des Prozesses verantwortlich ist (Organisationen, Rollen oder Personen)?

- Ist das Vorgehen in dem Prozess auf die Interessensgruppen (beispielsweise Kunden) ausgerichtet?

- Ist das Vorgehen im Prozess klar begründet?

- Gibt es neben der Prozessbeschreibung noch ausreichend Hilfsmittel für die Ausführung des Prozesses (Checklisten, Arbeitsanweisungen etc.)?

- Ist der Geltungsbereich des Prozesses eindeutig festgelegt?

- Sind die Beziehungen des Prozesses zu anderen Prozessen beschrieben bzw. definiert?

Die obige Frageliste ist nur exemplarisch und nicht vollständig. In Unternehmen werden Fragelisten mit bis zu 100 Fragen verwendet.

Das Lesen von umfangreichen Prozessdokumentationen und deren Abgleich mit langen Checklisten ist sehr ermüdend. Die Erfahrung zeigt, dass mit zunehmender Seitenzahl die Intensität der Prüfung nachlässt. Die ersten Seiten werden noch genau gelesen. Dann nimmt die Genauigkeit immer mehr ab. Um die Schwächen einer visuellen Begutachtung zu kompensieren, wurde eine stärker formalisierte Version des Reviews entwickelt, der „Walk Through“, der sich überwiegend auf das Prozessmodell bezieht. 


\subsubsection{Walk Throughs}

Ähnlich wie bei der Code Inspection in der Programmierung, wird beim Walk Through ein Prozess gemeinsam mit ausgewählten Prozessbeteiligten Schritt für Schritt besprochen. Um das schrittweise Durchgehen lebendiger zu gestalten, kann eine formale Prozessbeschreibung mithilfe eines praktischen Beispiels durchlaufen werden. Ein Prozessbeteiligter geht anhand eines konkreten Beispiels die Geschäftsprozessbeschreibung schrittweise durch. $\mathrm{Zu}$ jedem Prozessschritt stellt ein Experte gezielte Fragen, um die Effektivität der Prozessbeschreibung zu hinterfragen.

Es werden beispielsweise das Verständnis der Fachbegriffe, die fachliche Notwendigkeit sowie die Vollständigkeit der Prozessbeschreibung hinterfragt. Auf diese Weise wird die Prozessbeschreibung überprüft. Ein Walk Through wird mit etwa zwei bis vier Prozessbeteiligten durchgeführt, die verschiedene Benutzergruppen vertreten.

Die „Autoren“ der Prozessbeschreibung (beispielsweise Prozess-Manager), sollten sich dabei im Hintergrund halten, damit Kritik offen formuliert werden kann. Alle Kritikpunkte und Anregungen werden gesammelt, dokumentiert und anschließend mit den Prozess-Beteiligten ausgewertet. Diese Auswertung führt zu einer Überarbeitung des Prozesses. Das folgende Bild gibt einen Eindruck von einem Walk-Through-Workshop. Es zeigt auch, dass dies bei der Verwendung von Swim-Lane-Methoden im Rahmen der Modellierung zu einer aufwendigen Angelegenheit werden kann. Mit Swim Lanes können Prozessmodelle nicht hierarchisch beschrieben werden, sodass komplexe Modelle sehr viel Platz benötigen.

Die schrittweise Analyse eines Prozesses kann durch entsprechende Werkzeuge unterstützt werden. Das verwendete Werkzeug zeigt das Prozessmodell am Bildschirm, und der aktuelle Prozessschritt wird farblich hervorgehoben.

\subsubsection{Rollenspiele}

Die nächste Steigerung für eine erlebbare Überprüfung von Prozessmodellen sind Rollenspiele. Diese sind insbesondere dann gut einsetzbar, wenn kommunikationsorientierte Modellierungssprachen verwendet werden. Die Handelnden sind dann bereits identifiziert und diese Rollen werden dann geeigneten Personen zugeordnet. Von einem Spielleiter werden Prozessanstöße ausgelöst und die notwendigen Eingaben zur Verfügung gestellt. Diese Prozessinstanzen werden dann von den einzelnen Rolleninhabern gemäß den Prozessbeschreibungen abgearbeitet. Diese „Prozessabläufe“ werden von anderen Betroffenen beobachtet und die identifizierten Auffälligkeiten werden notiert. Nach der Abarbeitung von einigen Prozessinstanzen werden die Befunde bewertet und notwendige Anpassungen identifiziert.

Die Ausführung von Rollenspielen kann durch geeignete IT-Werkzeuge unterstützt werden. Die Rolleninhaber eines Rollenspiels erhalten ihre Rollenbeschreibung nicht auf Papier, sie werden mittels Software, die insbesondere die Ablauflogik implementiert, durch den Prozess geführt. Diese Software wird dabei unmittelbar und automatisch aus dem Prozessmodell generiert. Voraussetzung dafür ist dass die Semantik der verwendeten Prozessmodellierungssprache eindeutig definiert ist. Dies ist beispielsweise 
für BPMN bedingt und für EPKs gar nicht der Fall, jedoch bei S-BPM dank einer eindeutigen, formalen Semantik erfüllt.

Das folgende Bild (siehe Abb. 5.17) zeigt, wie eine IT-gestützte Validierung aussehen kann. Der Vorteil eines IT-gestütztes Rollenspiels ist, dass die Vorbereitungszeit sehr gering ist und das Prozesserleben schon sehr nahe an der späteren Prozessausführung liegt.

\subsubsection{Optimierung}

Nach der Prüfung der Effektivität (liefern die Prozesse überhaupt das gewünschte Ergebnis?), muss überprüft werden, ob das Ergebnis mit dem geringsten möglichen Einsatz von Ressourcen zustande kommt. Eine Optimierung durch manuelle Prüfung, Walk Throughs oder Rollenspiele ist nur sehr eingeschränkt möglich. Die hierbei gewonnenen Erkenntnisse liefern nur eine Annäherung für die Ermittlung des Ressourcenbedarfs bei einer angenommenen Anzahl von Prozessdurchläufen. Eine systematische Ermittlung des Ressourcen- und Zeitbedarfs ist nur durch eine Simulation möglich. Voraussetzung für eine Simulation ist allerdings, dass das Prozessmodell ausführbar ist.

Bei der Simulation von Geschäftsprozessen werden die von einem Prozess verarbeiteten Geschäftsereignisse zufällig gemäß einer angenommenen Wahrscheinlichkeitsverteilung

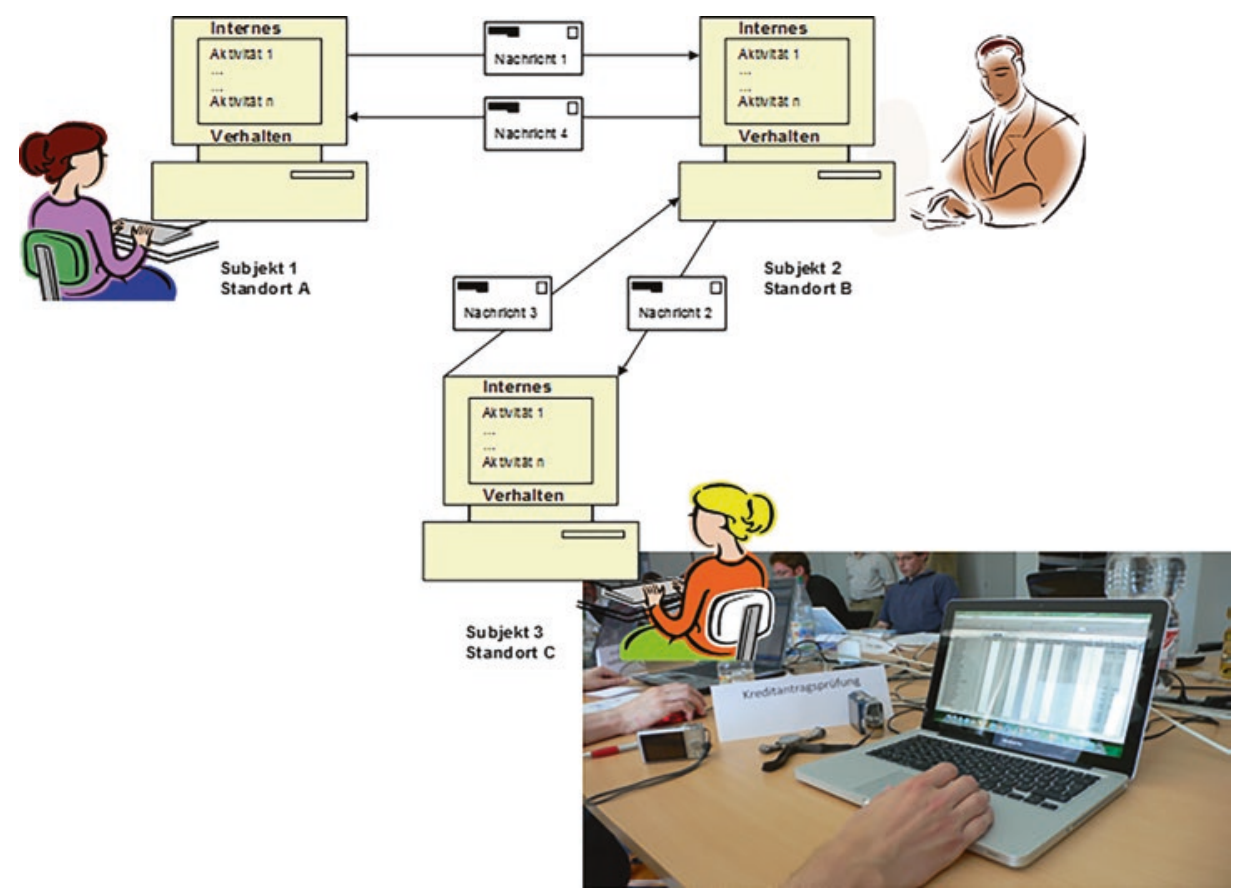

Abb. 5.17 Szenerie für ein IT-gestütztes Rollenspiel 
erzeugt. In der Regel ist dies die Exponentialverteilung mit einem vermuteten oder durch Beobachtung ermittelten Erwartungswert. Den einzelnen Arbeitsschritten werden die entsprechenden Ressourcen mit der benötigten Arbeitszeit zugeordnet. Die benötigte Arbeitszeit folgt in der Regel einer Normalverteilung mit aus Beobachtung ermittelten Erwartungswerten und Standardabweichungen.

Im Rahmen der Simulationsläufe werden Informationen über die Ablauffähigkeit von Prozessen, über Prozess-Schwachstellen und Ressourcenengpässe geliefert. Auf Basis der simulierten Prozesskennzahlen können bereits im Vorfeld kostenintensiver Prozessänderungen innerhalb eines Unternehmens verschiedene Alternativen bewertet und ein realitätsgetreues Benchmarking durchgeführt werden.

Moderne Werkzeuge und Simulationsmethoden ermöglichen die Analyse und Optimierung der Prozesse bezüglich der Kosten, der Durchlaufzeiten, der Auslastung oder der Engpässe. Zusätzlich bildet die Simulation der Geschäftsprozesse eine Ausgangsbasis zur Einführung der Prozesskostenrechnung anstelle der relativ ungenauen Zuschlagskalkulation. Die Gewinne bzw. Verluste der einzelnen Bereiche werden damit frühzeitig transparent.

Die Durchführung einer Simulationsuntersuchung setzt wie bereits erwähnt eine präzise Beschreibung des betrachteten Prozesses voraus. Dies bedeutet, dass zur Definition des Prozessablaufes eine formale Methode verwendet wird. Zusätzlich sind möglichst genaue Kenntnisse über die Wahrscheinlichkeitsverteilungen, deren Parameter und der untersuchten Kennzahlen notwendig. In der Praxis werden Simulationen wegen des damit verbundenen hohen Aufwandes nicht häufig eingesetzt, obwohl die gewonnenen Erkenntnisse überzeugend sein können.

\section{Literatur}

1. Seel, N. M. (1991). Weltwissen und mentale Modelle. Göttingen u. a.: Hogrefe.

2. Allee, V. (2003) The Future of Knowledge. Increasing Prosperity through Value Networks, Butterworth-Heinemann, Amsterdam.

3. Senge, P. (1990). The fifth discipline: The art and science of the learning organization. New York: Currency Doubleday.

4. Augl, M., \& Stary, C. (2015). Communication-and Value-Based Organizational Development at the University Clinic for Radiotherapy-Radiation Oncology. In S-BPM in the Wild (pp. 35-53). Springer International Publishing.

5. Augl, M., \& Stary, C. (2017). Stakeholders as Mindful Designers: Adjusting Capabilities Rather Than Needs in Computer-Supported Daily Workforce Planning. In Designing Healthcare That Works (pp. 95-112), Elsevier.

6. Fleischmann, A., Schmidt, W., Stary, C., \& Augl, M. (2013). Agiles Prozessmanagement mittels Subjektorientierung. HMD Praxis der Wirtschaftsinformatik, 50(2), 64-76.

7. Stary, C. (2014). Non-disruptive knowledge and business processing in knowledge life cycles-aligning value network analysis to process management. Journal of Knowledge Management, 18(4), 651-686. 
8. Giaglis, G. M. (2001). A taxonomy of business process modeling and information systems modeling techniques. International Journal of Flexible Manufacturing Systems, 13(2), 209-228.

9. Lin, F. R., Yang, M. C., \& Pai, Y. H. (2002). A generic structure for business process modeling. Business Process Management Journal, 8(1), 19-41.

10. Pinggera, J., Soffer, P., Fahland, D., Weidlich, M., Zugal, S., Weber, B., ... \& Mendling, J. (2015). Styles in business process modeling: an exploration and a model. Software \& Systems Modeling, 14(3), 1055-1080.

11. Recker, J., Rosemann, M., Indulska, M., \& Green, P. (2009). Business process modeling-a comparative analysis. Journal of the Association for Information Systems, 10(4), 1.

12. Zur Muehlen, M., \& Recker, J. (2013). How much language is enough? Theoretical and practical use of the business process modeling notation. In Seminal Contributions to Information Systems Engineering (pp. 429-443). Springer, Berlin, Heidelberg.

Open Access Dieses Kapitel wird unter der Creative Commons Namensnennung - Nicht kommerziell - Keine Bearbeitung 4.0 International Lizenz (http://creativecommons.org/licenses/ by-nc-nd/4.0/deed.de) veröffentlicht, welche die nicht-kommerzielle Nutzung, Vervielfältigung, Verbreitung und Wiedergabe in jeglichem Medium und Format erlaubt, sofern Sie den/die ursprünglichen Autor(en) und die Quelle ordnungsgemäß nennen, einen Link zur Creative Commons Lizenz beifügen und angeben, ob Änderungen vorgenommen wurden. Die Lizenz gibt Ihnen nicht das Recht, bearbeitete oder sonst wie umgestaltete Fassungen dieses Werkes zu verbreiten oder öffentlich wiederzugeben.

Die in diesem Kapitel enthaltenen Bilder und sonstiges Drittmaterial unterliegen ebenfalls der genannten Creative Commons Lizenz, sofern sich aus der Abbildungslegende nichts anderes ergibt. Sofern das betreffende Material nicht unter der genannten Creative Commons Lizenz steht und die betreffende Handlung nicht nach gesetzlichen Vorschriften erlaubt ist, ist auch für die oben aufgeführten nicht-kommerziellen Weiterverwendungen des Materials die Einwilligung des jeweiligen Rechteinhabers einzuholen.

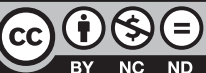




\section{Umsetzung}

Die bisherigen Ausführungen haben mit der Spezifikation effektiver und effizienter Prozesse die Grundlagen gelegt für deren Implementierung. Im Anschluss an diese vorbereitenden Aktivitäten befassen wir uns in der Folge mit der Umsetzung der Prozessspezifikation in einer Ausführungsumgebung und der Abwicklung von Prozessinstanzen im Echtbetrieb. Die Umsetzung einer Prozessbeschreibung in einen ausführbaren Prozess umfasst die Aktivitätsbündel der organisatorischen Implementierung, der IT-Implementierung und des Betriebs mit dem Monitoring. Abb. 6.1 zeigt die Einordung dieser Aktivitäten in das Prozessmanagementmodell.

In den folgenden Abschnitten werden, ausgehend von Überlegungen zur Dokumentation der erarbeiteten Prozessspezifikation, ausgewählte Methoden für die Aktivitätsbündel der organisatorischen und IT-Implementierung sowie für den Betrieb und das Monitoring von Prozessen vorgestellt.

Die Aktivitäten des Prozessmanagementmodells bieten den konzeptionellen Rahmen für die Umsetzung des Geschäftsprozessmanagements in ein Handlungssystem. Wie bereits in den vorangestellten Kapiteln diskutiert, stellen die Phasen zwar ein gewisses Ordnungskriterium dar, können aber grundsätzlich in beliebiger Reihenfolge durchlaufen werden. Jede der Phasen beinhaltet dabei ein Bündel an Aktivitäten, die typischerweise in ihr angewendet werden. Jeder Geschäftsprozess befindet sich in einem bestimmten Augenblick in einer bestimmten Phase - oder in anderen Worten, in einem bestimmten Zustand: er ist modelliert, er ist in Kraft gesetzt, er wird ausgeführt, er wird analysiert. Das Lebenszyklusmodell definiert damit einen Phasen- oder Zustandsraum für Geschäftsprozesse. 
Abb. 6.1 Einordnung der Umsetzung in das Prozessmanagementmodell

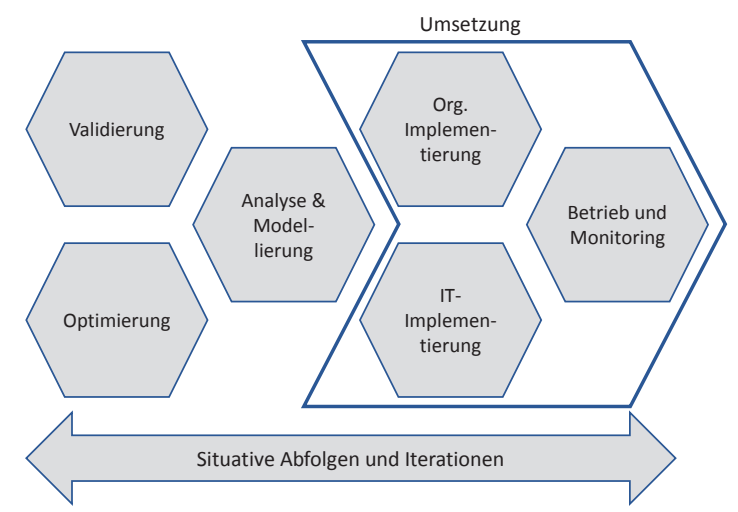

\subsection{Prozessdokumentation}

Da die Geschäftsprozesse eines Unternehmens definieren, wie Produkte und Dienstleistungen entwickelt, hergestellt und geliefert werden, ist es zweckmäßig eine Prozessdokumentation zu erstellen. Diese sollte naturgemäß allen Mitarbeitern zentral zur Verfügung gestellt werden. Die Dokumentation muss spätestens zu Beginn der Umsetzung (Implementierung) vorliegen. Die Geschäftsprozessdokumentation ist das Ergebnis der Aktivitätsbündel während der Vorbereitung.

Heutzutage bietet sich dazu die Form digitaler Dokumente an. Die Bereitstellung erfolgt dabei idealerweise über ein üblicherweise vorhandenes Intranet; damit kann auch in groben Zügen eine einfache Zugriffskontrolle gestaltet werden. Die Dokumente selbst können beispielsweise nicht veränderbare PDF-Dateien (eventuell auch digital signiert), oder HTML-Dateien, die über einen Browser betrachtet werden können, sein.

Für kleinere Organisationen und Unternehmen reicht es zumeist aus, wenn für die Erstellung und Wartung der Prozessdokumentation die üblicherweise verwendete Bürosoftware genutzt wird. Für umfangreichere Sammlungen erscheint die Nutzung spezifischer Software, sogenannter Business Prozess Management Systeme (BPMS), überlegenswert. Jedenfalls muss die gewählte Form die Menschen, die damit arbeiten, und die darauf aufbauenden Managementsysteme bestmöglich unterstützen. Auch sollten die Dokumente eine eindeutige Bezeichnung, eine Versionsnummer und ein Datum beinhalten. Soll eine Textverarbeitung benutzt werden, empfiehlt sich eine Formatvorlage, um ein einheitliches Erscheinungsbild und ebensolche Strukturen vorzugeben. Des Weiteren empfiehlt sich eine Liste mit allen Dokumenten inklusive Historie, um einen allgemeinen Überblick zur Verfügung zu stellen.

Verantwortlich für die Aktualität und Vollständigkeit einer Prozessdokumentation ist der Prozesseigner bzw. Prozesskoordinator. Das Prozess-Office gibt vor, was eine Prozessdokumentation beinhalten soll und stellt entsprechende Vorlagen und Erklärungen bereit. Falls ein dediziertes IT-System (BPMS) genutzt werden soll, kann 
die Schulung aller Mitarbeiter - abhängig davon, welche Rolle sie einnehmen - eindringlich angeraten werden. Im Sinne eines geplanten Veränderungsprozesses (Change Management), sollten die Mitarbeiter in angemessener Form auch in den Auswahl- oder Entwicklungsprozess eingebunden werden.

Folgende Inhalte einer Prozessdokumentation haben sich grosso modo bewährt; selbstverständlich sind nicht immer alle Punkte für jeden Geschäftsprozess relevant und können bei Bedarf auch weitere Punkte ergänzt werden:

- Ziel des Prozesses - ein kurzer beschreibender Text, in dem der Bezug des Prozesses zu übergeordneten Unternehmenszielen erläutert wird (Strategiebeitrag).

- Auslöser des Prozesses - Angabe, durch welches Ereignis eine Prozessinstanz gestartet wird und wer dieses Ereignis erzeugen kann.

- Input - Auflistung und Beschreibung der Informationen, Dokumente und auch physikalischen Artefakte, die als Input benötigt werden und von wem sie bereitgestellt werden.

- Output - Auflistung und Beschreibung der Informationen, Dokumente und physikalischen Artefakte, die vom Prozess erzeugt werden; eventuell auch Qualitätskriterien pro Kunde.

- Gültigkeitsbereich und organisatorische Rahmenbedingungen - gegebenenfalls Abgrenzung zu anderen Geschäftsprozessen, oder organisatorische Einschränkung (z. B. nur gültig für den Geschäftsbereich Großkunden).

- Definition der Begriffe und Abkürzungen - im Dokument verwendete Abkürzungen; Tipp: wenn man diese auch gleich in einem unternehmensweiten Verzeichnis konsolidiert sammelt, erhält man ein Glossar der wichtigsten Begriffe in einem Unternehmen, sowie einheitliche Definitionen in allen Prozessdokumentationen.

- Übersichtsbeschreibung des Prozessmodells - textuelle Darstellung der Handelnden/Rollen und der Prozessschritte.

- Prozessmodell - Ein Prozessmodell (visuelle Darstellung), erstellt in einer vereinbarten Modellierungssprache; für die Erstellung der Prozessmodelle sollte es einheitliche Regelungen geben, um einen Wildwuchs zu vermeiden (z. B. bezüglich Farben und Beschriftung von Notationselementen).

- Technische Rahmenbedingungen - Auflistung und Beschreibung technischer Hilfsmittel, die im Prozess benötigt werden. Darstellung der informationstechnischen Unterstützung bzw. Abhängigkeiten, z. B. der Verweis auf bestimmte Module eines ERP-Systems.

- Feedback Mechanismen - Darstellung, wie Prozessbeteiligte Probleme bei der Ausführung (z. B. falsch oder nicht ausreichend modellierte Logik) artikulieren oder Verbesserungsvorschläge einbringen können. Dies sollte eine im Geschäftsprozessmanagement implementierte Standardprozedur sein.

- Ausnahmen - eventuelle Ausnahmen vom Prozessmodell, d. h. Aktivitäten, die nicht im Prozessmodell berücksichtigt werden, da entsprechende Fälle nur selten vorkommen. 
- Schnittstellen mit anderen Prozessen - eine Darstellung, in welchen anderen Geschäftsprozessen der Output eines Prozesses benötigt wird (definiert damit den Prozesskunden bzw. dessen Erwartung).

- Kennzahlen (PPI) - Liste, Definition und Erklärung der vorgesehenen Prozesskennzahlen.

- Leistungsmessungen - Darstellung, wie und wann die Kennzahlen zu messen und zu berechnen sind. Verweis auf eventuell verfügbare andere Dokumente.

- Berichtswesen - Darstellung, in welcher Form und wann die Prozesskennzahlen an wen zu berichten sind; Verweis auf eventuell verfügbare andere Dokumente.

- Eskalationen - Definition von Prozeduren, die anlaufen sollen, sobald der Toleranzbereich von Kennzahlen verletzt wird.

- Audits und Compliance - Verweis auf entsprechende Richtlinien. Falls einzelne Aktivitäten in einem Geschäftsprozess aus Gründen der Compliance aufgenommen wurden, sollte dies dokumentiert werden, damit diese nicht im Rahmen einer Effizienzverbesserung eliminiert werden. Es empfiehlt sich, solche Aktivitäten in einer visualisierten Darstellung farblich zu markieren. Ferner hat sich in der Praxis als hilfreich erwiesen, (tabellarisch) zu dokumentieren, in welchen Geschäftsprozessen die Einhaltung bestimmter Normen und Gesetze notwendig ist. Ähnliche Überlegungen gelten für externe (z. B. Qualitätsnormen) oder interne Audits (z. B. Risikoanalysen).

\subsection{Verknüpfung von Elementen der Unternehmensarchitektur}

\subsection{1 Überblick}

Wie in Kap. 1 und 2 angesprochen, sind Geschäftsprozesse Teil der Unternehmensarchitektur. Unternehmensarchitekturen gehen auf die internen Aspekte und Strukturen eines Unternehmens ein. Sie sind im Wesentlichen Modelle der Interna eines Unternehmens und umfassen nicht nur organisatorische, sondern auch technische Aspekte, insbesondere die eingesetzte IT. Bei der Umsetzung der Prozessmodelle gilt es nun deren Bezug zu den vorhandenen Ressourcen herzustellen. Abb. 6.2 zeigt die einzelnen Stufen vom Prozessmodell zur ausführenden Prozessinstanz.

In einem Prozessmodell werden die Handelnden, die Handlungen, deren Reihenfolgen und die Objekte, auf denen die Handlungen ausgeführt werden, beschrieben. Handlungen (Aktionen, Aktivitäten) können von Menschen, Softwaresystemen, physikalischen Systemen oder einer Kombination aus diesen Grundtypen von Handelnden ausgeführt werden. Wir bezeichnen sie als Aufgabenträger. So kann ein Software-System die Aktion „Berechnung des Steuersatzes“ automatisch erledigen, während ein Mensch in Kombination mit einem Programm die Aktivität „Erfassen der Bestellung“ ausführt. Der Mensch tippt dabei die Bestelldaten über eine Bildschirmmaske ein. Die Software prüft die eingegebenen Daten auf Plausibilität und speichert sie ab. 


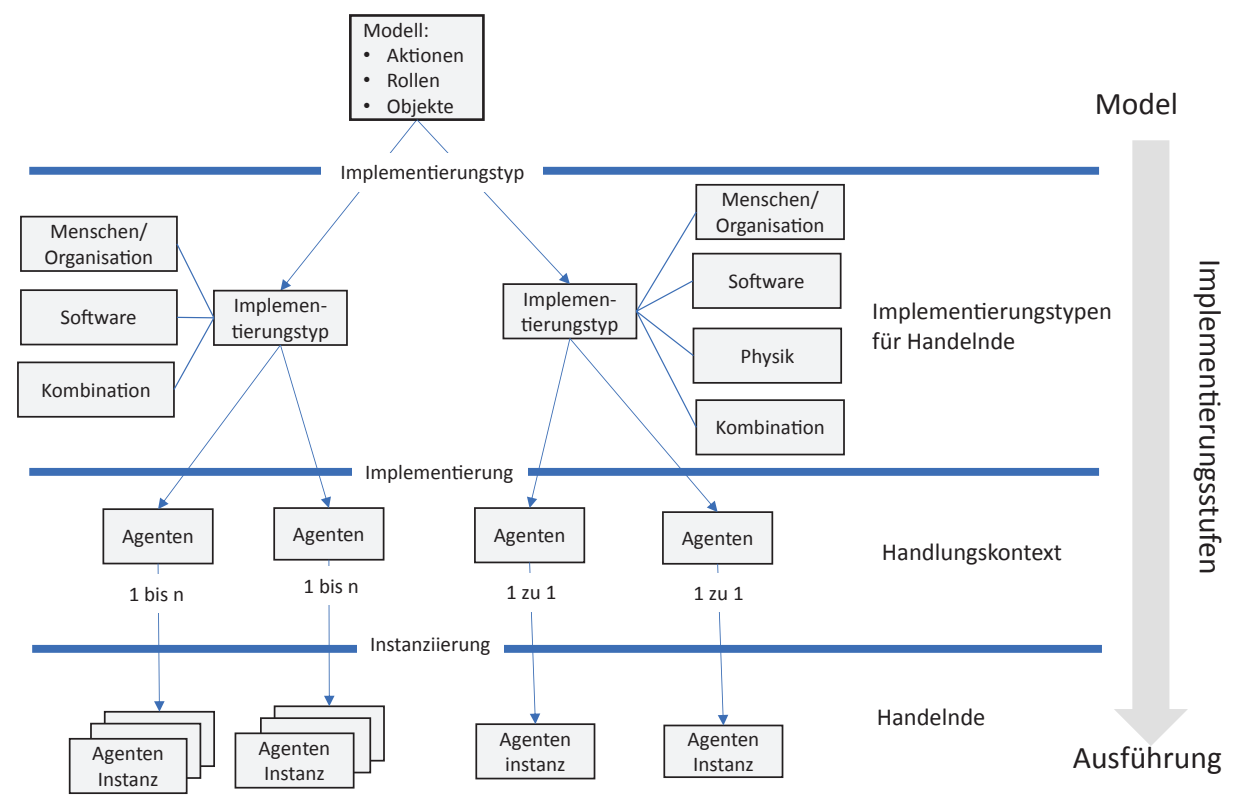

Abb. 6.2 Vom Prozessmodell zur Prozessausführung

Tätigkeiten können aber auch ausschließlich manuell ausgeführt werden, etwa wenn ein Lagerarbeiter einen Kommissionierungsauftrag auf Papier bekommt, diesen ausführt, auf dem Auftrag als ausgeführt kennzeichnet, und an den Lagermeister zurückgibt. Bei der Erstellung eines Prozessmodells ist oft noch nicht bekannt, welche Typen von Handelnden welche Aktionen ausführen. Deshalb kann es sinnvoll sein, zu Beginn einer Prozessbeschreibung davon zu abstrahieren, in dem man abstrakte Handelnde einführt. Eine Modellierungssprache sollte erlauben, solche Abstraktionen zu verwenden. Dies bedeutet, es muss bei der Definition der Prozesslogik noch keine Aussage gemacht werden, durch welchen Typ ein Akteur realisiert wird. In S-BPM repräsentieren die Subjekte abstrakte Handelnde. In BPMN können Pools oder Swim Lanes als abstrakte Handelnde interpretiert werden, während man in EPKs dafür Rollen verwenden kann.

In der Beschreibung der Ablauflogik eines Prozesses werden auch die einzelnen Aktivitäten noch unabhängig von ihrer Realisierung beschrieben. Beispielsweise wird bei einer Aktion „Erstellen eines Kommissionierungsauftrags“ nicht festgelegt, ob hier der Bearbeiter ein Papierformular oder eine Bildschirmmaske ausfüllt, oder ob ein Software-System diesen vollautomatisch erzeugt. Bei Aktionen wird also nicht beschrieben, mit welchen Mitteln etwas geschieht, sondern nur was geschieht.

Dies steht natürlich in Zusammenhang mit dem Implementierungstyp für den Handelnden als Aufgabenträgertyp. Sobald festgelegt wird, welche Typen von Handelnden den einzelnen Aktionen zugewiesen werden, ist auch die Art der Realisierung einer Aktivität definiert. Darüber hinaus ist zu definieren, auf welchem logischen oder physischen Objekt eine Aktion ausgeführt wird. Logische Objekte sind Datenstrukturen, 
deren Daten durch die Aktivitäten manipuliert werden. Papierformulare stellen eine Mischung zwischen logischen und physischen Objekten dar, während ein Werkstück, auf dem die Aktion ,Entgraten“ stattfindet, ein rein physisches Objekt ist. Es besteht also ein enger Zusammenhang zwischen dem Aufgabenträgertyp, den Aktionen und den zugehörigen Objekten, auf oder mit denen die Akteure Handlungen ausführen.

Ein Prozessmodell kann in verschiedenen Bereichen einer Organisation zum Einsatz kommen. Die Prozesslogik wird dann in den jeweiligen Bereichen unverändert übernommen. Es kann allerdings nötig sein, die einzelnen Handelnden und Handlungen unterschiedlich zu implementieren. So können in einer Umgebung bestimmte Handlungen von Menschen und in einer anderen die gleiche Aktivität von Software-Systemen ausgeführt werden. Wir bezeichnen solche verschiedenen Verwendungsumgebungen für ein Prozessmodell im Folgenden als Kontext. Für ein Prozessmodell kann es also variierende Kontexte geben, in denen die Realisierungstypen für Akteure und Aktionen voneinander abweichen können.

In BPMN kann der Modellierer für jeden Task separat festlegen, ob es sich um einen sogenannten Human Task, Service Task oder User Task handelt. User Tasks führen Menschen zusammen mit Software aus, wie z. B. das Ausfüllen eines Kommissionierungsauftrags am Bildschirm. Dies bedeutet, dass die Beschreibung des Implementierungstyps in BPMN einen Teil der Prozesslogik darstellt. Da in BPMN Pools und Swim Lanes als Handelnde interpretiert werden können, muss darauf geachtet werden, dass keine Widersprüche mit dem Implementierungstyp von Tasks innerhalb z. B. eines Pool entstehen. Legt der Designer beispielsweise fest, dass ein Pool nur durch Menschen ausgeführt wird, kann dieser Pool keine Service oder User Tasks enthalten. Da in BPMN die Definition des Implementierungstyps Bestandteil des Ablaufmodells ist, muss unter Umständen für jeden Kontext ein eigenes Prozessmodell erstellt werden.

In S-BPM werden Handelnde nicht einzelnen Aktivitäten zugeordnet, sondern der Typ des Handelnden einem gesamten Subjekt. Diese Zuordnung ist in S-BPM nicht Teil der Prozesslogik, sondern erfolgt je Prozess in einer separaten zweispaltigen Tabelle. In der linken Spalte findet sich der Subjektname und in der rechten der Implementierungstyp. Gibt es für ein Ablaufmodell mehrere Kontexte, so wird für jeden von ihnen eine eigene Zuordnungstabelle erstellt.

Die Zuordnung des Implementierungstyps bildet den Übergang zwischen der Prozesslogik und deren Implementierung. Anschließend muss noch definiert werden, welche Personen, Softwaresysteme und physikalische Systeme die Handelnden repräsentieren, und wie die einzelnen Aktionen konkret realisiert werden. In den folgenden Unterkapiteln werden diese Aspekte detailliert beschrieben.

\subsubsection{Menschen und Organisation}

Für jeden Kontext gilt es für alle Handlungen, an denen Menschen beteiligt sind, die jeweiligen Aufgaben- oder Handlungsträger, und damit konkret die ausführenden Personen oder Organisationseinheiten festzulegen. 
In Unternehmen und Verwaltungen gibt es Personen mit unterschiedlicher Ausbildung, Qualifikationen, Vorlieben und Interessen. Es gibt Kaufleute, Entwickler, Handwerker usw., welche die anfallenden Tätigkeiten erledigen. Organisationen kann man daher auch als strukturierte Ressourcenpools bezeichnen. Je nach Art und Umfang der Aufgaben bildet die Organisation Einheiten, in denen die jeweiligen Spezialisten dafür zusammengefasst werden. So existieren Abteilungen für den Einkauf mit Beschaffungsexperten, oder Entwicklungsabteilungen, die aus mehreren Entwicklungsingenieuren und sonstigen Fachleuten gebildet werden. Der Bezug zwischen den Personen und Organisationen in der Aufbauorganisation zu den abstrakten Handelnden in den Prozessen kann statisch oder dynamisch hergestellt werden.

\section{Statische Zuordnung}

Im einfachsten Fall gibt die bereits erwähnte zweispaltige Tabelle Aufschluss darüber, welchem im Prozess definierten Akteur (Spalte 1) welche Personen oder Organisationen bzw. Organisationseinheiten zugeordnet sind (Spalte 2). Die zweite Spalte kann auch eine Organisation bzw. Organisationseinheit enthalten. Dann sind ihre Mitglieder dem abstrakten Handelnden zugeordnet. Damit wird erreicht, dass bei Krankheit oder Urlaub eine beliebige Person aus der Organisation die im Prozess anfallenden Aufgaben übernehmen kann. Außerdem lässt sich so auch die Arbeitslast verteilen, wenn Handlungen aus mehreren Prozessabläufen (Prozessinstanzen) abgearbeitet werden müssen.

Bei BPMN können Pools, Swim Lanes und einzelne Aktionen separat einem Ausführenden zugeordnet werden. Hier ist darauf zu achten, dass bei der Verwendung aller Zuordnungsmöglichkeiten keine Inkonsistenzen entstehen. Wenn ein Software-System eine Swim Lane als Handelnder ausführt und in dieser Swim Lane eine Human Task liegt, so ist nicht klar, was dies bedeutet. Für das BPMN-basierte Werkzeug von Bonitasoft [1] wurden deshalb Actors eingeführt. Sie sind Platzhalter für Aufgabenträger, denen dann, ähnlich den Subjekten in der Subjektorientierung, konkret Handelnde zugeordnet werden.

Bei S-BPM wird jeweils ein komplettes Subjekt in die Organisation eingebettet, d. h. die Zuordnung gilt dann für alle Aktionen in einem Subjekt.

\section{Dynamische Zuordnung}

In vielen Prozessen kann die Zuordnung der Handelnden aus dem Prozess zu Personen und Organisationen nicht statisch festgelegt werden. So kann bei einem Geschäftsreiseantrag der Bearbeiter davon abhängen, ob es sich um eine Inlands- oder Auslandsreise handelt. Während die Ablauflogik in beiden Fällen gleich sein kann, unterscheiden sich möglicherweise die ausführenden Personen, beispielsweise, weil ein Mitarbeiter besondere Expertise für internationale Reisen mit Visaangelegenheiten besitzt.

In solchen Fällen ist es sinnvoll, die handelnden Personen oder Organisationseinheiten erst beim Ablauf der Prozesslogik beispielsweise in Abhängigkeit von Datenwerten in Geschäftsobjekten (hier im Reiseantrag) zu ermitteln und zuzuordnen. 
Für eine flexible, dynamische Zuordnung der Bearbeiter sind in der Regel Tabellen nicht mehr ausreichend, sondern es sind programmiersprachliche Konstrukte notwendig. Lavall et al. [2] zeigen, wie eine solche Sprache verwendet werden kann, um subjektorientierte Prozessmodelle in Organisationen einzubetten. Da in BPMN die Zuordnung der Bearbeiter über Pools, Lanes und Tasks möglich ist, gestaltet sich die Beschreibung der Zuordnung dort als sehr komplex. Verschiedene BPMN-basierte Werkzeuge wie Bonita [3] oder Activiti [4] integrieren Prozesse in die Organisation durch Programmierung in Java bzw. XML.

\subsubsection{Physikalische Infrastruktur}

Insbesondere in Fertigungsprozessen sind physikalische Systeme als Handelnde beteiligt. So können einer Maschine Rohteile über ein Transportsystem zugeführt werden, die Maschine bearbeitet die Rohteile und die bearbeiteten Teile werden zum nächsten Bearbeitungsschritt weiter transportiert. Wird eine solche Maschine als Subjekt oder Pool betrachtet, so werden die zugeführten Teile als zu empfangende und die abtransportierten Teile als zu sendende Nachrichten modelliert. Der Modellierer stellt den Bearbeitungsschritt als einzelnen Task in einem Subjekt oder Pool dar.

\subsubsection{IT-Infrastruktur}

Digitalisierung in der Wirtschaft bedeutet, Prozesse mit möglichst umfassender Unterstützung durch Software-Systeme umzusetzen. In entsprechenden Szenarien erledigen weitgehend Computer oder Maschinen die Tätigkeiten, menschliche Eingriffe werden soweit möglich und sinnvoll reduziert. Wesentliche Aspekte dabei sind:

- Die Steuerung des Prozessablaufs:

Die im Prozessablauf beschriebenen Handlungsfolgen werden automatisch durch Computerprogramme gesteuert.

- Die Ausführung von Handlungen:

Handlungen auf Datenobjekten können oft vollautomatisch durch entsprechende Computerprogramme ausgeführt werden.

Software-Systeme sind das Mittel zur Zusammenführung der verschiedenen Handlungstypen. So bindet beispielsweise eine Process Engine bei der Steuerung des Prozessablaufs die menschlichen und maschinellen Bearbeiter zur Laufzeit situationsgerecht in die Abarbeitung von Instanzen ein.

In den folgenden Ausführungen wird auf die Steuerung des Prozessablaufs und die Manipulation der zugehörigen Geschäftsobjekte durch Informationstechnologie eingegangen, jedoch noch ohne die Einbindung von Menschen oder physikalischen Systemen als Aufgabenträger näher zu betrachten. Diese ist Gegenstand von Abschn. 6.2.5. 


\begin{abstract}
Ablauf
Die Prozesslogik entspricht der Ablauflogik eines Computerprogramms. Die automatische vollständige Ausführung der Prozesslogik durch ein Computerprogramm ist nur bei stark strukturierten Prozessen möglich. Alle denkbaren Ablaufmöglichkeiten müssen abgedeckt sein. Es sind keine menschlichen Eingriffe vorgesehen. Um zu vermeiden, dass die beschriebene Prozesslogik von der Ausführungslogik des Computerprogramms abweicht, ist es hilfreich, wenn das Computerprogramm automatisch aus der Beschreibung der Prozesslogik abgeleitet werden kann. Dafür müssen folgende Voraussetzungen vorliegen:
\end{abstract}

- Die Syntax und Semantik der Sprache, in der die Prozesslogik beschrieben ist, muss präzise definiert sein.

- Die Beschreibung des Prozessablaufs muss in elektronischer Form vorliegen.

- Es muss ein Umsetzungsprogramm vorliegen, das die elektronische Form der Beschreibung der Prozesslogik liest und daraus, basierend auf der präzisen Semantik der Prozessbeschreibungssprache, ein Computerprogramm in einer geeigneten Programmiersprache erzeugt.

Die Bedeutung bereits heute weit verbreiteter verteilter Systeme nimmt mit der Entwicklung von Cloud und Edge Computing weiter zu. Dies kann bedeuten, dass auch Teile eines voll automatisierten Prozesses in einem verteilten System auf verschiedenen Rechnerknoten zur Ausführung kommen sollen. Da diese auf unterschiedlichen Technologien basieren können, müssen unter Umständen entsprechende Varianten für die automatische Umsetzung einer Ablaufbeschreibung in ein Computerprogramm verfügbar sein.

So können einzelne Subjekte einer S-BPM Beschreibung eines Prozesses auf unterschiedlichen Rechnerknoten eines verteilten Systems ablaufen. Dies kann bedeuten, dass der Programmcode für jedes Subjekt separat generiert werden muss. Vermeiden lässt sich dies, wenn der generierte Zielcode auf möglichst allen Knotentypen des verteilten Systems zur Verfügung steht, und dieses Zielsystem über ein Framework verfügt, durch das die auf verschiedenen Knoten laufenden Programmteile zusammenarbeiten können. Die Software-Komponenten synchronisieren ihre Zusammenarbeit in der Regel durch den Austausch von Nachrichten. Eine Programmiersprache, die dies ermöglicht, ist z. B. Java mit dem Framework AKKA, das den Nachrichtenaustausch über Rechnergrenzen hinweg erlaubt, auch ausgehend von S-BPM-Modellen [5].

In BPMN können einzelne Pools prinzipiell auf verschiedenen Rechnerknoten ausgeführt werden. Dies setzt voraus, dass eine Kommunikation zwischen den Pools über Rechnergrenzen hinweg möglich ist. Eine Recherche nach Möglichkeiten einer entsprechenden Codegenerierung für BPMN-Modelle im Frühjahr 2018 brachte keine Ergebnisse.

\title{
Aktivitäten und Daten
}

Bei vollständig digitalisierten Prozessen führen Computersysteme auch die im Prozessablauf enthaltenen Aktivitäten aus. Dies kann mittels Funktionen oder Services geschehen, 
die bereits in existierenden Anwendungsprogrammen verfügbar sind, und nur an den geeigneten Stellen in das Programm, das den Prozessablauf realisiert, eingefügt bzw. aufgerufen werden.

Für die Integration neu entwickelter Software für Aktivitäten kommen häufig Technologien zum Einsatz wie Web Services, REST-Schnittstellen oder einfache APIs. Datenbankzugriffe zur direkten Manipulation von Daten können abhängig von den Schnittstellen der verwendeten Datenbanksysteme analog eingebunden werden.

Bei der Verwendung von S-BPM gehören Daten zu einem Subjekt. Es ist nicht erlaubt, dass mehrere Subjekte auf gemeinsame Daten zugreifen. Möchten mehrere Subjekte die gleichen Daten z. B. in einer Datenbank nutzen, so muss diese in ein Subjekt , eingepackt“ werden. Dieses Subjekt nimmt dann die entsprechenden Anfragen von den anderen Subjekten entgegen, führt die gewünschte Aktion aus und schickt das Ergebnis an das anfordernde Subjekt zurück.

Aktivitäten zusammen mit ihren Daten werden in der Software-Technologie als Klassen bezeichnet. Mehrere solcher Klassen können zu Services zusammengeschlossen werden. In der IT wird dann von einer Service-orientierten Architektur gesprochen. Die Services werden entsprechend der Ablauflogik des Prozesses aufgerufen. Tasks in BPMN können durch Services realisiert werden. Analog dazu können die Funktionen innerhalb eines Subjekts implementiert werden.

Ein aktuelles Konzept zur Strukturierung von Software-Systemen sind Microservices [6]. Microservices beschreiben eine Architektur, bei der einzelne kleine fachliche Software-Bausteine unabhängig voneinander und, bei Bedarf, auf verschiedenen technischen Plattformen programmiert, getestet, abgenommen und bereitgestellt werden. Durch ihr Zusammenspiel über von der Programmiersprache unabhängige Schnittstellen lässt sich komplexe Anwendungssoftware realisieren. Dabei kommunizieren Microservices meist durch den Austausch asynchroner Nachrichten. Dies entspricht auch dem Konzept des Reactive Programming [7], dessen wesentliche Eigenschaft die Kopplung von Programmbausteinen durch asynchrone Nachrichten ist.

In BPMN können Pools als Microservices implementiert werden, falls alle Tasks in einem Pool vom gleichen Aufgabenträger ausgeführt werden.

Bei S-BPM sind Subjekte immer einem Aufgabenträger zugeordnet und kommunizieren über den asynchronen Austausch von Nachrichten. Deshalb entspricht ein subjektorientiert beschriebener Prozess mit Blick auf die IT-Architektur einer Microservice-orientierten Struktur, die den Anforderungen des Reactive Programming genügt.

\subsubsection{Kombinationen von Aufgabenträgern}

Tasks werden häufig nicht von einem einzigen Aufgabenträgertyp ausgeführt. Mit der zunehmenden Digitalisierung werden beispielsweise die Aufgaben, die ausschließlich Menschen ausführen, immer seltener. So erhält heute ein Lagerarbeiter seine Kommissionierungsaufträge häufig über ein Tablet, das mit einem IT-System verbunden 
ist. Nachdem er die Waren zusammengestellt hat, bestätigt er dies auch auf dem Tablet. Damit ist die Kommissionierungsaufgabe abgeschlossen. Das IT-System aktualisiert die Daten und stößt die Folgeaufgabe wie z. B. die Vorbereitung der Versanddokumente automatisch an. Die Kommissionierungsaufgabe wird also durch Mensch und IT als Typen von Aufgabenträgern ausgeführt, eine Kombination, die man in Geschäftsprozessen sehr häufig findet. Die IT übernimmt dabei zumindest die Steuerung der Prozesslogik und die Verwaltung der Daten, während Menschen Eingaben tätigen oder Maschinen bedienen.

Von Kleinrechnern mit entsprechender Software gesteuerte Maschinen lösen zunehmend Maschinen als rein mechanische Aufgabenträger ab. Diese eingebetteten Systeme (Embedded Systems) steuern die Mechanik und wickeln die Kommunikation mit anderen Maschinen oder übergeordneten Geschäftsanwendungen ab. Die Kommunikation mit anderen intelligenten Maschinen wird als horizontale Kommunikation und mit Geschäftsanwendungen als vertikale Kommunikation bezeichnet. Letztere verknüpft Produktionssysteme mit Geschäftsprozessen und ist ein wesentlicher Bestandteil der Industrie 4.0 Initiative.

In den folgenden Abschnitten wird nun detaillierter beschrieben wie die verschiedenen Aufgabenträger in die Implementierung der Prozessablauflogik integriert werden.

\section{Kombination Mensch-IT}

Die Kombination von Mensch und IT bei der Ausführung von Geschäftsprozessen ist der Kern deren Digitalisierung. Die IT übernimmt dabei zumindest die Steuerung der Prozesslogik, d. h. sie veranlasst entsprechend der Prozesslogik, dass die vorgesehenen Aufgaben von den jeweiligen Aufgabenträgern erledigt werden. Zusätzlich können Aufgaben von der IT direkt als Aufgabenträger ausgeführt werden, ohne dass Menschen eingebunden werden müssen. Kann eine Aufgabe nur durch menschliche Unterstützung erledigt werden, fordert die für die Ablauflogik zuständige Software über eine entsprechende Benutzungsschnittstelle den zuständigen menschlichen Aufgabenträger dazu auf. Dies kann zur Eingabe von Daten sein, aber auch zur Rückmeldung, dass der Benutzer eine manuelle Aktion ausgeführt hat.

IT-Systeme als Steuerungssoftware und als Handlungsträger für Aktivitäten übernehmen in der Regel auch die Speicherung der während einer Prozessausführung anfallenden Daten, sowohl Inhalte von Geschäftsobjekten, als auch Metadaten wie Zeitstempel für Beginn und Ende von Instanzen und Ausführungsschritten.

Eine umfassende Plattform zur Implementierung von Geschäftsprozessen, die Menschen und IT-Lösungen ausführen, wird als Workflow-Management-System bezeichnet. Die Workflow Management Coalition (WfMC) hat dafür ein Referenzmodell entwickelt. Abb. 6.3 zeigt die darin vorgesehenen Komponenten und Schnittstellen [8]. 


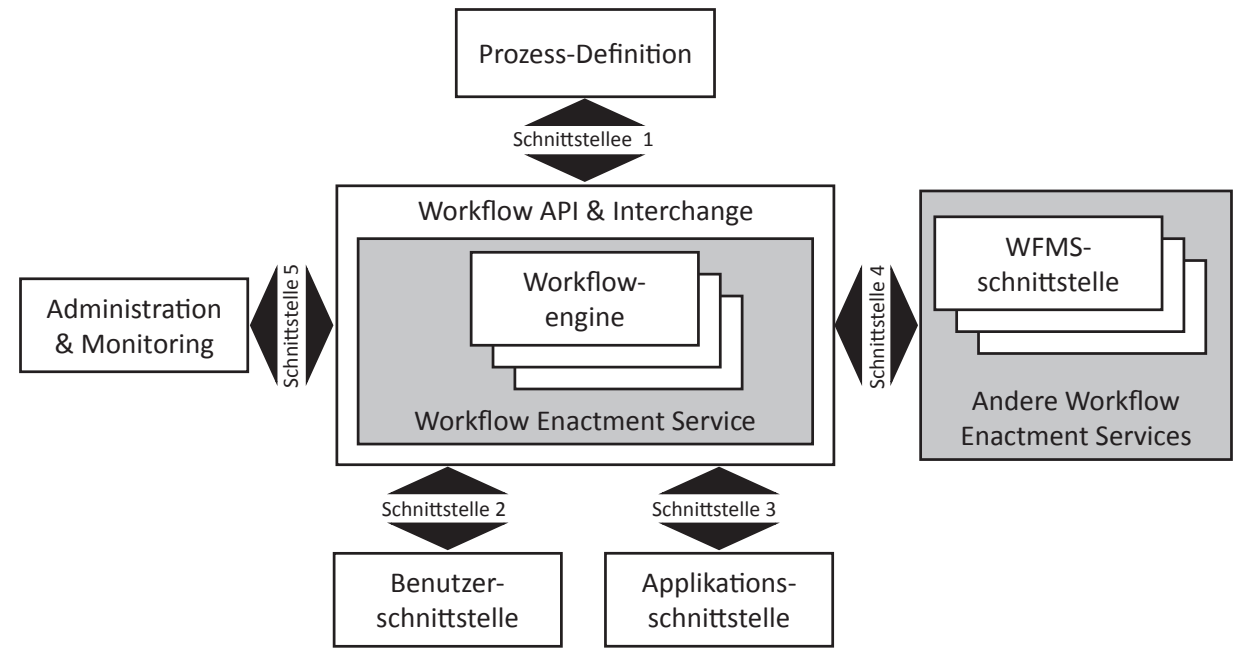

Abb. 6.3 Referenzmodell für Workflow-Management-Systeme (WFMS)

Die Schnittstellen zwischen den einzelnen Komponenten sind wie folgt definiert:

- Prozessdefinition (Schnittstelle 1)

Schnittstelle zwischen Prozessdefinition, Modellierungswerkzeugen und der Workflow Engine (Ausführungsumgebung)

- Benutzungsschnittstelle (Schnittstelle 2)

APIs für Clients, um Dienste von der Workflow Engine anzufordern, damit der Prozessfortschritt und die Aktionen kontrolliert werden können.

- Applikations-Schnittstelle (Schnittstelle 3)

APIs, die der Workflow Engine erlauben, Applikationen aufzurufen und zu nutzen.

- Workflow-Management-System-Schnittstelle (Schnittstelle 4)

Standard-Schnittstelle für den Austausch mit anderen Workflow-Systemen

- Administration und Monitoring (Schnittstelle 5)

Schnittstelle für Werkzeuge zur Kontrolle der Prozesse und zur Überwachung

Die wesentlichen Komponenten im Referenzsystem der WfMC sind der Workflow Enactment ${ }^{1}$ Service und die Workflow Engine. Die Aktionen und ihre Reihenfolge werden in der Prozessdefinition festgelegt und durch die Workflow Engine gesteuert.

In einem Unternehmen laufen in der Regel mehrere Instanzen eines Geschäftsprozesses gleichzeitig ab. Eine Prozessinstanz entsteht, wenn die Ausführung eines Geschäftsprozesses durch das dafür definierte Ereignis angestoßen wird. Prozessinstanzen

${ }^{1}$ Enactment steht für Umsetzung, Inkraftsetzung. 
folgen der einheitlichen Prozessbeschreibung, werden aber unabhängig voneinander ausgeführt.

Beispielsweise können zwei verschiedene Kunden eine Bestellung tätigen. Jeder dieser individuellen Kundenaufträge erzeugt eine eigenständig abzuwickelnde Instanz des Geschäftsprozesses „Auftragsbearbeitung“.

Das Management der einzelnen Instanzen übernimmt das Workflow Enactment System mithilfe von Workflow Engines. Eine Workflow Engine stellt die Ausführungsumgebung für eine Workflow-Instanz zur Verfügung. Ihre wesentlichen Aufgaben sind:

- Einordnung der Instanz in das organisatorische Umfeld

- Interpretation der Prozessdefinition

- Kontrolle der Prozessinstanzen

- Navigation zwischen sequenziellen oder parallelen Prozessaktivitäten

- Interpretation von Prozessdaten

- Identifikation der Benutzungsschnittstellen

- Verknüpfung mit anderen Programmen/Anwendungssystemen

- Verknüpfung mit anderen Workflowsystemen

- Übergeordnete Kontrollfunktion

Ein Workflow Enactment Service ist ein Dienst, der eine oder mehrere Workflow Engines startet, verwaltet und ausführt. Über Applikationsschnittstellen greifen Workflow-Systeme auf Funktionen von Anwendungssystemen zu, die in einem Prozess vorgesehene Aufgaben ausführen.

Ein Worklist Handler ist eine Komponente, welche die Benutzer in einen Prozess einbindet. Er kann Teil eines Workflow-Management-Systems sein, oder von einem Workflow-Experten definiert und programmiert werden. Durch den Worklist Handler wissen die beteiligten Aufgabenträger, in welcher Prozessinstanz sie welche Aufgaben zu erledigen haben.

Damit Benutzer bei der Bearbeitung von Prozessinstanzen eine einheitliche Arbeitsoberfläche haben, können Workflow-Funktionen in andere gebräuchliche Anwendungen eingebettet werden, beispielsweise in ein E-Mail-Programm. Für eine solche Integration muss zwischen dem Workflow Enactment Service und den anderen Anwendungen ein Kommunikationsmechanismus bestehen.

Die Referenzarchitektur sieht auch eine Schnittstelle für Administration und Monitoring vor. Dort anknüpfende Software dient der Überwachung des gleichzeitigen Ablaufs verschiedener Instanzen von mehreren Geschäftsprozessen. Dieses Monitoring bezieht sich zum einen auf die fachliche Ebene der Abwicklung der Geschäftsfälle, beispielsweise mit der Erfassung und Auswertung von Antwort- und Bearbeitungszeiten (vgl. Abschn. 6.3.3). Zum anderen sind auf der technischen Ebene die verwendeten IT-Systeme zu überwachen, etwa bezüglich Last- und Fehlverhalten.

Die meisten derzeit am Markt angebotenen Workflow-Systeme unterstützen nur die Prozessorchestrierung, d. h. einen einzigen Kontrollfluss von Aufgaben. Für BPMN 
bedeutet dies, dass ein Workflow-System nur die Funktionen in einem Pool ausführen kann. Werden in einer BPMN-basierten Prozessbeschreibung mehrere Pools verwendet, ist für jeden Pool eine eigene Workflow Engine nötig. Diese Workflow Engines müssen dann in der Regel durch Programmierung über die Schnittstelle 4 verbunden werden. Insbesondere wenn ein Prozess auf einer verteilten Infrastruktur ausgeführt werden soll, können solche Strukturen aufwendig und kostenintensiv werden, da viele Softwarehersteller Lizenzgebühren für jede installierte Instanz der Workflow Engine berechnen. Damit steigen neben den technischen Schwierigkeiten auch die Kosten.

Subjektorientierte Prozessbeschreibungen sind Prozesschoreografien, d. h. die Subjekte werden unabhängig voneinander parallel ausgeführt, ohne dass es eine zentrale Steuerung für alle Subjekte gibt. Zur Koordination ihrer einzelnen Tätigkeiten tauschen die Subjekte asynchron Nachrichten aus. Eine Ausführungsumgebung für subjektorientiert definierte Prozesse ist deshalb genau genommen ein sogenanntes Multiworkflow-System. Darin entspricht jedes Subjekt einer Orchestrierung, die von einem eigenen Workflow-System ausgeführt wird. Den asynchronen Nachrichtenaustausch zwischen mehreren Workflow-Systemen wird im subjektorientierten Prozessmanagement mithilfe des InputPool-Konzepts realisiert. Für S-BPM wurde eine Reihe von Multiworkflow-Systemen entwickelt [9].

\section{Kombination Physik-IT: Cyber Physical Systems}

In einem Prozess können Handelnde auch Maschinen sein, welche Aufgaben, rein mechanisch oder elektrisch gesteuert, vollautomatisch ohne menschliches Eingreifen erledigen.

Die Maschinensteuerung erfolgt mechanisch, elektromechanische oder, bei den bereits angesprochenen Embedded Systems, zunehmend rechner- und softwarebasiert. Sensoren kontrollieren den physikalischen Zustand von Maschine inkl. Umgebungsbedingungen, und Aktoren wie Stellmotoren, Schalter, Regler usw. greifen in den Betrieb der Maschine ein. Die Steuer-Software bestimmt weitgehend das Geschehen auf und an den intelligenten Maschinen sowie deren vertikale und horizontale Kommunikation mit anderen Systemen.

Maschinen kommunizieren untereinander nicht nur durch den Austausch von Daten, sondern auch durch das Weiterleiten und Empfangen von physikalischen Artefakten. Die Nachricht ,Zu entgratendes Werkstück“ kann dadurch realisiert sein, dass ein Werkstück von einer spanabhebenden Fertigungsmaschine automatisch weitertransportiert wird zu einer Maschine, die Kanten entgratet. Parallel dazu können bei der Entgratungsmaschine zusätzlich Nachrichten eintreffen, die eine nähere Beschreibung des Werkstücks enthalten sowie die Art der Entgratung spezifizieren.

Kombinationen aus physikalischen und informationstechnischen Komponenten werden als Cyber Physical Systems (CPS) bezeichnet. Da sie Produktions- und Geschäftsprozesse zusammenführen sind sie von besondere Bedeutung für Industrie 4.0-Anwendungen.

In BPMN kann der Modellierer einen Task, der von einer Kombination aus Mensch und IT ausgeführt wird, als Service Task modellieren, und eine intelligente Maschine 
als eigenen Prozess in einem Pool beschreiben. Gibt es eine entsprechende Anzahl von Maschinen, die in einer komplexen Fertigungssituation zusammenarbeiten, so kann die Verwendung von zahlreichen Pools in BPMN zu Darstellungsproblemen führen. Der Grund liegt in der horizontalen Anordnung der Pools, sodass bei einer größeren Anzahl die Nachrichtenkanten zwangsläufig Pools kreuzen müssen, was Unübersichtlichkeit bewirkt. In S-BPM wird eine Maschine dagegen immer als Subjekt modelliert, da erst bei der Implementierung entschieden wird, durch welche Art von Aufgabenträger das Subjekt ausgeführt wird.

Wird in einem Prozess ein physikalischer Aufgabenträger verwendet, kann es von diesem Prozess nicht beliebig viele parallele Instanzen geben. Eine physikalische Einheit kann nicht logisch aufgespalten werden, wie dies bei Aufgabenträgern der IT oder Menschen möglich ist. Auf diese Thematik werden wir im Abschn. 6.3.2 näher eingehen.

\section{Kombination Mensch-Physik-IT}

Auch beim Einsatz intelligenter Maschinen gibt es Szenarien, bei denen der Mensch bestimmte Aufgaben ausführt. Beispielsweise erhält eine Maschine das Werkstück über ein Transportsystem und die dazugehörigen Informationen als Nachricht. Die Maschine und ein Bediener führen die vorgesehenen Aufgaben gemäß den Angaben in den Begleitinformationen zusammen aus. Der Bediener bestätigt die Erledigung seiner Arbeiten über eine Benutzungsschnittstelle. Anschließend wird das Werkstück abtransportiert, und die aktualisierten Begleitinformationen wird an weitere involvierte Handelnde übermittelt.

In solchen Situationen ist es das Bestreben, den Anteil der menschlichen Arbeit weiter zu reduzieren und durch ausgefeiltere Mechanik oder verbesserte Embedded Systems zu ersetzen. Dabei muss es nicht zwingend notwendig sein, die Prozesslogik zu verändern.

\subsection{Betrieb und Monitoring}

\subsubsection{Inbetriebnahme}

Nach der Festlegung der Aufgabenträger und der Implementierung(sunterstützung) der Handlungen gilt es den Prozess in Betrieb zu nehmen.

Dazu müssen die Aufgabenträger vorbereitet werden, indem die physikalische und informationstechnische Infrastruktur aufgebaut und die menschlichen Aufgabenträger geschult werden. Informationstechnische Aufgabenträger werden mit den jeweiligen Programmen geladen und die Mechanik von Maschinen entsprechend eingestellt.

Bei der Inbetriebnahme ist es wichtig, die Verknüpfung mit anderen Prozessen herzustellen. In einem Unternehmen sind Prozesse in ein zusammenhängendes Netzwerk von Prozessen eingebunden. Es sollte keine isolierten Prozesse geben. Wird z. B. ein neuer Prozess Versandvorbereitung eingeführt, muss dieser verknüpft sein mit dem Prozess Auftragsannahme. Bei kommunikationsorientierten Prozessbeschreibungen geschieht dies einfach durch den Austausch von Nachrichten. Ein andere Möglichkeit 
sind gemeinsame Daten, d. h. Prozesse schreiben Daten, die andere Prozesse benötigen in eine gemeinsam genutzte Datenbank.

Nach den Vorbereitungsarbeiten sollte der Prozess als Gesamtkonstrukt getestet werden. Vorteilhaft dazu wäre eine Testumgebung, die ein möglichst realistisches Abbild der Einsatzumgebung darstellt. Allerdings ist diese Wunschsituation aus Kostengründen nicht oft gegeben.

Unabhängig davon empfiehlt es sich den Prozess Schritt für Schritt einzuführen. Dabei ist von Vorteil, wenn ein Prozess als ein lose gekoppeltes System von kommunizierenden Aufgabenträgern beschrieben und umgesetzt wurde. Die einzelnen Handlungsstränge für die Handlungsträger können Schritt für Schritt in Betrieb genommen werden. Da sie durch den asynchronen Nachrichtenaustausch nur lose miteinander gekoppelt sind, kann man andere Aufgabenträger zunächst einfach simulieren. Bei BPMN wird somit Pool für Pool in Betrieb genommen. Sind die Pools allerdings sehr komplex mit mehreren Swim Lanes, gestaltet sich die Inbetriebnahme ebenfalls als komplex. Bei der Verwendung von S-BPM kann ein System durch das schrittweise Hinzunehmen der einzelnen Subjekte aufgebaut werden.

Werden in BPMN nicht mehrere Pools verwendet, d. h. ein Prozess wird rein kontrollflussorientiert definiert und implementiert, kann dieser auch nur insgesamt in Betrieb genommen werden.

Bei Choreografien, d. h. bei der Verwendung von Subjekten bzw. mehrerer Pools kann der Prozess ausgeführt werden, obwohl z. B. bei einem Pool die Software noch fehlerhaft ist. Die an diesen Pool gesendeten Nachrichten können ersatzweise von Menschen entgegengenommen, bearbeitet und das Ergebnis als Nachricht zurückgesendet werden. Die anderen Pools nehmen die beschriebene Ablauflogik wahr (beobachtbares Verhalten), die aber noch nicht in der endgültigen Form implementiert ist.

\subsubsection{Prozessinstanzen}

Die konkrete Ausführung eines Prozesses wird als Prozessinstanz bezeichnet. Eine Prozessinstanz entsteht, wenn das Startereignis eintritt. Dies kann der Anruf eines Kunden sein, der ein Produkt bestellen möchte. In diesem Fall kreiert ein Telefonverkäufer explizit eine Instanz indem er den digitalen Bestellvorgang startet und die notwendigen Daten eingibt. Bei Bestellungen in einem Online-Shop erfasst der Kunde die Auftragsdaten selbst und erzeugt eine Instanz, sobald er den Kauf bestätigt. Anschließend durchläuft eine Instanz in beiden Fällen die gemäß der definierten Prozesslogik vorgesehenen Bearbeitungsschritte und -stellen.

$\mathrm{Da}$ in einem Unternehmen normalerweise zu einem bestimmten Zeitpunkt mehrere Bestellungen vorliegen, existieren gleichzeitig mehrere voneinander unabhängige Prozessinstanzen, die sich in verschiedenen Bearbeitungsstadien befinden. Mitarbeiter eines Unternehmens sind in der Regel in mehrere Prozessinstanzen eingebunden und führen darin jeweils die für sie vorgesehenen Aufgaben aus. Sie teilen sozusagen jeder Prozessinstanz eine Zeitscheibe ihrer Arbeitszeit zu. Analog geschieht dies bei 
IT-Systemen. Sowohl die Kapazität der Ressource Mensch als auch der IT-Systeme kann also in Zeitscheiben aufgeteilt werden, um mehrere Instanzen von gleichen oder unterschiedlichen Prozessen zu bearbeiten.

Bei physikalischen oder cyber-physikalischen Systemen ist dies nicht so ohne weiteres möglich. Ein solches System kann zu einem Zeitpunkt nur in genau einer Instanz involviert sein. Eine Maschine kann nicht mehrfach instanziiert werden, sie ist eben nur einmal vorhanden. Erst wenn eine Maschine eine Aufgabe oder Aufgabenfolge in einer Prozessinstanz vollständig abgeschlossen hat kann sie für eine andere Prozessinstanz tätig werden. $\mathrm{Zu}$ einem bestimmten Zeitpunkt ist ein physikalisches System deshalb genau einer Prozessinstanz zugeordnet.

Dieser Sachverhalt ist wichtig, wenn Prozesse, die problemlos instanziiert werden können, da sie keine physikalischen Aufgabenträger enthalten, mit Prozessen verknüpft werden, die physikalische Komponenten enthalten. Ein Fertigungssystem besteht nahezu vollständig aus physikalischen oder cyber-physikalischen Aufgabenträgern. Diese Prozesse werden zu Beginn instanziiert. Diese eine Instanz besteht dann bis die Fertigung abgeschaltet wird. Ein physikalischer Aufgabenträger kann nicht eine Zeitscheibe für eine Prozessinstanz 1 arbeiten, dann etwas für eine Prozess 2 tun, um danach wieder für die Prozessinstanz 1 weiterzuarbeiten. Die Aktionen eines physikalischen Aufgabenträgers für verschiedenen Prozessinstanzen wären nicht unabhängig voneinander. Soll beispielsweise ein Ventil für die Prozessinstanz 1 halb geöffnet werden und danach für die Prozessinstanz 2 ganz geöffnet werden dann wird natürlich auch für die Prozessinstanz 1 das Ventil ganz geöffnet, was aber nicht sein soll.

Wird in BPMN eine Maschine einem Task zugeordnet und kann der zugeordnete Pool mehrfach instanziiert werden, so muss die Maschine zur Laufzeit immer einer einzelnen Instanz zugeordnet werden. Die Maschine muss immer wissen, für welche Instanz sie tätig ist, um die richtigen Begleitdaten aus dem allen Instanzen gemeinsamen Speicher zu holen, um dann aus dem Werkstückbehälter das zu bearbeitende Werkstück zu entnehmen.

Bei S-BPM ist die Maschine dem entsprechenden Subjekt zugeordnet. Dieses empfängt das Werkstück und die Begleitdaten als Nachricht. Die Begleitdaten enthalten auch eine Identifikation der zugehörigen übergeordneten Prozessinstanz, in der Regel die Auftragsnummer. Damit weiß die Maschine, für welche Prozessinstanz sie nun arbeitet. Die Nachricht, mit der eine Maschine ihre Arbeit für beendet erklärt, geht an eine Subjektinstanz in der beauftragenden Prozessinstanz.

\subsubsection{Monitoring}

Prozessbeteiligte führen im Tagesgeschäft Geschäftsprozesse gemäß des modellierten Designs in der bei der Umsetzung geschaffenen Umgebung aus Personal- und technischen Ressourcen aus (vgl. Abschn. 6.2).

Jeder anfallende Geschäftsvorfall durchläuft als Instanz diese Ausführungsumgebung. Dabei zeichnen Transaktionssysteme wie Enterprise-Resource-Planning-Anwendungen 
(ERP) oder Workflow Engines das Verhalten der Instanz in Form von Einträgen in einem Log File (Event Log, Audit Log) auf. Ein Log-Datensatz enthält u. a. eine eindeutige Vorgangsidentifikation, Teilschrittidentifikation und Zeitstempel für Beginn und Ende. So entstehen Rohdaten für die Berechnung von Prozesskennzahlen (Process Performance Indicators, PPIs).

Verlässliche Managementinformation auf Basis solcher Kennzahlen ist die Voraussetzung für eine kontinuierliche Anpassung der Prozessgestaltung im Hinblick auf höhere Zielerreichungsgrade. Die periodische Ex-post-Auswertung über eine Vielzahl von Instanzen über längere Zeiträume wie Wochen, Monate, Quartale etc. dient dabei vorwiegend der Erkennung struktureller Verbesserungspotenziale, beispielsweise bezüglich planmäßigem Personaleinsatz, Ablauflogik oder Grad der IT-Abdeckung. Dieses traditionelle Monitoring und Reporting folgt dem Konzept der Business Intelligence mit dem Prinzip „Store and analyze“ und den Methoden des Data und Process Mining. Daraus resultierende Veränderungen sind eher mittel- und langfristiger Natur.

Um auch den steigenden Echtzeitanforderungen Rechnung zu tragen, wird das traditionelle Prozessmonitoring deshalb mittlerweile ergänzt durch das Business Activity Monitoring (BAM), welches ereignisgetrieben nahezu in Echtzeit Daten auswertet, zeitnah Ergebnisse meldet und damit kurzfristige, instanzbezogene Maßnahmen ermöglicht [10]. Beispiel wäre die bevorzugte weitere Bearbeitung der Bestellinstanz eines A-Kunden, wenn das System erkennt und meldet, dass diese an einem Messpunkt hinter dem dort üblichen Bearbeitungsfortschritt hinterherhinkt und deshalb der zugesagte Liefertermin nicht zu halten ist (predictive analysis).

BAM nutzt das Konzept des Complex Event Processing (CEP) mit dem Prinzip „Stream and analyze“ und Stream-Mining-Methoden. Dies bedeutet, dass das System im Strom von aufgezeichneten einzelnen Vorkommnissen (z. B. gesetzte Zeitstempel, passierte Messpunkte) permanent nach Mustern für komplexe Ereignisse sucht, die erst durch die Verknüpfung der einzelnen Ereignisse Relevanz für bestimmte Zwecke erlangen. Typisches Beispiel ist die Erfassung zweier Transaktionen mit derselben Kreditkarte in Hamburg und New York. Diese einfachen Einzelereignisse (low level events) werden im Ereignisstrom als normale Vorgänge registriert. Das CEP-System kombiniert sie erst zu einem komplexen Ereignis (complex event), wenn beide Transaktionen innerhalb eines kurzen Zeitraum stattfinden, im Beispiel etwa $3 \mathrm{~h}$. In diesem Fall würde ein Ereignismuster aus geografischem und Zeitabstand zur Klassifikation als komplexer Event „,angenommener Kreditkartenmissbrauch“ führen.

Business Activity Monitoring soll permanent und gleichzeitig eine Vielzahl von Datenquellen überwachen. $\mathrm{Zu}$ den Erzeugern von Ereignisdaten gehören Anwendungen, die Prozessinstanzen verarbeiten (z. B. ERP, CRM, Workflow Engines) sowie andere Informationen von innerhalb oder außerhalb des Unternehmens liefern (z. B. Umgebungsbedingungen, Wetter- und Verkehrsdaten). Zunehmend zählen dazu auch (Sensor)Daten, die smarte Geräten und Einrichtungen im Internet of Things produzieren. BAM analysiert und aggregiert die Datenflut mithilfe definierter Regeln und übermittelt Ergebnisse an berechtigte und interessierte Empfänger. 


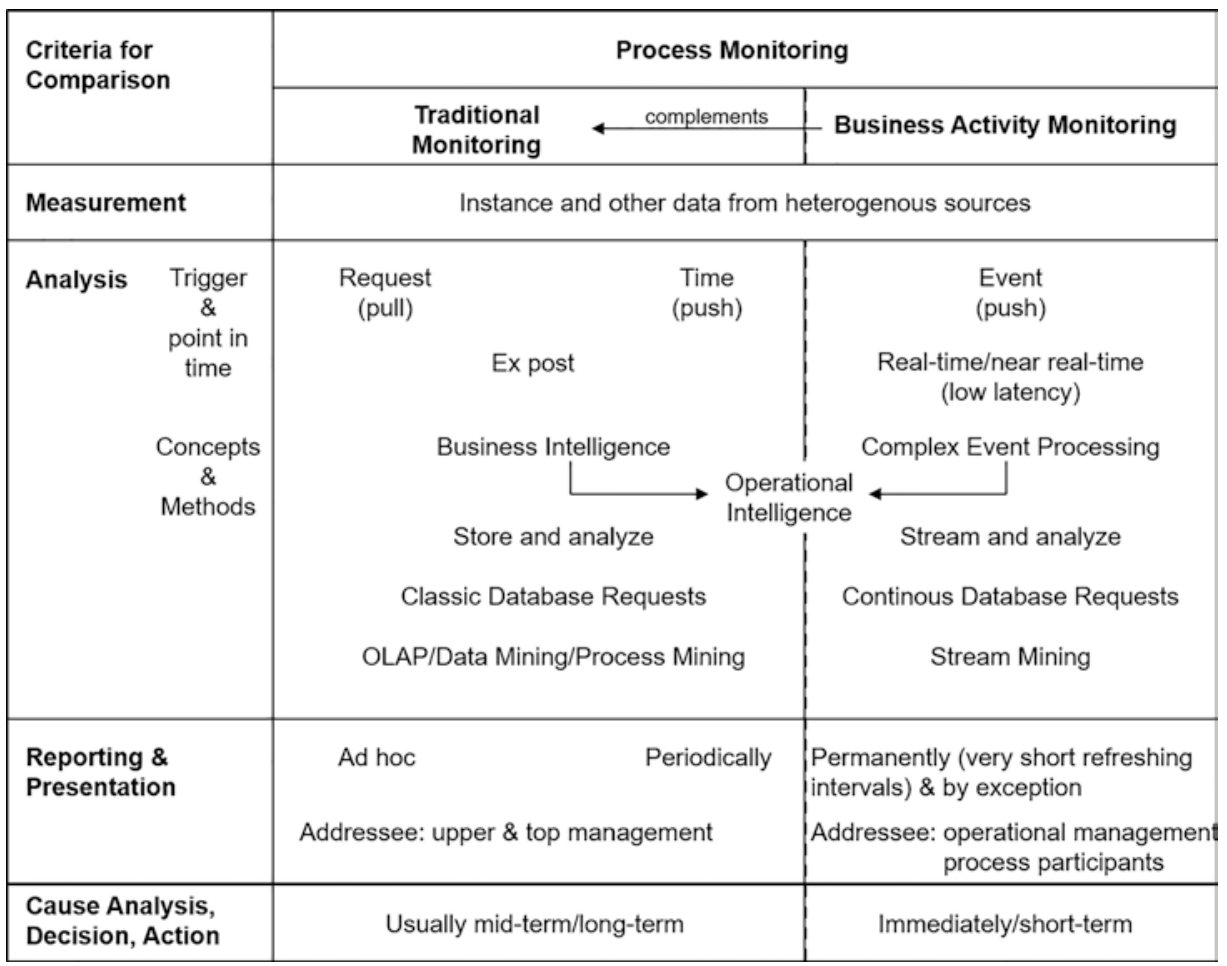

Abb. 6.4 Eigenschaften von traditionellem und Business Activity Monitoring

Abb. 6.4 stellt das traditionelle und das komplementäre Business Activity Monitoring nebeneinander. Als Vergleichsmerkmal dienen die in der linken Spalte aufgeführten Aktivitäten [10].

Zeitpunkt und Art der Verwertung der aufgezeichneten/registrierten Daten hängen von der Ausgestaltung des Monitoring und Reporting gemäß der Anwenderanforderungen ab. Beim Pull-Prinzip ruft der Anwender die von ihm gewünschte Auswertung zu einem beliebigen Zeitpunkt ab. Nach dem Push-Prinzip erzeugt das System zeitgesteuert z. B. täglich, wöchentlich, monatlich, quartalsweise zu festgelegten Zeiten Auswertungen und informiert den vordefinierten Empfängerkreis davon. Beim Überschreiten von Grenzwerten oder Toleranzschwellen für Process Performance Indicators einzelner Instanzen zur Laufzeit können, ebenfalls per Push, Alarmmeldungen an Verantwortliche übermittelt oder andere Vorgänge gestartet werden, z. B. ein umfangreicher Eskalationsprozess mit Korrekturmaßnahmen für den betreffenden Fall.

Als Präsentationsform für die Auswertungen dominieren Management Cockpits mit Instrumententafeln (Dashboards). Dort finden sich meist Darstellungen in Form von Tachometern, Ampeln und Balken- oder Kreisdiagrammen. Für platzsparende Anzeigen 


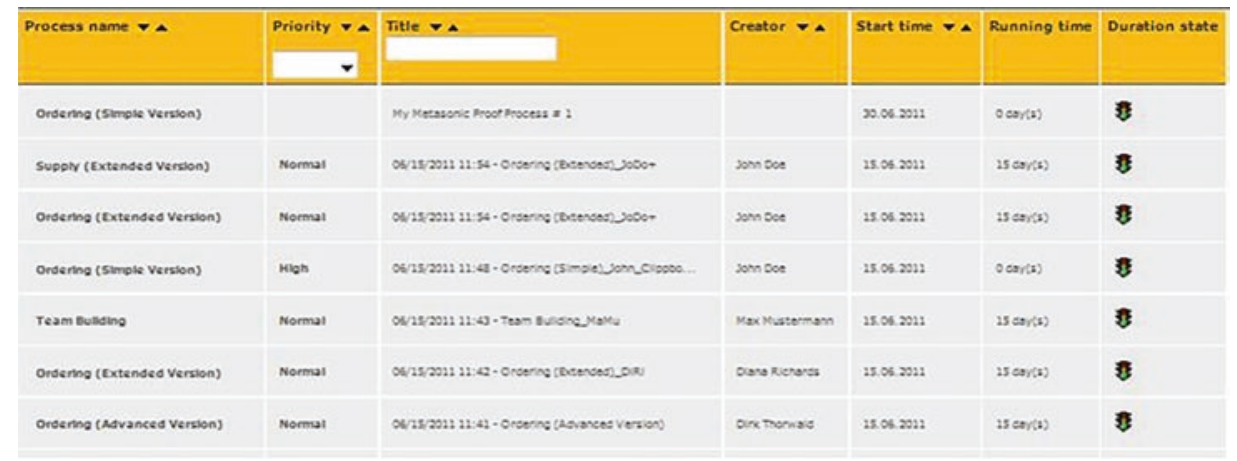

Abb. 6.5 Instanzbericht (Übersicht)
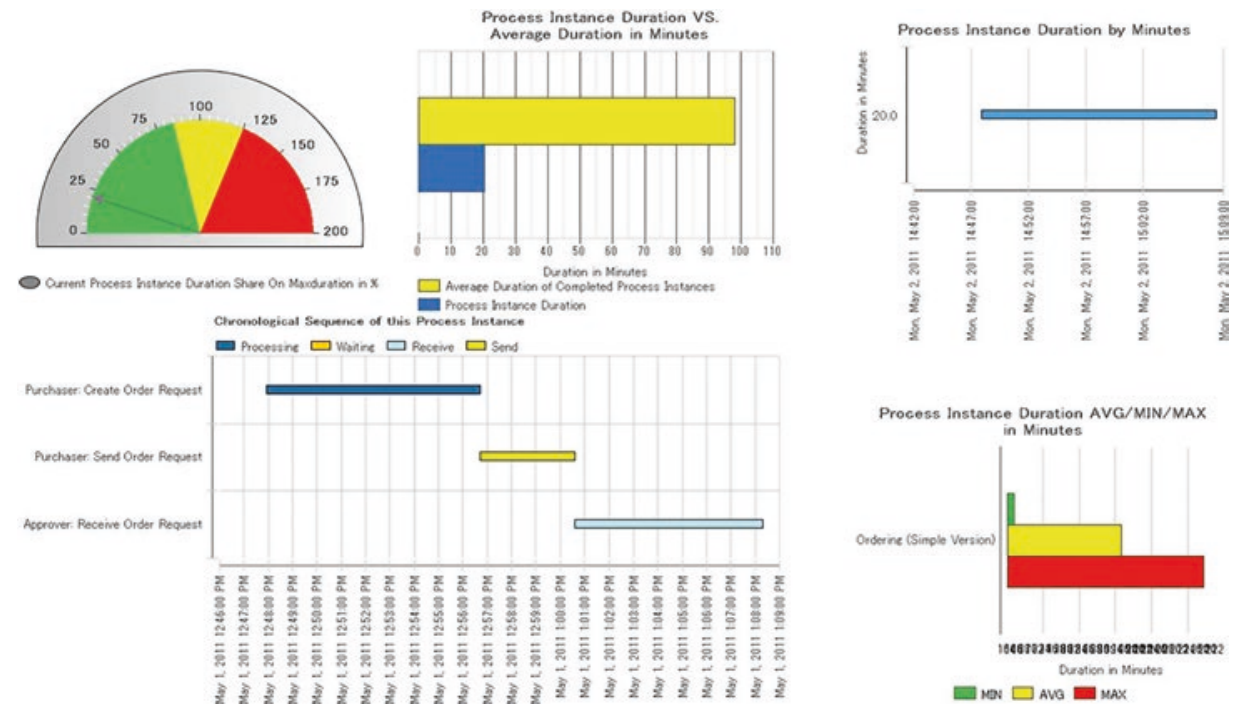

Abb. 6.6 Instanzbericht (Einzelne Instanz)

mit hoher Informationsdichte kommen auch Wortgrafiken wie Sparklines oder Bullet Graphs zum Einsatz. Beispiele für Auswertungen und ihre Darstellung sind:

- Durchlaufzeit von laufenden und abgeschlossenen Instanzen. Der Status wird in Ampelfarben ausgedrückt, je nach Abweichung von definierten Sollwerten bzw. Toleranzbereichen (vgl. Abb. 6.5).

- Durchlaufzeit einer laufenden Instanz (absoluter Wert und Vergleich mit Durchschnitt, chronologischer Ablauf und Dauer der Einzelschritte der Prozessbeteiligten (vgl. Abb. 6.6). 
- Abfolge der Prozessschritte einer laufenden Instanz mit Zeitstempeln für den Abschluss eines Schritts, d. h. für den Übergang von einem Zustand in den nächsten (vgl. Abb. 6.7).

- Anzahl von Instanzen eines Prozesses pro Zeiteinheit, minimale, durchschnittliche und maximale Bearbeitungszeit pro Prozessbeteiligtem und -schritt (vgl. Abb. 6.8).

\subsubsection{Process Mining}

Eine spezielle Form der Auswertung von Prozessdaten stellt das Process Mining dar. Dabei werden Log Files genutzt, um aus den Transaktionsdaten zentraler IT-Systeme (u. a. ERP, CRM und SCM) prozessbezogene Informationen zu extrahieren und so den tatsächlichen Prozessablauf visuell rekonstruieren und analysieren zu können. Dadurch können Erkenntnisse über das wirkliche Verhalten von abgewickelten Prozessinstanzen

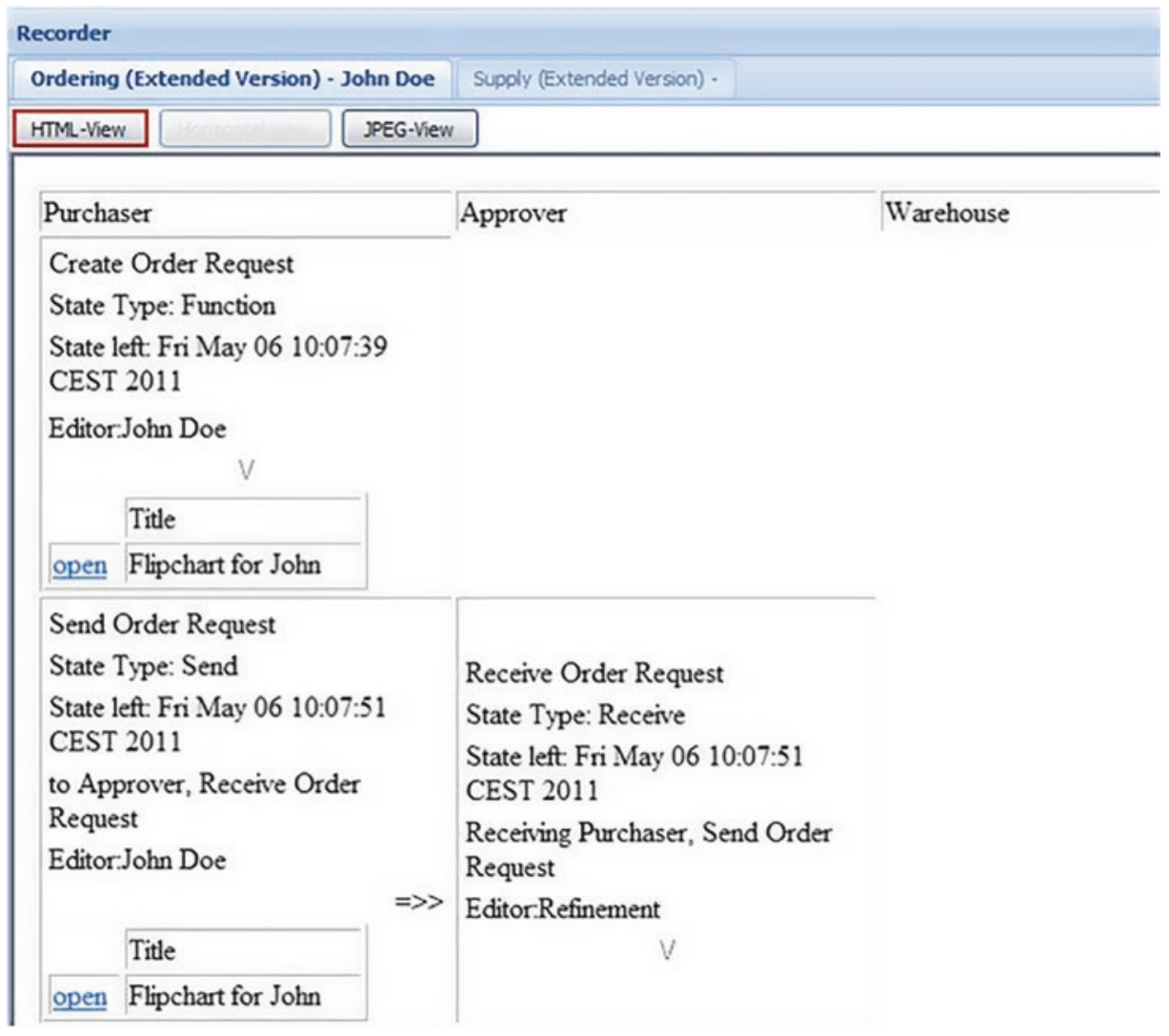

Abb. 6.7 Instanzbericht (aktueller Bearbeitungsstand) 
Started Process Instances (per Month)

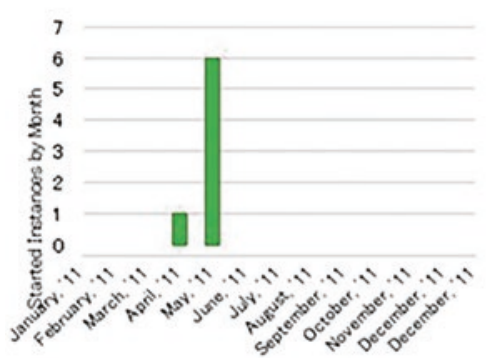

Processing Duration AVG/MIN/MAX

in Minutes by Subject

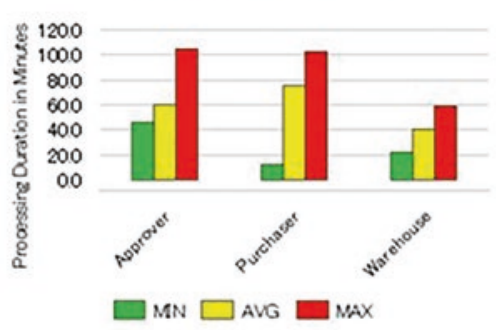

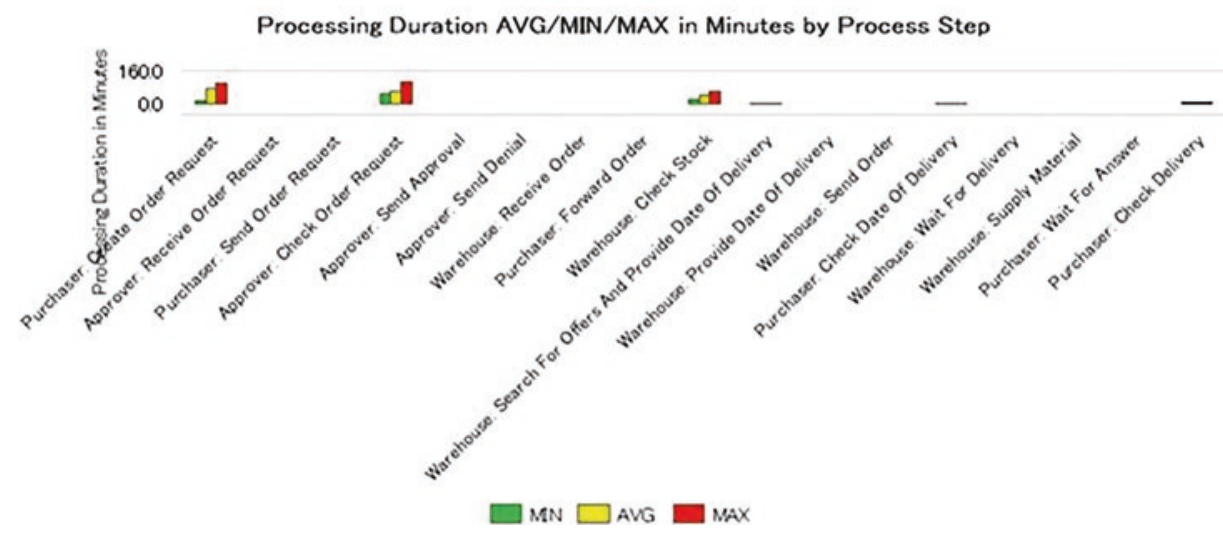

Abb. 6.8 Prozessbericht

gewonnen werden [11]. Häufig analysierte Unternehmensprozesse sind: Einkauf, Verkauf, Kreditoren-/Debitorenbuchhaltung, Ticketing-Systeme (z. B. im IT-Service Management).

Ein Event Log enthält sequenziell aufgezeichnete Ereignisse (trace) mit Attributen wie Fallnummer (caseID), Aktivität (activity), Zeitstempel (timestamp), ausführender Einheit (resource) und bearbeitete Geschäftsobjekte (data elements). Je nach Prozess können weitere Informationen dazu kommen, wie z. B. der Name eines Kunden oder Lieferanten, Bestellmenge und -wert, Liefermenge etc. In Abb. 6.9 ist ausschnittsweise ein Beispiel für ein Event Log dargestellt.

Mit Bezug solcher Protokolldaten für die Prozessausführung zu Prozessmodellen lassen sich drei wesentliche Arten von Process Mining identifizieren [11]:

- Entdeckung (Discovery): dabei rekonstruieren Algorithmen aus den Log-Daten ohne weitere Informationen den tatsächlichen Prozessablauf und dessen vielfältige Varianten. Damit lassen sich bereits gelebte, aber noch nicht dokumentierte Prozesse formal beschreiben.

- Konformität (Conformance): dabei vergleichen Algorithmen ein vorhandenes, gültiges Prozessmodell mit dem tatsächlichen Ablauf wie er aus den Log-Daten abgeleitet 


\begin{tabular}{|l|l|l|}
\hline CaselD & Activity & Timestamp \\
\hline 10001 & Create purchase order & 01-01-2009, 8:35 am \\
\hline 10001 & Print and send purchase order & 03-01-2009, 12:13 am \\
\hline 10001 & Goods receipt & $07-01-2009,07: 01 \mathrm{am}$ \\
\hline 10001 & Scan invoice & 09-01-2009, 2:00 pm \\
\hline 10001 & Book invoice & $10-01-2009,10: 30 \mathrm{am}$ \\
\hline 10002 & Create purchase requisition & $02-02-2009,1: 17 \mathrm{pm}$ \\
\hline 10002 & Create purchase order & $04-02-2009,9: 15 \mathrm{am}$ \\
\hline 10002 & Print and send purchase order & $07-02-2009,4: 41 \mathrm{pm}$ \\
\hline 10002 & Goods receipt & $27-02-2009,6: 53 \mathrm{am}$ \\
\hline 10002 & Scan invoice & $28-02-2009,1: 00 \mathrm{pm}$ \\
\hline 10002 & Book invoice & $13-03-2009,11: 59 \mathrm{am}$ \\
\hline 10003 & Scan invoice & $13-04-2009,10: 00 \mathrm{am}$ \\
\hline 10003 & Create purchase order & $17-04-2009,3: 47 \mathrm{pm}$ \\
\hline 10003 & Print and send purchase order & $17-04-2009,5: 30 \mathrm{pm}$ \\
\hline 10003 & Goods receipt & $27-04-2009,4: 23 \mathrm{pm}$ \\
\hline 10003 & Book invoice & $30-04-2009,8: 50 \mathrm{am}$ \\
\hline & & \\
\hline
\end{tabular}

Abb. 6.9 Ausschnitt aus Event Log (Abbildung übernommen mit Genehmigung der Celonis SE)

wird. Festgestellte Abweichungen können Anhaltspunkte für Optimierungen liefern und Missbrauch oder Verstöße gegen Compliance-Regeln aufdecken.

- Erweiterung (Enhancement): hier werden bestehende Modelle an die Realität der Vorgangsbearbeitung angepasst (Repair) oder dem Modell weitere Perspektiven wie Zeitdauern hinzugefügt (Extension).

Insbesondere die Überprüfung der Konformität sollte möglichst zur Laufzeit von Instanzen im Rahmen des Business Activity Monitoring durchgeführt werden, um Verstöße zeitnah zu entdecken und deren Folgen mildern zu können (z. B. Stoppen einer Überweisung bei Unregelmäßigkeiten bei der Zahlungsfreigabe).

Das folgende Beispiel ist der Process-Mining Lösung der Celonis SE (www.celonis.com) entnommen. Es zeigt ausgewählte Mining-Ergebnisse für einen Bestellabwicklungsprozess (Purchase to-Pay), der mit einem ERP-System abgewickelt wird. Die 
Analyse des dazugehörigen Event Logs für einen bestimmten Zeitraum hat folgende Information hervorgebracht (vgl. Abb. 6.10):

- 279.020 Instanzen des Prozesses mit einem Nettobestellwert von 539.180.072 €

- 107.688 Instanzen folgten dem normalen Ablauf des Prozesses (Happy Path), beginnend mit dem Erstellen einer Bestellanforderung und endend mit der Verbuchung der Rechnung (siehe Fallnummer 10.002 in Abb. 6.9). Die durchschnittliche Durchlaufzeit beträgt 27 Tage Diese Prozessvariante 1 deckt $39 \%$ aller Instanzen ab, daneben existieren 527 andere Varianten.

Erweitert man den Betrachtungsraum beispielsweise auf die sieben am häufigsten vorkommenden Prozessvarianten, sieht man, dass damit $83 \%$ aller Instanzen abgedeckt sind. Im Prozessgraphen sind jetzt auch die sieben Pfade mit den dazugehörigen Häufigkeiten sichtbar (vgl. Abb. 6.11). Alternativ können auch die durchschnittlichen Dauern der Wege und Zustandsübergänge angezeigt werden. Bezüglich des Ablaufs fällt außerdem auf, dass eine nicht geringe Zahl von Instanzen (18.938) mit dem Scan der Rechnung beginnt und erst dann eine Bestellung im System angelegt wird (siehe Fallnummer 10.003 in Abb. 6.9). Dies ist ein Indiz für sog. Maverick Buying, d. h. die Beschaffung von Fachabteilungen am Einkauf vorbei. Diesem i. d. R. unerwünschten Prozessverhalten kann im nächsten Schritt gezielt nachgegangen werden, um dessen Ursachen zu beseitigen.

Mit der Einblendung zusätzlicher Informationen lassen sich Prozesse tiefergehend untersuchen. Abb. 6.12 gibt beispielsweise Auskunft über die auf Monate verteilte Anzahl von Bestellungsinstanzen mit den jeweiligen Wertangaben sowie deren Verteilung auf Lieferanten.

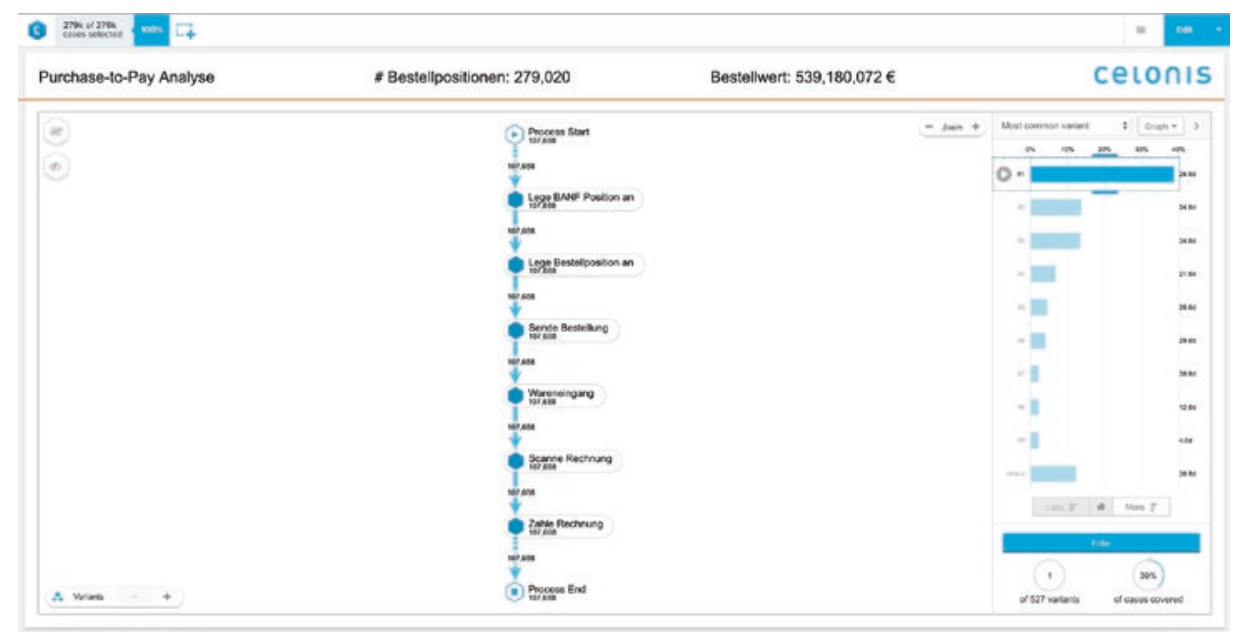

Abb. 6.10 Übersicht über Prozessvarianten (rechts) und Happy Path (links) (Screenshot des Celonis Viewer/Variant Explorer mit Genehmigung der Celonis SE) 


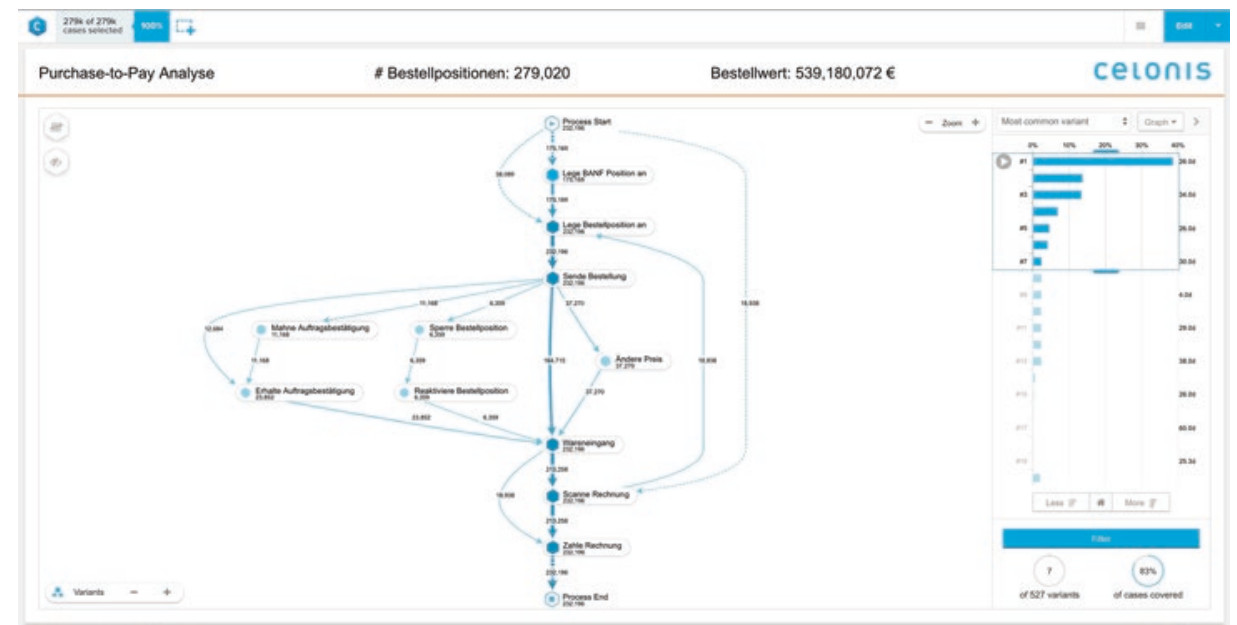

Abb. 6.11 Anzeige der sieben häufigsten Prozessvarianten (Screenshot des Celonis Viewer/ Variant Explorer mit Genehmigung der Celonis SE)

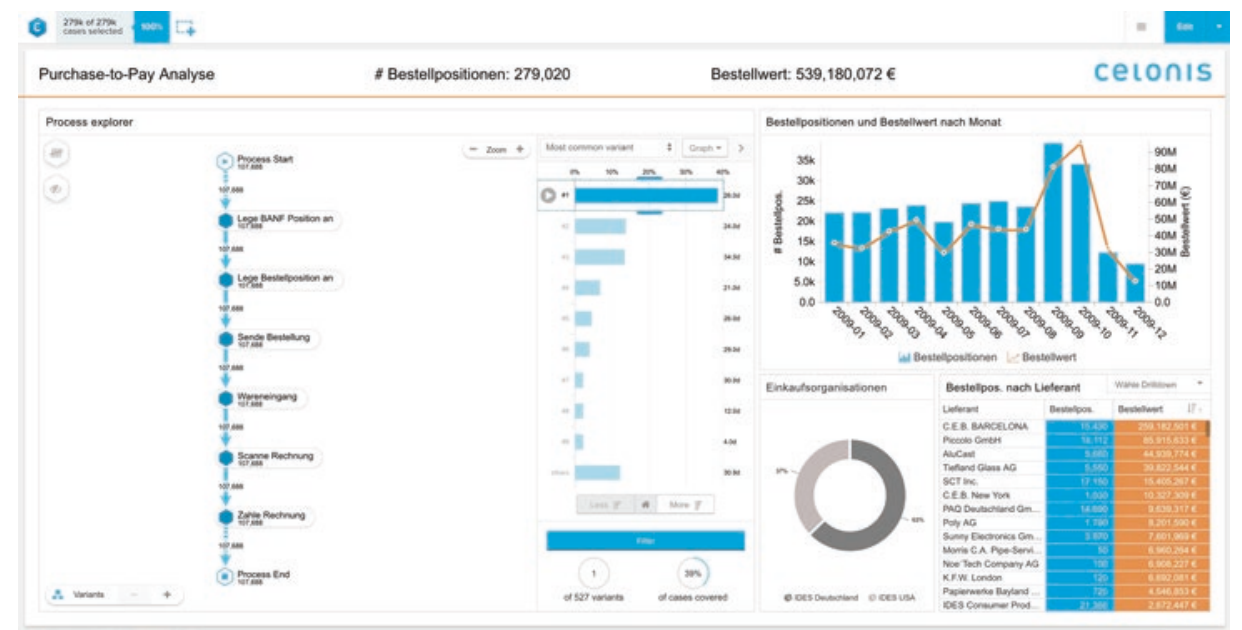

Abb. 6.12 Einblendung zusätzlicher Information (Screenshot des Celonis Viewer/Analysis mit Genehmigung der Celonis SE)

Die vorgestellten Analysen betreffen nur einen kleinen Ausschnitt der vielfältigen Nutzungsmöglichkeiten der Celonis-Umgebung. So können die Anwender über die vom System vorgesehenen Auswertungen hinaus auf Basis ihrer individuellen Datenmodelle eigene Auswertungen durch Rückgriff auf eine Vielzahl vorhandener Analysekomponenten (wie verschiedene Diagrammtypen, Prozesskennzahlen und OLAP-Tabellen) definieren. Außerdem erlaubt die Software die Ausführung eigener Prozessauswertungen mithilfe der Process Query Language (PQL). 
Zusätzliche Funktionen erlauben etwa die Analyse der Konformität des tatsächlichen mit dem im Modell vorgesehenen Ablauf (Konformität) oder die parallele Visualisierung und damit die Gegenüberstellung unterschiedlicher Verhalten desselben Prozesses beispielsweise in zwei Niederlassungen (Benchmarking).

Die Zukunft des Process Mining liegt in der Kombination der Prozessanalyse mit intelligenten Algorithmen. So integriert beispielsweise die Celonis Proactive Insights Engine (Pi) Machine Learning und Artificial Intelligence Techniken, und identifiziert für den Nutzer automatisch Schwachstellen im Prozess sowie deren Ursachen [12]. Darauf aufbauend erhält der Nutzer zudem intelligente Empfehlungen zur Prozessverbesserung.

\subsubsection{Kontinuierliche Wartung}

Ziel von Monitoring, Auswertung und Reporting des Prozessverhaltens ist es, Managementinformation zum Prozessverhalten zu erzeugen und bereitzustellen. Die Adressaten können diese analysieren, Ursachen für Abweichungen von Zielwerten erforschen und kurz-, mittel- und langfristige Handlungsbedarfe und Maßnahmen zur Restrukturierung daraus ableiten.

Die folgenden Ausführungen erläutern typische Veränderungen der Prozessgestaltung, welche sich positiv auf das Prozessverhalten im Sinne einer Optimierung auswirken sollen. Wir beziehen uns dabei exemplarisch auf das Kreditantragsbeispiel aus Abschn. 4.2.2.

- Parallelisieren und überlappte Ausführung. Logisch und technisch voneinander unabhängige Aktivitäten können ganz oder teilweise gleichzeitig und von verschiedenen Aufgabenträgern ausgeführt werden. Zwar kann die Zahl unterschiedlicher Aktivitäten durch die Aufspaltung ansteigen, die Parallelisierung bewirkt aber oft eine Prozessbeschleunigung. So könnte ein Prüfschritt bei der Kreditantragsbearbeitung in die dann parallel vorgenommene Bonitätsprüfung des Kunden und Werthaltigkeitsprüfung des Finanzierungsobjekts zerlegt werden.

- Zusammenfassen von Aktivitäten. Zusammenfassen als Gegenteil von Aufspaltung bedeutet, dass ursprünglich getrennt und von verschiedenen Aufgabenträgern bearbeitete Aktivitäten nun durch einen Aufgabenträger ausgeführt werden. Diese Reintegration von Aufgaben führt Arbeitsteilung zurück und verringert dadurch beispielsweise Schnittstellen. Die Zahl der Aktivitäten in einem Geschäftsprozess und seiner Darstellung nimmt durch die Zusammenfassung ab und die Anordnungsbeziehungen zwischen den Aktivitäten des Geschäftsprozesses ändern sich. Im Kreditantragsprozess könnte die Zusammenfassung der beiden Prüfschritte wieder sinnvoll sein, wenn die weiter unten beschriebene Automatisierung der Kundenbonitätsprüfung den Zeitgewinn durch Parallelisierung relativiert. 
- Änderung der Reihenfolge. Die Anordnungsbeziehungen zwischen den Aktivitäten, oder zwischen Gruppen oder Bündeln von Aktivitäten, können eventuell vertauscht werden, was zeitliche, kostenmäßige oder kapazitätsmäßige Vorteile haben kann. Beim Kreditantragsbeispiel sollte die Prüfung der Bonität der Bewertung des Finanzierungsobjekts vorgezogen werden, denn letztere ist hinfällig bzw. der ganze Prozess endet, wenn der Interessent nicht kreditwürdig ist. Eine Sequenz der Prüfungen steht im Gegensatz zu der Parallelisierungsoption. Um eine Entscheidung für eine der Varianten zu treffen, wäre die Information hilfreich, wie oft ein Antrag wegen mangelnder Bonität abgelehnt wird und damit im Falle der parallelen Objektprüfung Ressourcen verschwendet werden.

- Elimination von Aktivitäten. Verbale Diskussionen, Process Mining, Pfadanalyse und Simulationsexperimente geben Hinweise auf nicht benötigte Aktivitäten (tote Pfade), sehr selten ausgeführte Aktivitäten, auf Aktivitäten, bei denen kaum eine Wertschöpfung erzielt wird oder die unwirtschaftlich sind. Die Zahl der Aktivitäten eines Prozesses nimmt durch die Elimination ab und die Struktur der Anordnungsbeziehungen ändert sich. Beispiel für eine Elimination wenig wertschöpfender Aktivtäten könnte das in Abschn. 4.2.8.2 angesprochene Weglassen der zusätzlichen Genehmigung eines Kreditantrags über $200.000 €$ durch die Abteilungsleitung sein.

- Elimination oder Reduzierung von Zyklen. Wenn Geschäftstransaktionen Zyklen von Aktivitäten mehrfach durchlaufen, verlängert dies gewöhnlich die Durchlaufzeiten der Prozesse und führt oft zu Wartezeiten bei der Ausführung. Mit der Pfadanalyse können solche Zyklen identifiziert und lokalisiert und möglicherweise durch Änderung der Prozessgestaltung eliminiert werden. Bei der Kreditantragsbearbeitung kann es beispielsweise immer wieder zu Nachfragen der Sachbearbeiter beim Interessenten kommen, weil Informationen zur Beurteilung des Finanzierungsvorhabens fehlen. Sieht das elektronische Antragsformular Mussfelder und Anhänge für entsprechende Angaben vor, kann die Steuerung die Abgabe verhindern, so lange Information fehlt. Damit ist zwar nicht sichergestellt, dass der Antragsteller die richtige Information vollständig und valide übermittelt, aber die Wahrscheinlichkeit dafür steigt und Rückfrageschleifen dürften seltener nötig sein.

- Insourcing und Outsourcing. Unter Umständen ist es sinnvoll, in sich geschlossene Geschäftsprozesse oder einzelne Teile nicht in der eigenen Organisation, sondern von spezialisierten externen Dienstleistern ausführen zu lassen, welche dies z. B. aufgrund von Größenvorteilen (Economies of Scale) kostengünstiger und/oder schneller können. Damit lassen sich oft eigene Fixkosten durch variable Kosten für die Inanspruchnahme der Leistungen des Partners ersetzen. Beispielsweise könnte die Bank anstatt eigene Sachverständige für die Immobilienbewertung zu beschäftigen fallweise ein Architekturbüro damit beauftragen. Bei entsprechenden Voraussetzungen kann auch der umgekehrte Weg Vorteile bringen, etwa, wenn bisher ausgelagerte Aktivitäten z. B. wegen des Wegfalls von Schnittstellen und Transaktionskosten intern wirtschaftlicher organisierbar sind. 
- Automatisieren. Der technische Fortschritt eröffnet Möglichkeiten, manuelle Arbeitsschritte von IT-Anwendungen und Maschinen (z. B. Roboter) zu unterstützen oder komplett automatisiert ausführen zu lassen. Dies ist insbesondere für zeitintensive, wenig motivierende und fehlerträchtige Tätigkeiten relevant. Zur Realisierung von Automatisierungsoptionen gilt es, die Entwicklung und den Markt für entsprechende Technologien dauerhaft zu beobachten, um passende Lösungsbausteine identifizieren und diese in Prozessanpassungen einbeziehen zu können. Als Beispiel kann der parametrisierbare Webservice der SCHUFA für die Auskunft über Kunden dienen, dessen Integration nicht nur Automatisierung fördert, sondern auch ein Beispiel für teilweises Outsourcing darstellt.

- Reduktion von Schnittstellen. Arbeitsteilige Prozessabwicklung weist naturgemäß Schnittstellen auf organisatorischer und technischer Ebene auf. Sie involviert verschiedene Organisationseinheiten und externe Partner, die oft mit uneinheitlichen IT-Systemen und Arbeitsmitteln mitunter an verschiedene Medien gebundene Zwischenergebnisse erzeugen und austauschen. Folgen sind Medienbrüche und damit verbundene Doppelarbeit, Übertragungsfehler, Zeitverluste, Kosten usw. Eine Verringerung der Schnittstellen wirkt diesen Nachteilen entgegen. Sie lässt sich durch organisatorische Veränderungen wie die Reintegration oder Eliminierung von Aktivitäten und die Änderung der Zuordnung von Aufgaben zu Aufgabenträgern erzielen. Auf der technischen Ebene helfen integrierte IT-Systeme wie Enterprise-Resource-Planning-Software und Workflow-Anwendungen. Beim Kreditantragsbeispiel hat die teilweise Verlagerung der Unterschriftenkompetenz auf die Sachbearbeitungsebene deren Aufgabenzuschnitt und denjenigen der Abteilungsleitung verändert. Als Konsequenz konnten ein Ablaufzweig eliminiert und die dazugehörigen Schnittstellen reduziert werden.

$\mathrm{Zu}$ beachten ist, dass viele der genannten Optimierungsansätze interdependent sind, und deshalb immer Auswirkungen einer Maßnahme auf andere Faktoren der Gestaltung zu berücksichtigen sind. So kann beispielsweise das Outsourcing von Prozessteilen die Anzahl der Schnittstellen erhöhen und Aufwand für das Dienstleistungsmanagement verursachen. Diese Effekte wirken den Vorteilen der Auslagerung entgegen, sodass stets eine Abwägung nötig ist.

\section{Literatur}

1. Bonitasoft Documentation, https://documentation.bonitasoft.com/bonita/7.6/_application-andprocess-design Last access February 2018

2. A. Lawall, T. Schaller, D. Reichelt; Integration of Dynamic Role Resolution within the S-BPM Approach; S-BPM ONE-Running Processes; 5th International Conference, S-BPM ONE 2013; CCIS Springer Verlag 2013 
3. Bonitasoft Documentation, https://documentation.bonitasoft.com/bonita/7.6/_application-andprocess-design Last access February 2018

4. T. Rademakers; Activity in Action, Maning Publications, 2012 sowie Activity Documentation; https://www.activiti.org/docs, Last access February 2018

5. AKKA Documentation; https://akka.io/docs/, Last access February 2018

6. S. Newman; Building Microservices; O'Reilly; 2015

7. J. Bonér, D. Farley, R. Kuhn, and M. Thompson; The Reactive Manifesto; https://www.reactivemanifesto.org/; Last access February 2018

8. D. Hollingsworth; Workflow Management Coalition The Workflow Reference Model; Document Number TC00-1003 Document Status - Issue 1.1 19-Jan-95

9. A. Fleischmann et al.; An Overview to S-BPM oriented Tool Suites; in M. Mühlhäuser, C. Zebold (Eds.); S-BPM ONE - 9th International Conference; Published by ACM;2017; ISBN 978-1-4503-4503-1

10. Schmidt, W. (2013). Business Activity Monitoring, in: Rausch, P., Sheta, A., Ayesh, A. (Eds.), Business Intelligence and Performance Management - Theory, Systems and Industrial Applications, Springer UK, S. 229-242.

11. van der Aalst, W. (2016), Process Mining,- Data Science in Action, Springer, Berlin Heidelberg, S. $33 \mathrm{ff}$.

12. Veit F., Geyer-Klingeberg J., Madrzak J., Haug M. und Thomson J.P. (2017), The Proactive Insights Engine: Process Mining meets Machine Learning and Artificial Intelligence, Business Process Management Conference 2017, Barcelona.

Open Access Dieses Kapitel wird unter der Creative Commons Namensnennung - Nicht kommerziell - Keine Bearbeitung 4.0 International Lizenz (http://creativecommons.org/licenses/ by-nc-nd/4.0/deed.de) veröffentlicht, welche die nicht-kommerzielle Nutzung, Vervielfältigung, Verbreitung und Wiedergabe in jeglichem Medium und Format erlaubt, sofern Sie den/die ursprünglichen Autor(en) und die Quelle ordnungsgemäß nennen, einen Link zur Creative Commons Lizenz beifügen und angeben, ob Änderungen vorgenommen wurden. Die Lizenz gibt Ihnen nicht das Recht, bearbeitete oder sonst wie umgestaltete Fassungen dieses Werkes zu verbreiten oder öffentlich wiederzugeben.

Die in diesem Kapitel enthaltenen Bilder und sonstiges Drittmaterial unterliegen ebenfalls der genannten Creative Commons Lizenz, sofern sich aus der Abbildungslegende nichts anderes ergibt. Sofern das betreffende Material nicht unter der genannten Creative Commons Lizenz steht und die betreffende Handlung nicht nach gesetzlichen Vorschriften erlaubt ist, ist auch für die oben aufgeführten nicht-kommerziellen Weiterverwendungen des Materials die Einwilligung des jeweiligen Rechteinhabers einzuholen.

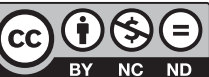




\section{Praxisbeispiel}

Das folgende Fallbeispiel zeigt, wie einige der beschriebenen Konzepte, Methoden und Sprachen angewendet wurden, um einen für ein konkretes Unternehmen wesentlichen Prozess zu verbessern. Die Dokumentation des real durchgeführten Projekts macht deutlich, dass sich die vorgestellten Aktivitäten nicht streng voneinander getrennt bearbeiten lassen, sondern sich in der Praxis oft überlappen und mit häufig wechselnden Schwerpunktbetrachtungen ineinander überfließen. Wir geben den Projektverlauf wieder, der von Christoph Moser stammt [1], und stellen mit den grau dargestellten Unterüberschriften jeweils den Bezug zu den passenden Ausführungen in Kap. 1 bis Kap. 6 und den betroffenen Aktivitätsbündeln in Abb. 7.1 her.

\subsection{Ausgangsituation}

Analyse: Blick auf die „Welt“ ENGEL ist ein traditioneller Hersteller von Spritzgießmaschinen aus Österreich und wurde 1945 von Ludwig Engel gegründet. Nach Einführung der ersten Spritzgießmaschine im Jahr 1952 hat sich ENGEL bis zum Jahr 2016 mit einem Gesamtumsatz von 1,36 Mrd. EUR zum Weltmarktführer entwickelt. Das vollständig eigentümergeführte Unternehmen beschäftigt weltweit ca. 5900 Mitarbeiter in 9 Produktionswerken und über 85 Niederlassungen [2]. ENGEL ist ein stark kundenorientiertes Unternehmen mit Fokus auf Flexibilität und Innovationen.

Festlegung des Key Performance Indicators ausgehend vom Geschäftsmodell und der Strategie Die starke Fokussierung auf die Kundenbedürfnisse und die fortschreitende Entwicklung hin zu kürzeren Lieferzeiten führten zur Definition eines unternehmensweiten Ziels: Reduzierung der Durchlaufzeit eines Prozesses für die Produktion aller Varianten einer Standardkomponente für Spritzgießmaschinen um $30 \%$. 
Abb. 7.1 Wechsel zwischen den Aktivitäten im Fallbeispiel

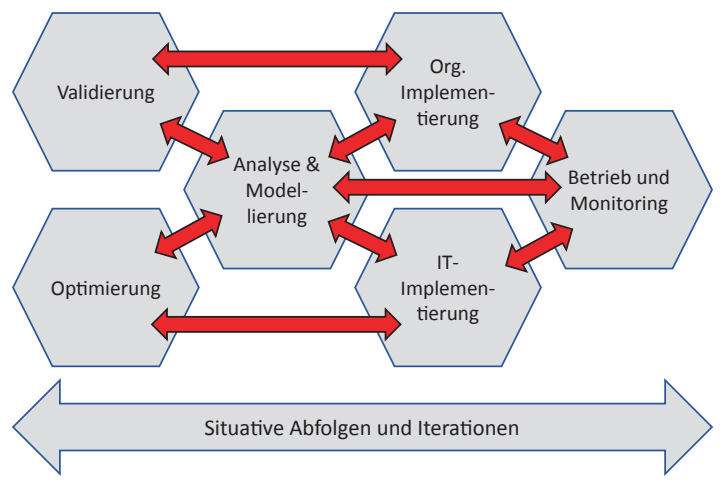

Ein Projektteam wurde geschaffen, um den bestehenden Prozess zu erheben, zu analysieren und die erforderlichen Verbesserungen zu implementieren. Der erste Schritt eines Optimierungsprozesses ist die Abbildung des Ist-Status, um detailliertere Kenntnisse über die Material- und Informationsflüsse sowie über alle Faktoren zu erhalten, die den betrachteten Prozess beeinflussen können. Zum Zeitpunkt des Projektstarts gab es kaum explizite Informationen zum Gesamtprozess, den detaillierten Prozessschritten oder den beteiligten Prozessakteuren.

Analyse und Modellierung Zu Beginn war nur bekannt, dass sich der Prozess über zwei Produktionsstandorte in zwei verschiedenen Ländern (Fabrik A und Fabrik B) erstreckt und sich auf drei Produktgruppen bezieht:

- Produkt 1: Der fertige Rahmen für die Spritzgussmaschine ist die Ausgangskomponente jeder Maschine. Rahmen werden in Fabrik A zusammengebaut und bestehen unter anderem aus Produkt 2. Die Gesamtdurchlaufzeit für Produkt 1 umfasst die Auftragsabwicklung, Produktion und Lieferung von Komponenten (Produkt 2) und Teilkomponenten (Produkt 3).

- Produkt 2: Der sogenannte ,rohe“ Rahmen mit Öltank. Diese Hauptkomponente ist noch mechanisch unbearbeitet und wird in Fabrik B aus Produkt 3 zusammengebaut. Es gibt mehrere Dutzend Varianten von Produkt 2, abhängig von den Anforderungen der Kunden.

- Produkt 3: Eine Art Bausatz aus Sägezuschnitten, welche in Fabrik A für die jeweilige Variante aus Stangenmaterial abgesägt werden.

Abb. 7.2 zeigt ein grobes Schema der Lieferkette. Der Auslöser des Prozesses (breaking point) ist der Auftrag der Fertigung der Spritzgießmaschine als internem Kunden (Customer) an Fabrik A. Diese sägt und liefert daraufhin die benötigten Teile (Produkt 3) für Produkt 2 und beauftragt Fabrik B mit dessen Fertigung. Das Zwischenprodukt wird anschließend an Fabrik A geliefert, dort zusammengebaut und als Produkt 1 an die Montagelinie der Spritzgießmaschine geliefert. 


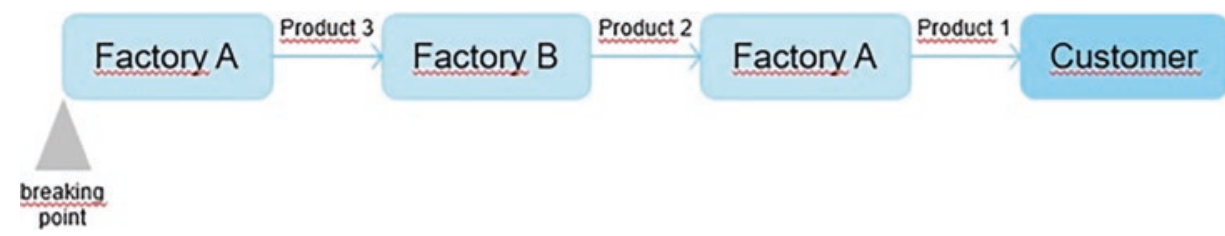

Abb. 7.2 Lieferkette zwischen den Werken

Der Prozess wird durch die Bestellungen gesteuert, die zwischen den Fabriken ausgetauscht werden. Wenn ein Auftrag in einer Fabrik eintrifft, wird er im ERP-System erfasst, und ein Fertigungsauftrag mit einem entsprechenden Lieferdatum erstellt. Erst nach dieser Erfassung ist der Auftrag produktiv wirksam. Wenn der Prozess bis zur Erfassung länger als 2 Arbeitstage dauert, trifft der Produktionsauftrag nicht rechtzeitig in der Produktion ein, da die Bestellvorlaufzeit auf zwei Arbeitstage festgelegt ist. In der Ausgangssituation wurden ca. $95 \%$ aller Bestellungen zwischen den Fabriken zu spät erfasst, d. h. zwischen der Ankunft des Auftrages in der Fabrik und der tatsächlichen Erfassung vergingen mehr als zwei Arbeitstage. Diese Aufträge mussten dann manuell mit enormem Mehraufwand bearbeitet werden, was zu einer internen Liefertreue für Produkt 2 von nur $39 \%$ führte. Daraus resultierende Probleme bei der Produktionsplanung in der Fabrik A und Verzögerungen bei der Produktion von Produkt 1.

Um das vorgegebene Ziel, die Durchlaufzeit um $30 \%$ zu reduzieren, zu erreichen wurde ein Zeitrahmen von nur 10 Wochen vorgegeben. Die zwei Fabriken lagen in zwei verschiedenen Ländern mit unterschiedlichen Sprachen.

Erste grobe Planung und Einordnung in die Enterprise Architecture: Keine grundlegenden Änderungen an der Organisation und der IT Der enge Zeitrahmen führte zu Einschränkungen für das Projekt: Neue Softwarelösungen oder Technologien können nicht eingeführt werden. Die Einführung solch weitreichender Veränderungen ist eine strategische Entscheidung und erfordert viel Personaleinsatz, Geld, Risikomanagement und Zeit. Dies bedeutete, dass Änderungen an den bestehenden Prozessen innerhalb der bestehenden Organisation und IT-Umgebung implementiert werden mussten. Erst nach Erreichen des ursprünglichen Projektziels können weitere, für zusätzliche Verbesserungen notwendige Maßnahmen umgesetzt werden.

\subsection{Durchgeführte Maßnahmen}

Prozessanalyse und Auswahl einer Modellierungssprache Mit Ausnahme einer oberflächlichen Beschreibung des Materialflusses zwischen den Fabriken standen uns keine expliziten Prozessinformationen zur Verfügung. Da eine ordnungsgemäße Prozessdokumentation für das Verständnis des Gesamtprozesses und die Identifizierung von Optimierungspotenzialen von grundlegender Bedeutung sind, bestand unser nächster 
Schritt in der Dokumentation und Analyse des bereits etablierten Produktionsprozesses mittels Wertstromanalyse (WSA) [3], Dem Standardwerkzeug zur Dokumentation von Produktionsprozessen in unserem Unternehmen.

Für eine erste Abbildung des Prozesses war es notwendig, ein geeignetes, repräsentatives Produkt auszuwählen, das die grundlegenden Produktionsschritte umfasst und den größten Teil des Materialflusses abdeckt. Wir kamen zu dem Schluss, eine Variante von Produkt 2 für eine erste Ist-Analyse zu verwenden. Diese Auswahl basierte auf einer ABC-Analyse aller Produktvarianten und der entsprechenden Arbeitspläne. Die ausgewählte Variante für Produkt 2 macht $30 \%$ der Gesamtproduktion aus, hatte die komplexesten Arbeitspläne und die längste Gesamtdurchlaufzeit der drei definierten Produktgruppen.

Modellierung eines Teils des Ist-Prozesses Durch die Verfolgung des Materialflusses auf Werksebene durch beide Fabriken, die Erhebung relevanter KPIs (Lagerstand, Produktionsdurchlaufzeiten, Kundentakt etc.) und die Befragung der verantwortlichen Mitarbeiter konnten wir ein Wertstrommodell (WSM) für Produkt 2 erstellen. Abb. 7.3 zeigt das WSM des Produktionsprozesses und seine hierarchische Struktur.

Ergebnis der Analyse Die Ergebnisse der Wertstromanalyse waren wie folgt:

- Der Produktionsprozess des Produkts 2 besteht aus zwei Hauptschritten: Herstellung des Basisrahmens und Herstellung des Öltanks. Sobald der Öltank fertiggestellt ist, wird er an den Rahmen montiert, um Produkt 2 herzustellen.

- Der Öltank und der Rahmen werden getrennt gefertigt. Dies bedeutet, dass es keine Abstimmung zwischen den beiden Produktionslinien gibt, sobald der Auftrag gestartet wurde.

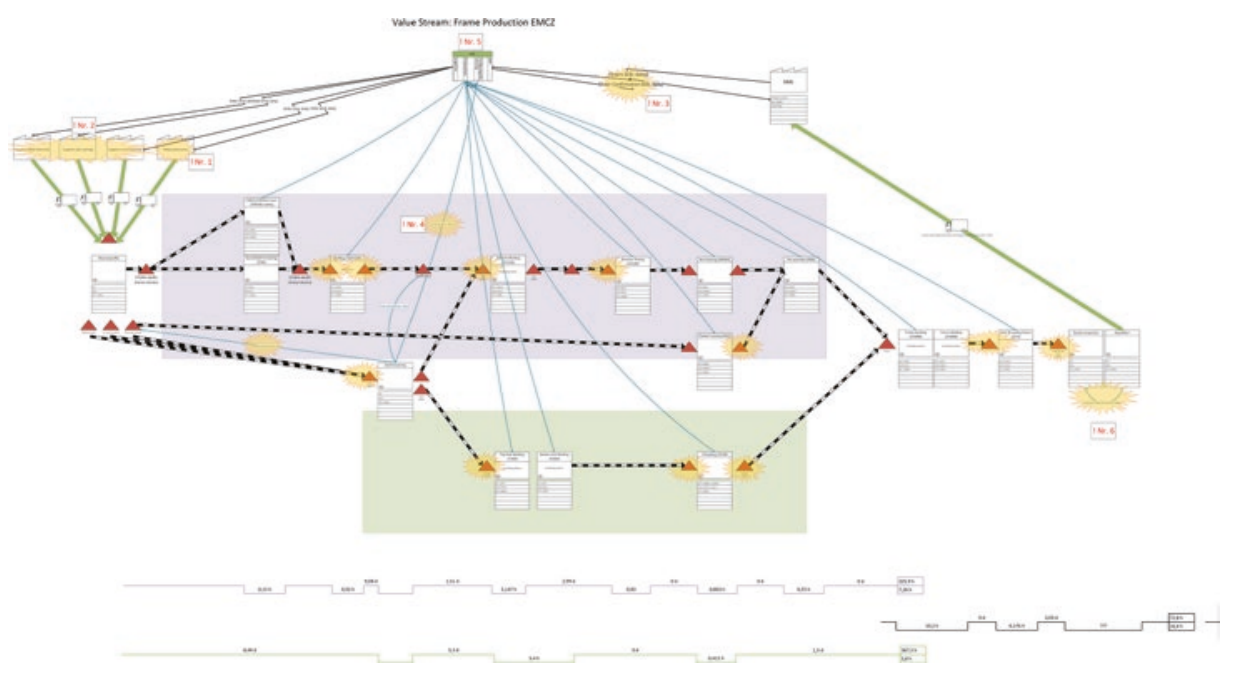

Abb. 7.3 Wertstromanalyse der Fertigung von Produkt 2 
- Aufgrund fehlender Produktionsplanung stapeln sich unfertige Bestände mit langen Wartezeiten. Nur ca. 10 \% der gesamten Durchlaufzeit ist Produktionszeit.

- Durch Optimierungen in der Produktionsplanung und den Arbeitsplänen kann die Durchlaufzeit verringert werden. Jedoch reichen die identifizierten Verbesserungen nicht aus, um das vorgegebene Ziel zu erreichen.

- Wir haben allerdings hohes Potenzial im Informationsfluss der Auftragsverarbeitung identifiziert.

Detaillierung des Prozessmodells Darüber hinaus wurden konkretere Informationen zum Bestellprozess zwischen den Fabriken gewonnen und die Prozessbeschreibung entsprechend erweitert:

- Die Nachfrage nach Produkt 1 stammt von Fabrik A, von der aus eine Bestellung für die erforderliche Variante von Produkt 2 an Fabrik B gesendet wird.

- Die Bestellung für Produkt 2 wird bearbeitet und die Beschaffung der erforderlichen Komponenten, darunter Produkt 3, beginnt.

- Fabrik B bestellt jetzt Produkt 3 von Fabrik A.

- Die Bestellung für Produkt 3 wird in Fabrik A erfasst, die Teile werden zugeschnitten und an Fabrik B verschickt.

- Sobald Produkt 3 in Fabrik B ankommt, beginnt die Produktion von Produkt 2 und das fertige Produkt 2 wird danach an Fabrik A geliefert.

- Sobald Produkt 2 in Fabrik A ankommt, beginnt die Fertigung von Produkt 1.

- Dieser Prozess ist für jede Maschine gleich, unabhängig von der Variante von Produkt 1.

Identifikation von Optimierungspotenzialen Durch die Wertstromanalyse konnten wir zwei Potenziale identifizieren: Die fehlende Produktionssynchronisierung und die nicht optimierte Auftragsabwicklung. Die Produktionssynchronisation ist allerdings direkt mit dem jeweiligen Produktionsplanungsprozess verknüpft. Bei einer kurzen Analyse des Planungsprozesses kamen wir zu dem Schluss, dass langfristige Verbesserungen nur möglich sind, wenn die Abläufe im Prozess Planung komplett reorganisiert und umstrukturiert werden und die Denkweise an sich verändert wird. Obwohl dies eine notwendige Änderung gewesen wäre, konnten wir sie im Zeitrahmen des Projekts nicht umsetzen. Wir haben uns daher auf den Bestellprozess und sein Optimierungspotenzial konzentriert, da wir es als möglichen „Quick Win“ für unser Projekt identifiziert haben.

Modellierung der noch fehlenden Aspekte Dies war der Einstiegspunkt für eine umfassende Prozesserhebung. Dem WSM fehlten jedoch noch relevante Prozessinformationen, um den Gesamtprozess und den entsprechenden Informationsfluss detailliert zu beschreiben, beispielsweise 
- keine Informationen über die Interaktionen zwischen den beteiligten Parteien

- keine Informationen darüber, welche Schritte im Prozess automatisiert sind und welche Schritte manuell durchgeführt werden

- keine konkreten Informationen zu den im SAP ${ }^{\circledR}$-ERP-System verwendeten Transaktionen

- keine Information über die Zeitlinien des Informationsflusses

- keine Überprüfung der bereitgestellten Informationen

Modellbildung, zusätzliche Modellierungssprache auswählen Da sich die WSA auf die Produktionsprozesse konzentriert und vor allem den Materialfluss zwischen den Prozessschritten beschreibt, haben wir schnell erkannt, dass wir einen anderen Ansatz benötigen. Wir mussten eine zusätzliche Methode verwenden, um den Informationsfluss in einem Detailgrad zu beschreiben, der es uns erlaubt eine genaue Analyse durchzuführen. Der nächste Schritt bestand darin, ein Prozessmodell der in den Prozess involvierten Personen und $\mathrm{SAP}^{\circledR}{ }^{\circledR}$-Systeme sowie deren jeweiligen Interaktionen bzw. des Informationsflusses zu erstellen.

Begründung warum subjektorientierte Modellierung hinzugenommen wurde Zur Ergänzung unseres bestehenden WSM haben wir das subjektorientiertes Geschäftsprozessmanagement eingeführt [4]. Wir wählten S-BPM aufgrund unserer vergangenen Erfahrungen und der Probleme, die wir mit Flussdiagrammen bzw. Swimlane-Diagrammen hatten, die gelegentlich neben der WSA verwendet werden. In früheren Anwendungen von Swimlanes lieferten die Prozessmodelle entweder einen Überblick über den Prozess, waren aber damit nicht detailliert genug für eine gründliche Prozessanalyse, oder die Prozessmodelle waren so detailliert, dass es sehr schwierig wurde, den Überblick zu behalten. Darüber hinaus sind Swimlane-Modelle unserer Erfahrung nach nicht dazu geeignet, die beteiligten Individuen und deren Abhängigkeit voneinander in transparenter Form, insbesondere bei mehr als fünf oder sechs Beteiligten, zu visualisieren.

Beschreibung von S-BPM und verwendete Werkzeuge Die Erfahrung hat gezeigt, dass die an den Prozessen beteiligten Personen, ihre individuellen Ansätze, ihr Wissen und ihre Erfahrungen eine entscheidende Triebfeder für die Prozesse eines Unternehmens sind und für erfolgreiche Prozesse unerlässlich sind [5, 6]. Die Art und Weise, in der der Informationsfluss zwischen den Prozessakteuren organisiert ist, hat einen wesentlichen Einfluss auf die Geschäftsprozessleistung [7].

S-BPM konzentriert sich speziell auf die Darstellung von Prozessen aus der Sicht der beteiligten Prozessakteure, sogenannte ,Subjekte“, und deren Interaktion in der Prozessumgebung. Ein Subjekt kann eine Maschine, ein IT-System oder eine Person sein. Subjekte sind abstrakte Handelnde ohne Angabe wie sie implementiert sind.

Die S-BPM Methode verwendet zwei Ebenen, um Prozesse zu beschreiben, das Subjekt Interaktionsdiagramm (SID: Subject Interaction Diagram) und das 
Subjektverhaltensdiagramm (SBD: Subject Behavior Diagram). Die Interaktion zwischen den Subjekten wird in dem SID visualisiert, welches die ausgetauschten Nachrichten zwischen den beteiligten Parteien (Subjekten) beschreibt. Das SID stellt eine Prozessübersicht bereit und hilft, die Rolle eines Subjekts im Gesamtprozess zu identifizieren.

Das Subjektverhaltensdiagramm beschreibt die einzelnen Prozessschritte eines beteiligten Probanden für dessen Rolle im Prozess. Das Verhalten eines Subjekts wird beschrieben durch drei unterschiedliche Aktionen (die sogenannten „Zustände“ in S-BPM), die es durchführt: „Senden“, „Empfangen“, und Ausführen einer internen Aufgabe (,Funktion“). Da der für einen Prozessakteur relevante Teil eines Prozesses innerhalb des jeweiligen Subjekts gekapselt ist, repräsentiert jedes SBD einen eigenständigen Handelnden innerhalb des Prozesses. Es ist nicht notwendig, den gesamten Prozess auf einmal oder in einer strikten Sequenz zu modellieren, z. B. wenn Informationen fehlen oder nicht verfügbar sind, oder ein Teil des Prozesses für die Untersuchung nicht relevant ist.

Detaillierte Modellierung mit S-BPM Die S-BPM-Notation besteht aus fünf Symbolen, die durch ihre Bedeutung und nicht durch die Form definiert sind, obwohl Empfehlungen in der Literatur existieren. Zwei Symbole werden für das SID verwendet, um ein Subjekt bzw. Handelnden (z. B. eine rechteckige Form) und die Wechselwirkung (z. B. einen Pfeil) darzustellen, und drei Symbole für das SBD, um jeden einzelnen Zustandstyp (Senden, Empfangen, Funktion) darzustellen (am häufigsten rechteckige Formen in verschiedenen Farben).

Ein Vorteil der beiden Ebenen und einer solchen einfachen Modellierungsnotation ist die Möglichkeit, Prozesse gleichzeitig in einem Top-Down- und/oder Bottom-Up-Ansatz $\mathrm{zu}$ modellieren, wobei die traditionell getrennten Bereiche Geschäftsprozessmanagement und Lean Production [8] in Abhängigkeit von den geforderten und unterschiedlichen Anforderungen zusammengeführt werden, je nach verfügbarer Detailtiefe der Informationen. Obwohl es für die Modellierung von S-BPM dedizierte Softwarelösungen gibt, haben wir unsere eigene MS Visio-Vorlage verwendet. Dies ermöglichte es uns, uns sofort auf die Modellierung und Analyse der Prozesse zu konzentrieren, ohne Ressourcen und Zeit in die Anwendung einer externen technischen Lösung zu investieren - ein häufiger Fehler in vielen Unternehmen, wenn Prozessmodellierung implementiert wird [9].

Durch detailliertere persönliche Interviews erstellten wir ein Modell der ersten Ebene des Prozesses, das SID. Das daraus resultierende Prozessmodell der Ist-Situation ergab, dass der Logistik- und Produktionsprozess wie erwartet sehr komplex ist. Ungefähr 40 involvierte Subjekte verteilten sich auf die Produktion, Produktionssteuerung und Logistik aller drei Produkte in beiden Fabriken. Die endgültige Version des resultierenden Ist-SID und die verwendete Notation sind in Abb. $7.4 \mathrm{zu}$ sehen. Das SID zeigt die allgemeine Kommunikationsstruktur des Prozesses und welche Subjekte miteinander welche Nachrichten austauschen. Die konkreten Namen der Subjekte oder der Inhalt der ausgetauschten Nachrichten sind für das weitere Verständnis der durchgeführten Maßnahmen nicht relevant. 


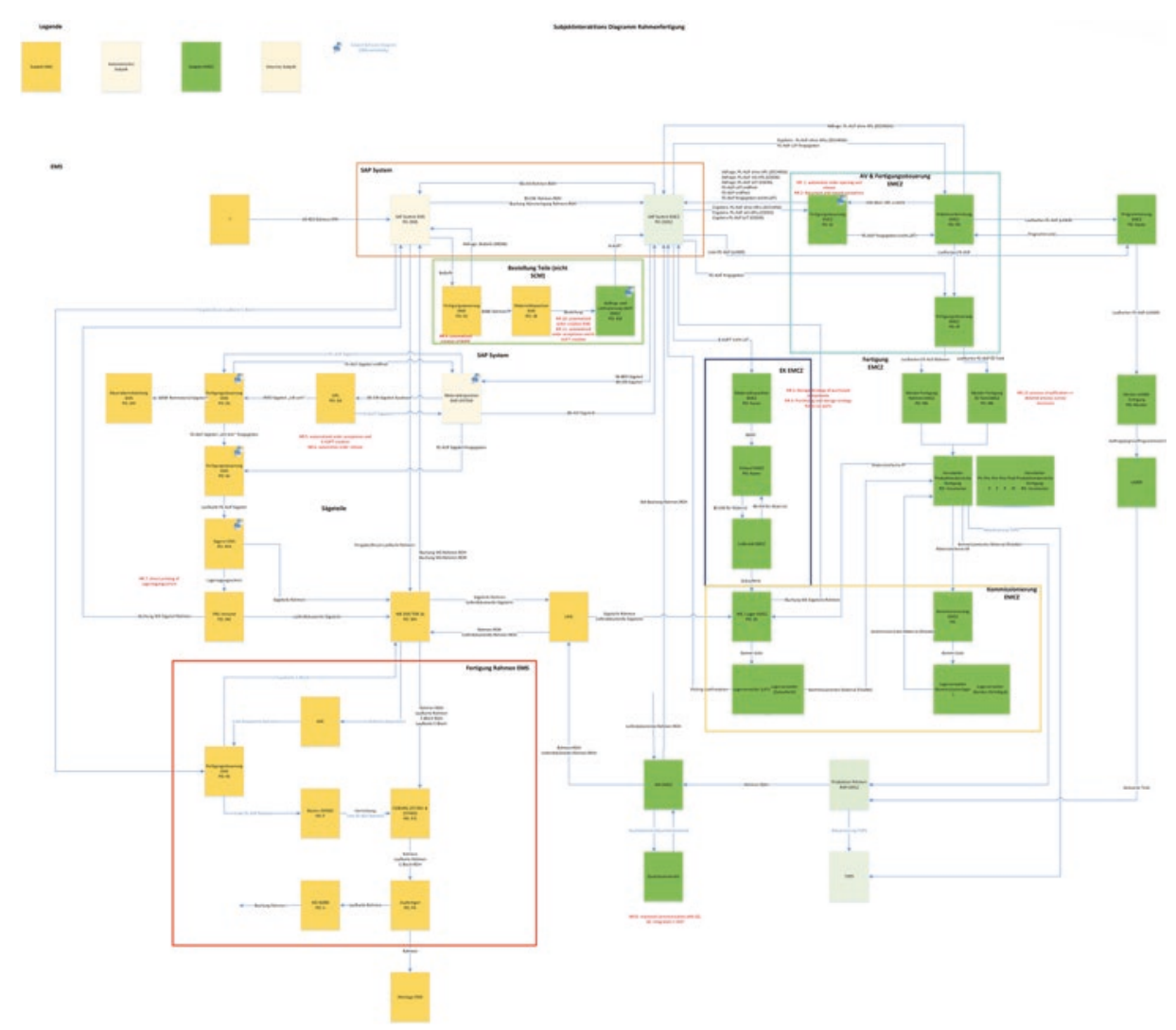

Abb. 7.4 Kommunikationsstruktur (Subject Interaction Diagram) der Produktion und des Bestellprozesses

Modellbildung und -analyse Die rechteckigen Formen repräsentieren die verschiedenen Subjekte, die an dem Prozess beteiligt sind, und die Pfeile repräsentieren die Nachrichten zwischen den Subjekten. Um zwischen den beiden Fabriken zu unterscheiden, haben wir die entsprechenden Subjekte farbig in Grün für Fabrik A und Orange für Fabrik B gekennzeichnet. Außerdem haben wir die Subjekte, welche SAP ${ }^{\circledR}$-Systeme darstellen mittels Schraffur gekennzeichnet, um die bereits digitalen Teile des bestehenden Prozesses hervorzuheben.

Obwohl von beiden Werken dasselbe ERP-System verwendet wird, teilen wir es für eine strukturiertere Visualisierung entsprechend den jeweiligen Abteilungen auf $\left(\right.$ SAP $^{\circledR}{ }_{-}$System A, SAP ${ }^{\circledR}{ }_{-}$System B und SAP ${ }^{\circledR}$-System A Disposition).

Identifikation von Optimierungsmöglichkeiten Aufgrund der Anzahl der involvierten Subjekte und der Komplexität des gesamten Prozesses wäre es nicht praktikabel gewesen, alle Subjektverhalten ohne einen definierten Rahmen für unsere nächsten Schritte zu erheben und zu modellieren. 
Um einen solchen Rahmen zu definieren, haben wir das nun vorhandene SID verwendet, um die Hauptknoten und Engpässe im Prozess für das entsprechende Produkt zu identifizieren und zu analysieren (Abb. 7.5).

Weiter Prozessmodellierung Ist-Analyse der IT-Unterstützung Der auffälligste Teil des Prozesses war die Auftragsabwicklung selbst. Die Abarbeitung der Bestellung von Produkt 1 durch Fabrik A, die Auftragsabwicklung und die Beschaffung von Produkt 2 durch Fabrik B sowie die Produktion von Produkt 3 in Fabrik A beschäftigte bis zu 12 Subjekte ( $3 \mathrm{SAP}^{\circledR}$-Systeme und 9 Personen) und nahm bis zu 15 Arbeitstage in Anspruch. Außerdem wurden nur $65 \%$ des Produkts 2 pünktlich fertiggestellt, weil die Auftragsabwicklung zu lange dauerte und die Aufträge im Produktionszentrum zu spät eingingen (ca. $95 \%$ aller Aufträge). Dies hatte direkte Auswirkungen auf die Produktion von Produkt 1 und auf die Prozessstabilität. Die Lieferzeiten konnten nur mit viel Aufwand in der Produktion eingehalten werden.

Wir haben uns entschieden, uns auf diesen Materialbeschaffungsprozess zu konzentrieren, da der Prozess selbst relativ zur Komplexität der bereitgestellten Komponenten

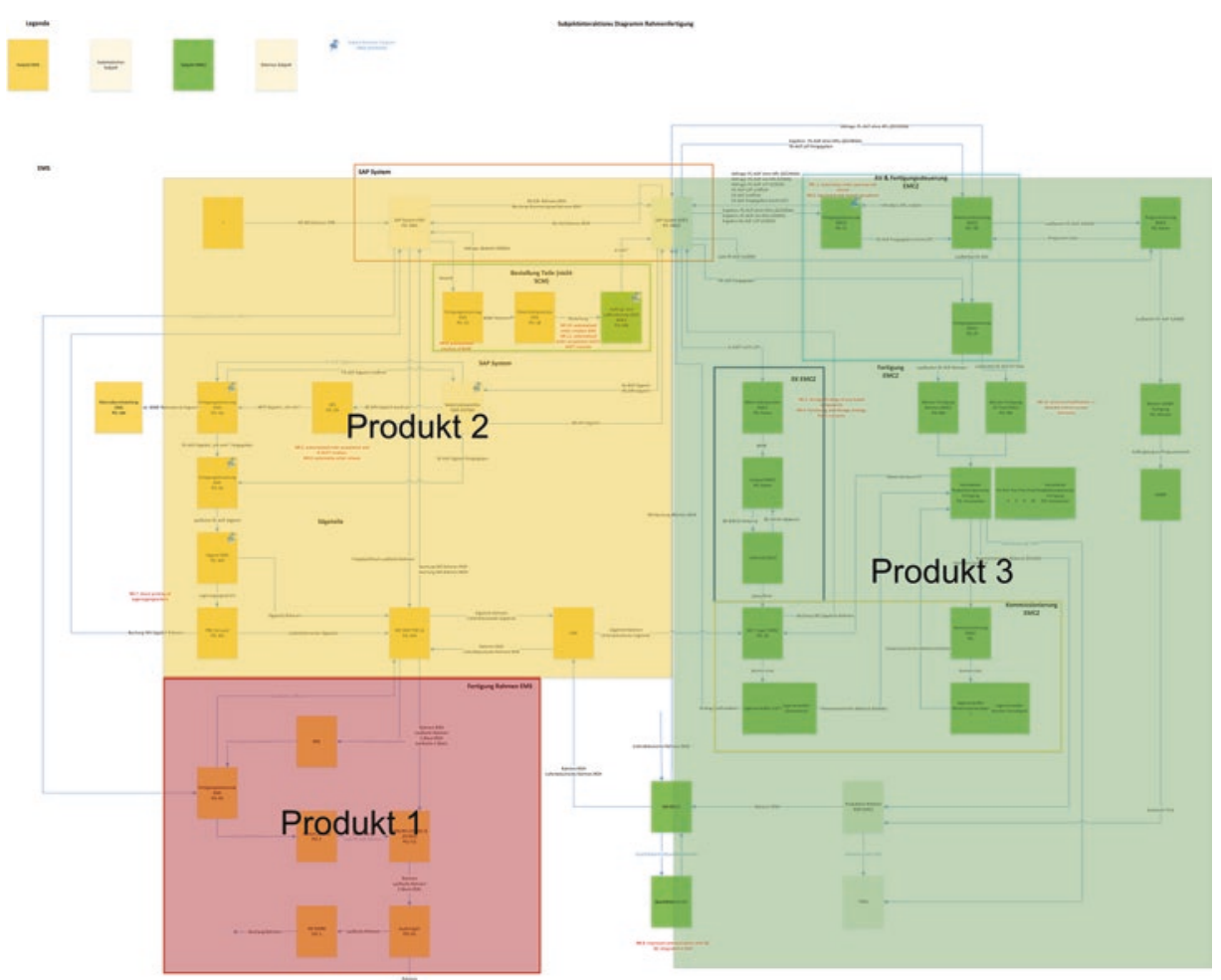

Abb. 7.5 Zusammenhang der Kommunikationsstruktur mit den jeweiligen Produkten 
(Produkt 3) sehr komplex und zeitaufwendig ist. Wir haben den Umfang unserer Prozesserhebung wie folgt definiert:

- Der Fokus liegt auf den Logistikabteilungen von Fabrik A und Fabrik B. Dazu gehört auch die Produktion von Produkt 3 in der Fabrik A, da diese organisatorisch direkt in die Logistik integriert ist und somit Teil des Prozesses und der Produktion von Produkt 2 in Fabrik B ist.

- Die Materialbeschaffung in Fabrik A und die tatsächliche Montage von Produkt 1 in Fabrik A sind nicht mehr Teil der Erhebung (siehe Abb. 7.5 für eine Visualisierung des Prozesses und der entsprechenden Produkte).

Validierung des Prozessmodells Wir untersuchten die relevanten Prozessschritte, indem wir die involvierten Mitarbeiter in Einzelinterviews befragten, die Mitarbeiter während des Prozesses begleiteten, und gleichzeitig für die Interviewpartner sichtbar die Subjektverhaltensdiagramme modellierten. So konnten wir den Prozessverlauf für Produkt 3 (siehe Pfeile in Abb. 7.6) detailliert beschreiben, detaillierte Informationen über das

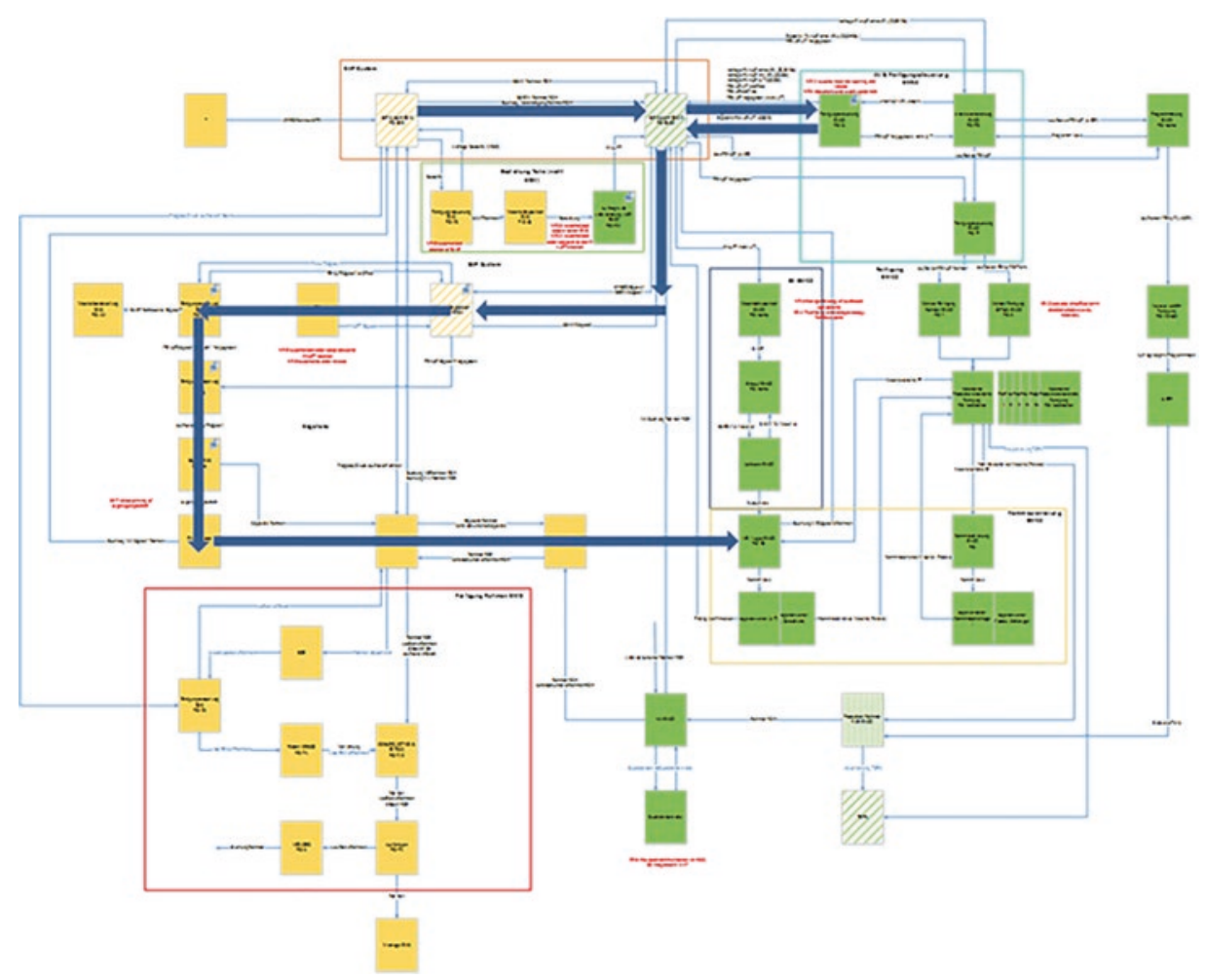

Abb. 7.6 Kommunikationsstruktur (SID) des Prozesses für die Produktion von Produkt 3 
SAP ${ }^{\circledR}$-ERP-System und die verwendeten Transaktionen dokumentieren und den Interviewpartnern ermöglichen, die Prozessmodellierung direkt zu begleiten und das Modell verifizieren.

Identifikation von Optimierungspotenzialen auf der Basis des Ist-Modells Nachdem die SAP $^{\circledR}$-Transaktionen in dem SID und dem SBD klar beschrieben wurden und einem Dummy-Auftrag durch das System gefolgt wurde, haben wir die verschiedenen Schritte des SAP $^{\circledR}$-Systems spezifiziert. Dies erlaubte es uns, zwischen automatisierten und manuellen Schritten zu unterscheiden, das überprüfte Prozessmodell zu verifizieren, und die tatsächlichen Prozessdurchlaufzeiten zu dokumentieren. Für das SBD verwendeten wir rechteckige Formen und verschiedene Farben, um die drei Zustände darzustellen; Rot für den Zustand „Senden“, Grün für den Zustand „Empfangen“ und Gelb für den Zustand „Funktion“ (siehe Abb. 7.7).

Abb. 7.7 visualisiert das Prozessverhalten eines Mitarbeiters, der die Bearbeitung von Fertigungsaufträgen in Fabrik B abwickelt. Dieser Mitarbeiter prüft, ob Produktionspläne für geplante Fertigungsaufträge vorliegen. Anschließend werden alle geplanten Produktionsaufträge mit verfügbaren Produktionsplänen nach einem definierten Regelwerk zusammengefasst und zur Produktion freigegeben. Die Mitarbeiter erledigen dies manuell für jeden Produktionsauftrag, bei mehreren tausend Bestellungen pro Tag. Produkt 3 allein verursacht insgesamt eine Arbeitsbelastung von ca. $7 \mathrm{~h}$ pro Tag.

Optimierung des Prozessmodells Der Gesamtaufwand für die gesamte Erhebung, alle Interviews und die Zeit, die für die Vervollständigung und Überprüfung der Prozessmodelle benötigt wurde, betrug ca. 200 geleistete Arbeitsstunden. Dies ist ein relativ geringer Aufwand im Vergleich zu anderen Prozessoptimierungsprojekten angesichts der Komplexität der Prozessmodelle und der untersuchten Tiefe bzw. Details.

Organisatorische Implementierung Mit dem nun vorhandenen detaillierten Wissen über die involvierten Subjekte haben wir anhand der gesammelten Daten und der im $\mathrm{SAP}^{\circledR}$-System dokumentierten Daten (Bestellzeiten, Lieferzeiten etc.) einen Zeitplan für den Prozess erstellt. Dieser Zeitplan beinhaltet alle Organisations- und Produktionsschritte und deren jeweilige Durchlaufzeiten. Beispielsweise betrug die Durchlaufzeit für eine der Produkt 1-Varianten, von der Bestellannahme in Fabrik B bis zur Lieferung des fertigen Produkts 1 an die Montagelinie in Fabrik A, ungefähr 30 Arbeitstage (vgl. Abb. 7.8).

Prozessoptimierungen In Zusammenarbeit mit den Mitarbeitern wurden die bestehenden Arbeitspläne überarbeitet, aktualisiert und verbessert. Dies führte zu verkürzten Durchlaufzeiten für die Arbeitsschritte sowie zu einer reduzierten Anzahl von Arbeitsschritten durch die Zusammenführung bestehender Schritte. In diesem Fall bedeutet eine reduzierte Anzahl von Arbeitsschritten weniger Subjekte als auch weniger Verhaltenszustände. Während der Analyse haben wir mehrere ähnliche Prozessschritte identifiziert, die in Fabrik A und Fabrik B unterschiedlich durchgeführt wurden. In einer Fabrik wurden notwendige 
Fertigungssteuerung EMCZ

PO: SJ

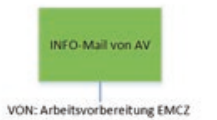

VON: Arbentsvorbere itung EMCZ info-Mall: ApL erstellit

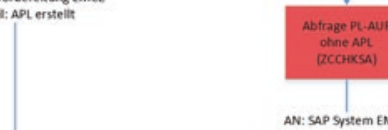

age PL.AuF
chine APL.

AN: SAP System EMCZ

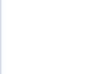

ohne APL (ZCORKSA)

$\checkmark$

liste Pl auf ohne

APts

VON-SAP Sintem facz VON-SAa system EMCZ 2 (n)

s (zCCHKsa)

$\downarrow$

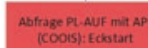

Tagesdatum $+2 d$
Tol

AN: SAP SYYStem EMCZ

Abfrage: (1)
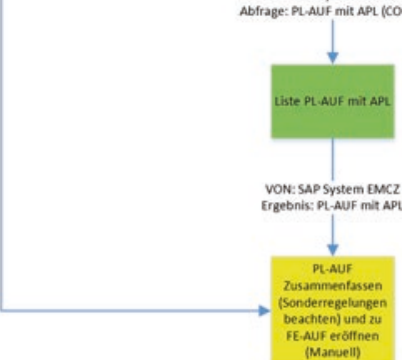

Nh. 2: document and rework

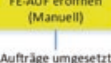

Auftràge umgesetzt

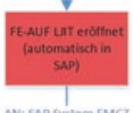

MN: sup Syitem EMC

FE-AUF eroffnet

$\downarrow$

Umgesetzte fE-AuF
Freigeben (manuelli)

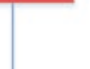

Nertigungssteverung FMc:

FE. Auf treigegeben

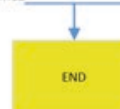

Abb. 7.7 Beispiel für eine Verhaltensbeschreibung eines Mitarbeiters

Prozessschritte manuell ausgeführt, jedoch automatisch vom SAP ${ }^{\circledR}$-System in der anderen Fabrik ausgeführt.

Darüber hinaus wurden vorhandene automatisierte $\mathrm{SAP}^{\circledR}$-Batch-Jobs unterbrochen, da die erforderliche manuelle Eingabe zwischen den Jobs fehlte. Diese Jobs wurden zu zwei definierten Zeiten während des Arbeitstages eingeplant. Wenn zu diesem Zeitpunkt die 


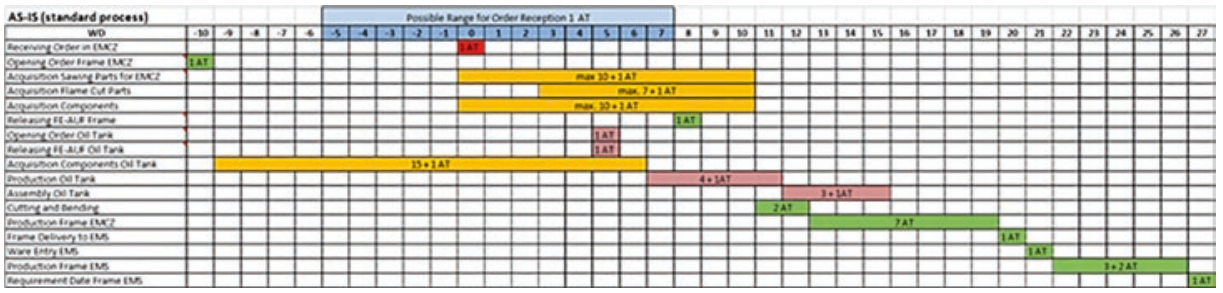

Abb. 7.8 Zeitverbrauch im Ausgangsprozess

manuelle Eingabe fehlte, musste die gesamte Bestellung bis zu einem gesamten Arbeitstag warten. Dies konnte bei jeder Bestellung mehrmals für verschiedene Jobs geschehen, was letztendlich zu einer Verzögerung von mehreren Arbeitstagen führen konnte.

IT-Implementierung Die beschriebenen Subjekte lieferten präzise definierte Prozesse,

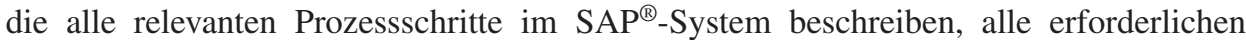
$\mathrm{SAP}^{\circledR}$-Transaktionen, wer diese Transaktionen ausführt, sowie die Interaktionen zwischen System und Mitarbeitern. Aufgrund dieser detaillierten Prozessdokumentation konnte unsere IT-Abteilung das relevante Subjektverhalten direkt implementieren, um neue standardisierte, digitalisierte und automatisierte Prozesse $\mathrm{zu}$ erstellen, Prozessschritte zu überarbeiten und den Verarbeitungsplan von bestehenden Batch-Jobs für beide Fabriken zu rationalisieren.

Dies beinhaltete Schritte wie Auftragsannahme, Auftragserfassung, Auftragseröffnung, Auftragsfreigabe in beiden Werken und Lieferung der Fertigungspapiere an die Produktion. Die automatische Bestellabwicklung ermöglichte die auftragsbezogene und zeitnahe Bearbeitung von Produkt 3 in der Fabrik A, was uns wiederum die Einführung von KANBAN-Beständen mit definierten kritischen Teilen, die Reduzierung des Lagerbestands unkritischer Teile, und den Versand von extern gekauften Teilen direkt in die Fertigung ermöglichte.

\subsection{Erzielte Ergebnisse}

Eines der erreichten Ergebnisse war eine neue Lagerstrategie und eine Neubewertung des Lagerbestandes. Dies ermöglichte, den gesamten Lagerbestand zu überarbeiten und ein KANBAN-System für kritische Teile von Produkt $3 \mathrm{zu}$ implementieren. Der neu geschaffene KANBAN-Bestand und der höhere Wert der betroffenen Teile führten zu einer Steigerung des gebundenen Kapitals um ca. $209 \%$. Dies hatte jedoch nur minimalen Einfluss auf den bestehenden Lagerwert von insgesamt etwa $10.000 €$. Die neue Strategie erhöhte die Verfügbarkeit und reduzierte die Lieferzeiten für alle zugekauften Teile.

Die Unterbrechungen bei der Herstellung von Produkt 2 aufgrund fehlender Teile konnten ursprünglich bis zu 15 Arbeitstage dauern. Nach den Änderungen waren alle 
benötigten Komponenten innerhalb eines Arbeitstages entweder direkt vor Ort oder über den Sicherheitsbestand beim Lieferanten verfügbar. Damit konnten eine signifikante Verbesserung der Prozessstabilität sowie eine Reduktion des Umlaufbestandes bei einem vergleichsweise geringen Anstieg des Lagerbestands erreicht werden.

Die Wertstromanalyse ist in unserem Unternehmen ein etabliertes Werkzeug zur Analyse von Produktionsprozessen. Obwohl die Literatur [10, 11] und unsere externen Berater oft die Möglichkeit der WSA hervorgehoben haben, nicht nur Materialflüsse, sondern auch Informationsflüsse zu beschreiben, wurden unsere Erwartungen nicht erfüllt, als wir versuchten, dies zu dokumentieren und zu visualisieren. Wenn die meisten relevanten Informationen verfügbar sind, können die administrativen Prozesse und der Informationsfluss mit einem Wertstrommodell beschrieben werden.

Erfahrungen mit Modellierungssprachen Aufgrund unserer Erfahrungen eignet sich ein WSM jedoch nicht für eine Darstellung des Informationsflusses mit teilweise abstrakten Informationen. S-BPM bot uns eine einfach zu lernende Modellierungsnotation, die dennoch sehr genaue und detaillierte Prozessmodelle liefern kann. Die involvierten Mitarbeiter konnten die S-BPM-Notation selbstständig verstehen, lesen und richtig interpretieren und begannen, ihre eigenen Prozessmodelle (das Subjektverhalten) ohne den Input der Methodenspezialisten zu verifizieren. Dies führte zu einer hohen Akzeptanz der Prozesserhebung und der folgenden Veränderungen im Prozess, da die Mitarbeiter direkt an der Dokumentation und den Optimierungsschritten beteiligt waren.

Betrieb und Monitoring Die vorgenommene Umstrukturierung und Digitalisierung zuvor manueller Prozessschritte führte $\mathrm{zu}$ einem standardisierten Prozess und einer Reduktion der beteiligten Subjekte von 12 auf 8 (siehe Abb. 7.9). Weniger Subjekte bedeuten weniger Schnittstellen im Prozess, was wiederum die Prozesskomplexität reduziert und die Prozessstabilität und -transparenz erhöht. Darüber hinaus wurden die Mitarbeiter von zeitraubenden und sich wiederholenden Aufgaben befreit.

Der erhöhte Digitalisierungsgrad und der neue geplante Ablauf führten zu einer neuen Prozessdurchlaufzeit von 2 Tagen (von ursprünglichen 5-10 Tagen) für die Auftragsabwicklung. Aufgrund des detaillierten und klar definierten Prozesses konnte unsere ITAbteilung die Prozessänderungen in der bestehenden Systemumgebung innerhalb von nur 3 Arbeitstagen umsetzen. Der Produktions- und Versandprozess für Produkt 3 konnte auf 3 Tage reduziert werden, und zwar von ursprünglichen 5-6 Tagen. Dies bedeutet, dass wir die Gesamtdurchlaufzeit von 11-15 Arbeitstagen von Produkt 3 um $87 \%$ auf 2 Arbeitstage reduzieren konnten. Diese Änderungen führten zu einer erhöhten Liefertermintreue für Produkt 3: Die Liefertreue stieg bereits vier Wochen nach der Umsetzung auf $89 \%$ und nach einem Jahr auf $97 \%$.

Gemessene Kennzahlen Der relativ hohe Zeitaufwand für die Auftragsabwicklung für Produkt 3 in der Anfangsphase führte dazu, dass die meisten Aufträge in der Fabrik A zu spät oder sehr kurzfristig ankamen. Die neu geschaffenen automatisierten 


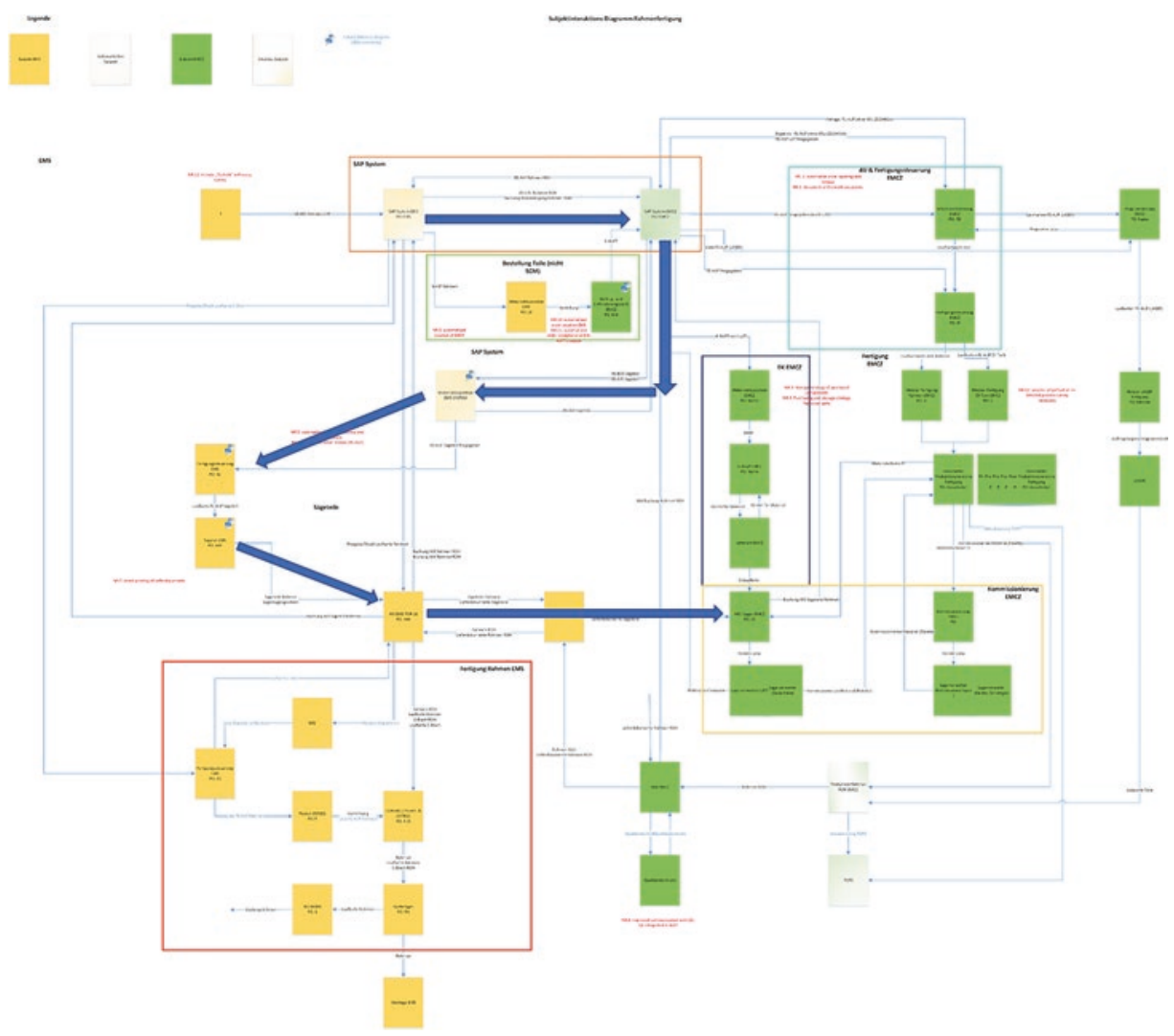

Abb. 7.9 Kommunikationsstruktur des aktualisierten Prozesses für Produkt 3

$\mathrm{SAP}^{\circledR}$-Prozesse führten zu einer schnelleren Bearbeitung von Bestellungen der Fabrik A innerhalb der Abteilungen von Fabrik B. Dies hatte eine kürzere Bestellzeit für Produkt 3 und einen früheren Produktionsstart anderer Komponenten, die für Produkt 2 erforderlich waren, zur Folge. Das Ergebnis war eine Reduktion von anfänglich $95 \%$ der Bestellungen, die zu spät registriert wurden, auf nur $12 \%$, wodurch wiederum die Prozessstabilität und Prozessqualität stark erhöht wurden und der Bedarf an Fehlersuche in beiden Fabriken reduziert werden konnte. Die Gesamtdurchlaufzeit des Produktionsund Bestellprozesses von Produkt 2 konnte um 7 Arbeitstage (ca. $38 \%$ ) verkürzt werden, von 19-23 Tagen auf 12-14 Tage.

Die Umwandlung von manueller Arbeit in automatisierte, digitalisierte Prozesse im $\mathrm{SAP}^{\circledR}$-System führte zu einer reduzierten Arbeitsbelastung der beteiligten Mitarbeiter von 5-6 h auf bis zu einer Stunde pro Tag. Die Mitarbeiter mussten nun die Bestellungen nur mehr für spezielle Komponenten bzw. Sonderfälle, die nicht vom SAP ${ }^{\circledR}$-System abgedeckt werden konnten, manuell bearbeiten. Die Auswirkungen dieser Änderungen addieren sich zu einer kalkulierten Prozesskostenreduktion von rd. $65.000 €$ pro Jahr. 
Die implementierten Verbesserungen und entsprechende Änderungen auf der Prozessebene reduzierten die Durchlaufzeiten für Produkt 2 und 3 und führten zu einer verkürzten Gesamtdurchlaufzeit für Produkt 1 von 26 bis 33 Arbeitstagen auf 18 bis 20 Arbeitstage. Dies entspricht einer Gesamtreduktion von ungefähr $60 \%$ für den gesamten Bestell- und Produktionsprozess (vgl. Abb. 7.10 und 7.11).

Abschließende Analyse der erreichten Ergebnisse Wir konnten nicht nur das gesteckte Ziel einer $30 \%$ igen Durchzeitverkürzung erreichen, sondern konnten diese Reduktion durch Digitalisierung und Automatisierung der Prozessschritte und des entsprechenden Informationsflusses mehr als verdoppeln. Dies führte in weiterer Folge zu einer Reduktion des Umlaufbestandes mit einem Gesamtwert von mehreren hunderttausend Euro über den gesamten Prozess.

Unsere Ergebnisse zeigen, dass es möglich ist, durch die Optimierung und Digitalisierung des Informationsflusses die Durchlaufzeit und die manuelle Arbeitsbelastung deutlich zu reduzieren. Der erhöhte Digitalisierungsgrad und die damit verbundene Prozesstransparenz kann dazu beitragen, weitere Verbesserungen zu erreichen und die Prozesse in zukünftigen Analysen besser zu verstehen [12]. Obwohl die Einführung spezialisierter S-BPM-Tools bewusst vermieden wurde, könnte deren Einführung dank der S-BPM-Methodik die Grundlage für eine noch umfassendere Digitalisierung der bestehenden Prozesse bieten. Die S-BPM-Methode und unterstützende Modellierungswerkzeuge ermöglichen eine direkte Transformation von Prozessmodellen in laufende Prozesse [4]. So könnte der Aufwand für die zukünftige Digitalisierung von Prozessen deutlich reduziert werden.

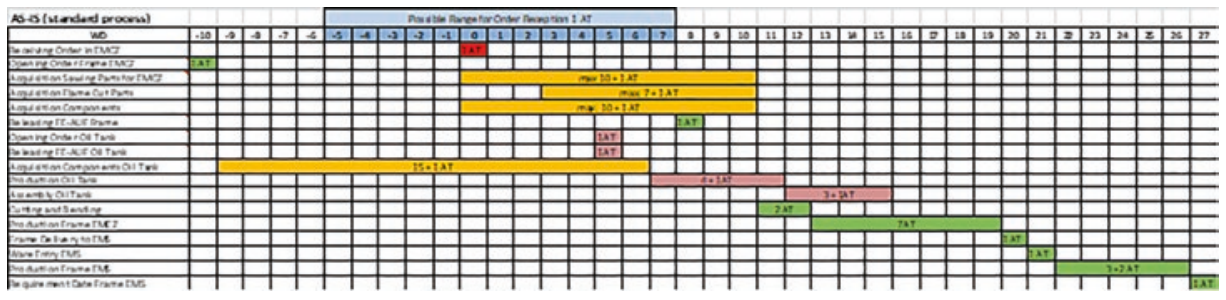

Abb. 7.10 Zeitbedarf im ursprünglichen Prozess

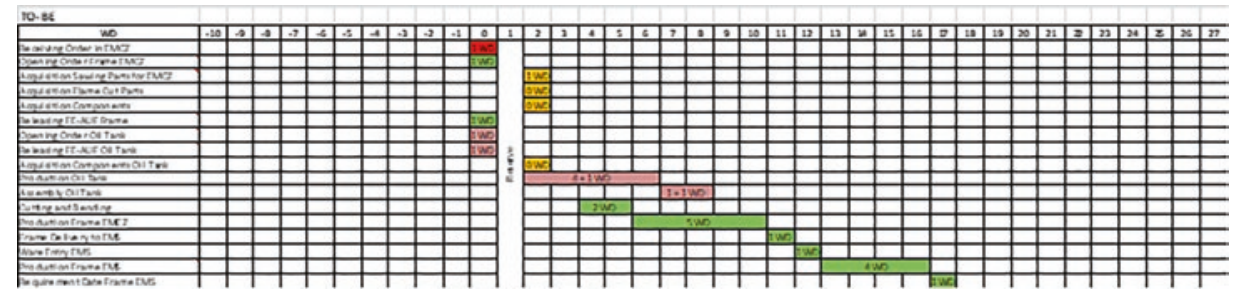

Abb. 7.11 Zeitbedarf im überarbeiteten Prozess 


\section{Literatur}

1. Moser, C., Riha, K. (2018) Digitalization of information-intensive logistics processes to reduce production lead times at ENGEL Austria GmbH. Extending value stream mapping with Subject-oriented Business Process Management, in Urbach, N., Röglinger, M. (Hrsg.) Digitalization Cases, Springer, Heidelberg

2. ENGEL AUSTRIA GmbH: Facts \& Figures. Retrieved Februar 20, 2018 http://www.engelglobal.com/en/uk/company/facts-figures.html

3. Rother M, Shook J (2004) Learning to See - Valuestream mapping to create value and eliminate muda. Lean Enterprise Institute; Erlach K (2010) Wertstromdesign. Der schlanke Weg zur Fabrik. (2. bearb. und erw. Aufl.). Berlin: Springer

4. Fleischmann A, Schmidt W, Stary C, Obermeier S, Börger E (2012) Subject-Oriented Business Process Management. Heidelberg: Springer

5. Liappas I, (2006) Vom Business zu den Prozessen. In A.-W. Scheer, H. Kruppke, W. Jost \& H. Kindermann, AGILITÄT durch ARIS Geschäftsprozessmanagement: Jahrbuch Business Process Excellence 2006/2007, Berlin Heidelberg: Springer: 43-56

6. Riempp G (2004) Integrierte Wissensmanagement-Systeme, Architektur und praktische Anwendung, Berlin Heidelberg: Springer

7. Kock N, Verville J, Danesh-Pajou A, DeLuca D (2009) Communication flow orientation in business process modeling and its effect on redesign success: Results from a field study. Decision Support Systems 46: 562-575

8. Kannengiesser U (2014) Supporting Value Stream Design Using S-BPM. In Proceedings of the 6th International Conference on Subject-oriented Business Process Management (S-BPM ONE 2014), Eichstätt, Germany, April: 22-23

9. Schmelzer H, Sesselmann W (2013) Geschäftsprozessmanagement in der Praxis - Kunden zufriedenstellen, Produktivität steigern, Wert erhöhen. München: Carl Hanser Verlag

10. Erlach K (2010) Wertstromdesign. Der schlanke Weg zur Fabrik. (2. bearb. und erw. Aufl.). Berlin: Springer

11. Wiegand B, Franck P (2004) Lean Administration I - So werden Geschäftsprozesse transparent. Aachen: Lean Management Institute

12. Parviainen P, Kääriäinen J, Tihinen $M$, Teppola S (2017) Tackling the digitalization challenge: how to benefit from digitalization in practice, International Journal of Information Systems and Project Management, Vol. 5, No. 1, 2017, 63-77 
Open Access Dieses Kapitel wird unter der Creative Commons Namensnennung - Nicht kommerziell - Keine Bearbeitung 4.0 International Lizenz (http://creativecommons.org/licenses/ by-nc-nd/4.0/deed.de) veröffentlicht, welche die nicht-kommerzielle Nutzung, Vervielfältigung, Verbreitung und Wiedergabe in jeglichem Medium und Format erlaubt, sofern Sie den/die ursprünglichen Autor(en) und die Quelle ordnungsgemäß nennen, einen Link zur Creative Commons Lizenz beifügen und angeben, ob Änderungen vorgenommen wurden. Die Lizenz gibt Ihnen nicht das Recht, bearbeitete oder sonst wie umgestaltete Fassungen dieses Werkes zu verbreiten oder öffentlich wiederzugeben.

Die in diesem Kapitel enthaltenen Bilder und sonstiges Drittmaterial unterliegen ebenfalls der genannten Creative Commons Lizenz, sofern sich aus der Abbildungslegende nichts anderes ergibt. Sofern das betreffende Material nicht unter der genannten Creative Commons Lizenz steht und die betreffende Handlung nicht nach gesetzlichen Vorschriften erlaubt ist, ist auch für die oben aufgeführten nicht-kommerziellen Weiterverwendungen des Materials die Einwilligung des jeweiligen Rechteinhabers einzuholen. 


\section{Sachverzeichnis}

A

Abbruch, 81, 108-111

Abbruch-Event, 110

Abbruchbedingung, 105

Ablauf, 6, 42, 56, 74, 79, 88, 112, 135, 137, $163,178,207,222,224$

detaillierter, 93

einer manuellen Prozessprüfung, 193

im holaren System, 185

paralleler, 73, 124

Ablaufdiagramm, 51

Ablauforganisation, 6

Ablaufsteuerung, 93, 98, 105

Abstimmung, 1, 4, 158, 160, 162, 163

kollaborative, 165

Abstract State Machines (ASM), 60

Actor, 154, 205

Ad-Hoc-Prozess, 105

ADM s. Architecture Development Method

Agent, 58, 64

Agentenkonzept, 64

Aggregation, 63

mehrstufige, 64

Agilität, 149

Akteur, 5, 20, 71, 86, 114, 132, 137, 162, 164, $165,168,187,190,203,205$

abstrakter, 136

externer, 188

menschlicher, 16, 71

technischer, 16

Aktion, 5, 51, 52, 56, 87, 154, 181, 202, 210 , 235

Aktivität, 10, 12, 45, 53, 77, 87, 101, 107, 108, 157, 170, 190, 203, 207, 220, 224
Ausführungsverhalten, 104

des Prozessmanagementmodells, 199

Elimination, 225

miteinander verknüpfte, 123

Modellierung, 103

ökonomische, 169

parallele, 72

Typen, 104

Aktivitätsbündel, 12, 130, 136, 139, 151, 152, 157, 199

Aktivitätsdiagramm, 72, 87, 88, 92, 124

Notationselemente, 87

Aktivitätsfluss, 127

Aktivsätze, 178, 181

Aktor, 26, 64, 212

Alternativanweisung, 60

Analyse, 24, 131, 157, 174, 176, 223, 229, 230, 232,244

Anforderungsbeschreibung, 179

Anwendungssystem, 40, 51, 84, 211

Arbeiter, 24

Arbeitsablauf, 24, 72

Arbeitsplanung, 25

Arbeitsprozess, 24, 103, 160, 161, 164, 165, 167

abzustimmender, 162

kollaborativer, 167

ArchiMate s. Architecture-Animate

Architecture-Animate (ArchiMate), 37, 42, 44

Architecture Development Method (ADM), 40

Architektur Integrierter Informationssysteme (ARIS), 37, 45, 61, 78

ARIS s. Architektur Integrierter Informationssysteme

A. Fleischmann et al., Ganzheitliche Digitalisierung von Prozessen,

https://doi.org/10.1007/978-3-658-22648-0 
Artikulation, 154, 158, 161-163

individuelle, 165

ASM s. Abstract State Machines

Assoziation, 62

Attribut, 22, 50, 62, 220

Attributtyp, 50

Aufbauorganisation, 13, 45, 67, 129, 136

Aufgabe, 2, 34, 52, 66, 93, 104, 110, 182, 209, 213

Aufgabenträger, 202, 204, 208, 213, 214, 224 physikalischer, 215

Aufgabenzuschnitt, 140, 226

Ausführung, 2, 13, 74, 84, 104, 107, 110, 207

überlappte, 224

Ausgabestelle, 54

Ausgangsstelle, 55

Ausnahme, 103, 108, 201

\section{B}

Balanced Scorecard, 31, 32

Bedingung, 54, 60, 74, 88, 93, 98, 182

Benutzerzentriertheit, 149, 150

Beschreibung, natürlich sprachliche, 178, 179

Beschreibungssprache, 8, 10 kommunikationsorientierte, 10

Betrieb, 14, 130, 138, 190, 199, 213, 242

Betriebswirtschaft, 3, 21, 27

Modelle, 28

Beziehungstyp, 50

BizDevOps, 155

Blackbox, 102

BPD s. Business Process Diagram

BPM s. Business Process Management

BPMN s. Business Process Model and Notation

BPMS s. Business-Process-ManagementSystem

Brainstorming, 147

Business Activity Monitoring (BAM), 216

Business-IT-Alignment, 155

Business Process, 2

Diagram (BPD), 93

Management (BPM), 2, 12, 133, 150, 154, 186

Management-System (BPMS), 2, 200

Model and Notation (BPMN), 10, 73, 87, 92, 100, 110, 124, 204
C

Calculus of Communicating System (CCS), $56-58$

CCS s. Calculus of Communicating System

Choreographie, 112, 214

Aktivität, 112

Diagramm, 93, 112

COBIT s. Control Objectives for Information and Related Technology

Communicating Sequential Process (CSP), 59

Compare/WP, 153, 161

Complex Event Processing (CEP), 216

Compliance, 2, 6, 36, 202, 221

Computerprogramm, 22, 23, 51, 72, 73, 207

Concept Map/Mapping, 163

Condition, 60

Control Objectives for Information and Related Technology (COBIT), 9, 36

Controlling, 130

CSP s. Communicating Sequential Process

Customer/User Experience Journey, 147, 151

Cyber Physical System (CPS), 10, 212

D

Daten, 16, 39, 45, 48, 49, 51, 61, 92, 120, 202, 208, 214, 217

Datenelement, 101, 120

Datenfluss, 85

Datenobjekt, 78, 89, 91, 96

Datensicht, 45, 46, 52, 78

Datenverarbeitung, 48, 51, 85

Deming-Zyklus, 9

Design, 152, 215

Design Thinking, 12, 129, 141, 147, 150

Digitalisierung, 5, 10, 16, 129, 206, 209, 242, 244

Diversitätsaspekte, 155

Durchführungskontext, 104

Durchlaufzeit, 15, 131, 139, 218, 244

DV-Konzept, 45

E

EAM s. Enterprise Architecture Management

Echtbetrieb, 138, 199

eEPK s. Prozesskette, ereignisgesteuerte mit Erweiterung 
Effektivität, 2, 133, 135, 152, 189, 192, 195, 196

Effizienz, 2, 7, 135, 152, 189, 192

EFQM s. European Foundation for Quality Management

Einbettung, 13

organisatorische, 136, 189

technische, 190

Eingabestelle, 54

Eingangsstelle, 55

Einheit, organisationale, 83

Empathie, 142, 145, 148, 150

Empfänger, 5, 27, 108, 114, 116, 119, 124, 172

Empfangsereignis, 101

Empfangszustand, 116

EN ISO 9001, 9, 34

Endereignis, 51, 94, 98, 99, 109

Endknoten, 87, 172

Endzustand, 55, 117

Engpass, 139

Enterprise Architecture Management (EAM), 9

Enterprise-Architekt, 42

Entität, 50, 52, 61, 182

Entitätstyp, 50

Entity Relationship Model (ERM), 45, 50, 61

Entscheidung, 49, 75, 77, 78, 80, 88, 89, 95, $117,134,167$

kaskadierte, 75

Entscheidungselement, 75, 78, 88

EPK s. Prozesskette, ereignisgesteuerte

Ereignis, 1, 5, 51, 78, 79, 81, 86, 93, 98, 101, 216

angeheftetes, 108

asynchrones, 185, 187

ausgelöstes, 107

eingetretenes, 107

sequenziell aufgezeichnetes, 220

Ereignis-Teilprozess, nicht-unterbrechender, 106

Ereignistyp, 105

ERM s. Entity Relationship Model

Error-Event, 110

Ersatzkennzahl, 8

Eskalationsereignis, 110

nicht-unterbrechendes, 110

European Foundation for Quality Management (EFQM), 9, 33

Event, 105, 111

angeheftetes, 107, 111 unterbrechendes, 109

Exchange Analysis, 171

Experte, 143, 154

Externalisierung, 160, 166

F

Fachkonzept, 46

Facilitator, 144, 145, 154, 162

Fehler, semantischer, 110

Fehlerereignis, unterbrechendes, 110

Fehlerkultur, 144

Flowchart, 72, 73, 78, 86, 124

Flussdiagramm s. auch Flowchart, 10, 25, 51, $61,73,234$

Folgezustand, 56, 116

Ford, Henry, 25

Fordismus, 24

Framework, 9, 36, 37, 207

für IT-Service-Management, 47

nach Zachman, 37

Führungsphilosophie, 16

Funktion, 45, 47, 51, 52, 61, 78, 81, 84, 86, $116,137,185,235,239$

sequenziell angeordnete, 91, 96

Funktionssicht, 45, 52, 61

Funktionszustand, 115, 116

G

Gateway, 93, 94

ereignisbasiertes, 99

paralleles, 94, 96, 109

zusammenführendes, 96

Geschäftsarchitektur, 6, 41

Geschäftsereignis, 1, 3, 196

Geschäftsmodell, 6, 7, 16, 28, 37, 67

Geschäftsobjekt, 16, 60, 66, 120, 191

Geschäftsprozess, 1, 28, 73, 77, 86, 130, 202, 210

Beschreibung, 5, 78

Definition, 11, 124

Dokumentation, 200

Management, 1, 16, 23, 35, 130, 168, 234, 235

Modellierung, 19, 65, 72, 87, 168

Monitoring, 131, 211

Qualitätskontrolle, 192

Geschäftsregel, 104, 131, 139, 190 
Geschäftsvorgänge, 115

Gesellschaft, 24, 26

Gesellschaftmodell, 26

Governance, 36

Governor, 154

Guarded Command, 60

\section{$\mathbf{H}$}

Habermas, Jürgen, 26, 27, 66

Handeln

kommunikatives, 26

Handeln, kommunikatives, 17, 24, 26

Handelnde, 1, 5, 48, 115, 133, 150, 178, 182, 202

abstrakte, 182, 234

Handlungsträger, 136, 158, 176, 204, 214

Hauptprozess, 106

Hierarchie, 28

Hilfsmittel, 1, 6, 8, 40, 146

Holomapping, 170

I

Impact Analysis, 172

Implementierung, 10, 12, 46, 157, 203

IT-bezogene, 137, 152, 199, 241

organisatorische, 130, 136, 199, 239

technische, 13, 154

Industrie 4.0, 10, 16, 209, 212

Information, 26, 48, 86, 131, 138, 151, 201, 225

prozessbezogene, 219

strategische, 171

Informationsfluss, 233, 242

Informationsobjekt, 42, 45, 51, 85

Informationssystem, 6, 36, 37, 45, 48, 53, 114

Informationstechnik, 16, 45, 213

Informationstechnologie (IT), 48, 73, 206

Implementierung, 12, 130, 137, 152, 199, 241

Infrastructure Library (ITIL), 9, 36, 47

Infrastruktur, 2, 47, 129, 206

Management, 9, 36

System, 11, 84, 137, 200, 208, 209, 219

Infrastruktur, physikalische, 206

Innovation, 143, 149, 155

Zielgruppe, 143

Input, 5, 119, 157, 172, 201
Inputpool, 119, 124

Instanz s. auch Prozessinstanz, 2, 13, 61, 130, 138, 191, 209, 210, 214, 222

Durchlaufzeit, 218

Interaktion, 35, 57, 59, 120, 163, 176, 234

Interaktionsdiagramm, 115, 122, 234

Interaktionsverhalten, 73

Internet of Things, 16, 216

ISO 9001 s. EN ISO 9001

IT s. Informationstechnologie

Iteration, 135, 141, 144, 148, 150

ITIL s. Informationstechnologie (IT) Infrastructure Library

K

Kanten, 51, 53, 54, 79, 170, 212

Kapazität, 136, 215

Kennzahl s. auch Prozesskennzahl, 7, 11, 13, 31, 131, 202

gemessene, 242

Key Performance Indicators (KPI), 7, 229 , 232

Klasse, 22, 61, 208

Kollaboration, 100, 108, 112

Kollaborationsdiagramm, 93

Kommunikation, 10, 26, 114

asynchrone, 119

Beschreibung, 115

horizontale, 209

nachrichtenbasierte, 108

Signale, 88

synchrone, 119

vertikale, 209

Kommunikationsmodellierung, 100, 118

Kommunikationsstruktur, 133, 235

Kommunikationsunterstützung, 73

Kommunikationsvorgang, 78, 92, 101, 114

Kompensationsaktivität, 105

Kompensierung, 110

Kompensierungsereignis, 110

Komposition, 64 parallele, 56

Konnektor, 51, 78, 79, 81

Kontext, 4, 49, 72, 136, 168, 204

Kontrollfluss, 72, 89, 94, 106, 182

KPI s. Key Performance Indicators

Kundenbedürfnis, 66, 150, 157, 229

Kundennutzen, 29, 65 
L

Lane s. auch Swim Lane, 93, 96, 100, 206, 214

Leistung, 3, 36, 129, 169, 177

abrechenbare, 78

ideelle, 175

materielle, 175

Leistungslohn, 25

Lernen, 159

modellbasiertes, 161

Liegezeit, 139

Link-Event, 107

Log File, 216, 219

Luhmann, Niklas, 17, 24, 26, 27, 66

M

Makrozyklus, 144

Management Cockpit, 217

Managementmodell, 17

Managementphilosophie, 16, 17

Marke, 54

Maschine, 5, 10, 16, 206, 209, 212, 215, 226

Mehrfachausführung parallele, 102 sequenzielle, 105

Mensch, 5, 10, 16, 24, 26, 142, 143, 159, 202, 209 und IT, 209 und Organisation, 204

Mensch-Maschine-Interaktion, 36

Menschenbild, 17, 24

Menschenmodell, 21

Methode, 10, 39, 62, 146-148, 161

Microservice, 208

Mikrozyklus, 144

Modell s. auch Geschäftsmodell, 2, 3, 19, 67

Agenten-/Aktoren-orientiertes, 64

der Betriebswirtschaft, 28

der Gesellschaft, 24

der Informatik, 48

der Wirtschaftsinformatik, 36

Eigenschaften, 21

formales, 22, 56, 178, 186

für Geschäftsprozesse, 65

grafisches, 22

materielles, 22

mentales, 4, 13, 22, 159

objektorientiertes, 61

verbales, 22

von Habermas, 66 von Luhmann, 66

Modellbildung, 3, 10, 19, 21, 61, 234

Modellierung, 2, 10, 37, 53

Modellierungssprache, 42,71

Auswahl, 187, 231

Erfahrungen, 242

kommunikationsorientierte, 195

Prozessanalyse, 231

Modelltyp, 45, 51

Monitored Function, 61

Monitoring, 12, 130, 138, 199, 211, 213, 215, 242

Multiagentensystem, 65

$\mathbf{N}$

Nachricht, 57, 59, 101, 108, 112, 116, 191

asynchrone, 57, 65, 208, 212

eingehende, 119

synchrone, 65

zwischen Objekten, 64

Nachrichtenaustausch, 59, 112, 115, 122, 212

Nachrichtenfluss, 93, 101, 114

Nebenläufigkeit, 58, 60

Notation, 58, 74, 78, 87, 103

grafische, 131

$\mathbf{O}$

Object Management Group (OMG), 62, 93

Objekt, 42, 50, 61, 64

logisches, 203

physisches, 203

Objektorientierung, 61

Objekttyp, 50

ODER-Konnektor, 78, 79, 83, 92

OMG s. Object Management Group

Operation, 45, 51, 60, 62, 64, 74, 87

alternative, 74

Optimierung, 131, 135, 139, 152, 157, 189, 192, 196

der Qualität, 32

des Prozessmodells, 239

Organigramm, 37, 45, 66, 177

Organisation, 1, 16, 27, 78, 168, 189, 205

komplexe, 66

Organisationseinheit, 13, 51, 100, 189, 204, 205

Organisationsentwicklung, 177

Organisationsgestaltung, 142 
Organisationssicht, 45, 53, 78

Organisationssoziologie, 27

Organisationsstruktur, 6, 13, 27

Organisationstheorie, 17, 24, 27

Organisationswissen, 136

Output, 52, 133, 173, 174, 201

\section{$\mathbf{P}$}

Parallelisierung, 107, 136, 224

Parallelität, 53, 55, 79, 185

Partition, 88, 92

PDCA (Plan-Do-Check-Act), 9

Performance Indicator, 8, 151

Persona, 145, 151

Petrinetz, 53

Petrinetzmodell, 55

Philosophie, 21, 23

$\pi$-Kalkül, 58

Point of View (POV), 146

Pool, 93, 96, 100, 106, 114, 204, 206, 212, 214

PPI s. Process Performance Indicators

Prädikat, 43, 60

Pragmatismus, 22

Process Mining, 216, 219, 225

Process Performance Indicators (PPI), 7, 131, 138, 202, 216, 217

Produktivsetzung, 136, 140

Programmablauf, 73

Programmablaufplan, 51, 73

Projekt, 12, 50

Prototyp, 141, 147

Prozess, 1, 7, 66, 78, 86, 93, 98, 99, 130, 212

agiler, iterativer, 151

Inbetriebnahme, 213

leerer, 56

Sequenzflüsse, 109

sequenzieller, 73

Prozessablauf, 86, 133, 192, 197, 206, 207, 220

paralleler, 87

Prozessarchitektur, 6, 191

Prozessaspekte, 65

Prozessausführung, 16, 120, 196, 220

Prozesschoreographie, 212

Prozessdefinition, 58, 123, 210

Prozessdigitalisierung, 6, 151, 152

Prozessdokumentation, 193, 194, 200, 231

Prozesseigner, 133, 200

Prozessgestaltung, 6, 135, 216, 224
Prozessinnovation, 129, 152, 155

Prozessinstanz, 2, 5, 6, 13, 105, 195, 210, 214

Prozesskennzahl, 7, 13, 15, 197, 216

Prozesskette, ereignisgesteuerte (EPK), 10, 45, $51,78,92$

mit Erweiterung (eEPK), 10, 51, 72, 78, 83, 85,86

Prozesslandkarte, 136

Prozesslogik, 5, 66, 123, 157, 204, 207, 209

Prozessmanagement s. auch Geschäftsprozessmanagement, 3, 15, 16, 36, 130, 150, 151,199

subjektorientiertes, 62, 212

Prozessmodell, 2, 6, 8, 13, 114, 135, 158, 178, $189,191,192,195,201,202,204$

Detaillierung, 233

kontrollflussorientiertes, 182

Optimierung, 239

subjektorientiertes, 115, 206

Validierung, 238

Prozessmodellierung, 13, 92, 99, 113, 186, 237

graphische, 72

Prozessorientierung, 16

Prozessparameter, 135

Prozessrealisierung, 5, 66

Prozessstrategie, 5, 66, 123, 157

Prozessziel, 131, 135

$\mathbf{R}$

Rahmenwerk, 37, 39, 45

Reactive Programming, 208

Relation, 22, 50

Ressource, 5, 30, 78, 196

Richtigkeit, semantische, 193

Rolle, 35, 47, 84, 132, 136, 137, 154, 171, 175, 176, 195

Rollenspiel, 148, 192, 195

Rules of Engagement, 145

S

Sachmittel, 66, 123

Schleife, 104, 112

Schnittstelle, 42, 136, 202, 210, 226

Schwachstelle, 131, 151, 155, 224

Scientific Management, 24

Semantik, 71, 74, 107, 114, 120, 123, 163, 164, 195, 207 
Sender, 27, 60, 108, 114, 119, 124, 173

Sendezustand, 116, 117, 119

Sequenz, 51, 96, 105, 130

Sequenzfluss, 93, 101, 107, 109

Service, 3, 10, 43, 47, 104, 140, 149, 207

Servitization, 151

Signal, 59, 88, 91, 98, 108

Simulation, 55, 114, 135, 196

Social BPM, 150

Soziologie, 21, 24

Spezifikation, 2, 71, 84, 87, 114, 120, 157, 160, 179,199

Split/Join, 88, 91, 94, 122

Sprache, 71, 77, 92, 114, 123, 150, 186

diagrammatische, 189

imperative, 59

natürliche, 158, 178, 179

Sprachelement, 72, 74, 75, 113

Startereignis, 66, 94, 98, 214

unterbrechendes, 106

Startknoten, 87

Startzustand, 54, 117

Stelle, 51, 54

Steuerungssicht, 45,52

Strategie, 7, 16, 31, 130, 229

Strukturelement

aktives, 42

passives, 42

Strukturierung, 3, 28, 45, 165

der Arbeitsschritte, 25

von Software-Systemen, 208

Subjekt, 20, 43, 48, 115, 121, 133, 150, 182, 186, 191, 206, 208, 212, 234

Verhalten, 117

Subprozess, 93, 103, 106, 110, 111

nicht-unterbrechender, 111

Symbol, 51, 98, 99, 189, 235

Synchronisation, 59, 136

System, 115, 190

cyber-physikalisches, 215

dynamisches, 55

eingebettetes, 209

elektrisches, 181

gesellschaftliches, 66

komplexes, 61

lebendes, 170

physikalisches, 181, 204, 206, 215

soziales, 24, 26, 27

soziotechnisches, 3, 16, 167, 176 technisches, 115

verteiltes, 207

T

T-Shape, 143, 155

Task, 93, 204, 208, 212

Tätigkeit, 1, 3, 13, 45, 51, 203

subjektive, 20

Taylorismus, 17, 24

Team, interdisziplinäres, 11, 143, 149, 154

Teilprozess, 98, 104, 108

Terminate-Event, 109

Terminierung, 99, 109

Terminierungselement, 74, 87

The Open Group Architecture Framework (TOGAF), 9, 37, 39

Timer, 94, 107, 108

Timer-Event, nicht-unterbrechendes, 109

TOGAF s. The Open Group Architecture Framework

Token, 54

Total Quality Management (TQM), 9, 32

TQM s. Total Quality Management

Transaktion, 35, 110, 170

intangible, 169

tangible, 169

Transaktionsbehandlung, 110

Transaktionsmodellierung, 105

Transaktionssubprozess, 110

Transition, 54

$\mathbf{U}$

UML s. Unified Modeling Language

UND-Konnektor, 78, 79, 81, 122

Unified Modeling Language (UML), 50, 62, 72,87

Unternehmen, 1, 16

eigentümergeführtes, 229

Instanzen, 210

Verhaltensweisen, 31

Unternehmensarchitektur, 6, 11, 36, 37, 39, 44, 67, 129, 202

Unternehmensarchitekturmanagement, 9

Unternehmensführung, 16, 28

Unternehmenskultur, 17

Unternehmensmodelle, 45 


\section{V}

Validierung, 12, 130, 131, 133, 152, 157, 189, 192, 193

des Prozessmodells, 238

Value Network, 11, 35, 170, 171, 175, 176

Value Network Analysis, 168, 176, 177

Verantwortlichkeit, 34, 73, 77, 83, 86, 88, 91, 93, 96, 163

Verbesserung, 28, 131, 135, 139, 230

inkrementelle, 129

kontinuierliche, 15

Vererbung, 62

Verhalten, 42

des Subjekts, 115, 117, 235

eines Prozesses, 56

von Führungskräften, 24

von Systemen, 53

Verhaltensdiagramm, 115

Verhaltensspezifikation, 133

Verkürzung, 22, 189

Verzweigung, 72, 74, 77, 89, 96

nicht-deterministische, 56

Vorgehensmodell, 8, 144

W

Wahrscheinlichkeitsverteilung, 135, 196

Walk Through, 195, 196

Wartung, 200

kontinuierliche, 224

Wertschöpfung, 10, 28, 36, 65, 168, 172, 175

Wertschöpfungskette, 46, 136, 169

Wertschöpfungsnetz, 136

Wertstromanalyse, 232, 242

Wertstrommodell, 232, 242
Wir-Denken, 143, 155

Wirklichkeit, 3, 19, 21, 61

Wirtschaftsinformatik, 23, 36

Wissensmanagement, 11, 168

Workflow

Engine, 5, 13, 136, 210, 216

System, 13, 211

$\mathbf{X}$

XOR-Konnektor, 78-80

$\mathbf{Z}$

Zachman-Framework, 9, 37, 39

Zeitstempel, 209, 216, 219, 220

Ziel, 7, 131, 192, 194, 201

bewegliches, 15

Zielgruppe, 28, 71, 143

Zielkonflikt, 140

Zielsetzung, 32, 192

Zielwert, 11, 13, 31, 131, 194

Zurückspulen, 110

Zustand, 54

angestrebter, 15

funktionsloser, 117

gewünschter, 41

nichtdeterministischer, 55

physikalischer, 212

Zustandsdiagramm, 115, 122

Zustandsmaschine, 60

Zustandstyp, 115, 235

Zustandsübergang, 54, 116

Zwischenereignis, 98, 99, 107 